\title{
61. 5
}

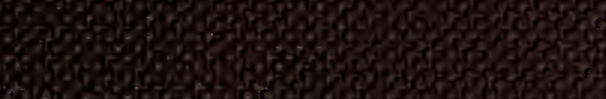

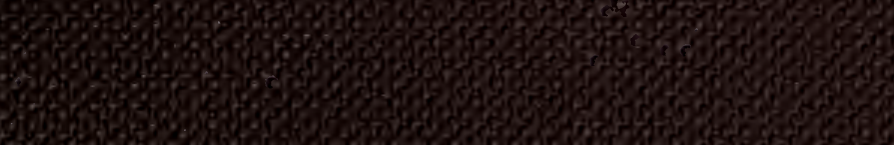

1.0.

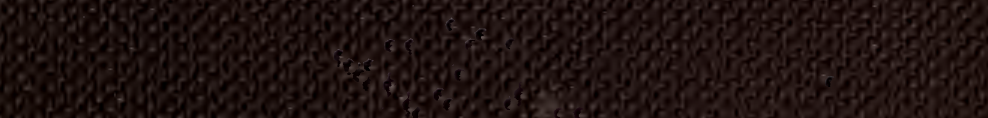

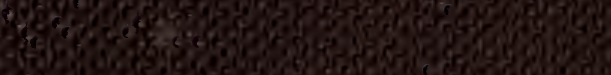
Noven

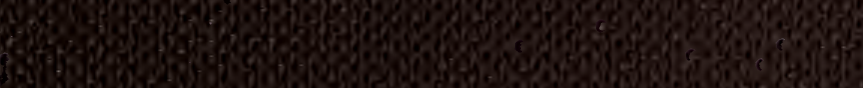
Wo

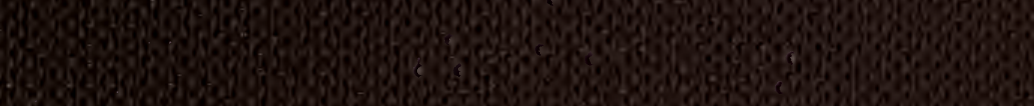

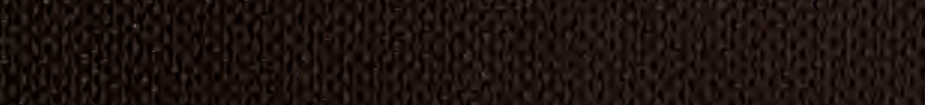

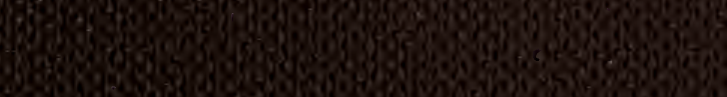




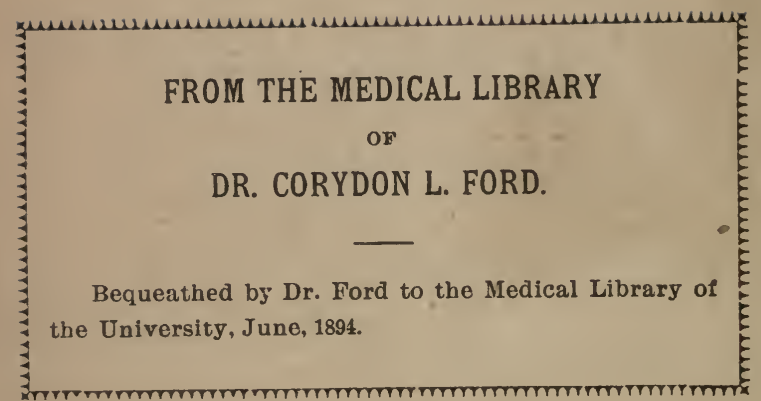



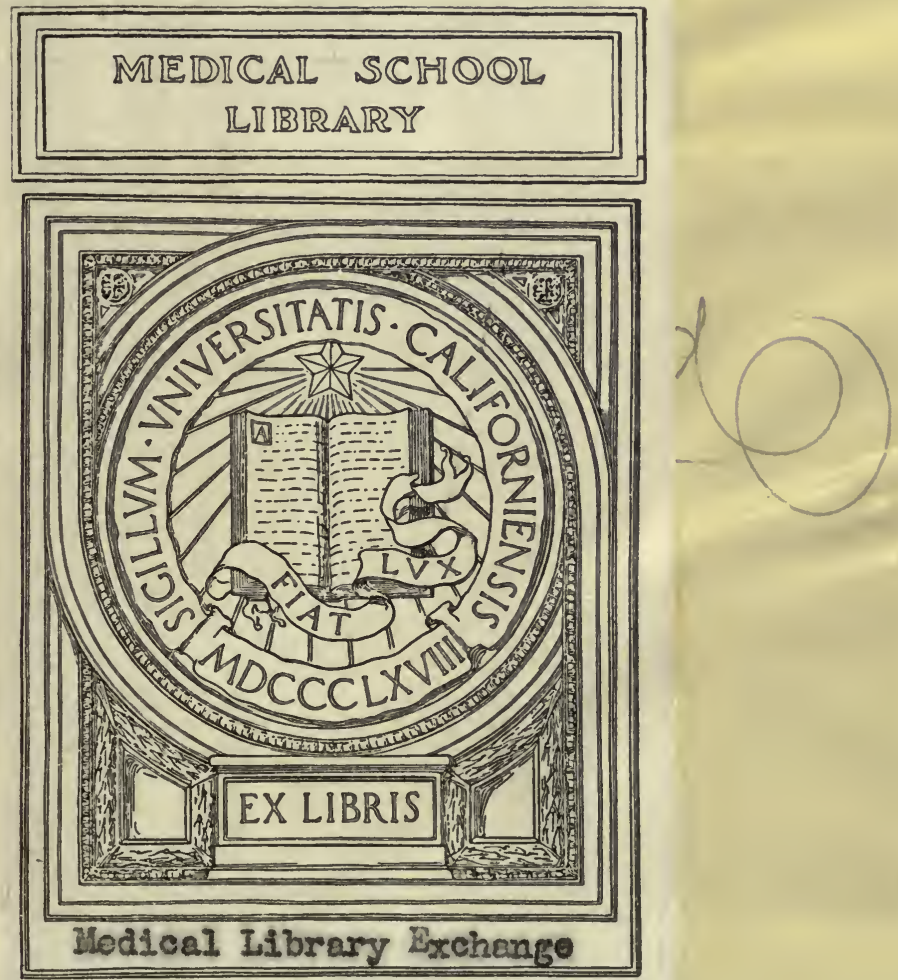

\section{University of ulehigan, Medical Iibrary}




$$
\bullet
$$

-

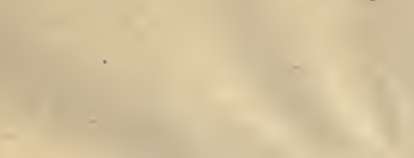

1

$-$

$+$
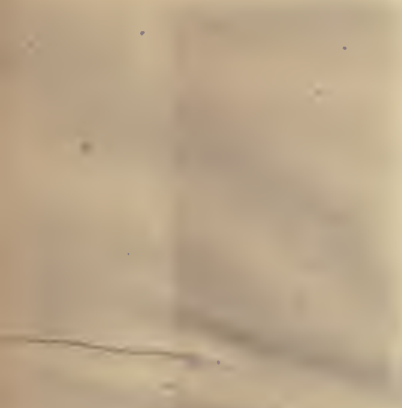

$=$

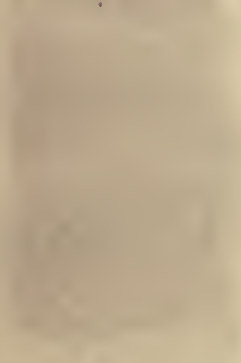


Digitized by the Internet Archive in 2007 with funding from Microsoft Corporation 



\section{THE}

\section{PHYSIOLOGY OF IIAN;}

DESIGNED TO REPRESENT

\section{THE EXISTING STATE OF PHYSIOLOGICAL SCIENCE,}

AS APPLIED

TO THE FUNCTIONS OF THE HUMAN BODY.

BV

AUSTIN FLINT, JR., II.D.,

PROFESSOR OF PHYRIOLOGY AND MICROSCOPY IN THE BELLEV UE HOSPITAL MEDICAL COLLEGR NEW YORK, AND IN THE LONE ISLAND COLLEGE HOSPITAL; FELLOW OF THE NEW YORK ACADEMY OF MEDICINE, MICROSCOPIST TO BELIEV UE HOSPITAI.

INTRODUCTION; THE BLOOD; CIROULATION; RESPIRATION.

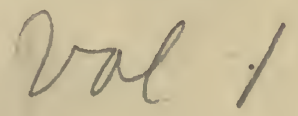

NEW YORK:

D. A P PLETON A N D C O MA N Y,

443 \& 445 BROADWAY.

1866. 
ENTERED, according to Act of Congress, in the year 1865 , by

D. APPLETON \& CO.,

In the Clerk's Office of the District Court of the United States for the Southern District of New York. 


\section{CHARLES ROBIN,}

THE FIRST PROFESSOR OF HISTOLOGT IN THE FACULTY OF SUEDICINE OF PARIS,

AS A TOKEN OF APPRECIATION OF THE

NUMEROUS ORIGINAL RESEARCHES AND DISCOVERIES,

PARTIOULARLY IN

HISTOLOGY AND PHYSIOLOGICAL CHEMISTRY,

BY WHIOH HE HAS CONTRIBUTED SO LARGELY TO BRLAG THE SOIENOE OF

PHYSIOLOGY TO ITS PRESENT CONDITION,

AND IN

GRATEFUL REMEMBRANCE OF MANY ACTS OF FRIENDSHIP, THIS WORK IS IXSCRIBED BY

THE AUTHOR. 



\section{P R E F A O E.}

Is entering upon the labor incident to the preparation of a work purporting to treat comprehensively of the physiology of man, the author appreciated the magnitude of the undertaking; and the special study which it necessarily demanded has not diminished that diffidence with which a student of any of the natural sciences puts forward a book which he hopes may add somewhat to existing knowledge, or fairly represent what is known in any particular department. In assuming so grave a responsibility, the author should be actuated by a sense of peculiar fitness for his task, as well as a conviction that literature demands such a work as he proposes to write. Without assuming these good and sufficient reasons, the author of the present volume pleads an earnest desire to advance the science of physiology and facilitate its study; and he indulges the hope that he may be instrumental in making the student and practitioner of medicine better acquainted with what must be conceded to be the basis of true pathology, and interest, to some extent, the general reader in the all-important subject of human physiology.

The plan of the present work involves a consideration of 
pure human physiology, and will embrace physiological chemistry and the anatomy of the tissues and organs of the body, only so far as necessary for the elucidation of the functions of the organism. Though, undoubtedly, the chemistry and general anatomy of the tissues and organs strictly belong to physiology, they present many points which have no bearing, that we are as yet able to comprehend, upon the functions. In the present condition of the science, a consideration of these would only encumber and obscure the history of the physiological processes. While it is undoubtedly true that every advance in physiological chemistry or histology will have its bearing, sooner or later, upon physiology, it is evident that discoveries in these departments must be multiplied and coördinated before their relations to the functions can be fully appreciated. Until then they are specially interesting only in a chemical and anatomical point of view. In the same way every discovery in physiology, no matter how unimportant it may at first appear in a practical point of view, will eventnally have its bearing upon practical medicine, surgery, or obstetries; yet it will not find its way into works on those subjects until its relations become apparent.

As an introduction to the study of physiology proper, a certain amount of knowledge of physiological chemistry is indispensable. It is in this direction that we are to look for advances which will enable us to comprehend the processes of nutrition, the end and object of all the regetative functions of the body. The introduction, then, is devoted to physiological chemistry. No attempt has been made to treat of this subject exhaustively, or to include a consideration of all the proximate principles which have been isolated and studied. As the general properties and relations of the different classes 
of proximate principles are by far the most important to us as physiologists, these have been specially dwelt.upon, and their relations to nutrition followed out as completely as possible, with our present knowledge. A consideration of the excrementitious proximate principles, being connected exclusively with excretion, has been deferred, to be taken up in connection with that function.

In treating of physiology proper, it has been the design of the author to present what is actually known regarding the functions of the body; and in order to facilitate their study, he has generally commenced the consideration of individual functions with a sketch of the physiological anatomy of the parts. This is the natural point of departure in the thorough investigation of any special function.

The science of physiology dates from the earliest periods in the history of medicine; and certain important physiological facts were demonstrated experimentally hundreds of years ago. While the author has regarded purely historical considerations, and discussions of mere theoretical questions, as unprofitable, he has attempted to give due credit to those who, by their experiments and observations, have contributed to bring the-science to its present condition. With this view, he has procured and consulted, as far as possible, accounts of original investigations; but from the poverty in physiological works of the public libraries to which he has had access, it has been necessary to depend to a certain extent on the exhaustive treatises on physiology published in other countries. Though, undoubtedly, he has been unable in all instances to give due credit to every observer, this has been attempted as far as possible.

It is an undoubted fact that nearly all the important 
developments in physiology have been the result of experiments upon living animals, by vivisections or otherwise, or accurate experimental observations upon the human subject. The great extension of this method of study is the cause of the rapid advances the science is making at the present day. For some years the author has been in the habit of employing vivisections in public teaching, and in this way has frequently verified the observations of the earlier as well as the more modern physiologists. A frequent repetition of experiments has often enabled him to reconcile the discordant results of the observations of others; and following out new questions which have presented themselves in the constant observation of the living organs, he has advanced some original views regarding certain of the functions. A new method is likewise presented for the analysis of the blood with reference to its organic constituents.

The plan of publication of the present work is one which is novel in this country, but which has been adopted abroad, particularly in France, in almost all elaborate treatises on physiology. It is to be issued in separate parts, each, however, forming a distinct treatise devoted to natural subdivisions of the subject. The volume now issued embraces an Introduction, the Blood, Circulation, and Respiration. The remaining volumes, three in number, will be issued yearly until the work is finished, and will likewise be severally complete in themselves. Simple and well-known anatomical and physiological points have not been illustrated by engravings, which have only been introduced where they seemed necessary to elucidate the text.

NEw Yonk, October, 1865. 


\section{O N T E N T S.}

\section{INTRODUCTION.}

General considerations-Vital properties of organized structures-Proximate principles-Inorganic principles-Organic non-nitrogenized principles-Organic nitrogenized principles, . . . . . . . . Page 13

\section{CHAPTER I.}

\section{THE BLOOD.}

General considerations-Transfusion-Quantity-Physical characters-OpacityTemperature-Specific gravity-Color-Anatomical elements of the bloodRed corpuscles-Chemical characters of red corpuscles-Development of red corpuscles-Formation of red corpuscles-Leucocytes, or white corpuscles-

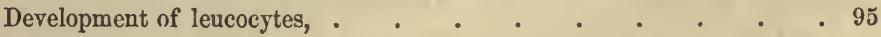

\section{CHAPTER II.}

\section{OONPOSITION OF THE BLOOD.}

General considerations-Methods of quantitative analysis-Fibrin-CorpusclesAlbumen-Inorganic constituents-Sugar-Fatty emulsion-Coloring matter of the serum-Urea and the urates-Cholesterine-Creatine-Creatinine, 127

\section{CHAPTER III.}

\section{OOAGULATION OF THE BLOOD.}

General considerations-Characters of the clot-Characters of the serum-Coagulating principle in the blood-Circumstances which modify coagulation-Coagulation of the blood in the organism-Spontaneous arrest of hemorrhageCause of coagulation of the blood-Summary of the properties and functions of the blood, 


\section{CHAPTER IV.}

\section{OIROULATION OF THE BLOOD.}

Discovery of the circulation-Physiological anatomy of the heart-Valves of the heart-Movements of the heart-Impulse of the heart-Succession of movements of the heart-Force of the heart-Action of the valves-Sounds of the heart-Cause of the sounds of the heart, . . . . Page 170

\section{CHAPTER V.}

\section{FREQUENCT OF THE HEART'S AOTION.}

Frequency of the heart's action-Influence of age-Influence of digestion-Influence of posture and muscular exertion-Influence of exercise-Influence of temperature-Influence of respiration on the action of the heart-Cause of the rhythmical contractions of the heart-Influence of the nervous system on the heart-Division of the pneumogastrics-Galvanization of the pneumogastrics-Causes of the arrest of action of the heart-Blows upon the epigastrium,

\section{CHAPTER VI.}

\section{CIROULATION OF THE BLOOD IN THE ARTERIES.}

Physiological anatomy of the arteries-Course of blood in the arteries-Elasticity of the arteries-Contractility of the arteries-Locomotion of the arteries and production of the pulse-Form of the pulse-Sphygmograph-Pressure of blood in the arteries-Hemodynamometer-Cardiometer-Differential cardiometer-Pressure in different parts of the arterial system-Iufluence of respi ration on the arterial pressure-Effects of hemorrhage-Rapidity of the current of blood in the arteries-Instruments for measuring the rapidity of the arterial circulation-Variations in rapidity with the action of the heart-Rapidity in different parts of the arterial system-Arterial murmurs, . . 240

\section{CHAPTER VII.}

\section{OIROULATION OF THE BLOOD IN THE OAPILLARIES.}

Distinction between capillaries and the smallest arteries and veins-Physiological anatomy of the capillaries-Peculiarities of distribution-Capacity of the capillary system-Course of blood in the capillaries-Phenomena of the capillary circulation-Rapidity of the capillary circulation-Relations of the capillary circulation to respiration-Causes of the capillary circulation-Influence of temperature on the capillary circulation-Influence of direct irritation on the capillary circulation, . . . . . . . . 278 


\section{CHAPTER VIII.}

\section{OIROULATION OF THE BLOOD IN THE VEINS.}

Physiological anatomy of the veins-Strength of the coats of the veins-Valves of the veins-Course of the blood in the veins-Pressure of blood in the veins-Rapidity of the venous circulation-Causes of the venous circulationInfluence of muscular contraction-Air in the veins-Function of the valvesVenous anastomoses-Conditions which impede the venous circulation-Regurgitant venous pulse, . . . . . . . . . Page 301

\section{CHAPTER IX.}

\section{PEOULIARITIES OF THE OIROULATION IN DIFFERENT PARTS OF THE SYSTEM.}

Circulation in the cranial cavity-Circulation in erectile tissues-Derivative circulation-Pulmonary circulation-General rapidity of the circulation-Time required for the passage through the heart of all the blood in the organismRelations of the general rapidity of the circulation to the frequency of the heart's action-Phenomena in the circulatory system after death, . 332

\section{CHAPTER X.}

RESPIRATION.

General considerations-Physiological anatorny of the respiratory organs-Respiratory movements of the larynx-Epiglottis-Trachea and bronchial tubesParenchyma of the lungs-Carbonaceous matter in the lungs-Movements of respiration-Inspiration-Yuscles of inspiration-Action of the diaphragmAction of the scaleni-Intercostal muscles-Levatores costarum-Auxiliary

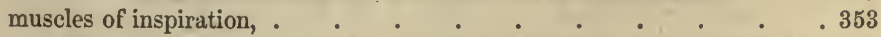

\section{CHAPTER XI.}

\section{MOVEMENTS OF EXPIRATION.}

Influence of the elasticity of the pulmonary structure and walls of the chestMuscles of expiration-Internal intercostals-Infra-costales-Triangularis sterni-Action of the abdominal muscles in expiration-Types of respirationAbdominal type-Inferior costal type-Superior costal type-Frequency of the respiratory movements-Relations of inspiration and expiration to each otherThe respiratory sounds-Coughing-Sneezing-Sighing-Yawning-Laughing-Sobbing-Hiccough-Capacity of the lungs and the quantity of air changed in the respiratory acts-Residual air-Reserve air-Tidal, or breathing air-Complemental air-Extreme breathing capacity-Relations in volume of the expired to the inspired air-Diffusion of air in the lungs, . . . 382 


\section{CHAPTER IV.}

\section{CIROULATION OF THE BLOOD.}

Discovery of the circulation-Physiological anatomy of the heart-Valves of the heart-Movements of the heart-Impulse of the heart-Succession of movements of the heart-Force of the heart-Action of the valves-Sounds of the heart-Cause of the sounds of the heart, . . . . Page 170

\section{CHAPTER V.}

\section{FREQUENOY OF THE MEART'S AOTION.}

Frequency of the heart's action-Influence of age-Influence of digestion-Influence of posture and muscular exertion-Influence of exercise-Influence of temperature-Influence of respiration on the action of the heart-Cause. of the rhythmical contractions of the heart-Influence of the nervous system on the heart-Division of the pneumogastrics-Galvanization of the pneumogastrics-Causes of the arrest of action of the heart-Blows upon the epigastrium,

\section{CHAPTER VI.}

\section{CIROULATION OF THE BLOOD IN THE ARTERIES.}

Physiological anatomy of the arteries-Course of blood in the arteries-Elasticity of the arteries-Contractility of the arteries-Locomotion of the arteries and production of the pulse-Form of the pulse-Sphygmograph-Pressure of blood in the arteries-Hemodynamometer-Cardiometer-Differential cardiometer-Pressure in different parts of the arterial system-Influence of respi ration on the arterial pressure-Effects of hemorrhage-Rapidity of the current of blood in the arteries-Instruments for measuring the rapidity of the arterial circulation-Variations in rapidity with the action of the heart-Rapidity in different parts of the arterial system-Arterial murmurs, . . 240

\section{CHAPTER VII.}

\section{CIRCULATION OF THE BLOOD IN THE OAPILLARIES.}

Distinction between capillaries and the smallest arteries and veins-Physiological anatomy of the capillaries-Peculiarities of distribution-Capacity of the capillary system-Course of blood in the capillaries-Phenomena of the capillary circulation-Rapidity of the capillary circulation-Relations of the capillary circulation to respiration-Causes of the capillary circulation-Influence of temperature on the capillary circulation-Influence of direct irritation on the capillary circulation, . . . . . . . . 278 


\section{CHAPTER VIII.}

OIROULATION OF THE BLOOD IN THE VEINS.

Physiological anatomy of the veins-Strength of the coats of the veins-Valves of the veins-Course of the blood in the veins-Pressure of blood in the veins-Rapidity of the venous circulation-Causes of the venous circulationInfluence of muscular contraction-Air in the veins-Function of the valvesVenous anastomoses-Conditions which impede the venous circulation-Re-

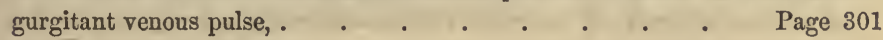

\section{OHAPTER IX.}

\section{PEOULIARITIES OF THE OIROULATION IN DIFFERENT PARTS OF THE SYSTEM.}

Circulation in the cranial cavity-Circulation in erectile tissues-Derivative circulation-Pulmonary circulation-General rapidity of the circulation-Time required for the passage through the heart of all the blood in the organismRelations of the general rapidity of the circulation to the frequency of the heart's action-Phenomena in the circulatory system after death, . . 332

\section{CHAPTER X.}

RESPIRATION.

General considerations-Physiological anatorny of the respiratory organs-Respiratory movements of the larynx-Epiglottis-Trachea and bronchial tubesParenchyma of the lungs - Carbonaceous matter in the lungs-Movements of respiration-Inspiration-Yuscles of inspiration-Action of the diaphragmAction of the scaleni-Intercostal muscles-Levatores costarum-Auxiliary muscles of inspiration, . . . . . . . . . . . . . 353

\section{CHAPTER XI.}

\section{MOVEMENTS OF EXPIRATION.}

Influence of the elasticity of the pulmonary structure and walls of the ehestMuscles of expiration-Internal intercostals-Infra-costales-Triangularis sterni-Action of the abdominal muscles in expiration-Types of respirationAbdominal type-Inferior costal type-Superior costal type-Frequency of the respiratory movements-Relations of inspiration and expiration to each otherThe respiratory sounds-Coughing-Sneezing-Sighing-Yawning-Laughing-Sobbing-Hiceough-Capacity of the lungs and the quantity of air changed in the respiratory acts-Residual air-Reserve air-Tidal, or breathing air-Complemental air-Extreme breathing capacity-Relations in volume of the expired to the inspired air-Diffusion of air in the lungs, . . $\quad$. 382 


\section{CHAPTER XII.}

\section{OHANGES WHICH THE ATR UNDERGOES IN RESPIRATION.}

General considerations-Discovery of carbonic acid-Discovery of oxygen-Composition of the air-Consumption of oxygen-Influence of temperature-Influence of sleep-Influence of an increased proportion of oxygen in the atmosphere-Temperature of the expired air-Exhalation of carbonic acid-Influence of age-Influence of sex-Influence of digestion-Influence of diet-Influence of sleep-Influence of muscular activity-Influence of moisture and temperature-Influence of seasons-Relations between the quantity of oxygen consumed and the quantity of carbonic acid exhaled-Exhalation of watery vapor-Exhalation of ammonia-Exhalation of organic matter-Exhalation

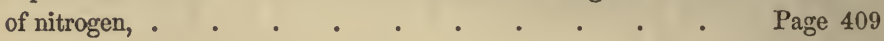

\section{CHAPTER XIII.}

\section{CHANges OF the BLOOD IN Respiration. (Hematosis.)}

Difference in color between arterial and venous blood-Comparison of the gases in renous and arterial blood-Observations of Magnus-Analysis of the blood for gases-Relative quantities of oxygen and carbonic acid in venous and arterial blood-Nitrogen of the blood-Condition of the gases in the bloodMechanism of the interchange of gases between the blood and the air in the lungs-General differences in the composition of arterial and venous blood, . . . . . . . . . . . . . . . . . . . . . .

\section{CHAPTER XIV.}

\section{RELATIONS OF RESPIRATION TO NUTRITION, ETO.}

Views of physiologists anterior to the time of Lavoisier-Relations of the consumption of oxygen to nutrition-Relations of the exhalation of carbonic acid to nutrition-Essential processes of respiration-The respiratory sense, or want on the part of the system which induces the respiratory movementsLocation of the respiratory sense in the general system-Sense of suffocation -Respiratory efforts before birth-Cutaneous respiration-Asphyxia, . 472 


\section{PHYSIOLOGY OF MAN.}

\section{INTRODUCTION.}

General considerations-Vital properties of organized structures-Proximate principles-Inorganic principles-Organic non-nitrogenized principles-Organic nitrogenized principles.

THE epoch of purely speculative reasoning, without the basis of established facts sufficient to justify any connected theories, belongs to the remote history of Natural Science. The ideas of the great philosophers of ancient times, who studied Nature by what may be called the intuitive method, have been gradually giving place to doctrines based on the observation and investigation of phenomena. Ages of observation and generalization of facts by the greatest intellects have put us but little beyond the threshold of the great domain of Science. But we have learned enough to know that all Nature is regulated by immutable laws. Students of her divine mysteries should be more than content if permitted to discover some of the truths, the development of which marks the scientific advancement of each succeeding age, though they may seem an insignificant portion of what is to be learned. It is only by accurate observation and generalization of a sufficient number of phenomena, that the laws of Nature are to be discovered. They are the creation 
of an infinite wisdom which never errs. We cannot hope to arrive at a knowledge of them by pure reasoning; or by assuming that they are in accordance with definite principles, too often the offspring of our own limited intellects. Nevertheless, it is a physiological attribute of the human mind to desire to press on in advance of observation, and to form theories, which may or may not be carried out by the succeeding development of actual knowledge. Theories which are not built upon false or imperfectly observed phenomena, are the pioneers of actual discovery. When theoretical preconceptions are justified and corrected by original observations and experiments, with the brain to conceive and the will to execute, man, in thus working out the great problems of Nature, is fulfilling one of the highest purposes of his existence.

With the few facts which were at first known, the ancient speculative philosophy professed to embrace the whole of natural science; but as discoveries were made in different departments, a division of labor became necessary. We now find different classes of scientific men, each working in a particular sphere; as in the lower zoological divisions, a single organ performs all the varied functions of nutrition, while in the higher orders, when the processes of life are more intricate and complicated, the system is divided up into elaborately-organized parts, each of which has an allotted office.

From the time of Galen may be said to date, as distinct from astronomy, chemistry (or rather alchemy), physics, \&c., the science which is now called Physiologr.

Physiology, from its etymology, signifies the science of Nature; but in the sense in which the term is now used, it may be defined to be the science of life. More elaborate definitions have been given, but they only qualify and explain the meaning of what we know as life.

A natural division of physiology is into animal and vegetable; and again, into the physiology of the inferior 
animals as compared with man, or comparative physiology, and the Physiology of Mav. 'The latter, which is the subject of the present work, is peculiarly interesting to the physician, as the basis of all accurate knowledge of the science of medicine.

In the early history of physiological science, the development of anatomy necessarily gave us much information concerning the functions of the body; and we now have to acknowledge our continual indebtedness to anatomical investigations, particularly those made with the aid of the microscope, for important advancements in physiology. In treating of the subject, it is impossible to neglect what is most appropriately called the physiological anatomy of parts, a knowledge of which alone enables us, oftentimes, to comprehend their functions. For example, we can scarcely conceive how the anatomy of the circulatory system could be clearly understood without giving us a knowledge of its physiology.

Chemistry, also, when the components of the body are, studied in such a way as not to destroy their properties as organic compounds, has a most important bearing on the advancement of physiology. As a striking example of this, we may take the discovery of the properties of the gases of the air and their relations to the blood by Lavoisier, which gave us the first definite ideas regarding the essential phenomena of respiration. We are now largely indebted to modern physiological chemistry for a knowledge of many of the essential phenomena of life, and look to a further development of this science for an elucidation of many important, but still obscure, questions connected with nutrition.

Certain physiological functions are in exact accordance with established physical laws; which are competent, for example, to explain the refraction in the structures of the eye, or the conduction of vibrations in the ear. Physical laws are involved in most of the phenomena of life, but are generally more or less modified by the peculiar properties of organized bodies. 
Many of the phenomena of life are made clear by a comparison of the physiology of man with that of the inferior animals, which is often simpler and more easily investigated.

As physiology is the natural and only correct basis of pathology, we frequently derive important information as to the functions of parts by studying the effects of disease, by which their functions are modified or abolished. The experiments thus performed by Nature on the human system are frequently more instructive than those which we make on the inferior animals.

As the complement to anatomy, human and comparative, organic chemistry, and pathology, we have as the most precious and fruitful means of physiological investigation, direct observation of the phenomena of life in man and the inferior animals, and experiments on animals by vivisections. The present condition of physiology is a testimony of the incalculable value of this method of study. Were it consistent with our plan to follow out the general development of the science from an historical point of view, we should find the names of Harvey, Aselli, Haller, Hales, Spallanzani, Edwards, Bichat, Bell, Majendie, and a host of others, bearing witness by their works to the value of vivisections in physiological investigations; to say nothing of the great observers of the present day, who are constantly adding to our knowledge. The field would be sterile indeed were it not for experiments on living animals; and the loss to the science which has for its object the alleviation of the sufferings of mankind, would have been incalculable, had physiologists been unwilling, from false motives of humanity, to inflict pain upon the lower animals, which is to a certain extent unavoidable in experimentation.

Physiological literature, in the great Elementa Physiologice of Haller, which belonged to a past generation, and the elaborate systematic works of Bérard, ${ }^{2}$ Longet, Müller,

${ }^{1}$ Bérard did not live to complete his great work on physiology. He died 
and other experimentalists of the present generation, furnishes abundant proof that the faculty of observation and the power of generalization are not necessarily inconsistent with each other.

It would be futile to attempt to point out all the difficulties and sources of error in experimentation on living animals. These must be overcome by the physiologist after he has become practically acquainted with them. It must be borne in mind, however, that we are interrogating Nature; and our sole aim must be to put our questions intelligently and interpret the answers correctly. She does not unfold her mysteries to the careless and inconsiderate observer. An accident may lead the reflecting student to frame a particular set of experiments, for the explanation of an unexpected phenomenon; but we should go to work with an idea of what we wish to know, always ready to correct or abandon our most cherished preconceived notions if we find they are not in accordance with facts. Experiments should not be isolated. A golden opportunity is thrown away if we stop short of the end in a legitimate series of investigations; for none are better fitted to go through the later steps of a natural series of experiments than they who have conceived and executed the first.

With the many varying conditions of the system which inevitably occur in living animals, it is almost unnecessary to add that an important observation should be repeatedly confirmed, and the answer to our experimental inquiries obtained, if possible, in different ways: It must be remembered that Nature never contradicts herself, and has no exceptions. $\mathrm{Her}$ laws are invariable; and if experiments are apparently contradictory, we must look for differences in the conditions

shortly after he had commenced the publication of the fourth volume in 1855 . The prolégomènes, and the sections on digestion, absorption, the blood, respiration, and circulation, are perhaps the most candid, exhaustive, and best considered essays on these subjects in any language. Science suffered a great loss when the author was thus cut off in the midst of his labors. 
animals ; it belongs to psychology to study and make known the faculties which separate him from them." :

Even without accidents, physiological death is a necessity of existence ; but nature has provided, as one of the most important attributes of organization, a means by which organized bodies may be perpetuated through all ages. In the fullydeveloped organism are produced two kinds of organic elements, the male and the female. These, when brought in contact with each other under proper conditions, are capable of being developed into a new being, similar in organization to, and designed to take the place of, the one which is to pass away. These new beings are generated in sufficient number to insure the perpetuation of the species.

The excrementitious products of the body during life, and the body itself after death, changed by the peculiar process of putrefaction, are returned to the earth and to the air, and contribute to the nutrition of the vegetable kingdom. The vegetables, in their turn, are consumed in the nutrition of animals. All the elements necessary to nutrition, except oxygen, are taken into the alimentary canal as food. Our food consists either of vegetables, or the flesh of animals that are nourished by vegetables.

\section{PROXIMATE PRINCIPLES.}

From the preceding general remarks, it is evident that physiology, to be systematically and properly studied, must be connected with physiological anatomy and chemistry. The physiological anatomy of special organs and systems naturally precedes the consideration of their functions; and in treating of the functions of other parts, more especially the nutritive and excrementitious fluids and the secretions, we are unavoidably led to consider fully their chemical constitution. There are, however, certain constituents of the body,

${ }^{1}$ Lovget, Traité de Physiologie, Paris, 1861, tome i., p. دxviii. 
a full consideration of which, in connection with special functions, would be out of place, as well as many points in physiological chemistry, showing the relations of the different elements to nutrition, etc.; hence is desirable, as an introduction to physiology proper, a brief review of the proximate principles of the economy. In this introduction it is not proposed to treat exhaustively of physiological chemistry. Such principles as will demand, from their connection with special functions, extended consideration in another place, are omitted or simply alluded to, as well as some which have a very unimportant or obscure function.

If we were to study the constitution of the body from a purely chemical point of view, it would be divided into elementary substances, or those which are absolutely incapable of further subdivision. In this way we should lose all distinction between organic matters and those which enter indifferently into the composition of all bodies in Nature, whether inert or endowed with vital properties. After having thus ascertained the ultimate constitution of the organism, we have learned all that is possible by this method; for we are already familiar with the properties and behavior of elementary matter, as obtained from the inorganic kingdom.

In physiological chemistry this method is inadmissible. The substances which are presented for our study in the living organism are endowed with vital properties. Their ultimate composition is of little consequence compared with a knowledge of the laws which regulate their behavior, not as elements, but as constituents of an elaborate vital organization.

We can separate from the organism of animals substances of a peculiar nature which are never found in the inorganic world. These demand our special consideration. If we attempt to study them by the ordinary chemical processes of analysis, they are destroyed and lose their properties as organic principles. 
Combined with these organic principles we always have a certain proportion of inorganic matters which may, it is true, be separated from them easily, and apparently without decomposition, but which are, notwithstanding, necessary to the peculiar properties by which we recognize organic substances. Their physiological union is so intimate that they may justly be considered as organic, though originating in the inorganic kingdom.

Chemistry recognizes fifty-nine elementary substances, of which some fifteen or eighteen enter into the constitution of the human body; but as physiologists, we must make a division of the body into component principles, without reference to the elementary substances themselves, but with a view to the form and condition of their existence in the organism. As we have seen that the distinguishing properties of organic principles are destroyed when they are reduced to their ultimate elements, it is evident that many or most of the principles into which the body is divided physiologically are compound substances.

From this point of view, the organism may be said to be composed of Immediate or Proximate Principles.

A Proximate Principle may be defined to be $a$ substance extracted from the body, which cannot be further subdivided without chemical decomposition and loss of its characteristic properties.

According to Robin and Verdeil, there exist from eightyfive to ninety distinct proximate principles in the human body. ${ }^{1}$

The distinction between proximate principles and chemical elements is apparent from the definition above giren. To illustrate this difference, however, we may take the following example. Chloride of sodium is an important proximate principle, and is composed of the chemical elements chlorine and sodium. As chloride of sodium, it has certain

${ }^{1}$ Robin and Verdeil, Chimie Anatomique et Physiologique, Paris, 1853, tome i., p. 128. 
properties, and is endowed with certain functions in the economy, which are, of course, entirely different from the properties of chlorine or sodium; the latter especially being only obtained in a state of chemical purity by a difficult and elaborate process of manipulation. As physiologists we have nothing to do with the properties of chlorine, or the rare metal sodium; we only wish to know as much as possible about the functions of these two bodies united to form common salt. Again, fibrin, a proximate principle found in the blood, may be reduced by chemical manipulations to a certain number of atoms of carbon, hydrogen, oxygen, nitrogen, and sulphur. But a knowledge of even the exact proportions of these ingredients would be of no practical benefit, if we were unacquainted with the general properties of fibrin and its uses in the economy. Salt cannot be subdivided into chlorine and sodium, nor fibrin into its elements, without chemical decomposition and loss of characteristic properties; but both of these substances can be extracted from the body in the condition in which they exist in the organism, and are therefore proximate principles.

A constituent of the body may be at the same time a chemical element and a proximate principle. An example of this is the free oxygen in solution in the blood. This enjoys, in the body, the properties of free oxygen, and may be extracted from the blood by mere displacement with another gas, or by the air-pump; a process quite different from the elaborate chemical manipulation which would be necessary to obtain oxygen by decomposition of fibrin, albumen, or any compound principle.

The principles which compose the body, with the exception of excrementitious substances, exist in our food; this being the only way in which material is supplied for the continual repair which is characteristic of living tissues. They are all introduced from without. Certain principles, such as water and the inorganic salts, are merely transitory in the interior of the body, and are discharged in the same form in 
which they enter. Others are consumed in the process of repair, and after having performed their functions, are thrown off as effete matters. Examples of the latter are fibrin and albumen, which are transformed first into the substance of the tissues, and then into urea, creatine, cholesterine, and other excrementitious matters, which are the result of the breaking down or wearing out of the tissues. Finally, there are certain principles, the sugars and fats for example, which have an important connection with the process of nutrition, and disappear in the system, but whose transformations we have not as yet been able to follow. These, besides being taken in as food, are manufactured by certain organs, and appear de novo in the economy.

Division of Proximate Principles.-In the division of proximate principles, we shall follow, with slight modifications, the classification of Robin and Verdeil. With reference solely to anatomical and physiological chemistry, the classification of these authors cannot be improved; but in treating of the whole subject of physiology, it will be conven-

- ient to take up certain of the elements in connection with the functions in which they play an important part. Oxygen and carbonic acid, for example, will be fully considered in connection with respiration; urea and cholesterine with excretion, \&c. Again, there are some whose function is apparently of so little importance, or so obscure, that, while they may be interesting in a chemical point of view, merely as copstituents of the body, it is not worth while to treat of them in connection with physiology.

The two great divisions of proximate principles which we propose, comprise :

First. Substances which enter into the normal constitution of the organized tissues, and those constituents of the fluids which are used in nutrition. 
SECOND. Substances which are the result of the wearing out of the tissues, and are not used in nutrition. ${ }^{1}$

The first division, which is the only one that will be taken up in this connection, may be subdivided, according to the classification of Robin and Verdeil, into three classes.

1. Inorganio Substances.-This class is of inorganic origin, definite chemical composition, and crystallizable.- The substances forming it are all introduced from without, and are all discharged from the body in the same form in which they entered. They never exist alone, but are always combined with the organic principles, to form the organized fluids or solids. This union is "atom-to-atom," and so intimate that they are taken up with the organic elements, as the latter are worn out and become effete, and are discharged from the body, though themselves unchanged. To supply the place of the principles thus thrown off, a fresh quantity is deposited in the process of nutrition. They give to the various organs important properties ; and, though identical with substances in the inorganic world, in the interior of the body they behave as organic substances. They require no special preparation for absorption, but are soluble and taken in unchanged. They are received into the body in about the same proportion at alk periods of life, but their discharge is notably diminished in old age ; giving rise to calcareous incrustations and deposits, and a considerable increase in the calcareous matter entering into the composition of the tissues. As examples of this class we may cite water, chloride of sodium, the carbonates, sulphates, phosphates, and other inorganic salts.

\section{Organic Non-Nitrogenized Substances.-This class of}

1 This division is composed of excrementitious matters, which will be fully considered when treating of excretion. It is included in the second class of proximate principles by Robin and Verdeil. 
proximate principles is of organic origin, definite chemical composition, and crystallizable. With the exception of the salts peculiar to the bile, which will be considered when we eome to treat of that fluid, pneumic acid, and one or two unimportant principles, they are distinguished by being composed of three elements, Carbon, Hydrogen, and Oxygen. As they thus contain hydrogen and carbon, to the exclusion of all other elements, except the almost universal principle, oxygen, they are frequently spoken of as Hydro-carbons. They are distinguished from other organic substances by the absence of nitrogen, which has given them the name of $\mathrm{Nom}$ nitrogenized or Non-azotized substances. They are introduced into the body as food, and are manufactured in the economy by special organs; but, unlike principles of the first class, with the exception of sugar and fat, which are discharged in the milk during lactation, are never discharged from the body in health. The principles of this class play an important part in development and nutrition. One of them, sugar, appears very early in fœtal life, formed first by the placenta, and afterwards by the liver; its formation by the latter organ continuing during life. Fat is a necessary element of food, and is also formed in the interior of the body. The exact influence which these substances have on development and nutrition is not known, but experiments and observation have shown that this influence is important. Many physiologists are of the opinion that principles of this class undergo direct oxidation or combustion in the lungs, and have the exclusive office of keeping up the animal temperature. At one time, indeed, they were generally spoken of as calorific elements; but in the present condition of science this exclusive view is not tenable; and we shall see, when treating of the subject of animal heat, that its production cannot be referred entirely to combustion of the hydro-carbons. The sugars and fats, lactic acid and the lactates, pneumic acid and the pneumates, the fatty acids and their combinations, constitute the most important principles of this class. 
3. Organic Nitrogenized Substances.-This class of proximate principles is of organic origin, indefinite chemical composition, and non-crystallizable. Substances forming this class are apparently the only principles which are endowed with vital properties, taking materials for their regeneration from the nutritive fluids, and appropriating them to form part of their own substance. Considered from this point of view, they are different from any thing which is met with out of the living body. They are all, in the body, in a state of continual change, wearing out and becoming effete, when they are transformed into excrementitious substances, which constitute the second grand division of proximate principles. The process of repair in this instance is not the same as in inorganic substances, which enter and are discharged from the body without undergoing any change. The analogous substances which exist in food, undergo a very elaborate preparation, by digestion, before they can even be absorbed by the blood-ressels; and still another change takes place when they are appropriated by the various tissues. They exist in all the solids, semi-solids, and fluids of the body, never alone, but always combined with inorganic substances. As a peculiarity of chemical constitution, they all contain nitrogen, which has given them the name of Nitrogenized or Azotized prineiples. As before intimated, they give to the tissues and fluids their vital properties. In studying their properties more fully, we shall see that they are by far the most important elements in the organism. The elaborate preparation which they require for absorption involves the most important part of the function of digestion. Their absolute integrity is necessary to the operation of the essential functions of many tissues, as muscular contraction, or conduction of nervous force. An exact knowledge of all the transformations which take place in their regeneration and the process by which they are converted into effete or excrementitious matters, would enable us to comprehend nutrition, which is the essence of physiology ; but as yet we know little 
of these changes, and consider ourselves fortunate in understanding a few of the laws which regulate them. As examples of principles of this class we may cite musculine, osteine, fibrin, albumen, and caseine.

\section{INORGANIC PRINCIPLES.}

The number of principles of this class, now well established as existing in the human body, is twenty-one. ${ }^{1}$ All substances which at any time exist in the body are proximate principles; but some are found in small quantities, are not always present, and apparently have no very important function. These will be passed over rapidly, as well as those which are so intimately connected with some important function as to render their full consideration in connection with that function indispensable. The following is a list of the inorganic principles, excluding those which are excrementitious, and one or two which are not yet well established:

\section{Table of Inorganic Principles.}

Proximate Principle.

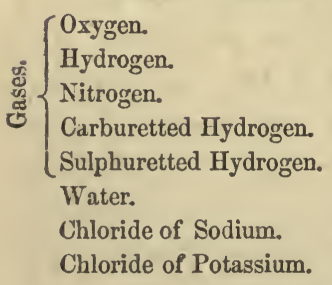

Where Found.

Lungs and Blood.

Gases of Stomach and Colon.

Lungs, Intestinal Gases, and Blood.

Lungs (expired air), Intestines.

Lungs (expired air), Intestines.

Universal.

Universal, except the enamel.

Muscles, Liver, Milk, Chyle, Blood, Mucus, Saliva, Bile, Gastric Juice, Cephalo-rachidian Fluid, and Urine.

1 Robin and Verdeil give twenty-nine; but of these, three (acid phosphate of soda, acid phosphate of lime, and ammonio-magnesian phosphate) are found only in the urine, and may be considered as coming under the head of excrements, with carbonic acid, which is one of the most important excretions; one (bicarbonate of lime) is abnormal; one (bicarbonate of potassa) is found only in certain of the inferior animals; and two (carbonate and bicarbonate of ammonia) are doubtful. 
Proximate Principle.

Phosphate of Lime (basic).

Carbonate of Lime.

Carbonate of Soda.

Carbonate of Potassa.

Phosphate of Magnesia.

Phosphate of Soda (neutral).

Phosphate of Potassa.

Sulphate of Soda.

Sulphate of Potassa.

Sulphate of Lime.

Hydrochlorate of Ammonia.

Carbonate of Magnesia.

Bicarbonate of Soda.
Where Found.

Universal.

Bones, Teeth, Cartilage, Internal Ear, Blood, Sebaceous Matter, and sometimes Urine.

Blood, Bone, Saliva, Lymph, Cephalo. rachidian Fluid, and Urine.

Blood, Bone, Lymph, and Urine.

Universal.

Universal.

Universal.

Universal, except Milk, Bile, and Gastric Juice.

Same as Sulphate of Soda.

Blood and Feces.

Gastric Juice, Saliva, Tears, and Urine.

A trace in the Blood and Sebaceous matter.

Blood (Liebig).

\section{The Gases.}

The gases (oxygen, hydrogen, nitrogen, carburetted hydrogen, sulphuretted hydrogen) ${ }^{1}$ exist both in a gaseous state, and in solution in some of the fluids of the body. Oxygen plays a most important part in the function of respiration; but the office of the others is by no means so essential. Nitrogen seems to be formed by the system in small quantity, is taken up by the blood and exhaled by the lungs; except during inanition, when the blood absorbs a little from the inspired air. It exists in greatest quantity in the intestinal canal. The carburetted and sulphuretted hydrogen, with pure hydrogen, are found in minute quantities in the expired air, and are also found in a gaseous state in the alimentary canal. From the offensive nature of the contents of the large intestine, we would suspect the presence of sulphuretted hydrogen in considerable quantity; but actual analysis has shown that the gas contained in the stomach and intestines,

1 Carbonic acid is here omitted, and will be treated of under the head of excretions. 
large as well as small, is composed chiefly of nitrogen, with hydrogen and carburetted hydrogen in about equal proportion, five to eleven parts per hundred, and but a trace of sulphuretted hydrogen. With the exception then of oxygen and carbonic acid, the latter being an excretion, the gases do not hold an important place among the proximate principles. At all events, their function, whether it be important or not, is but little understood.

\section{Water, HO.}

Water is by far the most important of the inorganic principles. ${ }^{2} \quad$ It is present at all periods of life, existing even in the ovum. It exists in all parts of the body ; in the fluids, some of which, as the lachrymal fluid and perspiration, contain little else, and in the hardest structures, as the bones, or the enamel of the teeth.

. In the solids and semi-solids it does not exist as water, but enters into their structure, assuming the consistence by which they are characterized. For example, we have water in the bones, teeth, and even in the enamel, not contained in the interstices of their structure, as in a sponge, but incorporated into the substance of the tissue. In these situations it is essentially water of composition. During the process of nutrition, water is deposited in the tissues with the other nutritive principles, as we have it incorporated in the substance of certain inorganic compounds in the process of crystallization, when it is known in chemistry as water of crystallization. In the interior of the body, water is thus incorporated in the substance of organic matters, which are

I In comparing principles which are essential to nutrition and to life, it is impossible to say that one is absolutely more important than another; still, writers are in the habit of making a distinetion in the importanee of necessary constituents of the body, chiefly with reference to their quantity and the extent of their distribution. When we come to organic principles, we shall see that thesc are manifestly the most important constituents of the living body, as giving to the tissues their vital properties. 
of indefinite chemical composition, and non-crystallizable, and we have no reason to be surprised, as physiologists, to find it entering into their composition in indefinite proportions, assuming the form and consistence of the organic substance. Our definition of a proximate principle is: "a substance extracted from the body, which cannot be further subdividéd without chemical decomposition." The union of water with the organic principles is chemical ; and though feeble, is not more so than the chemical union of elements in some compounds found in the inorganic world. The bicarbonates, for example, are formed by a union of two equivalents of carbonic acid with one of the base; but the second atom of carbonic acid is in so feeble a condition of union, that it is set free when the compound is placed under the receiver of an air-pump. It might be objected that water is combined with organic substances in an indefinite quantity, while the carbonic acid is present in definite proportion; but it must be remembered that indefinite proportions of all the constituents are characteristic of organic substances; and that the quantity of water existing, within certain limits, in indefinite proportions, only obeys the law which regulates the components which are universally recognized as existing in a state of chemical union. The only difference between water and the other constituents of an organic compound, is that the former is extracted with facility; as one atom of carbonic acid is extracted from the bicarbonates more easily than. the other. Studying the organism as physiologists, we must consider water as an integral constituent of the tissues, and not as merely absorbed by them.

All the organized structures contain a certain proportion of water, and this is necessary to the performance of all or any of their functions. If a normal muscle be considered as a contracting organ, and a nerve as a conducting organ, or albumen as a nutritious element, we must consider, as one of their constituents, water. It is necessary to the proper form, consistence, and function of these and all organized structures. In analysis of organic matters, when water is lost or driven off 
in our manipulations, the principle is not brought near a state of chemical purity, but is essentially and radically changed.

The quantity of water which each organic substance contains is important; and it is provided that this quantity, though indefinite, shall not exceed or fall below certain limits. The truth of this proposition is made evident from the following facts: In the first place, all organs and tissiues must contain a tolerably definite quantity of water to give them proper consistence. The evils of too great a proportion of water in the system, and consequently a diminution of solid elements, are well known to the practical physician. General muscular debility, loss of appetite, dropsies, and various other indications of imperfect nutrition, are among the results of such a condition; while a deficiency of water is immediately made known by the sensation of thirst, which leads to its introduction from without.

The fact that water never exists in any of the fluids, semisolids, or solids, without being combined with inorganic salts, and especially chloride of sodium, is one reason why its proportion in various situations is to a certain extent constant. The presence of these salts influences, in the semi-solids at least, the quantity of water entering into their composition, and consequently regulates their consistence. A very simple experiment shows this with reference to the chloride of sodium. If a piece of muscle be placed in a strong solution of common salt, as in salting meat, it becomes harder, and loses a portion of its water of composition; while exposed to the action of pure water, it absorbs a certain quantity and becomes softer. The nutrient fluid of the muscles during life contains water with just enough saline matter to preserve their normal consistence. This action of saline matters is even more apparent in the case of the blood corpuscles. If pure water be added to the blood, these bodies swell up and are finally dissolved; while if we add a strong solution of salt, they lose water, and become shrunken and corrugated; but their natural form and consistence can be restored, even after they have been completely dried, by 
adding water containing about the proportion of salt which exists in the plasma.

It seems clear, then, that water is a necessary element of all tissues, and is especially important to the proper constitution of organic nitrogenized substances; that it enters into the constitution of these substances, not as pure water, but always in connection with certain inorganic salts; that its proportion is confined within certain limits; and that the quantity in which it exists, in organic nitrogenized substances particularly, is regulated by the quantity of salts which enter, with it, into the constitution of these substances.

The quantities of water which can be driven off by a moderate temperature $\left(212^{\circ} \mathrm{Fahr}\right.$.) from the different fluids and tissues of the body, vary of course very considerably, according to the consistence of the parts. The following is a list of the quantities in the most important fluids and solids :

\section{Table of Quantity of Water.}

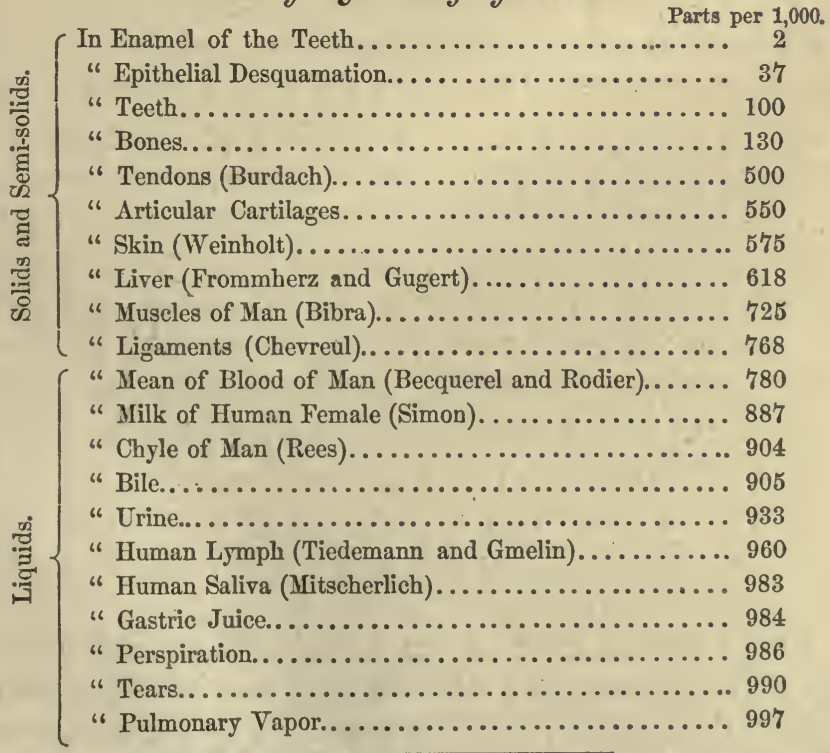

1 This table is made of selections from the table of Robin and Verdeil-taken from various authors. 
Function of Water.-After what has been stated respecting the condition in which water exists in the body, there remains but little to say concerning its function. As a constituent of organized tissues, it gives to cartilage its elasticity, to tendons their pliability and toughness; it is necessary to the peculiar power of resistance of the bones, and, as.we have already seen, it is necessary to the proper consistence of all parts of the body. It has other important functions as a solvent. Soluble articles of food are introduced in solution in water. The excrementitious matters, which are generally soluble in water, are dissolved by it in the blood, carried to the organs of excretion, and discharged in a watery solution from the body.

Origin and Discharge of Water.-It is evident that the great proportion of water is introduced from without in the fluids, and in the watery constituents of all kinds of food; but the theoretical views of some physiologists with regard to the hydrocarbons and their combustion, led to the supposition that water is also formed in the body by a direct union of oxygen and hydrogen. The true way of determining this point is to estimate all the water introduced into the organism, and compare this quantity with that which is discharged. The latter estimate, however, presents very great difficulties. $\Lambda \mathrm{s}$ water is continually given off in the form of vapor from the skin, and in the expired air, the quantities thus discharged are subject to great variations, dependent upon exercise, temperature, the state of the atmosphere, etc., and even if constant could be estimated with great difficulty. Experiments on this point have been undertaken by Sanctorius, Barral, Boussingault, and others; but they are not sufficiently com. plete to settle the question.

In the present state of our knowledge, we can only say that water is introduced with the fluid and solid elements of food, by the stomach, and that it escapes by the urine, feces, lungs, and skin. There is no direct evidence that any is pro- 
duced in the interior of the body. In the issue of water by the kidneys and skin, it has long been observed that, in point of activity, these two emunctories bear a certain relation to each other. When the skin is inactive, as in cold weather, the kidneys discharge a large quantity of water; when the skin is active, the quantity of water discharged by the kidneys is diminished. Certain therapeutical agents, also, can be made to act as diaphoretics by combining other measures which favor cutaneous action ; or as diuretics, by employing measures to diminish the action of the skin.

\section{Chloride of Sodium (Common Salt), NaCl.}

Chloride of sodium is next in importance, as an inorganic proximate principle, to water. It is found in the body at all periods of life, existing, like water, in the ovum. It exists in all the fluids and solids of the body, with the single exception of the enamel of the teeth. In the fluids, it seems to be simply in a state of solution, and can be recognized by the ordinary tests; in this respect we may class together the chlorides of sodium and potassium.

The quantity of chloride of sodium in the entire body has never been estimated; nor, indeed, has any accurate estimate been made of the quantity contained in the various tissues; for all the chlorides are generally estimated together. It exists in greatest proportion in the fluids, giving to some of them, as the tears and perspiration, a distinctly saline taste. The following table gives an idea of the quantity which has been found in some of the most important of the fluids and solids :

\section{Table of Quantity of Chloride of Sodium.}

In Blood, Human (Lehmann). . . . . . . . . . . . . . . 4 4 210

" Chyle (Lehmann)........................ 5.310

“ Lymph (Nasse)............................ 4. 120

" Milk, Human (Lehmann).................. 0.870 
Parts per 1,000 .

In Saliva, Human (Lehmann). . . . . . . . . . . 1.530

" Perspiration, Human (mean of three analyses, Piutti)... 3.433

"Urine (maximum) $\quad\{\ldots \ldots \ldots \ldots \ldots \ldots \ldots .7 .280$

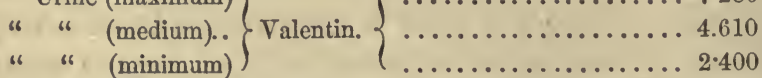

" Fecal Matters (Berzelius)................... 3.010

Function of Chloride of Sodium.-The function of this principle is undoubtedly important, but is not yet fully understood. It does not seem to enter into the substance of the organized solids and semi-solids as an important and essential element, but apparently exercises its chief function in the fluids. It certainly determines, to a great extent, the quantities of exudations, regulates absorption, and serves to maintain the albuminoids, especially those contained in the blood, in a state of fluidity. Albumen is coagulated by heat with much greater difficulty in a solution of chloride of sodium than when mixed with pure water. A strong solution of common salt is capable of dissolving casein, or of preventing the coagulation of fibrin. We have already alluded to the fact that it is the chloride of sodium particularly which regulates the quantity of water entering into the composition of the blood corpuscles, thereby preserving their form and consistence; and that it seems to perform an analogous function with reference to the other semi-solids of the body. With regard to the general function of this substance, the following proposition of Liebig is adopted by Robin and Verdeil, and a little reflection will show that it is sustained, as far as we know, by the facts:

"Common salt is intermediate in certain general processes, and does not participate by its elements in the formation of organs."

In the first place, the fluids of the body are generally intermediate in their functions, containing nutritious elements, which are destined to be appropriated by the tissues and organs, and worn-out elements, which are to be separated from the body. In the blood and chyle chloride of sodium is found in greatest 
abundance. When the nutrition of organs takes place, which consists in the fixation of new proximate principles, chloride of sodium is not deposited in any considerable quantity, but seems to regulate the general process, at least to a certain extent. In all civilized countries salt is used extensively as a condiment, and it undoubtedly facilitates digestion by rendering the food more savory, and increasing the flow of the digestive fluids; here, likewise, acting simply as an intermediate agent. There is nothing more general among men and animals than this desire for common salt. The carnivora crave it, and obtain it in the blood of animals; the herbivora frequent "salt licks" and places where it is found, and relish it when mixed with their food; while by man its use is almost universal. In the domestic herbivora the effect of a deprivation of this article is very marked, and has been made the subject of some very interesting experiments by Boussingault. This observer experimented upon two lots of bullocks, of three each, all of them, at the time the observations were commenced, being perfectly healthy and in fine condition. 'One of these lots he deprived entirely of salt, excepting what was contained in their fodder, while the other was supplied with the usual quantity. No marked difference in the two lots was noticed until between five and six months, when the difference in general appearance was very distinct. The animals receiving salt retained their fine appearance, while the others, though not diminished in flesh, were not as sleek and fine. At the end of a year the difference was very marked. The hides of those which had been deprived of salt were rough and ragged, their appearance listless and inanimate, contrasting strongly with the sleek appearance and vivacious disposition of the others. ${ }^{1}$ The experiments of Boussingault are the most conclusive that have ever been instituted with regard to the influence of chloride of sodium

3 Bodssumgaul, Mémoires de Chimie Agricole et de Physiologie, Paris, 1854, p. 271 et seq. 
upon nutrition. They indicate a certain deficiency in the nutrition of animals deprived of it, but not any considerable loss of weight. Before these observations were made, Dailly made upon twenty sheep analogous experiments, which were continued for three months. At the end of that time the lot which received salt presented a considerable excess of weight (about $22 \frac{8}{4}$ lbs.) over the others. ${ }^{1}$

It is a significant fact that the quantity of chloride of sodium existing in the blood is not subject to rariation, but that an excess introduced with the food is thrown off by the kidneys. The quantity in the urine, then, bears a relation to the quantity introduced as food, but the proportion in the blood is constant. This is another fact in favor of the view that the presence of a definite quantity of common salt in the circulating fluid is essential to the proper performance of the general function of nutrition.

Origin and Discharge of Chloride of Sodium.-This substance is always introduced with food in the condition in which it is found in the body. It is contained in the substance of all kinds of food, animal and vegetable; but in the herbivora and in man, this source is not sufficient to supply the wants of the system, and it is introduced, therefore, as salt. The quantity which is discharged from the body has been estimated by Barral $^{2}$ to be somewhat less than the quantity introduced, about one-fifth disappearing; but these estimates are not exactly accurate, for the amount thrown off in perspiration has never been directly ascertained. It exists in the blood in connection with the phosphate of potassa, and a certain amount is lost in a double decomposition which takes place between these two salts, resulting in the formation of chloride of potassium and phosphate of soda. It also is supposed to furnish the soda to all the salts which have a

2 Losget, Traité de Physiologic, tome i., p. 76.

${ }^{2}$ Cited by Robin and Verdeil. Chimie Anatomique et Physiologique, Paris, 1853, tome ii., p. 193. 
soda base, and a certain quantity, therefore, disappears in this way.

Existing, as it does, in all the solids and fluids of the body, it is discharged in all the excretions, being thrown off in the urine, feces, perspiration, and mucus.

\section{Chloride of Potassium, $\mathrm{KCl}$.}

Chloride of potassium, though not as important a proximate principle as the chloride of sodium, nor so generally distributed in the economy, seems to have an analogous function. It is found in the Muscles, Liver, Milk, Chyle, Blood, Mucus, Saliva, Bile, Gastric Juice, Cephalo-Rachidian Fluid, and Urine. It is exceedingly soluble, and in these situations exists in solution in the fluids.

Its quantity in these situations has not been accurately ascertained, as it has generally been estimated together with the chloride of sodium. In the muscles, it exists, however, in a larger proportion than common salt. In cow's milk, Berzelins ${ }^{1}$ has found 1.7 pts. per 1,000 ; Pfaff and Schwartz, 1.35 per 1,000 in cow's milk, and 0.3 per 1,000 in human milk. ${ }^{2}$

Of the function of this principle, little remains to be said after what has been stated with regard to the chloride of sodium. Their functions are probably identical, though the latter, from its greater quantity in the fluids, and its universal distribution, is by far the more important.

\section{Origin and Discharge of Chloride of Potassium.-This} substance has two sources; one in the food, existing, as it does, in muscular tissue, milk, etc., and the other in a chemical reaction between the phosphate of potassa and the chloride of sodium, forming the chloride of potassium and

${ }^{2}$ Robin and Verdeil, op. cit., tome ii., p. 205. 
the phosphate of soda. That this decomposition takes place in the body, is evident from the fact that the ingestion of a considerable quantity of common salt has been found, in the sheep, to increase the quantity of chloride of potassium in the urine, without having any influence on the amount of chloride of sodium. The chloride of potassium is discharged from the body in the urine and mucus.

\section{Phosphate of Lime, $3 \mathrm{CaO}, \mathrm{PO}_{5}$.}

Phosphate of Lime is found in all the solids and fluids of the body. As it is always united, in the solids, with organic substances as an important element of constitution, it is hardly second in importance to water. It differs in its functions so essentially from the chlorides of sodium and potassium, that they are hardly to be compared. It is insoluble in water, but held in solution in the fluids of the body by virtue of free carbonic acid, the bicarbonates, and the chloride of sodium. In the solids and semi-solids, the condition of its existence is the same as that of water; i.e. it is incorporated, particle to particle, with the organic substance characteristic of the tissue, and is one of its essential elements of composition. Nothing need be added here as to this mode of union in the body of organic and inorganic substances, after what has been said under the head of water.

The following table ${ }^{1}$ gives the relative quantity of phosphate of lime in various situations:

Table of Quantity of Phosphate of Lime.

Parts per 1,000 .

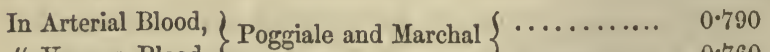

"Venous Blood, $\}$ Poggiale and Marchal $\left\{\begin{array}{cc}\ldots \ldots \ldots \ldots & 0 \\ \ldots \ldots \ldots \ldots & 0.760\end{array}\right.$

" Milk, Human (Pfaff and Schwartz)............. 2.500

" Saliva (Wright).................... 0.600

1 Selections from the table of Robin and Verdeil, op. cit. 
Parts per 1,000 .

In Urine (proportion to weight of ash, Fleitmann). . . . 25.700

" Excrements (Berzelius).................... 40.000

"Bone (Lassaigne)...................... 400.

"Vertebra of a rachitic patient (Bostock)......... 136.

"Teeth of Infant one day old. $) \quad\left(\ldots \ldots \ldots . .510^{\circ}\right.$

"Teeth of Adult.........

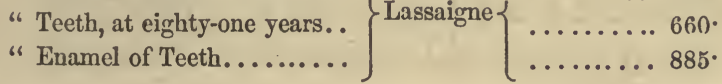

By this table it is seen that the phosphate of lime exists in very small quantity in the fluids, but is abundant in the solids. In the latter the quantity is in proportion to the hardness of the structure, the quantity in enamel being, for example, more than twice that in bone. The rariations in quantity with age are very considerable. In the teeth of an infant one day old, Lassaigne found 510 parts per 1,000 ; in the teeth of an adult, 610 parts; and in the teeth of an old man of eighty-one years, 660 parts. This increase in the calcareous elements of the bones, teeth, etc., in old age is very marked; and in extreme old age they are deposited in considerable quantity in situations where there existed but a small proportion in adult life. The system seems to gradually lose the property of appropriating to itself organic matters ; and though articles of food are digested as well as ever, the power of assimilation by the tissues is diminished. The bones become brittle, and fractures, therefore, are common at this period of life, when dislocations are almost unknown. Inasmuch as the real efficiency of organs depends on organic matters, the system actually wears out, and this progressive change finally unfits the various parts for the performance of their functions. An individual, if he escapes accidents and dies, as we term it, of old age, passes away thus by a simple wearing out of his organism.

Function of Phosphate of Lime.-This substance, as before remarked, enters largely into the constitution of the solids of the body. In the bones its function is most appa- 
rent. Its existence, in suitable proportion, is necessary to the mechanical office of these parts, giving them their power of resistance, without rendering them too brittle. It is more abundant in the bones of the lower extremities, which have to sustain the weight of the body, than in those of the upper extremities; and in the ribs, which are elastic rather than resisting, it exists in less quantity than in the bones of the arm.

The necessity of a proper proportion of phosphate of lime in the bones is made evident by cases of disease. In rachitis, where, as is seen by the table, its quantity is very much diminished, the bones are unable to sustain the weight of the body, and become deformed. Finally, when the phosphate of lime is deposited, they retain their distorted shape. The phosphate of lime may be extracted from the bones by maceration in dilute hydrochloric acid, which dissolves it, leaving only the organic substance. Bones treated in this way: though they retain their form, become very pliable; and a long slender one, like the fibula, may be actually tied into a knot.

Origin and Discharge of Phosphate of Lime.-The origin of this principle is exclusively from the external world. It enters into the constitution of our food, and is discharged with the feces, urine, and other matters thrown off by the body. Its quantity in the urine is exceedingly variable. Lecanu found from 0.43 个 to $29 \cdot 250$ grains thrown off by the kidneys during the twenty-four hours. ${ }^{1}$

\section{Carbonate of Lime, $\mathrm{CaO}, \mathrm{CO}_{2}$.}

Carbonate of lime exists in the Bones, Teeth, Cartilage, Internal Ear, Blood, Sebaceous Matter, and sometimes in the Urine. It exists as a normal constituent in the urine of some herbivora, but not in the carnivora, nor in man. It is most

${ }^{1}$ Lemmann, Physiological Chemistry, American Edition, vol. ii., p. 161. 
appropriately considered immediately after the phosphate of lime, because it is the salt next in importance in the constitution of the bones and teeth. In these structures it exists intimately combined with the organic matter, under the same conditions as the phosphates, and has analogous functions. In the fluids it exists in small quantity, and is held in solution by virtue of free carbonic acid and the chloride of potassium.

The carbonate of lime is the only example of an inorganic proximate principle existing uncombined, and in a crystalline form, in the body. In the internal ear it is found in this form, and has a function connected with audition.

According to Robin and Verdeil, it is possible that in chemical analyses a certain quantity may come from a decomposition by calcination of those salts of lime which contain a combustible acid. ${ }^{2}$. These authors give a table of the quantity of this substance in various of the solids and fluids of the body, fiom which we make the following selections :

\section{Table of Quantity of Carbonate of Lime.}

Parts per 1,000 .

In Bone, Human (Berzelius). . . . . . . . . . 113.00

" " " (Marchand)................. 102.00

" " " (Lassaigne)................. 76.00

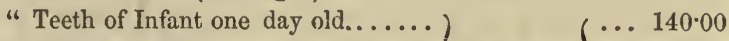

"Teeth of Adult............. $\}$ Lassaigne $\{\ldots 100 \cdot 00$

"Teeth of Old Man, eighty-one years $\}$... 10.00

" Urine of Horse (Boussingault)............ 10.82

Origin and Discharge of Carbonate of Lime.-Carbonate of lime is introduced into the body with our food, held in solution in water by the carbonic acid, which is always present in small quantity. It is also formed in the body, particularly in the herbivora, by a decomposition of the tartrates, ma- 
lates, citrates, and acetates of lime contained in the food. These salts, meeting with carbonic acid, are decomposed, and the carbonate of lime is formed. It is probable that in the human subject some of it is changed into the phosphate of lime, and in this form is discharged in the urine; but when and how this change takes place has not been definitely ascertained.

$$
\text { Carbonate of Soda, } \mathrm{NaO}, \mathrm{CO}_{2}+10 \mathrm{HO} \text {. }
$$

Carbonate of soda is found in the blood and saliva, giving to these fluids their alkalinity ; in the urine of the human subject, when it is alkaline without being ammoniacal; in the urine of the herbivora; in the lymph, cephalo-rachidian fluid, and bone. The analyses of chemists with regard to this substance are very contradictory, on account of its formation during the process of incineration; but there is no doubt that it is found in the above situations. The following table gives the quantities which have been found in some of the fluids and solids:

\section{Table of Quantity of Carbonate of Soda.}

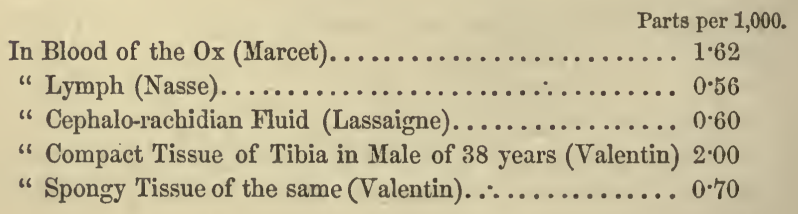

Function of Carbonate of Soda.-This substance has a tendency to maintain the fluidity of the fibrin and albumen of the blood, and assists in preserving the form and consistence of the blood corpuscles. Its function with regard to nutrition is rather accessory, like that of chloride of sodium, than essential, like the phosphate of lime in the constitution of certain structures. 
Origin and Discharge of Carbonate of Soda.-This substance is not introduced into the body as carbonate of soda, but is formed, as is the carbonate of lime in part, by a docomposition of the malates, tartrates, etc., which exist in fruits. It is discharged occasionally in the urine of the human subject, and a great part of it is decomposed in the lungs by the action of pneumic acid, setting free carbonic acid, which is discharged in the expired air.

\section{Carbonate of Potassa, $\mathrm{KO}, \mathrm{CO}_{2}$.}

This salt exists particularly in herbivorous animals. It is found in the human subject when subjected to a vegetable diet. Under the heads of function, origin, and discharge, what has been said with regard to the carbonate of soda will apply to the carbonate of potash.

\section{Carbonate of Magnesia, $\mathrm{MgO}, \mathrm{CO}_{2} \mathrm{HO}$, and Bicarbonate of Soda, $\mathrm{NaO}, \mathrm{CO}_{2}+\mathrm{HO}, \mathrm{CO}_{2}{ }^{\cdot}$}

It is most convenient to take up these two salts in connection with the other carbonates, though they are put at the end of the list of inorganic substances, as the least important. We know very little about them, chemically or physiologically. Traces of carbonate of magnesia have been found in the blood of man, and it exists normally in considerable quantity in the urine of herbivora. In the human subject it is discharged in the sebaceous matter.

Liebig has merely indicated the presence of bicarbonate of soda in the blood.

Phosphate of Magnesia, $3 \mathrm{MgO}, \mathrm{PO}_{5}+7 \mathrm{HO}$; Phosphate of Soda (neutral), $3 \mathrm{NaO}, \mathrm{PO}_{5}$; and Phosphate of Potassa, $2 \mathrm{KO}, \mathrm{PO}_{6}$.

${ }^{1}$ Formula of Graham, op. cit., p. 389. 
These salts are found in all the fluids and solids of the body, though not existing in a very large proportion, compared with the phosphate of lime, which we have already considered. In their relations to organized structures, they are analogous to the phosphate of lime; entering into the composition of the tissues, and existing there in a state of intimate combination. They are all taken into the body with food, especially by the carnivora, in the fluids of which they are found in much greater abundance than the carbonates; which latter, as we have already seen, are in great part the result of the decomposition by carbonic acid of the malates, tartrates, oxalates, etc.

With respect to their functions, we can only say that, with the phosphate of lime, they go to form, and are necessary constituents of, the organized structures.

They are discharged from the body in the urine and feees.

Sulphate of Soda, $\mathrm{NaO}, \mathrm{SO}_{3}+10 \mathrm{HO}$; Sulphate of Potassa, $\mathrm{KO}, \mathrm{SO}_{3}$; Sulphate of Lime, $\mathrm{CaO}, \mathrm{SO}_{3}+2 \mathrm{HO}$.

The sulphate of soda and the sulphate of potassa are identical in their situation, and apparently in their functions. They are found in all the fluids and solids of the body, excepting milk, bile, and gastric juice. Their origin in the body is from the food, in which they are contained in small quantity, and they are discharged in the trine. Their chief function appears to be in the blood, where they tend to preserve the fluidity of the fibrin and albumen, and the form and consistence of the blood corpuscles.

The sulphate of lime is found in the blood and feces. It is introduced into the body in solution in the water which is used as drink, and is discharged in the feces.

Its function is not understood, and is probably not very important. 


\section{Hydrochlorate of Ammonia, $\mathrm{NH}_{3}, \mathrm{HCl}$.}

This substance has simply been indicated by chemists' as existing in the gastric juice of ruminants, the saliva, tears, and urine. Some chemists make a rearrangement of its particles, calling it chloride of ammonium, when instead of $\mathrm{NH}_{3}, \mathrm{HCl}$, it would be $\mathrm{NH}_{4} \mathrm{Cl}$; but as the ammonium is hypothetical, the name we have used seems more appropriate.

It is discharged in the urine, in which it exists, according to Simon, ${ }^{1}$ in the proportion of $0 \cdot 41$ parts per 1,000 . Its origin and function are unknown.

Summary.-A review of the functions of the individual inorganic constituents of the body, excluding the gases, will show that they may be divided into two groups: one, which is composed of those substances, existing particularly in the solids and semi-solids, which are in a condition of molecular union with organic substances, merge their identity, as it were, into them, and become necessary constituents of the tissues; and the other, composed of substances which rather regulate, by their influence in endosmosis, or otherwise, the nutritive processes, do not seem to be indispensable constituents of the tissues, but have rather an accessory office to perform in the function of nutrition.

At the head of the first group we may place water; the absence of which involves destruction of the properties of the tissues, and even of the organic elements.

At the head of the second group we may place common salt; which is absolutely necessary to the functions of nutrition, though it does not seem to form an indispensable element of the tissues.

The first group, as we should naturally expect, forms a considerable proportion of the body, and the articles composing it are discharged in small quantity; as in the case of

1 Sruon, Animal Chemistry, with Reference to the Physiology and Pathology of Man, Philadelphia, 1846, p. 403. 
water, which composes two-thirds of the entire organism, aud yet only about four and a half pounds are discharged daily from the skin and lungs, and in the urine and feces.

The second group enters and is discharged from the body in considerable quantity, and very little remains in the organism; as common salt, which exists in the urine in a greater proportion than in any of the solids or other fluids.

The following are the organic substances which are apparently indispensable to the constitution of organized tissues :

Water.

Basic Phosphate of Lime.

Carbonate of Lime.

Phosphate of Magnesia.

" "Soda.

" "Potassa.

The following are those which appear to have an accessory office in nutrition :

Chloride of Sodium.
"6 Potassium.

Carbonate of Soda.

Bicarbonate of Soda.

Carbonate of Potassa.

" "Magnesia.

Sulphate of Soda.

" "Potassa.

The remaining two principles, sulphate of lime and hydrochlorate of ammonia, are so obscure in their function that they cannot be definitely put in either of the above groups.

ORGANIC NON-NITROGENIZED PRINCIPLES.

\section{(Hydro-Carbons.)}

The principles of this elass differ widely from inorganic substances. In the first place, they have a different origin, 
being formed exclusively in animal or vegetable bodies. They are of definite chemical composition, and crystallizable. The most important groups of this class, $i . e$. the sugars and fats, are composed of carbon, hydrogen, and oxygen, whence they are sometimes called Hydro-Carbons. They are distinguished from another class of organic substances by the fact that they do not contain nitrogen; which has given them the name of Non-nitrogenized Principles. They are in part introduced into the body as food, and in part formed in the economy by special organs. In the former instance, they undergo an elaborate preparation by digestion before they become part of the organism, differing in this respect from the inorganic principles, which are absorbed unchanged With the exception of butter and the sugar of milk, they are never discharged from the body in health, but disappear in the processes of nutrition. In this respect, also, they differ from the inorganic principles, all of which are discharged from the body, most of them in the condition in which they entered.

The most important principles of this class may be divided into two great groups, the Sugars and the Fats; in addition to which we have, lactic acid and the lactates, pneumic acid, pneumate of soda, the fatty acids and their combinations, and certain organic salts which are found in the bile.

\section{Sugars.}

The varieties of sugar with which we are most familiar, of which cane sugar may be taken as the type, are not found in the animal body, but belong to the vegetable kingdom. These, which form an important element of food, must be modified by digestion before they become proximate principles. For a long time it was supposed that sugar was an exclusively vegetable production and consumed by animals; but late experiments, especially those of Bernard, have shown that sugar is constantly produced by animals, presenting, in this instance, marked differences from 
the vegetable varieties. Vegetable sugar taken as food is changed so as to resemble animal sugar, before it is absorbed. In considering, then, the proximate principles of the body, we have only to do with the animal sugars.

There are two varieties of sugar manufactured in the economy. The first is constantly formed by the liver, and is found in this organ and the blood which circulates between it and the lungs. This variety is called Liver Sugar ; and, as it appears in the urine of diabetics, is sometimes known under the name of diabetic sugar. The second variety is only present in the organism during lactation. It exists in the milk, and is called Milk Sugar. We have also sugar resulting from the transformation by digestion of cane sugar and starch, which is called Glucose. This resembles the liver sugar very closely, and is, indeed, identical with it in composition, but differs from it in the fact that it ferments less easily.

The presence of sugar in the economy seems to be a necessity of existence. It, or starch which is readily converted into glucose, constitutes an important and necessary element of food. In early life large quantities are taken in with the milk. This, however, does not seem to be sufficient to supply the wants of the system, and we have it continually manufactured in the interior of the body ; but once formed, or introduced from without, it undergoes some transformation in nutrition, and is never discharged in health. Sugar is exceedingly soluble, and in the economy, exists in solution in the blood. Here it forms a union with the chloride of sodium, which masks, to a certain extent, some of its characteristic properties, such as the peculiar taste by which it is so readily recognized.

Composition and Properties. - The sugars are composed of carbon, hydrogen, and oxygen ; and it is noticeable that the hydrogen and oxygen always exist in equal proportions, or in the proportions which form water; a peculiarity affording an explanation of the transformation of one variety of sugar 
into another, which is effected in some instances with great facility.

Simon ${ }^{1}$ gives the following composition of the animal sugars in a crystalline form:

Liver Sugar and Grlucose, $\mathrm{C}_{12} \mathrm{H}_{14} \mathrm{O}_{14}$. Mith Sugar, $\mathrm{C}_{12} \mathrm{H}_{12} \mathrm{O}_{12}$.

On exposing either of these varieties of sugar to a dry heat, two atoms of water of crystallization are driven off, leaving the formula for liver 'sugar, $\mathrm{C}_{12} \mathrm{H}_{12} \mathrm{O}_{12}$, and for milk sugar, $\mathrm{C}_{12} \mathrm{H}_{10} \mathrm{O}_{10}$. From the relative composition of these varieties of sugar, it is seen that the addition of two atoms of hydrogen and oxygen, or water, to milk sugar, will transform it into glucose. This change actually takes place in digestion. The digestive fluids act also upon cane sugar $\left(\mathrm{C}_{12} \mathrm{H}_{11} \mathrm{O}_{11}\right)$ and starch $\left(\mathrm{C}_{12} \mathrm{H}_{10} \mathrm{O}_{10}\right)$, transforming them into glucose.

Animal sugars are distinguished from cane sugar by their different behavior in the presence of acids and alkalis. Cane sugar is converted into the animal variety by boiling for a few seconds with a dilute mineral acid, and is unaffected by boiling with an alkali; while the animal sugars are not affected by acids, and are transformed into a dark-brown substance, melassic acid, by boiling with an alkali.

If a solution of sugar be mixed with a little fresh yeast and kept for a few hours at a temperature of from $80^{\circ}$ to $100^{\circ}$ Fahr., a peculiar change takes place which is called fermentation. The sugar is decomposed into carbonic acid gas, which rises to the top in bubbles, and alcohol, which remains in the liquid. Some ferments, especially organic matters in process of decomposition, when they exist in a saccharine solution, have the property of inducing a change of the sugar into lactic acid $\left(\mathrm{C}_{6} \mathrm{H}_{6} \mathrm{O}_{6}\right)$, giving rise to what is called the lactic-acid fermentation. This process is peculiarly interest- 
ing in a physiological point of view, from the fact that much of the sugar which disappears in the economy is now thought to undergo this change.

A clear solution of sugar has a peculiar effect upon polarized light, being possessed of what is called a rotatory power. If a ray of polarized light be passed through a tube contain- ing simple water, its direction is unchanged; but if a saccharine solution be substituted, it is found to possess what is called molecular activity, and turns the ray to the right. The amount of deviation, which can easily be measured by an instrument constructed for this purpose by Biot and Soleil, called a polarimeter, indicates the quantity of sugar in the solution. The instrument above mentioned is sometimes used for quantitative analysis.

Tests for Sugar.-Reliable tests for determining the presence of sugar in the animal tissues and fluids are useful to the practical physician as well as the physiologist; for this substance frequently occurs in the urine, as a pathological condition, when it is essential to ascertain the fact of its presence, even if no attempt be made to determine its quantity. For this purpose a number of tests have been devised, which are most of them reliable and simple of application.

Moore's, or the Potash Test.-This test depends on the conversion of the animal sugars into melassic acid by boiling with a caustic alkali. It is employed in the following way: 'To a small portion of the suspected liquid in a test tube we add a little caustic potash in solution, and boil the mixture. If sugar be present, a brownish color will be produced, its intensity depending upon the quantity of sugar. This test is applicable only to glucose, grape sugar, and the animal varieties.

Trommer's Test.-This is one of the most delicate and convenient tests for sugar. It is employed in the following 
way: To the suspected liquid in a test tube, we add one or two drops of a moderately strong solution of sulphate of copper, and render the mixture distinctly alkaline by the addition of caustic potash in solution. On the addition of the alkali the mixture will assume a distinctly blue color, especially marked if sugar be present. On the application of heat, if sugar be present, a little before the liquid reaches the boiling point, a yellowish or reddish precipitate will begin to show itself in the upper part of the test tube, which as the heat continues will gradually extend through the whole of the liquid. If no sugar be present, the liquid will retain its clear blue color, unless the boiling be prolonged, when a black precipitate of the black oxide of copper is likely to appear. In this test, before the heat is applied, the copper is in the form of the sulphate of a protoxide $\left(\mathrm{CuO}, \mathrm{SO}_{3}\right)$, which is soluble; but on boiling in an alkaline solution, the sugar becomes oxidized, is transformed into an acid, the nature of which is not well determined, and the copper, losing an equivalent of oxygen, becomes reduced to the condition of a sub-oxide $\left(\mathrm{Cu}_{2} \mathrm{O}\right)$, which has a reddish or yellowish color, and is insoluble. This is the way in which the test is generally employed. Trommer recommended (1841), with special reference to examination of urine, to first add the solution of potash, then filter, and then add the solution of copper. If sugar be present, a reduction of the sub-oxide will take place spontaneously in a few hours, or may be produced immediately by boiling. This removes certain sources of obscurity in examining the urine, which result from a precipitate produced by the simple action of the potash, and not dependent on the presence of sugar.

If care be taken to employ the following simple precautions in the application of this test, it will be found the most reliable and simple of any that are in use for qualitative analysis.

The solution to be examined must be clear. A clear extract of the blood, muscles, or liver, is easily made in the 
following way: The blood, or tissue, finely divided, is boiled with a little water and sulphate of soda. In a few moments the organic and coloring matters will become coagulated, when it is to be thrown on a filter, and a clear extract will pass through. This extract will contain sulphate of soda, which is very soluble in hot water, but this does not interfere with the application of the test. The same result may be obtained by boiling with animal charcoal, enough being added to make a thin paste, and filtering; a process, however, which is more tedious and has no advantages over the one just described.

In testing the urine, a light flocculent precipitate will generally be obtained, though no sugar be present. With a little experience this may be distinguished from the deposit of sub-oxide of copper, by the fact that it is less highly colored, and appears in flakes after it finally settles to the bottom of the test tube, of a light grayish color; while the sub-oxide of copper settles to the bottom in the form of a heavy red powder. If there be any doubt as to the nature of the reaction, the urine may be purified in rarious ways before testing. A very simple, and perhaps the best method, is to make a paste with animal charcoal and filter. Robin recommends the following process: "To be certain of the presence of glucose, we free it (the liquid) from all reducing matters; 1st, adding to the urine an excess of the neutral acetate of lead, then filtering; $2 d$, adding to this clear filtered liquid, ammonia, until it is slightly alkaline, and filtering. We can then treat the second liquid with the reagents; and if it precipitates, it is certain that there is sugar in the urine." Another method is to evaporate the urine to the consistence of a syrup, extract this with alcohol, drive off the alcohol by evaporation, and dissolve the residue in water; when if sugar be present it will respond to the test.

${ }^{1}$ Dictionnaire de Médecine, etc., de P. H. Nysten, par E. Littré et Cn. Robin, Paris, 1858. " "Sucre." 
It is a curious fact that sugar added to healthy urine, even in large quantity, will not respond to Trommer's test, on account of organic matters, which interfere with the reduction of the copper. The cause of this interference we do not understand; but in diabetes, the organic substances, whatever they may be, are not present, or at least do not interfere with the application of tests for sugar.

Another precaution to be adopted is to add a small quantity, two or three drops only, of the solution of sulphate of copper, especially if we suspect the sugar to be present in small quantity; for if too much be added, a portion only of the oxide of copper will be reduced, and that which remains, by its blue color, may obscure the reaction.

Barreswill's Test.-For those engaged in physiological investigations, when it is desired to roughly estimate the quantity of sugar in any clear extract, and when the test is to be employed very frequently, Barreswill's solution is convenient. This is simply a solution of tartrate of copper and caustic potash. The reaction with this fluid is precisely the same as in Trommer's test. It has seemed to me, if there be any difference, that the reduction takes place more promptly with the sulphate of copper, but that the tartrate will detect a smaller quantity of sugar. The advantage of Barreswill's test is, that but a single fluid is to be added to the suspected solution. The only disadvantage is, that the solution is liable to alteration if kept more than a few days or weeks. After standing for a certain time, a yellowish sediment is deposited, and the fluid will no longer reduce in the presence of sugar. Its properties may be renewed by adding a little potash and filtering; but in delicate observations, it is always better to use a solution which has not undergone alteration.

In employing this test, we add to the suspected fluid enough of the solution to give the whole a distinctly blue color, and boil ; if sugar be present, we have a reduction of the yellowish sub-oxide of copper as in Trommer's test. 
The solution may be prepared according to the following formula, reduced to grains from the formula given by Bernard : ${ }^{1}$

Of bitartrate of potash, 3 vi. gr. xxiij.

Of crystallized carbonate of soda, $3 \mathrm{v}$. gr. ix.

Dissolve in 3 vss. of water; add to the solution 3 iij. gr. li. of sulphate of eopper, and boil; allow the mixture to cool and add $3 \mathrm{v}$. gr. ix. of potash dissolved in $\xi i v$. of water. Add water till the whole measures $\overline{3}$ xvii.

Maumenés Test.-Böttger's Test.-The first of these tests is employed by saturating strips of some woollen tissue, . such as flannel, with a strong solution of bichloride of tin, and drying. One of these strips is moistened with the suspected liquid, and dried quickly by the heat of a fire or lamp. If sugar be present, the strips will assume a brownish or black tint.

Böttger's test depends upon the reduction of a salt of bismuth, analogous to the reduction of the copper in Trommer's test. It is employed in the following way: We add to the suspected liquid a few drops of a weak solution of the nitrate of bismuth in nitric acid, render the whole alkaline by the addition of a solution of carbonate of soda, and boil for three or four minutes. If sugar be present, the bismuth will be reduced, and form a dark precipitate. Neither of these tests presents any advantage over Trommer's test, which is the one most generally employed.

Fermentation Test.-With the exception of actual extraction, this is the most certain test for sugar, and should always be employed when the other tests leave any doubt with regard to its presence. It depends on a property of sugar whereby it is decomposed into alcohol and carbonic acid in the presence of certain ferments, at a moderately elevated temperature. The test is applicable to all varieties of

${ }^{1}$ Bernard, Legons de Physiologie Expérimentale, Paris, 1855, p. 34. 
SUGARS.

sugar; but it must be remembered that milk sugar ferments slowly and with difficulty. In its application, all that is necessary is to add a few drops of fresh yeast, and keep the suspected liquid for a few hours at a temperature of frow $80^{\circ}$ to $100^{\circ}$ Fahr. The mixture should be placed in some apparatus by which the gas which forms may be collected and analyzed. To effect this, we may fill a large test tube and invert it in a small shallow vessel ; or if there be but a small quantity of liquid, we may use a very simple and convenient apparatus described by Bernard. This is simply a large test tube fitted with a good cork, perforated to allow the passage of a small tube which extends to the bottom. This tube may be turned up at the lower end, and bent above so as to permit the escape of the liquid as the gas is formed. The whole is completely filled with the suspected solution, to which have been added a few drops of fresh yeast, and kept at a temperature of $80^{\circ}$ to $100^{\circ} \mathrm{Fahr}$. If sugar be present, bubbles of gas will soon begin to appear, which will collect at the top and force a portion of the liquid out by the small tube. If no gas has appeared at the end of four or six hours, it is certain that no sugar is present. This test is conclusive, if proper care be taken in its application; and to insure accuracy, it is well to test the yeast with a saccharine solution to demonstrate its activity, and test it also with pure water, to be sure that it contains no sugar. We may then demonstrate that the gas produced is carbonic acid by removing the cork and inserting a lighted taper, which will be immediately extinguished, or passing it into another vessel and agitating with line-water, which will be rendered milky by the formation of the insoluble carbonate of lime. The alcohol remains in the liquid, from which it may be separated by careful distillation.

Measures for demonstrating the composition of the gas and the presence of alcohol in the liquid are by no means necessary in the ordinary application of the test. The distinct formation of gas in the liquid is generally sufficient evidence of the presence of sugar. 
Tomula.-Another test of the presence of sugar is the growth of the Torulce cerevisice. After diabetic urine has stood for some time at a moderate temperature, a delicate scum will form upon the surface, which, on microscopic examination, will be found to consist of a vegetable growth, presenting a number of oval joints irregularly connected. These are called Tomulce. After a time they break up and fall to the bottom of the ressel, as minute oval spores. This appearance is observed even when a small quantity of sugar is present.

Various modes of procedure have been described for the determination of the quantities of sugar. In general terms it may be stated that the copiousness of the precipitate in Trommer's test, and the amount of gas evolved in the fermentation test, give some idea of the quantity of sugar present. For directions for accurate quantitative analysis the reader is referred to works on organic chemistry.

Origin and Functions of Sugar.-Sugar is an important element of food at all periods of life. In the young child it is introduced in considerable quantity with the milk. In the adult it is introduced partly in the form of cane sugar, but mostly in the form of starch, which is converted into sugar in the process of digestion. With the exception of milk sugar, which is present only during lactation, all the sugar in the body exists in a form resembling glucose, into which milk sugar, cane sugar, and starch are all converted, either before they are absorbed, or as they pass through the liver. In addition to these external sources of sugar, it is continually manufactured in the economy by the liver, whence it is taken up by the blood passing through this organ. It disappears from the blood in its passage through the lungs. Sugar is found then in the economy constantly, in the substance of the liver, in the blood coming from the liver, and in the blood of the right side of the heart; and after the ingestion of saccharine or anylaceous 
articles of food, in the blood of the portal vein. It is not found in other organs, nor does it normally exist in the arterial blood.

During the first three or four months of fœtal life sugar is formed by the placenta, and exists in all the fiuids of the fotus, in greater quantity even than after birth. At the third or fourth month the liver begins to take on this function, which is gradually lost by the placenta. The constant production of this principle in the economy, even in the early months of foetal life, is significant of the importance of its function.

The function of sugar and its mode of disappearance in the economy are not yet well understood. Its early formation in large quantity, when the processes of nutrition are most active, seems to point to an important office in the performance of this general function. Its presence is undoubtedly necessary at all periods of life; for its formation never ceases in health. Bernard has attempted to show that its presence in the animal fluids favors cell development, but has hardly succeeded in establishing this fully."

It has been claimed that the sugars and fats are for the purpose of keeping up the animal temperature, and are oxidized or undergo combustion in the lungs. This view was afterwards modified by Liebig and others, who supposed that the oxidation takes place in the general system. This theory will be discussed more fully in the chapter on animal heat. Here we can only say that, while there are many circumstances which, taken by themselves, might lead to such a conclusion, the production of heat in the body is closely connected with the general process of nutrition, of which the disappearance of oxygen and formation of carbonic acid are but a single one of many important changes. We have not yet sufficient ground for the supposition that the substances under consideration are directly and exclusively acted upon by oxy-

${ }^{1}$ Bervard, Leģons de Physiologie Expérimentale, Paris, 1855, p. 247 et seq. 
gen in the organism. The term calorific elements, which is sometimes applied to them, cannot therefore be accepted. When we endeavor to substitute for this theory a definite explanation of the uses of sugar in the economy, we find ourselves at a loss ; but it must be remembered that we are yet far from having a complete knowledge of the functions of the body, particularly those connected with the intimate processes of nutrition.

In the present state of science, we are only justified in saying that sugar is important in the process of development and nutrition, at all periods of life. The precise way in which it influences these processes is not fully understood.

Sugar disappears from the blood in its passage through the lungs, in great part, probably, by conversion into lactic acid. This change has been demonstrated in the blood of a diabetic patient; all the sugar contained in the blood being thus changed in less than twenty minutes. ${ }^{1}$

Sugar is never discharged from the body in health, with the single exception of the sugar of milk in the female during lactation. Under certain diseased conditions of the system its production by the liver is exaggerated, so that a certain quantity passes through the lungs, exists in the arterial blood, and appears in the urine, constituting the very serious affection called diabetes mellitus.

\section{Fats.}

Fatty or oily matters exist in both the animal and regetable kingdoms. Those which are most interesting to us as physiologists are the varieties found in animals, which constitute an important group of proximate principles. Both vegetable and animal fats are important elements of food.

In the animal economy fat exists in three varieties, which are called, respectively, Oleine, Murgarine, and Stearine. In certain situations are found some of the fatty acids and

${ }^{1}$ Robin and Verdert, Chimie Anatomique, tome ii., p. 553. 
their combinations, but they exist in minute quantity, and their function is comparatively unimportant.

Composition and Properties.-In their ultimate composition, fats bear a certain resemblance to the sugars. Like them they are composed of carbon, hydrogen, and oxygen; but the two latter elements do not exist, as in sugar, in the proportions to form water. From this difference we should be led to suspect, what is really the fact, that the different varieties of fat are not mutually convertible.

The fat which exists in the body is a mixture of the three varieties above mentioned, and is found in the ordinary adipose tissue, and in the substance of certain tissues in the form of minute globules or granules. It is not found in any great quantity in the blood, except after digestion of a full meal. It exists in the chyle in a state of extremely minute subdivision and suspension. It exists in the milk, also in a state of minute subdivision, but presenting some slight differences from the ordinary fatty matter of the economy.

Robin and Verdeil give, as the ultimate composition of Stearine, $\mathrm{C}_{71} \mathrm{H}_{70} \mathrm{O}_{8}$. The other varieties are separated from their union with each other with great difficulty, and have not yet been obtained in a state of sufficient purity for ultimate analysis. The reaction of all the varieties of fat is neutral.

Fat, in greater or less quantity, is found in all the tissues of the body, with the exception of the substance of the bones, the teeth, and the elastic and inelastic fibrons tissue. It always consists of a mixture of the three varieties in varying proportions, but, with one or two exceptions, is never combined with any other of the proximate principles. In the adipose tissue proper, it is enclosed in little cells which are called the adipose vesicles. In all other situations it is in the form of microscopic globules or granules. As it is thas distinct from other elements, it may be always recognized in the organism by the naked eye or the microscope. In the ner- 
vous matter there exists a phosphorized fat, the composition and properties of which are not very well understood, in union with organic matter. A minute quantity of fat exists in combination with the organic matter of the blood corpuscles.

The fats are insoluble in water and in the animal fluids, with the exception perhaps of the bile, which holds a small quantity in solution by virtue of its saponaceous constituents. They are all very soluble in ether and hot alcohol, and but slightly soluble in cold alcohol. The varieties which are solid at the temperature of the body, stearine and margarine, are easily dissolved by oleine, which is liquid.

The most marked distinction between the varieties of fat is in their consistence. Oleine is liquid at the temperature of the body, and even at the freezing point of water. Margarine is liquid at or above the temperature of $118^{\circ}$, and stearine at the temperature of $143^{\circ} \mathrm{Fahr}$. The difference in, the consistence of adipose tissue of different animals depends upon the relative proportion of the various kinds of fat.

Saponification.-When fat is boiled for a certain time with an alkali, in the presence of water, it undergoes a peculiar decomposition which is called saponification. A portion of the water is appropriated, and the fat is converted into glycerine and an acid. The acid is called oleic, margaric, or stearic acid, as it is formed from oleine, margarine, or stearine. In this process the glycerine remains uncombined, and the acid unites with the alkali to form what is commonly known as a soap.

This kind of decomposition is called saponification by a base; but technically, saponification is regarded as any process by which a fat is decomposed into its acid and glycerine. This may be effected by passing the vapor of water through fat which has been raised to a temperature of $572^{\circ} \mathrm{Faln}$. The action of the strong acids is also to decompose fat. When a small quantity of acid is used, it unites with the glycerine; 
when a large quantity is used, it unites with the fatty acid. The process of formation of glycerine and fatty acids involves the fixation of a certain quantity of water; so that the combined weights of the glycerine and acid exceed that of the fat originally employed. ${ }^{1}$ It is thought by some that this acidification of fat takes place to a certain extent in digestion; however this may be, it is not an essential part of the digestive process.

Emulsion.-When liquid fat is violently shaken up with water, it is minutely subdivided, and an opaque milky mixture is the result. But this is momentary, the two liquids separating almost immediately from each other when they are no longer agitated. There are certain fluids, however, which have the property of holding fat permanently in a state of minute subdivision and suspension, forming what is called an emulsion. Out of the body, mucilaginous fluids and white of egg have this property. In the body, we find as examples of emulsions the chyle, which is formed by the action of the pancreatic juice upon the fatty elements of food, and milk, which is composed of butter held in suspension by the water and caseine. The property of forming emulsions with certain liquids is one of the most interesting attributes of the fats, as it is in this form only that it can find its way from the alimentary canal into the general system.

Origin and Functions of Fat.-One source of fat in the economy is the food. It constitutes an important article of diet, existing in animal food in the form of adipose tissue, and mingled to a certain extent with the muscular tissue. Vegetable oil also is quite a common article of food. When introduced in the form of adipose tissue, the fat is freed from its vesicles by the action of the gastric juice, is generally

1 Regnadrt, Cours Élémentaire de Chimie, Paris, 1853, tome iv., p. 414. 
melted at the temperature of the body, and floats in the form of oil on the alimentary mass. It passes then into the small intestines unchanged, is emulsified by the pancreatic juice, and absorbed by the lacteals. A small quantity of fat is absorbed by the radicles of the portal vein. After a full meal, the blood of a carnivorous animal frequently contains enough fatty emulsion to form a thick white pelicle on cooling.

The question as to the possibility of the formation of fat in the organism may be now considered as definitely settled. It has been shown by Liebig, Boussingault, and others, that in young animals especially, the fat in the body cannot all be accounted for by that which has been taken in as food added to that which the body contained at birth. The experiments of Boussingault, ${ }^{1}$ on this point, on young pigs, are very conclusive, and demonstrate that fat must be produced somewhere in the organism. Bernard ${ }^{2}$ has shown that an emulsive substance, which he regards as fat in combination with organic nitrogenized matters, is produced by the liver, and is taken up by the blood of the hepatic vein. He believes that it is produced at the expense of the amylaceous or saccharine elements of food.

It is very certain that the generation or deposition of fat in the body may be influenced very considerably by diet, and the conditions of the system. This is daily exemplified in the inferior animals, and is true, though it is not perhaps as universal, in the human subject. It has been found that a diet consisting largely of fatty, amylaceous, and saccharine principles favors the accumulation of fat, while an exclusively nitrogenized diet is unfavorable to it, and will produce emaciation, if rigidly followed. Muscular activity, it is well known, is unfavorable to the accumulation of fat; which may account in a measure for its greater relative quantity in the female. In some individuals, especially when its ac- .

1 Boussingault, Chimie Agricole, Paris, 1854.

\& Bernard, Legons de Physiologie Expérimentale, Paris, 1855, p. 154 et seq. 
cumulation is excessive, there is an hereditary tendency to fat. Organs which are in process of atrophy from disease, or other causes, are apt to be the seat of a deposit of fatty granules; as the muscular fibres, which, in many diseases characterized by rapid emaciation, are found to be the seat of fatty degeneration.

There are certain situations where fat never exists, as in the eyelids and scrotum; and others where it is always found, even in extreme emaciation, as in the orbit and around the kidneys. Ordinarily, fat is pretty well distributed throughout the body, having a tendency to accumulate, however, beneath the skin, and in the omentum, where its presence is least likely to interfere with the function of parts, and where it serves to maintain the uniform temperature of the body, and particularly of the delicate abdominal organs.

The average relative quantity of fat in the human body has been calculated by Burdach to be five parts per hundred. In the body of a man weighing $176^{\circ}$ pounds, he found 8.8 pounds of fat.

In certain parts fat has an important mechanical function. It serves as a soft bed for delicate organs, as the eye and kidney. It is a bad conductor, and thus prevents the loss of heat by the organism. This is very important in some warm-blooded animals, as the whale, in which the loss of heat would be very great were it not for the immensely thick layer of fat just beneath the skin. It is important in filling up the interstices between the muscles, bones, vessels, \&c.

Fat, like sugar, has undoubtedly an important office in connection with the general processes of development and nutrition. We have not yet arrived at an accurate knowledge of the changes which it undergoes as it is used up by the economy; for with the single exception of butter in the milk,

${ }^{1}$ Burdach, Traité de Physiologie, Paris, 1837, tome viii., p. 80. Translated from the German by Jourdan. 
it is never discharged from the body in health. We have already alluded to the view that the sugars and fats are respiratory or calorific elements, which undergo oxidation in respiration, and are immediately concerned in the production of animal heat. One of the arguments in favor of this function of fat has been that in cold climates, where there is a greater demand for the generation of heat by the system, fat is a more common and more abundant article of diet. This is undoubtedly true, but other principles are consumed in greater quantity, and the general process of nutrition, of which the production of heat is but a single phenomenon, is intensified. There is not sufficient ground for supposing that fat has any such exclusive function. Its office is connected with the general process of nutrition; and its various transformations in connection with this function, we have as yet been unable to follow.

Fatty Acids and Soaps.-In addition to the fatty substances just described, the following fatty acids, free, and united with bases to form soaps, have been found in the blood:

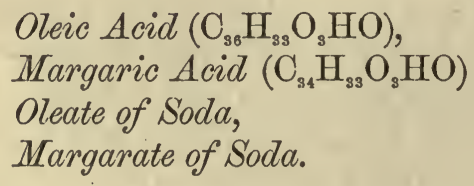

Oleic and margaric acids have been detected in minute quantities in a free state in the blood and bile. Their function is unknown. The oleate and margarate of soda are found in sinall quantity in the blood, bile, and lymph. They serve to hold in solution the small quantity of the fatty acids and fats which exists in these fluids. The function of all these substances is comparatively unimportant. In the blood of the ox, Robin and Verdeil have found a small quantity of stearic acid and the stearate of soda.

Odorous Principles.-It is well known that the perspira- 
tion of certain parts, as the axilla and sometimes the feet, has a distinct odor. This is supposed to be due to combinations of volatile fatty acids with soda and potassa. Most of the inferior animals have a distinctive odor, which may generally be readily recognized, and is always strongly developed in the blood by the addition of sulphuric acid. Barreul gives the following conclusions as the result of an extended series of observations on this subject :

" 1 . That the blood of every species of animal contains a principle peculiar to each one. 2. This principle, which is very volatile, has an odor like that of the perspiration. 3. The volatile principle is in a state of combination in the blood, and while this combination exists it is not appreciable. 4. When this combination is destroyed, the principle of the blood becomes volatile, and from that time it is not only possible, but very easy to recognize the animal to which it belongs. 5. In each species of animal the odorous principle is manifested with greater intensity in the male than in the female. 6. The combination of this odorous principle is in a state of solution in the blood which permits it to be developed either in the blood entire, in the defibrinated blood, or in the serum. 7. Of all the means employed for setting free the odorous principle of the blood, concentrated sulphuric acid is that which succeeds the best. It suffices to add onethird or one-half of the volume of blood employed, and a few drops of blood is sufficient." ${ }^{1}$

\section{Lactic Acid-Pneumic Acid-Pneumate of Soda.}

Lactic acid may be formed by what is called the lactic acid fermentation of sugar's, particularly sugar of milk. This kind of action is induced by the presence of certain organic ferments, or by organic nitrogenized matter in process of decomposition. This principle does not exist, as was at one

${ }^{2}$ Robis and Verdiel, op. cit., tome iii., p. 90. 
time supposed, in fresh milk, but only after it has become sour. Its composition $\left(\mathrm{C}_{6} \mathrm{H}_{5} \mathrm{O}_{5}+\mathrm{HO}\right)$ assimilates it to the sugars, and indicates how it may be formed theoretically from them by transposition of their atoms ; milk sugar having for its composition $\mathrm{C}_{12} \mathrm{H}_{12} \mathrm{O}_{12}$, which is also the formula for anhydrous glucose.

It is a constant constituent of the gastric juice, and is indispensable to the digestive properties of this secretion.

Lactic acid has been demonstrated by Liebig in the juice of muscular tissue.

Sources and Function.-This principle may be formed, in minute quantity, in the intestines, from the saccharine and amylaceous articles of food; but it is in greatest part produced in the economy as an element of secretion. It is thought that a great portion of the sugar which passes in the blood from the liver to the lungs is converted into lactic acid. If this be the case, it unites with bases and is almost immediately decomposed and lost. Lactates in the blood are very readily converted into carbonates, as has been shown by the experiments of Lehmann, ${ }^{2}$ who took into the stomach half an ounce of dry lactate of soda, and in thirteen minutes his urine had an alkaline reaction from the presence of carbonates. Alkalinity of the urine from this cause is often produced by the ingestion of combinations of the vegetable acids in fruits, etc.

The most marked function of lactic acid is in the gastric juice, and will be considered under the head of digestion.

Pneumic Acid and Pneumate of Soda.-Pneumic acid was discovered and extracted from the tissue of the lungs by Verdeil in 1851. ${ }^{3}$ Its ultimate composition is not given. According to this author, it exists in the lungs of the mam-

${ }^{1}$ Lermann, Physiological Chemistry, Philadelphia, 1855, vol i., p. 90.

2 Ibid, p. 97.

${ }^{8}$ Robiv and Verdeil, op. cit., tome ii., p. 466. 
malia at all periods of life. He extracted about three-foarths of a grain from the perfectly healthy lungs of a female who was guillotined. It has not been found in other situations.

Its function is connected with respiration. The carbonates and bicarbonates of the blood, in passing through the lungs, are in part decomposed by pneumic acid, a certain portion of the carbonic acid in the expired air being evolved in this way.

Pneumate of Soda is produced by the action of pneumic acid upon the carbonates of soda in the blood, and is found in the blood which passes through the lungs. It is not discharged from the body, undergoing in the system some transformation with which we are unacquainted.

\section{ORGANIC NITROGENIZED PRINCIPLES.}

Principles of this class differ essentially from all the other constituents of the body. They are the only elements endowed with what are called vital properties, and upon them depend all the phenomena which characterize living structures. This important fact cannot be too fully insisted upon.

All the vital phenomena which take place in the body depend primarily upon organic nitrogenized principles, which are the only elements in the organism endowed with life.

By a tissue or fluid endowed with life is meant:

A combination of proximate principles which has the property, under certain conditions, of appropriating materials for its nourishment and regenerating itself, to repair the continual destruction or waste to which all living bodies are subject.

This, which is the great process of Nutrition, is going on from the beginning to the end of life; its phenomena are distinct from those which take place in inert compounds, and are called vital. Take, for example, the nutritive processes which take place in the muscles or the bones. In common with all parts of the body, these tissuea are continually undergoing waste. The circumstances under 
which they can supply this waste, or regenerate themselves by the appropriation of suitable materials, involve contact with the circulating blood. They take materials from this fluid and change them into their own substance. This process takes place only in living bodies, and is unknown in the inorganic kingdom. As it is the great characteristic of life, its accomplishment being the end and object of all the functions of the organism, the study of these organic principles is manifestly of the greatest importance. We shall find that their properties are peculiar to themselves, and their chemical study must necessarily be eminently physiological. To arrive at any definite idea of their properties, the methods of study which have been generally employed by chemists must be discarded, as by these they are reduced to inorganic elements, and treated simply as combinations of inert substances. They must be studied as nearly as possible in the condition in which they exist in the body; which is necessarily the condition in which they are capable of manifesting their characteristic vital phenomena.

These principles are found in all the fluids, semi-solids, and solids of the body, except the excrementitious fluids. ${ }^{1}$ The nutritive fluids contain several. In each tissue an organic principle is found which presents certain peculiarities more or less distinctive. They are all formed in the organism, and, with the exception of the milk, a little mucus, desquamated epidermis and epithelinm, and an almost inappreciable quantity exhaled by the lungs and skin, are never discharged unchanged from the body, in health. They assume the consistence of the part in which they are found; being, therefore, fluid, semi-solid, and solid. They constitute by far the greater part of the organism; but their quantity in the whole body has never been accurately estimated. Their reaction is neutral. As a peculiarity of chemical composition, they all contain nitrogen; whence they are called

1 The excrementitious fluids contain coloring matters, which Robin and Verdeil put in this class, but which do not seem to be endowed with vital properties. 
Nitrogenized Principles. They all closely resemble one of the most important and certainly the most earefully studied of their number, namely, albumen; whence they are sometimes called Albuminoids. They were regarded by Mulder as compounds of a theoretical radical or base which he called Proteine, and after this chemist are sometimes called Proteine compounds.

Composition and Properties.-1. Studied, as they generally have been, from a purely chemical point of view, they are regarded by many as solid substances in solution in the fluids, in a condition approximating to this in the semi-solids, and of course as solid in the solids, like the bones and teeth. This view is erroneous, as we shall see that some are naturally fluid, some are semi-solid, and some are solid. In this condition they have been found to consist of Carbon, Hydrogen, Oxygen, Nitrogen, with sometimes a little Sulphur and Phosphorus. The coloring matters contain in addition a small proportion of Tron. By ultimate analysis they have been found to be of indefinite chemical composition, ${ }^{1}$ which, indeed, we would be led to expect from the state of continual change in which they exist in the body. By the method employed in arriving at their ultimate composition, even before analysis, they are completely destroyed as organic principles by desiccation, and rendered incapable of exhibiting any of their characteristic properties. The composition of their dry residue only is thus given, while in reality they all contain more or less water, which enters into their composition, and deprived of which they cannot be called organic substances. The proportion of water is to some extent variable, but confined within tolerably narrow limits. ${ }^{2}$

2. The organic principles never exist alone, but always in

1 Robin and VerderL, op. cit., tome iii., p. 147.

${ }^{2}$ For a further discussion of this important subject, see an article by the author in the American Journal of the Medical Sciences, October, 1863, On the Organic Nitrogenized Principles of the Body, with a New Method for their Estimation in the Blood. 
combination with inorganic substances, which, though perhaps not absolutely necessary to the properties by which they are recognized out of the body, are essential in the performance of their vital functions in the economy. Under these circumstances the organic and inorganic principles are so closely united, that the latter may be said to acquire, by virtue of this union, vital properties. Though unaltered, the inorganic are discharged with the worn-out organic substances, and, combined with fresh organic matter, are deposited in the tissues in the process of regeneration.

3. The organic principles which are naturally fluid may be coagulated, but under no circumstances do they assume a definite or erystalline form. We should be led to expect this from the fact that they have no absolutely fixed composition. When the liquids of this class are thus solidified, they are not precipitated from a solution, but are made to assume a new form, still retaining their water of composition. When exposed to evaporation, whether they be fluid or semisolid, their water may be driven off, and they are said to be desiccated. They can be made to assume their water of composition again by simple contact, as they have in a high degree the property of hygrometricity. Both these properties are peculiar to organic substances.

4. When exposed to a very elevated temperature, that which has been considered by chemists as the organic substance proper is volatilized and driven off; leaving the inorganic substances, which always enter into its composition.

5. In their natural condition, the organic principles have no very distinct odor; but when exposed for some time to a moderate heat, certain odorous or empyreumatic substances are produced. This change is peculiar to organic matters, and takes place in the process of cooking. When these elements are used as food, this process serves a useful purpose, rendering them more agreeable to the taste, and facilitating their digestion.

6. One of the great distinctive properties of organic prin- 
ciples, out of the body, is putrefaction. In contact with the air, at a moderate temperature, they undergo decomposition into carbonic, lactic, and butyric acids, and ammonia. When this change has once commenced, it has been found by Wurtz to continue in a vacuum. ${ }^{1} \quad$ Putrefaction is a process peculiar to organic substances. By it they are transformed into substances which are used in the nutrition of vegetables; and as vegetables are eventually consumed by animals, the animal matter is not lost, but returns again through this channel, so that the two kingdoms are continually interchanging elements. Organic matters in putrefaction are capable of setting up the same process in other articles of this class by simple contact, neither giving up nor taking away any chemical elements. They are then called ferments, and this action is said to be catalytic. As before remarked, this constitutes one of the most important characteristics of organic matters; one, indeed, which enables us to recognize them when they exist in quantities too minute for chemical analysis, as in exhalations from the pulmonary and cutaneous surfaces.

Proteine.-In 1838, just after the promulgation of the theory of regetable organic radicals by Liebig and Dumas, Mulder attempted to show that the organic animal substances were all compounds of a radical which he called Proteine. This theory was pretty generally received, and gave to organic matters the name of Proteine Compounds, by which they are sometimes known. He treated albumen, fibrin, and caseine with alcohol and ether to remove the fats, and with hydrochloric acid to remove inorganic salts; dissolved them, thus purified, in a solution of potash, and precipitated with acetic acid a substance said to possess always the same characters, which he called proteine; and which, by union with a certain quantity of sulphur and phosphorus, was capable of forming fibrin, albumen, and caseine. But the analyses

${ }^{1}$ Cited by Robin and Verdeil, op. cit., tome iii., p. 142. 
of different chemists have shown that proteine itself has an indefinite chemical composition, hardly any two formulæ being the same. It is essentially an artificial product; and with the views we have taken of the composition of organic substances, there is not the slightest reason to suppose that it plays the part of a base or radical for a group of definite compounds. It is not a distinct chemical substance, for its composition is indefinite; nor a proximate principle, for it is produced artificially and by decomposition. We must therefore reject the theory that it serves as the radical of a definite series, and discard the name of Proteine Compounds, as applied to organic principles.

Catalysis.-Catalysis, or catalytic action, is a name given to a certain process which we do not as yet understand. The word was introduced by Berzelius in 1835, and applied to certain actions or affinities brought into play in inorganic bodies by the mere presence of another substance, the latter not undergoing any chemical alteration. It is now applied to all chemical changes which are induced by the simple presence of any substance, like the particular class of substances called ferments, in which the substance inducing this action undergoes no chemical change.' Fermentation, which was considered in treating of sugar, is an example of catalysis; the sugar being decomposed into carbonic acid and alcohol from the fact of the mere presence of yeast, which has nothing to do, chemically, with the process. Putrefaction, which we have just considered, is an example of catalysis; for a small quantity of any animal substance in a state of putrefaction is capable, by its presence, of setting up the same process in other principles of this class. Nutrition, and to a certain extent digestion, are examples of catalysis; for in the repair of the system, certain materials are taken from the blood by the tissues, and by the latter changed into different substances, as musculine for the muscles, osteine for the bones, etc.; and in digestion, the organic elements which are dissolved 
are changed by the presence of certain organic substances in the digestive fluids. Any process set up by the mere presence of substances, which themselves undergo no chemical change, or the transformation of one variety of organic matter into another from the mere fact of contact, is called catalysis.

The general properties we have mentioned are possessed by all organic principles; which, indeed, differ from each other very little in their general characters, and even in ultimate composition. Those which go to form the tissues are endowed with identical vital properties. Robin and Verdeil give seventeen distinct substances belonging to this class, of which four are coloring matters. ${ }^{1}$ But three of these principles have been carefully studied with reference to their ultimate composition; but their composition, which is indefinite, and not necessary to their vital properties, is of little physiological interest. The number of equivalents of the various ultimate elements is entirely arbitrary, as these principles enter into no definite combinations.

Table of Organic Principles. Name.

Where Founci.

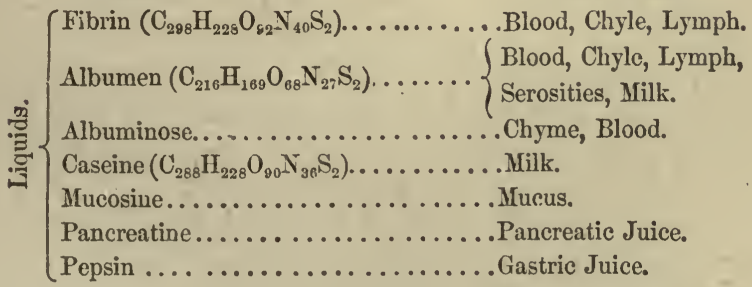

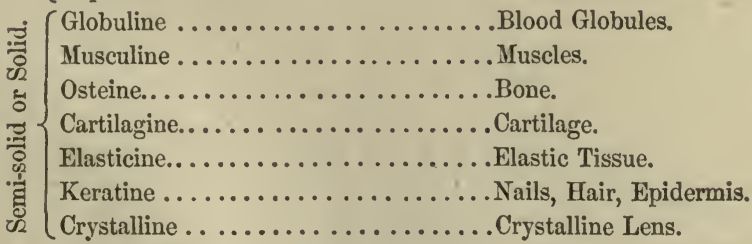

3 These authors do not consider that pepsin has been fully established as a distinct proximate principle. Its distinctive properties seem to be sufficiently woll marked, and it has therefore been included in the list. 


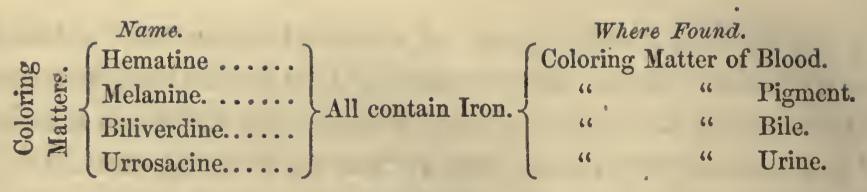

Fïbrin.

Fibrin is found in the blood, lymph, and chyle. In the first-named fluid it exists in considerable quantity, but in the last two it is much less abundant. Its quantity has been estimated by chemists in all the above-mentioned fluids, but the analyses which are generally given represent dried fibrin, and give us no definite idea of its quantity in the form in which it naturally exists. The quantity of fibrin in the blood, estimated by the author by a process in which it is not exposed to desiccation, is between 8 and 9 parts per $1000{ }^{2}$ This proportion is undoubtedly quite variable within the limits of health. According to Becquerel and Rodier, ${ }^{2}$ its quantity is considerably increased during gestation, and is greater in adults than in very young or very old persons. As a general rule, it is more abundant in arterial than in venous blood, and is often entirely absent from the blood of the hepatic and renal veins. No constant difference in quantity has been established in the sexes, and its proportion appears to bear no definite relation to the vigor of the individual.

It appears in the blood at about the fifteenth day of intrauterine life, and exists constantly from that time.

The composition of fibrin is given in the table. It contains carbon, hydrogen, oxygen, nitrogen, and a little sulphur. The proportion of these substances, however, is indefinite, and the formula, like that of all the principles of this class, is entirely arbitrary, as it enters into no definite combinations, and consequently has no combining equivalent. Its ultimate composition is comparatively unimportant, for it

${ }^{2}$ See article in $\Lambda \mathrm{m}$. Jour., loc. cit. Though the ordinary methods of analysis do not give the real quantities of fibrin, they give important results with regard to the comparative quantities in different situations.

2 Becquerer and Rodier, Traité de Chimie Pathologique, p. 101 et seq. 
gives us no indication of the properties by which it is recognized, nor of its functions; and, indeed, has been found to differ little, if at all, from the composition of musculine or albumen, the properties of which are very different.

Fibrin may be easily extracted from the fluids in which it exists. Perhaps the best mode of procedure is to whip the fluid, freshly drawn, with a bundle of twigs or broom corn. In this way the fibrin may be quickly and completely separated. It is then freed from foreign matters, such as blood-corpuscles, by washing under a stream of water, at the same time kneading with the fingers:

Fibrin is not, as is supposed by many, a solid substance in solution in the liquids in which it is found. It is naturally liquid and mingled with the watery elements. After coagulation it contains a certain proportion of water, capable, it is true, of being driven off by evaporation, but nevertheless water of composition, deprived of which it loses the properties by which we recognize it as fibrin.

Properties of Fibrin.-The striking peculiarity by which fibrin is recognized is its spontaneous coagulability. All the fluids in which it is contained, when drawn from the body or placed under abnormal conditions, become more or less coagulated, and their coagulating principle is called fibrin. It is this substance, therefore, which gives to the blood its peculiar and important property of coagulability. The condition under which fiorin coagulates seems to be that of stasis. Whenever it is drawn from the body, or in the ressels, when circulation becomes arrested, it assumes, after a variable time, a semi-solid consistence. The cause of this remarkable phenomenon was obscure until the essay of Richardson on the "Cause of the Coagulation of the Blood" appeared in 1856. By a series of carefully conducted experiments, this observer demonstrated that the blood contains a small quantity of free ammonia, which has the power of maintaining the fibrin in its liquid condition. This ammonia is being continually devel- 
oped in the system, is taken up by the circulating blood and exhaled by the lungs. When the circulation is arrested in any part, of course the blood takes up no more ammonia; and as that which it contained is gradually exhaled through the tissues, arrest of the circulation in any part for a certain time is followed by coagulation of the fibrin. When blood is drawn from the vessels, the exhalation of ammonia is rapid, and coagulation takes place very readily. Some other chemical substances, such as the carbonate of soda, have the power of maintaining the fluidity of the fibrin.

Fibrin does not coagulate into a homogeneous mass, but forms minute microscopic filaments, or fibrils, which afterwards contract for ten or twelve hours, so that the clot at the end of that time is much smaller than immediately after coagulation.

We recognize only as fibrin that liquid organic principle which coagulates whenever removed from its natural condition. By coagulation its form only is changed, not its weight, and we must consider, therefore, the water which is contained in the coagulated mass as water of composition.

Pure coagulated fibrin is a grayish-white substance, composed of microscopic fibrils, and possessing considerable strength and elasticity. It is insoluble in water and in the serum of the blood, but dissolves slowly in solutions of caustic alkalis. It swells, assumes a jelly-like consistence, and is finally partially dissolved in a very feeble mixture of hydrochloric acid and water. Iike all principles of this class, it decomposes at a moderate temperature in contact with the air and moisture.

Organization of Fibrin.--The question of the organization of accidentally effused and coagulated fibrin has occupied the attention of pathologists a great deal, and some are of opinion that it is capable of becoming part of the organized living structure. This supposition had its origin in an assumed identity between fibrin and reparative lymph, or, 
as it is sometimes called, coagulable lymph, which repairs losses of tissue. As the process of repair of parts after destruction must be considered as analogous to, and almost identical with, ordinary nutrition, the above question, which is so important in pathology, is one of great physiological interest.

The conditions under which the organization of fibrin has been assumed to have taken place, are in clots remaining after vascular extravasations, and fibrinous exudations upon inflamed surfaces. The most important information is to be derived from a study of the anatomical characters of such effusions. By the microscope, and all means of investigation which are at our command, it is impossible to distinguish in these effusions any thing but fibrin. There are no bloodvessels, nerves, nor any anatomical elements which would lead us to suppose them capable of self-regeneration, that distinctive property of all organized tissues; and, in addition, these are never developed. The changes which these effusions undergo are retrograde in their character; and the fibrin, if it be not absorbed, remains as a foreign substance. The fibrillation which takes place is by no means an evidence of even commencing organization; for in effusions into the tissues it soon disappears, and if the effusion be not too large, the mass breaks down and is finally absorbed. When, on the other hand, effusion of organizable lymph takes place, the process is very different. It is elaborated, indeed, rather than effused; first appearing as a homogeneous fluid, in which fibro-plastic nuclei, then fibres, are developed, and in some instances blood-ressels, lymphaties, and nerves. According to Robin, plastic lymph does not even contain fibrin ${ }^{1}$ much less are the two identical. The process of organization is slow and gradual, and in no case does it take place from the blood, or elements of the blood, suddenly or accidentally effused.

There can be no doubt that effused and coagulated

${ }^{1}$ Dictionnaire de Nysten, par Robin et Litrné, Paris, 1858. " "Lymph Plastique." 
fibrin is incapable of organization; and it may be further stated as a general law that no single proximate principle, nor mere mechanical mixture of proximate principles, effused into any part of the body, ever acts in any other way than as a foreign substance.

In certain instances of morbid action, effusions take place, either on the surfaces of membranes, or between two opposing surfaces, attaching them to each other by bridles or adhesions, which actually become organized. This occurs most frequently in serous membranes, and the structure thus formed is entirely different from coagulated fibrin, which has no connection with the parts, except that of contiguity. Both of these formations have been included in the term, false membranes; but Robin makes a very proper distinction between them, calling the one, which is merely coagulated fibrin, like the membrane of croup, false membranes, or pseudo-membranes; and the others membranes of new formation, or neo-membranes. The former consist simply of the fibrin, which nature has been unable to remove by absorption ; and the latter, of regularly elaborated anatomical elements, endowed with the properties of self-regeneration common to all organized structures.

Origin and Function of Fibrin.-The fibrin of the blood has its direct origin, in part at least, from the albumen, by the catalytic transformation which so often takes place in principles of this class. It has been noticed that when fibrin is increased in the blood, albumen is diminished. In some experiments presented to the Society of Biology of Paris by Dr. Brown-Séquard, it was shown that defibrinated blood injected into the arteries of a criminal just after death, on being returned by the veins, coagulated, and presented a notable quantity of fibrin. ${ }^{2}$ The remote origin of fibrin is from the organic nitrogenized elements of food; which, after having undergone the catalytic changes incident to digestion,

${ }^{1}$ Robin and Verdeil, op. cit., tome iii., p. 269. 
are absorbed and transformed into albumen. As albumen exists in the lymph and chyle, it is probable that in these fluids fibrin is produced in the same way as in the blood.

A very important office of fibrin is to give coagulability to the blood. This will be taken up more fully hereafter. At present we need only say that by virtue of this property spontaneous arrest of hemorrhage after division or rupture of small ressels is effected. In its natural liquid condition, in intimate union with albumen and certain inorganic matters which cannot be separated from it without incineration, fibrin constitutes one of the two peculiar organic principles of the plasma of the blood. It is brought in contact with the tissues in the capillary ressels, and probably takes part in the catalytic changes which constitute nutrition, being transformed into the peculiar organic element of each part. In this way it disappears forever as fibrin, and is only discharged from the body after the tissue has undergone the transformations which result in excrementitious products.

Simon, Lehmann, Bernard, and others have noticed the remarkable fact that the blood of the hepatic and renal veins generally contains no fibrin. The liver and kidneys seem to have the power of destroying this principle. Its transformations in these organs we have not been able to follow.

\section{Albumen.}

Albumen is found in the blood, lymph, chyle, intermuscular fluid, secretions of serous membranes, and in small quantity in the milk. It is most abundant in the blood, constituting the most important organic constituent of the plasma. Its proportion has heen estimated in the various situations in which it is found, but, as in the case of fibrin, this has been done after complete desiccation, and the results thus obtained are far from representing the real quantities. In some analyses designed to give the quantity of moist albumen in the blood, we have found a proportion in a healthy specimen of 329.82 parts per 1000 . The proportion will 
undoubtedly be found to vary considerably within the limits of health, and, as a rule, it bears an inverse ratio to the quantity of fibrin. No constant difference in the quantity of albumen in the sexes has been established. The quantity is greater in the well-nourished and vigorous, than in anemic and feeble subjects.

Albumen is found in the organism at all periods of life, existing even in the ovum.

In ultimate composition albumen has been found by chemists to differ very little, if at all, from fibrin. Like the other principles of this class, the proportions of its ultimate elements are indefinite.

Albumen may be extracted from the fluids in which it is contained by simple coagulation. The most convenient method of separating it is to add to the liquid a quantity of absolute alcohol, and immediately filter. In operating upon the serum, we have found that about twice its volume of alcohol will coagulate all the albumen. It may then be collected on a filter, and its weight will represent the proportion of this principle in its natural condition.

Like fibrin, albumen is naturally fluid, and in this condition-and this condition only-forms the important organic principle of the fluids in which it is contained.

Properties of Albumen.-Liquid albumen has certain properties which serve to distinguish it from other principles of the same class. In a neutral mixture it is coagulated completely by a temperature of $167^{\circ} \mathrm{Fahr}$. The same result follows the addition of the strong mineral acids, alcohol, and some of the metallic salts. It is distinguished from caseine by the fact that it is not coagulated by the vegetable acids. Coagulated albumen is a grayish-white substance, always combined with inorganic matter, which cannot be separated without incineration, insoluble in water, but soluble in a weak solution of a caustic alkali. In an alkaline solution it is no longer coagulable by heat. Becquerel has found that albumen has 
the property of deviating the plane of polarization to the left. He has employed a polarizing apparatus like the one used by Biot in the examination for sugar, for the purpose of estimating the quantity of albumen in a watery mixture, and found that "each minute of deviation corresponds to 18 decigrammes (29.77 grains) of dried albumen in 1,000 cubic centimetres ( 1.76 pints) of water." 1 This instrument he calls an albuminimeter. A current of galvanism passed through a mixture containing albumen produces coagulation, which has been attributed to a decomposition of certain salts which are combined with it and maintain its fluidity.

Some organic principles almost identical with albumen in chemical reactions, are found to possess very different vital properties. One of these is the organic principle of the gastric juice, which, like albumen, is coagulable by heat, alcohol, and the metallic salts, but exerts a peculiar and distinctive action in the digestion of certain articles of food.

Tests for Albumen.-As a pathological condition, albumen sometimes exists in the urine, and it becomes important clinically to be able to determine this fact by the application of tests. These require certain precautions for their successful application. They depend upon its property of coagulation.

If a solution containing albumen be exposed to heat in a test tube, as the temperature rises a slight cloudiness or opacity in the upper part of the liquid occurs, which gradually extends through the whole mass, until, at a temperature of about $167^{\circ}$, a precipitate more or less abundant is produced, which is entirely insoluble. If albumen be very abundant, the whole mass may become solidified, and we may have all shades between this and the slight opacity produced by a very minute quantity. In the latter case

1 Becquerel and Rodier, Traité de Chimie Pathologique, Paris, 1854, p. 53. 
coagulation is not complete until the liquid has been brought to the boiling point. It must be remembered, however, that albumen is not coagulated by heat in an alkaline solution. In testing the urine for albumen by heat, if the liquid be alkaline it must be neutralized with a little acetic acid; otherwise there will be no coagulation, even if albumen be present in abundance. There may also arise a source of error from the precipitation by heat of an excess of earthy phosphates. This precipitate is distinguished from albumen by the fact that it is dissolved by a few drops of hydrochloric acid, while coagulated albumen is not changed. Coagulated albumen in urine is redissolved by the addition of a little potash, which has no effect upon an opacity produced by the phosphates.

Another test is the addition to the suspected solution of a strong mineral acid; when, if albumen be present, coagulation will take place. There is only one source of error in the application of this test to the urine. If the urates be present in very large quantity, we may have a deposit of uric acid, giving an opacity something like that produced by coagulated albumen. This error may be avoided by adding an excess of nitric acid, which will clear up the mixture if the deposit be due to the presence of urates, but has no effect upon albumen. In such a case, also, no turbidity is produced by heat. When uric acid is deposited, the turbidity makes its appearance more slowly than when albumen is present. Various acid mixtures have been proposed as tests for albumen, but they seem to possess no advantages over nitric acid, which is the one most generally employed.

The tests by heat and nitric acid are sufficient to determine the presence or absence of albumen in any clear fluid, if applied with the precautions above indicated. We may employ, however, coagulation by alcohol, or the albuminimeter of Beequerel ; but the latter, like the saccharometer of Biot and Soleil, is little used on account of the 
expense of the instrument, and a certain dexterity which is necessary for its exact application.

Origin and Function of Albumen.-The albumen of the blood has its origin from a catalytic transformation of the products of digestion of the albuminoid elements of food. It forms the great organic nutrient element of the blood. As we have already seen, it seems to be used in the formation of the fibrin. In nutrition, it undergoes catalytic transformations which result in the peculiar organic principles of the various tissues. In the circulating blood there seems to be a union of the fibrin and albumen which is necessary to the nutritive properties of the latter. Bernard has shown ${ }^{1}$ that the albumen of white of egg injected into the veins of an animal is incapable of assimilation, and is therefore rejected by the kidneys. The same result follows the injection of fresh serum, even from an animal of the same species; but the blood itself, containing both albumen and fibrin, can be injected without the appearance of albumen in the urine, showing that in this state it is capable of being used in nutrition.

In the passage of the blood through the liver, it has been found that a small quantity of albumen disappears; but, as in the case of fibrin, we have not been able to follow its transformations. With the exception of the minute quantity which is discharged in the milk during lactation, albumen is never discharged from the body in health. After being appropriated by the tissues in the process of nutrition, it undergoes changes in the wearing out of the system, which convert it into exerementitious matter.

\section{Albuminose.}

This principle is intermediate between the organic nitrogenized elements of food and the albumen of the blood. It is found in the blood in very small quantity after digestion,

${ }^{2}$ Bernard, Lecons sur les Propriétés Physiologiques et les Altératians $P a$. thologiques des Liquides de l'Organisme, Paris, 1859, tome i., p. 467. 
almost immediately undergoing transformation into albumen. It is also contained in the stomach and small intestines during digestion. It is naturally fluid, like albumen and fibrin.

In its behavior to reagents, albuminose presents certain differences from albumen. It is coagulated by alcohol and many metallic salts, but is not coagulable by heat, and only imperfectly by nitric acid. It is coagulated by a small quantity of acetic acid, but the coagulum is dissolved in an excess of this agent, the latter peculiarity distinguishing it from caseine, which is coagulated by acetic acid in any quantity. Mialhe states that albuminose is more endosmotic, or passes through membranes with much greater facility than albumen, which he says is absolutely non-endosmotic. This property favors its introduction into the blood.

Albuminose has its origin from the organic nitrogenized elements of food, which are not only liquefied by the digestive fluids, but undergo a catalytic transformation into this substance. By virtue of its endosmotic properties, it passes into the blood-vessels, and is there converted into albumen. Mialhe, who first described this substance under the name of albuminose, has shown that, injected into the veins of an animal, it becomes assimilated, and does not pass away in the urine. ${ }^{1}$

\section{Caseine.}

This organic principle is peculiar to the milk, and therefore exists in the body only during lactation. Like fibrin and albumen, it is naturally fluid.

Caseine may be easily extracted by the following process, which is recommended by Robin and Verdeil." "We add to the milk a few drops of acetic acid, which precipitates the caseine accompanied by the fats. The coagulum separated

${ }^{1}$ Mralue, Chimie Appliquée a la Physiologie, Paris, 1856, p. 125.

${ }^{2}$ Op. cit., tome iii., p. 341. 
from the liquid, then washed, is redissolved in a solution of carbonate of soda; this solution separates from the fat which floats on the top, and can be completely removed at the end of twelve hours of repose. The liquid thus freed from fat is acidified by a few drops of hydrochloric acid, and the caseine is precipitated perfectly pure." Obtained by this process, it is perfectly white, and insoluble in water, resembling pot cheese.

Caseine has certain marked properties by which it is distinguished from albumen. It is not coagulable by heat; is coagulable by the feeble vegetable, as well as the mineral acids, and by rennet. This latter substance is obtained from the fourth stomach, or abomasus, of sucking ruminating animals, and is the milk almost reduced to caseine, and mixed with the gastric fluids. It is salted and dried, and in this condition used in making cheese. Added to the milk in the proportion of fifteen to twenty grains to a quart, it produces complete coagulation. According to Robin and Verdeil, caseine is precipitated by the metallic salts, with which it forms combinations not to be distinguished from like combinations of albumen. ${ }^{1}$ It is a curious fact that caseine is sometimes coagulated almost instantly during thunder storms. This phenomenon we cannot fully explain; but the immediate cause of the coagulation is the transformation of some of the sugar of milk into lactic acid. Caseine retains its fluidity in the milk by union with the carbonate of soda; and when coagulated spontaneously, it may be restored to its liquid condition by the addition of this salt, which does not render the fluid alkaline, but seems to enter into combination with the organic substance.

Caseine has its origin in the albumen of the blood, by a catalytic process which takes place in the mammary glands. In its liquid condition it constitutes the important organic element of the milk. It is taken into the stomach of the 
infant, converted into albuminose, which it resembles very closely, and absorbed by the blood, where it is converted into fibrin and albumen, and contributes to the nutrition of the system. At this period it constitutes almost the only nitrogenized element of food. It is the only proximate principle of this class, with the exception of a little mucosine and the coloring matter of the urine and bile, which is discharged from the body in health.

\section{Pancreatine.}

This is the organic principle peculiar to the pancreatic juice. Bernard was the first to describe its properties, both chemical and physiological. ${ }^{1}$ Before the appearance of his admirable monogragh on the pancreas it was confounded with albumen; but we shall see that it possesses properties by which it may be distinguished as readily as caseine.

Pancreatine exists in the pancreatic juice in large quantity. It is naturally fluid, but very riscid. It is coagulated by heat, the strong acids, and alcohol, but is unaffected by the feeble vegetable acids. It is distinguished from albumen by the fact that it is completely coagulated by an excess of sulphate of magnesia. Its distinctive physiological character is its powerful digestive action upon certain elements of food, and its property of forming an instantaneous, complete, and very fine emulsion with liquid fats.

Pancreatine has its origin from the albumen of the blood by a catalytic change which takes place in the pancreas. It gives to the pancreatic juice its digestive properties.

\section{Pepsin.}

Pepsin is the organic principle of the gastric juice. It is hardly to be distinguished from albumen, except by its physiological action in digestion. The principle which has been extracted by various processes from the mucous membrane

1 Bernard, Mémoire sur le Pancréas, Paris 1858. 
of the stomach, particularly after commencing putrefaction, cannot be regarded as pure pepsin. It is undoubtedly necessary to the digestive action of the gastric juice, which loses its physiological properties when this substance has been coagulated by heat and separated by filtration. Its properties will be more fully considered under the head of digestion.

\section{Mucosine.}

This is the organic principle of the general secretion of mucous membranes, presenting, however, some differences in different situations. In its general properties it closely resembles albumen; indeed, what is generally taken as the type of pure albumen, the white of egg, should strictly be called mucosine, as it is the secretion of the mucous membrane of the Fallopian tubes, and almost identical with some specimens of pure mucus, such as the secretion at the neck of the uterus during gestation. It is coagulated by heat, strong acids, and the metallic salts. It is formed from the blood by the mucous follicles; and, as a small quantity of mucus is discharged from the body, forms one exception to the general law that organic nitrogenized principles are never discharged from the body in health.

\section{Semi-sotid or Sotid Principles.}

Most of the liquid elements which we have just considered have been found to be connected, directly or indirectly, with the nutrition of the body. Those which we now have to consider are all directly formed from the organic principles of the blood, and constitute the organic portion of the economy. Here is found to be the final destination of fibrin and albumen in nutrition; for the organic principles constitute the vital elements of all the tissues, and are nourished exclusively by these elements of the blood. We include here the blood corpuscles, which must be regarded as organized bodies, nourished like any of the tissues. The following are the prin- 
ciples in this group which are well established, and have been studied to a greater or less extent:

Globuline,
Crystalline,
M.usculine,
Osteine,
Cartilagine,
Elasticine,
Keratine.

Globuline.-This is a semi-solid organic principle, con- stituting the greater portion of the blood corpuscles. It is soluble in water, from which it is coagulated by a temperature a little below the boiling point. Excepting that when mixed with water it requires a much higher temperature for its coagulation, it has nearly the same properties as albumen.

Like the rest of these principles, it exists in a state of intimate molecular union with inorganic elements; but, exceptionally in this case, is united with a small quantity of fat. In this condition it goes to form the organized structure of the blood corpuscles.

Crystalline.-This is a semi-solid organic principle, peculiar to the crystalline lens. It presents most of the characters of globuline, but is coagulated at a little lower temperature, though higher than is required to coagulate albumen.

Musculine.-This semi-solid organic principle is peculiar to the muscular tissue. It is immediately dissolved at the ordinary temperature by a mixture of ten parts of water with one of hydrochloric acid. It may be precipitated from this solution by neutralizing the acid, and the precipitate is redissolved by an alkali. It is always united with a consider- 
able quantity of inorganic salts, in which the phosphates predominate.

Musculine, in combination with inorganic substances, goes to form the muscles; but in addition, is interesting as being by far the most important and abundant nitrogenized element of food. It is the great source of the fibrin and albumen of the blood of man and of the carnivorous animals.

Osteine.-This organic principle, naturally solid, is peculiar to the bones. If the earthy matter of bone be dissolved out with dilute hydrochloric acid, the residue is nearly pure osteine. By boiling with water it is transformed into gelatine, a soluble substance differing in many respects from osteine. According to the experiments of Majendie, fresh bones possess considerable nutritive power, which is entirely destroyed by prolonged boiling. It enters into combination with large quantities of earthy salts, to form the bones.

Cartilagine.-This principle holds the same relation to cartilage as osteine does to bone. By prolonged boiling it is transformed into a substance resembling gelatine, called by Müller chondrine. This presents many points of difference from gelatine, which renders it probable that the transformation of cartilage into bone, does not merely consist in the deposition of calcareous matter, but also the substitution of a new organic principle.

Elasticine.-This is the organic principle of the yellow elastic tissue and the investing membrane of the muscular fibres. According to Robin and Verdeil it is slowly dissolved by sulphuric, nitric, and hydrochloric acids, and these solutions, diluted with water, are not precipitated by alkalis. It is possessed of great strength and elasticity.

Keratine.-This is an organic principle, found in the nails and hair, about which we know very little. It differs from 
the other principles in the fact that it is not dissolved, but decomposed by potash, giving off ammoniacal vapor.

\section{Coloring Matters.}

These substances have been classed with the organic nitrogenized principles, from the fact that they contain nitrogen; but they do not seem to be endowed with the vital properties which characterize this class, with the exception perhaps of hematine and melanine. As a peculiarity of chemical constitution, they all contain iron, which is molecularly united with their other elements. The following are the principles of this group:

\section{Hematine, Melanine, Biliverdine, Urrosacine.}

Hematine.-This is the red coloring matter of the blood, and exists, intimately united with globuline, in the blood corpuscles. The iron which it contains can be readily demonstrated, even in a single drop of blood, by the following process : To a small quantity of blood in a watch-glass we add a drop of nitric acid, then evaporate slowly over a lamp, when fumes of nitrous acid are driven off, the iron takes oxygen and is converted into a per-oxide. If we then add a drop of the sulpho-cyanide of potassium, we produce the characteristic red color of the sulpho-cyanide of iron. Separated from the blood, hematine is soluble in ether and boiling alcohol, but insoluble in water and in acids.

We do not exactly understand the mode of formation of hematine, but pathology teaches us that it is an essential principle. of the blood. In certain cases of anemia, when there is extreme pallor and consequently deficiency of hematine, the administration of iron in any form induces the formation of this substance, restores the normal constitution of 
the circulating fluid, and relieves the general effects of the deficiency of coloring matter; an effect which cannot be produced by the most nutritious articles of food. Hematine is probably destroyed in the organism, and furnishes material for the formation of the other coloring matters.

Melanine.-This substance resembles hematine, containing, however, a smaller proportion of iron. It is of a brownish color, and is found in all parts of the body where pigment exists; such as the choroid, iris, hair, or epidermis. It exists in the form of granulations, either free or enclosed in epithelial cells. In all probability it is formed by a transformation of'hematine.

Biliverdine.-This is a greenish-yellow coloring matter peculiar to the bile. Extracted from the bile, it is insoluble in .water, but soluble in alcohol or ether. It contains iron in nearly the same proportion as hematine.

Biliverdine is formed from hematine, enters into the constitution of the bile, is discharged into the small intestine, and, after undergoing certain modifications, is discharged from the body in the feces.

Urrosacine.-This is the principle which gives the amber color to the urine. After extraction, it is insoluble in water, but soluble in alcohol or ether. It exists in the urine in very small quantity, and is formed in the kidney, in all probability at the expense of the hematine. Urrosacine and biliverdine are the two coloring matters discharged from the body.

Summary.-A review of the individual properties of the organic nitrogenized principles shows great differences in their physiological, and very slight differences in their purely chemical characters. It is a fact too apparent to require argument, that their chemical history is of little importance compared to a study of their vital properties. In fact researches into their ultimate composition, with the excep- 
tion that they have shown them all to contain nitrogen, are almost without value. Without exception they are all in a state of intimate molecular union with inorganic matter, and in this union inorganic compounds become endowed with life; that is, the inorganic parts of the body, as the calcareous elements of bone, taken up by the blood with the worn-out organic principles and undergoing constant waste, are capable of self-regeneration.

The vitality thus imparted to inorganic matters, and the fact that neither the organic nor inorganic elements are alone capable of engaging in the phenomena of life, cannot be too fully insisted upon. Both are taken into the body as food, are digested, assimilated, and finally discharged, always in combination; the organic principles changed, and converted into excrementitious substances, and the inorganic principles unchanged.

The readiness with which the organic principles are converted one into the other by catalysis must also be appreciated, as well as the constant operation of this process in all the phenomena of life. Even albumen, taken in as food, must be converted into albuminose, and again into albumen, before it is capable of building up the tissues; and all the nitrogenized articles of food are converted into the same substance, regenerating the blood, and through it the body.

In the economy we find two great divisions of organic elements: one, which is nutritive, and the other, which forms the great part of the tissues. By simple contact, the plastic, or nutritive, principles are mysteriously converted into the varied elements of the organism, and take with them the inorganic elements necessary to the proper constitution of the parts.

It is only with a just appreciation of these general principles that we are able to study intelligently the special functions of respiration, circulation, digestion, absorption, secretion and excretion, which are all tributary to the complicated function of nutrition. 


\title{
CHAPTER I.
}

\author{
THE BLOOD.
}

General considerations-Transfusion-Quantity-Physical characters-0pacityTemperature-Specific gravity-Color-Anatomical elements of the bloodRed corpuscles-Chemical characters of red corpuscles-Development of red corpuscles-Formation of red corpuscles-Leucocytes, or white corpusclesDevelopment of leucocytes.

Iv all ages, even before physiology became known as a distinct science, the importance of the blood in the animal economy has been recognized; and with the progress of knowledge this great nutritive fluid has been shown to be more and more intimately connected with the phenomena of life. It is now known to be the most abundant and highly organized of the animal fluids; providing materials for the regeneration of all parts, without exception, receiving the products of their waste and conveying them to proper organs, by which they are removed from the system. These processes, on the one hand, require constant regeneration of its constituents, and on the other, constant purification by the removal of effete matters. As it has been found desirable to preface our study of general physiology with a history of proximate principles, showing the chemical and vital properties of what may be considered as the permanent constituents of the body, so before considering individual functions, all of which bear finally on the great process of nutrition, we should have an accurate knowledge of the anatomy and chemistry 
of what is most appropriately called the great nutritive fluid. It has been said that all parts are dependent on the blood for nourishment. Those tissues in which the processes of nutrition are active are supplied with blood by vessels; but some less highly organized, like the epidermis, hair, cartilage, etc., which are sometimes called extra-vascular because they are not penetrated by blood-vessels, are none the less dependent upon the fluid under consideration; imbibing, as they do, nourishment from the blood of adjacent parts.

It must be remembered that in nutrition the tissues are active, selecting, appropriating, and modifying material which is simply furmished by the blood; and as the real vital force which governs these processes resides in the tissues, tendencies of the system, such as the tubercular, scrofulous, or cancerous diatheses, which lead to disordered nutrition, must have their seat in the solids, and not in the circulating fluid. The first cause of these conditions may lie in a disordered state of the blood, from bad nourishment, from the introduction of poisons, such as malaria, or the emanations from persons affected with contagious diseases, and under some circumstances the elimination of these poisons may be effected through the blood; but when they exist in the blood, they either become fixed in the system, or are thrown off. We must regard most of the morbid actions which are dependent on diathesis, as the result of a vice in the tissue itself, not the blood with which it is supplied. It is none the less essential to health, however, that the blood should have its proper constitution.

The final importance of the blood in the processes of nutrition is evident; and in animals in which nutrition is active, death is the immediate result of its abstraction in large quantity. Its immediate importance to life can be beautifully demonstrated by experiments upon inferior animals. If we take a small dog, introduce a canula through the right jugular vein into the right side of the heart, adapt to it a syringe, and suddenly withdraw a great part of the blood 
from the circulation, immediate suspension of all the vital processes is the result. If we then return the blood to the system, the animal is as suddenly revived. ${ }^{1}$ To perform this experiment satisfactorily, we must accurately adjust the capacity of the syringe to the size of the animal. Carefully performed, it is very striking.

Transfusion.-Certain causes, one of which is diminution in the force of the heart after copious hemorrhage, prevent the escape of all the blood from the body, even after division of the largest arteries; but after the arrest of the vital functions which follows copious discharges of this fluid, life may be restored by the injection into the vessels of the same blood, or the fresh blood of another animal of the same species. This observation, which was first made on the inferior animals, has been applied to the human subject; and it has been ascertained that in patients sinking under hemorrhage, the introduction of even a few ounces of fresh blood will restore the vital forces for a time, and sometimes permanently. The operation of transfusion, which consists in the introduction of the blood of one individual into the vessels of another, was performed upon animals in the middle of the seventeenth century, and was soon after attempted in the human subject. So great was the enthusiasm with which some regarded these experiments, that it was even thought possible to effect a renewal of youth by the introduction of young blood into the veins of old persons; and it was also proposed to cure certain diseases, such as insanity, by an actuai renewal of the circulating fluid. These ideas were not without apparent foundation. It was stated in 1667 , that a dog, old and deaf, had his hearing improved and was apparently rejurenated by transfusion of blood from a young animal. A year later Denys and Emmerets published the case of a maniac who was restored to health by the transfusion of eight ounces

${ }^{1}$ Bersard, Leçons sur les Liquides de l'Organisme, tome i., p. 44. 
of blood from a calf; and another case was reported of a man who was cured of leprosy by the same means. But a reaction followed. The case of insanity, which was apparently cured, suffered a relapse, and the patient died during a second operation of transfusion. ${ }^{1}$ It is almost unnecessary to say that these extravagant expectations were not realized. In fact some operations were followed by such disastrous consequences, that the practice was forbidden by law in Paris in 1668 , and soon fell into disuse.

Transfusion, with more reasonable applications, was revived in the early part of this century (1818) by Blundell, who, with others, demonstrated its occasional efficacy in desperate hemorrhage, and in the last stages of some diseases, especially cholera. There are now quite a number of cases on record where life has been saved by this means; and oftentimes, when the result has not been so happy, the fatal event has been considerably delayed. In a case which occurred at New Orleans, when the system was prostrated by an obscure affection and life became nearly extinct, about seven ounces of blood in all were transfused in three operations, within two hours, with the palpable effect of prolonging life for from twelve to sixteen hours. ${ }^{2} \quad$ Bérard had collected from various sources thirteen observations of hemorrhage, which would have been fatal, in which life was permanently restored by the injection of a few ounces of healthy human blood. In all but two of these cases the hemorrhage was uterine."

${ }^{1}$ Bérard, Cours de Physiologie, tome iii., p. 209 et seq.

${ }^{2}$ In this case the patient suffered extrcme prostration after the delivery of a seven and a half months' child. This continued for a few days, and at the time of transfusion, the pulse was 140 and very feeble; respirations six to eight per minute; nostrils compressed at each inspiration; surface cool ; countenance Hippocratic, and the coma so profound that the patient could not be aroused. After each transfusion the lips became more florid, the nostrils dilated in inspiration, and the surface became warmer. The patient lived twenty-four hours after the first operation. The blood was taken from the arm of a healthy male and transfused immediately into the median cephalic vein.

${ }^{3}$ BérARD, op. cit., tome iii., p. 219 et seq. 
Sinee this time a great many experiments on transfusion in animals have been performed, with very interesting results. Provost and Dumas ${ }^{1}$ have shown, that while an animal may be restored afterhemorrhage by the transfusion of defibrinated blood, no such effect follows the introduction of the serum; showing that the vivifying influence in all probability resides in the corpuscles. These observers have also shown, that though an animal may be temporarily revived by the injection of defibrinated blood from an animal of a different species, death follows the operation in a few days. ${ }^{2}$ Brown-Séquard has shown that in parts detached from the body, after nervous and muscular irritability have disappeared, these properties may be restored for a time by the injection of fresh blood. ${ }^{3}$ He also reports a curious experiment in which blood was passed from a living dog into the carotid of a dog just dead from peritonitis. The animal was so far revived as to sustain himself on his feet, wag his tail, etc., and died a second time, twelve and a half hours after. In this experiment insufflation was employed in addition to the transfusion. ${ }^{4}$

It may then be considered established, that in animals, after hemorrhage, life may be restored by injecting the blood, defibrinated or not, of an animal of the same species, provided it be introduced slowly, without admixture with air, and not in too great quantity. If, however, the blood of an animal of a different species be used, life will be restored but for a short time. Death oceurs after the transfusion of blood in this instance, only when the animal receiving it is exsanguine, and the blood of an animal of a different species is substituted. If the animal be not exsanguine, a little blood can be superadded to the mass from an animal of different species without this result, as is shown by the experiments

${ }^{1}$ BÉRard, op. cit., tome iii., p. 219.

${ }^{2}$ Minne-Edwards, Leģons sur la Physiologie et l'Anatomie Comparée, tome i, p. 322 et séq.

${ }^{3}$ Journal de la Physiologie, tome i., p. 106.

${ }^{4}$ 1bid., p. 668. 
already alluded to, of transfusion of the blood of a calf into the veins of a man.

In the human subject, especially after hemorrhage, the vital powers are sometimes restored by careful transfusion of human blood, with the above precautions; remembering that a very small quantity, three or four ounees, will sometimes be sufficient.

Quantity of Blood.-The determination of the entire quantity of blood contained in the body is a question of great interest, and has long engaged the attention of physiologists, without, however, absolutely definite results. Among those who have experimented on this point, may be mentioned Allen-Moulins, Herbst, Fried. Hoffmann, Valentin, Blake, Lehmann and Weber, and Vierordt. ${ }^{1}$ The fact that the labor's of these eminent observers have been so far unsuccessful in determining definitely the entire quantity of blood, shows the difficulties which are to be overcome before the question can be entirely settled. The chief difficulty lies in the fact that all the blood is not discharged from the body on division of the largest vessels, as after decapitation; and no perfectly accurate means have been devised for estimating the quantity which must always remain in the vessels. The estimates of experimenters present the following wide differences. Allen-Moulins, who was one of the first to study this question, estimates the quantity of blood at one-twentieth the weight of the entire body. The estimate of Herbst is a little higher. Hoffmann estimates the quantity at one-fifth the weight of the body. These observers estimated the quantity remaining in the system after opening the vessels, by mere conjecture. Valentin was the first who attempted to overcome this difficulty by experiment. For this purpose

1 The reader is referred to the works of Longet (Physiologie, Paris, 1861, tome i., p. 705 et seq.) and Milne-Edwards (Physiologie, Paris, 1857, tome i., p. 308 et $s(q$.), for a more extended account of the various experiments which have been made with a view of determining the entire quantity of blood in the body. 
he employed the following process. He took first a small quantity of blood from an animal for purposes of comparison; then injected into the vessels a known quantity of a saline solution, and taking another specimen of blood some time after, he ascertained by evaporation the proportion of water which it contained, compared with the proportion in the first specimen. He reasoned that the excess of water in the second specimen over the first would give the proportion of the water introduced, to the whole mass of blood; and as the entire quantity of water introduced is known, the entire quantity of blood could be deduced therefrom. Suppose, for example, that the excess of water in the second specimen should be one part to ten of the blood, it would show that one part of water had been mixed with ten of the blood; and if we had injected in all five ounces of water, we would have the whole quantity of blood ten times that, or fifty ounces.

This method is open to the objection that it is impossible to take note of the processes of imbibition and exhalation which are constantly in operation. Taking it for what it is worth, the estimates, applied to the human subject, give the weight of blood as $\frac{23}{100}$ that of the body.

Blake estimated the quantity of blood by an analogous process, injecting a known quantity of sulphate of alumina into the vessels, estimating its proportion in a specimen of blood, and from that deducing the entire quantity. He gives the proportion of blood in dogs as from one-ninth to onethird the weight of the body. The objection we have mentioned applies also to these experiments.

The following process, which is, perhaps, least open to sources of error, was émployed by Lehmann and Weber, and applied directly to the human subject, in the case of two decapitated criminals. These observers estimated the blood remaining in the body after decapitation, by injecting the ressels with water until it canne through nearly colorless. It was carefully collected, evaporated to dryness, and the dry residue assumed to represent a certain quantity of blood; the 
proportion of dry residue to a definite quantity of blood having been previously ascertained. If we could be certain that only the solid matter of the blood was thus removed, the estimate would be tolerably accurate. As it is, we may consider it as approximating very nearly to the truth. We quote the following account of these observations:

"My friend, Ed. Weber, determined, with my coöperation, the weights of two criminals both before and after their decapitation. The quantity of blood which escaped from the body was determined in the following manner: Water was injected into the vessels of the trunk and head, until the fluid escaping from the reins had only a pale red or yellow color; the quantity of the blood remaining in the body was then calculated, by instituting a comparison between the solid residue of this pale-red aqueous fluid, and that of the blood which first escaped. By way of illustration, I subjoin the results yielded by one of the experiments. The living body of one of the criminals weighed 60,140 grammes $(132 \cdot 7$ pounds), and the same body after decapitation 54,600 grammes; consequently 5,540 grammes of blood had escaped. $28 \cdot 560$ grammes of this blood yielded $5 \cdot 36$ grammes of solid residue; 60.5 grammes of sanguineous water collected after the injection, contained 3.724 grammes of solid substances; 6,050 grammes of the sanguineous water that returned from the veins were collected, and these contained $37 \cdot 24$ grammes of solid residue, which corresponds to 1,980 grammes of blood; consequently, the body contained 7,520 grammes (16.59 pounds), 5,540 escaping in the act of decapitation, and 1,980 remaining in the body; hence, the weight of the whole blood was to that of the body nearly in the ratio of $1: 8$. The other experiment yielded a precisely similar result.

"It cannot be assumed that such experiments as these possess extreme accuracy, but they appear to have the advantage of giving in this manner the minimum of the blood contained in the body of an adult man; for although some solid substances, not belonging to the blood, may be taken up by 
the water from the parenchyma of the organs permeated with capillary vessels, the excess thus obtained is so completely counteracted by the deficiency caused by the retention of some blood in the capillaries, and in part by transudation, that our estimate of the quantity of blood contained in the human body may be considered as slightly below the actual quantity."

In extreme obesity, the weight of the blood would not bear a natural ratio to that of the body; but from the data which we have at our command, we may state the proportion in a well-formed man to be about 1 to 8 , or the whole quantity of blood at from 16 to 20 pounds aroirdupois. The quantity of blood undoubtedly varies in the same individual in different conditions of the system; and these variations are fully as important, in a physiological point of view, as the entire quantity.

Prolonged abstinence has a notable effect in diminishing the mass of blood, as indicated by the small quantity which can be removed from the body, under these circumstances, with impunity. It has been experimentally demonstrated ${ }^{2}$ that the entire quantity of blood is considerably increased during digestion. Bernard drew from a rabbit weighing about $2 \frac{1}{5} \mathrm{lbs}$., during digestion, over $10 \frac{1}{2}$ ounces of blood without producing death; while he found that the removal of half that quantity from an animal of the same size, fasting, was followed by death. In Burdach, ${ }^{3}$ we find a case reported by Wrisberg, of a female criminal, very plethoric, from whom 21 lbs. $7 \frac{3}{4}$ ounces of blood flowed after decapitation. As the relations of the quantity of blood to the digestive function are so important, it is unfortunate that in the observations of Lehmann and Weber, the conditions of the system in this

${ }^{1}$ Lemuanv, Physiological Chemistry, Philadelphia, 1855, vol. i., p. 638. The weights of the body and the entire quantity of blood have been reduced from grammes to pounds avoirdupois.

${ }^{2}$ Bernard, Liquides de l'Organisme, tome i., p. 419.

Op. cit., tome vi., p. 119. 
respect were not noted; a circumstance which would have added materially to their value.

It is thus evident that the quantity of blood in the body is considerably increased during digestion; but as to the extent of this increase, we cannot yet form any definite idea. It is only shown that there is a very marked difference in the effects of hemorrhage in animals, during digestion and fasting.

The reaction of the blood, which has been determined after the globules have separated so as to allow the application of test paper to the clear plasma, has been found to be uniformly alkaline.

\section{Physical Characters of the Blood.}

Opacity.-One of the first physical characters of the blood which attract our attention is its opacity. This depends upon the fact that it is not a homogeneous fluid, but composed of two distinct elements : a clear plasma, and corpuscles, which are nearly as transparent, but which have a different refractive power. If both of these elements had the same refractive power, the mixture would present no obstacle to the passage of light; but as it is, the rays, which are bent or refracted in passing from the air through the plasma, are again refracted when they enter the corpuscles, and again when they pass from the corpuscles to the plasma, so that they are lost, even in a thin layer of the fluid. This loss of light in a mechanical mixture of two transparent liquids of unequal refractive power can be demonstrated by the following simple experiment. If to a little chloroform, colored red, clear water be added in a test-tube, these liquids remain distinct from each other, and are both transparent; but if we agitate them violently, the chloroform is temporarily subdivided into globules and mixed with the water; and as they refract light differently, the mixture is opaque.

Odor. - The blood has a faint but characteristic odor. This 
may be developed more strongly by the addition of a few drops of sulphuric acid, when an odor, peculiar to the animal whose blood we are examining, becomes very distinct.

Temperature.-The temperature of the blood is generally given as $98^{\circ}$ to $100^{\circ} \mathrm{Fahr}$., but recent experiments have shown that it varies considerably in different parts of the circulatory system, independently of exposure to the refrigerating influence of the atmosphere. By the use of very delicate registering thermometers, Bernard has succeeded in establishing the following facts with regard to the temperature in various parts of the circulatory system in dogs and sheep :

1. The blood is warmer in the right than in the left cavities of the heart.

2. It is warmer in the arteries than in the veins, with a few exceptions.

3. It is generally warmer in the portal vein than in the abdominal aorta, independently of the digestive act.

4. It is constantly warmer in the hepatic than in the portal veins.

He found the highest temperature in the blood of the hepatic vein, where it ranged from $101^{\circ}$ to $107^{\circ}$. In the aorta it ranged from $99^{\circ}$ to $105^{\circ}$.

We may assume, then, in general terms, that the temperature of the blood in the deeper vessels is from $100^{\circ}$ to $107^{\circ}$ Fahrenheit. ${ }^{1}$

Specific Gravity of the Blood.-According to Becquerel and Rodier, who, perhaps, are as high authority as any on this subject, the specific gravity of defibrinated blood is firom 1055 to $1063 .^{2}$ It is somewhat less in the female than in the male.

${ }^{1}$ These facts were taken from the lectures of Bernard, "Sur les Liquides de l'Organisme," Paris, 1859, in two volumes. The first volume is deroted to the blood, and the subject of temperature is very thoroughly investigated.

${ }^{2}$ Becquerel and Rodrer, Traité de Chimie Pathologique, Paris, 1854. 
Color of the Blood.- The color of the blood is due to the corpuscles. In the arterial system it is uniformly red. In the veins it is dark blue and sometimes almost black. This difference in color between the blood in the arterial and in the venous system, was a matter of controversy at the time of Harvey. By the discoverer of the circulation, the difference, which is now universally known and admitted, as regards most of the veins, was supposed to be merely accidental, and dependent on external causes. Fifty years later, Lower ${ }^{1}$ demonstrated the change of color in the blood as it passes through the lungs, and associated it with the true cause, viz., the absorption of oxygen. The color in the veins, however, is not constant. Many years ago, John Hunter observed, in a case of syneope, that the blood drawn by venesection was bright red $;^{2}$ and more recently Bernard has demonstrated that in some veins the blood is nearly, if not quite, as red as in the arterial system. The color of the renous blood depends upon the condition of the organ or part from which it is returned. The red color was first noticed by Bernard in the renal veins, where it contrasts very strongly with the black blood in the vena cava. He afterwards observed that the redness only existed during the functional activity of the kidneys; and when, from any cause, the secretion of urine was arrested, the blood became dark. He was led from this observation to examine the venous blood from other glands; and directing his attention to those which he was able to examine during their functional activity, particularly the salivary glands, found the blood red in the veins during secretion; but becoming dark as soon as secretion was arrested. These observations may be easily verified by opening the abdomen of a living animal so as to expose the emulgent reins, introducing a canula into the ureter so as to be able to note the flow or arrest of the urine. As long as the urine

${ }^{1}$ Lower, Tractatus de Corde item de Motu \& Colore Sanguinis, Amstelodami, 1669, p. 180.

3 The Works of John Hunter, Philadelphia, 1840, vol. iii., p. 93. 
continues to flow, the blood in these ressels will be bright red; but when secretion becomes arrested, as it soon does after exposure of the organs, it presents no difference from the blood in the vena cava. In the sub-maxillary gland, by the galvanization of a certain nerve, which he calls the motornerve of the gland, Bernard has been able to produce secretion, and by the galvanization of another nerve, to arrest it; in this way changing at will the color of the blood in the vein. It has been found by the same observer that division of the sympathetic in the neck, which dilates the vessels and increases the supply of blood to one side of the head, produces a red color of the blood in the jugular. He has also found that paralysis of a member by division of the nerve has the same effect on the blood returning by the veins. ${ }^{1}$

The explanation of these facts is evident when we reflect upon the reasons why the blood is red in the arteries and dark in the veins. Its color depends upon the corpuscles; and as the blood passes through the lungs it loses carbonic acid and gains oxygen, changing from black to red. In its passage through the capillaries of the system, in the ordinary processes of nutrition, it loses oxygen and gains carbonic acid, changing from red to black. During the intervals of secretion, the glands have just enough blood sent to them for their nutrition; and the ordinary interchange of gases takes place, with the consequent change of color; but during their functional activity, the blood is supplied in greatly increased quantity, in order to furnish the watery elements of the secretions. Under these circumstances it does not lose oxygen and gain carbonic acid in any great quantity, as has been demonstrated by actual analysis, ${ }^{2}$ and consequently experiences no change in color. When filaments of the sympathetic are divided, the vessels going to the part are dilated, and the supply of blood is increased to such an extent, that a

${ }^{1}$ Branard, op. cit.

${ }^{2}$ Unpublished lectures delivered by Bernard in the College of France during the summer of 1861 . 
certain proportion passes through without parting with its oxygen, a fact which has also been demonstrated by analysis, and consequently retains its red color. The explanation in cases of syncope is probably the same; though this is merely a supposition. Even during secretion, a certain quantity of carbonic acid is formed in the gland, which, according to Bernard, is carried off in solution in the secreted fluid. ${ }^{1}$

It may be stated in general terms that the color of the blood in the arteries is bright red; and in the ordinary veins, like the cutaneous or muscular, it is dark blue, almost black. It is red in the veins coming from glands during secretion, and dark during the intervals of secretion.

\section{Anatomical Elements of the Blood.}

In 1661, the celebrated anatomist, Malpighi, in examining the blood of the.hedgehog with the feeble and imperfect lenses at his command, discovered little floating particles which he mistook for granules of fat, but which were the blood-corpuscles. He did not extend his observations in this direction; but a few years later (1673), Leeuwenhoek, by the aid of simple lenses of his own construction, varying in magnifying power from 40 to 160 diameters, first saw the corpuscles of human blood, which he minutely described in a paper published in the Philosophical Transactions, in 1674. To him is generally ascribed the honor of the discovery of the blood-corpuscles. About a century later, William Hewson ${ }^{2}$ described another kind of corpuscles in the blood, which are much less abundant than the red, and which are now known under the name of white globules, or as they have lately been called by Robin, leucocytes.

Without following the progress of microscopic investiga-

${ }^{2}$ Bernard, op. cit., tome i., p. 346.

${ }^{2}$ The Works of William Hewson, F. R. S., Sydenham Society edition, London, 1846. 
tions into the constitution of the blood, it may be stated that it is now known to be composed of a clear fluid, the Plasma, or liquor sanguinis, holding certain corpuscles in suspension. These corpuscles are

1. Red Corpuscles; by far.the most abundant, constituting about one-half of the mass of blood.

2. Leucocytes, or White Corpuscles; much less abundant, existing only in the proportion of one to several hundred red corpuscles.

3. Granules; exceedingly minute, called, by MilneEdwards, globulins, and by Kölliker, elementary granules. These are few in number, and are undoubtedly fatty particles from the chyle. They are to be regarded as accidental constituents of the blood.

Red Corpuscles.-These little bodies give to the blood its red color and its opacity. They are true organized structures, containing organic-nitrogenized and inorganic elements molecularly united, and, as an exception to the general rule, a little fatty matter in union with their organic principle. Like other organized structures, they are constantly undergoing decay, and are capable of self-regeneration. They constitute about one-half the mass of blood, and, according to the observations of all who have investigated this subject, are more abundant in the male than in the female; this constituting, perlaps, the only constant difference in the composition of the blood in the sexes.

The form of the blood-corpuscles is peculiar. They are flattened, bi-concave, circular disks, with a thickness of from one-fourth to one-third of their diameter. Their edges are rounded, and the thin central portion occupies about one-half of their diameter. Their consistence is not much greater than that of the plasma. They are very elastic, and if deformed by pressure, immediately resume their original shape when the pressure is removed. Their specific gravity is somewhat greater than that of the plasma. 
The peculiar form of the blood-corpuscles gives them a very characteristic appearance under the microscope. When examined with a magnifying power of from 300 to 500 diameters, those which present their flat surfaces have a shaded centre, when the edges are in focus. Before we were in possession of the perfect instruments now used in microscopic investigation, this spot was supposed to be a nucleus having a constitution different from the rest of the corpuscle. Now this is understoord to be an optical effect, the result of the form of the corpuscle; their bi-concavity rendering it impossible for the centre and edges to be exactly in focus at the same instant, so that when the edges are in focus the centre is dark, and when the centre is bright the edges are shaded.

As the blood-corpuscles are examined by the microscope by transmitted light, they are quite transparent, and of a pale amber color. It is only when they are collected in masses that they present the red tint characteristic of blood as it appears to the naked eye. This yellow or amber tint is characteristic. A pretty good idea of it may be obtained by largely diluting blood in a test tube and holding it between the eye and the light.

In examining blood under the microscope, the corpuscles are seen in many different positions; some flat, some on their edges, etc. This assists us in recognizing their peculiar form.

It has been observed by microscopists that the bloodcorpuscles have a remarkable tendency to arrange themselves in rows like rouleaux of coin. This has attracted universal attention, and for a long time was not satisfactorily explained. Robin has lately given us what seems to be the true explanation of this phenomenon. ${ }^{1}$ This observer has shown that

${ }^{2}$ Roвin, Sur quelques Points de l'Anatomie et de la Physiologie des Globules liouges du Sang. Journa? de la Plysiologie de l'Homme et des Animaux, Paris, 1858, tome i., p. 295. 
shortly after removal from the vessels, there exudes from the corpuscles an adhesive substance which smears their surface and causes them to stick together. Of course the tendency is to adhere by their flat surfaces. In examining a specimen of blood under the microscope, the presence of this adhesive exudation may be demonstrated by employing firm and gradual pressure on the glass cover, when the adherent corpuscles_may be separated in some instances, and with oblique light we can sometimes see a little transparent filament between them, which draws them together, as it were, when the pressure is removed. This phenomenon is due to a post mortem change, but it occurs so soon, that it presents itself in nearly every specimen of fresh blond which we examine, and is therefore mentioned in connection with the normal characters of the blood-corpuscles.

Dimensions.-The diameter of the blood-corpuscles has a more than ordinary anatomical interest; for, varying perhaps less in size than in other anatomical elements, they are rather taken as the standard by which we form an idea of the size of other microscopic objects. The diameter usually given is $\frac{1}{3} \frac{1}{5} \overline{0}$ of an inch. The exact measurement given by Robin is .0073 of a millimètre ${ }^{1}$ or $\frac{1}{3} \frac{1}{4} \overline{7}$ of an inch. It is stated by some authors that the size of the corpuscles is very variable, even in a single specimen of blood. I have repeatedly measured them with the eye-piece micrometer of Nachet, and found a diameter of $\frac{1}{3} \frac{1}{5} \overline{0}$ of an inch. Very few are to be found which vary from this measurement. Kölliker, who gives their average diameter as $\frac{-1}{3} \frac{1}{0}-$ of an inch, states that " at least ninety-five out of every hundred corpuscles are of the same size." 2

We cannot leave the subject of the size of the blood-corpuscles without a notice of the measurements in the blood of

${ }^{1}$ Loc. Cit.

${ }^{2}$ KöLLIKER, Manual of Microscopic Anatomy, London, 1860, p. 519. 
different animals. This point is interesting from the fact that it is often an important question to determine whether a given specimen of blood be from the human subject or one of the inferior animals. Comparative measurements also have an interest on account of a relation which seems to exist in the animal scale between the size of the blood-corpuscles, and muscular activity. In all the mammalia, with the exception of the camel and lama, in which they are oval, the blood-corpuscles have the same anatomical characters as in the human subject; the only difference is in size. In only two animals, the elephant and sloth, are they larger than in man; in all others they are smaller, or of nearly the same diameter. By reference to the table it will be seen that in some animals the corpuscles are very.much smaller than in man; and by accurate measurement, we are enabled to distinguish their blood from the blood of the human subject. But in forming an opinion on this subject, it must be remembered that there is some variation in the size of the corpuscles of the same animal. We can easily distinguish the blood of the human subject, or of the mammals generally, from that of birds, fishes, or reptiles; for in these classes of animals the corpuscles are oval and contain a granular nucleus.

Milne-Edwards has attempted to show, by a comparison of the diameter of the blood-corpuscles in different species, that their dimensions are in inverse ratio to the muscular activity of the animal. ${ }^{2}$ Reference to the table will show that this relation holds good to a certain extent, while there certainly exists none between the size of the corpuscle and the size of the animal. In deer, which are remarkable for their muscular activity, the corpuscles are very small, $\frac{1}{50} \overline{0} \overline{0}$ of an inch; while in the sloth they are $\frac{1}{2} \frac{1}{8} \overline{0} \overline{0}$, and in the ape, which is comparatirely inactive, $\frac{1}{3} \frac{1}{4} \overline{0}$. But, on the other hand, in the dog, which is quite active, we have a corpuscle

'Mrlne-Edwards, Legons sur la Physiologie et l'Anatomie Comparée, tome i., p. 57 et seq. 
of $\frac{1}{3} \frac{1}{5} \overline{0}$ of an inch, and in the ox, which is certainly not so active, the diameter of the corpuscle is $\frac{1}{4} \frac{1}{2} \overline{0}$ of an inch. Though this relation between the size of the blood-corpuscles and muscular activity is not invariable, it is certain that the higher we go in the great classes of animals, the smaller the blood-corpuscle becomes; the largest being found in the lowest orders of reptiles, and the smallest in the mammalia. In the blood of the invertebrates, with a few exceptions, ${ }^{1}$. we find no colored corpuscles.

\section{Table of Measurements of Red Corpuscles.}

This table is taken from the table of Mr. Gulliver, published in the Sydenham edition of Hewson's Works, page 237. Nearly five hundred measurements were made by Mr. Gulliver; and of these, one hundred of the most important have been selected. It will be observed that the diameter of the human blood-corpuscle is greater than that generally given. It must be borne in mind that all these measurements are mere approximations; but as such they are useful, as showing the relations of the corpuscles in different animals, and enabling us to distinguish the blood of the human subject from that of some of the inferior animals; a question which is often of vital importance. The measurements are all given in fractions of an English inch; and in making the selections, the common names of the animals have been substituted for the technical names given in the original.

MAMMALS.

\section{Corpuscles Circular.}

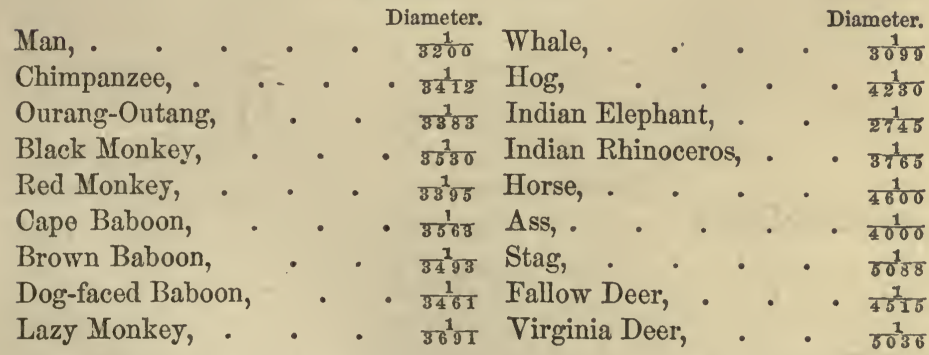

1 Note sur l'Existence de Globules du Sang colorés chez plusieurs d'animaux invertébrés. Par le Docteur CH. Rovget. Journal de Physiologie, \&c., 1859, tome ii., p. 660. In this article Dr. Rouget cites a number of invertebrate animals, in the blood of which he has found corpuscular elements. This is opposed to the general idea that corpuscles exist only in the blood of the vertebrates. 


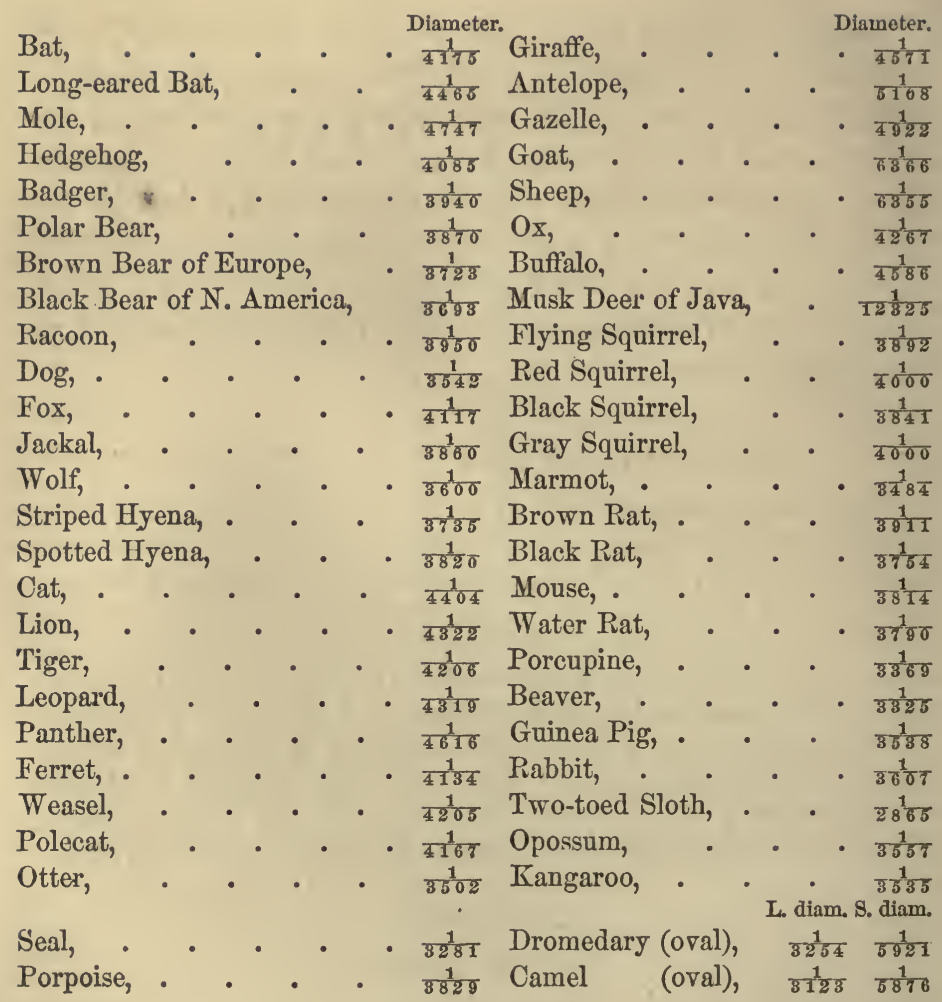

BIRDS.

Corpuscles Oval.

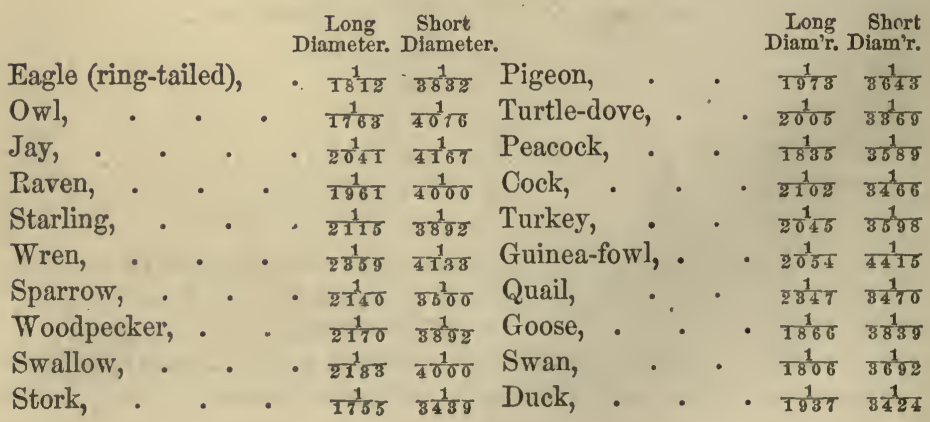


REPTILES.

Corpuscles Oval.

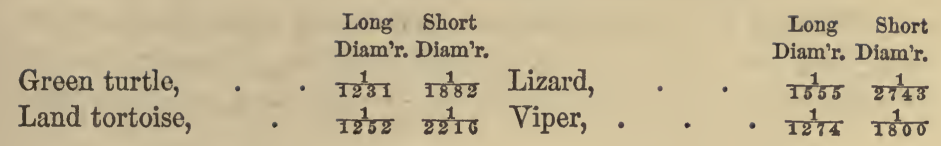

AMPHIBLA.

Corpuscles Oval.

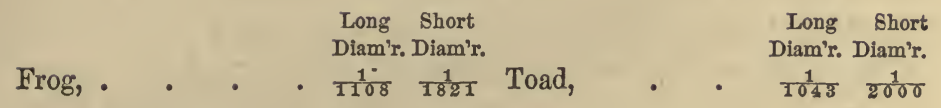

FISHES.

Corpuscles Oval.

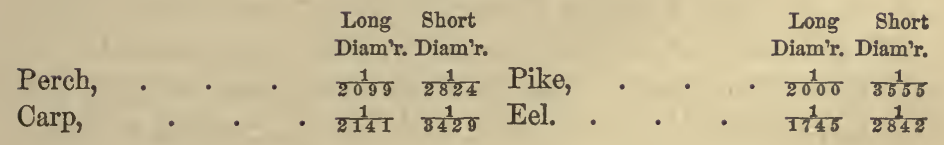

Post-mortem Changes of Blood-Corpuscles.-In examining the fiesh blood under the microscope, after the specimen has been under observation a short time, the corpuscles assume a peculiar appearance, from the development on their surface of very minute rounded projections, like the granules of a raspberry; indeed they are said by the French to become framboisées, which expresses the appearance very well. A little later, when they have become desiccated to a certain extent, they present a shrunken appearance, and their edges become serrated. Under these circumstances, their original form may be restored by adding to the specimen a liquid of the density of the serum. When they have been completely dried, as in blood spilled upon clothing or a floor, months or even years after, they can be made to assume their characteristic form by being carefully moistened with an appropriate fluid. This property is taken advantage of in examinations of old spots supposed to be blood; and if the manipu- 
lations be carefully conducted, the corpuscles may be reeognized without difficulty by the microscope. ${ }^{1}$

If pure water be added to a specimen of blood under the microscope, the corpuscles will first swell up, become spherical, and are finally lost to view by solution. The same effect follows almost instantaneously on the addition of acetic acid.

Structure.-The structure of the blood-corpuscles is very simple. They are perfectly homogeneous, presenting, in their normal condition, no nuclei nor granules, and are not provided with an investing membrane. A great deal has been said by anatomists concerning this latter point, and many are of the opinion that they are cellular in their structure, being composed of a membrane, with viscid, semi-fluid contents. Without going fully into the discussion of this point, it may be stated that few have assumed actually to demonstrate this membrane; but they have, for the most part, inferred its existence from the fact of the swelling, and as they term it, bursting on the addition of water ; and particularly, as it seems to me, to make the blood-corpuscles obey the theoretical laws of cell-development and nutrition laid down by Schwann. Their great elasticity, the persistence with which they preserve their bi-concave form, and their general appearance, would rather favor the idea that they are homogeneous bodies of a definite shape, than that they have a cell-wall with semi-fluid contents; especially as the existence of a membrane has been inferred rather than demonstrated. Their mode of nutrition is like that of any other anatomical elements: They are continually bathed in a nutritive fluid, the plasma, and as fast as their substance becomes worn out and effete, new material is supplied. In this way they undergo the same changes as other anatomical elements. When destroyed, or removed from the body in

${ }^{1}$ For full directions for the examination of blood stains, the reader is referred to an article on the medico-legal examination of spots of blood by Robin, in the Buffalo Medical Journal, 1857-'58. Vol. xiii., p. 555. 
hemorrhages, new corpuscles are gradually developed, until their quantity reaches the normal standard. Thus in the anemia which follows considerable loss of blood, the color gradually returns with the development of the corpuscles.

Chemical Characters.-In all chemical analyses of the blood-corpuscles, the proportions of dried constituents only are given. As we have seen in treating of organic-nitrogenized elements, such estimates give no idea of the actual proportions of the organic constituents of fluids or tissues. We must consider the corpuscles as organized bodies, consisting almost entirely of globuline, with which are combined a small quantity of hematine, or coloring matter, fat, and certain inorganic salts, from which it cannot be separated withont decomposition. The chemical characters of globuline have already been considered. ${ }^{1}$ The iron which the blood contains is regarded as existing in the hematine. Its presence can readily be demonstrated in a single drop of blood by adding nitric acid and evaporating, which reduces it to the condition of a per-oxide, when a red color is produced on the addition of the sulpho-cyanide of potassium. The iron is molecularly united with the other constituents, probably as iron, and not as an oxide, as has been supposed by some. ${ }^{2}$ The fat which is found in the corpuscles forms an exception to the general law regulating the condition of this principle in the tissnes, namely, that it is always uncombined with

1 Vide page 90.

${ }^{2}$ Crystals have long been observed in blood under certain circumstances. Sir Ererhard Home first observed them in the clots of aneurismal sacs in 1830. Since then they have been described by Scherer, Virchow, and others, and by many are supposed to be pure hematine, or the normal coloring matter of the red corpuscles. Robin and Verdeil, who have studied them very closely, do not consider these crystals as constituting a proximate principle, but as formed by an alteration of the hematine, consisting in the substitution of water for the iron. By careful analysis, these observers have failed to detect any iron entering into their composition. They are treated of in their "Chimie Anatomique," under Hornatö̈dine. Op. cit., tome iii., pp. 376 and 430, and Nysten's Dictionary, 1858. Hcematoïdine. 
other principles, existing as adipose tissue or in granules. Here it is molecularly united with the other elements.

In accordance with the invariable law, that the organic nitrogenized elements of the body are combined with inorganic principles, we find entering into the composition of the blood-corpuscles certain inorganic salts. These all exist in the plasma in about the same proportions as in the corpuscles. In short, as we shall see when we take up the composition of the entire blood, the corpuscles differ from the plasma only in the fact that they contain coloring matter and globuline, instead of fibrin and albumen, and that the fat is united with the organic matter instead of being in distinct granules. In all other respects their composition is nearly identical. We can thus appreciate how favorable their constitution and situation are for their nutrition at the expense of elements furnished by the plasma. ${ }^{1}$

Development of the Blood-Corpuscles.-Very early in the development of the ovum the blood-ressels appear, consti-

${ }^{2}$ Lehmann gives the following table showing the comparative composition of the corpuscles and plasma; the organic matters being desiccated.

1000 parts of Blood-Corpuscles contain:

Water, ....................... 688.00

Solid constituents, ................. $312 \cdot 00$

Specific Gravity, 1.0885.

Hematine, ........................16 16 \%

Globuline and cell-membrane, ........ 282*22

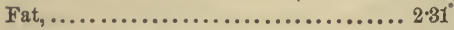

Extractive matters, ................. $2 \cdot 60$

Mineral substances (without iron), ..... $8 \cdot 12$

Chlorine, ............................1.686

Sulphuric Acid, . .................. 0.066

Phosphoric Acld, ..................1184

Potassium, ....................... 8 ${ }^{\bullet 828}$

Sodium, ........................... 1.052

Oxygen, ........................... 0.667

Phosphate of Lime, ................ 0.114

Phosphate of Magnesia, ............. 0.073

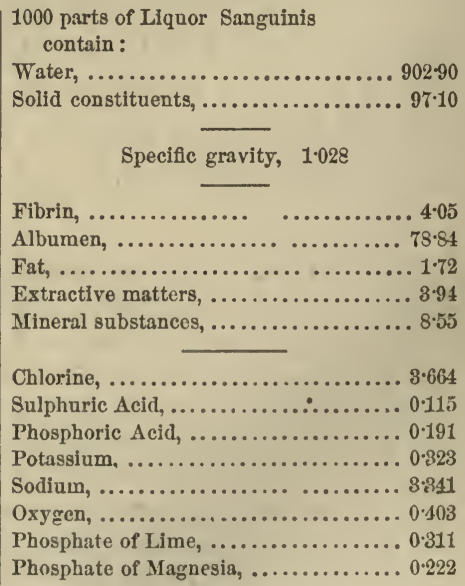
contain :

Water, ....................... 902990

Solid constituents, .............. $97 \cdot 10$

Specific gravity, 1.028

-Physiological Chemistry. Philadelphia, 1855; vol, i., p. 546. 
tuting what is called the area vasculosa. At first the vessels are filled with a colorless fluid, which soon becomes yellow, and when the embryo is about one-tenth of an inch in length, becomes red, and the corpuscles make their appearance. From this time until the sixth to the eighth week, they are from 30 to 100 per cent. larger than in the adult. Most of them are circular, but some are ovoid, and a few are globular. At this period, nearly all of them are provided with a nucleus; but from the first, there are some in which this is wanting. The nucleus is from $\frac{1}{7000}$ to $\frac{1}{8000}$ of an inch in diameter, globular, granular, and insoluble in water and acetic acid. As development advances, these nucleated corpuscles are gradually lost; but even at the fourth month we may still see a few remaining. After this time they present no anatomical differences from the blood-corpuscles in the adult.

In many works on physiology and microscopic anatomy, we find accounts of the development of the red corpuscles from the colorless corpuscles, or leucocytes, which are supposed to become disintegrated, their particles becoming developed into red corpuscles; but there seems to be no sufficient evidence that such a process takes place. The red corpuscles appear before the leucocytes are formed; ${ }^{1}$ and it is only the fact that the two varieties coexist in the bloodvessels which has given rise to such a theory. It is most reasonable to conisider that the red corpuscles are formed by a true genesis in the sanguineous blastema. We can offer no satisfactory explanation of the process by which the tissues. are formed from their blastema, nor can we explain the way in which the blood-corpuscles, which are true anatomical elements, take their origin. There is furthermore no sufficient evidence that any particular organ or organs have the function of producing the blood-corpuscles. Hewson supposed that they were formed in the spleen: Kölliker is of the opinion that they are destroyed in the spleen. It is

${ }^{2}$ Longet, Traité de Physiologie, tome i., p. 715. 
regarded by some as a necessity that there should be an organ for the destruction of the corpuscles, and one for their formation. Regarding them, as we certainly must, as organized bodies which are essential anatomical elements of the blood, it is difficult to imagine what reasons, based on their function, should lead physiologists to seek so persistently after an organ for their destruction. The hypothesis that they are used in the formation of pigment seems hardly sufficient to account for this.

In the present state of our science, the following seem to be the most rational views with regard to the development and nutrition of the blood-corpuscles :

1. At their first appearance in the ovum, they are formed by no special organs, for no special organs exist at that time, but appear by genesis in the sanguineous blastema.

2. When fully formed, they are regularly organized anatomical elements, subject, to the same laws of gradual molecular waste and repair as any of the tissues.

3. They are generated de novo in the adult, when diminished in quantity by hemorrhage or otherwise, and under these circumstances they are probably formed in the liquor sanguinis by the same process by which they take their origin in the ovum.

Function of the Blood-Corpuscles.-Though the fibrin and albumen of the plasma of the blood are essential to nutrition, the red corpuscles are the parts most immediately necessary to life. We have already seen, in treating of transfusion, that life may be resstored to an animal in which the functions have been suspended from hemorrhage, by the introduction of fresh blood; and while it is not necessary that this blood should contain fibrin, it has been shown by the experiments of Provost and Dumas and others, that the introduction of serum, without the corpuscles, has no restorative effect. When all the arteries leading to a part are ligated, the tissues lose their properties of contractility, sen- 
sibility, \&c., which may be restored, however, by supplying it again with the vivifying fluid. We shall see when we come to treat of the function of Respiration, that one great distinction between the corpuscular and fluid elements of the blood, is the great capacity which the former have for absorbing gases. Direct observations have shown that blood will absorb 10 to 13 times as much oxygen as an equal bulk of water. This is dependent almost entirely on the presence of the red corpuscles. ${ }^{1}$ As all the tissues are continually absorbing oxygen and giving off carbonic acid, a property which is immediately essential to a continuance of vitality, a great function of the corpuscles is to carry this principle to all parts of the body. In the present state of our knowledge, this is the only well-defined function which can be attributed to the red corpuscles, and it undoubtedly is the principal one. They have an affinity, though not so great, for carbonic acid, which, after the blood has circulated in the capillaries of the system, takes the place of the oxygen. In some experiments performed a few years ago on the effects of hemorrhage and the location of the "besoin de respirer," it was shown that one of the results of removal of blood from the system, was a condition of asphyxia, dependent upon the absence of these respiratory elements. ${ }^{2}$ The following may then be stated as the principal function of the red corpuscles of the blood:

They are respiratory organs; taking up the greater part of the oxygen which is absorbed by the blood in its passage through the lungs, and conveying it to the tissues, where it is given up, and its place supplied by carbonic acid.

Leucocytes, or White Corpuscles of the Blood.-In addition to the red corpuscles of the blood, this fluid always contains a number of colorless bodies, globular in form, in the sub-

${ }^{2}$ Robin and VERDeIL, op. cit., tome ii., p. 32.

'See an artiele by the Author in the American Journal of the DLedical Sciences, October, 1861. 
stance of which are embedded a greater or less number of minute granules. These have been called by Robin, Leucocytes. This name seems more appropriate than that of white or colorless blood-corpuscles, inasmuch as they are not peculiar to the blood, but are found in the lymph, chyle, pus, and various other fluids, in which they were formerly known by different names. All who have been in the habit of examining the animal fluids microscopically, must have noticed the great similarity existing between the corpuscular elements found in the above-mentioned situations. As microscopes have been improved, and as investigations have become more exact, the varieties of corpuiscles have been narrowed down. Now it is pretty generally acknowledged that the corpuscles found in mucus and pus are identical; also that there is no difference between the white corpuscles found in the lymph, chyle, and blood; and finally, the recent investigations of Robin have shown that all of these bodies, which were formerly supposed to present marked distinctive characters, belong to the same class, presenting but slight differences in different situations. The description which will be given of the white corpuscles of the blood, and the effects of reagents upon them, will answer, in the main, for all that are grouped under the name of Leucocytes. ${ }^{3}$

Leucocytes are normally found in the Blood, Lymph, Chyle, Semen, Colostrum, and Vitreous Humor. Pathologically they are found in the secretion of mucous membranes, after the slightest irritation, and in inflammatory products, when they are called pus corpuscles.

In examining a specimen of blood with the microscope, we immediately notice the marked difference between the leucocytes and red corpuscles. The former are globular, with a smooth surface, but rendered somewhat opaque by

1 For a full account of the Anatomy and Physiology of these bodies, the reader is referred to an elaborate article on this subject by Robin in the Journal de la Physiologie, tome ii., p. 41, and the article "Leucocyte" in Nysten's Dictionary, Paris, 1858. 
the presence of more or less granular matter, white, and larger than the red corpuscles.

In examining the circulation under the microscope, we are struck with the adhesive character of the leucocytes as compared with the red corpuscles. The latter circnlate with wonderful rapidity in the centre of the vessel, while the leucocytes have a tendency to adhere to the sides, moving along slowly, and occasionally remaining for a time entirely stationary, until they are swept along by a change in the direction or force of the current.

Their size varies somewhat, even in any one fluid, as the blood. Their average diameter may be stated as $\frac{1}{2500}$ of an inch. It is in pus, where they exist in greatest abundance, that their microscopic characters may be studied with greatest advantage. In this fluid, after it is discharged, the corpuscles sometimes present remarkable deformities. They become polygonal in shape, and sometimes ovoid; occasionally presenting projections from their surface, which give them a stellate appearance. These alterations, however, are only temporary; and after from twelve to twentyfour hours, they resume their globular shape. On the addition of acetic acid they swell up, become transparent with a delicate outline, and present in their interior one, two, three, or even four rounded nuclear bodies generally collected in a mass. This is rather to be considered as a coagulation of a portion of the corpuscle, than a nucleus brought out by the action of the acid, which renders the corpuscle transparent; though in some it is seen through the granules without the addition of any reagent. This appearance is produced, though more slowly, by the addition of water.

Leucocytes vary considerably in their external characters in different situations. Sometimes they are very pale and almost without granulations, while at others they are filled with fatty granules, and are not rendered clear by acetic acid. As a rule, they increase in size and become granular when confined in the tissues. In colostrum, when they are 
called colostrum corpuscles, they generally undergo this change. ${ }^{1} \quad$ As the result of inflammatory action, when they are sometimes called inflammatory or exudation corpuscles, leucocytes frequently become much hypertrophied, and are filled with fatty granules. They always retain, however, general characters by which they may be recognized.

Development of Leucocytes.-These corpuscles appear in the blood-vessels very early in fotal life, before the lymphatics can be demonstrated. They arise in the same way as the red corpuscles, by genesis from materials existing in the vessels. They appear in lymphatics, before we come to the lymphatic glands, and in the fœetus anterior to the development of the spleen, and also on the surface of mucous membranes; so they cannot be considered as produced exclusively by these glands, as has been supposed. There is no organ nor class of organs in the body specially charged with their formation; and though frequently a result of inflammation, this process is by no means necessary for their production. Robin ${ }^{2}$ has carefully noted the phenomena of their development in recent wounds. The first exudation consists of clear fluid, with a few red corpuscles; then a finely granular blastema. In from a quarter of an hour to an hour, pale transparent globules, $\frac{1}{8000}$ to $\frac{1}{6000}$ of an inch in diameter, make their appearance, which soon become finely granular, and present the ordinary appearance of leucocytes. They are thus developed, like other anatomical elements, by

${ }^{1}$ Colostrum is the discharge from the mammary glands, occurring during the first few days after delivery, which precedes the full establishment of the lacteal secretion. It is a serous fluid, rather clear, which presents, on microscopical examination, a few milk globules, large drops of oil, rounded masses of small fatty granules, and enlarged and granular leucocytes, called colostrum corpuseles, as well as those which have undergone no alteration. These gradually disappear, as the secretion is established, and their place is supplied by the milk globules. (See "Colostrum," Nysten's Dictionary, by Littré and Robin; Paris, 1858.)

${ }^{2}$ Loc. cit. 
organization of the necessary elements furnished by a blastema, and not by the action of any special organ or organs.

The quantity of leucocytes compared to the red corpuscles can only be given approximately. It has been estimated by counting under the microscope the red corpuscles and leucocytes contained in a certain space. Moleschott ${ }^{1}$ gives the proportion as 1:335; others at from $1: 300$ to $1: 500$. It has been found by Dr. E. Hirt, of Zittau, whose observations have been confirmed by others, that the relative quantity of leucocytes is much increased during digestion. He found in one individual a proportion of 1: 1800 before breakfast; an hour after breakfast, which he took at 8 o'clock, 1 : 700 ; between 11 and 1 o'clock, 1: 1500; after dining at 1 o'clock, 1:400; two hours after, 1: 1475; after suipper at 8 P.M., $1: 550$; at $11 \frac{1}{2}$ P. M., $1: 12000^{2}$ The leucocytes are much lighter than the red corpuscles, and when the blood coagulates slowly, are frequently found forming a layer on the surface of the clot, which is called the "buffy coat."

Numerous observers, among whom may be mentioned Donné, Kölliker, Gray, and Hirt, ${ }^{3}$ have noticed a great increase in the number of leucocytes in the blood coming from the spleen, and have supposed that they are chiefly manufactured in this organ. It is inconsistent with the mode of development of these corpuscles to suppose that any special organ is exclusively engaged in their production; and their persistence in animals after extirpation of the spleen shows that they are developed in other situations.

The function of the leucocytes is not understood. The supposition that they break down and become nuclei for the development of red corpuscles, which at one time obtained, is a pure hypothesis, and has no basis in fact.

${ }^{1}$ Kölliker, Manual of Microscopic Anatomy, London, 1860, p. э21.

2 Milne-Edwards, Lecons sur la Physiologie et l'Anatomie Comparée, tome i., p. 350 .

${ }^{3}$ Ibid., p. 353. 
Elementary Corpuscles. - Little granules are found in the blood, especially during digestion, which, as they were supposed to take part in the formation of the white corpuscles, have been called elementary granules or corpuscles. They are little fatty particles of the chyle which come from the thoracic duct, and are not positively known to have any connection with the formation of the other corpuscular elements of the blood. 


\section{CHAPTER II.}

\section{COMPOSITION OF THE BLOOD.}

General considerations-Methods of quantitative analysis-Fibrin-CorpusclesAlbumen-Inorganic constituents-Sugar-Fatty emulsion-Coloring matter of the serum-Urea and the Urates-Cholesterine-Creatine-Creatinine.

Assuming, as we certainly must, that the blood furnishes material for the nourishment of all the tissues and organs, we expect to find entering into its composition all the proximate principles existing in the body which undergo no change in nutrition, like the inorganic principles, and organic matters which are capable of being converted into the organic elements of every tissue. Furthermore, as the products of waste are all taken up by the blood before their final elimination, these also should enter into its composition. With these great principles in our minds, it is unnecessary to insist upon the importance of accurate proximate analyses of the circu. lating fluid. . It is not many years that our knowledge of the laws of nutrition and destructive assimilation have enabled us to appreciate the full importance of the blood; but it has been so palpable that this fluid is necessary to life, that the older physiologists made numberless futile attempts to obtain some clear idea of its composition. We have only to go back to the beginning of the present century to find the first analyses of the blood which were attended with any degree of success. In 1808, Berzelius analyzed the serum of the 
human blood, indicating eertain proportions of albumen, lactate of soda, muriate of soda, etc.; he was followed by Marcet in 1811, by whom his observations were confirmed. In 1823, Provost and Dumas published their elaborate researches into the composition of the blood, which seemed to give an impulse to investigations in this direction, and were soon followed by the analyses of Andral and Gavarret, Lehmann, Simon, Becquerel and Rodier, Denis, and a host of others, whose labors have made us comprehend some of the most important laws which regulate the general processes of nutrition.

Notwithstanding the immense amount of labor bestowed by the most eminent chemists of the day upon the quantitative analysis of the blood, and the great physiological interest attaching to every advance in our knowledge in this direction, the difficulties in the way are so great, that even now there are no analyses which give the exact quantities of each of its inorganic constituents. This is owing to the great difficulty in the analysis of any fluid in which inorganic and organic principles are so closely united; for there is no more delicate problem in analytical chemistry than the determination of the presence and quantities of inorganic substances united with organic matter. Of the animal fluids which are easily obtained, the blood, from the large proportion of different organic principles which enter into its composition, presents the greatest difficulties to the analytical chemist. Another difficulty presents itself in the necessity of a proximate, and not an ultimate analysis. It is not sufficient to give the amount of certain chemical elements which the blood contains; we must ascertain the amount of these elements in the state of union with each other to form proximate principles.

Analyses have shown that the constituents of the blood may be divided into:

1. Inorganic Constituents.-These exist in a state of intimate and molecular union with the organic-nitrogenized ele- 
ments. Their presence is indicated by the appropriate tests applied to the residue of the blood after incineration, which show the well-known reactions of the chlorides, sulphates, phosphates, and carbonates, with sodium, potassium, lime, magnesia, and iron. In addition we have certain gases (oxygen, nitrogen, and carbonic acid), which may be extracted by the air-pump or by displacement.

2. Organic, Non-nitrogenized Constituents. - These are the sugars and fats; which are separated from the other elements without much difficulty, and may be recognized by their peculiar properties.

3. Organic, Nitrogenized Constituents.-These constitute the greater part of the blood, and are inseparably connected, in their functions, and as a condition of existence, with the inorganic principles. They may be extracted by processes already described in treating of fibrin, albumen, 'and globuline, and recognized by their peculiar properties.

Most of the constituents of the blood are found both in the corpuscles and plasma. It is difficult to determine the different constituents of these two parts of the blood. It has been shown, however, by Schmidt, of Dorpat, that the phosphorized fats are more abundant in the globules, while the fatty acids are more abundant in the plasma. The salts with a potash base have been found by the same observer to exist almost entirely in the corpuscles, and the soda salts are four times more abundant in the plasma than in the corpuscles, ${ }^{2}$ All the iron exists in the red corpuscles.

The proportions of the various constituents of the blood are subject to certain variations. These points, with their relations to the tissues in the processes of nutrition, have been so fully taken up in the consideration of Proximate Principles, that they do not demand special notice in this

${ }^{1}$ Milne-Edwards, Leçons sur la Physiologie, ete., tome i., p. 225. 
connection. In addition to the nutritive principles, we have entering into the composition of the blood, urea, cholesterine, urate of soda, creatine, creatinine, and other substances, the characters of which are not yet fully determined, belonging to the class of Excrementitious Principles. Their consideration comes more appropriately under the head of Excretion, and they will be fully taken up in the chapter devoted to that subject. Though a knowledge of the exact proportions of the various elements of the blood is not necessary in order to appreciate the relations of this fluid to the tissues, the great interest which is attached to this line of investigation, and the important advantages which we may look for in the future from extended inquiry in this direction, lead ns to discuss at some length the methods which have been employed by physiological chemists in quantitative analyses, with some of the results which have already been obtained.

\section{Quantitative Analysis of the Blood.}

The methods which have been, and are now, commonly employed for quantitative analysis of the blood vary very little from the process recommended by Provost and Dumas in 1823. They are based upon the supposition that the organic constituents, fibrin and albumen, are solid substances in solution in the watery elements, and that all the water of the blood is to be attributed to the serum. As we have shown in treating of organic substances that this view of their condition in the fluids is erroneons, and that the desiceated materials obtained from the blood do not represent the real quantities of its organic elements, a new method of analysis, based on the view that these principles are naturally fluid, seems necessary. The same process has been employed for the estimation of the proportion of corpuscles. Here the error is too manifest to require discussion. It is evident that the blood-corpuscles are semi-solid bodies which become altered by desiccation; and an estimate which does not give 
their weight in their natural moist condition, gives us no idea of their real proportion. So apparent has this been to physiological chemists, that attempts have been made by Denis, Schmidt, Vierordt, Figuier, and others to estimate the moist corpuscles; but in attempting to attain extreme accuracy, these observers have almost entirely failed, and their ideas of the real proportion of the corpuscles are merely conjectural. These-remarks only apply to researches into the organic constituents of the blood. The analyses with reference to the inorganic elements, though they have not yet shown us the exact proportion of each one of them, are of course accurate as far as they go.

The various processes for analysis of the blood now employed by chemists do not differ very much. As one of the best, we may take that recommended by Becquerel and Rodier, who are perhaps as high authority on this subject as any. Their process, which we give in its essential particulars, has an advantage over most others in simplicity.

Two specimens of blood are taken and carefully weighed; one of them is defibrinated, the fibrin collected, dried, and weighed, which gives the proportion of fibrin. The other is set aside to coagulate. A known weight of the defibrinated blood is then evaporated to dryness, and the proportion of dry residue carefully estimated. The residue is then calcinated to give the proportions of inorganic constituents, which remain after the organic matters have become volatilized. After the blood set aside to coagulate has separated into clot and serum, a definite quantity of the serum is evaporated to dryness and the residue estimated. As the dry residue of the defibrinated blood contains the solid matters of the serum + the dried corpuscles-the proportion per 1,000 parts of the solid matters of the defibrinated blood-the proportion per 1,000 parts of the solid matters of the serum, would give the proportion of corpuscles.

We thus have obtained the proportions of water, of inorganic matter, of corpuscles, and of fibrin. The next step is 
to estimate the albumen, fatty, and extractive matter. For this purpose we desiccate a known quantity of serum, carefully pulverize the dry residue, and treat it repeatedly with boiling water till it has washed out all soluble matters. These are undetermined extractive matters, and free salts in solution in the serum. The residue, thus treated with boiling water, is desiccated and treated several times with boiling alcohol, which dissolves all the fatty substances. The insoluble residue is then dried and weighed, and represents pure albumen, which, it will be remembered, is not affected by boiling water or alcohol. The loss after treating with boiling alcohol gives the quantity of fatty matters. The proportions of inorganic matters are obtained by analysis of the residue after incineration. It is unnecessary to describe the complicated and difficult manipulations involved in this process. ${ }^{1}$

1 The above is condensed from Becquerel and Rodier, "Traité de Chimie Pathologique appliquée à la Médecine Pratique," Paris, 1854, page 21 et seq. As the result of analyses of the blood of twenty-two healthy persons, they give the following table, page 86 . The list of inorganic salts is taken from pages 65,66 , and 67 .

Denstty of tile BLood .................. 1060

COMPOSITION.

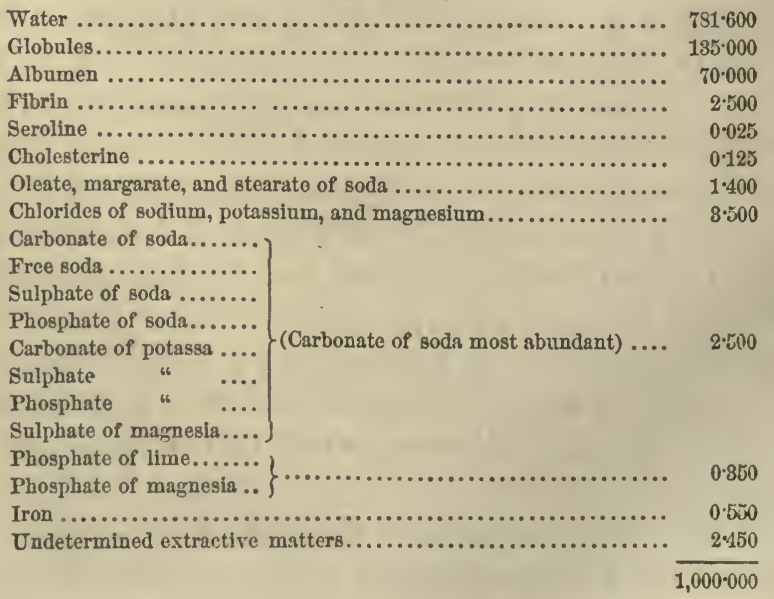


The above process is perhaps as simple and reliable as any; but of course each chemist has some slight modifications. By some the globules are estimated by drying the clot after coagulation and deducting the weight of the fibrin. Some recommend to expose the fibrin after desiccation to incineration, and deduct the weight of the residue of inorganic matter. All of the processes, however, are materially the same, and differ but little from that employed by Provost and Dumas. As before remarked, the results, as regards the fatty and inorganic constituents of the blood, are as accurate as possible with our present means of investigation; and the comparative results, in analyses of the blood for fibrin, albumen, and corpuscles in health and disease, which have crowned the labors of Andral and Gavarret, Becquerel and Rodier, and a number of others, are of permanent value. But a glance at the process, and the quantities given for the fibrin, albumen, and corpuscles, indicate that the whole is inconsistent with our ideas of the condition under which these substances exist in the body. Microscopic examination shows that at least one-half the mass of the blood consists of corpuscles, while analysis gives only 135 parts per 1,000. The fibrin of the blood is sufficient to entangle, as it coagulates, all the corpuscles, and with them form the clot; yet we are told that its proportion is 2.5 parts per 1,000 . We boil the serum, the albumien changes from a fluid to a semi-solid condition, and the whole mass is solidified; yet the estimate of its proportion is 70 parts per 1,000 . The fact is that these estimates give us only the dry residue of the organic principles; and to form an idea of their actual proportion, we should estimate them, if possible, with their water of composition, and united with the inorganic salts, which cannot be separated from them without incineration and consequent destruction.

With this end in view, and forwant of a better process, we may employ the following mode of analysis, which is easy of application, and sufficiently accurate for all practical purposes. ${ }^{1}$

${ }^{1}$ See an article by the author, on The Organic Nitrogenized Principles of the 
The blood to be analyzed is taken from the arm, and received into two carefully weighed vessels. The quantity in each vessel may be from two to four ounces. One of the specimens is immediately whipped with a small bundle of broom-corn, previously moistened and weighed, so as to collect the fibrin; and after the fibrin is completely coagulated, the whole is carefully weighed, deducting the weights of the vessel and broom-corn, which gives the weight of the specimen of blood used. The other specimen is set aside to coagulate.

The first specimen is used in the estimation of the fibrin and corpuscles; the second is set aside to coagulate, and is used to estimate the albumen. It is important to cover the vessels as soon as the blood is drawn, for, as has been demonstrated by Beequerel and Rodier, blood exposed to the air loses weight rapidly by evaporation. ${ }^{1}$

We now pass the first specimen of blood through a fine sieve to collect any fibrin that may not have become attached to the wisp, strip the fibrin from the wisp, and wash it under a stream of water. This may be done very rapidly if we cause the water to flow through a small strainer, by which it is broken up into a number of little streams, and knead the fibrin with the fingers, doing this over a sieve so as to catch any particles that may become detached. In this way it may be freed from the corpuscles in five or ten minutes. The fibrin is then freed from most of the adherent moisture by bibulous paper, and weighed as soon as possible. By the following formula we estimate the proportion per 1,000 parts of blood :

Weight of blood used : Weight of fibrin : : 1,000 : Fibrin per 1,000 .

The next step is to estimate the corpuscles. For this purpose a portion of the defibrinated blood, which is carefully

Body, with a New Method for their Estimation in the Blood, American Journal of the Medical Sciences, October, 1863.

${ }^{1}$ Op. cit., p. 31. 
weighed, is mixed with twice its volume of a saturated solution of sulphate of soda, and thrown upon a filter which has been carefiully weighed and moistened with distilled water, and .also, just before receiving the mixture of blood and sulphate of soda, with the saline solution. The fluid which passes through should be about the color of the serum; if a tew corpusles pass at first, the liquid should be poured back until it becomes clear. The funnel is then covered, and the fluid allowed to separate, the blood-corpuscles being retained on the filter. The filter and funnel are then plunged several times into a vessel of boiling water, by which all the sulphate of soda which remains is washed out, and the corpuscles are coagulated without changing in weight. The funnel should be again covered and the water allowed to drip from the filter, after which it is weighed, deducting the weight of the moist filter previously obtained, which gives us the weight of the corpuscles. We obtain the proportion of corpuscles to 1,000 parts of blood by the following formula:

Defibrinated blood used : Corpuscles : : Defibrinated blood per 1,000 : Corpuscles per 1,000.

The next step is to estimate the quantity of albumen in the serum, and thence its proportion in the blood. For this purpose we first ascertain the quantity of serum in 1,000 parts of blood, which is done by subtracting the sum of the fibrin and corpuscles per 1,000 from 1,000. Having done this, and waited ten or twelve hours for specimen No. 2 to separate completely into clot and serum, we take a small quantity of the serum, about half an ounce, weigh it carefully, and add suddenly twice its volume of absolute alcohol. The albumen will be thrown down in a grumous mass, and the whole is thrown upon a filter, which has been previously moistened with alcohol and weighed. The funnel is immediately covered, and the fluid separates from the albumen very rapidly. We ascertain that no fluid albumen passes through the filter by testing the fluid with nitric acid. After 
the filter has ceased to drip, it is weighed, and the weight of the albumen ascertained by deducting the weight of the filter. The proportion of albumen to 1,000 parts of blood is obtained by the following formula:

Serum used : Albumen : : Serum per 1,000 : Albumen per 1,000 .

The above process, which has been described in detail in the hope that it may be employed by others in analysis of the blood for its organic constituents, has at least the advantage of simplicity and facility of application. As regards accuracy, having repeatedly made analyses of different portions of the same fluid with almost identical results, it has seemed sufficiently exact for all practical purposes. As an example we may mention an analysis of two equal portions of defibrinated blood (34.20 grammes) for corpuscles; one specimen gave 16.40 , and the other 16.43 grammes. This part of the process would seem more open to the objeetion of inaccuracy than any, yet the difference of the result in the two analyses is so slight that it may be disregarded. Repeated examinations of different specimens of the same serum for albumen were followed by identical results. ${ }^{1}$ While the exceeding accuracy which is desired by chemists, and is necessary in many analyses, is not attainable in such examinations as these, it is not even desirable; for as physiologists we must see that even an approximation of the proportions of the organic matters, as they really exist, is better than the most accurate estimate of their dry residue. In taking the weights, the only point is to do it rapidly and avoid loss by evaporation. If this be borne in mind, and care be taken in different examinations to weigh the principles at the same stage of the operation, the simplicity of the process should make it valuable in comparative analyses of the blood in different conditions of the system.

In estimating the proportion of fibrin, the ordinary ${ }^{1}$ American Journal of the Medical Sciences, loc. cit. 
method is followed, with the exception that the weight of the moist fibrin is taken instead of the dry residue.

In estimating the corpuscles, after a number of trials, the process recommended by Figuier was adopted, with a similar modification. Figuier dried the corpuscles after separating them from the serum by filtration, taking advantage of the property of sulphate of soda, which retains them on the filter. He employed this method to separate the corpuseles completely, and investigate their chemical constitution. ${ }^{1}$

In estimating the albumen, the object was, as in the case of the other principles, to obtain.it as nearly as possible in its natural condition, simply changing its form from fluid to semi-solid, without adding any thing which would decompose it, or unite with it. For this purpose absolute alcohol seemed better than heat, nitric acid, the galvanic current, or any other agents by which it is coagulated.

If the different organic principles be incinerated, the ash will present the characteristic reactions of the chlorides, sulphates, phosphates, etc., inorganic principles, which, as we have already seen, cannot be separated from the organic constituents of the body without destruction of the latter.

The blood of a healthy male, æt. 27 years, weight 170 pounds, who had never suffered from disease, taken from the arm at 1 P. .r., the last meal having been taken at 8 . . .., furnished the proportions of organic constituents given in the following table. To complete the table, the proportions of inorganic principles, fats, etc., were taken from the analyses of Becquerel and Rodier, to which reference has already been made. The proportion of water is estimated by subtracting the sum of the solid and semi-solid constituents from the entire weight of the blood. ${ }^{2}$

1 Sur une Méthode nouvelle pour l'Analyse du Sang, et sur la Constitution chimique des Globules sanguins. Par M. L. Figurer. (Ann. de Chim. et de Phys., 1844, $3^{\mathrm{m} \theta}$ série, tome xi., p. 506.)

${ }^{2}$ Further details of experiments on this subject are contained in the article, to which reference has been made, in the "American Journal," October, 1863. 


\section{Composition of the Blood.'}

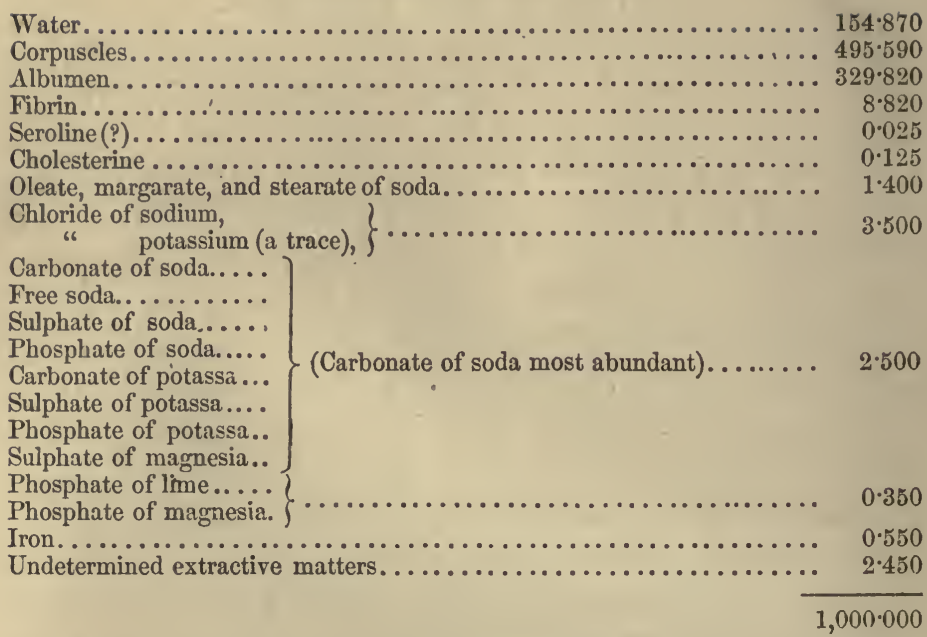

There exist in the blood certain well-determined principles not given in the above table, some of which have great physiological importance; and it is to be expected that further investigations will reveal others, among what are now called extractive matters, an acquaintance with which will materially advance our pathological, as well as our physiological knowledge of this fluid. The developments of the last few years with regard to urea and cholesterine lead us to look for the discovery of new principles, variations from the normal proportions of which will, perhaps, be found to constitute important pathological conditions. In both a physiological and pathological point of view, there is much to be done in this line of investigation.

Aside from the gases, we are now acquainted with the

1 For purposes of comparison, the fibrin, albumen, and corpuscles were desiccated and weighed, giving the following proportions of dry residue:

Fibrin, $\quad 2 \cdot 50$ parts per 1,000 of fresh blood.

Albumen, 71.53 do. do.

Corpuscles, $125^{\circ} 00$ do. do. 
following additional principles in the blood, which are either constant or temporary constituents : Sugar, Fatty Emulsion, a Coloring Matter peculiar to the serum, Urea, Uric Acid in combination, Cholesterine, Creatine, and Creatinine.

Sugar.-Bernard ${ }^{1}$ showed in 1848 that sugar always exists in the blood of the hepatic veins and the right side of the heart. It is manufactured by the liver, and disappears in the lungs. When its production is most active, as in full digestion, it may exist in small quantity in the arterial blood. Ordinarily it is only to be found in the blood between the liver and the lungs, except when it exists in the blood of the portal vein, after the ingestion of saccharine or starchy matters.

Fatty Emulsion.-After a full meal with an abundance of fat, the blood contains a considerable proportion of fatty emulsion. Bernard ${ }^{2}$ has shown, also, that the blood of the hepatic veins contains an emulsive substance which is formed by the liver. We have already seen that the blood corpuscles contain a certain proportion of fatty matter in a state of molecular union with the organic nitrogenized principles.

Coloring Matter of the Serum.-The serum has a yellowish color, more or less intense, which is dependent upon a peculiar coloring matter. This has never been isolated, but is thought by some to be identical with the coloring matter of the bile, ${ }^{3}$ a supposition, however, which does not seem very probable.

${ }^{1}$ Recherches sur une Nouvelle Fonction du Foie considéré comme Organe Producteur de Matière Sucrée chez THomme et les Animaux. Thèse. Paris, 1853.

${ }^{2}$ See page 64.

s Becquerer and Rodier, Recherches sur la Composition du Sang dans Tétat de Santé et dans l'état de Maladie, Paris, 1844. 
Urea and the Urates.-In 1823 Provost and Dumas ${ }^{1}$ discovered urea in the blood of animals from which the kidneys had been removed; which was the first experimental demonstration that this principle is formed in the system and eliminated by, not manufactured in, the kidneys. It was demonstrated in healthy blood by Marchand, ${ }^{2}$ in 1838, and since then has been recognized as one of its normal constituents, though existing in very minute quantity. These observations have been confirmed by numerous French, German, and English physiologists. The urate of soda also exists in small quantity in the blood, and possibly the hippurate of soda. The reason why the proportion of these principles is so small, is that they are eliminated by the proper organs as soon as formed.

Cholesterine.-This substance was demonstrated in the blood by Denis in $1830 .^{3}$ It is now known to exist in this fluid in considerable quantity. It is most abundant in the blood coming from the nervous centres, where it is produced in great part, and is diminished in the passage of the blood through the liver. ${ }^{1}$

A substance was described by Boudet in 1833, in the blood, which he called Seroline. Its existence in the blood is problematical. ${ }^{\circ}$

Creatine and Creatinine.-Verdeil and Marcet have demonstrated the presence of these substances in the blood. Their proportion is very small, and has not been determined. They undoubtedly have the same relation to the system as urea and cholesterine.

1 Annales de Chimie et de Physique, 1821, tome xviii., p. 280.

2 Annales des Sciences Naturelles, 1838, 2me série, tome x., p. 46.

${ }^{3}$ Robin and Verdeil, op. cit., tome ii., page 63.

4 See an article by the author on a New Excretory Function of the Liver, American Journal of the Mcdical Sciences, October, 1862.

${ }^{b}$ Ibid.

${ }^{3}$ Robin and Verdeil, Chimie Anatomique, tome ii., pp. 480 and 439 
A consideration of abnormal or accidental constituents of the blood, such as poisonous or medicinal substances, does not belong to its physiological history. It is hardly necessary to mention certain substances, the existence of which is doubtful, such as lactic acid, copper, magnesia, etc. 


\section{CHAPTER III.}

COAGULATION OF THE BLOOD.

General considerations-Characters of the clot-Characters of the serum-Coagulating principle in the blood-Circumstances which modify coagulation-Coagulation of the blood in the organism-Spontaneous arrest of hemorrhageCause of coagulation of the blood-Summary of the properties and functions of the blood.

THe remarkable property in the blood of spontaneous coagulation has been commonly recognized as far back as we can look into the history of physiology; and since the immortal discovery of Harvey, which naturally gave an impulse to investigations into the properties of the circulating fluid, there have been few subjects connected with the physiology of the blood which have excited more universal interest. At first, the ideas with regard to the cause of this phenomenon were entirely speculative. The first definite experiments on record were performed by Malpighi and published in 1666. He was followed by Borelli, Ruysch, and a host of others who hold conspicuous places in the history of our science; among whom may be mentioned Hunter, Hewson, Müller, Thackrah, J. Davy, Magendie, Nasse, and Dumas. While much labor has been expended on this subject, the final cause of coagulation cannot even now be said to be settled beyond question.

The blood retains its fluidity while it remains in the vessels, and circulation is not interfered with. It is then com- 
posed, as we have seen, of clear plasma, holding corpuscles in suspension; but these little bodies do not differ much from the plasma, either in consistence or specific gravity, and give to the fluid only a slight degree of viscidity. Shortly after the circulation is interrupted, or after blood is drawn from the vessels, it coagulates or "sets" into a jelly-like mass. In a few hours we find that contraction has taken place, and a clear, strâw-colored fluid has been expressed, the blood thus separating into a solid portion, the crassamentum or clot, and a liquid, which is called serum. The serum contains all the elements of the blood except the red corpuscles and fibrin, which together form the clot. Coagulation takes place in the blood of all animals, commencing a variable time after its removal from the vessels. In the human subject, according to Nasse, ${ }^{1}$ when the blood is received into a moderately deep, smooth vessel, the phenomena of coagulation present themselves in the following order:

First; a gelatinous pellicle forms on the surface, which occurs in from 1 minute and 45 seconds to 6 minutes; in from 2 to 7 minutes a gelatinous layer has formed on the sides of the vessel; the whole mass becomes of a jelly-like consistence in from 7 to 16 minutes. Contraction then begins, and if we watch the surface of the clot we will see little drops of clear serum making their appearance. This fluid increases in quantity, and in from 10 to 12 hours separation is complete. The clot, which is heavier, sinks to the bottom of the vessel, unless it contain bubbles of gas, or the surface be very concave. In most of the warm-blooded animals the blood coagulates more rapidly than in man. It is particularly rapid in the class of birds, in some of which it takes place almost instantaneously. Observations have shown that coagulation is more rapid in arterial than in venous blood. In the former the proportion of fibrin is notably greater. 
The relative proportions of the serum and clot are very variable, unless we include in our estimate of the serum that portion which is retained between the meshes of the clot. ${ }^{2}$ As the clot is composed of corpuscles and fibrin, and as these in their moist state represent in general terms about one-half of the blood (see table; page 138), it may be stated that after coagulation, the actual proportions of the clot and serum are about equal. If we take simply the serum which separates spontaneously, we have a large quantity when the clot is densely contracted, and a very small quantity when it is loose and soft. ${ }^{2}$

Characters of the Clot.-On removing the clot, after the separation of the serum is complete, it presents a gelatinous consistence, and is more or less firm, according to the degree of contraction which has taken place. As a general rule, when coagulation has been rapid, the clot is soft and but slightly contracted. When, on the other hand, coagulation has been slow, it contracts for a long time, and is much denser. When coagulation is slow, the clot frequently presents what is known as the cupped appearance, having a concave surface, a phenomenon which merely depends on the extent of its contraction. It also presents a marked differ-

${ }^{1}$ It is estimated by Milne-Edwards that the clot retains, in most instances, one-fifth of the entire volume of serum. Legons sur la Physiologie, etc., tome i, p. 124.

2 According to Thackrah the fcllowing are the periods required for the coagulation of the blood in some of the inferior animals:

$\begin{array}{llccccc}\text { Horse, Blood coagulates in from } & 5 & \text { to } & 13 & \text { minutes } \\ \text { Ox, " } & \text { " } & \text { " } & 2 & 12 & \text { " } \\ \text { Dog, " } & \text { " } & \text { " } & \frac{1}{2} & 3 & \text { " } \\ \text { Sheep, " } & \text { " } & \text { " } & \frac{1}{2} & 1 \frac{1}{2} & \text { " } \\ \text { Hog, " } & \text { " } & \text { " } & \frac{1}{2} & 1 \frac{1}{2} & \text { " } \\ \text { Rabbit, " } & \text { " } & \text { " } & \frac{1}{2} & 1 \frac{1}{2} & \text { " } \\ \text { Lamb, " } & \text { " } & \text { " } & \frac{7}{2} & 1 & \text { " } \\ \text { Duck, " } & \text { " } & \text { " } & 1 & 2 & \text { " } \\ \text { Fowl, " } & \text { " } & \text { " } & \frac{1}{2} & 1 \frac{1}{2} & \text { " } \\ \text { Pigeon, " } & \text { " } & \text { almost instantaneously. }\end{array}$


ence in color at its superior portion. The blood having remained fluid for some time, the red corpuscles settle, by virtue of their greater weight, leaving a colorless layer on the top. This is the buffy coat so frequently spoken, of by authors. The buffed and cupped appearance of the clot has been supposed to indicate an inflammatory condition of the circulating. fluid; inasmuch as the quantity of fibrin is generally increased in inflammation, and the greater the quantity of fibrin the more rapid is the gravitation of the red corpuscles. Though this frequently presents itself in the blood drawn in inflammations, it is by no means pathognomonic of this condition, and is liable to occur whenever coagulation is slow, or retarded by artificial means. It is always present in the blood of the horse. Examined microscopically, the buffy coat presents fibrils of coagulated fibrin with some. of the white corpuscles of the blood. On removing a clot of venous blood from the serum, the upper surface is florid from contact with the air, while the rest of it is dark; and on making a section, if the coagulation has not been too rapid, the gravitation of the red corpuscles is apparent. The section, which is at first almost black, soon becomes red from contact with the atmosphere. The clot from arterial blood has a dark-red color. If the clot be cut into small pieces, it will undergo further contraction, and express a part of the contained serum. "If the clot be washed under a stream of water, at the same time kneading it with the fingers, we may remove almost all the red corpuscles, leaving the meshes of fibrin, which, on microscopic examination, will present the fibrillated appearance to which we have already referred. This is a method sometimes employed for the extraction of the fibrin. It was in this way that fibrin was isolated by Malpighi; who made the first experiments which rendered it probable that coagulation of the blood depended upon this principle. In a few days, as the result of putrefaction, the clot softens, mixes with the serum, and the blood regains its fluidity. 
Characters of the Serum.-After coagulation, if the serum be carefully removed, it is found to be a fluid of a color varying from a light amber to quite a deep, but clear, red. This depends upon a peculiar coloring matter, distinct from hematine, but which has never been isolated. The specific gravity of the serum is somewhat less than that of the entire mass of blood; being, according to Becquerel and Rodier, about 1,028. ${ }^{1}$ It contains all the principles found in the plasma, or. liquor sanguinis, with the exception of the fibrin. It can hardly be called a physiological fluid, as it is formed only after coagulation of the blood, and never exists isolated in the body. The effusions which are commonly called serum, though they resemble this fluid in some particulars, are not identical with it, being formed by a process of transudation rather than separation of the blood, as in coagulation. We have already seen that; in the body, fibrin and albumen are in combination, and that the organic principle of the serum (albumen) when injected into the ressels of a living animal does not beenme assimilated, but is rejected by the kidneys. The serum must not, therefore, be confounded with the plasma or liquor sanguinis, which is the natural clear portion of the blood.

Coagulating Principle in the Blood.-Acquainted, as we are, with the properties of fibrin, it is evident that this principle is the agent which produces coagnlation of the blood. In fact, whatever coagulates spontaneously is called fibrin, and whatever requires some agent to produce this change of consistence is called by another name. But before the properties of fibrin were fully understood, the question of the coagulating principle was a matter of much discussion. ${ }^{2}$ Malpighi was probably the first to isolate this principle;

1 Op. cit., p. 86.

2 An admirable historical review of the theories and discoveries relating to the properties of fibrin and the coagulation of the blood is to be found in $\mathrm{Mr}$. Gulliver's introduction to the Sydenham edition of the works of William Hewson London, 1846, p. 25 et seq. 
which he did by washing the clot in a stream of water, which removed the corpuscles and left a whitish fibrous network. His experiments are set forth in an article in which he attempted to show that the so-called polypi of the heart were formed of fibrin, though it was not then called by that name. These observations were soon confirmed by others, and finally Ruysch extracted fibrin from his own blood and the blood of the pig by whipping with a bundle of twigs, and thereby prevented its coagulation. 'This is the method now most commonly employed for the separation of fibrin. It then became a question whether this substance existed as a fluid in the liquor sanguinis, or was furnished by the corpuscles after the removal of blood from the vessels. This was decided by Hewson, whose simple and conclusive experiments, published in 1771 , leare no doubt that coagulation of the blood is due to fibrin, and that this principle is entirely distinct from, and independent of, the corpuscles. This observer, taking advantage of the property possessed by certain saline substances of preventing the coagulation of the blood, was the first to separate the liquor sanguinis from the corpuscles. He mixed fresh blood with a little sulphate of soda, which prevented coagulation, and after the mixture had been allowed to stand for a time, the corpuscles gravitated to the bottom of the vessel. The clear fluid was then decanted, and diluted with twice its quantity of water, when the fibrin became coagulated. ${ }^{1}$ Another experiment is still more conclusive; and as the credit of having first. separated the corpuscles from the plasma and demonstrated the coagulability of the latter is by some ascribed to Müller, we will give it in the author's own words :

"Immediately after killing a dog, I tied up his jugular veins near the sternum, and hung his head over the edge of the table, so that the parts of the veins where the ligatures were might be higher than his head. I looked at the veins

1 The Works of William Hewson, F. R. S., Sydenham edition, p. 12. 
from time to time, and observed that they became transparent at their upper part, the red particles subsiding. I then made a ligature upon one vein, so as to divide the transparent from the red portion of the blood; and opening the vein, I let out the transparent portion, which was still fluid, but coagulated soon after. On pressing this coagulum, I found it contained a little serum. The othèr vein I did not open till after the blood was congealed, and then I found the upper part of the coagulum whitish like the crust in pleuritic blood." 1

Nothing could more conclusively demonstrate that coag- ulation of the blood depends upon a coagulating principle existing in the liquor sanguinis, than this simple experiment. It also beautifully illustrates the formation of the buffy-coat.

The facts thus demonstrated by Hewson were confirmed by Müller in 1832. He succeeded in separating the plasma from the corpuscles in the blood of the frog by simple filtration; first diluting it with a saccharine solution. 'The great size of the corpuscles in this animal prevents their passage through a filter, and the clear fluid which is thus separated soon forms a colorless coagulum. ${ }^{2}$

From these observations it is evident that the coagulation of the blood is due to the presence of fibrin in the liquor sanguinis. Coagulation of this principle first causes the whole mass of blood to assume a gelatinous consistence; and by virtue of its contractile properties it soon expresses the serum, but the red corpuscles are retained. One of the causes which operate to retain the corpuscles in the clot is the adhesive matter which covers their surface after they escape from the vessels, which produces the arrangement in rows like piles of coin, which we liave'already noted under the head of microscopic appearances. This undoubtedly prevents those

2 The Works of William Hewson, F. R. S., Sydenham edition, p. 32.

2 J. Mueller, Manuel de Physiologie, trad. par Jourdan, Paris, 1851, tome i., p. 96 . 
which are near the surface from escaping from the clot during its contraction.

\section{Circumstances which modify Coagulation out of the Body.}

The conditions which modify coagulation of the blood have been closely studied by Hewson, Davy, Thackrah, Robin and Verdeil, and others. They are, in brief, the following:

Blood flowing slowly from a small orifice is more rapidly coagulated than when it flows in a full stream from a large orifice. If it be received into a shallow vessel, it coagulates much more rapidly than when received into a deep vessel. If the vessel be rough, coagulation is more rapid than if it be smooth and polished. If the blood, as it flows, be received on a cloth or a bundle of twigs, it coagulates almost instantaneously. In short, it appears that all circumstances which favor exposure of the blood to the air, hasten its coagulation. The blood will coagulate more rapidly in a vacuum than in the air.

Coagulation of the blood is prevented by rapid freezing, but afterwards takes place when the fluid is carefully thawed. Between $32^{\circ}$ and $140^{\circ} \mathrm{Fahr}$, elevation of temperature increases the rapidity of coagulation. ${ }^{1}$ Experiments are impracticable above $140^{\circ}$, as we, are then likely to have coagulation of the albumen. According to Richardson, agitation of the blood in closed ressels retards, and in open vessels hastens coagulation. ${ }^{2}$

Various chemical substances retard or prevent coagulation. Among them we may mention: solutions of potash and of soda; carbonate of soda; carbonate of ammonia; carbonate of potash; ammonia; sulphate of soda. In the menstrual flow the blood is kept fluid by mixture with the abundant secretions of the vaginal mucous membrane.

${ }^{1}$ Rrchandsox, The Cause of the Coagulation of the Blood. Astley Cooper Prize Essay for 1856, p. 140 et seq.

${ }^{2}$ Ibid., p. 228. 


\section{Coagulation of the Blood in the Organism.}

The blood coagulates in the vessels after death, though less rapidly than when removed from the body. As a general proposition it may be stated that this takes place in from twelve to twenty-four hours after circulation has ceased. Under these circumstances it is found chiefly in-the venous system, as the arteries are generally emptied by post mortem contraction of their muscular coat. Coagula are found, however, in the left side of the heart and in the aorta, but they are much smaller than those found in the right side of the heart and the large veins. These coagula present the general characters we have already described. They are frequently covered by a soft whitish film, analogous to the buffy coat, and are dark in their interior.

It was supposed by John Hunter that coagulation of the blood did not take place in animals killed by lightning, hydrocyanic acid, or prolonged muscular exertion, as when hunted to death; but it appears from the observations of others that this view is not correct. J. Davy reports a case of death by lightning where a loose coagulum was found in the heart twenty-four hours after. In this case decomposition was very far advanced, and it is probable that the coagula had become less firm from that cause. His observations also show that coagulation occurs after poisoning by hydrocyanic acid, and in animals hunted to death. ${ }^{1}$

Coagulation in different parts of the vascular system is by no means unusual during life. In the heart we sometimes find coagula which bear evidence of having existed for some time before death. These were called polypi by some of the older writers, and are often formed of fibrin almost free from red corpuscles. They generally occur when death is very gradual, and the circulation continues for some time with

${ }^{1}$ Dr. JoHN DAvr, Researches Physiological and Anatomical, vol. ii., p. 70 et seq. 
greatly diminished activity. It is probable that a small coagulum is first formed, from which the corpuscles are washed away by the current of blood; that this becomes larger by further depositions, until we have large vermicular masses of fibrin, attached, in some instances, to the chordæ tendineæ. Clots formed in this way may be distinguished from those formed after death by their whitish color, dense consistence, and the closeness with which they adhere to the walls of the heart. Cases have been reported by Richardson and others, where concretions of this kind extended from the cavities of the heart far into the large vessels. It is also stated by Richardson ${ }^{1}$ that they sometimes become partly organized, and connected with the tissue of the heart; but we have seen that accidental deposits of a proximate principle, like fibrin, never become transformed into organized structures.

We need only enumerate some of the other circumstances under which the blood coagulates in the vessels, as this subject belongs rather to pathology. than to physiology. Coagulation may be said, in general terms, to occur as a condition of stasis. When a ligature is applied to an artery, the vessel becomes filled with a coagulum up to the site of the first branch which is given off, whatever be its situation. In applying the ligature, the delicate inner coat is ruptured, and the shreds, which curl up in the interior of the vessel, soon become covered with a layer of coagulated blood, which thickens until the whole vessel is filled. In cases in which the flow of blood becomes arrested, or very much retarded, as in varicose veins of the extremities, the enlarged veins in hemorrhoids, etc., these vessels may become obliterated by the formation of a clot. In some aneurisms, the retardation of the blood-current produces spontaneous cure by the deposition of successive layers of fibrin next the walls of the dilated vessel. A knowledge of this fact has been made use of in the treatment of aneurism by compression of the artery which 
supplies it with blood. Many cases are on record, where this has been continued for a number of hours, and a cure effected.

Bodies projecting into the caliber of a blood-ressel soon become coated with a layer of fibrin. Rough concretions about the orifices of the heart frequently induce the deposition of little masses of fibrin, which sometimes become detached, and are carried to various parts of the circulatory system, as the lungs or brain, plugging up one or more of the smaller vessels. These masses have been called by Virchow, emboli, and have been traced by him, in some instances, from the heart to the situations above mentioned. The experiment has been made of passing a thread through a small artery, allowing it to remain for a few hours, when it is found coated with a layer of coagulated fibrin.

Blood generally coagulates when it is effused into the areolar tissue, or any of the cavities of the body; though, effused into the serous cavities, the tunica vaginalis for example, it has been known to remain fluid for days and even weeks, and coagulate when let out by an incision. In the Graafian follicles, after the discharge of the ovum, we generally have the cavity filled with blood, which forms a clot, and is slowly remored by the process of absorption.

Coagulation thus takes place in the vessels as the resuit of stasis, or very great retardation of the circulation, and in the tissues or cavities of the body, whenever it is accidentally effused. In the latter case, it is generally removed in the course of time by absorption. This takes place in the following way: First, we have disappearance of the red corpuscles, or decoloration of the clot, and the fibrin is then the only element which remains. This becomes reduced from a fibrillated to a granular condition, softens, finally becomes amorphous, and is absorbed; though when the size of the clot is considerable, this may occupy weeks, and even months, and may never be completely effected. Effused in this manner, the constituents of the blood act as foreign 
bodies; the corpuscles cease to be organized anatomical elements capable of self-regeneration, break down, and are absorbed. The fibrin which remains undergoes the same process; the stages through which it passes being always those of decay, and not of development. In other words, it is incapable of organization.

\section{Office of the Coagulation of the Blood in Arresting} Hemorrhage.-The property of the blood under consideration has a most important office in the arrest, of hemorrhage. The effect of an absence or great diminution of the coagulability of the circulating fluid is exemplified in instances of what is called the hemorrhagic diathesis; a condition in which slight wounds are apt to be followed by alarming, and it may be fatal, hemorrhage. This condition of the blood is not characterized by any symptoms excepting the obstinate flow of blood from slight wounds, and may continue for years. In a case which came under the observation of the author a few years since, excision of the tonsils was followed by bleeding, which continued for several days, and was arrested with great difficulty. On inquiry it was ascertained that the patient, a young man about twenty years of age, in other respects perfectly healthy, had been subject from early life to persistent hemorrhage from slight wounds. In reviewing the functions of fibrin, we find that apparently its most important office is in the arrest of hemorrhage. The degree of coagulability of the blood depends on the quantity of fibrin, but its proportion has not been shown to bear any definite relation to the vigor of the individual, nor to the processes of nutrition generally. The necessary and constant variations in the organic elements of the blood, which are the result of insufficient alimentation, exhausting discharges, or diseases characterized by impoverishment of this fluid, are observed in the albumen and red corpuscles, and not in the fibrin. By this it must not be understood that the quantity of tibrin is not variable. It has 
been found, for example, by Andral and Gavarret to be pretty generally increased in the phlegmasiæ; but it bears no relation to the richness of the blood. Its proportion is not increased always in plethora and diminished in anemia; and in fact it has been found by Nasse to be increased in animals suffering from hunger." After hemorrhage, which diminishes the corpuscles and albumen, the fibrin is generally increased; so that the fact of loss of blood, diminishing the force of the heart and increasing the tendency to coagulation, has an influence in the arrest of the flow.

Circumstances which accelerate coagulation have a tendency to arrest hemorrhage. It is well known that exposure of a bleeding surface to the air has this effect. The way in which the ressel is divided has an important influence. A clean cut will bleed more freely than a ragged laceration. In division of large vessels this difference is sometimes marked. Cases are on record where the arm has been torn off at the shoulder-joint, and yet the hemorrhage was, for a time, spontaneously arrested; while we know that division of an artery of smaller size, if it be ent across, would be fatal if left to itself. Under these circumstances the internal coat is torn in shreds, which retract, their curled ends projecting into the caliber of the vessel, and have the same effect on the coagulation of blood as a bundle of twigs. In laceration of such a large vessel as the axillary artery, the arrest cannot be permanent, for as soon as the system recovers from the shock, the contractions of the heart will force out the coagulated blood which has closed the opening.

In our study of the functions of the body we shall continually see evidences that Nature, not content with simply providing for the ordinary wants of the system, has made provision for extraordinary occurrences and accidents. A striking example of this is the function of fibrin. All the ordinary operations of the body go on perfectly well in a

${ }^{1}$ Robin and Verderl, Chimie Anatomique, tome iii., p. 205. 
person affected with the hemorrhagic diathesis, in whose blood the fibrin is wanting; and, as we have already seen in treating of transfusion, the vivifying effects of defibrinated blood are equal to those of blood which contains all its constituents ; yet it is provided that in hemorrhage the blood solidifies and closes the opening in the vessels, if they be not too large. She often makes attempts to cure aneurisms, or hemorrhoids, by the same process; and hence does not obliterate the vessels by an organized substance, which would be capable of selfregeneration and always remain as part of the body, but throws out a temporary plug, which is destined to be removed, partially, if not completely, by absorption. The process of coagulation of the fibrin of the blood is essentially different from that of gradual effusion of plastic lymph by which injuries are repaired. Individuals suffering under the hemorrhagic diathesis, are not deprived of the power of repairing injuries by means of plastic exudations from the blood, though the blood contains no fibrin, and hemorrhage is not arrested until the process of repair has closed the openings in the vessels, or we have closed them by the effect of our styptics. We likewise see that in the lower animals who have not the means of artificially arresting hemorrhage, its spontaneous arrest is more effectually provided for by a more rapid coagulation of the blood.

From the foregoing considerations it is evident that the remarkable phenomenon of coagulation of the blood, which has so much engaged the attention of physiologists, has rather a mechanical than a vital function; for its chief office is in the arrest of hemorrhage. Coagulation never takes place in the organism, unless the blood be in an abnormal condition with respect to circulation. Here its operations are mainly conservative; but as almost all conservative processes are sometimes perverted, clots in the body may be productive of injury, as in the instances of cerebral apoplexy, clots in the heart occurring before death, the detachment of emboli, etc. 
Cause of the Coagulation of the Blood.-Though the phenomena of coagulation, and the circumstances which modify it, especially as occurring in the organism, are of more practical importance than any thing else, the study of these phenomena naturally leads us to inquire into the reason why fibrin thus changes its form. When we say that this principle is endowed with the property of spontaneous coagulability, we do not express what is strictly the fact. It remains fluid until it is placed in abnormal conditions, when, without the application of heat, or any chemical reagents, it coagulates; but so long as it remains in the circulating blood, lymph, or chyle, coagulation does not take place. This property, which has been so long recognized, has been the subject of many speculations as to its cause, and some experiments ; but until the last few years the experiments have done nothing but familiarize us with the actual phenomena which take place, and left the cause, as before, entirely a matter of speculation. Under these circumstances it will not be found very profitable to discuss the old theories on the subject. Our object in the historical review of physiological questions is to show the gradual development of truth, as facts have been accumulated by different observers, which those last in the field have been able to coördinate, rather than to exhume hypotheses which have fallen before actual observation. On no subject have hypotheses been more vague and unsatisfactory, and more readily disproved by experiment, than with regard to the cause of coagulation of the fibrin. The idea that exposure to the air is the cause of coagulation, which was held by Hewson, is disproved by the simple fact that coagulation takes place in a vacuum. The vital theory of Hunter, which was adopted by most physiologists of his time, is too indefinite for discussion at the present day, and really expresses utter want of knowledge on the subject. The theory that motion is the cause of the fluidity of fibrin in the body, is disproved by the fact that violent agitation of the blood out of the body does not prevent coagulation. 
On the other hand, we are not justified, with Robin and Verdeil, in abandoning the subject with the assertion that it is "as vain to seek after the cause of this fact as to inquire why fibrin exists, why sulphate of copper is blue, etc." ; assuming that fibrin coagulates merely because it has the property of coagulation, as albumen is coagulated by heat, or caseine by acetic acid. An extension of this method in physiology would put an end to all generalization, restricting the operations of the intellect to the mere observation of phenomena.

Circulating in the organism, the plasma contains, molecularly united with each other and uniformly distributed in the fluid, fibrin, albumen, salts, and volatile substances. Albumen retains its fluidity out of the body, until heat or some coagulating agent is applied; but by employing a current of galvanism, which we know changes the condition of the inorganic substances in the serum, something is taken away which causes albumen to coagulate, or which, when it existed unchanged, retained albumen in its fluid condition. Is it not possible that the blood while circulating may contain a substance capable of keeping fibrin fluid, the evolution of which out of the body is the cause of coagulation? We are particularly led to ask this question, as we are acquainted with many substances which possess this property when added to blood drawn from the ressels; such as carbonate of soda, ammonia, etc. This idea forms a fit basis for experimental inquiry, by a study of the substances evolved by the blood during coagulation in the form of vapor. If it be objected that no coagulation takes place in the vessels, while an opportunity for volatilization is constantly presented in the lungs in normal circulation, it must be remembered that the blood is continually washing out, as it were, in the course of circulation, matters formed in the various parts of the organism; and substances which are continually discharged by the lungs, skin, kidneys, ete., are necessarily as continually taken

${ }^{2}$ Robiv and VERDEIL, op. cit., tome iii., p. 210. 
up by the blood in the system. From this point of view it does not seem entirely unprofitable to look after the cause of the coagulation of the blood. It was with such an idea as this that almost the first definite experiments which we have on the cause of coagulation, were performed. These constitute the basis of the Astley Cooper prize essay for 1856, and if they be not sufficient to convince all physiologists, must be acknowledged to settle many points with reference to the question under consideration. Dr. Richardson has here given us the only definite and probable explanation of this phenom'enon that has ever been presented.'

The views of Richardson, and the experiments on which they are based, are briefly the following:

Taking as a point of departure the fact, which, as we have already seen, is sufficiently proven, that all circumstances which facilitate the separation of volatile elements from the blood hasten coagulation, Richardson attempted to show that the volatile substances which thus escape, if retained, or if made to pass through blood, will retard or arrest coagulation. His experiments on the prevention of exhalation are very satisfactory. The jugular vein is laid bare; a portion of it, filled with blood, is included between two ligatures, then separated from the body and drawn under mercury in a $U$ tube, the vein being allowed to remain in the bend of the tube for from nine to twenty-four hours. At the end of this time it is removed, the blood let out, and exposed to the air. In a number of experiments he found the blood entirely fluid when drawn from the vein immediately after removal from beneath the mercury, while it coagulated firmly in a few minutes after exposure to the air. ${ }^{2}$ This simple experiment we have repeated with the same result. It shows conclusively that coagulation of the blood is not a consequence of simple rest, or lowering of temperature, and that it is not kept fluid in the organism by any vital influence.

${ }^{1}$ Richardson, The Cause of the Coagulation of the Blood, London, 1858.

2 Ibid., p. 204 et seq. 
The next experiments, which bear directly on the subject under consideration, were made with reference to the important question, whether the volatile substances escaping from coagulatirig blood, if passed through fresh blood, would have the effect of retarding or preventing coagulation. The experiments on this point are likewise conclusive. The apparatus which is used consists of two wide-mouthed bottles, capable of holding about two ounces, and a Wolffe's bottle capable of holding about three pounds. The small bottles, fitted with perforated corks, are half filled, and the large bottle nearly filled, with fresh blood. A tube connected with a small bellows is introduced into one of the small bottles, passing nearly to the bottom, while a second perforation in the cork is fitted with a short tube which simply allows the escape of air or vapor. The latter is connected with a tube passing nearly to the bottom of the Wolffe's bottle through one of the necks, while the other is fitted with a short tube to permit the escape of the vapor. The vapor is then made to pass through the blood in the third bottle by a long tube reaching to the bottom. If air be now gently forced through the apparatus by the bellows, the vapor from the mass of blood (about two pounds is used) in the large bottle will pass through the third, which contains but an ounce of blood. In an experiment of this kind performed by Richardson, " the blood through which the air was first passed coagulated in two minutes; that in the Wolffe's bottle coagulated in three minutes; while the blood in the third bottle, which for a time receired a full charge of the vapor, retained its red color and its fluidity for eight minutes and a half; as long, in fact, as any vapor conld be sent through it. When the vapor failed, and air only began to circulate, this blood coagulated feebly, the fibrin separating and floating on the top." 1

These experiments apparently have but one explanation. As the blood when drawn from the body may sometimes be 
kept fluid by preventing the escape of volatile substances, and the rapor of coagulating blood forced through another specimen of blood prevents coagulation so long as it continues to pass, something is given off from the blood which, when contained in this fluid, has the power of retaining fibrin in its fluid state. Having gone thus far in the investigation, the next point is to subject the vapor of blood to analysis, and ascertain, if possible, what substance or substances it contains which, when retained in the blood, or introduced, have the power of keeping it fluid.

This was the next step in Richardson's investigations. He found that blood-vapor contained, among other things, ammonia. This he detected by passing blood-vapor through hydrochloric acid and afterwards testing it with the perchloride of platinum, forming the ammonio-chloride of platinum. He also obtained crystals of the chloride of ammonium, by allowing the vapor to pass over a glass slide moistened with hydrochloric acid. He demonstrated in this way the presence of ammonia in the exhalation from the blood of the human subject, as well as the inferior animals. He also demonstrated by numerous experiments that ammonia mixed with blood, or the vapor passed through it, will prevent coagulation; while the passage of air and the various gases has the effect of hastening, rather than retarding this process. It was further demonstrated that ammonia is constantly discharged by the organism, particularly by the lungs; and, of course, must be as constantly produced in the tissues, and taken up by the blood in the course of the circulation. ${ }^{1}$

The points above enumerated certainly seem to be ex-

In the discussion of Richardson's views, we have attempted to connect the great experimental links in his chain of evidence. His admirable and laborious treatise contains details of 399 experiments; and though a summary is given at the end of each chapter, and a summary at the conclusion, much labor is neccssary on the part of the reader to separate those which are important from the great mass of minor facts, and appreciate the proofs of the doctrines advanced. This, as it seems to me, has had the effect of causing the views of Dr. Richardson to receive far less attention at the hands of physiologists than they really merit. 
perimentally proven. The experiments cited show conclusively that as blood coagulates, out of the body, a vapor is given off which contains some substance capable of preserving the fluidity of the fibrin; and that ammonia, which is a constituent of this vapor, has this property. But the rigid requirements of our science render it necessary, in order to establish the fact that the evolution of ammonia is the sole and constant cause of coagulation, to show how ammonia is given off under all the varied circumstances under which coagulation of the blood is known to take place. In other words, it must be demonstrated that the evolution of ammonia in coagulation is not a coincidence, occurring, it may be, pretty generally, but a necessity. The fact that ammonia added to blood prevents coagulation is not sufficient evidence of this; for, as we have seen, other substances, such as carbonate of soda, have the same effect.

Are there any circumstances under which coagulation of blood takes place, where ammonia is not, and cannot be, given off? There are observations which seem to answer this question in the affirmative; and it becomes necessary now to carefully study, with reference to this point, all the varied conditions under which the blood will coagulate.

The view that coagulation of the blood is due to the evolution of ammonia explains perfectly how this process is hastened by exposure to air, by a moderately high temperature, by a vacuum, by the blood flowing slowly in a simall stream, and in brief, the various circumstances which modify coagulation out of the body. Its evolution from the blood by the lungs is not incompatible with the fact of the fluidity of the blood in the body, for it is taken up from the tissues as fast as it is eliminated. Some instances, however, of coagulation in the body, and some experiments on coagulation out of the body, when, as is thought, ammonia is not and cannot be evolved, seem opposed to the view advanced by Richardson.

It is easy to understand, adopting the views of Richard- 
son, why the blood coagulates in the body after death. Under the circumstances in which it is then placed, the escape of volatile substances, though retarded, is evidently not prevented. Thus when the body is opened shortly after death, we may find the blood perfectly fluid, coagulating, however, shortly after it is removed from the vessels and exposed to the air. During life, when circulation is arrested or much retarded, the blood will coagulate; but here there is the same opportunity presented for the escape of volatile matter. As ammonia is undoubtedly received by the blood in the course of circulation, arrest of circulation in any part of the vascular system prevents the blood therein contained from receiving its constant supply. As it has been shown that out of the body the evolution of ammonia always accompanies coagulation, we must infer simply that coagulation in the body, under the above-mentioned circumstances, is attended with the evolution of this principle, for the conditions here do not admit of direct experimentation, situated as the blood is in the midst of tissues, from which volatile substances are also evolved. It is not proper, however, to shut our eyes to the fact that blood effused into the tissues and into the cavities, during life, has been known to remain fluid for days and even weeks, when there are no circumstances which we can appreciate as modifying or preventing the gradual evolution of ammonia. But we know that there are many animal products, such as the vaginal mucus, etc., which prevent coagulation; and in these instances, which are not very frequent, it has not been shown that some influence of this kind was not brought to bear on the process. It is a curious fact, also, that leech-drawn blood remains fluid in the body of the animal. Richardson has verified this fact, but says that he can offer no satisfactory explanation. He observed also that the blood flowing from the leech-bite presented the same persistent fluidity, which explains the well-known fact that the insignificant wound gives rise to considerable hemorrhage. On this point he has made the following curious experiment: 
"After the leech was removed from the arm, the wound it had produced continued to give out blood very freely. I caught the blood thus flowing at different intervals, allowing it to trickle into teaspoons of the same size and shape. The results were curjous. The blood which was received into the first spoon, and which was collected immediately after the removal of the leech, was dark, and showed the same feebleness of coagulation as the blood taken from the leech itself. Another portion of blood, received into a second spoon five minutes later, coagulated in twenty-five minutes with moderate firmness. A third portion of blood, caught ten minutes later still, coagulated in eight minutes; while at the end of half an hour the blood which still flowed from the wound coagulated firmly, and in fine red clots, in two minutes. Ultimately the blood coagulated as it slowly oozed from the wound, so that the wound itself was sealed up."

The existence of projections into the caliber of vessels, or, as was done by Simon, the passage of a fine thread through an artery or vein, will determine the formation of a small coagulum upon the foreign substance, while the circulation is neither interrupted nor retarded. These facts demand explanation, but all we can say with regard to them is, that in the present state of our knowledge explanation is difficult, if not impossible. As before remarked, the process, under these circumstances, cannot be subjected to direct experiment, as in the case of the blood coagulating out of the body.

Since the publication of Richardson's essay, various experiments on coagulation out of the body have been made which are claimed to disprove his views. Dr. John Davy has reported some experiments on the coagulation of blood in the common fowl, in which he attempts to show that the process is not attended with the evolution of ammonia, and furthermore, that ammonia mixed with the blood will not prevent coagulation." It is well known that the blood of

${ }^{1}$ Op. cit., p. 207.

2 John Davr, M.D., Physiological Researches, London, 1863, p. 384 et seq. 
birds is remarkable for the rapidity of its coagulation, and is therefore not so well adapted to experiments relative to the circumstances which attend this process as the blood of animals in which coagulation is less rapid. The experiments referred to are imperfect, and no attempt is made to invalidate the accuracy of the observations of Richardson on the blood of mammals and the human subject.

The most recent experiments on this subject are by Joseph Lister, published in a lecture on "Coagulation of the Blood," in the "London Lancet," February, 1864. The view entertained by Mr. Lister is, that the blood is kept fluid in the organism by its contact with living parts; and that all other contact, especially that of inorganic bodies, produces a tendency in this fluid to coagulate. The power of retaining the fluidity of the blood he supposes to reside particularly in the coats of the blood-vessels, but he further says: "I think it probable, though not yet proved, that all living tissues have these properties with reference to the blood." 1 The ammonia theory he considers entirely fallacious, and ascribes coagulation either to the contact of animal tissues after death, when their vital property of maintaining the fluidity of the blood slowly disappears, or the contact of ordinary matter. ${ }^{2}$

Various experiments are cited in support of the view thus briefly given. In one of them, the author, by an ingenious mechanism, draws the blood into an apparatus consisting of a tube in which it is effectually secluded from the air, and which allows the fluid to be stirred with a little wire which is provided with projecting spokes. In one experiment the tube was filled with blood, which did not come in contact with the air, and the blood stirred with the wire. In thirtyseven minutes the wire was removed and found enveloped in a mass of clot. In another experiment, "Receiving blood from the throat of a bullock into two similar wide-mouthed

${ }^{1}$ London Lancet, American republication, Feb. 1864, p. 91.

2 This view, as stated by Mr. Lister, was entertained by $\Lambda$ stley Cooper, Thackrah, Brucke, and others. 
bottles, I immediately stirred one of them with a clean ivory rod for ten seconds very gently, so as to avoid the introduction of any air, and then left both undisturbed. At the end of a certain number of minutes, I found that, while the blood which had not been disturbed could be poured out as a fluid, with the exception of a thin layer of clot on the surface and an incrustation on the interior of the vessel, the blood in the other vessel, which had been stirred for so brief a period, was already a solid mass." "

Other experiments are brought forward, modifications of the one already mentioned as performed by Simon, showing that incrustations will form on the surface of foreign substances introduced into the vessels; and that after death their introduction will induce coagulation in the entire vessel much sooner than it would otherwise have taken place.

The idea of simple contact with living tissues preventing coagulation hardly merits discussion. It is well known that coagulation frequently takes place during life, almost always following arrest of the circulation. After division of the vessels, the blood, in contact with living parts, performs its conservative function in the arrest of hemorrhage. There is certainly something very curious in the effect of the contact of foreign substances, and the experiments on this point are very striking. Why is it that a coagulum forms upon a fine thread or a needle passed through a vessel; or on the wire with which the blood in Mr. Lister's apparatus was stirred, though there was no exposure to the air? And why did the blood, which was only gently stirred for a few seconds with a smooth ivory rod, coagulate so much more rapidly than that which was undisturbed?

These are questions which we must acknowledge our inability to answer. The phenomena cannot be satisfactorily explained by the supposition that ammonia is evolved; but on the other hand, this is not a sufficient reason for rejecting the fact, experimentally demonstrated, that, out of the or-

\footnotetext{
${ }^{1}$ Op. cit., p. 83.
} 
ganism, ammonia, a substance capable of maintaining the fluidity of the fibrin, is given off from coagulating blood. We may suppose that ammonia separates itself from one portion of the blood, and is retained in another. An experiment by Richardson gives color to this supposition, for in one experiment on the passage of blood-vapor through blood, he found that the lower part coagulated while the upper part remained fluid; and on examination, ascertained, in explanation of this, that the tube which carried the vapor into the blood did not extend to the bottom of the vessel. ${ }^{1}$

The effect of foreign bodies on coagulation is not more inexplicable than the operation of inert substances in certain chemical processes; as the action of the oxide of manganese in the formation of oxygen from the chlorate of potash ; or, to take a process more like the one under consideration, the formation of crystals on threads and projections in vessels, or the escape of electricity from points. Examples of this kind in the organic world are numerous, and we are content to say that these facts are entirely beyond explanation, in the present state of our knowledge. We should hardly be surprised, then, at our inability to explain the tendency which the presence of foreign bodies has to induce the deposition of so congulable a substance as fibrin. The theory that coagulation of the blood is always, or even generally, due to the contact of foreign substances, or tissues which have lost their vital properties and act as foreign substances, must be rejected as opposed to experiment and observation. When, as happens in the interior of the body, the blood coagulates under circumstances when the process will not admit of direct experimentation as far as the evolution of volatile substances is concerned, the best we can do is to apply, as far as possible, the facts which are proven with regard to coagulation out of the body, when the phenomena can be minutely studied. Here, at least in the human subject and in mammals, it seems demonstrated to be due to the evolution of ammonia. 


\section{Summary of the Properties and Functions of the Blood.}

The blood, constituting as nearly as can be estimated oneeighth of the weight of the body, is the great nutritive fluid; its presence being necessary to life, and its normal constitution and circulation essential to the performance of all the functions.

Anatomically, its most important elements are a clear plasma and the red corpuscles, these existing in about equal proportions. The corpuscles are intimately connected with the function of respiration. Their chief office seems to be to carry oxygen from the lungs to the tissues. Their presence is immediately essential to life, and their normal proportion essential to health. They are organized anatomical elements, capable of self-regeneration from principles contained in the plasma. They contain all the principles which exist in the plasma, with the difference that the fibrin and albumen of the latter are replaced by globuline, and a coloring matter, hematine, is superadded. The plasma seems to be the part chiefly employed in the nourishment of the tissues, some of which, as cartilage, do not receive any of the corpuscular elements of the blood.

Chemically, the plasma contains all the elements which are necessary for the regeneration of all parts of the body. These are continually being used up in nutrition, but are replaced by the absorption of articles of food after they have undergone the preparation of digestion. In the deposition of new matter in the regeneration of the tissues, the organic and inorganic constituents of the plasma are deposited together ; the inorganic elements of the tissues receiving, as it were, the vital properties of self-regeneration, which we suppose to reside particularly in organic principles, from the fact of their molecular union with these organic principles.

Of the organic constituents, albumen constitutes by far the greater proportion, and is the one chiefly used in the 
nutrition of the organic nitrogenized elements of the tissues. Its diminution in the blood to any considerable extent determines defective nutrition. It is probable that all the other organic. nitrogenized principles are formed from it.

In the blood, part of the albumen is transformed into fibrin, which exists in small quantity, and does not appear to bear any relation to nutrition. Its peculiar property of spontaneous coagulation gives it a most important conservative function in the arrest of hemorrhage. Ammonia, which is contained in the blood, has the property of maintaining its fluidity; but on exposure to air, or in rupture of vessels, we have an escape of ammonia, and the fibrin by its coagulation reduces the whole mass of blood to a semi-solid consistence. The proportion of fibrin in the blood bears no relation to the function of nutrition. Its occasional absence only induces obstinate hemorrhage on the division of vessels, even of very small size.

Fat, which exists in small quantity in the blood, and sugar, which exists only in certain parts of the circulatory system, disappear in the organism in a way which is not at present understood. They are concerned in, and necessary to, the processes of nutrition; but the exact nature of their function is unknown.

The inorganic constituents of the body are found in varying proportions in the plasma, and have varied functions. Their presence tends to preserve the proper constitution of the corpuscles, which are dissolved and lost in pure water.

The water which does not enter into the constitution of the albumen and fibrin serves to hold the various salts in solution, and cannot vary much in quantity from a certain standard.

Some of the inorganic salts, the chlorides particularly, seem to regulate, to a certain extent, the processes of nutrition, are found most abundantly in the fluids, and apparently do not form a very essential portion of the tissues themselves. A tendency to an excess in the blood is relieved by discharge 
from the system, and a diminution is accompanied by certain indefinite disorders in the general processes of nutrition.

The alkatine carbonates have a tendency to preserve the fluidity of the fibrin.

Some of the inorganic salts, such as the phosphate of lime, are important elements entering into the constitution of the various tissues. They are most abundant in the solids and semi-solids. of the body; and when their introduction with food is prevented, we have certain definite changes in the constitution of some of the tissues, as softening of the bones in animals deprived of the phosphate of lime.

As already remarked, the inorganic principles are necessary to, and participate in the performance of the vital functions of organic principles.

In addition to these elements, the blood contains large quantities of carbonic acid, which is eliminated by the lungs, and small quantities of other excrementitious matters, such as urea, the urates, cholesterine, creatine, creatinine, and ammonia (which is perhaps an excretion), their proportion being kept down by their constant removal by the proper eliminating organs. Their increase in the blood from any cause produces toxic effects, which, as regards some, urea and cholesterine for example, are easily recognized. 


\section{CHAPTER IV.}

\section{CIRCULATION OF THE BLOOD.}

Discovery of the circulation-Physiological anatomy of the heart-Valves of the heart-Movements of the heart-Impulse of the heart-Succession of move ments of the heart-Force of the heart-Action of the valves-Sounds of the heart-Cause of the sounds of the heart.

HARver discovered the circulation of the blood in 1616 , taught it in his public lectures in 1619, and in 1628 published the "Exercitatio Anatomica de Motu Cordis et Sanguinis in Animalibus." It is justly said by Flourens, in his elegant little work on the discovery of the circulation, that from this discovery dates the epoch of modern physiology, when tradition began to give place to observation. When we reflect that it is through the medium of the blood that all the processes of life take place; that all tissues are nourished by it, and all fluids formed from it; that it gives fresh material to every part, and takes away that which is worn out; that it carries oxygen to every part of the system, and gires to each structure its vital properties; we can form some idea of the state of physiology before anything was known of the circulation. This moinentous discovery, from the isolated facts bearing upon it which were observed by numerous anatomists, to its grand culmination with Harvey, so fully illustrates the gradual development of most great physiological truths, that it does not seem out of place to begin our study of the circulation with a rapid sketch of its history. 
The facts bearing upon the circulation which were developed before the time of Harvey were chiefly of an anatomical character. Hippocrates and his contemporaries distinguished two kinds of vessels, arteries and veins; but they regarded the former as air-bearing tubes, as their name implies, in communication with the trachea. Galen, by a few simple experiments upon living animals, demonstrated the error of this view. He showed that blood issued from divided arteries, and demonstrated its presence in a portion of one of these ressels included between two ligatures in a living animal. His ideas, however, of the mode of communication between the arteries and veins were entirely erroneous, believing, as he did, in the existence of numerous small orifices between the ventricles.

In 1553, Michael Servetus, who is generally regarded as the discoverer of the passage of the blood through the lungs, or the pulmonary circulation, described in a work on theology the course of the blood through the lungs, from the right to the left side of the heart. This description, complete as it is, was merely incidental to the development of a theory with regard to the formation of the soul, and the development of what were called animal and vital spirits (spiritus). The same year, by order of Calvin, Servetus was burned alive at Geneva, and nearly every copy of his work was committed to the flames. But one or two copies of this work are now in existence. One is in the library of the Institute of France, and bears evidence, in some pages which are partially burned, of the fate which it so narrowly escaped. ${ }^{1}$

A few years later, Columbo, professor of anatomy at Padua, and Cesalpinus, of Pisa, also described the passage of the blood through the lungs, though probably without any knowledge of what had been written by Servetus. To Cesalpinus is attributed the first use of the expression, circulation

${ }^{1}$ The physiological portion of the Christianismi Restitutio of SERverus has been extracted from the original by Flourexs, and is published in his little woris entitled Histoire de la Découverte de la Circulation du Sang, Paris, 1854. 
of the blood. He also remarked that after ligature or compression of veins, the swelling is always below the point of obstruction. These ideas, the importance of which is evident now that we understand the circulation, passed into oblivion. They were unknown to investigators during the succeeding century, and were only brought to light after the discoveries of Harvey had become widely disseminated. From this point of view they can hardly be called discoveries, taking no place in science, and their authors not considering them definite enough, or of sufficient importance, to be fully insisted upon.

A great discovery, preparatory to that of the circulation, was made by Fabricius ab Aquapendente, professor at Padua, who, in the words of Flourens, had a double glory: "He discovered the valves of the veins, and he was the master of Harvey." Valves had been described by Etienne in the portal vein, by Cananius in the azygos vein, and Eustachi had discovered the valve which bears his name and the valves of the coronary veins; but to Fabricius is generally ascribed the honor of the discovery of the valvular system in the veins. ${ }^{2}$ This was demonstrated to Harvey at Padua, though Fabricius does not appear to have had any detinite idea of their function. It is possible that this anatomical fact may have directed the mind of Harvey in his first speculations on the circulation. Shortly after his return from Padua in 1602, he advanced beyond the study of inanimate parts by dissections, and investigated animated nature by

${ }^{1}$ Bérard, (Cours de Physiologie, tome iv., p. 34) quotes a passage from Piccolomini, an Italian anatomist, in which the valves of the veins are mentioned: "* * * quod est, in mediis venis reconditas esse innumerabiles pene valvas, quemadmodum in orificiis vasorum cordis. Ho venarum valvce maxime conspicuce sunt in divisione ramorum vence cavae" (Anatomica Prcelectionces, Romce, 1586, p. 412). It is the assertion, undoubtedly made in good faith, in the great work of Fabricius, that the valves had never before been seen, which has led many physiologists to regard him as the discoverer; especially when this fact is taken in connection with their demonstration by Fabricius to Harvey, to whom is due the sole credit of having pointed out their function. 
means of vivisections. As is evident when we consider the state of science at that time, anatomists had long been preparing the way for the discovery of the circulation, though they knew little of the functions of the parts they described. The conformation of the heart and vessels, and even the arrangement of the valves of the veins, did not lead them to suspect the course of the blood; but a few well conceived experiments on living animals have made it appear so simple, that we now wonder it remained unknown so long. Furthermore, these experiments made it evident that there was a communication at the periphery between the arteries and the veins.

In the work of Harvey are described, first, the movements of the heart, which he exposed and studied in living animals. He describes minutely all the phenomena which accompany its action; its diastole, when it is filled with blood, and its systole, when the fibres of which the ventricles are composed contract simultaneously, and "by an admirable adjustment all the internal surfaces are drawn together, as if with cords, and so is the charge of blood expelled with force." From the description of the action of the ventricles, he passes to the auricles, and shows how these, by their contraction, fill the ventricles with blood. By experiments upon serpents and fishes, he proved that the blood fills the heart from the veins, and is sent out into the arteries. Exposing the heart and great vessels in these animals, he applied a ligature to the veins, which had the effect of cutting off the supply from the heart so that it became pale and flaccid; and by removing the ligature the blood could be seen flowing into the organ. When, on the contrary, a ligature was applied to the artery, the heart became unusually distended, which continued as long as the obstruction remained. When the ligature was removed, the heart soon returned to its normal condirion. ${ }^{1}$

The descriptions given by Harvey were the result of nu-

${ }^{1}$ The Works of William Harvey, M. D. Sydenham Edition, p. 53. 
merous experiments upon living animals; exposing the heart of cold-blooded animals, in which the movements are comparatively slow; studying also the action of this organ in warm-blooded animals, after its movements had become enfeebled. As we shall see when we come to describe the movements of the heart, nothing can exceed the simplicity and accuracy of the descriptions of Harvey, which are universally acknowledged to be correct in almost every particular.

Harvey completed his description of the circulation, by experiments showing the course of the blood in the arteries and veins, and the uses of the valves of the veins. These experiments are models of simplicity and pertinence. First, he showed that a ligature tightly applied to a limb prevented the blood from entering the artery and arrested pulsation. The ligature then relaxed, and applied with moderate tightness so as to compress only the superficial veins, allowed the blood to pass into the part by the arteries, but prevented its return by the veins, which consequently became excessively congested. The ligature being removed, the veins soon emptied themselves, and the member regained its ordinary appearance. ${ }^{1}$

He observed the "knots" in the veins of the arm when a ligature is applied, as for phlebotomy, and showed that the space between these knots, which are formed by the valves, could be emptied of blood by pressing toward the heart, and would not fill itself while the finger was kept at the lower extremity. It was impossible, by pressure with the fingers, to force the blood back through one of the valves. ${ }^{2}$

By such simple, yet irresistibly conclusive experiments, was completed the chain of evidence establishing the fact of the circulation of the blood. Truly is it said that here commenced an epoch in the study of physiology; for then the scientific world began to emancipate itself from the ideas of the ancients, which had held despotic sway for two 
centuries, and study Nature for themselves by means of experiments.

Though Harvey described so perfectly the course of the blood, and left not a shadow of doubt as to the communication between the arteries and reins, it was left to others to actually see the blood in movement and follow it from one system of vessels to the other. In 1661, Malpighi saw the blood circulating in the vessels of the lung of a living frog, in examining it with magnifying glasses; and a little later, Leeuwenhoek saw the circulation in the wing of the bat. The great discovery was then completed.

Enough has been said in the preceding historical sketch to give a general idea of the course of the great nutritive fluid, and the natural anatomical and physiological divisions of the circulatory system. There is a constant flow from the central organ to all the tissues and organs of the body, and a constant return of the blood after it has passed through these parts. But before the blood, which has thus been brought back, is fit to return again to the system, it must pass through the lungs and undergo the changes which constitute the process of Respiration. In some animals, like fishes, the same force sends the blood through the gills, and from them through the system. In others, like the reptiles, a mixture of aërated and non-aërated blood takes place in the heart, and the general system never receives blood that has been fully arterialized. But in man and all warm-blooded animals, the organism demands blood that has been fully purified and oxygenated by its passage through the lungs, and here we find the first great and complete divisions of the circulation into the pulmonary and systemic, or, as they have been called, the lesser and greater circulation. The heart in this instance is donble; having a right and left side which are entirely distinct from each other. The right heart receives the bloud as it is brought from the system by the reins, and sends it to the lungs; the left heart receives the blood from 
the lungs and sends it to the system. ${ }^{1}$ It must be borne in mind, however, that though the two sides of the heart are distinct from each other, their action is simultaneous; and in studying the motions of this organ, we will find that the blood is sent simultaneously from the right side to the lungs, and from the left side to the system. It will not be necessary, therefore, to separate the two circulations in our study of their mechanism; for the simultaneous action of both sides of the heart enables us to study its functions as a single organ, and the constitution and operations of the two kinds of vessels do not present any material differences.

For convenience of study, the circulatory system may be divided into heart and vessels, the latter being of three kinds: the arteries, which carry blood from the heart to the system; the capillaries, which distribute the blood more or less abundantly in different parts of the system; and the veins, which return the blood from the system to the heart. The function of each of these divisions may be considered separately.

\section{Action of the Heart.}

Physiological Anatomy of the Heart.-The heart of the human subject is a pear-shaped, muscular organ, situated in the thoracic cavity, with its base about in the median line, and its apex at the fifth intercostal space, midway between the median line and a perpendicular dropped through the left nipple. Its weight is firom 8 to 10 ounces in the female, and from 10 to 12 ounces in the male. It has four distinct cavities : a right and a left auricle, and a right and a left ventricle. Of these, the ventricles are the more capacious. The heart is held in place, or may be said to be attached, by the great vessels, to the posterior wall of the thorax, while the apex is free and eapable of a certain degree

${ }^{1}$ In some animals, as the dugong, the division between the two sides of the heart is very marked. The heart is really double, having two points, the two sides joined together only at the base. 
of motion. The whole organ is enveloped in a fibrous sac called the pericardium, lined by a serous membrane which is attached to the great vessels at the base and reflected over its surface. This sac is lubricated by a drachm or two of fluid, so that the movements are normally accomplished without any friction. The serous pericardium does not present any differences from serous membranes in other situations. The cavities of the heart are lined by a smooth membrane, called the endocardium, which is continuons with the lining membrane of the blood-vessels.

The right auricie receives the blood from the venæ caræ and empties it into the right ventricle. The auricle presents a principal carity or sinus, as it is called, with a little appendix, called, from its resemblance to the ear of a dog, the auricular appendix. It has two large openings for the vena cara ascendens and the vena cava descendens, with a small opening for the coronary vein, which brings the blood from the substance of the heart itself. It has, also, another large opening, called the auriculo-ventricular opening, by which the blood flows into the ventricle. The walls of this carity are quite thin as compared with the rentricles, measuring about one line. They are constituted of muscular fibres which are arranged in two layers; one of which, the external, is common to both auricles, and the other, the internal, is proper to each. These muscular fibres, though involuntary in their action, belong to the striped, or what is termed voluntary, variety, and are similar in structure to the fibres of the ventricles. The fibres of the auricles are much fewer than those of the ventricles. . Some of them are looped, arising from a cartilaginous ring which separates the auricles and ventricles, and passing over the auricles; and other's are circular, surrounding the auricular appendages and the openings of the veins, extending, also, a short distance along the course of these vessels. One or two valvular folds are found at the orifice of the coronary vein, preventing a reflux of blood; but there are no valves at the orifices of the venæ cavæ. 
The left auricle receives the blood which comes from the lungs by the pulmonary veins. It does not differ materially in its anatomy from the right. It is a little smaller, and its walls are thicker, measuring about a line and a half. It has four openings by which it receives the blood from the four pulmonary veins. These openings are not provided with ralves. Like the right auricle, it has a large opening by which the blood flows into the left ventricle. The arrangement of the muscular fibres is essentially the same as in the right auricle.

In adult life the cavities of the auricles are entirely distinct from each other. Before birth they communicate by a large opening, the foramen ovale, and the orifice of the inferior vena cava is provided with a membranous fold, the Eustachian valve, which serves to direct the blood from the lower part of the body through this foramen into the left auricle. After birth the foramen ovale is closed, and the Eustachian valve gradually disappears.

The ventricles, in the human subject and in warm-blooded animals, constitute the bulk of the heart. They have a capacity somewhat greater than that of the auricles, and are provided with thick muscular walls. It is by the powerful action of this portion of the heart that the blood is forced, on the one hand, to the lungs and back to the left side, and on the other, through the entire system of the greater circulation to the right side. It was supposed by Legallois ${ }^{1}$ that the capacity of the right ventricle. was considerably greater than the left, while the more recent observations of Bouillaud ${ }^{2}$ on the human heart seem to show that there is no great difference betweenen the two sides in this regard. The most recent and conclusive observations on this subject are those of Hiffelsheim and Robin. ${ }^{3}$ In these experiments the cavities were filled with an injection of wax, and the estimates

${ }^{1}$ Legallors, Euvres, Paris, 1824, tome i., p. 331.

${ }^{2}$ J. Boullnaud, Traité Clinique des Maladies du Coeur, précédé de Recherches nouvelles sur l'A Anutomie et la Physiologie de cette Organe, Paris, 1841, tome i., p. 54.

a Journal de l'Anatomie et de la Physiologie, Paris, juillet, 1864, p. 413. 
made by calculating the amount of liquid displaced by the moulds of the different cavities. Care was taken to make the injection in animals before cadaveric rigidity set in, or after it had passed away in the human subject. The comparative results obtained by these observers are the most interesting, for the cavities were undoubtedly distended by the injection to their extreme capacity, and contained more than they ever do during life. They found the capacity of the right auricle from $\frac{1}{10}$ to $\frac{1}{3}$ greater than that of the left. The capacity of the right ventricle was from $\frac{1}{10}$ to $\frac{1}{3}$ greater than that of the left, but more frequently there was less disparity between the two ventricles than between the auricles. The capacity of each ventricle exceeded that of the corresponding auricle by from $\frac{1}{4}$ to $\frac{1}{3}$. Nine times out of ten, this predominance of the ventricle was more marked on the left side. The absolute capacity of the left ventricle, according to these observations, is from 143 to 212 cubic centimeters, which is about 4.8 to 7 ounces. This is much greater than most estimates, which place the capacity of the various cavities, moderately distended, at about 2 ounces. The estimates of Volkmann and Valentin are about equal to those we have cited.

In spite of the disparity in the extreme capacity of the various cavities, the quantity of blood which enters the carities is necessarily equal to that which is expelled. This is given in the "Cyclopædia of Anatomy and Physiology". (vol. ii., p. 585) as a little more than two ounces. There are no means of estimating with exactness the quantity of blood discharged with each ventricular contraction; and we find the question rather avoided in works on physiology. All we can say is, that from observation on the heart during its action, it never seems to contain much more than half the quantity in all its cavities that it does when fully distended by injection; but it is the right cavities which are most dilatable, and probably the ordinary quantity of blood in the left ventricle is within one-fifth or one-sixth of its extreme capacity. 
The cavities of the rentricles are triangular or conoidal ; the right being broader and shorter than the left, which extends to the apex. The inner surface of both cavities is marked by peculiar ridges and papillæ, which are called the columnac carnece. Some of these are mere fleshy ridges projecting into the cavity; others are columns attached by each extremity and free at the central portion; and others are papillæ giving origin to the chordoe tendinea, which are attached to the free edges of the auriculo-ventricular valves. These fleshy columns interlace in every direction, and give the inner surface of the cavities a reticulated appearance. This arrangement evidently facilitates the complete emptying of the ventricles during their contraction.

The walls of the left ventricle are uniformly much thicker than the right. Bouillaud found the average thickness of the right rentricle at the base to be $2 \frac{1}{2}$ lines, and the thickness of the left ventricle at the corresponding part 7 lines.

The arrangement of the muscular fibres constituting the walls of the ventricles is more regular than in the auricles, and their course enables us to explain some of the phenomena which accompany the heart's action. The direction of the fibres cannot be well made ont unless the heart has been boiled for a number of hours, when part of the intermuscular tissue is dissolved out, and the fibres can be easily separated and followed. Without going into a minute description of their direction, it is sufficient to state, in this connection, that they present two principal layers: a superficial layer common to both ventricles, and a deep layer proper to each. The superficial fibres pass obliquely from right to left from the base to the apex; here they take a spiral course, become deep, and pass into the interior of the organ to form the columnæ carneæ. These fibres envelop both ventricles. They may be said to arise from cartilaginous rings which surround the auriculo-ventricular orifices. The external surface of the heart is marked by a little groove which indicates the division between the two ventricles. 
The deep fibres are circular, or transverse, and surround each ventricle separately.

The muscular tissue of the heart is of a deep red color, and resembles, in its gross characters, the tissue of ordinary voluntary muscles; but, as already intimated, it presents certain peculiarities in its minute anatomy. The fibres are considerably smaller and more granular than those of ordinary muscles. They are, moreover, connected with each other by short inosculating branches, while in the voluntary muscles each fibre runs from its origin to its insertion enveloped in its proper sheath, or sarcolemma. In the FT. 1.

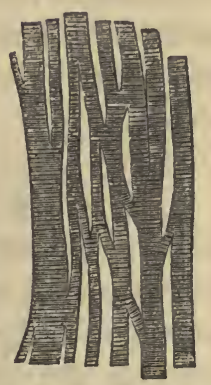

Anastomosing muscular fibres from the human heart. (After Köl. ker.)

heart the fibres have no sarcolemma." These peculiarities, particularly the inosculation of the fibres, favor the contraction of the ventricular walls in every direction, and the complete expulsion of the contents of the cavities with every systole.

Each ventricle has two orifices: one by which it receives the blood from the auricle, and the other by which the blood passes from the right side to the lungs, and from the left side to the system. All of these openings are provided with valves, which are so arranged as to allow the blood to pass in but one direction.

Tricuspid Valve.-This valve is situated at the right auriculo-ventricular opening. It has three curtains, formed of a thin but resisting membrane, which are attached around the opening. The free borders are attached to the chordæ tendineæ, some of which arise from the papillæ on the inner surface of the ventricle, and others directly from the walls of

${ }^{1}$ Roвin states (Dictionnaire de Médecine, etc., de P. H. Nysten, onzième édition par E. Littre et Ch. Robin. Cour.) that the fibres of the heart have no sarcolemma, which I believe to be the fact, though Kölliker (Manual of Microscopıc Anatomy, London, 1860, p. 477) says: "Their sarcolemma is very delicate, or even may not be demonstrable at all, except by the aid of reagents." 
the ventricle. When the organ is empty, these curtains are applied to the walls of the ventricle, leaving the auriculoventricular opening free; but when the ventricle is completely filled, and the fibres contract, they are forced up, their free edges become applied to each other, and the opening is closed.

Pulmonic Valves.-These valves, also called the semilunar or sigmoid valves of the right side, are situated at the orifice of the pulmonary artery. They are strong membranous pouches, with their convexities, when closed, looking towards the ventricle. They are attached around the orifice of the pulmonary artery, and are applied very nearly to the walls of the ressel when the blood passes in from the ventricle; but at other times their free edges meet in the centre, forming an effectual barrier to regurgitation. In the centre of the free edge of cach valve is a little corpuscle called the corpuscle of Arantius ; and just above these points of attachment, the artery presents three little dilatations, or sinuses, called the sinuses of Valsalva. The corpuscles of Arantius have been supposed to facilitate the closure of the valves by slightly removing them from the walls of the vessel, so that the blood may get behind them. This, however, is probably not their function. They aid in the adaptation of the valves to each other, and the effectual closure of the orifice.

Mitral Valve.-This valve, sometimes called the bicuspid, is situated at the left auriculo-ventricular orifice. It is called mitral from its resemblance, when open, to a bishop's mitre. It is attached to the edges of the opening, and its free borders are held in place when closed by the chordæ tendiner of the left side. It presents no material difference from the tricuspid valve, with the exception that it is divided into two curtains instead of three.

Aortic Valves. - These valves, also called the semilunar 
or sigmoid valves of the left side, present no difference from the valves at the orifice of the pulmonary artery. They are situated at the aortic orifice.

The physiological anatomy of the tricuspid and mitral valves may be stadied, by cutting away the auricles so as to expose the auriculo-ventricular openings, introducing a pipe into the pulmonary artery and aorta, after destroying the semilunar valves, and then forcing water into the ventricles by a syringe or from a hydrant. In this way the play of the valves will be beautifully exhibited.

We can study the action of the semilunar valves, by cutting away enough of the ventricles to expose them, and forcing water into the ressels. These experiments give an idea of the immense strength of the valres; for they can hardly be ruptured by a force which is not sufficient to rupture the vessels themselves.

\section{Movements of the Heart.}

In studying the phenomena which accompany the action of the heart, we shall follow the course of the blood, beginning with it as it flows from the vessels into the auricles. The dilatation of the cavities of the heart is called the diastole, and their contraction the systole. When these terms are used without any qualification, they are understood as referring to the ventricles; but they are also applied to the action of the auricles, as the auricular diastole or systole, which, as we shall see, is distinct from the action of the ventricles.

A complete revolution, so to speak, of the heart consists in the filling and emptying of all its cavities, during which they experience an alternation of repose and activity. As these phenomena occupy, in many warm-blooded animals, a period of time less than one second, it will be appreciated that the most careful study is necessary in order to ascertain their exact relations to each other. When the heart is exposed in a living animal, the most prominent phenomenon 
is the alternate contraction and relaxation of the veptricles; but this is only one of the operations of the organ. In any of the class of mammals the anatomy and action of the vascular system are to all intents and purposes the same as in the human subject; and though the exposure of the heart by opening the chest modifies somewhat the force and frequency of its pulsations, the various phenomena follow each other in their natural order, and present essentially their normal characters. The operation of exposure of the heart may be performed on a living animal without any great difficulty; and if we simply take care to keep up artificial respiration, the action of the heart will continue for a considerable time. ${ }^{1}$ We may keep the animal quiet by the administration of ether, or by poisoning with woorara, the latter agent acting upon the motor nerres, but having no effect upon the heart. Having opened the chest, we see the heart enveloped in its pericardium, regularly performing its functions; and on slitting up and removing this covering, the various parts are completely exposed. The right ventricle and auricle, and a portion of the left ventricle, can be seen without disturbing the position of the parts; but the greater part of the left auricle is concealed. As both auricles and ventricles act together, the parts of the heart which are exposed are sufficient for purposes of study.

Action of the Auricles.-Excepting the short time occlpied in the contraction of the auricles, these cavities are continually receiving blood on the right side from the system, by the venæ cavæ, and on the left side from the lungs, by the pulmonary veins. This continues until their cavities are completely filled, the blood coming in by a steady current; and during the repose of the heart, the blood is also flowing

${ }^{2}$ For a full description of the operations for exposing the heart in living animals, the reader is referred to an article by the author in the American Journal of the Medical Sciences, October, 1861, entitled Experimental Researches on points connected with the Action of the Heart and with Respiration. 
through the patent auriculo-ventricular orifices into the ventricles. When the auricles have become fully distended, they contract quickly and with considerable power (the auricular systole), and force the blood into the ventricles, effeeting the complete diastole of these cavities. During this contraction, the blood not only ceases to flow in from the veins, but some of it is regurgitated, as the orifices by which the vessels open into the auricles are not provided with valves. The size of the auriculo-ventricular orifices is one reason why the greater portion of the blood is made to pass into the ventricles; and furthermore, during the auricular systole, the muscular fibres which are arranged around the orifices of the veins constrict them to a certain extent, which tends to diminish the reflux of blood. There can be no doubt that some regurgitation takes place from the auricles into the veins, but this prevents the possibility of over-distention of the ventricles.

It has been shown by experiments that the systole of the auricles is not immediately necessary to the performance of the circulation. M. Marey, ${ }^{1}$ in a recent work on the circulation, cites an experiment of Chauveau in which the contractility of the auricles was temporarily exhausted by prolonged irritation; nevertheless the ventricles continued to act and keep up the circulation.

Action of the Ventricles.-Immediately following the contraction of the auricles, which has the effect of producing complete distention of the ventricles, we have the contraction of the ventricles. This is the chief active operation performed by the heart, and is generally spoken of as the systole. As we should expect from the great thickness of the muscular walls, the contraction of the ventricles is very much more powerful than that of the auricles. By their action, the blood is forced from the right side to the lungs by the pulmonary artery, and from the left side to the system by the aorta. Regurgitation into the auricles is effectually prevented by the

${ }^{1}$ Marex, Circulation du Sang, Paris, 1863, p. 36. 
closure of the tricuspid and mitral valves. This act aceomplished, the heart has a period of repose, the blood flowing into the auricles, and from them into the ventricles, until the auricles are filled, and another contraction takes place.

Locomotion of the Heart.-The position of the heart after death, or during the repose of the organ, is with its base directed slightly to the right, and its apex to the left side of the body; but with each rentricular systole, it raises itself up, the apex is sent forward, and moved a little from left to right. The movement from left to right is a necessary consequence of the course of the superficial fibres. The fibres on the anterior surface of the organ are longer than those on the posterior surface, and pass from the base, which is comparatively fixed, to the apex, which is movable. From this anatomical arrangement the heart is moved upwards and forwards. Their course, from the base to the apex, is from right to left; and as they shorten, the apex is of necessity slightly moved from left to right.

The locomotion of the entire heart forwards was observed by Harvey in the case of the son of the Viscount Montgomery. The young man, aged about nineteen years, suffered a severe injury to the chest, resulting in an abscess, which on cicatrization left an opening into which Harvey could introduce three fingers and the thumb. This opening was directly over the apex of the heart. The action of the portion of the heart thus exposed is described by Harvey in the following words :

"We also particularly observed the movements of the heart, riz.: that in the diastole it was retracted and withdrawn; whilst in the systole it emerged and protruded; and the systole of the heart took place at the moment the diastole or pulse in the wrist was perceived. To conclude, the heart struck the walls of the chest, and became prominent at the time it bounded upward and underwent contraction on itself."

2 Harvey, op. cit., p. 384. 
The locomotion of the heart takes place in the direction of its axis, and is due to the sudden distention of the great vessels at its base. These vessels are eminently elastic, and as they receive the charge of blood from the ventricles, become enlarged in every direction, and consequently project the entire organ against the walls of the chest. This movement is somewhat aided by the recoil of the ventricles as they discharge their contents. The displacement of the heart during its systole has long been observed in vivisections, and may be demonstrated in any of the mammals. The most interesting observations on this point are those of Chauveau and Faivre, which were made upon a monkey. In this animal, in which the position of the heart is very much the same as in the human subject, the locomotion of the organ was fully established. ${ }^{1}$

Twisting of the Heart.-The spiral course of the superficial fibres would lead us to look for another phenomenon accompanying its contraction, namely, twisting. If we attentively watch the apex of the heart, especially when its action has become a little retarded, there is a palpable twisting of the point upon itself from left to right with the systole, and an untwisting with the diastole.

Hardening of the Heart.-If the heart of a living animal be grasped by the hand, it will be observed that at each systole it becomes hardened. The fact that it is composed almost exclusively of fibres resembling very closely those of the voluntary muscles, explains this phenomenon. Like any other muscle, during contraction it is sensibly hardened.

Shortening and Elongation of the Heart.-The foregoing phenomena are admitted by all writers on physiology, and

${ }^{1}$ Nouvelles Recherches expérimentales sur les Mouvements et les Bruits normaux du Cour, envisagés au point de vue de la Physiologie Médicale. Par A. Cuauveau et J. Faìvre. Paris, 1856, p. 24. 
can easily be observed; but the change in length of the heart during its systole has been, and is now, a matter of discussion. All who have studied the heart in action have observed changes in length during contraction and relaxation; but the contemporaries of Harvey were divided as to the periods in the heart's action which are attended with elongation and shortening. Harvey himself is not absolutely definite on this point. In one passage he says, in describing the systole, " that it is everywhere contracted, but especially towards the sides, so that it looks narrower, relatively longer, more drawn together." "In his description of the case of the Viscount Montgomery, who suffered from ectopia cordis, he states that during the systole, the heart "emerged and protruded." " Vesalius, Riolan, Fontana, and some others, contended for elongation during the systole; but Haller, Steno, Lancisi, and Bassuel contended that it shortened. The view generally entertained at the present day is that the heart becomes shorter during its systole; but there are some eminent authorities who hold an opposite opinion. Among the latter may be mentioned Drs. Pennock and Moore, who made a great number of experiments on the action of the heart in sheep and young calves. These experiments were made in Philadelphia in 1839, and it was apparently demonstrated that the heart elongated to such a marked degree, that the distance could be measured with a shoemaker's rule. In one experiment (a ewe one year old), the elongation was a quarter of an inch." Of all the writers of systematic works on physiology, Prof. Dalton is the only one, as far as we know, who accepts this view. ${ }^{4}$ The experiments of this observer appa-

${ }^{2}$ HARver's Works, published by the Sydenham Society, p. 21.

${ }^{2}$ Ibid., p. 384.

'Hope, on the Heart. American Edition by Pexwock, Philadelphia, 1846, p. 59.

${ }^{4}$ Dalton, A Treatise on Human Physiology, Philadelphia, 1864, third edition, pp. 275, 276. The heart of the eel is said by Haller to elongate during its ventricular systole, though this is denied by Fontana (Mémoires de Haller, Lau- 
rently confirm those of Drs. Pennock and Moore. Some experiments made by the author a few years ago, published in the "American Journal of Medical Sciences," Oct. 1861, had apparently the same result. There is no doubt that the point of the heart is protruded during the ventricular systole, as the experiments referred to conclusively prove; but the author was led by the perusal of recent experiments by Chauveau and Faivre, to recognize the fact that this protrusion is probably due to other causes than the elongation of the ventricles, and that during the systole the ventricles are shortened. The experiment cited by these eminent physiologists is very simple and conclusive. It is made by suddenly cutting the heart ont of a warm-blooded animal, and watching the phenomena which accompany the few regular contractions which follow. They found that the ventricles invariably shortened during the systole. This could easily be appreciated by the eye, but more readily if the point. of the organ were brought just in contact with a plane surface at right angles, when at each contraction it is unmistakably observed to recede. ${ }^{1}$ This experinent we have lately repeated before the class of the Bellevue Hospital Medical College, and have satisfied ourselves of its accuracy. A large Newfoundland pup, about nine months old, was poisoned with woorara, artificial respiration was kept up, and the heart exposed. After showing the protru. sion of the point and the apparent elongation while in the

sanne, 1760, tome iii., p. 224); but in experimenting on the organ after excision, the position in which it is held is important. If, for example, we take the heart of a turtle between the thumb and finger and hold it with the point upwards, the ventricle is so thin and flabby that it will become flattened during the intervals of contraction, and the point will be considerably elevated at each systole; but if we reverse the position and allow the point to hang down, it will be drawn up and the ventricle will shorten with the systole.

${ }^{1}$ Chauveau et Faivre, op. cit., p. 14. These observers show the shorten ing of the heart during its systole by holding it by the great vessels with the point down. It is more free from sources of error to observe the phenomena as the heart lies on a flat surface. 
chest, the organ was rapidly removed, placed upon the table, and confined by two long needles passed through the base, pinning it to the wood. It contracted for one or two minutes; and at each systole, the ventricles were manifestly shortened. The point was then placed against an upright, and it receded with each systole about an eighth of an inch. This phenomenon was apparent to all present.

In another experiment, performed a few weeks later, the heart, which had been exposed in the same way, was examined in situ by pinning it with two needles to a thin board passed under the organ. The presence of these needles did not seem to interfere with the heart's action, and at each ventricular systole the point evidently approached the base. To render this absolutely certain, a knife was fixed in the wood at right angles to and touching the point during the diastole, and a small silver tube was introduced through the walls into the left ventricle. At each contraction, a jet of blood spurted out through the tribe, and the point of the heart receded from the knife about an eighth of an inch. The animal experimented upon was a dog a little above the medium size.

These simple experiments demonstrate that, in the dog at least, the ventricles shorten during their systole. The arrangement of the muscular fibres is too nearly identical in the heart of the warm-blooded animals to leave room for doubt that it also shortens in the human subject.

The error which has arisen in this respect, and which obtained in our former experiments, is due to the locomotion and protrusion of the entire organ, so as to make the point strike against the chest. A little reflection indicates the mechanism of this phenomenon. During the intervals of contraction, the great vessels, particularly the aorta and pulmonary artery, which attach the base of the heart to the posterior wall of the thorax, are filled, but not distended, with blood; at each systole, however, these ressels are distended to their utmost capacity; their elastic coats permit of con- 
siderable enlargement, as can be seen in the living animal, and this enlargement, taking place in every direction, pushes the whole organ forward. We have also considerable locomotion of the heart from recoil. It is for this reason that, observing the heart in situ, the ventricles seem to elongate, and an instrument applied to it apparently indicates removal of the apex from the base. It is only when we examine the heart firmly fixed, or contracting after it is removed from the body, that we can appreciate the actual changes which occur in the length of the ventricles. ${ }^{1}$

In addition to these marked changes in form, position, etc., which the heart undergoes during its action, we observe, on careful examination, that the surface of the ventricles becomes marked with slight longitudinal ridges during the systole. This was not observed by Harvey, but is mentioned by Haller. ${ }^{2}$

Impulse of the Heart.-Each movement of the heart produces an impulse, which can be readily felt and sometimes seen, in the fifth intercostal space, a little to the left of the median line. Vivisections have demonstrated that the impulse is synchronous with the contraction of the ventricles. If the hand be introduced into the chest of a living animal, and the finger placed between the point of the heart and the walls of the thorax, every time we have a hardening of the point the finger will be pressed against the side. If the impulse of the heart be felt while the tinger is on the pulse, it is evident that the heart strikes against the thorax at the time of the distention of the arterial system. The impulse is due to the locomotion of the ventricles. In the words of Harvey,

1 The observations of Fontana on the shortening of the heart are very conclusive. He constructed a little instrument consisting of two vertical rules, sliding on a horizontal bar like a shoemaker's measure, one of which was applied to the base, and the other just grazed the apex. He estimated the shortening of the heart in a lamb at about two Paris lines (Mém. de Haller, tome iii., p. 225).

${ }^{2}$ Elementa Physiologia, vol. i., p. 389. 
"the heart is erected, and rises upwards to a point, so that at this time it strikes against the breast and the pulse is felt externally." In the case of the son of the Viscount Montgomery, already referred to, Harvey gives a most graphic deEcription of the manner in which the heart is "retracted and withdrawn" during the diastole, and "emerged and protruded" during the systole.

Succession of the Movements of the Heart.-We have already followed, in a general way, the course of the blood through the heart, and the successive action of the various parts; but we have yet to consider these points more in de tail, and ascertain if possible the relative periods of activity and repose in each portion of the organ.

The great points in the succession of morements are readily observed in the hearts of cold-blooded animals, where the pulsations are very slow. In examining the heart of the frog, turtle, or alligator, the alternations of repose and activity are very strongly marked. During the intervals of contraction, the whole heart is flaccid, and the rentricle is comparatively pale; we then see the auricles slowly filling with blood; when they have become fully distended, they contract and fill the ventricle, which in those animals is single; the ventricle immediately contracts, its action following upon the contraction of the auricles as if it were propagated from them. When the heart is filled with blood, it has a dark red color, which contrasts strongly with its appearance after the systole. This operation may occupy from ten to twenty seconds, giving an abundance of time for observation. The case is different, however, with the warm-blooded animals, in which the anatomy of the heart is nearly the same as in man. Here a normal revolution may occupy less than a second, and it is evident that the varied phenomena we have just mentioned are followed with the utmost difficulty. In spite of this rapidity of action, it can be seen that a rapid contraction 
of the auricles precedes the ventricular systole, and that the latter is synchronous with the impulse.

Various estimates have been made of the relative time occupied by the auricular and ventricular contractions. This interesting point has been carefully studied by MCM. Chauveau and Faivre, by auscultating the heart exposed in a living animal, and establishing, by the touch, the relations between the contractions of its different parts and the heart sounds. These observers made a great number of experiments upon horses and dogs, in which the pulse was not more accelerated than the pulse of the human subject. As the result of these observations, the following numbers are given as representing the rhythm of the movements of the heart in man: Auricular systole, 6 ; Ventricular systole, 10 ; Diastole, $8 .^{1}$ Though this estimate is perhaps better than any we had before, it is evident from the way in which it was arrived at that it can be nothing more than an approximation; for it is impossible to estimate accurately, by the stethoscope and the touch, operations which follow each other with such rapidity.

This question has been at last definitely settled by the late observations of Marey, who has constructed some very ingenious instruments for registering the form and frequency of the pulse. He devised a series of most interesting experiments, in which he was enabled to register simultaneously the pulsations of the different divisions of the heart, and has succeeded in establishing a definite relation between the contractions of the auricles and ventricles. The method of M. Narey enables us to determine, to a small fraction of a second, the duration of the contraction of each of the divisions of the heart.

The method of transmitting the movement from the heart to a registering apparatus is very simple. It consists of two little elastic bags connected together by an elastic tube, the whole closed and filled with air. A pressure, like the pres-

1 Ciraureau et Faivre, op. cit., p. 18. These authors represent the rhythm by musical notes, which have been reduced to the numbers given above. 
sure of the fingers, upon one of these bags produces, of course, an instantaneous and corresponding dilatation of the other. If we suppose one of these bags to be introduced into one of the cavities of the heart, and the other placed under a small lever, so arranged on a pivot as to be sensible to the slightest impression, it is evident that any compression of the bag in the heart would produce a corresponding change in volume in the other, which would be indicated by a movement of the lever. M. Marey has arranged the lever with its short arm on the elastic bag, and the long arm, provided with a pen, moving against a roll of paper which passes along at a uniform rate. When the lever is at rest and the paper set in motion, the pen will make a horizontal mark; but when the lever ascends and descends, a corresponding trace will be made, and the duration of any movement can readily be estimated by calculating the rapidity of the motion of the paper. The bag which receives the impression is called by Marey the initial bag, and the other, which is connected with the lever, is called the terminal bag. The former may be modified in form with reference to the situation in which it is to be placed. .

The experiments of M. Marey, with reference to the relations between the systole of the auricles, the systole of the ventricles, and the impulse of the heart, were performed upon horses in the following way:

A sound is introduced into the right side of the heart through the jugular vein, an operation which is performed with certainty and ease. ${ }^{1}$ This sound is provided with two initial bags, one of which is lodged in the right auricle, while

${ }^{1}$ Catheterization of the cavities of the heart, especially upon the right side, is an operation familiar to physiologists. With a double canula, such as is described by Marey (p. 61), of the requisite dimensions and with the proper curres, it must be easy to lodge the bags respectively in the auricle and ventricle; especially in an animal of large size like the horse. $\mathbf{A}$ tube is easily introduced into the right side of the heart, in the dog, through the external jugular. M. Marey gives full details of every step of the operation, and there can be no doubt of the facility and accuracy with which it may be performed. 
the other passes into the ventricle. The bags are connected with distinct tubes which pass one within the other, and are connected by elastic tubing with the registering apparatus. At each systole of the heart the bags in its cavities are compressed, and produce corresponding movements of the levers, which may be registered simultaneously.

To register the impulse of the heart, an incision is made over the point where the apex beat is felt, through the skin

FIG. 2.

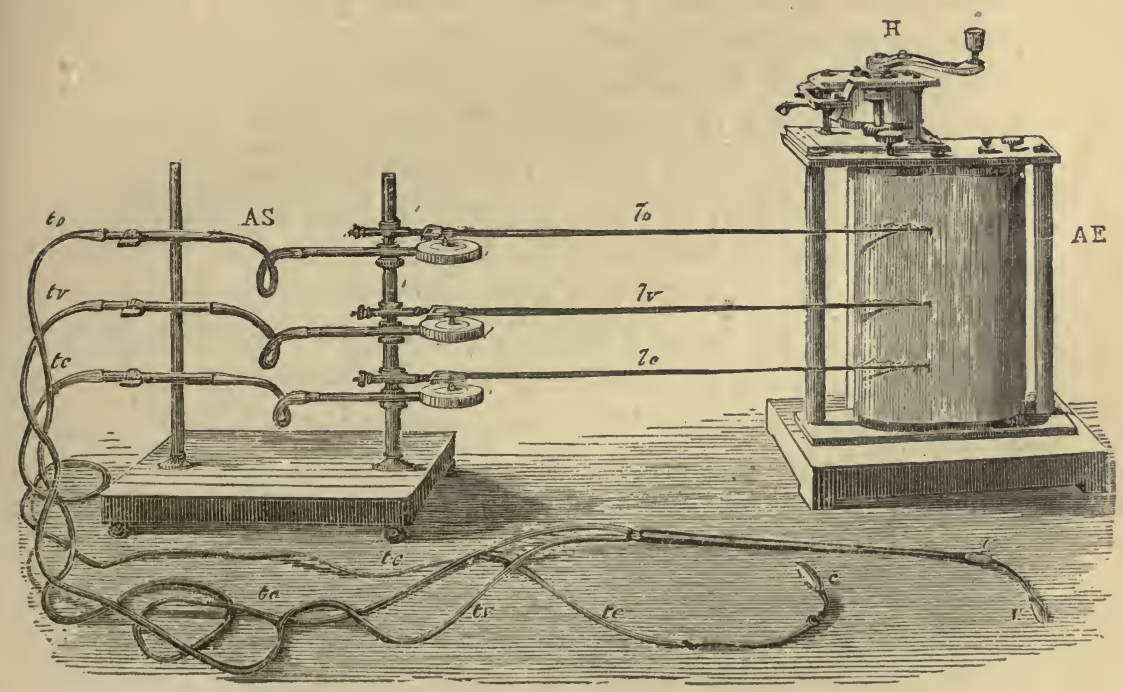

Figure representing the "cardiographe" of Marey. "The instrument is composed of two principal elements: A E, the registering apparatus and $\mathbf{A} \mathbf{S}$, the sphygmographic apparatus, that is to say, which receives, transmits, and amplifies the movements which are to be studied." The compression exerted upon the bag $c$, which is placed over the apex of the heart between the intercostal muscles, is conducted by the tube tc, which is filled with air, to the first lever. The eompression exerted upon the bags $o$ and $v$, in the double sound, is conducted by the tubes $t o$ and $t v$ to the two remaining levers. The movements of the levers are registered simultaneously by the cylinders A E. (MAREx, Sur la Circulation du Sang, Paris, 1863, p. 54.)

and external intercostal muscle. A little bag, stretched over two metallic buttons separated by a central rod, is then carefully secured in the cavity thus formed, and connected by an 
elastic tube with the registering apparatus. All the tubes are provided with stop-cocks, so that each initial bag may be made to communicate with its lever at will. When the operation is concluded, and the sound firmly secured in place by a ligature around the rein, the animal experiences no inconvenience, is able to walk about, eat, \&c., and there is every evidence that the circulation is not interfered with. The cylinders which carry the paper destined to receive the traces are arranged to move by clock-work at a given rate. The paper may also be ruled in lines, the distances between which represent certain fractions of a second.

Fig. 2, taken from the work of Marey, represents the apparatus reduced to one-sixth of its actual size. Two of the levers are connected with the double sound for the right auricle and ventricle, and one is connected with the bag destined to receive the impulse of the heart.

In an experiment upon a horse, every thing being carefully arranged in the way indicated, the elock-work was set in motion, and the movements of the three levers produced traces upon the paper which were interpreted as follows:

1. The paper was ruled so that each division represented one-tenth of a second. The traces formed by the three levers indicated four revolutions of the heart. The first revolution occupied $1_{10}^{\frac{1}{10}}$ sec., the second $1_{10}^{2}$ sec., the third $1_{10} \frac{1}{10}$ sec., and the fourth 1 sec.

2. The auricular systole, as marked by the first lever, immediately preceded the ventricular systole, and occupied about two-tenths of a second. The eleration of the lever indicated that it was much more feeble than the ventricular systole, and sudden in its character; the contraction, when it had arrived at the maximum, being immediately followed by relaxation.

3. The ventricular systole, as marked by the second lever, followed immediately the auricular systole, and occupied, about four-tenths of a second. The almost vertical direction of the trace, and the degree of elevation, showed that it 
was sudden and powerful in its character. The abrupt descent of the lever showed that the relaxation was almost instantaneous.

4. The impulse of the heart, as marked by the third lever, was shown to be absolutely synchronous with the ventricular systole. ${ }^{1}$

Condensing the general results obtained by Marey, which are of course subject to a certain amount of variation, we have, dividing the action of the heart into ten equal parts, three distinct periods, which occur in the following order :

Auricular Systole.-This occupies two-tenths of the heart's action. It is feeble compared with the ventricular systole, and relaxation immediately follows the contraction.

Ventricular Systole.-This occupies four-tenths of the heart's action. The contraction is powerful, and the relaxation sudden. It is absolutely synchronous with the impulse of the heart.

Diastole.-This occupies four-tenths of the heart's action.

Force of the Heart.-There are few points in physiology on which opinions have been more widely divergent, than on the question of the force employed by the heart at each contraction. Borelli, who was the first to give a definite estimate of this force, put it at 180,000 pounds ; while the calculations of Keill give only 5 ounces." These estimates, however, were made on purely theoretical grounds. Borelli estimated the force employed by the deltoid in sustaining a given weight held at arm's length, and formed his estimate of the

${ }^{1}$ Marey, op. cit., p. 68 et seq. I have preferred to give the general significance of the three traces obtained by Marey, rather than reproduce the traces themselves, which present certain minor characters which might confuse the reader. Nothing could be more distinct than the illustration of the particular points above enumerated; and there can be no other opinion than that these observations settle the question of the rhythm of the heart's action in the animals on which the experiments were performed.

${ }^{2}$ James KeIll, M.D., Fissays on Several Parts of the Animal EEconomy, London, 1717, pp. $87,91$. 
power of the heart by comparing the weight of the organ with that of the deltoid. Keill made his estimate from a calculation of the rapidity of the current of blood in the arteries. Hales was the first to investigate the question experimentally, by the application of the cardiometer. $\mathrm{He}$ showed that the pressure of blood in the aorta could be measured by the height to which the fluid would rise in a tube connected with that vessel, and estimated the force of the left ventricle by multiplying the pressure in the aorta by the area of the internal surface of the ventricle. The cardiometer has undergone various improvements and modifications, but this is the principle which is so extensively made use of at the present day, in estimating the pressure of the blood in different parts of the circulatory system. First we have the improvement of Poiseuille, who substituted a $U$ tube partly filled with mercury, for the long straight tube of Hales; and then the various forms of cardiometers constructed by Magendie, Bernard, Marey, and others, which will be more fully discussed in connection with the arterial circulation. These instruments have been made use of by Marey, with very good results, in investigating the relative force exerted by the different divisions of the heart.

Hales estimates, from experiments upon living animals, the height to which the blood would rise in a tube connected with the aorta of the human subject, at 7 feet 6 inches, and gives the area of the left ventricle as 15 square inches. From this he estimates the force of the left ventricle at 51.5 pounds. ${ }^{1}$

Though this estimate is only an approximation, it seems based on more reasonable data than any other.

The apparatus of Marey for registering the contractions of the different eavities of the heart enabled him to ascertain, also, the comparative force of the two ventricles and the right auricle; the situation of the left auricle as yet precluding the possibility of introducing a sound into its eavity.

${ }^{2}$ Stephex Hales, B.D., F.R.S., \&c., Statical Essays : Containing Hremastaticks, \&c., London, 1733. Vol. II., p. 40. 
By first subjecting the bags to known degrees of pressure, the degree of elevation of a lever may be graduated so as to represent the degrees of the cardiometer. In analyzing traces made by the left ventricle, right ventricle, and right auricle, in the horse, Marey found that, as a general rule, the comparative force of the right and left ventricles is as 1 to $3 .^{1}$

The force of the right auricle is comparatively insignificant, being in one case, as compared with the right ventricle, only as 1 to 10 .

Action of the Valves.-We have already indicated the course of the blood through the cavities of the heart, and it has been apparent that the necessities of the circulation demand some arrangement by which the current shall always be in one direction. The anatomy of the valves which guard the orifices of the ventricles gives an idea of their function; but we have yet to consider the precise mechanism by which they are opened and closed, and the way in which regurgitation is prevented.

In man and the warm-blooded animals, there are no valves at the orifices by which the veins open into the anricles. As has already been seen, compared with the ventricles, the force of the auricles is insignificant; and it has furthermore been shown by experiment that the ventricles may be filled with blood, and the circulation continue, when the auricles are entirely passive. Though their orifices are not provided with valves, the circular arrangement of the fibres about the veins is such, that during the contraction of the auricles the openings are materially narrowed, and regurgitation cannot take place to any great extent. The force of the blood flowing into the auricles likewise offers an obstacle to its return. There is really no valvular apparatus which operates to prevent regurgitation from the heart into the veins; for the valvular folds which are so numerous in the 
general venous system, and particularly in the veins of the extremities, do not exist in the venæ cavæ.

The continuous flow of blood from the veins into the auricles, the feeble character of their contractions, the arrangement of the fibres around the orifices of the vessels, and the great size of the auriculo-ventricular openings, are conditions which provide sufficiently well for the flow of blood into the ventricles.

Auriculo-Ventricular Valves.-After the ventricles have become completely distended by the auricular systole, they take on their contraction; which, it will be remembered, is very many times more powerful than the contraction of the auricles. They have to force open the valves which close the orifices of the pulmonary artery and aorta, and empty their contents into these vessels. To accomplish this, at the moment of the ventricular systole, there is an instantaneous and complete closure of the auriculo-ventricular valves, leaving but one opening through which the blood can pass. That these valves close at the moment of contraction of the ventricles is demonstrated by the experiments of Chauveau and Faivre, who introduced the finger through an opening into the auricle, and actually felt the valves close at the instant of the ventricular systole. ${ }^{1}$

This tactile demonstration, and the fact that the first sound of the leart, which is produced in great part by the closure of the auriculo-ventricular valves, is absolutely synchronous with the ventricular systole, leave no doubt as to the mechanism of the closure of these valves.

It is probable that as the blood flows into the ventricles the valves are slightly floated out, but they are not closed until the ventricles contract. A German physiologist, Baumgarten, ${ }^{2}$ has attempted to show that the valves are closed by the contraction of the auricles, basing this opinion upon the fact that when the auricles are cut away, and fluid is poured

${ }^{1}$ Op. cit., p. 21.

2 Milise-Edwards, op. cit., tome iv., p. 31. 
through the auriculo-ventricular opening, the valves are floated up, and finally closed when the ventricle is completely filled. This experiment we have repeated and found to be correct; but in this way we are far from fulfilling the ratural conditions of the circulation. In the natural action of the heart, the blood flows from the auricles in a large stream, which opens the valves and applies them to the walls of the ventricles. This is quite different from the action of a small stream, which may insinuate itself between the lips of the valves, and force them up by reacting from the ventricle. If the semilunar valves be exposed, and the artery closed, a stream of water poured from the ventricles will close the valves; and yet we could hardly say that in the natural course of the circulation the valves at the arterial orifices are closed by the ventricular systole.

These experiments do not throw any doubt upon the fact that the auriculo-ventricular valves are closed by the pressure of blood against them during the ventricular systole.

If a bullock's heart be prepared by cutting away the auricles so as to expose the mitral and tricuspid valves, securing the nozzles of a double syringe in the pulmonary artery and aorta, after having destroyed the semilumar valves, and if fluid be injected simultaneously into both ventricles, the play of the valves will be exhibited. The mitral valve effectually prevents the passage of the fluid, its edges being so accurately approximated that not a drop passes between them; but when the pressure is considerable, a certain quantity of fluid passes the tricuspid valve. There is, indeed, a certain amount of insufficiency at the right auriculo-ventricular orifice, which does not exist on the opposite side.

This fact was first pointed out by Mr. T. King, ${ }^{1}$ and is called by him the "safety-valve function of the right ventricle." The advantage of this slight insufficiency is apparent on a little reflection. The right ventricle sends its blood to the

${ }^{3} \mathrm{~K}$ iNG, An Essay on the Safety-valve Functions of the Right Ventricle of the Human Heart. Guy's Hospital Reports, 1837, vol. ii. p. 104. 
lungs, where, in order to facilitate the respiratory processes, the walls of the capillaries are very thin. The lungs themselves are exceedingly delicate, and an effusion of blood, or considerable congestion, would be liable to be followed by serious consequences. To prevent this, the right ventricle is not permitted to exert all its force, under all circumstances, upon the blood going into the pulmonary artery; but when the action of the heart is exaggerated from any canse, the lungs are relieved by a slight regurgitation, which takes place through the tricuspid valve. The lungs are still further protected by the sufficiency of the mitral valve, which provides that no regurgitation shall take place into their substance from the left heart. In the systemic circulation the capillaries are less delicate; extravasation of blood would not be followed by any serious results, and the circulating fluid is made to pass through a considerable extent of the elastic vessels, before it begins to be distributed in the tissues. 'It is evident that on the left side there is no necessity for such a provision, and it does not exist.

Aortic and Pulmonic Valves.-The action of the semi'unar valves is nearly the same upon both sides. In the intervals of the ventricular contractions, they are closed, and prevent regurgitation of blood into the ventricles. The systole, however, overcomes the resistance of these valves, and forces the contents of the ventricles into the arteries. During this time the valves are applied, or nearly applied, to the walls of the vessel; but as soon as the ventricles cease their contraction, the constant pressure of the blood, which, as we shall see hereafter, is very great, instantaneously closes the openings.

The action of the semilunar valves can be seen by cutting away a portion of the ventricles in the heart of a large animal, securing the nozzles of a double syringe in the aorta and pulmonary artery, and forcing water into the vessels. In performing this experiment, it will be noticed that while the 
aortic semilunar valves oppose the passage of the liquid so effectually that the aorta may be ruptured before the valves will give way, a considerable degree of insufficiency exists, under a bigh pressure, at the orifice of the pulmonary artery. There is at this orifice a safety-valve function as important as that ascribed by King to the tricuspid valve. It is evident that the slight insufficiency at the pulmonic orifice may be even more directly important in protecting the lungs than the insufficiency of the tricuspid valve. The difference in the sufficiency of the semilunar valves on the two sides is fully as marked as between the auriculo-ventricular valves, and it is surprising that since the observations of King, this fact has not attracted the attention of physiologists. ${ }^{1}$

It is probable that the corpuscles of Arantius, which are situated in the middle of each valvular curtain, assist in the accurate closure of the orifice. The sinuses of Valsalva, situated in the artery behind the valves, are regarded as facilitating the closure of the valves by allowing the blood to pass easily behind them.

Sounds of the Heart.-If the ear be applied to the præcordial region, it will be found that the action of the heart is accompanied by certain sounds. A careful study of these sounds, and their modifications in disease, has enabled the practical physician to distinguish, to a certain extent, the conditions of the heart. This increases the purely physiological interest which attaches to the audible manifestations of the action of the great central organ of the circulation.

The appreciable phenomena which attend the heart's action are connected with the systole of the ventricles. It is this which produces the impulse against the walls of the thorax, and, as we shall see further on, the dilatation of the arterial system, called the pulse. It is natural, therefore, in

1 This observation was first made, and the fact publicly demonstrated, in the course on physiology at the Bellevue Hospital Medical College, session of $1864-' 65$. 
studying these phenomena, to take the systole as a point of departure, instead of the action of the auricles, which we cannot appreciate without vivisections; and the sounds, which are two in number, have been called first and second, with reference to the systole.

The first sound is absolutely synchronous with the apex beat. The second sound follows the first without any appreciable interval. Between the second and first sounds there is an interval of silence.

Some writers have attempted to represent the sounds of the heart, and their relations to each other, by certain syllables, as, "lubb-dup or lubb-tub "," but it seems unnecessary to attempt to make a comparison, which can only be appreciated by one who is practically acquainted with the heartsounds, when the sounds themselves can be so easily studied.

Both sounds are generally heard with distinctness over any part of the præcordia. The first sound is heard with its maximum of intensity over the body of the heart, a little below and within the nipple, between the fourth and fifth ribs, and is propagated with greatest facility downwards, towards the apex. The second sound is heard with its maximum of intensity at the base of the heart, between the nipple and the sternum, about the locality of the third rib, and is propagated upwards, along the course of the great vessels.

The rhythm of the sounds bears a certain relation to the rhythm of the heart's action, which. we have already discussed; the difference being, that we here regard the heart's action as commencing with the systole of the ventricles, while in following the action of different parts of the organ, we followed the course of the blood, and commenced with the systole of the auricles. Laennec, the father of auscultation, was the first to direct special attention to the rhythm of these sounds, though they had been recognized by Harvey, who compared them to the sounds made by the passage of fluids

${ }^{1}$ C. J. B. Wrlliams, in Dunglison's Human Physiology. Philadelphia, 1856, rol. i., p. 393. 
along the osophagus of a horse when drinking. ${ }^{1}$ He divides a single revolution of the heart into four parts: the first two parts are occupied by the first sound; the third part by the second sound; and in the fourth part there is no sound. ${ }^{2}$ He regards the second sound as following immediately after the first. Some authors have described a "short silence" as occurring after the first sound, and a "long silence" after the second. The short silence, if appreciable at all, is so indistinct that it may practically be disregarded.

Attempts have been made to improve upon this division of Laennec, by dividing the heart's action into three equal parts, as is done by M. Beau ; the first being occupied by the first sound, the second by the second sound, and the third, silence. This hardly needs discussion. M. Beau bases this division upon a theory of the production of the sounds which, though pretty generally discussed by physiologists, is, as far as we have seen, adopted by none, and is so entirely opposed to facts that it hardly demands comment. It is evident to any one who has heard the sounds of the heart, that the first is longer than the second.

Most physiologists regard the duration of the first sound as a little less than two-fourths of the heart's action, and the second sound as a little more than one-fourth. When we come to consider the mechanism of the production of the two sounds, we shall see that if our views on that point be correct, the first sound should occupy the period of the ventricular systole, or four-tenths of the heart's action, the second sound about three-tenths, and the repose three-tenths.

The first sound is relatively dull, low in pitch, and made up of two elements: one, a valvular element, in which it resembles in character the second sound; the other, an element which is due to the action of the heart as a muscle. It has been ascertained that all muscular contraction is at-

Op. cit., p. 32.

${ }^{2}$ Laennec, Traité de l'Auscultation Médiate, Paris, 1837, tome iii., p. 48.

${ }^{3} \mathrm{BEad}$, Traité expérimentale et clinique d'Auscultation, Paris. 1856, p. 228. 
tended with a certain sound. To this is added an impulsion element, which is produced by the striking of the heart against the walls of the thorax.

The second sound is relatively sharp, high in pitch, and has but one clear, element, which we have already alluded to as valvular.

Cause of the Sounds of the Heart.-There is now scarcely any difference of opinion respecting the cause of the second sound of the heart. The experiments of Rouanet, published in 1832, settled beyond a doubt that it was due to a closure of the aortic and pulmonary semilunar valves. In his essay upon this subject, Rouanet acknowledges his indebtedness, for the first suggestion of this explanation, to $\mathrm{Mr}$ Carswell, who was at that time prosecuting his studies in Paris. $^{.}$

The experiments by which this is demonstrated are as simple as they are conclusive. First we have the experiments of Rouanet, who imitated the second sound by producing sudden closure of the aortic valves by a column of water. We then have the experiments, even more conclusive, of the British Commission, in which the semilunar valves were caught up by curved hooks introduced through the vessels of a living animal, the ass, with the result of abolishing the second sound, and substituting for it a hissing murmur. When the instruments were withdrawn, and the valves permitted to resume their action, the normal sound returned. ${ }^{2}$

It is unnecessary to discuss the various theories which have been advanced to explain the second sound, as it is now generally acknowledged to be due to the sudden closure of the

${ }^{1}$ Cyclopcedia of Anatomy and Physiology, vol. ii., p. 617. In this article, we find Ir. Elliott, of Carlisle, alluded to as having stated in his thesis, published in 1831 , "that the second sound of the heart is dependent upon the rush of blood from the auricles into the ventricles during their diastole, and also upon the sudden flapping inward of the sigmoid valves at the origin of the large arteries by the refluent blood."

2 Ibid., p. 618. 
semilunar valves at the orifices of the aorta and pulmonary artery. We remark, however, that the sound is heard with its maximum of intensity over the site of these valves, and is propagated along the great vessels, to which they are attached. It also occurs precisely at the time of their closure; $i$. e., immediately following the ventricular systole.

The cause of the first sound of the heart has not, until within a few years, been as well understood. It was maintained by Rouanet, that this sound was produced by the sudden closure of the auriculo-ventricular valves; but the situation of these valves rendered it difficult to demonstrate this by actual experiment. We have already seen, that while the second sound is purely valvular in its character, the first sound is composed of a certain number of different elements ; but auscultatory experiments have been made by which all but the valvular element are eliminated, and the character of the first sound made to resemble that of the second. Conclusive observations on this point were made a few years ago by Dr. Flint, constituting part of an essay which received the prize of the American Medical Association in 1858. ${ }^{1}$

The following facts were developed in this essay:

1. If a folded handkerchief be placed between the stethoscope and integument, the first sound is divested of some of its most distinctive features. It loses the quality of impulsion, and presents a well-marked valvular quality.

2. In many instances, when the stethoscope is applied to the præcordia, while the subject is in a recumbent posture, and the heart by force of gravity is removed from the anterior wall of the thorax, the first sound becomes purely valvular in character, and as short as the second.

3. When the stethoscope is applied to the chest a little distance from the point where the first sound is heard with its maximum of intensity, it will present only its valvular element.

1 Austin Flint, Prize Essay on the Hoart-Sounds in Health and Disease. Transactions of the American Medical Association, 1858. 
These facts, to which we may add the modifications of the first sound in disease, so as to leave only the valvular element, taken in connection with the fact that the first sound oecurs when the ventrieles contract, and neeessarily aceompanies the closure of the aurieulo-ventricular valves, show pretty conclusively that these valves produce at least a certain element of the sound. In further support of this opinion, we have the fact that the first sound is heard with its maximum of intensity over the site of the valves, and is propagated downwards along the ventricles, to which the valves are attached.

Actual experiments are not wanting to confirm the above view. Chauveau and Faivre ${ }^{1}$ have sueceeded in abolishing the first sound by the introduction of a wire ring through a little opening in the auricle into the auriculo-ventricular orifice, so arranged as to prevent the closure of the valves. When this is done, the first sound is lost; but on taking it out of the opening, the sound returns. These observers also abolished the first sound by introducing a small curved tenotomy-knife through the auriculo-ventricular orifice, and dividing the chordæ tendineæ. In this experiment a loud rushing murmur took the place of the sound. We have already alluded to the experiment of intradueing the finger through an opening in the auriele; if this be done, and the heart be auscultated at the same time, the valves will be felt striking against the finger in unison with the first sound.

The above observations and experiments settle beyond question the fact that the closure of the auriculo-ventricular valves produces one element of the first sound.

The other elements which enter into the composition of the first sound are not as prominent as the one we have just considered, though they serve to give it its prolonged and "booming" character. These elements are, a sound like that produced by any large muscle during its contraction, called 
by some the muscular murmur, and the sound produced by the impulse of the heart against the walls of the chest.

The muscular sound has been recognized by Wollaston, Laennec, and others, and by Laennec was supposed to be the sole cause of the first sound of the heart. This observer attributed the first sound to the muscular action of the ventricles, and the second to the action of the auricles. There can be no doubt but that this is one of the elements of the first sound; and it is this which gives it its prolonged character, when the stethoscope is applied over the body of the organ, as the sound produced in muscles continues during the whole period of their contraction. Admitting this to be an element of the first sound, we can understand how its duration must necessarily coincide with the ventricular systole. We can appreciate, also, how all but the valrular element is eliminated when the stethoscope is moved from the body of the heart, the muscular sound not being propagated as completely as the sound made by the closure of the valves.

The impulse of the heart against the walls of the thorax also contributes to produce the first sound. This is demonstrated by noting the difference in the sound when the subject is lying upon the back, and when he is upright; or by interposing any soft substance between the stethoscope and the chest, or by auscultating the heart after the sternum has been removed. Under these circumstances the first sound loses its booming character, retaining, however, the muscular element, when the instrument is applied to the exposed organ. It was thought by Magendie that the shock of the heart against the chest was the sole cause of the first sound. ${ }^{1}$ This observer maintained that when the sternum is removed in a living animal, the first sound cannot be heard over the heart. This, however, is not the fact; and though the element of impulsion enters into the composition of the first sound, the view that it is the sole cause of this sound is not tenable.

The first sound of the heart is complex. It is pro-

1 Milne-Enwards, Leģons sur la Physiologie, etc., tome iv., p. 3S. 
duced by the sudden closure of the auriculo-ventricular valves at the beginning of the ventricular systole; to which are superadded the muscular sound, due to the contraction of the muscular fibres of the heart, and the impulsion sound, due to the shock of the organ against the walls of the thorax.

The second sound is simple. It is produced by the sudden closure of the aortic and pulmonary semilunar valves, immediately following the ventricular systole.

It is of the greatest importance, with reference to pathology, to have a clear idea of the currents of blood throngh the heart, with their exact relation to the sounds and intervals.

At the commencement of the first sound, the blood is foreibly thrown from the ventricles into the pulmonary artery on the right side and the aorta on the left, and the auriculo-ventricular valves are suddenly closed. During the entire period occupied by this sound, the blood is flowing rapidly through the arterial orifices, and the auricles are receiving blood slowly from the venæ cavæ and the pulmonary veins.

While the second sound is produced, the ventricles having become suddenly relaxed, the recoil of the arterial walls, acting upon the column of blood, immediately closes the semilunar valves upon the two sides. The auricles continue to dilate, and the ventricles are slowly receiving blood.

Immediately following the second sound, during the first part of the interval the auricles become fully dilated; and in the last part of the interval immediately preceding the first sound, the auricles contract, and the ventricles are fully dilated. This completes a single revolution of the heart. 


\section{CHAPTER V.}

\section{FREQUENCY OF THE HEART'S ACTION.}

Frequency of the heart's action-Influence of age-Influence of digestion-Influence of posture and muscular exertion-Influence of exercise-Influence of temperature-Influence of respiration on the action of the heart-Canse of the rhythmical contractions of the heart-Influence of the nervous system on the heart-Division of the pneumogastrics-Galvanization of the pneumogastrics-Causes of the arrest of action of the heart-Blows upon the epigastrium.

Frequency of the Heart's Action.-Physicians have always attached the greatest importance to the frequency of the action of the heart, as one of the great indications of the general condition of the systern. The variations which are met with in health, dependent upon age, sex, muscular activity, the condition of the digestive system, etc., point to the fact that the action of the heart is closely allied to the various functions of the economy, and readily sympathizes with their derangements. As each ventricular systole is followed by an expansion of the arteries which is readily appreciated by the touch, it is more convenient to study the succession of these movements by exploring the vessels, than by examination of the heart itself. Leaving out certain of the qualities of the pulse, this becomes an exact criterion of the acts of the heart.

The number of pulsations of the heart is not far from seventy per minute in an adult male, and from six to ten 
more in the female. ${ }^{1}$ There are individual cases where the pulse is normally much slower or more frequent than this, a fact which must be remembered when examining the pulse in disease. It is said that the pulse of Napoleon I. was only forty per minute. Dr. Dunglison mentions a case which came under his own observation, in which the pulse was on an average thirty-six per minute. The same author states that the pulse of Sir William Congreve was never below one hundred and twenty-eight per minute, in health. ${ }^{2}$ It is by no means unfrequent to find a healthy pulse of a hundred or more per minute.

Influence of Age and Sex.-In both the male and female, observers have constantly found a great difference in the rapidity of the heart's action at different periods of life. The observations of Dr. Guy on this point are very numerous, and were made with the utmost care with regard to the conditions of the system, at the time the pulse was taken, in each case. All were taken at the same hour, and with the subject in a sitting posture.

Dr. Guy found the pulsations of the heart in the foetus to be pretty uniformly 140 per minute. At birth the pulse is 136. It gradually diminishes during the first year to abont 128. The second year the diminution is quite rapid, the tables of Dr. Guy giving 107 as the mean frequency at two years of age. After the second year, the frequency progressively diminishes until adult life, when it is at its minimum, which is about 70 per minute. It is a common, but erroneous, impression that the pulse diminishes in frequency in old age. On the contrary, numerous observations show that at the latter periods of life the movements of the heart become slightly accelerated, ranging from 75 to 80 .

During early life there is no marked and constant differ-

${ }^{2}$ Most of the facts which will be referred to with regard to the frequency of the pulse are taken from the article of Dr. Guy (Pulse) in Todd's Cyclopædia of Anatomy and Physiology.

2 Iuman Physiology. Philadelphia, 1856, vol. i., p. 445. 
ence in the rapidity of the pulse in the sexes; but towards the age of puberty, the development of the sexual peculiarities is accompanied with an acceleration of the heart's action in the female, which continues even into old age. The difference at different ages is shown in the following table, compiled by Milne-Edwards from the observations of Dr. Guy : ${ }^{3}$

AGES.

Males.

Females.

Average Pulsations.

Average Pulsations.

\begin{tabular}{|c|c|c|c|c|c|c|c|c|c|c|c|c|c|c|c|}
\hline & & & & & & & & & & & & & & & \\
\hline rom & $a 2$ to & $07 \mathrm{y}$ & rears & & • & . & & 97 & & & • & & & . & 98 \\
\hline "6 & 8 & 14 & 66 & - & • & & . & 84. & & & . & & - & & 94 \\
\hline 6 & 14 ' & " 21 & " & & - & . & & 76 & . & & . & & & & 32 \\
\hline “ & 21 & 28 & " & . & . & • & . & 73. & & & . & & . & & \\
\hline "6 & 28 ' & “ 35 & 6 & & . & . & & 70 & $\bullet$ & & & & & - & \\
\hline 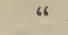 & 35 & " 42 & "6 & . & & & 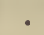 & 68 & & & & & . & & \\
\hline 36 & 42 & " 49 & " & & . & . & & 70 & . & & • & & & - & \\
\hline “ & 49 ، & “ 56 & 6 & - & . & & . & 67. & & & • & & . & & \\
\hline “ & $56 ،$ & “6 63 & “" & & . & . & & 68 & & & & & & - & \\
\hline " & 63 ، & $\because 70$ & 6 & • & . & & $x$ & 70 & & & . & & . & & \\
\hline "6 & 70 & & 6 & & . & . & & 67 & & & & & & • & \\
\hline "? 7 & 77 & " 84 & " & $0^{\circ}$ & & & & 71. & & & & & & & \\
\hline
\end{tabular}

Influence of Digestion.-The condition of the digestive system has a marked influence on the rapidity of the pulse. According to observations cited by Milne-Edwards, ${ }^{2}$ there is an increase in the pulse of from five to ten beats per minute after each meal. Prolonged fasting diminishes its frequency from twelve to fourteen beats. Alcohol first diminishes, and afterwards accelerates, the pulse. Coffee is said by the same author to accelerate the pulse in a marked degree. It has been ascertained that the pulse is accelerated to a greater degree by animal than by vegetable food. These variations have long been recognized by physiologists.

Influence of Posture and Muscular Exertion.-It has been observed that attitude has a very marked influence upon the rapidity of the action of the heart. Experiments of a

${ }^{1}$ Leçons sur la Physiologie, tome iv., p. 62.

2 Loc. cit. 
very interesting eharacter have been made by Dr. Guy and others, with a view to determine the difference in the pulse in different postures. In the male, there is a difference of about ten beats between standing and sitting, and fifteen beats between standing and the reeumbent posture. In the female, the variations with position are not so great. The average given by Dr. Guy is, for the male: standing, 81 ; sitting, 71; lying, 66 ;-for the female: standing, 91 ; sitting, 84 ; lying, 80 . This is given as the average of a large number of observations. There were a few instances, however, in which there was seareely any variation with posture, and some in which the variation was much greater than the average. In the inverted posture, the pulse was found to be reduced about fifteen beats.

The question at onee suggests itself whether the acceleration of the pulse in sitting and standing may not be due, in some measure, to the muscular effort required in making the change of posture. This is answered by the further experiments of Dr. Guy, in which the subjects were placed on a revolving board, and the posture changed without any muscular effort. The same results as those cited above were obtained in these experiments; showing that the difference is due to the position of the body alone. In a single observation, Dr. Guy found the pulse, standing, to be 89 ; lying, 77; difference, 12. With the posture elanged without any muscular effort, the results were: standing, 87; lying, 74; difference, 13.

Various theoretical explanations of these variations have been offered by physiologists; but Dr. Guy seems to have settled experimentally that the acceleration is due to the muscular effort required to maintain the body in the sitting and standing positions. The following are the results of experiments which bear eonclusively on this point, in which it is shown that when the body is carefully supported in the erect or sitting posture, so as to be maintained without muscular effort, the pulse is less frequent than when the subject is 
standing; and furthermore that the pulse is accelerated, in the recumbent posture, when the body is only partially supported :

"1. Difference between the pulse in the erect posture, without support, and leaning in the same posture, in an average of twelve experiments on the writer, 12 beats; and on an average of eight experiments on other healthy males, 8 beats.

" 2 . Difference in the frequency of the pulse in the recumbent posture, the body fully supported, and partially supported, 14 beats, on an average of tive experiments.

" 3 . Sitting posture (mean of ten experiments on the writer), back supported, 80 ; unsupported, 87 ; difference, 7 beats.

" 4 . Sitting posture with the legs raised at right angles with the body (average of twenty experiments on the writer), back unsupported, 86; supported, 68; difference, 18 beats. An average of fifteen experiments of the same kind on other healthy males gave the following numbers: back unsupported, 80 ; supported, 68 ; a difference of 12 beats." "

Influence of Exercise.-It is a fact generally appreciated that muscular exertion increases the frequency of the pulsations of the heart; and the experiments just cited show that the difference in rapidity, which is by some attributed to change in posture (some positions, it is fancied, offering fewer obstacles to the current of blood than others), is in reality due to muscular exertion. Every one knows that the action of the heart is much more rapid after violent exertion, such as running, lifting, etc. Experiments on this point date from quite a remote period. Bryan Robinson, who published a treatise on the "Animal Economy" in 1734,

${ }^{1}$ Tonn's C'yclopredia of Anatomy and Physiology, vol. iv., p. 188. There is an apparent contradiction between these results, and results of the experiments with the "revolving board." It is probable, however, that the subjects experimented upon with the board were simply placed in the erect posture without muscular effort, but maintained themselves in position without any aid. 
states, as the result of observation, that a man in the recumbent position has 64 pulsations per minnte; after a slow walk, 78; after walking a league and a half in an hour, 100 ; and 140 to 150 after running with all his might. ${ }^{2}$ This general statement, which has been repeatedly verified, shows the powerful influence of the muscular system on the heart. The fact is so familiar that it need not be further dwelt upon.

The influence of sleep upon the action of the heart reduces itself almost entirely to the proposition, that during this condition, we have an entire absence of muscular effort, and consequently the number of beats is less than when the individual is aroused. It has been found that there is no difference in the pulse between sleep and perfect quiet in the recumbent posture. This fact obtains in the adult male; but it is said by Quetelet that there is a marked difference in females and young children, the pulse being always slower during sleep. ${ }^{2}$

Influence of Temperature.-The influence of extremes of temperature upon the heart is very decided. The pulse may be doubled by remaining a very few minutes exposed to extreme heat. Bence Jones and Dickinson have ascertained that the pulse may be very much reduced in frequency, for a short time, by the cold douche. ${ }^{3} \quad$ It has also been remarked that the pulse is habitually more rapid in warm than in cold climates.

Though many circumstances materially affect the rapidity of the heart's action, they do not complicate, to any great extent, our examinations of the pulse in disease. In cases which present considerable febrile movement, the patient is generally in the recumbent pasture. The variations induced by violent exercise are easily recognized, while those dependent upon temperature, the condition of the digestive system, etc., are so slight that they may practically be disregarded.

${ }^{2}$ Milne-Edwards, Legons sur la Physiologie, tome iv., p. 68.

${ }^{2}$ Ibid.

3ournal de la Physiologie, 1858, tome i., p. 72. 
It is necessary to bear in mind, however, the variations which exist in the sexes, and at different periods of life, as well as the possibility of individual peculiarities, when the action of the heart may be extraordinarily rapid or slow.

Influence of Respiration upon the Action of the Heart.The relations between the functions of circulation and respiration are very intimate. One function cannot go on without the other. If circulation be arrested, the muscles, being no longer supplied with fresh blood, soon lose their contractile power, and respiration ceases. We shall also find that circulation is impossible if respiration be permanently arrested. When respiration is imperfectly performed, the action of the heart is slow and labored. All physicians are familiar with the slow, full pulse, indicating labored action of the heart, which occurs in profound coma. The effects of arrest of respiration are marked in all parts of the circulatory system, arteries, capillaries, and veins ; but the disturbances thus produced all react upon the heart, and the modifications which take place in the action of this organ are of the greatest interest and importance.

If the heart be exposed in a living animal, and artificial respiration be kept up, though the pulsations are increased in frequency and diminished in force, after a time they become perfectly regular, and continue thus as long as air is adequately supplied to the lungs. Under these circumstances we have the respiration entirely at our command, and can study the effects of its arrest upon the heart with the greatest facility. If we arrest respiration, we observe the following changes in the action of the heart:

For a few seconds pulsations go on as usual; but in about a minute they begin to diminish in frequency. At the same time the heart becomes engorged with blood, a condition which rapidly increases. For a time its contractions are competent to discharge the entire contents of the left ventricle into the arterial system, and a cardiometer applied to an 
artery will indicate a great increase in the pressure of blood, and a corresponding increase in the movements of the mercury will be noted at each action of the heart; indicating that the organ is acting with an abnormal vigor. If respiration be still discontinued, the engorgement becomes intense, the heart at each diastole being distended to its utmost capacity. It now becomes incapable of emptying itself; the contractions become very unfrequent, perhaps three or four in a minute, and are progressively enfeebled. The organ is dark, almost black, owing to the circulation of renous blood in its substance. If respiration be not resumed, this distention continues, the contractions become less frequent and more feeble, and in a few minutes entirely cease.

The arrest of the action of the heart, under these circumstances, is chiefly mechanical. The unaërated blood passes with diffieulty through the capillaries of the system, and as the heart is constantly at work, the arteries become immensely distended. This is proven by the great increase in the arterial pressure, while these vessels are full of black blood. If we now elosely examine the heart and great vessels, we are able to note distinctly the order in which they become distended. These phenomena were particularly noticed and described by Prof. Dalton, and they demonstrate conclusively that in asphyxia the obstruction to the circulation commences, not in the lungs, as is commonly supposed, but in the capillaries of the system, and is propagated backwards to the heart through the arteries.

"The obstacle to the passage of venous blood through the eapillaries, therefore, is partial, not complete. But it is still sufficient to produce an inmediate backward engorgement of the arterial system. Then the aorta becomes distended at its origin, and the left ventricle and left auricle in succession, being unable to relieve themselves of blood through the arterial system, become distended in a similar manner. During this time the same kind of engorgement takes place in the pulmonary artery and the right cavities of the heart; though 
the distention of the pulmonary artery is never so excessive as that of the aorta, either because there is less obstacle to the passage of venous blood through the lungs than through the general capillaries, or because the injecting force of the right ventricle is less than that of the left, or because less blood is supplied by the eapillaries to the veins, and by the veins to the right side of the heart. In either case the principal accumulation is certainly in the arterial system."

The distention of the heart in asphyxia is therefore due to the fact that unaërated blood cannot circulate in the systemic capillaries. When thus distended, its muscular fibres become paralyzed, like any muscle after a severe strain.

If respiration be resumed at any time before the heart's action has entirely ceased, the organ in a few moments resumes its normal function. We first notice a change from the dusky hue it has assumed to a vivid red, which is owing to the circulation of arterial blood in its capillaries. The distention then becomes gradually relieved, and for a few moments the pulsations are abnormally frequent. If we now open an artery, it will be found to contain red blood. An instrument applied to an artery will show a diminution of arterial pressure and force of the heart's action, if the arrest of respiration has been carried only far enough to moderately distend the heart; or an increase in the pressure and force of the heart, if its action has been nearly arrested. A few moments of regular insufflation will cause the pulsations to resume their normal character and frequency.

In the human subject, the effects of temporary or permanent arrest of respiration on the heart, are undoubtedly the same as those observed in experiments upon the warm-blooded animals. In the same way, also, it is possible to restore the normal action of the organ, if respiration be not too long suspended, by the regular introduction of fresh air into the lungs. The numerous examples of animation restored by

${ }^{1}$ Dalton, Lectures on the Physiology of the Circulation, published in the Buffalo Medical Journal and New York Review, Lecture IIJ., April, 1860. 
artificial respiration, in drowning, etc., particularly by what is known as the Marshall Hall method, are evidence of this fact. In cases of asphyxia, those measures by which artificial respiration is most effectually maintained have been found most efficient.

Certain individuals have the power of temporarily arresting the action of the heart by a voluntary suspension of respiration. The most remarkable case of this kind on record is that of Colonel Townshend, which is quoted in many works on physiology. ${ }^{1} \quad$ Col. T. was said to be able to arrest respiration and the action of the heart so completely as to simulate death. When in this condition, the pulse was not perceptible at the wrist nor over the præcordia, a mirror held before the mouth was not tarnished, and he was to all appearances dead. On one occasion, in the presence of several medical gentlemen, he remained in this condition for half an hour ; afterwards the functions of respiration and circulation becoming gradually reëstablished. This, to say the least, is a very remarkable ease, but is credited by many physiologists.

\section{Cause of the Rhythmical Contractions of the Heart.}

The phenomena attending the action of the heart present few difficulties in their investigation, compared with the study of the eause of the regular contractions and relaxations, which commenee early in fotal development, to terminate only with life. This interesting question has long engaged the attention of physiologists, and has been the subject of numerous experiments and speculations. It would be idle to follow the varions theories which have been offered to account for this constant movement, except as a subject of purely historical interest; for many of them are based upon a very imperfect knowledge of the phenomena of the circu-

${ }^{1}$ Dexglisox, Human Physiology, Philadelphia, 1856. Eighth edition, vol. i., p. 405 . 
lation, and have fallen to the ground, as science has advanced. At the present day, though we are perhaps as far as ever from a knowledge of the actual cause of the regular movements, we are pretty well acquainted with the various conditions which modify and regulate them, and have arrived at a limit of knowledge which there seems little prospect of exceeding. The enthusiastic dreamers of past ages hoped to discover the seat of the soul and arrive at the principle of life, but we are as much in the dark as were they with regard to the cause of the various vital phenomena. We know, for example, how to induce contraction in a living muscle, or one which is just separated from the organism and has not yet lost what we call its vital properties, but we must confess our utter ignorance when we ask ourselves why it acts in response to a stimulus. The wonderful advances we have made in chemistry and microscopic anatomy do not disclose the vital principle; and when we come to examine the various conditions under which the heart will continue its contractions, we are arrested by the impossibility of fathoming the mystery of the cause of contraction. The heart is, anatomically, very much like the voluntary muscles; but it has a constant function to perform, and will act without any palpable excitation, while the latter, which have an occasional function, act only under the influence of a natural stimulus like the nervous force, or artificial irritation. The movements of the heart are not the only examples of, what seems to be, spontaneous action. The ciliated epithelium is in motion from the beginning to the end of life, and will continue for a certain time even after the cells are detached from the organism. This motion cannot be explained, unless we call it an explanation to say that it is dependent on vital properties.

But if we are yet ignorant of the absolute cause of the rhythmical contraction of the heart, we are pretty well acquainted with the influences which render its action regular, powerful, and sufficient for the purposes of the economy. It 
will facilitate our comprehension of this, to compare this action with that of the ordinary voluntary muscles.

In the first place, every one knows that the action of the heart is involuntary. We can neither arrest, retard, nor accelerate its pulsations by a direct effort of the will. In this statement we of course except those examples of arrest by the stoppage of respiration, or acceleration by violent exercise, etc. In this respect the heart differs from certain muscles, like the muscles of respiration, which act involuntarily, it is true, but whose action may be temporarily arrested or accelerated by a direct voluntary effort.

The last-mentioned fact gives us the precise difference between the heart and all other striped muscles. All of them, in order to contract, must receive a stimulus, either natural or artificial. The natural stimulus comes from the nervous centres, and is conducted by the nerves. If the nerves going to any of the respiratory muscles, for example, be divided, the muscle is paralyzed, and will not contract without some kind of irritation. Connection with the nervous system does not seem necessary to the action of the heart, for it will contract, especially in the cold-blooded animals, some time after its removal from the body.

When a muscle has been removed from the body, and is subjected to a stimulus, such as galvanism, mechanical or chemical irritation, it is thrown into contraction; but if carefully protected from irritation, will remain quiescent. Contraction in this instance is evidently produced by the application of the stimulus ; but the question arises, Why does the muscle thus respond to stimulation? This is a question which it is impossible to answer satisfactorily, but one concerning which our ideas, since the time of Haller, have assumed a definite form. This great physiologist called the property which causes the muscle thus to contract, irritability; which is nothing more nor less than an unexplained property inherent in the muscle, and continuing as long as it retains its absolute physical and chemical integrity. More than a hun- 
dred years ago, Haller described certain tissues of the body which possessed this "irritability," such as the muscles, stomach, bladder, etc., and the different degrees of irritability with which each one was endowed. ${ }^{1}$ He applied this theory to the action of the heart, which he considered as the part endowed with irritability to the highest degree. His theory of the action of the heart was that its rhythmical contraction depended upon the irritability inherent in its muscular fibres. He was far from denying the various influences which modified this action, but regarded its actual power of contraction as independent. It will be interesting to review some of the facts which were established by Haller, and by numerous physiologists who have since investigated this subject, and see how far this riew of the independence of the contractile power of the heart accords with the present state of our knowledge.

Experiments have shown that the heart will pulsate for a time when removed from all connection with other parts of the organism. ${ }^{2}$ In the cold-blooded animals, in which the irritability of the tissues remains for some time after death, this is particularly marked. It is not the blood in the cavities of the heart which causes it to contract, for it will pulsate when its cavities have been emptied. It is not the con-

${ }^{1}$ Haller, Mémoires sur la Nature Sensible et Irritable des Parties du Corps Animal, Lausanne, 1756 , tome i. These views with regard to the cause of the action of the heart were first advanced by Haller in 1739 in commentaries on the "Institutes" of Boerhaave (Mém. de Haller, p. 87).

${ }^{2}$ Numerous instanees of contractions of the heart in cold-blooded animals continuing for a very long time after excision, are on reccrd. Dr. Dunglison, in his work on Physiology (op. cit., vol. i., p. 408), mentions several instances where the heart pulsated for from ten to twenty-four hours after removal from the body. The most remarkable examples of this prolonged action were in the heart of the sturgeon. In one instance, in an experiment on a large alligator, we found the heart pulsating, in situ, twenty-eight hours after the animal bad been killed by the injection of a solution of woorara. The heart was then exeised, and continued to beat during a long series of experiments, until it was arrested by powerful compres. sion with the hand, after it had been filled with water and the vessels tied. 
tact of the air, for the heart will pulsate in a vacuum. ${ }^{1}$ The heart does not receive its irritability from the nervous system, for, when removed from the body, it has no connection with the nervous system; and it is not probable that it receives any influence from sympathetic ganglia which have lately been discovered in its substance, for detached portions of the heart will pulsate, and the contractions of the organ will continue in animals poisoned with woorara, which is known to paralyze the motor system of nerves.

It is unnecessary to refer to the various experiments which have demonstrated the independence of the contractions of the heart. They are of such a simple nature that they may be verified by any one who will take the trouble to excise the heart of a frog or turtle, place it under a small bellglass so that it will not be subject to possible irritation from currents of air, and watch its pulsations. In such an observation as this, it is evident that for a certain time contractions, more or less regular, will take place; and the experiments referred to above show that they take place without any external influence. In short, it is evident that the muscular fibres of the heart possess an irritability, by virtue of which they will contract intermittently for a time, though no stimulus be applied; as ordinary striped muscular fibres have an irritability, by virtue of which they will respond, for a time, to the application of a stimulus.

It is manifestly necessary that the action of the heart should be constant, regular, and powerful; and when we say that the irritability inherent in its muscular tissue is such that it will contract for a time without any external stimulus, we by no means assume that this is the canse of its physiological action. It is only an important and incontestable property of the muscular fibres of the heart, and its regular action is dependent upon other circumstances.

In the first place, we have to inquire what makes the action of the heart constant. The answer to this is, that the

1 Joms ReID, in Cyclopadia of Anatomy and Physiology, vol. ii., p. 611. 
changes of nutrition, by which, through the blood circulating in its substance, the waste of its tissue is constantly supplied, preserves the integrity of the fibres, and keeps them, consequently, in a condition to contract. This is true, likewise, of the ordinary striped muscular fibres. If the supply of blood be cut off from the substance of the heart, especially in the warm-blooded animals, the organ soon loses its irritability. This was beautifully shown by the experiments of Erichsen. This observer, after exposing the heart in a warmblooded animal and keeping up artificial respiration, ligated the coronary arteries, thus cutting off the greatest part of the supply of blood to the muscular fibres. He found, as the mean of six experiments, that the heart ceased pulsating, though artificial respiration was continued, in $23 \frac{1}{2}$ minutes. After the pulsations had ceased, they could be restored by removing the ligatures and allowing the blood to circulate again in the substance of the heart. ${ }^{1}$ The same is true of the irritability of ordinary muscles, as has been lately shown by the experiments of Dr. Brown-Séquard, though the continuous action of the heart undoubtedly causes these phenomena to be more marked and rapid. If we take a muscle which has just lost its irritability and will no longer respond to the most powerful stimulus, and inject fresh blood by the artery supplying it, the irritability will be immediately restored."

In the second place, the regular and powerful contraction. of the heart is provided for by the circulation of the blood through its cavities. Though the heart, removed from the body, will contract for a time without a stimulus, it can be made to contract during the intervals of repose by an irritant, such as the point of a needle, or a feeble eurrent of galvanism. For a certain time after the heart has ceased to contract spontaneously, contractions may be induced in this way. This can easily be demonstrated in the heart of any

1 London Mcdical Gazette, July 8, 1842.

${ }^{2}$ Brown-Séquard, Propriétés et Usages du sang rouge et du sang noir, Journal de la Physiologie, 1858, tome i., p. 95 et seq. 
animal, warm or cold-blooded. This irritability, which is manifested, under these circumstances, in precisely the same way as in ordinary muscles, is different in degree in different parts of the organ. Haller and others have shown that it is greater in the cavities than on the surface; for long after irritation applied to the exterior fails to excite contraction, the organ will respond to a stimulus applied to its interior. The experiments of Haller also show that fluids in the cavities of the heart have a remarkable influence in exciting and keeping up its contractions. This observation is of much interest, as showing conclusively that the presence of blood is necessary to the natural and regular action of the heart. We quote one of the experiments on this point performed upon a cat:

"* * * The superior vena cava having been divided, and the inferior ligated, and the pulmonary artery opened, and the right ventricle emptied by a sufficient compression, and the aorta ligated, all with promptitude, I saw the right auricle repose first, the right ventricle continued to beat for some time in unison with the left ventricle, and its walls descended toward the middle line of the heart: but this ventricle did not delay to lose its movement the first. As for the other ventricle, which could no longer empty itself into the aorta, it was filled with blood, and its movement continued for four hours. * * * $\|_{1}$

This experiment was confirmed by numerous others. It will be observed that one side of the heart was made to cease its pulsations, while the other side continued to contract, by simply removing the blood from its interior; which conclusively proves that, though the heart may act for a time independently, the presence of blood in its cavities is a stimulus capable of prolonging its regular pulsations. Schiff has gone still further, and succeeded in restoring the pulsations in the heart of a frog, which had ceased after it had been

${ }^{1}$ Haller, Mémoires sur la Nature Irritable et Sensible des Parties, etc., tome i., p. 363. 
emptied, by introducing a few drops of blood into the auricle. ${ }^{1}$ Our own experiments upon the hearts of alligators and turtles show that when removed from the body and emptied of blood, the pulsations are feeble, rapid, and irregular; but that when filled with blood, the valves being destroyed so as to allow free passage in both directions between the auricles and ventricle, the contractions become powerful and regular. In these experiments, when water was introduced instead of blood, the pulsations became more regular, but were more frequent and not as powerful as when blood was used. ${ }^{2} \quad$ These experiments show also that the action of the heart may be affected by the character, particularly the density, of the fluid which passes through it, which may explain its rapid and feeble action in anemia.

It seems well established that the heart, though capable of independent action, is excited to contraction by the blood as it passes through its cavities. A glance at the succession of its movements, particularly in the cold-blooded animals, where they are so slow that the phenomena can be easily observed, will show how these contractions are induced. If we look at the organ as it is in action, we see first a distention of the auricle; this is immediately followed by a contraction filling the ventricle, which in its turn contracts. Undoubtedly the tension of the fibres, as well as the contact of blood in its interior, acts as a stimulus; and as all the fibres of each cavity are put on the stretch at the same instant, they contract simultaneously. The necessary regular distention of each cavity thus produces rhythmical and forcible contractions; and the mere fact that the action of the heart alternately empties and dilates its cavities, insures regular pulsations as long as blood is supplied, and no disturbing influences are in operation.

The muscular fibres of the heart are endowed with

2 Mrlue-Edwards, op. cit., tome iv., p. 126.

${ }^{2}$ Action of the Heart and Respiration, American Journal of the Medical Sciences, Oct. 1861. 
an inherent property, called irritability, by virtue of which they will contract for a certain time without the application of a stimulus. Irritability, manifested in this way, continues so long as, by the processes of nutrition, the fibres are maintained in their integrity. The muscular tissue, however, may be thrown into contraction, during the intervals of repose, by the application of a stimulus, a property which is enjoyed by all muscular fibres. The irritability manifested in this way is much more marked in the interior than on the exterior of the organ. Blood in contact with the lining membrane of the heart acts as a stimulus in a remarkable degree, and is even capable of restoring irritability after it has become extinct. The passage of blood through the heart is the natural stimulus of the organ, and may be said to be the cause of its regular pulsations, though it by no means endows the fibres with their contractile properties.

\section{Influence of the Nervous System on the Heart.}

The movements of the heart, as we have seen, are not directly under the control of the will; and observations on the human subject, as well as on living animals, have shown that the organ is devoid of general sensibility. The latter fact was demonstrated in the most satisfactory manner by Harvey in the case of the Viscount Montgomery. In this case the heart was exposed; and Harvey found that it could be touched and handled without even the knowledge of the subject. This has been verified in other instances in the human subject. Its physiological movements are capable of being influenced in a remarkable degree through the nervous system, notwithstanding this insensibility, and in spite of the fact that the muscular fibres composing it are capable of contraction when removed from all connection with the body, and that the regular pulsations can be kept up for a long time by the mere passage of blood through its cavities. The influence thus exerted is so great, that some eminent au- 
thorities held the opinion that the cause of the irritability of the organ was derived from the nerves. One of the most distinguished advocates of this opinion was Legallois. This observer arrested the action of the heart of the rabbit by suddenly destroying the spinal cord, from which he drew the conclusion that the heart derived its contractile power from the cerebro-spinal system. ${ }^{1}$ The experiments which we have already cited, showing the continuance of the heart's action after excision, disprove this so completely, that it was not thought worth while to discuss this view while treating of the cause of its rhythmical contraction. The same may be said with regard to the experiments of Brachet, in which he endeavored to prove that the contractility of the heart is derived from the cardiac plexus of the sympathetic system of nerves. The fact that the heart does not depend for its contractility upon external nervous influence may be regarded as long since definitely settled; but within a few years the discovery in its substance of ganglia belonging to the sympathetic system has revived, to some extent, the view that its irritability is derived from nerves.

It is not necessary to follow out all the experiments which combine to demonstrate the incorrectness of this view. Bernard, by a series of admirably conceived experiments upon the effects of the woorara poison, has succeeded in demonstrating the distinction between muscular and nervous irritability. ${ }^{2}$ In an animal killed with this remarkable poison, the functions of the motor nerves are entirely abolished; so that galvanization, or other irritation, does not produce the slightest effect. Yet the muscles retain their irritability, and if artificial respiration be kept up, the circulation will continue for a long time. The heart, by this means, seems to be isolated firom the nervous system as completely as if it were excised; and galvanization of the pneumogastric nerves in

${ }^{1}$ Legallois, Euvres, tome i., p. 97.

Bernard, Legons sur les Effets des Substances Toxiques et Médicamentereses, Paris, 1857. 
the neck, which, in a living animal, will immediately arrest its action, has no effect. On the other hand, poisoning by the sulpho-cyanide of potassium destroys the muscular irritability, and leaves the nerves intact. By these experiments, which we have frequently repeated, we can completely separate the nervous from the muscular irritability, and show their entire independence of each other; and there is every reason to suppose that the heart, like the other muscles, does not derive its contractility from any other system.

It is evident, however, that the heart is often powerfully influenced through the nerves. Sudden and violent emotions will occasionally arrest its action, and have been known to produce death. Palpitations are to be accounted for in the same way. Some of the modifications which we have already considered, depending on exercise, digestion, etc., are effected through the nerves; and it is through this system that the heart, and all the important organs of the body, are made to a certain extent mutually dependent. It becomes interesting, and highly important, then, to study their influences, and follow out, as clearly as possible, the action of the nerves which are distributed to the heart.

The anatomical connections of the heart with the nervous centres are mainly through the sympathetic and the pneumogastric nerves. We can study the influence of these nerves to most advantage in two ways: first, by dividing them and watching the effect of depriving the heart of their influence; and second, by exciting them by means of a feeble current of galvanism. It is well known that in an animal just killed the "nervous force" may be closely imitated by galvanism, which is better than any other means of stimulation, as it does not affect the integrity of the nerves, and the amount of the irritation may be easily regulated. ${ }^{1}$

1 We shall not discuss the effects upon the heart of sudden destruction of the great nervous centres. It has been shown that the heart becomes arrested when the brain is crushed, as by a blow with a hammer, when the medulla oblongata or the spinal cord is suddenly destroyed; and even the crushing of a font, in the frog, 
Experiments on the influence of the sympathetic nerves upon the heart are not quite as satisfactory as we might desire. Brachet asserts that the action of the heart is immediately arrested by destroying the cardiac plexus.' With regard to this observation, we must take into account the difficulty of making the operation, and the disturbance of the heart consequent upon the necessary manipulations; circumstances which take away much of its value. It has been shown pretty conclusively, however, that stimulation of the sympathetic in the neck has the effect of accelerating the pulsations of the heart. ${ }^{2}$ The extreme difficulty of dividing all the branches of the sympathetic going to the organ leaves a doubt as to whether such an operation would definitely abridge its action.

We have next to consider the influence of the pneumogastrics upon the heart. Experiments on these nerves are made with greater facility than on the nerves of the sympathetic system, and the results are much more satisfactory. Like all the cerebro-spinal nerves, the influence generated in the nerrous centre from which they take their origin is conducted along the nerve, and manifested at its distribution. When they are divided, we may be sure that, as far as they are concerned, all the organs which they supply are cut off from nervous influence; and when galvanized in their course, we imitate or exaggerate the influence sent from the nervous centre.

The invariable effect on the heart of division of the pneumogastric nerres in the neck is an increase in the frequency, and diminution in the force, of its pulsations. One or two

has been known to produce this effect. In fine, this may be done by any extensive injury to the nervous system; but this fact does not teach us much with regard to the physiological influences of the nerves. For example, while crushing of the brain arrests the heart, the brain may be removed from a living animal, and the heart will beat for days. Experiments upon the influence of the medulla oblongata and spinal cord are by no means satisfactory.

${ }^{1}$ Cyclopcedia of Anatomy and Physiology, vol. ii., p. 612.

2 Milde-Edwards, Physiologie, tome iv., p. 156, note. 
writers have denied this fact, but it is confirmed by the testimony of nearly all experimenters. To anticipate a little in the history of the pneumogastric nerves, it may be stated that while they are exclusively sensitive at their origin, they receive after having emerged from the cranial cavity a number of filaments from various motor nerves. That they influence certain muscles, is shown by their paralysis after division of the nerves in the neck; as, for example, the arrest of the movements of the glottis.

Having this double property of motion and sensation, and being distributed in part to an organ composed almost exclusively of muscular fibres, which, as we have seen, is not endowed with general sensibility, we should expect that their section would arrest, or at least diminish, the frequency of the heart's action. What explanation, then, can we offer for the fact that this seems actually to excite the movements of the heart? We will be better prepared to answer this question after we have studied the effects of galvanization of the nerves in a living animal, or one in which the action of the heart is kept up by artificial respiration.

Numerous experiments have been made with reference to the effects on the heart of galvanic currents, both feeble and powerful, passed through the pneumogastrics before division, of currents passed through the upper and lower extremities after division, etc., a full detail of which belongs properly to the physiological history of the nervous system. In this connection, a few of these facts only need be stated.

It has been shown by repeated experiments, which we have frequently confirmed, that a moderately powerful current of galvanism passed through both pneumogastrics will arrest the action of the heart, and that the organ will cease its contractions as long as the current is continued. This experiment has been performed upon living animals, both with and without exposure of the heart. The arrest is not due to violent and continued contraction of the muscular fibres; on the contrary, the heart is relaxed, its ventricles are 
flaccid, and its fibres are for the time paralyzed. The question then arises whether this action is directly exerted on the heart through the nerves, or whether an influence is conveyed to the nervous centre, and transmitted to the heart in another way. This is settled by the experiment of dividing the nerves and galvanizing alternately the extremities connected with the heart and those connected with the nervous centres. It has been ascertained that galvanization of the extremities connected with the heart arrests its action, while galvanization of the central extremities has no such effect. Another interesting fact also shows that the influence exerted upon the heart is through the motor filaments of the pneumogastrics. It has been shown by Bernard, in a very curious series of experiments which we will not fully discuss in this connection, that the woorara poison paralyzes only the motor nerves, leaving the sensory nerves intact. If we expose the heart and pneumogastric nerves in a warm-blooded animal poisoned with this agent, and continue the pulsations by keeping up artificial respiration, we find that the most powerful current of galvanism passed through the pneumogastrics has no effect upon the heart. The effect of a feeble current of galvanism upon the motor nerves is so like the operation of the natural stimulus, or nervous force, that for a time many physiologists regarded the two forces as identical. Though this view is not received at the present day, it is an admitted fact that by galvanism we imitate in the closest manner the natural action of the motor nerves, and this has become a most valnable means of investigation into the physiology of the nervous system.

Though galvanization of the pneumogastries arrests the action of the heart in nearly all animals, there are some in which this does not take place, as in birds; a fact which is stated by Bernard, ${ }^{2}$ but for which he offers no explanation. In some experiments instituted on this subject a few years

' Bernard, Physiologie et Pathologie du Système Nerveux,Paris, 1858, tome ii., p. 394. 
ago on alligators, we noticed a singular peculiarity which throws some light on the question we are now considering. Desiring to demonstrate to the class at the New Orleans School of Medicine the action of the heart in this animal, an alligator six feet in length was poisoned with woorara, and the heart exposed. The animal came under the influence of the poison in about thirty minutes, when the dissection was commenced, and was quite dead when the heart was exposed. The pneumogastrics were then exposed and galvanized, with the effect of promptly arresting the action of the heart. This observation was verified in another experiment. We were at first at a loss to account for the absence of effect of the woorara on the motor filaments of the pneumogastric nerves ; but on reflection thought it might be due to slow absorption of the poison in so large a cold-blooded animal. With a view of ascertaining whether there is any difference in the promptness with which different nerves in the body are affected by this agent, we made the following experiment upon a dog. The animal was brought under the influence of ether, and the heart, the pneumogastrics, and the sciatic nerve were exposed. Galvanization of the sciatic produced muscular contraction, and of the pneumogastrics arrested the heart promptly. A grain of woorara, dissolved in water, was then injected under the skin of the thigh. One hour after the injection of the woorara, the sciatic was found insensible to galvanism, but the heart could be arrested by galvanization of the pneumogastrics, though it required a powerful current. A weaker current diminished the frequency, and increased the force, of its pulsations. ${ }^{1}$ In this experiment, the operation of opening the chest undoubtedly diminished the activity of absorption of the poison, and consequently retarded its effects upon the nervous system. Taken in connection with

1 This increase in the force of the heart, which accompanied the diminution in the frequency of its pulsations, consequent upon feeble galvanization of the pneumogastrics, was constantly observed in many experiments. The force of the pulsations was measured by the cardiometer. 
the observations on alligator's, it shows that the motor nerves are not all affected at the same time, and that the pneumogastries resist the action of this pecnliar poison after the motor nerves generally are paralyzed. This shows a conservative provision of Nature which guards particularly the important influence exerted by these nerves upon the heart. ${ }^{1}$

Our knowledge of the inherent properties of the muscular fibres of the heart, and the effects of the passage of blood through its eavities, which together are competent to keep up for a time regular pulsations without the intervention of the nervous system, taken in connection with the facts just stated, concerning the influence of section or galvanization of the pneumogastric nerves, enables us to comprehend pretty well the influence of these nerves on the heart. They undoubtedly perform the important function of regulating the force and frequency of its pulsations.

Hardly any reflection is necessary to convince us of the importance of such a function, and how it must of necessity be accomplished through the pneumogastrics. It is important, of course, that the heart should act at all times with nearly the same force and frequency. We have seen that the inherent properties of its fibres are competent to make it contract, and the necessary intermittent dilation of its cavities makes these contractions assume a certain regularity; but the quantity and density of the blood are subject to very considerable variations within the limits of health, which, without some regulating influence, would undoubtedly cause variations in the heart's action, so considerable as to be injurious. This is shown by the comparatively inefficient and palpitating action of the heart when the pneumogastrics are divided. These nerves convey to the heart a constant influence, which we may compare to the insensible tonicity imparted to voluntary muscles by the general motor system.

${ }^{i}$ For details of these experiments the reader is referred to an article by the author, on the Action of the Heart and Respiration, in The American Journal of Medical S'ciences, Oct., 1861. 
For we know that when a set of muscles on one side is paralyzed, as in facial palsy, their tonicity is lost, they become flaccid, and the muscles on the other side, without any effort of the will, distort the features.

We can imitate an exaggeration of this force by a feeble current of galvanism, which makes the pulsations of the heart less frequent and more powerful; or exaggerate it still more by a more powerful current, which arrests the action of the heart altogether.

Phenomena are not wanting in the human subject which verify these views. Causes which operate through the nervous system frequently produce palpitation and irregular action of the heart. Cases are not uncommon in which palpitation habitually occurs after a full meal. There are instances on record of immediate death from arrest of the heart's action from fright, anger, grief, or other severe mental emotions. Syncope from these causes is by no means uncommon. In the latter instance, when the heart resumes its functions, the nervous shock carried along the pneumogastrics is only sufficient to arrest its action temporarily. When death takes place, the shock is so great that the heart never recovers from its effects. ${ }^{1}$

Summary of certain Causes of Arrest of the Action of the Heart.

In warm-blooded animals, the heart's action speedily ceases after it is deprived of its natural stimulus, the blood. It is not from experiments on the inferior animals alone that we derive proof of this fact. It is well known that in profuse hemorrhage in the human subject, the contractions of the

- An explanation of the influence of the pneumogastric nerves on the heart, very like the one we have given, is made by Longet (Traite de Physiologie, Paris, 1861, tome i., p. 785); but this author assumes that the pneumogastrics and the sympathetic have an antagonistic action, the former moderating, and the latter accelerating the heart's action. 
heart are progressively enfeebled, and, when the loss of blood has proceeded to a certain extent, are permanently arrested. Cases of transfusion after hemorrhage show that when blood is introduced, the heart may be made to resume its pulsations. The same result takes place in death by asthenia; and cases are on record where life has been prolonged, as in hemorrhage, by transfusion of even a small quantity of healthy blood. - These facts have been demonstrated on the inferior animals by experiments already cited. The experiment of Haller, in which the action of the right side of the heart of a cat was arrested by emptying it of blood, while the left side, which was filled with blood, continued to pulsate, showed that the absence of blood in its cavities is competent of itself to arrest the heart. The experiments of Erichsen, who paralyzed the heart by ligating the coronary arteries, and Schiff, who produced a local paralysis by ligating the vessel going to the right ventricle, show that the heart may also be arrested by cutting off the circulation of blood in its substance. Both of these causes must operate in arrest of the heart's action in hemorrhage.

The mechanical causes of arrest of the heart's action are of considerable pathological importance. The heart, in common with other muscles, may be paralyzed by sufficient mechanical injury. A violent blow upon the deltoid paralyzes the arm; a serere strain will paralyze the muscles of an extremity; in the same way excessive distention of the cavities of the heart will arrest its pulsations. This is shown by arrest of the circulation in asphyxia. We have already seen, that under these circumstances the heart is incapable of forcing the unaërated blood through the systemic capillaries; it finally becomes enormously strained and distended, and consequently paralyzed. The same result follows the application of a ligature to the aorta. This effect may be produced, also, in the cold-blooded animals, in which, if the heart be left undisturbed, the pulsations will continue for a long time. The following experiment illustrating this point 
was performed upon the heart of an alligator six feet in length :

The animal was poisoned with woorara, and twenty-eight hours after death the heart, which had been exposed and left in situ, was pulsating regularly. It was then removed from the body, and after some experiments on the comparative force, etc., of the pulsations, when empty, and when filled with blood, was filled with water, the valves having been destroyed so as to allow free passage of the fluid through the cavities, and the vessels ligated. "The ventricles, still filled with water confined in their cavity, were then firmly compressed with the hand, so as to subject the muscular fibres to powerful compression. From that time the heart entirely ceased its contractions, and became hard, like a muscle in a state of cadaveric rigidity." "

This experiment shows how completely and promptly the heart, even of a cold-blooded animal, may.be arrested in its action by mechanical injury.

Cases of death from distention of the heart are not infrequent in practice. It is well established that the form of organic diseuse which most frequently leads to sudden death is that in which the heart is liable to great distention. WVe refer to disease at the aortic orifice. In other lesions, there is not this tendency; but when the aortic orifice is contracted, or the valves are insufficient, any great disturbance of the circulation will cause the heart to become engorged, which is liable to produce a fatal result.

Most persons are practically familiar with the distressing sense of suffocation which frequently follows a blow upon the epigastrium. A few cases are on record of instantaneous death following a comparatively slight blow in this region. We had an opportunity in the winter of 1854-'55 of witnessing an autopsy in a case of this kind. A young mulatto man, employed as a waiter at the Louisville Hotel, received a blow in the epigastrium, while frolicking, which

${ }^{1}$ American Journal, Oct. 1861, p. 352. 
produced instantaneous death. On post-mortem examination no lesion was discovered. Though these cases are rare, they are well known, and the effects are generally attributed to injury of the solar plexus. The distress is precisely what would occur from sudden arrest of the heart's action; for it is the blood charged with oxygen and sent by the heart to the system, which supplies the wants of the tissues, and not the simple entrance of air into the lungs; and arrest of the circulation of arterial blood, from any cause, produces suffocation as completely as though the trachea were ligated. This fact is clearly proven by experiments in the article referred to above. It is a question whether the arrest of the heart, if this be the pathological condition, be due to concussion of the nervous centre, or to the direct effects of the blow upon the organ itself. Our present data do not enable us to answer this question definitely, but rather incline us to the opinion that in such accidents the symptoms are due to direct injury of the heart. An additional argument in favor of this view is founded on our knowledge of the mode of operation of the sympathetic system. The effects of stimulation or irritation of this system are not instantaneously manifested, as is the case in the cerebro-spinal system, but are developed slowly and gradually.

As far as we have been able to learn by experiment, the nerrous influences which arrest the action of the heart operate through the pneumogastrics. As we have just seen, we can closely imitate this action by galvanism. The causes of arrest in this way are numerous. Among them may be mentioned, sudden and severe bodily pain and severe mental emotions. With the exceptions of arrest of the heart from loss of blood and from distention, from whatever cause it may occur, stoppage of the heart takes place through the nervous system. It may be temporary, as in syncope, or it may be permanent; examples of which, though rare, are sufficiently well authenticated. 


\section{CHAPTER VI.}

CIRCULATION OF THE BLOOD IN THE ARTERIES.

Physiological anatomy of the arteries-Course of blood in the arteries-Elasticity of the arteries-Contractility of the arteries-Locomotion of the arteries and production of the pulse-Form of the pulse-Sphygmograph-Pressure of blood in the arteries-Hemodynamometer-Cardiometer-Differential cardiometer-Pressure in different parts of the arterial system-Influence of respiration on the arterial pressure-Effects of hemorrhage-Rapidity of the current of blood in the arteries-Instruments for measuring the rapidity of the arterial circulation-Variations in rapidity with the action of the heart-Rapidity in different parts of the arterial system-Arterial murmurs.

Is man and in all animals possessed of a double heart, each contraction of this organ forces a charge of blood from the right ventricle into the pulmonary artery, and from the left ventricle into the aorta. We have seen how the valves which guard the orifices of these vessels effectually prevent regurgitation during the intervals of contraction. There is, therefore, but one direction in which the blood can flow in obedience to this intermittent force; and the fact that in the smallest arteries there is an acceleration in the current coincident with each contraction of the heart, which disappears when the action of the heart is arrested, shows that the ventricular systole is the prime cause of the arterial circulation.

This part of the physiology of the circulation is not as simple as we might at first be led to suppose. The arteries have the important function of supplying nutritive matter to all the tissues, of furnishing to the glands materials out of 
which the secretions are formed, and in short are the avenues of supply to every part of the organism. The supply of blood regulates, to a considerable extent, the process of nutrition, and has an important bearing on the general and special functions. The physiological processes necessarily demand considerable modifications in the quantity of arterial blood which is furnished to parts at different times. For example, during secretion, the glands require twice or three times as much blood as in the intervals of their action. The force of the heart, we have seen, varies but little within the limits of health, and the conditions necessary to the proper distribution of blood in the economy are regulated almost exclusively by the arterial system. These vessels are not inert tubes, but are endowed with elasticity, by which the circulation is considerably facilitated, and with contractility, by which the supply to any part may be modified, independently of the action of the heart. Sudden flushes or pallor of the countenance are examples of the facility with which this may be effected. It is evident, therefore, that the properties of the coats of the arteries are of great physiological importance. We will then commence the study of this division of the circulatory system with a consideration of its physiological anatomy.

\section{Physiological Anatomy of the Arteries.}

The ressels which carry the venous blood to the lungs are branches of a great trunk which takes its origin from the right ventricle. They do not differ in structure from the vessels which carry the blood to the general system, except in the fact that their coats are somewhat thinner and more distensible. The aorta, branches and ramifications of which supply all parts of the body, is given off from the left ventricle. Just at the origin, behind the semilunar valves, the aorta has three sacculated pouches, called the sinuses of Valsalva. Beyond this point the ressels are cylindrical. As we recede 
from the heart, the arteries branch, divide, and subdivide, until they are reduced to microscopic size. The branches, with the exception of the intercostal arteries, which make nearly a right angle with the thoracic aorta, are given off at an acute angle. $\Lambda$ s a rule, the arteries are nearly straight, taking the shortest course to the parts which they supply with blood; and while the branches progressively diminish in size, but few are given off between the great trunk and the minute vessels which empty into the capillary system. Haller counted but twenty branches of the mesenteric artery between the aorta and the capillaries of the intestines. ${ }^{2}$ So long as a vessel gives off no branches, its caliber does not progressively diminish; as the common carotids, which are as large at their bifurcation as they are at their origin. There are one or two instances in which ressels, though giving off numerous branches in their course, do not diminish in size for some distance; as the aorta, which is as large at the point of division into the iliacs, as it is in the chest; and the vertebral arteries, which do not diminish in caliber until they enter the foramen magnum. ${ }^{2}$ With these exceptions, as we recede from the heart, the caliber of the vessels progressively diminishes.

It has long been remarked that the combined caliber of the branches of an arterial trunk is much greater than that of the main vessel; so that the arterial system, as it branches, increases in capacity.

The arrangement of the arteries is such that the requisite supply of blood is sent to all parts of the economy by the shortest course, and with the least expenditure of force from the heart. Generally the vessels are so situated as not to be exposed to pressure and consequent interruption of the current of blood; but in certain situations, as about some of the joints, there is necessarily some liability to occasional com-

1 Cyclopcedia of Anatomy and Physiology, vol. i., p. 220; and Haller, Ele. menta Physiologia, tomus i., sec. i., § 17.

${ }^{2}$ Ibid. 
pression. In some situations, also, as in the vessels going to the brain, particularly in some inferior animals, it is necessary to moderate the force of the blood enrrent, on account of the delicate structure of the organs in which they are distributed. Here Nature makes a provision in the shape of anastomoses; by which, on the one hand, compression of a vessel simply diverts, and does not arrest, the current of blood, and on the other hand, the current is rendered more equable and the force of the heart moderated.

The arteries are provided with membranous sheaths, of greater or less strength, as the vessels are situated in parts more or less exposed to disturbing influences or accidents.

Researches into the minute anatomy of the arteries have shown that they are possessed of three pretty well marked coats. As these vary very considerably in arteries of different sizes, in their description, it is convenient to divide the vessels into three classes.

1. The largest arteries; in which are included all that are larger than the carotids and common iliacs.

2. The arteries of medium size; that is, between the carotids and iliacs and the smallest.

3. The smallest arteries; or those less than $\frac{1}{15}$ to $\frac{1}{12}$ of an inch in diameter. ${ }^{1}$

The largest arteries are endowed with great strength and elasticity. Their external coat is composed of white or inelastic fibrous tissue. According to Kölliker, this coat is no thicker in the largest vessels than in some of the vessels of medium size. In some medium-sized vessels it is actually thicker than in the aorta. This is the only coat which is vascular.

The middle coat, on which the thickness of the vessel de-

1 This is essentially the division made by Kölliker (Manual of Human Microscopic Anatomy, London, 1860, p. 485). Some anatomists make five or even more coats to the arteries. The three coats are pretty well marked, each possessing distinctive properties. The numerous coats which are sometimes given are, many of them, simple layers of the same tissue. The division into three coats is more simple and physiological. 
pends, is composed chiefly of the yellow elastic tissue. This tissue is disposed in numerous layers. First we have a thin layer of ramifying elastic fibres, and then a number of layers of elastic membrane, with numerous oval longitudinal openings, which has given it the name of the "fenestrated membrane." According to Kölliker, between the layers of this membrane are found a few unstriped or involuntary muscular fibres, but Robin states that muscular fibres are only found in arteries of medium size. ${ }^{1}$ Muscular fibres, if they exist at all in the largest arteries, are very few, and of little physiological importance. The middle coat of the largest arteries gives them their yellowish hue, and the elasticity for which they are so remarkable.

The internal coat of the largest arteries does not differ materially from the lining membrane of the rest of the arterial system. It is identical in structure with the endocardium, the membrane lining the cavities of the heart, and is continued through the entire vascular system. It is a thin homogeneous membrane, covered with a layer of elongated epithelial scales, with oval nuclei, their long diameter following the direction of the ressel.

The arteries of medium size possess considerable strength, some elasticity, and very great contractility. In the outer and inner coats we do not distinguish any great difference between them and the largest arterics, even in thickness. The essential difference in the anatomy of these vessels is found in the middle coat. Here we have a continuation of the elastic elements found in the largest vessels, but relatively diminished in thickness, and mingled with the fusiform involuntary muscular fibres, arranged at right angles to the course of the ressel. These fibres are found in the inner layers of the middle coat, and, according to Robin, only in arteries smaller than the carotids and primitive iliacs. In arteries of medium size, like the femoral, profunda femoris, radial, or ulnar, they exist in numerous layers. There is no

\footnotetext{
${ }^{2}$ Roвis, in Nysten's Dictionnaire de Médecine, 1858. Artère.
} 
distinct division, as regards the middle coat, between the largest arteries and those of medium size. As we recede from the heart, muscular fibres gradually make their appearance between the elastic layers, progressively increasing in quantity, while the elastic element is diminished.

In the smallest arteries the external coat is thin, and disappears just before the vessels empty into the capillary systern; so that the very smallest arterioles have only the inner coat and a layer of muscular fibres.

The middle coat is composed of circular muscular fibres, without any admixture of elastic elements. In vessels $\frac{1}{100}$ of an inch in diameter, we have two or three layers of fibres: but as we near the capillaries, and as the vessels lose the external fibrous coat, these fibres have but a single layer.'

The internal coat presents no difference from the coat in other vessels, with the exception that the epithelium is less distinctly marked, and is lost near the capillaries; the membrane being studded with longitudinal oval nuclei.

A tolerably rich plexus of vessels is found in the external coats of the arteries. These are called the vasa vasorum, and come from the adjacent arterioles, having no direct connection with the vessel on which they are distributed. A few vessels penetrate the external layers of the middle coat, but none are erer found in the internal coat.

Nervous filaments, principally from the sympathetic system, accompany the arteries, in all probability, to their remotest ramifications; though they have not yet been demonstrated in the smallest arterioles. These are not distributed in the walls of the large vessels, but rather follow them in their course; their filaments of distribution being found in those vessels in which the muscular element of the middle coat predominates. When we come to treat of the physiology of the organic system of nerves, we shall see that the "vaso-

1 The structure of the smallest arteries can be beautifully exhibited in fresh mieroscopic preparations of the pia mater, in which the various points to which we have alluded can be easily studied. 
motor" nerves play an important part in regulating the function of uutrition.

Course of the Blood in the Arteries.-At every pulsation of the heart, all the blood contained in the ventricles, excepting, perhaps, a few drops, is forced into the great vessels. We have already studied the valvular arrangement by which the blood, once foreed into these vessels, is prevented from returning into the ventricles during the diastole. The sketch we have given of the anatomy of the arteries has prepared us for a complexity of phenomena in the circulation in these vessels, which would not obtain if they were simple, inelastic tubes. In this case the intermittent force of the heart would be felt equally in all the vessels, and the arterial circulation would be subject to no modifications which did not come from the action of the central organ. As it is, the blood is received from the heart into vessels endowed, not only with great elasticity, but with contractility. The elasticity, which is the prominent property of the largest arteries, moderates the intermittency of the heart's action, providing a continuous supply to the parts; while the contractility of the smallest arteries is capable of increasing or diminishing the supply in any part, as may be required in the various functions.

Elasticity of the Arteries.-This property, particularly marked in large vessels, has long been recognized. If, for example, we forcibly distend the aorta with water, it may be dilated to more than double its ordinary capacity, and will resume its original size and form as soon as the pressure is removed. This simple experiment teaches us, that if the force of the heart be sufficient to distend the great vessels, their elasticity during the intervals of its action must be continually foreing the blood toward the periphery. The fact that the arteries are distended at each systole is abundantly proven by actual experiment; though the immense capacity of the arterial system, compared with the small 
charge of blood which enters at each pulsation, renders the actual distention of the vessels less than we should be led to expect from the force of the heart's contraction. The most satisfactory experiments on this subject are those of Poiseuille. ${ }^{1} \quad$ This observer illustrated the dilatation of the arteries in the following way: Having exposed a considerable extent of the primitive carotid in a horse, he enclosed a portion in a tin tube filled with water and connected with a small upright graduated tube of glass. The openings around the artery, as it passed in and out of the apparatus, being carefully sealed with tallow, it is evident that any dilatation of the vessel would be indicated by an elevation of the water in the graduated tube. This experiment invariably showed a marked dilatation of the artery with each contraction of the heart.

"We remark that the dilatation is not very considerable; thus it is not easy to recognize it by simple inspection, in an artery of even the caliber of that which occupies us, after we have it exposed." "

It being fully established that the arteries are dilated with each ventricular systole, it becomes important to study the influence of their elasticity upon the current of blood. Division of an artery in a living animal exhibits one of the important phenomena due to the elastic and yielding character of its walls. We observe, even in vessels of considerable size, as the carotid or femoral, that the flow of blood is not intermittent, but remittent. With each ventricular systole there is a sudden and marked impulse ; but during the intervals of contraction, the blood continues to flow with considerable force. As we recede from the heart, the impulse becomes less and less marked; but it is not entirely lost, even in the smallest vessels, the flow becoming constant only in the capillary system. That the force of the heart is absolutely intermittent, is shown by the following experiment:

1 Poiseunle, Recherches sur l'Action des Artères dans la Circulation Artérielle, Journal de la Physiologie, 1829, tome ix., p. 44.

2 Ibid., p. 48. 
If the organ be exposed in a living animal, and a canula be introduced through the walls into one of the ventricles, we have a powerful jet at each systole, but no blood is discharged during the diastole. The same absolute intermittency of the current will be seen if the aorta be divided. It is evident that we must look to the arteries themselves for the force which produces a flow of blood in the intervals of the heart's action. The conversion of the intermittent current in the largest vessels into a nearly constant flow in the smallest arterioles is effected by the physical property of elasticity. This may be illustrated in any elastic tube of sufficient length. If we connect with a syringe a series of rubber tubes progressively diminishing in caliber, and discharging by a very small orifice, and inject water in an intermittent current, if the apparatus be properly adjusted, the fluid will be discharged at the end of the tube in a continuous stream. Nearer the syringe, the stream will be remittent; and directly at the point of connection of the syringe with the tube, the stream will be intermittent. The intermittent impulse may be said, in this case, to be progressively absorbed by the elastic walls of the tube. Each impulse first distends that portion of the tube nearest to it, and further on, the distention is diminished, until it becomes inappreciable. If the syringe be connected with two tubes, one elastic and the other inelastic, the current will be either remittent or continnous in the one, and intermittent in the other.

This modification of the impulse of the heart has great physiological importance; for it is evidently essential that the current of blood, as it flows into the delicate capillary vessels, should not be alternately intermitted, and impelled with the full power of the ventricle. After all, it is in the capillaries that the blood performs its functions, and here we should have a constant supply of the fluid in proper quantity and in proper condition to meet the nutritive requirements of the parts. 
The elasticity of the arteries favors the flow of the blood toward the capillaries by a mechanism which is easily understood. The blood discharged from the heart distends the elastic vessel, which reacts, after the distending force ceases to operate, and compresses its fluid contents. This reaction would have a tendency to force the blood in two directions, were it not for an instantaneous closure of the valves, which makes regurgitation impossible. The influence then can only be exerted in the direction of the periphery ; and, if we can imagine as divided an action which is propagated with such rapidity, the reaction of that portion of the vessel immediately distended by the heart, distends a portion farther on, which in its turn distends another portion, and so the wave passes along until the blood is discharged into the capillaries. In this way we can see that in vessels removed a sufficient distance from the heart, the force exerted on the blood by the reaction of the elastic walls is competent to produce a very considerable current during the intervals of the heart's contraction.

This theoretical view is fully carried out by the following simple and conclusive experiment of M. Marey. He connected two tubes of equal size, one of rubber and the other of glass, with the stop-cock of a large vase filled with water. The elastic tube was provided with a valve near the stopcock, which prevented the reflux of fluid, and both were fitted with tips of equal caliber. When, by alternately opening and closing the stop-cock, water was allowed to flow into these tubes in an intermittent stream, it was found that a greater quantity was discharged by the elastic tube; but an equal quantity was discharged by both tubes when the stop-cock was left open, and the fluid allowed to pass in a continuous stream. ${ }^{2}$

This simple experiment shows that not only does the elasticity of the arteries convert the internittent current in the largest vessels into a current more and more nearly contin-

${ }^{1}$ Marey, Circulation du Sang, Paris, 1863, pp. 128, 131. 
uous as we approach the periphery, but that when reflux is prevented, as it is by the semilunar valves, the resiliency of the arteries assists the circulation.

Contractility of the Arteries.-It is a fully established anatomical fact that the medium-sized and smallest arteries contain contractile or muscular elements; and it is also a fact, proven by actual experiment, that as a consequence of the condition of these fibres, the ressels undergo considerable variation in their caliber. The opinions of the older physiologists on this question have only an historical interest, and will not, therefore, be discussed. Among the more recent investigations on this subject, we have the experiments of $\mathrm{Cl}$. Bernard and Schiff, which have been repeatedly confirmed, showing that through the nervous system the muscular coats of arteries may be readily made to contract or become relaxed. If the sympathetic be divided in the neck of a rabbit, in a very few minutes the arteries of the ear on that side are notably dilated. If the divided extremity of the nerve be feebly galvanized, the vessels soon take on contraction, and may become smaller than on the opposite side. These experiments demonstrate, in the most conclusive manner, the contractile properties of the small arteries, and give us an idea how the supply of blood to any particular part may be regulated. The vessels may be most effectually excited through the nervous system; and it is on account of the difficulty in producing marked results by direct irritation, that the older physiologists were divided on the subject of their "irritability."

The contractility of the arteries has great physiological importance. As their function is simply to supply blood to the various tissues and organs, it is evident that when the ressels going to any particular part are dilated, the supply of blood is necessarily increased. This is particularly important in the glands, which, during the intervals of secretion, receive a comparatively small quantity of blood. Bernard 
has shown, by a beantiful series of experiments, which will be more particularly alluded to on the subject of secretion, that galvanization of what he calls the motor nerve of a gland dilates the vessels, largely increases the supply of blood, and induces secretion; while galvanization of the sympathetic filaments contracts the vessels, diminishes the supply of blood, and arrests secretion. The pallor of parts exposed to cold, and the flush produced by heat, are due, on the one hand, to contraction, and on the other to dilatation of the small arteries. Pallor and blushing from mental emotions are examples of the same kind of action.

The ulterior effects on nutrition, which result from dilatation of the vessels of a part, are of great interest. When the supply of blood is much increased, as in section of the sympathetic in the neck, nutrition is exaggerated, and the temperature is raised beyond that of the rest of the body.

The idea, which at one time obtained, that the arteries were the seat of rhythmical contractions, which had a favorable influence on the current of blood, is entirely erroneous. ${ }^{1}$ It is hardly necessary to repeat that the prime cause of the arterial circulation is the force of the ventricles. We have seen that the elasticity of the arteries produces a flow during the intervals of the heart's action, and the question now arises whether the force thus exerted is simply a return of the force required to expand the vessels, which has been borrowed, as it were, from the heart, or is something superadded to the force of the heart. The experiment of Marey, already alluded to, settles this question. When water was forced in an intermittent current into two tubes, one elastic and the other inelastic, but discharging by openings of equal size, by far the greater quantity was discharged by the elastic tube. A little reflection will show how the

${ }^{1}$ Schiff has noticed rhythmical contractions in the superficial arteries of the ear in the rabbit, and some other animals; but this phenomenon is exceptional, and the movements do not appear to furor the current of blood. (Milne-Edwards, Physiologie tome, iv., p. 217.) 
action of the elastic arteries must actually assist the circulation. The resiliency of the vessels is continually pressing their contents toward the periphery, as regurgitation is rendered impossible by the action of the semilunar valves. The dilatation of the vessels with each systole, of course, admits an increased quantity of blood; and it has been experimentally demonstrated, that the same intermittent force exerted on an inelastic tube, will discharge a less quantity of liquid from openings of equal caliber.

Superadded, then, to the force of the heart, we must recognize, as a cause influencing the flow of blood in the arteries, the resiliency of the vessels, especially those of large size.

Thus it will be seen that the arteries are constantly kept distended with blood by the heart, and by virtue of their elasticity and the progressive increase in the capacity of this system as they branch, the powerful contractions of the central organ only serve to keep up an equable current in the capillaries. The sinall vessels, by virtue of their contractile walls, regulate the distribution of the blood; acting as the guards or sentinels of the process of nutrition, and, in fact, all the numerous functions in which the blood is concerned. Obeying the commands transmitted through the sympathetic nervous system, they allow the passage to every part of the proper quantity of the nutritive fluid at the proper time.

Locomotion of the Arteries and Production of the Pulse.At each contraction of the heart, the arteries are increased in length, and many of them undergo a considerable locomotion. This may be readily observed in vessels which are tortuous in their course, and is frequently very marked in the temporal artery in old persons. The elongation may also be seen if we watch attentively the point where an artery bifurcates, as at the division of the common carotid. It is simply the mechanical effect of sudden distention; which, while it 
increases the caliber of the vessel, causes an elongation even more marked.

The finger placed over an exposed artery, or one which lies near the surface, experiences a sensation at every beat of the heart, as though the vessel were striking against it. This has long been observed, and is called the pulse. Ordinarily it is appreciated when the current of blood can be subjected to a certain amount of obstruction, as in the radial, which can readily be compressed against the bone. In an artery imbedded in soft parts, which yield to pressure, the actual dilatation of the vessel being very slight, pulsation is felt with difficulty, if at all. When obstruction is complete, as in ligation of a vessel, the pulsation above the point of ligature is very marked, and can be readily appreciated by the eye. The explanation of this exaggeration of the movement is the following: Normally, the blood passes freely through the arteries, and produces, in the smaller vessels, very little movement or dilatation; but when the current is obstructed, as by ligation, or even compression with the finger, the force of the heart is not sent through the vessel to the periphery, but is arrested, and therefore becomes more marked and easily appreciated. In vessels which have become undilatable and incompressible from calcareous deposit, the pulse cannot be felt. The character of the pulse indicates, to a certain extent, the condition of the heart and vessels. We have spoken, when treating of the heart, of the varying rapidity of the pulse, as it is a record of the rapidity of the action of this organ; but it remains for us to consider the mechanism of its production, and its various characters.

Under ordinary circumstances, the pulse may be felt in all arteries which are exposed to investigation; and as it is due to the movement of the blood in the vessels, the prime (ause of its production is the contraction of the left ventricle. The late very interesting experiments of M. Marey have shown that the impulse given to the blood by the heart is 
not felt in all the vessels at the same instant. By ingenious contrivances, which will be described further on, this observer has succeeded in registering simultaneously the impulse of the heart, the pulse of the aorta, and the pulse of the femoral artery. He has thus ascertained that the contraction of the ventricle is anterior to the pulsation of the aorta, and the pulsation of the aorta precedes the pulse in the femoral. ${ }^{2}$ This only confirms the views of other physiologists, particularly Weber, who described this progressive retardation of the pulse as we recede from the heart, estimating the difference between the ventricular systole and the pulsation of the artery in the foot, at one-seventh of a second. ${ }^{2}$ The observations of M. Marey are particularly referred to as being the most conclusive.

It is evident from what we know of the variations which occur in the force of the heart's action, the quantity of blood in the vessels, and from the changes which may take place in the caliber of the arteries, that the character of the pulse must be subject to numerous variations. Many of these may be appreciated simply by the sense of touch. We find writers treating of the soft and compressible pulse, the hard pulse, the wiry pulse, the thready pulse, etc., as indicating various conditions of the circulatory system. The character of the pulse, aside from its frequency, has always been regarded as of great importance in disease; and the variations which occur in health form a most interesting subject for physiological inquiry.

Form of the Pulse.-It is evident that few of the characters of a pulsation, occupying as it does but a seventieth part of a minute, can be ascertained by the sense of touch

${ }^{1}$ Marey, Circulation du Sang, p. 197. In an article published in the Journal de la Physiologie, 1859, tome ii., p. 267, Marey took ground against the progressive retardation of the pulse in arteries removed from the heart; but in his last work the fact is admitted, and seems proven beyond a doubt.

${ }^{2}$ Milde-Edwards, Physiologie, tome iv., p. 188. 
alone. This fact has been appreciated by physiologists; and within the last few years, in order to accurately study this important subject, instruments for registering the impulse felt by the arterial system have been constructed, to enable us to accurately analyze the dilatation or movements of the vessels. The idea of such an instrument was probably suggested by the following simple observation: When the legs are crossed, with one knee over the other, the beating of the popliteal artery will produce a marked movement in the foot. If we could apply to an artery a lever provided with a marking point in contact with a slip of paper moving at a definite rate, this point would register the movements of the vessel, and its changes in caliber. The first physiologist who put • this in practice was Vierordt, who constructed quite a complex instrument, so arranged that the impulse from an accessible artery, like the radial, was conveyed to a lever, which marked the movement upon a revolving cylinder of paper. This instrument was called a "sphygmograph." The traces made by it were perfectly regular, and simply marked the extremes of dilatation, exaggerated, of course, by the length of the lever, and the number of pulsations in a given time. The latter can, of course, be easily estimated by more simple means; and as the former did not convey any very definite physiological idea, the apparatus was regarded rather as a curiosity than an-instrument for accurate research.

The principle on which the instrument of Vierordt was constructed was correct, and it only remained to construct one which would be easy of application, and-produce, a trace representing the shades of dilatation and contraction of the vessels, in order to lead to important practical results. These indispensable conditions are fully realized in the sphyginogragh of M. Marey, to whose researches on the circulation we have repeatedly referred. The instrument simply amplifies the changes in the caliber of the ressel, without deforming them ; and though its application is, perhaps, not so easy as to make it generally useful in practice, in the hands 
of Marey it has given us a definite knowledge of the physiological character of the pulse, and its modifications in certain

Fra. 8.

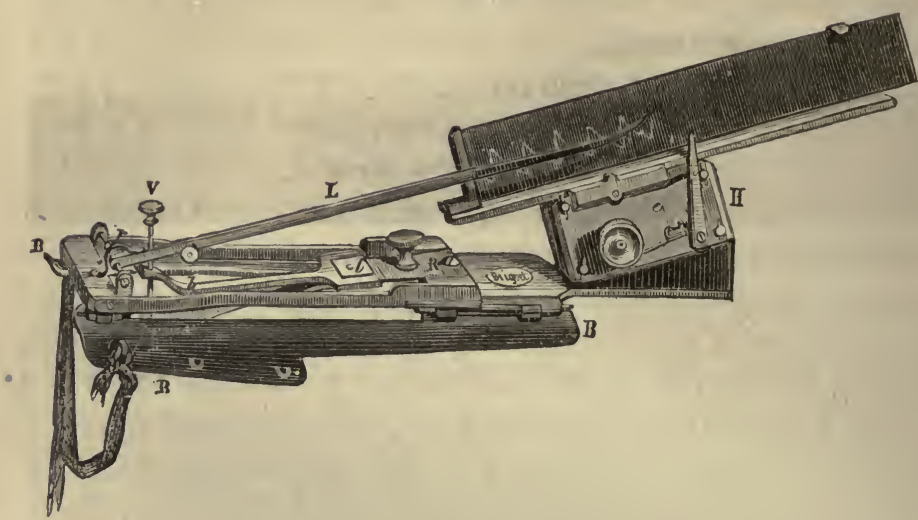

Sphygmograph of Marey. The apparatus is securely fixed on the forearm, so that the spring under the screw $\mathrm{V}$, is directly over the radial artery. The movements of the pulse

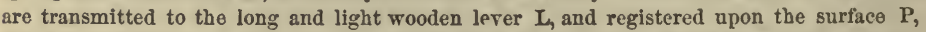
which is moved at a known rate by the clock-work $H$. The apparatus is so adjusted that the movements of the vessel are accurately amplifled and registered by the extreme point of the lever. (Marex, Recherches, etc. Journal de la Physiologie, Avril, 1860, tome lii., p. 244.)

diseases; information which is exceedingly desirable, and could not be arrived at by other means of investigation. In short, its mechanism is so accurate that, when skilfully used, it gives on paper the actual 'form of the pulse.'

FIG. 4.

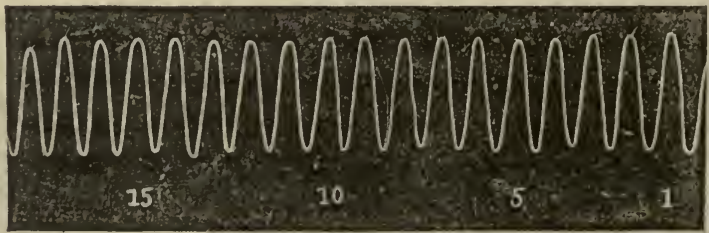

Trace of Vlerordt (Ibid.).

This instrument, applied to the radial artery, gives a trace very different from that obtained by Vierordt, which 
was simply a series of regular elevations and depressions. A comparison of the traces obtained by these two observers gives an idea of the defects which have been remedied by Marey; for it is evident that the dilatation and contraction of

Fig. 5.

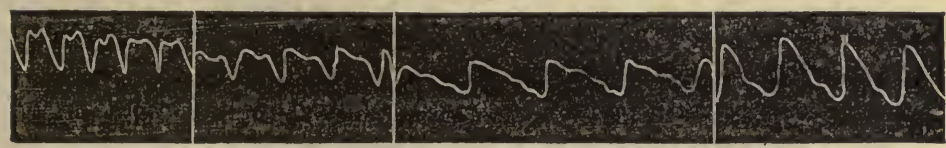

Trace of Marey. Portions of four traces taken in different conditions of the pulse (Ibid.)

the arteries cannot be as regular and simple as would be inferred merely from the trace made by the instrument of Vierordt.

Analyzing the traces of Marey, we see that there is a dilatation following the systole of the heart, marked by an elevation of the lever, more or less sudden, as indicated by the angle of the trace, and of greater or less amplitude. The dilatation, having arrived at its maximum, is followed by contraction; which may be slow and regular, or may be, and generally is, interrupted by a second and slighter upward movement of the lever. This second impulse varies very much in amplitude. In some rare instances it is nearly as marked as the first, and may be appreciated by the finger, giving the sensation of a double pulse following each contraction of the heart. This is called the dicrotic pulse.

As a rule, the first dilatation of the vessel is sudden, and indicated by an almost vertical line; this is followed by a slow reaction, indicated by a gradual descent of the trace, which is not, however, absolutely regular, but marked by a slight elevation indicating a second impulse.

The amplitude of the trace, or the distance between the highest and lowest points marked by the lever, depends upon the amount of constant tension of the vessels. Marey has found that the amplitude is in an inverse ratio to the tension; which is very easily understood, for when the arteries are little distended, the force of the heart must be more marked in its 
effects than when the pressure of blond in them is very great. Any circumstance which facilitates the flow of blood from the arteries into the capillaries, will, of course, relieve the tension of the arterial system, lessen the obstacle to the force of the heart, and increase the amplitude of the pulsation; and vice versâ. In support of this view, Marey has found that cold applied to the surface of the body, contracting, as it does, the smallest arteries, increases the arterial tension and diminishes the amplitude of the pulsation; while a moderate elevation of temperature produces an opposite effect.

In nearly all the traces given by Marey, the descent of the lever indicates more or less oscillation of the mass of blood. The physical properties of the larger arteries render this inevitable. As they yield to the distending influence of the heart, reaction occurs after this force is taken off, and, if the distention be very great, gives a second impulse to the blood. This is quite marked, unless the tension of the arterial system be so great as to offer too much resistence. One of the most favorable conditions for the manifestation of dicrotism is diminished tension, which is always found coexisting with a very marked exhibition of this phenomenon.

The delicate instrument employed by Marey enabled him to accurately determine and register these various phenomena, by observations on arteries of the human subject and animals; and by means of an ingeniously constructed "schema," representing the arterial system by elastic tubes, and the left ventricle by an elastic bag, provided with valves, acting as a syringe, he satisfactorily established the conditions of tension, etc., necessary to their production. In this schema, the registering apparatus, simpler in construction than the sphygmograph, could be applied to the tubes with more accuracy and ease.

He demonstrated, by experiments with this system of tubes, that the amplitude of the pulsations, the force of the central organ being the same, is greatest when the tubes are moderately distended, or the tension of fluid is low, and vice 
versâ. He demonstrated, also, that a low tension favors dicrotism. In this latter observation he diminished the tension by enlarging the orifices by which the fluid is discharged from the tubes, imitating the dilatation of the small vessels, by which the tension is diminished in the arterial system. He also demonstrated that an important and essential element in the production of dicrotism, is the tendency to oscillation of the fluid in the vessels, between the contractions of the heart. This can only occur in fluid which has a certain weight, and acquires a velocity from the impulse; for when air was introduced into the apparatus, dicrotism could not be produced under any circumstances, as the fluid did not possess weight enough to oscillate between the impulses. Water produced a well-marked dicrotic impulse under favorable circumstances; and with mercury, the oscillations made two, three, or more distinct impulses.

By these experiments he proved that the blood oscillates in the vessels, if this movement be not suppressed by too great pressure, or tension. This oscillation gives the successive rebounds that are marked in the descending line of the pulse, and is capable, in some rare instances, when the arterial tension is very slight, of producing a second rebound of sufficient force to be appreciated by the finger. ${ }^{1}$

${ }^{3}$ In treating of the form of the pulse, of course including dicrotism, from a purely physiological point of view, we have given an analysis of the physiological portion of the late work of Marey (Physiologie Médicale de la Circulation du Sang, Paris, 1863). To portions of this work relating to the action of the heart, sounds, etc., we have already referred. As is evident from our sketch of the instruments for registering the pulse, the author referred to is the only one who has produced a trace correctly representing the shades of locomotion and dilatation of the arteries; and by his brilliant and ingenious experiments, which cannot be too highly praised, he has settled many important points, and given a precious means of investigation to other physiologists. He has opened a new field for study of the pathological changes in the form of the pulse; but before we can advance far in this direction, we must become familiar with all the modifications which occur in health, an end which as yet is by no means fully attained. The construction of a sphygmograph was a problem of great delicacy, and a ccrtain amount of practical experience with the instrument has convinced us of 
Without treating of the variations in the character of the pulse in disease, due to the action of the muscular coat, we will consider some of the external modifying influences which come within the range of physiology. The smallest vessels and those of medium size possess to an eminent degree what is called tonicity, or the property of maintaining a certain continued amount of contraction. This contraction is antagonistic to the distending force of the blood, as is shown by opening a portion of an artery included between two ligatures, in a living animal, when the contents will be forcibly discharged and the caliber of that portion of the vessel very much diminished. Too great distention of the vessels by the pressure of blood seems to be prevented by this constant action of the muscular coat; and thus the conditions are maintained which give the pulse the character we have just described.

By excessive and continued heat, the muscular tissue of the arteries may be dilated so as to offer less resistance to the distending force of the heart. Under these circumstances, the pulse, as felt by the finger, will be found to be larger and softer than normal. Cold, either general or local, has a precisely opposite effect; the arteries become contracted, and the pulse assumes a harder and more wiry character. Usually, prolonged contraction of the arteries is followed by relaxation, as is seen in the full pulse and glow of the surface which accompany reaction after exposure to cold.

It has been found, also, that there is a considerable differ-

the accuracy of results to be obtained when it is used with skill and care; but the very perfection and nicety of the instrument present almost insurmountable difficulties in the way of its use by the general practitioner. Results, regarding the amplitude of pulsations especially, should be received with great caution, from the extreme difficulty of adjusting the lever so as to give the maximum of the impulse. It does not appear, however, how these drawbacks to the general use of the instrument can be obviated; for its construction leaves nothing to be desired, and the delicacy of its adjustment, like that of a fine balance, is indispensable. In the hands of Marey, its results, we conceive, are to be fully ao cepted. 
ence in the caliber of the arteries at different periods of the day. The diameter of the radial has been found very much greater in the evening than in the morning, ${ }^{1}$ producing, naturally, a variation in the character of the pulse. We learn from these physiological variations, how in disease, when they become more considerable, they may give important information with regard to the condition of the system.

\section{Pressure of Blood in the Arteries.}

The reaction of the elastic walls of the arteries during the intervals of the heart's action gives rise to a certain amount of constant pressure, by which the blood is continually forced toward the capillaries. The discharge of blood into the capillaries has a constant tendency to diminish this pressure; but the contractions of the left ventricle, by forcing repeated charges of blood into the arteries, have a compensating action. By the equilibrium between these two agencies, a certain degree of tension is maintained in the arteries, which is called the arterial pressure.

The first experiments with regard to the extent of the arterial pressure were made by Hales an English physiologist, more than a hundred years ago. ${ }^{2}$ This observer, adapting a long glass tube to the artery of a living animal, ascertained the height of the column of blood which could be sustained by the arterial pressure. In some experiments on the carotid of the horse, the blood mounted to the height of from eight to ten feet. Hales was not fully acquainted with the influences capable of modifying the arterial pressure, and his estimates of the normal tension in these vessels were not entirely correct. It is now ascertained that the pressure in the arteries will sustain a column of about six feet of water, or six inches of mercury, and is subject to considerable vari-

1 Milne-Edwards, op. cit., tome iv., p. 222.

${ }^{2}$ Hales, Statical Essays, London, 1733, vol. ii., Hoemastaticks. 
ations, depending upon the condition of the heart and vessels, the quantity of blood, respiration, muscular exercise, etc.

All experiments on the arterial pressure are made on the principle of the experiment of Hales, which, with reference simply to the constant pressure in the arteries, is as useful as those of later date, and much more striking. The only inconvenience is in the manipulation of the long tube, but this may be avoided by setting it in a strip of wood, when it can be easily handled. If a large artery, as the carotid, be ex-

Fig. 6.

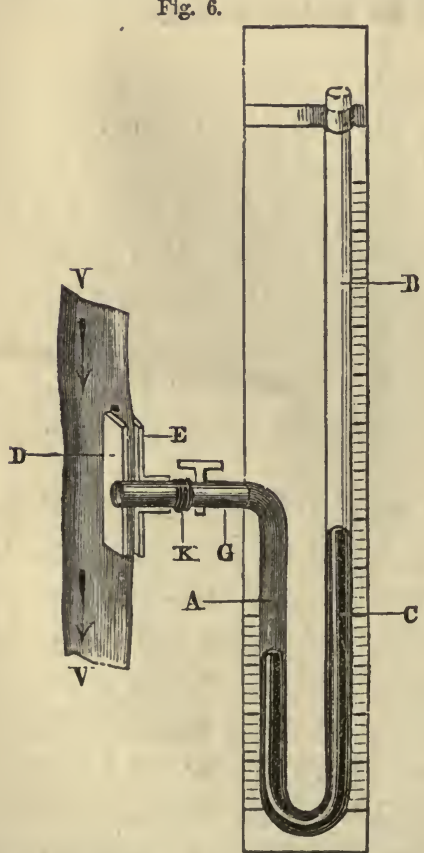

Hemodynamometer of Poisenille, modiffed by Ludwig, Spengler, and Valentin. The instrument is connected with the ressel $\mathrm{V} V$, in sach a manner that the circulation is not interrupted. The elevation of the mercury in the branch B C indicates the amount of pressure. (BÉCLARD, Phy(iologie, Paris, 1859, p. 204.) posed in a living animal, and a metallic point, connected with a vertical tube of small caliber and from seven to eight feet long by a bit of elastic tubing, be secured in the ressel, the blood will rise to the height of about six' feet, and remain at this point almost stationary, indicating by a slight pulsatile movement the action of the heart. On carefully watching the level in the tube, in addition to the rapid oscillation eoincident with the pulse, another oscillation will be observed, which is less frequent, and which corresponds with the movements of respiration. The pressure, as indicated by an elevation of the fluid, is slightly increased during expiration, and diminished during inspiration. ${ }^{2}$

${ }^{1}$ In all these experiments on the arterial or cardiac pressure, it is necessary to fill part of the tube, or whatever apparatns we may use, with a solution of carbonate of soda, in order to prevent coagulation of the blood as it passes out of the ressels. 
The experiment with the long tube gives us the best idea of the arterial pressure, which will be found to vary from five and a half to six feet of blood, or a few inches more of water. The oscillations produced by the contractions of the heart are not very marked, on account of the immense friction in so long a tube; but this is favorable to the study of the constant pressure in the arteries. It has been found that the estimates above given do not vary very much in animals of different sizes. Bernard found the pressure in the carotid of a horse little more than in the dog or rabbit. In the larger animals it is the force of the heart which is increased, and not to any considerable extent the constant pressure in the vessels. ${ }^{1}$

The experiments of Hales were made with a view of calculating the force of the heart, and were not directed particularly to the conditions and rariations of the arterial pressure. It is only since the experiments performed by Poiseuille with the hemodynamometer, in 1828, that we have any reliable data. Fig. 7.

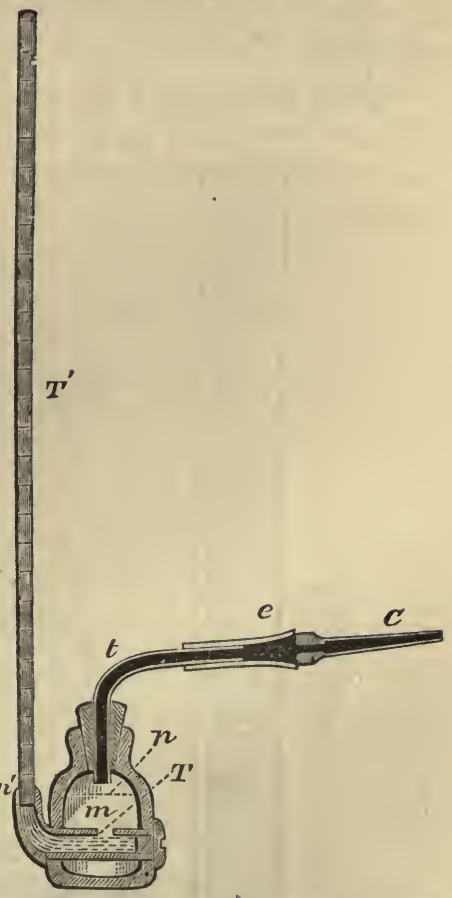

Section of the cardiometer of Magendie, as modified by Bernard. A strong glass bottle is perforated at each side, and fitterl with an iron tube, with an opening, $T$, by which the mercury enters. One end of the iron tube is closed, and the other is bent upwards and connected with the graduated glass tube T', which has a caliber of from $\frac{3}{5} \frac{1}{2}$ to $\frac{1}{8}$ of an inch. The bottle is filled with mercnry until it rises to $n^{\prime}$ in the tube which is marked 0 . The cork is perforated by the tube $t$, which is connected by a rubber tube with the point $\mathrm{C}$, which is introduced into the vessel. (Branard, Liquides de l'Organisme, Paris, 1859, tome i., p. 167.)

on this latter point. ${ }^{2}$ Poiseuille's instrument for measuring the force of the blood is a simple graduated $U$ tube, half

${ }^{1}$ Bernard, Liquides de l'Organisme, Paris, 1859, tome i., p. 172.

${ }^{2}$ Porsetille, Recherches sur la Force du Cour Aortique, Paris, 1828. 
filled with mercury, with one arm bent at a right angle, so that it can easily be connected with the artery. The pressure of the blood is indicated by a depression in the level of the mercury on one side, and a corresponding elevation on the other.

This instrument is generally considered as possessing

Fig. 8 .

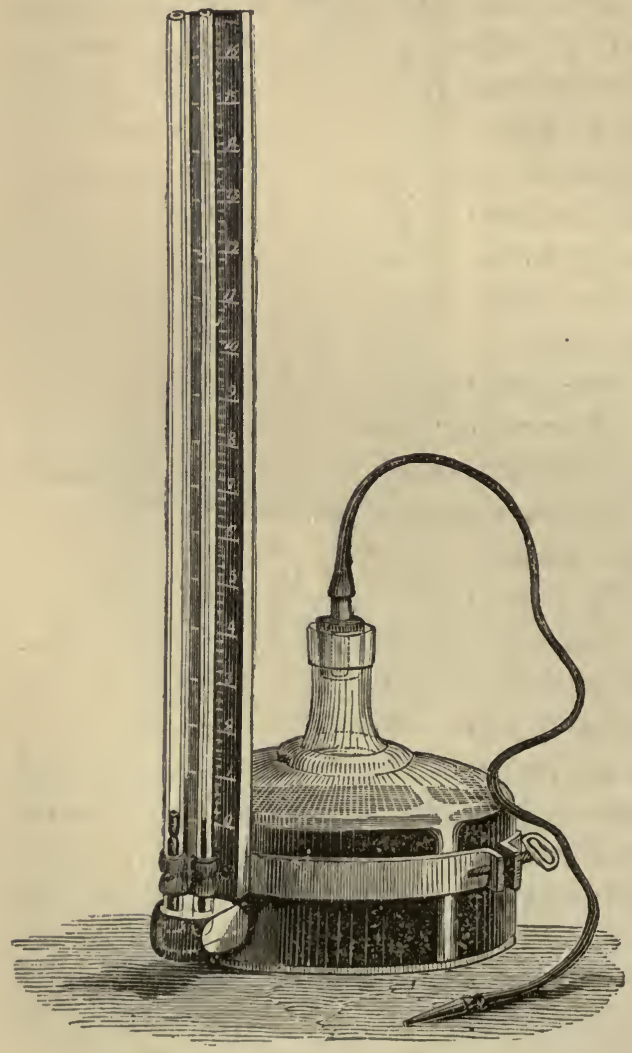

Compensating Instrument of Marey. great advantages over the long glass tube; but for estimating simply the arterial pressure, it is much less useful, as it is more sensitive to the impulse of the heart. For the study of the cardiac pressure, it has the disadvan: tage, in the first place, of considerable friction; and again, the weight of the column of mercury produces an extent of oscillation by its mere impetus, greater than that which would actually represent the force of the heart. ${ }^{1}$

An important improvement in

${ }^{1}$ Ludwig devised a means of registering the oscillation in the hemodynamometer of Poiseuille. He used a $U$ tube of considerable size, and placed a float on the surface of the mercury, to which a peneil was attached The point 
the hemodynamometer was made by Magendie. This apparatus, the cardiometer, in which Bernard has made some important modifications, is the one now generally used. It consists of a small but thick glass bottle, with a fine graduated glass tube about twelve inches in length, communicating with it, either through the stopper, or by an orifice in the side. The stopper is pierced by a bent tube which is to be connected with the blood-vessel. The bottle is filled with mercury so that it will rise in the tube to a point which is marked zero. It is evident that the amount of pressure on the mercury in the bottle will be indicated by an elevation in the graduated tube; and, moreover, from the fineness of the column in the tube, we avoid some of the inconveniences which are due to the weight of mercury in the hemodynamometer, and also have less friction.

This instrument is appropriately called the cardiometer, as it indicates most accurately, by the extreme elevation of the mercury, the force of the heart; but it is not as perfect in its indications of the mean arterial pressure, as in the abrupt descent of the mercury during the diastole of the heart, the impetus causes the level to fall considerably below the real standard of the constant pressure. Marey has succeeded in correcting this difficulty in what he calls the "compensating" instrument; which is constructed on the following principle: Instead of a simple glass tube which communicates with the mercury in the bottle, as in Magendie's cardiometer, he has two tubes: one of which is like the one already described, and represents oscillations produced by the heart; the other is larger, and has at the lower part a constriction of the caliber which is there reduced to capillary fineness. This tube is designed to give the mean arterial pressure. The

of the pencil, brought in contact with a revolving cylinder covered with paper, produced a trace of the oscillations. By analysis of this trace he arrived at the mean pressure in the arteries. This instrument was called the kymographion. It has never been much used in investigation, and is entirely superseded by the cardiometer of the present day. 
constriction in the tube offers such an obstacle to the rise of the mercury that the intermittent action of the heart is not felt, the mercury rising slowly to a certain level, which is constant, and varies only with the constant pressure in the vessels.

We have only an approximative idea of the average pressure in the arterial system in the human subject, deduced firom experiments on animals. It has already been stated to be equal to about six feet of water, or six inches of mercury.

The most interesting questions connected with this subject are: the comparative pressure in different parts of the arterial system, the influences which modify the arterial pressure, and its influence on the pulse. These points have all been pretty fully investigated by experiments on animals, and on systems of elastic tubes arranged to represent the vessels.

Pressure in Different Parts of the Arterial System.The experiments of Hales, Poiseuille, Bernard, and others, seem to show that the constant arterial pressure does not vary in arteries of different sizes. These physiologists have experimented particularly on the carotid and crural, and have found the pressure in these two vessels about the same. From their experiments, they conclude that the force is equal in all parts of the arterial system. The experiments of Volkmann, however, have shown that this conclusion has been too hasty: With the registering apparatus of Ludwig, he has taken the pressure in the carotid and metatarsal arteries, and has always found a considerable difference in favor of the former. ${ }^{1} \quad$ In an experiment on a dog, he found the pressure

${ }^{1}$ For comparing the pressure in different vessels and in different animals, Berrard has devised an instrument which he calls the differential hemodynamometer. It consists of a graduated $U$ tube so arranged that both arms may be simultaneously conneeted with separate vessels. If the pressure be equal in the two ressels with which it is connected, the level of the mereury will not be affeeted; but an inequality of pressure will be marked by a depression of the mercury in the arm corresponding to the ressel in which the pressure is the more powerful. With this instrument, Bernard assumes to have demonstrated that the constant pressure is equal in all parts $c_{e}^{e}$ the arterial system, the foree of the heart, 
equal to 172 millimetres in the carotid, and $165 \mathrm{~mm}$. in the metatarsal. In an experiment on a calf, the pressure was 116 $\mathrm{mm}$. in the carotid, and $89 \mathrm{~mm}$. in the metatarsal; and in a rabbit, $91 \mathrm{~mm}$. in the carotid, and $86 \mathrm{~mm}$. in the crural: ${ }^{1}$

These experiments, which seem to have been performed with great care, show that the pressure is not absolutely the same in all parts of the arterial system; that it is greatest in the arteries nearest the heart, and gradually diminishes as we near the capillaries. The difference is very slight, almost inappreciable, until we come to vessels of very small size; but here the pressure is directly influenced by the discharge of blood into the capillaries.

The cause of this diminution of pressure in the smallest vessels is the proximity of the great outlet of the arteries, the capillary system; for, as we shall see further on, the flow into the capillaries has a constant tendency to diminish the pressure in the arteries. It is obvious that this influence can only be felt in a very marked degree in the vessels of smallest size. ${ }^{2}$

Influence of Respiration.-It is easy to see, in studying the arterial pressure with any of the instruments we have described, that there is a marked increase with expiration, and a diminution with inspiration. The fact that expiration will increase the force of the jet of blood from a divided artery has long been observed, and accords perfectly. with the above statement.

ouly, diminishing in the smaller vesscls. The instrument by no means possesses the delicacy of the apparatus used by Volkmann, in giving the mean pressure. (Liquides de l'Organisme, tome i., p. 209 et seq.)

${ }^{1}$ Mulne-Edwards, op. cit., tome iv., p. 234.

2 This view is fully sustained by physical laws. If fluid be discharged from a reservoir by a long horizontal tube of uniform caliber, the pressure, as indicated by vertical tubes at different points, will be found to diminish regularly from the height of the fluid in the reservoir to the orifice of discharge. An instrument of this kind, which is called a piezometer, shows the apparent plyysical necessity of a progressive diminution in pressure in the arterial system, as we pass from the heart to the capillaries. 
In tranquil respiration, the influence upon the flow of blood is due simply to the mechanical action of the thorax. With every inspiration the air-cells are enlarged, as well as the blood-vessels of the lungs; the air rushes in through the trachea, and the movement of the blood in the veins near the chest is accelerated. At the same time the blood in the arteries is somewhat retarded in its flow from the thorax, or at least does not feel the expulsive influence which follows with the act of expiration. The mean of the arterial pressure at that time is at its minimum. With the expiratory act, the air is expelled by compression of the lungs, the flow of blood into the thorax by the veins is retarded to a certain extent, while the flow of blood into the arteries is favored. This is strikingly exhibited in the augmented force, with expiration, in the jet from a divided artery. Under these circumstances the arterial pressure is at its maximum.

In perfectly tranquil respiration, the changes due to inspiration and expiration are very slight, marked by a difference of not more than half an inch to an inch in the cardiometer. When the respiratory movements are exaggerated, the oscillations are very much more marked.

Interruption of respiration is followed by a very great increase in the arterial pressure. This is due, not to causes within the chest, but to obstruction to the circulation in the capillaries. We are already aware of the influence which the flow of blood into the capillaries is constantly exerting upon the arterial pressure. This tendency to diminish the quantity of blood in the arteries, and consequently the pressure, is constantly counteracted by the blood sent into the arteries by the contractions of the heart. In interruption of the respiratory function, the non-aërated blood passes into the arteries, but refuses to pass through the capillaries; and as a consequence, the arteries are abnormally distended, and the arterial pressure is enormously increased. If respiration be permanently arrested, the arterial pressure becomes, after a time, diminished below the normal standard, and ultimate- 
ly abolished, on acconnt of the stoppage of the action of the heart. If respiration be resumed before the heart has become arrested, the pressure soon returns to its normal standard.

Muscular effort considerably increases the arterial pressure. This is due to two eauses. - In the first place, the chest is generally compressed, favoring the flow of blood into the great vessels. In the second place, muscular exertion produces a certain amount of obstruction to the discharge of blood from the arteries into the capillaries. Numerous experiments upon animals have shown a great increase in pressure in the struggles which occur during severe operations. Bernard has shown that galvanization of the sympathetic in the neck and irritation of some of the cerebro-spinal nerves increase the arterial pressure, probably from their effects on the muscular coats of some of the arteries, causing them to contract, and thereby diminishing the total capacity of the arterial system. ${ }^{1}$

Effects of Hemorrhage.-Diminution in the quantity of blood has a remarkable effect upon the arterial pressure. If, in connecting the instrument with the arteries, we allow even one or two jets of blood to escape, the pressure will be found diminished perhaps one-half, or even more. It is hardly necessary to discuss the mechanism of the effect of the loss of blood on the tension of the vessels, but it is wonderful how soon the pressure in the arteries regains its normal standard after it has been lowered by hemorrhage. As it depends upon the quantity of blood, as soon as the ressels absorb the serosities in sufficient quantity to repair the loss, the pressure is increased. This takes place in a very short time, if the loss of blood be not too great.

Experiments on the arterial pressure with the cardiometer have verified the fact stated in treating of the form of the pulse, namely, that the pressure in the vessels bears an inverse ratio to the distention produced by the contractions of the heart.

${ }^{1}$ BerNakd, Liquides de l'Organisme, tome i. 
In the cardiometer, the mean height of the mercury indicates the constant, or arterial, pressure, and the oscillations, the distention produced by the heart. It is found that when the pressure is great, the extent of oscillation is small, and vice versâ. It will be remembered that the researches of Marey demonstrated that an increase of the arterial pressure diminishes the amplitude of the pulsations, as indicated by the sphygmograph, and that the amplitude is very great when the pressure is slight.

It is also true, as a general rule, that the force of the heart, as indicated by the cardiometer, bears an inverse ratio to the frequency of its pulsations.

Summary. - The arterial pressure, due to the distention of the arteries, and the reaction of their elastic walls continually forcing the blood toward the capillaries, is equal to about six feet of water or six inches of mercury. It is increased by any thing which favors the flow of blood into the great vessels, like the expiratory act, or by any thing which obstructs the flow from the arterioles into the capillaries, like muscular effort, contraction of the muscular coat of the smallest arteries, or non-aëration of the blood. It is diminished by any considerable diminution in the quantity of the circulating fluid, or by any thing which facilitates the passage of blood through the capillaries.

\section{Rapidity of the Current of Blood in the Arteries.}

Though this is not a question of great physiological importance, it is a point of some interest. It has long engaged the attention of physiologists, and has lately been made the subject of some curious and ingenious experiments. Passing over the speculations and calculations from imperfect physical data of the older physiologists, which led to no definite results, we find the first experiments on this subject made by Volkmann, with an instrument called the hemodromometer. This apparatus consists of a $U$ tube, graduated, and so ar- 
ranged that when the instrument is connected with the artery of a living animal, the current may be instantaneously direeted through the graduated tube, and by a stop-watch, the length of time occupied in passing from one extremity to the other accurately measured. Observations with this instrument, on the rapidity of the circulation in the carotid of the $\mathrm{dog}$ and horse, show that the blood moves at the rate of from 10 to 13 inehes per second. The rapidity is diminished in the smaller vessels, being but 2.2 inches per second in the metatarsal artery of a horse, and 10 inches in the carotid. ${ }^{1}$

The results thus obtained cannot be received as absolutely exact. The blood is diverted from its natural course, and must experience a certain diminution in velocity from the curves in the tubes. It is also evident that the normal current is not uniform; that it is much more rapid immediately after the systole of the heart, than during the diastole; and, as has been demonstrated by Marey, the blood in the arteries undergoes a certain oscillation. The experiments of Volkmann give an approximative idea of the mean rapidity, it is true, but they are far from exhibiting the natural current, with the variations corresponding to the movements of the heart.

A few years later (1858), an instrument was devised by Vierordt, which seemed to embody the right principle, but it was not sufficiently sensitive to accomplish all that was de-

- The experiments of Volkmann and Hüttenheim, published in 1846, are referred to, and the instrument described and delineated, in most works on physiology. When the instrument is first connected with the artery, the blood passes through a straight tube, and is not deviated from its course. The current is diverted into the graduated $U$ tube by two stop-cocks which are arranged so that they may be turned simultaneously. Before it is applied, the apparatus is filled with warm water, so as to prevent the entrance of air into the vessels.

The following are the results obtained by Volkmann in experiments on dogs and horses:

In the $\mathrm{dog}$, carotid . . . 10.7 inches per second. do. do. • • • 13 " 13 " "

In the horse, carotid . . . . " 10 " " "

do. metatarsal artery - . " $2 \cdot 2$. " " -Longet, Traité de Physiologie, Paris, 1861, tome i., p. 848. 
sired. It consisted of a little square box made of glass, with au opening at each end, by which it was to be connected with the artery. This is filled with water, and contains a pendulum, which is struck by the current of blood. The deviations of the pendulum are marked on a scale. After this has been applied to an artery, and the extent of movement of the pendulum noted, it is removed from the vessel and connected with an elastic tube, in which a current of water is made to pass with a degree of rapidity which will produce the same deviation as occurred when the instrument was connected with the blood-vessel. The rapidity of the current in this tube may be easily calculated by receiving the fluid in a graduated vessel, and noting the time occupied in discharging a given quantity. By this means we ascertain the rapidity of the current of blood. By means of a needle attached to the pendulum, the oscillations could be registered on a revolving cylinder of paper, and the mean velocity taken.

With this instrument, Vierordt estimated the mean velocity of blood in the carotid at $10 \cdot 2$ inches per second. Chauveau, who invented an instrument which we will describe presently, found the instrument of Vierordt not sufficiently sensitive, and requiring so much care and precaution in its use as to essentially diminish the value of its results.

The best instrument for measuring the rapidity of the circulation in the arteries was devised by Chauveau, of the Veterinary School at Lyons. ${ }^{1}$ This will give, by calculation, the actual rapidity of the circulation; and, what is more interesting, it marks accurately the rapid variations in velo. city, with reference to the heart's action.

The instrument to be applied to the carotid of the horse consists of a thin brass tube, about $1 \frac{1}{2}$ inch in length, and of the diameter of the artery (about $\frac{3}{8}$ of an inch), which is

1 M.M. A. Chatveau, G. Bertolus et L. Laroyenne, Vitesse de la Circulation dans les Artires du Cheval. Journal de la Physiologie, Paris, 1860, tome iii., p. 695. 
provided with an

Fig. 9.

oblong longitudinal opening, or window, near the middle, about two lines long and one line wide. A piece of thin vulcanized rubber is wound around the tube, and firmly tied, so as to cover this opening. Through a transverse slit in the rubber is introduced a very light

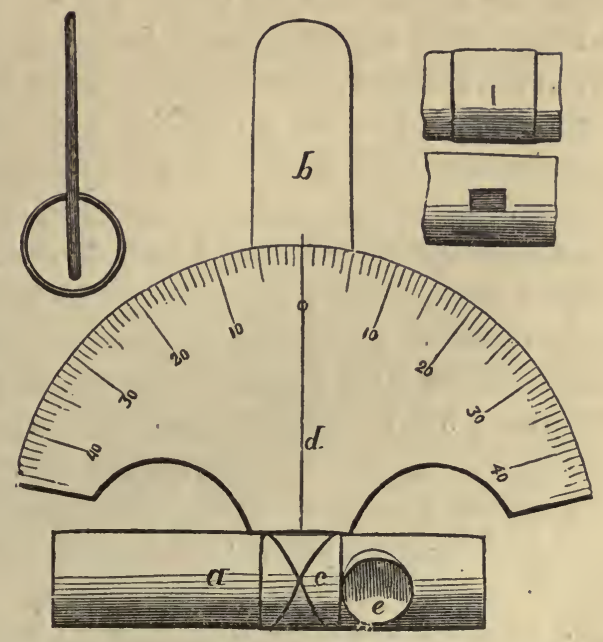
metallic needle, Chaureau's instrument for measuring the rapidity of the flow of an inch alood in the arteries. The instrument viewed in face $-a$, the an inch and a half tube to be fixed in the vessel; $b$, the dial which marks the exin length and flat- tent of movement of the needie $d ;$; $e$ a lateral tube for the tened at its lower part. This is made to project about half way into the caliber of the tube. A flat semicircular piece of metal, divided into an arbitrary scale, is attached to the tube, to indicate the deviations of the point of the needle.

The apparatus is introduced carefully into the carotid of a horse, by making a slit in the vessel, introducing first one end of the tube, directed toward the heart, then allowing a little blood to enter the instrument, so as to expel the air, and, when full, introducing the other end, securing the whole by ligatures above and below.

When the circulation is arrested, the needle should be vertical, or mark zero on the scale. When the flow is established, a deviation of the needle occurs, which varies in extent with the rapidity of the current.

Having removed all pressure from the vessel, so as to allow the current to resume its normal character, the deviations 
of the needle are carefully noted, as they occur with the systole of the heart, with the diastole, etc. After withdrawing the instrument, it is applied to a tube of the size of the artery, and we measure the rapidity of the current required to carry the needle to the points noted, which may be done by the same calculation used in graduating the apparatus of Vierordt. ${ }^{\prime}$

This instrument is on the same principle as the one constructed by Vierordt, but in sensitiveness and aecuracy is much superior. In the hands of Chauveau, the results, particularly those with regard to rariations in the rapidity of the current, are very interesting.

Rapidity of the Current in the Carotid.-It has been found that three currents, with different degrees of rapidity, may be distinguished in the carotid:

1. At each ventricular systole, we have, as the arerage of the experiments of Chauveau, the blood moving in the carotids at the rate of twenty $\frac{4}{10}$ inches per second. After this the rapidity quickly diminishes, the needle returning quite or nearly to zero, which would indicate complete arrest.

2. Immediately succeeding the ventricular systole, we have a second impulse given to the blood, which is synchronous with the closure of the semilunar valves, the blood moving at the rate of eight $\frac{6}{10}$ inches per second. This Chauveau calls the dicrotic impulse.

3. After the dicrotic impulse, the rapidity of the current gradually diminishes, until, just before the systole of the heart, it becomes almost nil. The average rate after the dicrotic impulse is five $\frac{9}{10}$ inches per second.

These experiments give us, for the first time, correct notions of the rapidity and variations of the flow of blood in the larger vessels; and it is seen that they correspond in a

1 In graduating the apparatus, Chauveau uses warm water. It would be more accurate to use defibrinated blood, or a fluid of equal density. 
remarkable degree with the experiments of Marey on the form of the pulse. Marey showed that there is a marked os. cillation of the blood in the vessels, due to a reaction of their elastic walls, following the first violent distention by the heart; that at the time of closure of the semilunar valves, the arteries experience a second, or dicrotic, distention, much less than the first; and following this, there is a gradual decline in the distention until the minimum is reached. Chauveau shows by experiments with his instrument, that corresponding to the first dilatation of the vessels, the blood moves with immense rapidity; following this, the current suddenly becomes nearly arrested; this is followed by a second acceleration in the current, less than the first; and following this we have a gradual decline in the rapidity to the time of the next. pulsation.

\section{Rapidity in Different Parts of the Arterial System.--} From the fact that the arterial system increases in capacity as we recede from the heart, we should expect to find a corresponding diminution in the rapidity of the flow of blood. There are, however, many circumstances, aside from simple increase in the capacity of the vessels, which undoubtedly modify the blood current, and render inexact any calculations on purely physical principles; such as the tension of the blood, the conditions of contraction or relaxation of the smallest arteries, etc. It is therefore necessary to have recourse to actual experiment to arrive at any definite results on this point. The experiments of Volkmann showed a great difference in the rapidity of the current in the carotid and metatarsal arteries, the average being 10 inches per second in the carotid, to 2.2 inches in the metatarsal. The same difference, though not quite as marked, was found by Chauveau between the carotid and the facial.

The last-named observer also noted an important modification in the character of the current in the smaller vessels. As we recede from the central organ, the systolic impulse 
becomes rapidly diminished, being reduced in one experiment about two-thirds; the dicrotic impulse becomes very feeble or even abolished; but the constant flow is very much increased in rapidity. This fact coincides with the ideas already advanced, with regard to the gradual conversion, by virtue of the elasticity of the vessels, of the impulse of the heart into, first, a remittent, and, in the very smallest arteries, a nearly constant current.

The rapidity of the flow in any artery must be subject to constant modifications due to the condition of the arterioles which are supplied by it. When these little vessels are dilated, the artery of course empties itself with greater facility, and the rapidity is increased. Thus the rapidity bears a relation to the arterial pressure; as, independently of a diminution in the entire quantity of the circulating fluid, variations in the pressure depend chiefly on causes which facilitate or retard the flow of blood into the capillaries. A good example of enlargement of the capillaries of a particular part, is in mastication, when the salivary glands are brought into activity, and the quantity of blood which they receive is greatly increased. Chauveau found an immense increase in the rapidity of the flow in the carotid of a horse during mastication. The enlargement of the vessels of the glands during their function has been conclusively proven by the experiments of Bernard.

It must be remembered that in all parts of the arterial system the rapidity of the current of blood is constantly liable to increase from dilatation of the smiall vessels, and diminution from their contraction.

\section{Arterial Murmurs.}

In the largest vessels, we can frequently hear with the stethoscope the sounds conducted from the heart. In addition, we ean hear, in all except the smallest vessels, a peculiar blowing sound, called the bruit de souffle, which is 
produced by the pressure of the end of the instrument on the artery. The following is the mechanism of the production of this sound: The pressure of the instrument produces a constriction in the vessel, and more or loss obstruction to the current of blood. As the blood flows from this constricted portion into that just beyond, where of course the vessel is relatively larger and the current is somewhat retarded, the relatively small and forcible stream produces an unusual and irregular current, which is accompanied by a certain sound. It has been proven by the experiments of Chauveau and Marey with elastic tubes, that this sound is always produced when any part of the apparatus is dilated so that the fluid passes from the tube into a sort of sac. In this way aneurismal murmurs are accounted for. The sounds which are heard in the arteries, and are not dependent upon compression with the stethoscope, depend upon conditions, the consideration of which belongs to pathology. 


\section{CHAPTER VII.}

\section{CIRCULATION OF THE BLOOD IN THE CAPILLARIES.}

Distinction between capillaries and the smallest arteries and veins-Physiological anatomy of the capillaries-Peculiarities of distribution-Capacity of the capillary system-Course of blood in the capillaries-Phenomena of the capillary circulation-Rapidity of the capillary circulation-Relations of the capillary circulation to respiration-Causes of the capillary circulation-Influence of temperature on the capillary circulation-Influence of direct irritation on the capillary circulation.

BEFORE entering upon the study of the capillary circulation, let us define what we mean by the capillary vessels, as distinguished from the smallest arteries and veins. From a strictly physiological point of view, the capillaries should be regarded as commencing at the point where the blood is brought near enough to the tissues, to enable them to separate the elements necessary for their regeneration, and give up the products of their physiological decay. With our present knowledge, it is impossible to assign any limit where the vessels cease to be simple carriers of blood; and it does not seem probable that it will ever be known to what part of the vascular system the processes of nutrition are exclusively confined. The divisions of the blood-vessels must be, to a certain extent, arbitrarily defined, and we șhould feel at liberty to adopt the views of any reliable observer with regard to the kind of vessels which are to be considered as capillaries. The most simple, and what seems to be the most phys- 
iological view, is that the capillaries are the vessels which have but a single, homogeneous tunic; for in these the blood is brought in closest proximity to the tissues. Vessels which are provided, in addition, with a muscular, or muscular and fibrous coats, are to be regarded as either small arteries, or venous radicles. This view is favored by the character of the currents of blood, as seen in microscopic observations on the circulation in transparent parts. Here an impulse is observed with each contraction of the heart, until we come to vessels which have but a single coat, and are so narrow as to allow the passage of but a single line of blood-corpuscles.

Physiological Anatomy of the Capillaries.-If the arteries be followed out to their minutest ramifications, they will be found progressively diminishing in size as they branch, and their coats, especially the muscular, becoming thinner and thinner, until at last they present an internal structureless coat, provided with oval longitudinal nuclei; a middle coat formed of but a single layer of circular muscular fibres, the oval nuclei of which are at. right angles to the nuclei of the internal coat; and an external coat composed of a very thin layer of longitudinal fibres of the white inelastic tissue. Robin calls these the third variety of capillary vessels; but they are large, $\frac{1}{400}$ to $\frac{1}{200}$ of an inch in diameter, become smaller as they branch, and - undoubtedly possess the property of contractility, which is particularly marked in the arterial system. Following the course of the vessels, when they are reduced in size to about $\frac{1}{800}$ of an inch, the external fibrous coat is lost, and the vessel then presents only the internal structureless coat, and the single layer of muscular fibres. These are called by Robin, capillaries of the second variety. They become smaller as they branch, and finally lose the muscular coat, and have then but the single amorphous tunic, with its longitudinal nuclei. These, the capillaries of the first variety of Robin, ${ }^{1}$ we shall consider as the true capillary ressels.

${ }^{1}$ Dictionnaire de Médecine, etc., Paris, 1858 (C'apillaire). This dirision of the 
The true capillary vessels present the following characteristics :

1. Simplicity of Structure.-They have but the single amorphous coat, from $\frac{251}{2500}$ to $\frac{1}{12500}$ of an inch thick, the continuation of the lining membrane of the larger vessels; not provided with an epithelial lining, but presenting, imbedded in its thickness; a number of oval nuclei with their long diameters in the direction of the axis of the vessel.

2. Small Diameter of the Vessels.-Their diameter is generally as small or smaller than that of the blood-corpuscles; so that these bodies always move in a single-line, and must become deformed in passing through the smallest vessels; recovering their natural shape, howerer, when they pass into vessels of larger size. The capillaries are smallest in the nervous and muscular tissue, retina, and patches of Peyer, where they have a diameter of from $\frac{1}{6000}$ to $\frac{1}{4000}$ of an inch. In the mucous layer of the skin, and in the mucous membranes, they are from $\frac{1}{4000}$ to $\frac{1}{2400}$ of an inch in diameter. They are largest in the glands and bones, where they are from $\frac{1}{3000}$ to $\frac{1}{2000}$ of an inch in diameter. ${ }^{1}$ : These measurements indicate the size of the vessel, and not its caliber. Taking out the thickness of their walls, it is only the very largest of them which will admit of the passage of a blood-disk without a change in its form.

3. Peculiarities of Distribution.-Unlike the arteries, which grow smaller as they branch, and simply carry blood by the shortest course to the parts, and the veins, which become larger as we follow the course of the blood by union with each other, the capillaries form a true plexus of vessels of nearly uniform diameter, branching and inosculating in

eapillaries into thrce varicties, the first with a homogeneous coat, the second with the addition of the muscular coat, and the third with the muscular and fibrous coat, was made by Henle, and is, perhaps, the one most generally adopted. Kölliker gives the division we have adopted, regarding as true capillaries only those ressels which have a single coat. The others he ealls "vessels of transition."

'KöLliker, MFanual of Human Microscopic Anatomy, London, 1860, p. 500. 
every direction, distributing blood to the parts, as their physiological necessities demand. This inosculation is peculiar to these vessels, and the plexus is rich in the tissues, as a general rule, in proportion to the activity of their nutrition. Though their arrangement presents certain differences in different organs, the capillary vessels have everywhere the same general characteristics, the most prominent of which are uniform diameter and absence of any positive direction.

The network thus formed is very rich in the substance of the glands, and in the organs of absorption; but the vessels are only disteuded with blood during the physiological activity of these parts. In the lungs the meshes are particularly close. In other parts the vessels are not so abundant, presenting great variations in different tissues. In the muscles and nerves, in which nutrition is very active, the supply is much more abundant than in other parts, like fibro-serous membranes, tendons, etc., whose functions are rather passive." In none of the tissues do we find capillaries penetrating the anatomical elements, as the ultimate muscular or nerrous fibres. Some tissues receive no blood, at least they contain no vessels which are capable of carrying red blood, and are nourished by imbibition of the nutrient plasma of the circulating fluid. Examples of these, which are called extra-vascular, aré cartilage, nails, hair, etc.

The foregoing -anatomical sketch gives an idea of how near the blood is brought to the tissues in the capillary system, and how, once conveyed there by the arteries, and the supply regulated by the action of the muscular coat of the smaller vessels, the blood is distributed for the purposes of nutrition, secretion, absorption, exhalation, or whatever func-

1 The arrangement of the capillaries in different tissues and organs has generally been ascertained by minute injections. In studying injected preparations, however, it must be borne in mind that when injected, the elastic and yielding vessels are distended to their extreme capacity, and the capillaries, therefore, occupy a space much greater than is natural. In injections of the liver, for example, the capillaries seem to constitute the bulk of the organ, and we are at a loss to understand how the cells, ducts, etc, find place between their meshes. 
tion the part has to perform. This will be still more apparent when we come to consider the course of the blood in the capillaries, and the immense capacity of this system, as compared with the arteries or veins.

The capacity of the capillary system is immense. It is only necessary to consider the prodigious vascularity of the skin, mucous membranes, or muscles, to realize this fact. In injections of these parts, it seems, on microscopic examination, as though they contained nothing but capillaries. In preparations of this kind, the elastic and yielding coats of the capillaries are distended to their utmost limit. Under some circumstances, in health, they are much distended with blood, as the mucous lining of the alimentary canal during digestion, the whole surface presenting a vivid red color, indicating the great richness of the capillary plexus. Various estimates of the capacity of the capillary, as compared with the arterial system, have been made, but they are simply approximative, and there seems to be no means by which an estimate, with any pretentions to accuracy, can be formed. The various estimates which are given are founded upon calculations from microscopic examinations of the rapidity of the capillary circulation, as compared with the arteries. In this way Donders estimates the entire capacity of the capillary system as 500 , and Vierordt as 800 times that of the arterial system. It must be evident to any one who has witnessed the capillary circulation under the microscope, that the conditions under which the animal under examination is placed are liable to interfere with the current of blood; and the periodical congestion of certain parts, the fugitive flushes of the skin, the condition of the smallest arteries induced by changes of temperature, exercise, etc., make it evident that the current of blood is liable to great variations. It is impossible to strictly apply to the capillary circulation in the various parts of the human subject, observations on the wing of a bat, or the mesentery of a cat. We must consider, then, 
these estimates as mere suppositions; and they are given for what they are worth.

With the older physiologists, the contractility of the capillaries was a subject of discussion. Some went so far as to suppose that these little ressels were the seat of rhythmical contractions which materially assisted the flow of the blood. In microscopic examinations, irritation or stimulation is seen to produce contraction of the smallest arteries; but there is no evidence that the capillaries, which have a single amorphous coat, have any such property. They undergo, while under observation, considerable alterations in caliber; but this is due, in all probability, to differences in the pressure of blood in their interior. The capillaries can only be considered as endowed with elasticity, which enables them to react upon their contents, when there is any diminution in pressure. In the vascular system, contractility disappears with the muscular fibre-cells which form the middle coat of the arterioles.

\section{Course of the Blood in the Capillaries.}

The phenomena of the capillary circulation are only observable with the aid of the microscope. It was not granted to the discoverer of the circulation to see the blood moving through the capillaries, and he never knew the exact mode of communication-between the arteries and veins. After it was pretty generally acknowledged that the blood did pass from the arteries to the veins, it was disputed whether it passed in an intermediate system of vessels, or became diffused in the substance of the tissues, like a river flowing between numberless little islands, to be collected by the venous radicles and conveyed to the heart. Accurate microscopic investigations have now demonstrated the existence, and given us a clear idea of the anatomy, of the intermediate vessels. In 1661, the celebrated anatomist, Malpighi, first saw the movement of the blood in the capillaries, in the lungs of a frog. Since that time, physiologists have studied 
the circulation in various transparent parts in the inferior animals, as the web of the frog's foot, the tongue of the frog, the lungs of the frog and of the water-newt, the mesentery of very young rats or mice, the wing of the bat, etc. The most convenient situation is the tongue or the web of the frog. Here may be studied, not only the movement of the blood in the true capillaries, but the circulation in the smallest arteries and veins; the variations in caliber of these vessels, especially the arterioles, by the action of their muscular tunic; and indeed the action of ressels of considerable size. This has been a most valuable means of studying the circulation in the capillaries, as contrasted with the small arteries and veins; the only one, indeed, which could give us any definite idea of the action of these vessels.

Before taking up the causes of the eapillary circulation, and the various physical or vital laws which are involved, we will deseribe the phenomena which are observed with the aid of the microscope.

\section{Phenomena of the Capillary Circulation.-The magnifi-} cent spectacle of the capillary circulation, first observed by Malpighi, in the lungs, and afterwards by Leeuwenhoek, Spallanzani, Haller, Cowper, and others, in other parts, has ever since been the delight of the physiologist. We see the great arterial rivers, in which the blood flows with wonderful rapidity, branching and subdividing, until the blood is brought to the superb network of fine capillaries, where the corpuscles dart along one by one; the fluid being then collected by the veins, and carried in great currents to the heart. This exhibition, to the student of Nature, is of inexpressible grandeur; and our admiration is not diminished when we come to study the phenomena in detail. We find here a subject as interesting as was the action of the heart when first seen by Harvey, involving some of the most important phenomena of the circulation. It can be seen how the arterioles regulate the supply of blood to the tissues; how the blood 
distributes itself by the capillaries; and finally, having performed its office, how it is collected and carried off by the veins. ${ }^{1}$

In studying the circulation under the microscope, the anatomical division of the blood into corpuscles and a clear plasma is observed. This is peculiarly evident in cold-blooded animals, the corpuscles being comparatively large, and floating in a plasma which forms a distinct layer next the walls of the vessel. The white corpuscles, which are much fewer than the red, are generally found in the layer of plasma.

In vessels of considerable size, as well as the capillaries, the corpuscles, occupying the central portion, move with much greater rapidity than the rest of the blood, leaving a layer of clear plasma at the sides, which is nearly immovable. This curious phenomenon is in obedience to a physical law regulating the passage of liquids through capillary tubes for which they have an attraction, such as exists, for example, between the blood and the vessels. In tubes reduced to a diameter approximating to that of the capillaries, the attractive force exerted by their walls upon a liquid, causing it to enter

${ }^{1}$ Various methods of preparing the animal for examination have been employed. The one we have found most convenient, in examining the circulation in the frog, is to break up the medulla with a needle, an operation which does not interfere with the circulation, and attach the animal by pins to a thin piece of cork, stretching the web over an orifice in the cork, to allow the passage of light, and securing it with pins through the toes. The membrane is then moistened with water, and covered with thin glass, and if the general surface be kept moist, the circulation may be studied for hours. (See "Phenomena of the Capillary Circulation," an inaugural thesis, by the author, American Journal of the Medical Scienccs, July, 1857.) By gently inflating the lungs with a small blow-pipe, securing them by a ligature passed around the larynx beneath the mucous membrane, and opening the chest, the circulation may be examined in this situation. It may be studied in the tongue (which presents a magnificent vicw of the circulation as well as the nerves and muscular fibres) by drawing it out of the mouth, and spreading it into a thin sheet, securing it with pins. The circulation may be studied in the mesentery of a small warm-blooded animal, like the mouse, by fixing it upon the frog-plate, opening the abdomen, and drawing out the membrane; but not as well or as conveniently as in the tongue or web of the frog. 
the tube to a certain distance, called capillary attraction, becomes an obstacle to the passage of fluid in obedience to pressure. Of course, as the diameter of the tube is reduced, this force becomes relatively increased, for a larger proportion of the liquid contents is bronght in contact with it. When we come to the smallest arteries and veins, and still more the capillaries, the capillary attraction is sufficient to produce the immovable layer, called the "still layer" by many physiologists, and the liquid only moves in the central portion. The plasma occupies the position next the walls of the vessels, for it is this portion of the blood which is capable of wetting the tubes. The transparent layer was observed by Malpighi, Haller, and all who have described the capillary circulation. Poiseuille recognized its true relation to the blood-current, and explained the phenomenon of the still layer by physical laws, which had been previously established with regard to the flow of liquids in tubes of the diameter of from $\frac{1}{25}$ to $\frac{1}{8}$ of an inch, but which he had succeeded in applying to tubes of the diameter of the capillaries. ${ }^{2}$

A red corpuscle occasionally becomes involved in the still layer, when it moves slowly, turning over and over, or even remains stationary for a time, until it is taken up again and carried along with the central current. A few white corpuscles are constantly seen in this layer. They move along slowly, and apparently have a tendency to adhere to the walls of the vessel. This is due to the adhesive character of the surface of the white corpuscles as compared with the red, which can easily be observed in examining a drop of blood between glass surfaces, the red corpuscles moving about with great facility, while the white have a tendency to adhere.

Great differences exist in the character of the flow of blood in the three varieties of vessels which are under observation. In the arterioles, which may be distinguished from the capillaries by their size and the presence of the muscular

${ }^{1}$ Poiseoille, Recherches sur les Causes du Mrouvement ảu Sang dans les Vaisseaux Capillaires, p. 144 ct seq. 
and fibrous coats, the movement is distinctly remittent, even in their most minute ramifications. The blood moves in them with much greater rapidity than in either the capillaries or veins. They become smaller as they branch, and carry the blood always in the direction of the capillaries. The veins, which are relatively larger than the arteries, carry the blood more slowly, and in a continuous stream, from the capillaries toward the heart. In both these vessels the current is frequently so rapid, that the form of the corpuscles cannot be distinguished. Only a portion of the white corpuscles occupy the still layer, the rest being carried on in the central current.

The circulation in the true capillaries is sui generis. Here the blood is distributed in every direction, in vessels of nearly uniform diameter. The vessels are generally so small as to admit but a single row of corpuscles, which move almost like beings endowed with volition. In a single vessel, a line of corpuscles may be seen moving in one direction at one moment, and a few moments after taking a directly opposite course. Spallanzani, in one of his observations, describes the following phenomenon. Two single rows of corpuscles, passing in two capillary vessels of equal size, were directed toward a third capillary vessel, formed by the union of the two others, which would itself admit but a single corpuscle. The corpuscles in one of these vessels seemed to hold back until those from the other had passed in, when they followed in their turn. ${ }^{2}$ When the circulation is natural, the movement in the capillaries is always quite slow compared with the movement in the arterioles, and is continuous. Here, at last, the impulse of the heart is lost. The corpuscles do not neces sarily circulate in all the capillaries which are in the field of view. Certain vessels may not receive a corpuscle for some time, but after a while one or two corpuscles become engaged in them, and a current is finally established. Many interesting little points are noticed in examining the circulation

${ }^{1}$ Spallanzani, Expériences sur la Circulation Paris, 1808, p. 177. 
for a length of time. A corpuscle is frequently seen caught at the angle where a vessel divides into two, remaining fixed for a time, distorted and bent by the force of the current. It soon becomes released, and, as it enters the vessel, regains its original form. In some of the vessels of smallest size, the corpuscles are slightly deformed as they pass through.

The scene is changed with every different part which is examined. In the tongue, in addition to the arterioles and venules, with the rich network of capillaries, dark-bordered nerve-fibres, striated muscular fibres, and parement epithelium can be distinguished. In the lungs, the view is very beautiful. Large, polygonal air-cells are observed, bounded by capillary vessels, in which the corpuscles move with extreme rapidity. It has been observed that the larger vessels are crowded to their utmost capacity with corpuscles, leaving no still layer next the walls, such as is seen in the circulation in other situations.

When the circulation has been for a long time under observation, as the animal becomes enfeebled, very interesting changes in the character of the flow of blood take place. The continuous stream in the smallest vessels diminishes in rapidity, and after a while, when the contractions of the heart have become infrequent and feeble, the blood is nearly arrested, even in the smallest capillaries, during the intervals of the heart's action, and the current becomes remittent. As the central organ becomes more and more enfeebled, the circulation becomes intermittent: the blood receiving an impulse from each contraction, but remaining stationary during the intervals. At this time, the corpuscles cease to occupy exclusirely the central portion of the vessels, and the clear layer of plasma next their, walls, which was observed in the normal circulation, is no longer apparent. Following this, there is actual oscillation in the capillaries. At each contraction of the heart, the blood is forced onwards a little distance, but almost immediately returns to about its former position. This phenomenon has long been observed, 
and is explained in the following way: As the heart has become enfeebled, the contractions are so infrequent and ineffectual, that during their intervals the constant flow in the capillaries is entirely arrested ; for the arterial pressure, which is its immediate cause, and which is maintained by the successive charges of blood sent into the arteries at each ventricular systole, is lost. But as the blood is contained in a connected system of closed tubes, the feeble impulse of the heart is propagated through the vessels and produces a slight impulse, even in the smallest capillaries, which dilates them and forces the fluid a little distance. As soon, however, as the heart ceases to contract, the current is arrested, and the blood, meeting with a certain amount of obstruction from the fluid in the small veins, which are still further removed from the heart, is made to return to its former position.

This phenomenon continues for a short time only, for the heart soon loses its contractility, and the circulation in all the vessels is permanently arrested.

Rapidity of the Capillary Circulation.-The circulation in the capillaries of a part is subject to such great variations, and the differences in different situations are so considerable, that it is impossible to give any definite rate which will represent the rapidity of the capillary circulation. It is for this reason that it has been found impracticable to estimate the eapacity of the capillary, as compared with the arterial, system. The rapidity of the flow of blood is by no means as great as it appears in microscopic examinations; being, of course, exaggerated in proportion to the magnifying power employed. It is, nevertheless, to microscopic investigations that we are indebted for the scanty information we possess on this subject. The estimates which have been made by varions observers refer generally to cold-blooded animals, and have been arrived at by simply calculating the time occupied by a blood-corpuscle in passing over a certain distance. Hales, who was the first to investigate this question, estimated that 
in the frog a corpuscle moved at the rate of an inch in ninety seconds. ${ }^{1}$ The estimates of Weber and Valentin are considerably higher, being about $\frac{x}{50}$ of an inch per second. Volkmann calculated the rapidity in the mesentery of the dog, which would approximate more nearly to the human subject, and found it to be about $\frac{1}{30}$ of an inch per second. ${ }^{3}$ Vierordt made a number of curious observations upon himself, by which he professed to be able to estimate the rapidity of the circulation in the little vessels of the eye. He states that when the eye is fatigued, and sometimes when the nervous system is disordered, compression of the globe in a certain way will enable one to see a current like that in a capillary plexus. This he believes to be the capillary circulation, and by certain calculations he formed an estimate of its rapidity, putting it at from $\frac{1}{40}$ to $\frac{1}{28}$ of an inch. The latter figure accords pretty nearly with the observations of Volkmann upon the dog. ${ }^{3}$ How far these observations are to be relied upon it is impossible to say. Certainly no great importance would be attached to them if they did not, in their results, approximate to the estimates of Volkmann, which probably represent, more nearly than any, the rapidity in the capillaries of the human subject.

After what has been said of the variations in the capillary circulation, it is evident that the foregoing estimates are by no means to be considered exact.

\section{Relations of the Capillary Circulation to Respiration.-} In treating of the influence of respiration upon the action of the heart, the arterial pressure, pulse, etc., it has already been stated that non-aërated blood cannot circulate freely in the capillaries. Various ideas with regard to the effects of asphyxia upon the circulation have been advanced, which will be again discussed in connection with respiration. The

${ }^{1}$ Statical Essays, containing Homastaticks, London, 1733, p. 68.

${ }^{2}$ Minne-Edwards, Legons sur la Physiologie, Paris, 1859, tome iv., p. 286.

Ibid. 
fact is evident, that arrest of respiration produces arrest of circulation. This is ordinarily attributed to an impediment to the passage of blood through the lungs, when they no longer contain the proper quantity of oxygen. This view is entirely theoretical, and has been disproved by experiments dating more than half a century ago. In 1789, Goodwyn advanced the theory that, in asphyxia, the blood passes through the lungs, but is incapable of exciting contractions in the left ventricle." Bichat, in his celebrated essay "Sur la Vie et la Mort," 1805, proved by experiment that black blood passes through the lungs in asphyxia, and is found in the arteries. His theory was that non-aërated blood, cireulating in the capillaries of the nervous centres, arrests their function, thus acting indirectly upon the circulation; and that finally the heart itself is paralyzed by the circulation of black blood in its substance.

Dr. John Reid, in an article "On the Cessation of the Vital Actions in Asphyxia," 2 describes an experiment in which a hemodynamometer applied to the femoral artery of a dog indicated increase in the arterial pressure during the first moments of asphyxia, followed finally by a depression in the mercury. He found a corresponding diminution in the pressure in the vein of the opposite side. "This was so unlooked for-at first sight so inexplicable, and so much at variance with my preconceived notions on the subject," says the author, "that I was strongly inclined to believe there must be some source of fallacy; but after repeating the experiment more than twenty times, and invariably with the same results, I was at last compelled to admit its accuracy." This he surmises is due to "an impediment to the passage of the venous blood through the capillaries of the systemic circulation." In his conclusions at the end of the article, how-

I P. Bérard, Cours de Physiologie fait à la Faculté de Médccine de Paris, 1851, tome iii., p. 444.

2 Joun Reid, M. D., Physiological, Anatomical, and Pathological Researches, Edinburgh, 1848, p. 26. (Article extracted from the Edinburgh Mfectical and Surgical Journal, April, 1841.) 
ever, he takes no account of the results of this experiment, which point conclusively to arrest of blood in the capillary system, and the conclusions with regard to the effect of asphyxia upon the circulation are substantially those of Bichat.

The immediate effects of asphyxia upon the circulation are referable to the general capillary system. This fact was demonstrated by experiments on the frog published in $1857 .{ }^{2}$ In these experiments, the medulla oblongata was broken up, and the web of the foot submitted to microscopic examination. This operation does not interfere with the circulation, which may be observed for hours without difficulty. The cutaneous surface was then coated with collodion, care only being taken to aroid the web under observation. The effect on the circulation was immediate. It instantly became less rapid, until, at the expiration of twenty minutes, it had entirely ceased. The entire coating of collodion was then instantly peeled off. Quite a rapid circulation immediately commenced, but it soon began to decline, and in twenty minutes had almost ceased. In another observation, the coating of collodion was applied without destroying the medulla. The circulation was affected in the same manner as before, and ceased in twenty-five minutes.

These experiments, taken in connection with observations on the influence of asphyxia upon the arterial pressure, conclusively show that non-aërated blood cannot circulate freely in the systemic capillaries. ${ }^{2}$ Venous blood, however, can be forced through them with a syringe, and even in asphyxia it filters slowly through, and if air be admitted to the lungs before the heart has lost its contractility, the circulation is restored.

No differences in the capillary circulation have been no-

1 See article by the author, entitled "Phenomena of the Capillary Circulation," American Journal of the Medical Sciences, July, 1857.

2 In these experiments, ether had previously been freely applied to the surface to render it certain that the effects on the circulation were not the result of this ingredient of the collodion. 
ticed accompanying the ordinary acts of inspiration and expiration.

Causes of the Capillary Circulation.-The contractions of the left ventricle are evidently capable of giving an impulse to the blood in the smallest arterioles, for a marked acceleration of the current accompanying each systole can be distinguished in all but the true capillaries. It has also been shown by experiments after death, that blood can be forced through the capillary system and returned by the veins by a force less than that exerted by the heart. This, however, cannot rigidly be applied to the natural circulation, as the smallest arteries are endowed during life with contractility, which is capable of modifying the blood current. ${ }^{1}$ Dr. Sharpey adapted a syringe, with a hemodynamometer attached, to the aorta of a dog just killed, and found that fresh defibrinated blood could be made to pass through the double capillary systems of the intestines and liver, by a pressure of three and a half inches of mercury. It spurted out at the vein in a full jet under a pressure of five inches. In this observation, the aorta was tied just above the renal arteries. The same pressure, the ligature being removed, forced the blood through the capillaries of the inferior extremities. ${ }^{2}$ This is much less than the arterial pressure, which is equal to from five and a half to six inches of mercury.

It is thus seen that the pressure in the arteries which forces the blood toward the capillaries is competent, unless opposed by excessive contraction of the arterioles, not only to cause the blood to circulate in these vessels, but to return it to the heart by the veins. This fact is so evident, that it is un-

${ }^{1}$ As showing the difference between the ressels immediately after death, and after they have lost all their vital properties, we may refer to an observation of Bérard (op. cit., p. 776), in which he found it impossible to inject, with a solidifiable fluid, parts of the body immediately after amputation. Water passed with facility, but alcohol or vinegar could not be forced through.

2 Tond and Bowmax, The Physiological Anatomy and Physiology of Man, Philadelphia, 1857 , p. 678. 
necessary to discuss the views of Bichat, and some others, who supposed that the action of the heart had no effect upon the capillary circulation. It must be admitted that this is its prime cause; and the only questions to be considered are, first, whether there be any reason why the force of the heart should not operate on the blood in the capillaries, and second, whether there be any force in these vessels which is superadded to the action of the heart.

The first of these questions is answered by microscopic observations on the circulation. A distinct impulse, following each ventricular systole, is observed in the smallest arteries. The blood flows from them directly and freely into the capillaries; and there is not the slightest ground for the supposition that the force is not propagated to this system of vessels.

Various writers have supposed the existence of a " capillary power," which they have regarded as of greater or less importance in producing the capillary circulation. The views of some are purely theoretical, but others base their opinion on microscopic observations. These views do not demand an extended discussion. There is a force in operation, the action of the heart, which is capable of producing the capillary circulation; and there is nothing in the phenomena of the circulation in these vessels, which is inconsistent with its full operation. Under these circumstances, it is unphilosophical to invoke the aid of the currents produced in capillary tubes in which liquids of different characters are brought in contact, or a "capillary power" dependent upon a vital nutritive attraction between the tissues and the blood, unless we do it on the basis of phenomena observed in the capillaries when the action of the heart is suppressed. When the heart ceases its action, movements in the capillaries are sometimes due to the contractions of the arteries, a property which has already been fully considercd. Movements which have been observed in membranes detached from the body are due to the mere emptying of the 
divided vessels or simple gravitation. It must be remembered that in microscopic examinations, the movements which are observed are immensely exaggerated by the magnifying power, and we receive, at first sight, an erroneous idea of their rapidity. The morements of the blood in detached membranes, due merely to gravity, have been so satisfactorily explained by the experiments of Poiseuille, that it is deemed unnecessary to refer to the observations of those who have attributed this phenomenon to other causes. ${ }^{2}$

Dr. Dowler, of New Orleans, made some experiments on the circulation in patients dead with yellow fever, in which he found that the blood would flow in a tolerably full stream from a punctured vein a few minutes after death. This he attributes to an independent action of the capillaries, which continues for a time after the action of the heart has ceased." These observations are met by the following experiment performed years before by Magendie. ${ }^{3} \quad$ A ligature was passed around the thigh of a dog, leaving only the crural artery and vein. A ligature was then applied to the vein, and a small opening made below it in the vessel, from which the blood escaped in a jet. On compressing the artery, the flow of blood was not immediately ariested in the vein, but continued to gradually diminish in force until it stopped after a few moments. On examining the artery below the point of compression, it was found contracted, and completely emptied of blood, while the vein was full below the puncture. The pressure being removed from the artery, the blood commenced to flow from the vein, and a jet was soon established as before. When the artery was slightly compressed, so as to allow the passage of a small quantity of blood, not enough to distend the vessel, the blood flowed from the vein, but no longer in a jet. This experiment shows that when

${ }^{1}$ Porsedille, Recherches sur les Causes du Mouvement du Sang dans les Vaisseaux Capillaires, 1835, p. 127.

${ }^{2}$ Doxglison, Human Physiology, Philadelphia, 1851, vol. i., p. 420.

${ }^{3}$ Magendie, Précis Élémentaire de Physiologie, Paris, 1836, tome ii., p. 390. 
an artery supplying a part with blood is removed from the influence of the heart, the vessel will contract and force its contents into the vein. This affords the most rational explanation of the phenomena observed by Dr. Dowler. When the blood is allowed to enter slowly, so as not to distend the vessel, though it be supplied to the capillary system, it does not there undergo any propelling influence, competent, at any time, to increase the rapidity of the flow from the vein.

Physiologists who, like Bichat,' have been unable to explain the local variations in the capillary circulation without the intervention of a force resident in these vessels or the surrounding tissues, have not appreciated the action of the arterioles. These little vessels are endowed to an eminent degree with contractility and, by the contractions and relaxations of their muscular walls, regulate the supply of blood to the capillaries of individual parts. Their action is competent to produce all the variations which are observed in the capillary circulation.

It is evident, then, that the arterial pressure, which is itself derived from the action of the heart, is competent to produce the circulation of the blood, as we observe it, with all its variations, in the capillary vessels; that there is no evidence of the intervention of any other force, but, on the contrary, microscopic observations and experiments on the arteries and veins, thus far, show that there is no other force in operation. ${ }^{2}$

Loc. cit.

${ }^{2}$ It has been asserted that there is a circulation of the blood in the area vasculosa, the first blood-vessels that are developed, before the heart is formed; but there are no definite and reliable observations which show that there is any regular movement of the blood, which can be likened to the circulation as it is observed after the development of the heart, anterior to the appearance of a contractile central organ. Another example of what is supposed to be circulation without the intervention of the heart is in cases of acardiac foetuses. Monsters without a heart, which have undergone considerable development and which present systems of arteries, capillaries, and veins, have been described. All of these, however, are accompanied by a twin, in which the development of the circulatory system is 


\section{Influence of Temperature on the Capillary Circulation.-} Within moderate limits, a low temperature, induced by local applications, has been found to diminish the quantity of blood sent to the capillaries, and retard the circulation; while a high temperature increases the supply of blood and accelerates its current. The mechanism of this is beautifully shown by the experiments of Poiseuille. This observer found that when a piece of ice was applied to the web of a frog's foot, the mesentery of a small warm-blooded animal, or any part in which the capillary circulation can be observed, the quantity of corpuscles circulating in the arterioles became very much diminished, "those which carried two or three rows of corpuscles giving passage to but a single row." The circulation in the capillaries first became slower, and then entirely ceased in parts. On removing the ice, in a very few minutẹs the circulation regained its former characters.

If, on the other hand, the part be covered with water at $104^{\circ}$, the rapidity of the current in the capillaries is so much

perfect. The most remarkable ease of this kind is one reported by Dr. Houston in the Dublin Journal of Medical Science (1836, vol, x., p. 204). In this ease there was a perfect twin, but two distinct cords and sets of membranes. Dr. Houston supposed that the circulation in this monster was carried on by "capillary power" alone. In these cases, as has been shown by Astley Cooper and Lallemand (Edinburgh Medical and Surgical Journal, 1844, vol. 1xii., p. 156 et $\varepsilon e q$.$) , there is a free anastomosis of the vessels of the two foetuses in the placenta.$ Some have supposed, from the fact that the veins of the monster are not provided with valves, that in it the circulation is from the veins to the arteries, or is inverted. It is not exactly elear how the circulation is carried on in an acardiac foetus. Undoubtedly the heart of one child may influence the circulation in the umbilical vessels of the other, in cases of twins; for Lallemand has observed (loc. cit.), after the birth of one child, the cord having been divided, a regular pulsatile flow from the placental extremity of the cord, as from a divided artery; but we find on careful examination of the case reported by Dr. Houston, and an article on the case by Dr. G. Calvert Holland (Edinburgh Med. and Surg. Journal, loc. cit.), no sufficient evidence that the circulation was carried on by any "capillary power." Not being able to regard as facts these grounds, on which some have based their belief in the existence of a force in the circulation which is independent of the heart's action, we have abstained from their discussion in treating of the causes of the capillary circulation. 
increased, that we can hardly distinguish the form of the corpuscles. ${ }^{1}$

Influence of Direct Irritation upon the Capillary Circulation.-Experimental researches on the effects of direct irritation of the capillaries, in parts where the circulation can be observed microscopically, have been quite numerous since Thompson studied the effects of saline solutions on the web of the frog's foot in 1813. ${ }^{2}$ The most noticeable papers on this subject are those of Dr. Wilson Philip ${ }^{3}$ and Mr. Wharton Jones." The latter paper, which received the Astley Cooper prize for 1850, is based on very extended and carefully conducted observations, in which the author, by means of various irritants, succeeded in producing very curious and interesting phenomena, which he regarded as inflammatory. It is not our object to discuss the nature of inflammation, or to treat of the changes in the character of the capillary circulation which are supposed to attend this condition, as this subject is eminently pathological ; but it must be remembered, in considering the effects of direct irritation on the capillary circulation, that the phenomena thus observed in coldblooded animals cannot be taken as absolutely representing the characters of inflammation in the human subject. When an irritation is applied to a transparent part, the phenomena observed may be due to many causes, as the direct effects upon the contractile elements of the blood-vessels, the reflex action through the nervous system, and the direct influence of the application upon the constitution of the blood. Saline or other fluids are competent to modify, to a very considerable extent, the composition of the blood, separated from it only by the thin, permeable walls of the vessels; and the

${ }^{1}$ Poisedille, op. 'cit., p. 158 et seq.

2 Thospsos, Lectures on Inflammation, Edinburgh, 1813.

Medico-Chirurgical Transactions, 1823, vol. xii.

4 Guy's Hospital Reports, vol. vii., 1851, On the State of the Blood and the Blood-vessels in Inflammation, ascertained by Expcriments, Injections, and Obser. vations by the Microscope, by T. Wharton Jones, F. R. S. 
phenomena which follow their application are necessarily very complex. The process of iuflammation is by no means completely understood, but it is pretty generally acknowledged to be a modification of nutrition, in a way that we are as yet ignorant of. We are hardly prepared to admit that this modification, whatever it may be, can be induced under our very eyes, simply by the application of irritants. With these views, microscopic researches on the "state of the blood and blood-vessels in inflammation" do not assume the importance which is attributed to them by many authors.

Keeping this in mind, we may state the following as a summary of the phenomena which have been observed in the capillary circulation, as the result of irritation applied to transparent parts :

The application of the irritant is immediately followed by constriction of the arterioles, and diminution in the rapidity of the current in them as well as in the capillaries.

This constriction of the vessels is but momentary, if a powerful irritant, like a very strong solution of salt, be used. It is followed by a dilatation of the ressels, and an increase in the rapidity of the circulation.

Soon after the vessels have become dilated, the rapidity of the circulation is progressively diminished, until oscillation of the blood in the vessels takes place, which occurs when the circulation is about to cease. This oscillation finally gives place to complete stagnation. The vessels become crowded with blood, so that the transparent layer next their walls is no longer observed. In this condition, it has been often noticed that the proportion of colorless corpuscles is increased.

Following the contraction and subsequent dilatation of the vessels, there is stasis, and engorgement of the parts which have been exposed to irritation. If the irritation be discontinued, this condition is gradually relieved, and the blood resumes its normal current.

In inflammation, as it is observed in the conjunctiva and 
other vascular parts, there is unquestionably congestion of the vessels; but there is no positive evidence of stagnation of blood in the parts as a constant occurrence. The circulation seems, indeed, to be more active than in health. With regard to the microscopic phenomena just mentioned, the contraction of the arterioles is simply the effect of a stimulus upon their muscular coats; and dilatation takes place probably in consequence of the excessive contraction, for it has been shown that this condition of the muscular fibres is pretty constantly followed by unusual relaxation. It has never yet been determined how far the stasis of the blood is due to an osmotic action of solutions employed in observations of this kind. 


\title{
CHAPTER VIII.
}

\author{
CIRCULATION OF THE BLOOD IN THE VEINS.
}

Physiological anatomy of the veins-Strength of the coats of the veins-Valves of the veins-Course of the blood in the veins-Pressure of blood in the veins-Rapidity of the venous circulation-Causes of the venous circulationInfluence of muscular contraction-Air in the veins-Function of the valvesVenous anastomoses-Conditions which impede the venous circulation-Regergitant venous pulse.

Physiological Anatomy of the Veins.-The blood, distributed to the capillaries of all the tissues and organs by the arteries, is collected from these parts in the veins and carried back to the heart. In studying the anatomy of the capillary system, or in observing the passage of the blood from the capillaries to larger vessels, in parts of the living organism which can be submitted to microscopic examination, it is seen that the capillaries, vessels of nearly uniform diameter and anastomosing in every direction, give origin, so to speak, to a system of vessels, which, by union with others as we follow their course, become larger and larger, and carry the blood away in a uniform current. These are called the venules, or venous radicles. They are the peripheral radicles of the numerous vessels which transport the blood, after it has served the purposes of nutrition or secretion, to the central organ.

The venous system may be considered, in general terms, as divided into two sets of vessels : one, which is deep, and 
situated in proximity to the arteries; and the other, which is superficial, and receives for the most part the blood from the cutaneous surface. The entire eapacity of these ressels, as compared with the arteries, is very great. As a general rule, each vein when fully distended is larger than its adjacent artery. Many arteries are accompanied by two veins, as the arteries of the extremities; while certain of them, like the brachial or spermatic, have more than two. Added to these is the superficial system of veins which have no corresponding arteries. It is true that some arteries have no corresponding veins, but examples of this kind are not sufficiently numerous to diminish, in any marked degree, the great preponderance of the veins, both in number and volume. It is impossible to give an accurate estimate of the extreme capacity of the veins as compared with the arteries; but from the best information we have, it is several times greater. Borelli estimated that the capacity of the veins was, to the eapacity of the arteries, as 4 to 1 ; and Haller, as $2 \frac{1}{4}$ to 1 . The proportion is very variable in different parts of the body. In some situations the capacity of the veins and arteries is about equal; while in others, as in the pia mater, according to the researches of Hirschfeld, the veins will contain six times as much as the arteries. ${ }^{1}$

In attempting to compare the quantity of blood normally circulating in the veins, with that contained in the arteries, such variations in the venous system at different times and in different parts, both in the quantity of blood, rapidity of circulation, pressure, etc., are found, that a definite estimate is impossible. It would be unphilosophical to attempt an approximate comparison, as the variations in the venous circulation constitute one of its greatest and most important physiological peculiarities, which must be fully appreciated in order to form a just idea of the function of the veins.

${ }^{1}$ BÉrard, Cours de Physiologie, Paris, 1855, tome iv., p. 7. The circulation in the erectile tissues will be separately considered, and no account is now taken of the relative capacity of veins and arteries in them. 
The arteries are always full, and their tension is subject to comparatively slight variations. Following the blood into the capillaries, there are the immense variations in the circulation with varying physiological conditions of the parts, which we have already noted. As should naturally be expected, the condition of the veins varies with the changes in the eapillaries, from which the blood is taken. In addition to this, there are independent variations, as in the erectile tissues, in the veins of the alimentary canal during absorption, in veins subject to pressure, etc.

Following the veins in their course, it is observed that anastomoses with each other form the rule and not the exception, as in the arteries. There are always a number of channels by which the blood may be returned from a part ; and if one vessel be obstructed from any cause, the current is simply diverted into another. The veins do not present a true anastomosing plexus, such as exists in the capillary system, but simply all arrangement by which the blood can easily find its way back to the heart, and by which the vessels may accommodate themselves to the immense variations in the quantity of fluid contents to which they are liable. This, with the peculiar valvular arrangement in all but the veins of the cavities, provides against obstruction to the flow of blood through, as well as from, the capillaries, in which it seems essential to the proper nutrition and function of parts, that the quantity and course of the blood should be regulated exclusively through the arterial system. Special allusion to the different venous anastomoses belongs to descriptive anatomy. Physiologically, the communication between the different veins is such, that the blood can always find a way to the heart, and once fairly out of the capillaries, it cannot react and influence the circulation of fresh blood in the tissues.

Collected in this way from all parts of the body, the blood is returned to the right auricle, from the head and upper extremities, by the superior vena cava, from the trunk and 
lower extremities, by the inferior vena cava, and from the substance of the heart, by the coronary veins.

Structure and Properties of the Veins.-The structure of the veins is somewhat more complex and difficult of study than that of the arteries. Their walls, which are always much thinner than the walls of the arteries, may be divided into quite a number of layers; but for convenience of pbysiological description, we shall regard them as presenting three distinct coats. These have properties which are tolerably distinctive, though not as much so as the three coats of the arteries.

The internal coat is a continuation of the single coat of the capillaries and the internal coat of the arteries. It is a simple, homogeneous membrane, somewhat thinner than in the arteries, lined by a delicate layer of epithelium.

The middle coat is divided by some into two layers: an internal layer, which is composed chiefly of longitudinal fibres; and an external layer, in which the fibres have a circular direction. These two layers are intimately adherent, and are quite closely attached to the internal coat. The longitudinal fibres are composed of the white fibrous tissue mingled with a large number of the smallest variety of the elastic fibres. This layer contains a large number of capillary vessels (vasa vasorum). The circular fibres are composed of the elastic tissue, some of the same variety as found in the longitudinal layer, some of medium size, and some in the form of the "fenestrated membrane." In addition, there are white inelastic fibres interlacing in every direction and mingled with capillary blood-vessels, and the unstriped or involuntary muscular fibres, which are always circular in their direction. The muscular fibres are relatively much less numerous than in the arterial system. They are most abundant in the superficial veins.

The external coat is generally composed simply of the white fibrous tissue, like the corresponding coat of the arteries. In the largest veins, particularly those of the abdominal 
cavity, this coat contains a layer of longitudinal unstriped muscular fibres. In the veins near the heart, are found a few striated fibres, which are continued on to the reins from the auricles. In some of the inferior animals, as the turtle, these fibres are quite thick, and pulsation of the veiris in the immediate vicinity of the heart is very marked.

In nearly all veins, the external coat is several times thicker than the internal. This is most marked in the larger veins, in which the middle coat, particularly the layer of muscular fibres, is very slightly developed.

In what are called the venous sinuses, and in the veins which pass through bony tissue, we have only the internal coat, to which are superadded a few longitudinal fibres, the whole closely attached to the surrounding parts. As examples of this, may be mentioned the sinuses of the dura mater, and the veins of the large bones of the skull. In the first instance, there is little more than the internal coat of the vein firmly attached to the surrounding layers of the dura mater. In the second instance, the same thin membrane is adherent to bony canals formed by a layer of compact tissue. The veins are much more closely adherent to the surrounding tissues than the arteries, particularly when they pass between layers of aponeurosis. This fact has been pointed out by Bérard $^{1}$ as very general, and is one to which he attaches considerable physiological importance. He considers that this arrangement serves to keep the veins open and give them additional strength.

The above peculiarities in the anatomy of the veins indicate considerable differences in their properties, as compared with the arteries. When a vein is cut across, its walls fall together, if not supported by adhesions to surrounding tissues, so that its ealiber is nearly or quite obliterated. The yellow elastic tissue, which gives to the larger arteries their great thickness, is very scanty in the veins, and the thin walls collapse when not sustained by liquid in the interior of

${ }^{2}$ Op. cit., tome iv., p. 9. 
the vessels. Whenever the veins remain open after section, it is on account of their attachment to surrounding tissues, and is not due to the walls of the vessels themselves.

Though with much thinner and apparently much weaker walls, the veins, as a rule, will resist a greater pressure than the arteries. Observations on the relative strength of the arteriês and veins were made by Hales, ${ }^{1}$ but the most extended experiments on the subject were made by Clifton Wintringham, in $17400^{2}$ This observer ascertained that the inferior vena cava of a sheep, just above the opening of the renal veins, was ruptured by a pressure of 176 pounds, while the aorta at a corresponding point yielded to a pressure of 158 pounds. The strength of the portal vein was even greater, supporting a pressure of nearly 5 atmospheres, bearing a relation to the vena cava of 6 to 5 ; yet these ressels had hardly one-fifth the thickness of the arteries. In the lower extremities in the human subject, the veins are much thicker and stronger than in other situations, a provision against the increased pressure to which they are liabitually subjected in the upright posture. Wintringham noticed one singular exception to the general rule just given. In the vessels of the glands, and of the spleen, the strength of the arteries was much greater than that of the veins. The splenic vein gave way under a pressure of little more than one atmosphere, while the artery supported a pressure of more than six atmospheres.

A little reflection on the influences to which the venous and arterial circulation are subject will enable us to understand the physiological importance of the great difference in the tenacity of the two varieties of vessels. It is true that in the arterial system the constant pressure is greater than in

${ }^{1}$ Slatical Essays, vol. ii., p. 154 et seq. These observations are not very satisfaetory. In a ease where the strength of the earotid and jugular were compared, in a mare, the carotid sustained the greater pressure; but it is stated that the jugular had been weakened by repeated renesections.

${ }^{2}$ Bérard, op. cit., tome iv., p. 24 et seq. 
the veins; but it is nearly the same in all the ressels, and the immense extent of the outlet into the eapillaries provides against any very great increase in pressure, so long as the blood is in a condition which enables it to pass into the capillaries. The muscular fibres of the left ventricle. have but a limited power, and when the pressure in the arteries is such, as it sometimes is in asphyxia, as to close the aortic valves so firmly that the force of the ventricle will not open them, it cannot be increased. At the same time it is being gradually relieved by the capillaries, through which the blood slowly filters, even when completely unaërated. With the veins it is different. The blood has a comparatively restricted outlet at the heart, and is received by the capillaries from all parts of the system. The vessels are provided with numerous valves, which render a general backward action impossible. Thus, restricted portions of the venous system, from pressure in the vessels, increase of fluid from absorption, accumulation by force of gravity, and other causes, may be subjected to great and sudden variations in pressure. 'The great strength of these vessels enables them ordinarily to suffer these variations without injury; though varicose veins in various parts present examples of the effects of repeated and continued distention.

The veins possess a considerable degree of elasticity, though this property is not as marked as it is in the arteries. If we include between two ligatures a portion of a vein distended with blood, and make a small opening in the vessel, the blood will be ejected with some force, and the vessel becomes very much reduced in ealiber.

It has been proven by direct experiment that the veins are endowed with that peculiar contractility which is eharacteristic of the action of the unstriped muscular fibres. On the application of galvanic or mechanical excitation, they contract slowly and gradually, the contraction being followed by a correspondingly gradual relaxation. There is never any rhythmical or peristaltic movement in the veins, which is 
competent to assist the circulation. ${ }^{1} \quad$ The only regular movements which occur are seen in the vessels in immediate proximity to the right auricle, which are provided with a few fibres similar to those which exist in the walls of the heart.

Nerves, chiefly from the sympathetic system, have been demonstrated in the walls of the larger veins, but have not been followed out to the smaller ramifications.

Valves of the Veins.-The discovery of the valves of the veins has already been alluded to in connection with the history of the discovery of the circulation. They had undoubtedly been observed in various parts of the venous system by Cananius, and found very generally distributed throughout this system by Piccolomini, the last named anatomist having published an account of them in 1586; but Fabricius, the greatest anatomist of his day, had the good fortune to demonstrate them to his illustrious pupil William Harvey, whose immortal discovery indicated their physiological importance. Being ignorant of the observations of his predecessors on this subject, Fabricius announced himself as their discoverer, and is generally so regarded. In all parts of the venous system, except, in general terms, in the abdominal, thoracic, and cerebral cavities, there exist little membranous semilunar folds, resembling the aortic and pulmonic valves of the heart. When distended, the convexities of these valves look toward the periphery. In the great majority of instances the valves exist in pairs, but are occasionally found in groups of three. They are formed of the delicate lining membrane of the veins, with the internal or longitudinal layer of the middle coat. Some transverse fibres are found around the base of the valves, and a few muscular fibres have been

1 This statement applies particularly to the human subject. Schiff has noticed rhythmical contractions of the vcins in the ear of a rabbit (Losget, Traite de Physiologie, Paris, 1861, tome i., p. 876), and Mr. Wharton Jones has observed the same phenomenon in the wing of the bat (Tond and Bowsan, Physiological Anatomy, Am. ed. 1857, p. 703, note). There is no evidence that this is gencral, or that it has any influence in favor of the circulation. 
traced into their folds. There exists, also, a fibrous ring following the line of attachment of the valvular curtains to the vein, which renders the vessel much stronger and less dilatable here than in the spaces between the valves. The valves are by far the most numerous in the veins of the lower extremities. They are generally situated just below the point where a small vein empties into one of larger size, so that the blood, as it passes in, finds an immediate obstacle to passage in the wrong direction. The situation of the valves may be readily observed in any of the superficial veins. If the flow of blood be obstructed, little knots will be formed in the congested vessels, which indicate the position and action of the valves. The simple experiment of Harvey, already referred to, presents a striking illustration of the action of the valves. When the vein is thus congested and knotted, if the finger be pressed along the vessel in the direction of the blood current, a portion situated between two valves may be emptied of blood; but it is impossible to empty any portion of the vessel by pressing the blood in the opposite direction. On slitting open a rein, we observe the shape, attachment, and extreme delicacy of structure of the valves. When the vessel is empty, or when fluid moves toward the heart, they are closely applied to the walls; but if liquid or air be forced in the opposite direction, they project into its caliber, and by the application of their-free edges to each other, effectually prevent any backward current. Fabricius noted the following peculiarity in the arrangement of the valves. When closed, the application of their free edges forms a line which runs across the vessel; it is found that in successive sets of valves these lines are at right angles to each other, so that if in one set, this line has a direction from before backwards, in the sets above and below the lines run from side to side.

There are certain exceptions to the general proposition that the veins of the great cavities are not provided with valves. Valves are found in the portal system of some of the inferior animals, as the horse. They do not exist, how- 
ever, in this situation in the human subject. Generally, in following out the branches of the inferior vena cava, no valves are found until we come to the crural vein; but oceasionally there is a double valve at the origin of the external iliac. In some of the inferior animals, there exists constantly a single valvular fold in the rena cava at the openings of the hepatic, and one at the opening of the renal vein. This is not constant in the human subject. ${ }^{1}$ Valves are found in the spermatic, but not in the ovarian veins. A single valvular fold has been described by Dr. J. H. Brinton, at the opening of the right spermatic into the vena cava. ${ }^{2}$ There are two valves in the azygos vein near its opening into the superior vena cava. There is a single valve at the orifice of the coronary vein. There are no valves at the openings of the brachio-cephalic into the superior vena cava; but there is a strong double valve at the point where the internal jugular opens into the brachio-cephalic. Between this point and the capillaries of the brain, the vessels are entirely deprived of valves, except in very rare instances, when one or two are found in the course of the jugular.

In addition to the double, or more rarely triple, valves which have just been described, there is another variety, found in certain parts, at the point where a tributary vein opens into a main trunk. This consists of a single fold which is attached to the smaller vessel, but projects into the larger. Its action is to prevent regurgitation, by the same mechanism as the ileo-cæcal valve prevents the passage of matter from the large into the small intestine. These valves are much less numerous than the first variety.

${ }^{1}$ Dr. Crisp, of England, has described valves in the splenic veins in some of the inferior animals. In one of the mesentcrie veins of the reindeer, he showed forty-two pairs of valves (New York MYedical Journal, April, 1865, p. 67).

${ }^{2}$ Description of a Valve at the Termination of the Right Spermatic Vein in the Vena Cava, with Remarks on its Relations to Varicocele. By Jonn H. Brinton, M. D. American Journal of the Medical Sciences, July, 1856. The presence of this valve, according to Dr. Brinton, explains the more frequent occurrence of varieocele on the right side. 
The reins form a system which is adapted to the return of blood to the heart in a comparatively slow and unequal current. Distention of certain portions is provided for; and the ressels are so protected with valves, that whatever influences the current must favor its flow in the direction of the heart. It is a system which is calculated to receive the blood from the parts after it has become unfit for nutrition, and pass it in the requisite quantity to the lungs, through the right side of the heart, for regeneration.

Course of the Blood in the Veins.-The experiments of Hales and Sharpey, showing that defibrinated blood can be made to pass from the arteries into the capillaries and out at the veins by a pressure less than that which exists in the arteriai system, and the observations of Magendie upon the circulation in the leg of a living dog, showing that ligation of the artery arrest's the flow in the vein, points which have already been fully discussed in treating of the causes of the capillary circulation, have established, beyond question, the fact that the force exerted by the left ventricle is sufficient to account for the venous circulation. The heart must be considered the prime cause of all movement in these vessels. Regarding this as definitely ascertained, there remain to consider, in the study of the course of the blood in the veins, the character of the current, the influence of the vessels themselves, and the question of the existence of forces which may assist the vis a tergo from the heart, and circumstances which may interfere with the flow of blood.

As a rule, in the normal circulation, the flow of blood in the veins is continuous. The intermittent impulse of the heart, which progressively diminishes as we recede from this organ, but is still felt even in the smallest arteries, is lost, as we have seen, in the capillaries. Here, for the first time, the blood mores in a constant current; and as the pressure in the arteries is continually supplying fresh blood, that which has 
circulated in the capillaries is forced into the venous radicles in a steady stream. As the supply to the eapillaries of different parts is regulated by the action of the small arteries, and as this supply is subject to great variations, there must necessarily be corresponding variations in the intensity of the current in the veins, and the quantity of blood which these vessels receive. As we should anticipate, then, the venous circulation is subject to very great variations arising from irregularity in the supply of blood, aside from any action of the vessels themselves, or any external disturbing influences. A great variation in the venous current is observed in the veins which collect the blood from the intestinal canal. During the intervals of digestion, these vessels carry a comparatively small quantity of blood; but during digestion, they are laden with the fluids received by absorption, and the quantity is immensely increased.

It often happens that a vein becomes obstructed from some cause which is entirely physiological, as the action of muscles. The immense number of veins, as compared with the arteries, and their free communications with each other, provide that the current, under these circumstances, is simply diverted, passing to the heart by another channel. When any part of the venous system is distended, the vessels react on the blood, and exert a certain influence on the current, always pressing it toward the heart, for the valves oppose the flow in the opposite direction.

The intermittent action of the heart, which pervades the whole arterial system, is generally absorbed, as it were, in the passage of the blood through the capillaries; but when the arterioles of any part are very much relaxed, the impulse of the central organ may extend to the veins. Bernard has shown this in the most striking manner, in his well-known experiments on the circulation in the glands. ${ }^{1}$ When the glands are in physiological activity, the quantity of blood

${ }^{2}$ Bernard, Liquides de l' Organisme, Paris, tome i., p. 301 ; and Journal de lAnatomie ct de la Physiologie, Septembre, 1864, p. 507 et seq. 
which they receive is very much increased. It is then furnished to supply material for the secretion, and not exclusively for nutrition. If the vein be opened at such a time, it is found that the blood has not lost its arterial character, that the quantity which escapes is much increased, and the flow is in an intermittent jet, as from a divided artery. This is due to the relaxed condition of the arterioles of the part, and the phenomenon thus observed is the true venous pulse. What thus occurs in a restricted portion of the circulatory system may take place in all the veins, though in a less marked degree. Physicians have frequently noticed, after the blood has been flowing for some time, in the operation of venesection, that the color changes from black to red, and the stream becomes intermittent, often leading the operator to fear that he has pricked the artery. In all probability the phenomenon is due to the relaxation of the arterioles, as one of the effects of abstraction of blood, producing the same condition that has been noted in some of the glands during their functional activity. The hypothesis that it is due to an impulse from the adjacent artery is not admissible. Except in the veins near the heart, any pulsation which occurs is to be attributed to the force of the heart, transmitted with unusual facility through the capillary system. A nearly uniform current, however, is the rule, and a marked pulsation the rare exception. - Mr. T. M. King, in an article on the "Safety-Valve of the Human Heart," 1 discussing the forces which concur to produce the venous circulation, mentions the fact that in some individuals, after a full meal, pulsation can be observed in the veins of the hand or the median veins of the forehead. This phenomenon is very delicate, and, to make it more apparent, he employed a thread of black sealing wax about two inches long, which was fixed across the vein of the back of the hand with a little tallow, so as to make a long and excessively light lever, capable of indicating a very slight movement in the vessel. In this way he dem-

${ }^{1}$ Guy's Hospital Reports, 1837. 
onstrated pulsation in the veins of the hand, and also in the arm, foot, and leg. These movements are very slight, and are generally only appreciable by some such delicate means of investigation. This is a strong argument in opposition to the opinion of those who regard the action of the heart as inoperative in the veins. In certain cases of disease, Mr. King has noted very marked pulsation in the veins of the back of the hand, and other vessels far removed from the heart.

Pressure of Blood in the Veins.-The pressure in the veins is always much less than in the arteries. It is exceedingly variable in different parts of the venous system, and in the same part at different times. As a rule, it is in inverse ratio to the arterial pressure. Whatever favors the passage of blood from the arteries into the capillaries has a tendency to diminish the arterial pressure; and, as it increases the quantity of blood which passes into the veins, must increase the venous pressure. The great capacity of the venous system, its numerous anastomoses, the presence of valves which may shut off a portion from the rest, are circumstances which involve great variations in pressure in different vessels. It has been ascertained by Volkmann, and this has been confirmed by others, that as a rule the pressure is diminished as we pass from the periphery toward the heart. In an observation on the calf, he found that with a pressure of about 6.5 inches of mercury in the carotid, the pressure in the metatarsal vein was $1 \cdot 1$ inch, and but 0.36 in the jugular. ${ }^{1}$ The pressure is, of course, subject to certain variations. Muscular effort has a marked influence on the force of the circulation in certain veins, and, consequently, in these vessels produees an elevation in the pressure. As the reduced pressure in the veins is due in a measure to the great relative capacity of the venous system, and the free communications between the vessels, it would seem that if it were possible to reduce the

${ }^{1}$ Mrlse-Edwards, Legons sur la Physiologie, Paris, 1859, tome iv., p. 32 ?. 
capacity of the veins in a part, and force all the blood to pass to the heart by a single vessel corresponding to the artery, the pressure in this vessel should be greatly increased. Poiseuille has shown this to be the fact by the experiment of ligating all the veins coming from a part, except one, which had the rolume of the artery by which the blood was supplied, forcing all the blood to return by this single channel. This being done, he found the pressure in the vein immensely increased, becoming nearly equal to that in the artery. ${ }^{1}$

Rapidity of the Venous Circulation.-It is impossible to fix upon any definite rate as representing the rapidity of the current of blood in the veins. It will be seen that various circumstances are capable of increasing very considerably the rapidity of the flow in certain veins, and that under certain conditions the current in some parts of the venous system is very much retarded. Undoubtedly the general movement of blood in the veins is very much slower than in the arteries, from the fact that the quantity of blood is greater. If it be assumed that the quantity of blood in the veins is double that contained in the arteries, the general average of the current would be diminished one-half. As we near the heart, however, the flow becomes more uniform, and progressively increases in rapidity.

As the effect of the heart's action upon the venous circulation is subject to so many modifying influences through the small arteries and capillaries, and as there are other forces influencing the current, which are by no means uniform in their action, with our present knowledge, estimates of the general rapidity of the venous circulation, or the variations in different vessels, would be founded on mere speculations.

\section{Causes of the Venous Circulation.}

In the veins, the blood is farthest removed from the influence of the contractions of the left ventricle; and though

' Bérard, Cours de Plysiologie, Paris, 1855, tome iv., p. 21. 
these are felt, there are many other causes which combine to carry on the circulation, and many influences by which it is retarded or obstructed.

The great and uniform force which operates on the circulation in these vessels is the vis a tergo. We have repeatedly referred to the entire adequacy of the arterial pressure, propagated through the capillaries, to account for the movement of blood in the veins, provided there be no very great obstacles to the current. There are no facts which lead us to doubt the operation of this force as the prime cause of the venous circulation; and the only question which arises is whether there be any force exerted in the capillaries themselves which is superadded to the force of the heart. In discussing the capillary circulation, there has been found no direct proof of the existence of a distinct "capillary power" influencing the movement of blood in these ressels; and eonsequently all the vis a tergo operating on the circulation in the reins must be attributed to the action of the left ventricle.

The other forces which concur to produce movement of blood in the veins are:

1. Muscular action, by which many of the veins are at times compressed, thus forcing the blood toward the heart, regurgitation being prevented by the action of the valves.

2. A suction force exerted by the action of the thorax in respiration; operating, however, only on the veins in the immediate neighborhood of the chest.

3. A possible influence in the contraction of the coats of the vessels themselves. This is marked in the veins near the heart, in some of the inferior animals.

4. The force of gravity, which operates only on vessels which carry blood from above downward to the heart; and a little suction force which may be exerted upon the blood in a small vein as it passes into a larger vessel in which the current is more rapid.

The obstacles to the venous circulation are: Pressure sufficient to obliterate the caliber of a vessel, when, from the 
free communication with other vessels, the current is simply diverted into another channel; the expulsive efforts of respiration; the contractions of the right side of the heart ; and the force of gravity, which operates, in the erect posture, on the current in all excepting the veins of the head, neck, and parts of the trunk above the heart.

Influence of Muscular Contraction.-That the action of muscles has a considerable influence on the current of blood in the veins situated between them, and in their substance, has long been recognized. It is exemplified in the operation of venesection, when it is well known that the jet from the vein may be very much increased in force by contraction of the muscles below the opening. This action is so marked, that the parts of the venous system which are situated in the substance of muscles have been compared by Chassaignac to a sponge full of liquid, vigorously pressed by the hand. ${ }^{2}$ It must always be remembered, however, that though the muscles are capable of acting on the blood contained in veins in their substance with great vigor, the heart is fully capable of producing the venous circulation without their aid; a fact which is exemplified in a striking manner in the venous circulation in paralyzed parts.

It has been shown by actual observations with the hemodynamometer, that muscular action is capable of immensely increasing the pressure in certain veins. The first definite experiments on this subject were made by Magendie, who showed a pressure of over two inches of mercury produced by a general muscular contraction, on the passage of a galvanic current from a needle plunged into the cervical region of the spinal marrow to one fixed in the muscles of the thigh. ${ }^{2}$ The experiments of Bernard have shown this more accurately. This physiologist found that the pressure in the jugular of a horse, in repose, was $1 \cdot 4$ inch ; but the action of the muscles in

1 BÉrARD, op. cit., tome iv., p. 57.

'Magende, Phénomènes Physiques de la Vie, Paris, 1842, tome iii., p. 163. 
raising the head increased it to a little more than five inches, or nearly four times. ${ }^{1}$ These observations show at once the great variations in the venous current, and the important influence of muscular contraction on the circulation.

In order that contractions of muscles shall assist the venous circulation, two things are necessary :

1. The contraction must be intermittent. This is always the case in the voluntary muscles. It is a view entertained by many that each muscular fibre relaxes immediately after its contraction, which is instantaneous, and that a certain period of repose is necessary before it can contract again. However this may be, it is well known that all active muscular contraction, as distinguished from the efforts necessary to maintain the body in certain ordinary positions, is intermittent, and not very prolonged. Thus the veins, which are partly emptied by the compression, are filled again during the repose of the muscle.

2. There should be no possibility of a retrograde movement of the blood. This condition is fulfilled by the action of the valves. Anatomical researches have shown that these valves are most abundant in veins situated in the substance of or between the muscles, and that they do not exist in the veins of the cavities, wlrich are not subject to the same kind of compression. It is thus that the blood is prevented from passing backward toward the capillary system; and when the caliber of a vein is reduced by compression, part of its contents must be forced toward the heart. This action of the valves constitutes their most important function.

Milne-Edwards alludes to an important physiological bearing of the acceleration of the venous circulation by contractions of muscles, on their nutrition. ${ }^{2}$ It is apparently necessary that the supply of blood should be increased in a muscle, in proportion to and during its activity; for at that

'Bersard, Legons sur la Physiologie et la Pathologie du Système Nevveux, Paris, 1858, tome i., p. 285.

- Legons sur la Physiologie, tome iv., p. 310. 
time its destructive assimilation is undoubtedly augmented, and there is an increased demand on the blood to supply the waste. It is apparently a provision of Nature that the activity of a muscle, facilitating the passage of blood in its veins, and consequently its flow from the capillaries, induces an increased supply of the nutrient fluid. As the development of tissues is generally in proportion to their vascularity, this may account for the increase in the development of muscles, which is the invariable result of continued exercise.

Force of Aspiration from the Thorax.-During the act of inspiration, the enlargement of the thorax, by depression of the diaphragm and elevation of the ribs, affects the movements of fluids in all the tubes in its vicinity. The air rushes in by the trachea and expands the lungs, so that they follow the movements of the thoracic walls. The flow of blood into the great arteries is somewhat retarded, as is indicated by the diminution in the arterial pressure ; and finally, the blood in the great veins passes to the heart with greater facility, and in increased quantity. This last-mentioned phenomenon can be easily observed, when the veins are prominent, in profound or violent inspiration. The veins at the lower part of the neck are then seen to empty themselves of blood during the inspiration, and become distended during expiration, producing a sort of -pulsation which is synchronous with respiration. This can always be observed after exposure of the jugular in the lower part of the neck in an inferior animal. After this operation, if we cause the animal to make violent respiratory efforts, the vein will be almost emptied and collapsed with inspiration, and turgid with expiration. The movements of the veins near the thorax have long been observed and described with tolerable accuracy. By the following simple yet conclusive experiment, the regular action of the suction force was demonstrated by Magendie. Having introduced a gum-elastic sound into the jugular vein of a dog, and passed it down to the right auricle, he saw "that the 
blood flowed from the extremity of the sound only in the moment of expiration. We obtain results entirely analogous if we introduce the sound into the crural vein, directing it toward the abdomen." "As several contractions of the right auricle occur between two acts of respiration, it is shown by this experiment that, during inspiration, the suction force is sufficient to counterbalance the contractions of the auricle, which would otherwise force a certain quantity of blood through the sound, as it does during expiration; for then we have a jet synchronous with the beats of the heart. Catheterization of the right side of the heart is now quite a common experiment ; and we have frequently observed the variations in the flow of blood from a sound introduced through the jugular, which were mentioned by Magendie. The suction force is still more strikingly exhibited in this operation by the entrance of air, which is frequently drawn into the heart during a violent inspiration.

The influence of aspiration on the circulation in the veins was still more minutely studied in 1825 by Barry, whose most important experiments have been repeated, with some modifications, by Poiseuille. Barry introduced through the jugular of a horse a bent tube of glass, one extremity being passed into the right cavities of the heart, or the vena cava, and the other into a vessel containing a colored liquid. He found that with each act of inspiration the liquid mounted up in the tube, demonstrating the operation of a notable suction force. The obscrvations and experiments of Barry were made on quite an extended scale, but many of his conclusions were not entirely warranted. He studied, for example, the effect of preventing the entrance of air into the chest by the trachea, and found that this increased the suction force very considerably, as indicated by the greater elevation of liquid in the tube with each inspiratory effort; but he supposed

${ }^{1}$ Magendie, Influences des Mouvements de la Poitrine et des Efforts sur la Circulation du Sang. Journal de Physiologie Expérimentale, Paris, 1821, tome i., p. 136. 
that this force from the thorax was felt ${ }^{-}$in the entire venous system, an opinion which, as we shall see, the most simple observations have shown to be entirely erroneous. ${ }^{1}$ As this force is not felt throughout the whole of the venous system, it becomes a question of interest to determine how far its influence extends, and why it is restricted to certain vessels. Like the action of the muscular system on certain veins, it is simply superadded to the force of the heart, the latter being entirely competent to keep up the venous circulation. A proof that it is not essential is seen in the fact that the circulation is effected in animals which do not inspire, but swallow their air, and in the fœetus, before any movements of respiration take place.

Direct observations on the jugulars show conclusively that the influence of inspiration cannot be felt much beyond these vessels. They are seen to collapse with each inspiratory act, a condition which limits this influence to the veins near the heart. The flaccidity of the walls of the veins will not permit the extended action of any suction force. If a portion of a vein removed from the body be attached to the nozzle of a syringe, and we attempt to draw a liquid through it, though the suction force be applied very gently, when the vessel has any considerable length, its walls will be drawn together. In the circulation, the veins are moderately distended with blood by the vis a tergo, and, to a certain extent, supported by connections with surrounding tissues, so that the force of aspiration is felt farther than in any experiment on vessels removed from the body. The blood, as it approaches the thorax, impelled by other forces, is considerably accelerated in its flow ; but it is seen by direct observation, that beyond

\footnotetext{
${ }^{1}$ Barry, Recherches Expérimentales sur les Causes du Mouvement du Sang dans les Veines, Paris, 1825, p. 12 et seq.

${ }^{2}$ In many animals that take the air into the lungs by an act like that of deglutition, there are regular pulsations in the reins near the heart, which are quite abundantly provided with muscular fibres like those found in the heart. It is a question whether this does not take the place of the suction force from the chest, which operates in other animals.
} 
a certain point, and that very near the chest, ordinary aspiration has no influence, and violent efforts rather retard than favor the current.

In the liver, the influence of inspiration becomes a very important element in the production of the circulation. This organ presents a vascular arrangement which is exceptional. The blood, distributed by the arteries in a capillary plexus in the mucous membrane of the alimentary canal and in the spleen, instead of being returned directly to the heart by the veins, is collected into the portal vein, carried to the liver, and there distributed in a second set of eapillary vessels. It is then collected in the hepatic veins, and earried by the vena cava to the heart. This double capillary plexus between the left and right sides of the heart has been cited as an argument against the fact that the left ventricle is capable of sending the blood through the entire circuit of the vascular system. The three hepatic veins open into the inferior vena cava near the point where it passes the diaphragm, where the force of aspiration from the thorax would materially assist the current of blood. On following these vessels into the substance of the liver, it is found that their walls are so firmly adherent to the tissue of the organ, that, when cut across, they remain patulous; and it is evident that they remain open under all conditions. The thorax can therefore exert a powerful influence upon the hepatic circulation; for it is only the flaccidity of the walls of the vessels which prevents this influence from operating throughout the entire venous system.

Though this must be a very important element in the production of the circulation in the liver, the fact that the blood circulates in this organ in the foetus before any movements of the thorax take place, shows that it is not absolutely essential. All of the influences which we have thus far considered are merely supplementary to the action of the great central organ of the circulation.

A further proof, if any were needed, of the suction force 
of inspiration is found in an accident which is not infrequent in surgical operations in the lower part of the neck. When the veins in this situation are kept open by a tumor, or by induration of the surrounding tissues, an inspiratory effort has occasionally been followed by the entrance of air into the circulation; an accident which is liable to lead to the gravest results. This occurs only when a divided vein is kept patulous; and the accident proves both the influence of inspiration on liquids in the veins near the chest, and its restriction to the ressels in this particular situation by the flaccidity of their walls. The conditions under which this occurs may be imitated in the lower animals by introducing a tube through the vein into the thorax; when, with a violent act of inspiration, air will be drawn in, and the curious and startling effects upon the circulation may be observed.

A full discussion of the subject of air in the veins, which is of great pathological interest, does not belong to the domain of physiology. The blood is capable of dissolving a certain quantity of atmospheric air; and a small quantity, very gradually introduced into a vein, can be disposed of in this way. When, however, a considerable quantity suddenly finds its way into the venous system, the patient, or animal, experiences a sense of mortal distress, and almost immediately falls into a state of insensibility. A peculiar whistling sound is heard when the air passes in ; and if the ear be applied to the chest, we distinguish the labored efforts of the heart, accompanied by a loud churning sound. On opening the chest after death, the right cavities of the heart are invariably found distended with air and blood; the blood being frothy and florid. Generally the left side of the heart is nearly or quite empty.

The production of death from air in the veins is purely mechanical. The air, finding its way to the right ventricle, is mixed with the blood in the form of minute bubbles, and passed into the pulmonary artery. Once in this vessel, it is impossible for it to pass through the capillaries of the lungs, 
and death by suffocation is the inevitable result, if the quantity of air be large. It is because no blood can pass through the lungs, that the left cavities of the heart are usually found empty.

Certain cases of entrance of air into the veins in surgical operations, though presenting the most alarming immediate symptoms, have terminated in recovery. In these instances, the quantity of air is not sufficient to completely block up the pulmonary capillaries, and it is gradually absorbed by the blood.

Air injected into the arteries produces no such serious effects as air in the veins. It is arrested in the capillaries of certain parts, and in the course of time is absorbed without having produced any injury.

Aside from the pressure exerted by the contraction of muscles, and the force of aspiration from the thorax, the influences which assist the venous circulation are very slight. As far as the action of the coats of the vessels themselves is concerned, their contraction, it must be remembered, is slow and gradual, like the contraction of the arteries; and it is hardly possible that in the general venous system it should operate at all on the blood-current, beyond the simple influence of the reduction of the caliber of the vessel. There is a slight contraction in the venæ cavæ, in the immediate proximity of the heart, which is very much more extended in many of the lower vertebrate animals, and may be mentioned as having an influence, very insignificant it is true, on the flow of blood from the great veins.

In the veins which pass from above downwards, the force of gravity favors the flow of blood. This is seen by the turgescence of the veins of the neck and face, when the head is kept for' a short time below the level of the heart. If the arm be elevated above the head, the veins of the back of the hand will be much reduced in size, from the greater facility with which the blood passes to the heart; while they are 
distended when the hand is allowed to hang by the side, and the blood has to mount up against the force of gravity.

In the extreme irregularity in the rapidity of the circulation in different veins, it must frequently happen that a vessel empties its blood into another of larger size, in which the current is more rapid. In such an instance, as a physical necessity, the more rapid current in the larger vessel exerts a certain suction force on the fluid in the vessel which joins with it.

\section{Function of the Valves.}

With our present knowledge, it is difficult to comprehend how any anatomist could have accurately described the valves of the veins, and yet be ignorant of their function; and the fact that their use was not understood before the description of the circulation by Harvey, shows the greatness of this as a discovery, and the shallow character of any pretence that men of science had any idea of the motion of the blood before his time.

With our present knowledge of the course of the blood, it is evident that the great function of the valves is in presenting an obstacle to the reflux of blood toward the capillary system; and it only remains to study the conditions under which they are brought into action.

There are two distinct conditions under which the valves of the veins may be closed. One of them is the arrest of circulation, from any eause, in veins in which the blood has to mount against the force of gravity ; and the other, compression of veins, from any cause (generally from muscular contraction) which tends to force the blood from the vessels compressed into others, when the valves offer an obstruction to a flow toward the capillaries, and necessitate a current in the direction of the heart.

In the first of these conditions, the valves are antagonistic to the force of gravity, and, when the caliber of any vessel is 
temporarily obliterated, aid in directing the current into anastomotic vessels. It is but rarely, however, that they act thus in opposition to the force of gravity; and it is only when many of the veins of a part are simultaneously compressed that they aid in diverting the current. When a single vein is obstructed, it is not probable that the valves are necessary to divert the current into other vessels, for this would take place in obedience to the vis a tergo; but when many veins are obstructed in a dependent part, and the avenues to the heart become insufficient, the numerous valves divide the columns of blood, so that the pressure is equally distributed through the extent of the vessels. For it must be remembered, the strength of the walls diminishes as we pass from the larger veins to the periphery, and the smallest vessels, which, were it not for the valves, would be subjected to the greatest amount of pressure, are least calculated to bear distention. This is but an occasional function which the valves are called upon to perform ; and it is evident that their influence is only to prevent the weight of the entire column of blood, in vessels thus obstructed, from operating on the smallest veins and the capillaries. It cannot make the labor of the heart, when the blood is again put in motion, any less than if the column were undivided, as this organ must have sufficient power to open successively each set of valves, when, of course, they cease to have any influence whatsoever.

It is in connection with the intermittent compression of the reins that the valves have their principal and almost constant function. Their situation alone would lead to this supposition. They are found in greatest numbers throughout the muscular system, having been demonstrated by Sappey in the smallest venules. They are also found in the upper parts of the body, where they certainly do not operate against the force of gravity, while they do not exist in the cavities, where the venous trunks are not subject to compression. It has already been made sufficiently evident that the action of 
muscles seconds most powerfully the contractions of the heart. The vis a tergo from the heart is, doubtless, generally sufficient to turn this influence of muscular compression from the capillary system, and the valves of the veins are open; but they stand ready, nevertheless, to oppose any tendency to regurgitation.

In the action of muscles, the skin is frequently stretched over the part, and the cutaneous veins are somewhat compressed. This may be seen in the hand, by letting it hang by the side until the veins become somewhat swollen, and then contracting the muscles, when the skin will become tense and the veins very much less prominent. Here the valves have an important action. The compression of the veins is much greater in the substance of and between the muscles than in the skin; but the blood is forced from the muscles into the skin, and the valves act to prevent it from taking a retrograde course. The fact that the contraction of muscles forces blood into the veins of the skin may be seen by surrounding the upper part of the forearm with a moderately tight ligature, which will distend the cutaneous veins below. If we now contract the muscles vigorously, the veins below will become sensibly more distended and knotted; showing, at once, the passage of blood into the skin, and the action of the valves.

When a vein is distended by the injection of air, or a liquid, forced against the valves, it is observed that at the point where the convex borders of the valves are attached, the vessel is not dilated as much as at other parts. This is due to the fact that the valves are bordered with a fibrous ring, which strengthens the vessel, and prevents distention at that point, which would separate the free borders of the valves and render them insufficient.

A full consideration of the venous anastomoses belongs to descriptive anatomy. Suffice it to say, in this connection, that they are very numerous, and provide for a return of the blood to the heart by a number of channels. The azygos vein, the 
veins of the spinal canal, and veins in the walls of the abdomen and thorax, connect the inferior with the superior vena cava. Even the portal vein has lately been shown to have its communications with the general venous system. Thus, in all parts of the organism, temporary compression of a vein only diverts the current into some other vessel, and permanent obliteration of a vein produces enlargement of communicating branches, which soon become sufficient to meet all the requirements of the circulation.

\section{Conditions which impede the Venous Circulation.}

Influence of Expiration.-The influence of expiration on the circulation in the reins near the thorax, is directly opposite to that of inspiration. As the act of inspiration has a tendency to draw the blood from these vessels into the chest, the act of expiration has a tendency to force the blood out from the vessels of the thorax, as the air is forced out by the trachea, and opposes a flow in the opposite direction. The effect of prolonged and violent expiratory efforts is very marked; being followed by deep congestion of the veins of the face and neck, and a sense of fulness in the head, which may become very distressing. The opposition to the venous current generally extends only to vessels in the immediate vicinity of the thorax, or, it may be stated in general terms, to those veins in which the flow of blood is assisted by the movements of inspiration; but, while the inspiratory influence is absolutely confined to a very restricted circuit of vessels, the obstructive influence of very violent and prolonged expiration may be extended very much further, as is seen when the vessels of the neck, face, and conjunctiva become congested in prolonged vocal efforts, blowing, etc.

The mechanism of this is not what we might at first be led to suppose; namely, a mere reflux from the large trunks of the thoracic cavity. Were this the case, it would be necessary to assume an insufficiency of certain valves, which 
does not exist. In extreme congestion, reflux of blood may take place to a certain extent in the external jugular, for this vessel has but two valves, which are not competent to prevent regurgitation: ${ }^{1}$ but the chief cause of congestion is due, not to regurgitation, but to accumulation from the pe- riphery, and an obstruction to the flow of blood into the great vessels.

It is in the internal jugular that the influence of expiration is most important, both from the great size of the vessel in the human sulject, as compared with the other vessels, and from the importance and delicacy of the parts from which it collects the blood. At the opening of this vessel into the innominate vein, is a pair of strong and perfect valves, which effectually close the orifice when there is a tendency to regurgitation. These valves have attracted much attention among physiologists, since the discovery of the circulation has made it evident how important they might be in protecting the brain from reflux of blood. When the act of expiration arrests the onward flow in the veins near the thorax, these valves are closed, and effectually protect the brain from congestion by regurgitation. The blood accumulates behind the valves, but the free communication of the internal jugular with the other veins of the neck relieves the brain from congestion, unless the effort be extraordinarily violent and prolonged.

The above remarks with regard to the influence of expiration are applicable to vocal efforts, violent coughing or sneezing, or any violent muscular efforts, such as straining, in which the glottis is closed.

Regurgitant Venous Pulse.-In the inferior animals, like the $\operatorname{dog}$, if the external jugular be exposed, a distention of the vessel is seen to accompany each expiratory act. This is sometimes observed in the human subject, when respiration is exaggerated, and has been called improperly the venous pulse. There is no sufficient obstacle to the regurgitation of

${ }^{1}$ Gray, Descriptive Anatomy, Philadelphia, 1859, p. 404. 
blood from the thorax into the external jugular, and distinct pulsations, synchronous with the movements of respiration, may be produced in this way.

In some forms of cardiac disease affecting the right side, a pulsation, synchronous with the heart's action, has also been noticed. This is always confined to the jugular, and must not be connected with the slight pulsations which sometimes occur in the veins of the extremities. It is due to a regurgitant impulse from the right side of the heart; and generally, to the action of the right ventricle, propagated into the veins on account of pathological insufficiency of the tricuspid valves. Two distinct pulsations accompanying each act of the heart have been occasionally observed: one immediately preceding, and the other coinciding with, the ventricular systole. In a case of this kind, post-mortem examination revealed contraction of the right auriculo-ventricular orifice, as well as insufficiency of the tricuspid valves. ${ }^{2}$ The relation of the pulsation of the jugular to the action of the heart showed that the first impulse was produced by the contraction of the right auricle, and the second by the contraction of the right ventricle.

It is evident that there are various other circumstances which may impede the venous circulation. Accidental compression may temporarily arrest the flow in any particular vein. When the whole volume of blood is materially increased, as after a full meal, with copious ingestion of liquids, the additional quantity of blood accumulates chiefly in the venous system, and proportionately diminishes the rapidity of the venous circulation.

The force of gravity also has an important influence. It is much more difficult for the blood to mount from below up to the heart, than to flow downwards from the head and neck. The action of this is seen if comparison be made between the circulation in the arm elevated above the head and hanging by the side. In the one case the veins are read.

${ }^{1}$ Fuist, Diseases of the Meart, Philadelphia, 1859, p. 147. 
ily emptied, and contain but little blood; and in the other the circulation is more difficult, and the vessels are moderately distended. The walls of the veins are thickest, and the valves most numerous, in parts of the body which are habit. ually dependent. The influence of gravity is exemplified in the production of varicose veins in the lower extremities. This disease is frequently induced by occupations which require constant standing; but the exercise of walking, aiding the renous circulation, as it does, by the muscular effort, has no such tendency. 


\section{CHAPTER IX.}

PECULIARITIES OF THE CIRCULATION IN DIFFERENT PARTS OF THE SYSTEM.

Circulation in the cranial cavity-Circulation in erectile tissues-Derivative circulation-Pulmonary circulation-General rapidity of the circulation-Time required for the passage through the heart of all the blood in the organismRelations of the general rapidity of the circulation to the frequency of the heart's action-Phenomena in the circulatory system after death.

Circulation in the Cranial Cavity.-In the encephalic cavity, there are certain peculiarities in the anatomy of some of the vessels, with exceptional conditions of the blood, as regards atmospheric pressure, which have been considered capable of essentially modifying the circulation. In the adult, the cranium is a closed, air-tight box, containing the incompressible cerebral substance, and blood; conditions which are widely different from those presented in other parts of the system. On this account, some have gone so far as to consider any change in the quantity of circulating fluid in the brain, a physical impossibility. ${ }^{\text {. }}$ Pathological facts in oppo-

1 A number of years ago, there was considerable interest excited in the discussion of the possibility of an increase or diminution in the quantity of blood in the brain under any circumstances. Monro, Abercrombie, and Dr. Kellie supposed the quantity of blood in the brain to be invariable; Dr. Kellie assuming to hare proved this position by experiments which showed (according to his conclusions at least) no diminution in the quantity of blood in the brain in animals killed by hemorrhage, and no increase in the quantity in auimals killed by a ligature around the neck. He made other observations on this subject which it is un- 
sition to such a view are so numerous and well established, that the question does not demand extended discussion. It is well known, that in certain cases the vessels of the brain and its membranes are found engorged with blood, and in others containing a comparatively small quantity; but it is nevertheless true that there are anatomical peculiarities in these parts, the effects of which on the circulation present important and interesting points for study.

In the brain, the venous passages which correspond to the great veins of other parts, are sinuses between the folds of the dura mater, and are but slightly dilatable. In the perfectly consolidated adult head, the blood is not subjected to atmospheric pressure as in other parts, and the semi-solids and liquids which compose the encephalic mass cannot increase in size in congestion, and diminish in anemia. Notwithstanding these conditions, the undoubted fact remains that examinations of the vessels of the brain after death show great differences in the quantity of blood which they contain. The question then arises as to what is displaced to make room for the blood in congestion, and what supplies the place of the blood in anemia.

An anatomical peculiarity, which has not yet been considered, offers an explanation of these phenomena. Magendie has shown by observations on living animals, confirmed by dissections of the-human body, that between the pia mater and the arachnoid of the brain and spinal cord there exists a

necessary to enumerate. These experiments were fully reviewed by Dr. George Burrows, who shows by his quotations from Dr. Kellie that they proved nothing of the kind. Dr. B. repeated the experiments on rabbits, and demonstrated that great variations exist in the quantity of blood in the brain, when the animals are killed in different ways. He showed that the blood-vessels are engorged when the head is left dependent for a number of hours, and that they contain but little blood when it is elevated. Certain of Kellie's experiments, cited by Dr. Burrows, show that the difference is in the conclusions, and not in the experimental facts. For a full discussion of this subjeet, the reader is referred to the work of Dr. Burrows on Disorders of the Cerebral Circulation, \&c. (American reprint), Philadelphia, 1848. 
liquid, the cephalo-rachidian fluid, which is capable of passing from the surface of the brain to the spinal canal, and communicates with the fluid in the ventricles. ${ }^{1} \quad$ This he has conclusively demonstrated to be situated, not between the layers of the arachnoid, as was supposed by Bichat, but between the inner layer of this membrane and the pia mater. The communication between the cranial cavity and the spinal canal is very free. This was demonstrated by exposing the dura mater of the brain and of the cord, making an opening in the membranes of the cord, so as to allow the liquid to escape (which it does in quite a forcible jet), when pressure on the membranes of the brain not only accelerated the flow, but pressed out a quantity of the liquid after all that would escape spontaneously had been evacuated.

It is easy to see one of the physiological nses of this liquid. When the pressure of blood in the arteries leading to the brain is increased, or when there is an obstacle to its return by the veins, more or less congestion takes place, and the blood forces the liquid from the cranial into the spinal cavity; the reverse taking place when the supply of blood to the brain is diminished. The functions of all highly organized and vascular parts seem to require certain variations in the supply of blood; and there is no reason to suppose that the brain, in its varied conditions of activity and repose, is any exception to this general rule, though the physiological conditions of its vascularity are not easily studied.

In some late experiments by Mr. Durham on the physiology of sleep, the comparative vascularity of the meninges of the brain at different times has been studied in animals, by removing a portion of the skull with a trephine, and supplying its place by a watch-glass cemented to the edges of the bone with Canada balsam. In these experiments, the author demonstrates that the vessels are much more congested dur-

1 Magendie, Joumal de Physiologie, 1825, tome v., p. 27 et seq., and 1827, tome vii., p. 66 et seq. Sur un Liquide qui se trouve dans le Crane et le Canal Vertébral de l'Homme et des Animaux Mammif̣ères. 
ing the activity of the brain, than during the suspension of its functions in sleep. The blood-ressels of the meninges were exposed freely to view by the operation, and were examined by the microscope, with a low power, as well as with the naked eye. ${ }^{1}$ Dr. Hammond has lately published an interesting paper on sleep and insomnia, in which the observations of Mr. Durham are fully confirmed, leaving no doubt that the vessels within the cranial cavity are subject to considerable physiological variations in tension. These observations were published in $1865,{ }^{2}$ though they were made before the article of Mr. Durham appeared.

Physiologists, even before the time of Haller, had noticed alternate movements of expansion and contraction in the brain, connected with the acts of respiration. This is observed in children before the fontanels are closed, and in the adult when the brain is exposed by an injury or a surgical operation. The movements are, an expansion with the act of expiration, which, in violent efforts, is sometimes so considerable as to produce protrusion, and contraction with inspiration. Magendie also studied these movements, which he explained in the following way: ${ }^{3}$ With the act of expiration, the flow of blood in the arteries is favored, and the current in the veins is retarded. If the effort be violent, the valve at the opening of the internal jugular may be closed. This act would produce an expansion of the brain, not from reflux by the veins, but from the fact that the flow into the chest is impeded, and the blood, while passing in more freely by the arteries, is momentarily confined. With inspiration, the flow into the thorax is materially aided, and the brain is in some degree relieved of this expanding force.

${ }^{1}$ Arthor E. Derham, The Physiology of Sleep. Guy's Hospital Reports, 1860, p. 149.

${ }^{2}$ Wy. A. Hammomd, M.D., On Slcep and Insomnia. New York Medical Journàl, 1865, vol. i., Nos. 2 and 3.

${ }^{3}$ Journal de Physiologie, tome i., p. 132. De l'Influence des Mouvements de la Poitrine et des Efforts sur la Circulation du Sang. 
Robin has lately noted a peculiarity in the small vessels of the brain, spinal cord, and pia mater, which is curious, but the physiological function of which is not yet apparent. These vessels are surrounded by a thin, amorphous sheath, which has a diameter of from $\frac{1}{1200}$ to $\frac{1}{400}$ of an inch greater than that of the vessel itself. Between this and the bloodvessel is a transparent liquid. This structure, which has been observed in no other part of the circulatory system, is regarded by its discoverer as the commencement of the lymphatics of the nervous centres. What effect this disposition of the ressels may have upon the facility with which they may become dilated or contracted, it is difficult to determine.

Circulation in Erectile Tissues.-In the organs of generation in both sexes there exists a tissue which is subject to great increase in volume and rigidity, when in a state of what is called erection: The parts in which the erectile tissue exists are, in the male, the corpora cavernosa of the penis, the corpora spongiosa, with the glans penis; and in the female, the corpora cavernosa of the clitoris, the gland of the clitoris, and the bulb of the restibule. In addition, Rouget has lately demonstrated the presence of true erectile tissue in the body of the uterus, and in a bulb annexed to the ovary of the human female, but states that it is not found in the inferior animals. He has shown by injections that the uterus is capable of erection like the penis. ${ }^{2}$ In some other parts, such as the nipple and the mucous membrane of the vagina, which are sometimes described as erectile, the peculiar vascular arrangement which is characteristic of true erectile tissues is not found. In the nipple, the hardness which follows gentle stimulation is simply the result of contraction of the smooth muscular fibres with which this part

${ }^{1}$ Roвis, Sur une Tunique Appartenante en propre aux Capillaires EncéphaloRachidiens. Journal de la Physiologie, etc., Oct. 1859, tome ii., p. 54.3.

${ }^{2}$ Rocget, Recherches sur les Organes Érectiles de la Femme, etc. Journal de la Physiologie, Paris, 1858, tome i., pp. 320, 479, 735. 
is largely supplied, and is analogous to the elevations in the follicles of the skin from the same cause, in what is called goose-flesh. In the vagina, congestion may occur, as in other mucous membranes, but there is no proper erection.

The vascular arrangement in erectile organs, of which the penis may be taken as the type, is peculiar to them, and not found in any other part of the circulatory system. Taking the penis as an example, the arteries, which have an unusually thick muscular coat, after they have entered the organ, do not simply branch and divide dichotomously, as in most other parts, but send off large numbers of arborescent branches, which immediately become tortuous, and are distributed in the cavernous and spongy bodies in numerous anastomosing vessels, with but a single thin homogeneous coat, like the true capillaries. These vessels are larger, even, than the arterioles which supply them with blood, some having a diameter of from $\frac{1}{25}$ to $\frac{1}{17}$ of an inch. ${ }^{\text {i }}$ The cavernous bodies have an external investment of strong fibrous tissue of considerable elasticity, which sends bands, or trabeculæ, into the interior, by which it is divided up into cells. The trabeculæ are composed of fibrous tissue mixed with a large number of smooth muscular fibres. These cells lodge the blood-vessels, which ramify in the tortuous manner already indicated, and finally terminate in the veins. ${ }^{2} \quad$ The anatomy of the corpora spongiosa is essentially the same; the only difference being that the fibrous envelope and the trabeculæ are more delicate, and the cells are of smaller size.

Without going fully into the mechanism of erection, which comes more properly under the head of generation, it may be stated in general terms that during sexual excite-

1 RoBin, Observations sur la Constitution du Tissu Érectile, Paris, 1865.

${ }^{2} \mathrm{~J}$. Müller professed to have discovered a peculiarity in the arteries of erectile tissues consisting in arborescent diverticula from the main vessel, with blind extremities. These he called the helicine arteries. (Manuel de Physiologie. Trad. par Jourdan, Paris, 1851, tome i., p. 181.) Rouget in his admirable article (loc. cit.) has gone over the experiments of Müller, and shown conclusively that the so-called helicine arteries do not exist; and that the appearances described by Müller are due to imperfect filling of the vessels by the injection. 
ment, or when erection oceurs from any cause, the thick muscular walls of the arteries of supply relax, and allow the arterial pressure to distend the eapacious vessels lodged in the cells of the cavernous and spongy bodies. This produces the characteristic change in the volume and position of the organ. It is evident that erection depends upon the peculiar arrangement of the blood-vessels, and is not simply a congestion, such as could oceur in any vaseular part. During erection, there is not a stasis of blood; but if it continue for any length of time, the quantity which passes out of the part by the veins must be equal to that which passes in by the arteries. If return by the veins were prevented, gangrene would inevitably supervene, an occurrence which sometimes takes place when the root of the penis has become constricted, and is not speedily relieved. Erection may be produced in the dead body, by preventing reflux by the veins, and filling the vessels contained in the cells of the cavernous and spongy bodies by injection. It has been shown by Müller that the penis may be made rigid by an injection at a pressure about equal to the pressure of blood in the arteries. ${ }^{1}$

The mechanism of erection of the clitoris, and other erectile parts, is essentially the 'same as in the penis. It is seen that in this condition, circulation is by no means arrested; and the tortuous vessels are filled with blood by an enlargement in the caliber of the small arteries of supply.

Rouget has shown that the body of the uterus possesses an erectile tissue as perfect as that of the penis; and that after death the organ may be made to change its form and position by injecting the vessels, when it inereases in size about one-half, rising up, and becoming rigid and erect in the cavity of the pelvis. ${ }^{2}$

This relaxation of the muscular coats of the arteries only exists for a time; tonic contraction occurs, the supply of blood is diminished, and the organ returns to its ordinary condition.

${ }^{1}$ J. Muller, op. cit., tome i., p. 182.

${ }^{2}$ Rouget, op. cit., pp. 338, 339. 
Under stimulation, the muscular fibres in the covering and trabeculæ of the corpora cavernosa and spongiosa may contract, force the blood from the parts, and produce a certain amount of rigidity, with diminution in size. This is frequently seen under the influence of cold, which is a powerful excitant of the unstriped muscular fibres.

Derivative Circulation.-In some parts of the circulatory system, there exists a direct communication between the arteries and the reins, so that all the blood does not necessarily pass through the minute ressels which have been described as true capillaries. This peculiarity has been closely studied by M. Suquet, who was first led to investigate the subject by noticing that by injecting a very small quantity of fluid, entirely insufficient to fill all the capillaries of a member, it was returned by certain of the veins. On using a black, solidifiable injection, he found that there were certain parts of the upper and lower extremities and the head which became colored by the injection, while other parts were not penetrated. Following this out by dissection, he showed that, in the upper extremity, the skin of the fingers and part of the palm of the hand, and the skin over the olecranon, is provided with ressels of considerable size, which allowed the fluid injected by the axillary artery to pass directly into some of the veins, while in other parts the veins were entirely empty. Extending his researches to the lower extremity, he found analogous communications between the vessels in the knee, toes, and parts of the sole of the foot. He also found communications in the nose, cheeks, lips, forehead, and ends of the ears, parts which are particularly liable to changes in color from congestion of vessels. ${ }^{1}$

${ }^{1}$ J. P. Suquet, De la Circulation du Sang dans les Membres et dans la Tîte de l'Homme, Paris, 1860, p. 55. Though all the physiological deductions in this memoir do not seem justifiable, the anatomical facts are undoubted. The preparations have been examined by a commission, of which M. Robin was a member, which confirmed the statements of M. Suquet. (Oral communication from MI. Robin.) 
It is evident that; under certain circumstances, a larger quantity of blood than usual may pass through these parts without necessarily penetrating the true capillaries and thus exerting a modifying influence upon nutrition. The changes which are liable to occur in the quantity of blood, in the force of the heart's action, etc., may thus take place without disturbing the circulation in the eapillaries, a provision which the functions of the parts would seem to demand. ${ }^{1}$

Pulmonary Circulation.-The vascular system of the lungs merits the name, which is frequently applied to it, of the lesser circulation. The right side of the heart acts simultaneously with the left, but is entirely distinct from it, and its muscular walls are very much less powerful. The pulmonary artery has thinner and more distensible coats than the aorta, and distributes its blood to a single system of capillaries, which are located very near the heart. We have seen that the orifice of the pulmonary artery is provided with valves which prevent regurgitation into the ventricle. In the substance of the lungs, the pulmonary artery is broken up into capillaries, most of them just large enough to allow the passage of the blood-corpuscles in a single row. These vessels are provided with a single coat, and form a very close network surrounding the air-cells. From the capillaries, the blood is collected by the pulmonary veins, and conveyed to

${ }^{2}$ Before the publication of the researches of Suquet, Todd and Bowman mentioned the possibility of direct communications between the arteries and veins in many parts of the body, and the probable existence of such communications in some of the bones.

"It is not improbable that further research may detect a direct communication between arteries and veins, even in tissues, the greatest part of which is furnished with a true capillary plexus. In the cancellated structure of bone, and the diploe of the cranial bones, it seems highly probable that the arteries communicate immediately with the veins at many points. Mr. Paget (Lectures on Inflammation) describes a direct communication between the arteries and veins of the wing of the bat, without any intermediate capillary plexus."-Todd and Bowman, Physiological Anatomy and Physiology of Man, American edition, Philadelphia, 1857, p. 662 . 
the left auricle. There is no great disparity between the arteries and veins of the pulmonary system as regards capacity. The pulmonary veins in the human subject are not provided with valves.

The blood in its passage through the lungs does not meet with the resistance which is presented in the systemic circulation. This fact we have often noticed in injecting defibrinated blood through the lungs of an animal just killed. We have also observed that an injection passes through the lungs as easily when they are collapsed as when they are inflated. The anatomy of the circulatory system in the lungs and of the right side of the beart shows that the blood must pass through these organs with comparative ease. The power of the right ventricle is evidently less than half that of the left, and the pulmonary artery will sustain a much less pressure than the aorta.

The two sides of the heart act simultaneously; and while the blood is sent by the left ventricle to the system, it is sent by the right ventricle to the lungs. Some physiologists have endeavored to measure the pressure of blood in the pulmonary artery. The only experiments which have not involved opening the thoracic cavity, an operation which must interfere materially with the pressure of blood in the pulmonary artery, as it does with the general arterial pressure, are those of Chauveau and Faivre. ${ }^{1}$ These observers measured the pressure by connecting a cardiometer with a trocar introduced into the pulmonary artery of a living horse, through one of the intercostal spaces, and found it to be about onethird as great as the pressure in the aorta; an estimate which corresponds pretty nearly with the comparative power of the two ventricles, as deduced from the thickness of their muscular walls.

Anatomy teaches us that the capillaries of the lungs have exceedingly delicate walls; and it is evident that rupture of these vessels from excessive action of the heart would lead to

${ }^{1}$ Lovger, Traité de Physiologie, Paris, 1861, tome i., pp. 886, 887. 
grave results. It has already been noted that on the right side the lungs are protected by an insufficiency of the auriculo-ventricular valves, which does not exist on the left side, allowing of a certain degree of regurgitation when the heart is acting with unusual force, and thus relieving, to a certain extent, the pulmonary system. This was pointed out by Mr. King of London, and called the safety-valve function of the right ventricle. ${ }^{1}$ We have noticed, in the heart of the ox, a like disparity between the aortic and pulmonic semilunar valves. If these be exposed on both sides by cutting away portions of the ventricles, and a current of liquid be forced against them through the vessels, the aortic valves will be found to entirely prevent the passage of the liquid, even under very great pressure, while the pulmonic valves permit regurgitation under a very inconsiderable pressure. A little reflection will make it evident that when the heart is acting with undue foree it is quite as important to relieve the lungs by a certain amount of regurgitation from the pulmonary artery, as by insufficiency of the trieuspid valves. This insufficiency is important, both at the auriculo-ventricular and pulmonic orifices, in protecting the delicate structure of the lungs from the variations in force to which the action of both ventricles is constantly liable.

On microseopic examination of the circulation in the lower animals, as the frog, the movement of blood in the capillaries of the lungs does not present any differences from the capillary circulation in other parts; except that the vessels seem more crowded with corpuscles, and there is no "still layer" next their walls.

There are no forces of any moment which are superadded to the action of the right ventricle, in the production of the arterial, eapillary, or venous circulation in the lungs; but there are certain conditions which may obstruct the flow of blood through these parts. We have already noted the effect of introduction of air into the veins, in blocking up the capil-

4 Guy's Hospital Reports, 1837. 
laries of the lungs, and preventing the passage of blood. It is a view pretty generally entertained, that in asphyxia the non-aëration of the blood obstructs the pulmonary circulation. We have already considered this subject rather fully in treating of the general effects of arrest of respiration on the circulation. The celebrated experiments of Bichat demonstrated the passage of black blood through the lungs in asphyxia, and its presence in the arterial system. The experiments of Dalton and others have shown that in this condition, the obstruction to the circulation occurs first in the systemic capillaries, and the distention is propagated backward throngh the great vessels and left cavities of the heart to the right side. When the heart is exposed in a living animal, and artificial respiration is kept up, arrest of respiration produces engorgement and labored action of both sides. There are no observations which show that increase of pressure in the pulmonary artery is the first and immediate result of asphyxia. It is true, that after death the right side of the heart is engorged; but it is well known, from observations after death, and experiments on living animals, 'that the tonic contraction of the arteries is competent to empty the blood into the veins; and the facts just stated regarding the insufficiency of the pulmonic semilunar valves explain how the right side of the heart may become engorged as the result of obstruction to the blood-current in the left side. Established facts seem to show that asphyxia does not primarily affect the pulmonary circulation; but that it is possible for venous blood to pass through the lungs without undergoing arterialization.

\section{General Rapidity of the Circulation.}

Several questions of considerable physiological interest arise in connection with the general rapidity of the circulation:

1. It would be interesting to determine, if possible, what

1 See experiments by MaGende on the causes of the circulation in the reins. Précis Élémentaire de Physiologie, Paris, 1833, tome ii., p. 391. 
length of time is occupied by the blood in its passage through the entire circuit of both the lesser and greater circulations.

2. What is the time required for the passage of the entire mass of blood through the heart?

3. What influence has the number of pulsations of the heart on the general rapidity of the circulation?

The first of these questions is the one which has been most satisfactorily answered by experiments on living animals. In 1827, Hering, ${ }^{1}$ a German physiologist, performed the experiment of injecting into the jugular vein of a living animal a harmless substance, which could be easily recognized by its chemical reactions, and noted the time which elapsed before it could be detected in the blood of the vein of the opposite side. This gave the first correct idea of the rapidity of the circulation; for though the older physiologists, such as Haller, Hales, and Keill, had studied the subject, their estimates were founded on calculations which had no accurate basis, and gave very different results. The experiment of Hering is often roughly performed as a physiological demonstration; and we have thus had frequent occasions to verify, in a general way, its accuracy. If, for example, we expose both jugulars of a dog, inject into one a solution of ferro-cyanide of potassium in water, and draw a specimen of blood from the other with as little loss of time as possible, it will be found, that in twenty or thirty seconds after the injection, the salt has had time to pass from the jugular to the right heart, thence to the lungs and left heart, from this through the capillaries of the head and face back to the jugular on the opposite side. Its presence can be determined by the distinct blue color produced on the addition of tilı perchloride of iron to the serum, if the specimen be allowed to stand, or a clear extract of the blood may be made by boiling with a little sulphate of soda and filtering, treating the colorless liquid thus obtained with the salt of iron.

${ }^{1}$ Minde-Edwards, Legons sur la Physiologie, tome iv., p. 362, note. 
The experiments of Hering were evidently conducted with great care and accuracy. He drew the blood at intervals of five seconds after the commencement of the injection, and thus, by repeated observations, ascertained pretty nearly the rapidity of a circuit of blood in the animals on which he experimented. Others have taken up these investigations, and introduced some modifications in the manipulations. Vierordt collected the blood as it flowed, in little vessels fixed on a disk revolving at a known rate, which gave a little more exactness to the observations $;{ }^{1}$ but the method is essentially the same as that employed by IIering, and the results obtained by these two observer's nearly correspond.

The length of time occupied by a portion of blood in making a complete circuit of the vascular system, in the human subject, is only to be deduced from observations on the inferior animals; but before this application is made, it will be well to examine the objections, if any exist, to the experimental procedure above described.

The only objection which could be made is, that a saline solution, introduced into the torrent of the circulation, would have a tendency to diffuse itself throughout the whole mass of blood, it might be, with considerable rapidity; and that this fact is opposed to the proposition that the salt, when detected in a specimen of blood drawn from a given vessel, is simply carried there by the force of the blood-current. This objection to the observations of Hering has been made. by Matteucci, and is considered by him as fatal to their accuracy. ${ }^{2}$ It certainly is an element which should be taken into account; but from the definite data which have been obtained concerning the rapidity of the arterial circulation, and the inferences which are unavoidable with regard to the rapidity of the venous circulation, it would seem that the saline solution must be carried on by the mere rapidity of the arterial flow to the capillaries, which are very short, taken up

1 Milne-Edwards, loc. cit.

${ }^{2}$ Matredcci, Phénomènes Physiques des Corps Vivants, p. 326 et seq. 
from them, and carried on by the veins, and thus through the entire circuit, before it has had time to diffuse itself sufficiently to interfere with the observation. It is not apparent how this objection can be overcome, for a substance must be used which will mix with the blood, otherwise it could not pass through the eapillaries. The objection made by Matteucci, especially as it does not appear how the difficulty can be obviated, seems an unnecessary refinement; for the question itself is not one of vital importance, on which depends an important physiological principle, but simply one to which a tolerably close approximation of the exact truth is a sufficient answer. It is interesting to know that the varied and complicated actions which we have been studying effect a single complete circuit of the blood in about half a minute; but it makes no great difference whether it be four or five seconds more or less. In this statement, we must not be understood as denying the value of the closest possible accuracy in physiological investigations; but it is evident that this accuracy is important in proportion to the importance of the question, in itself, and in its physiological relations.

There seems no reason why, with the above restrictions, the results obtained by Hering should not be accepted, and their application, as far as possible, made to the human subject.

Hering found that the rapidity of the circulation in different animals was in inverse ratio to their size, and in direct ratio to the rapidity of the action of the heart.

The following are the mean results in certain of the domestic animals, taking the course from jugular to jugular, when the blood passes through the lungs and through the capillaries of the face and head:

In the Horse, the circulation is accomplished in $27 \cdot 3$ seconds.

$\begin{array}{lllrl}\text { " Dog, " } & \text { " } & \text { " } & 15 \cdot 2 & \text { " } \\ \text { " Roat, } & \text { " } & \text { " } & 12 \cdot 8 & \text { " } \\ & & \text { " } & 6 \cdot 9 & \text { " } 1\end{array}$

4 Milne-Edwards, loc. cit. Vierordt found the mean rapidity in the horse 
Applying these results to the human subject, taking into account the size of the body and the rapidity of the heart's action, the duration of the circuit from one jugular to the other is estimated at 21.4 seconds, and the general average throngh the entire system at 23 seconds. This is simply approximative; but the results in the inferior animals may be received as very nearly, if not entirely, accurate.

An estimate of the time required for the passage of the whole mass of blood through the heart is even less definite than the estimate of the general rapidity of the circulation. To arrive at any satisfactory result, it is necessary to know the entire quantity of blood in the body, and the exact quantity which passes through the heart at each 'pulsation. If we divide the whole mass of blood by the quantity discharged from the heart with each systole of the ventricles, we ascertain the number of pulsations required for the passage of the whole mass of blood through the heart; and, knowing the number of beats per minute, can ascertain the length of time thus occupied.

The objection to this kind of estimate is the inaccuracy of the data respecting the quantity of blood in the system, and the quantity which passes through the heart with each pulsation. Nevertheless, an estimate can be made, which, if it be not entirely accurate, cannot be very far from the truth.

The entire quantity of blood, according to estimates which seem to be based on the most reliable data, is about one-eighth the weight of the body, or eighteen pounds in a man weighing one hundred and forty-five. The quantity discharged at each ventricular systole is estimated by Valentin at five ounces, and by Volkmann at six ounces. ${ }^{1}$ In

to be 28.8 seconds. In experimenting on the crural vein, this observer found that the circulation in the lower extremities, probably from the greater length of the vessels, occupied from one to three seconds more than in the head.

${ }^{1}$ Topn and Bowmax, Physiological Anatomy, American edition, 1857, p. 704. 
treating of the capacity of the different cavities of the heart, it has been noted that the left ventricle, when fully distended, contains from five to seven ounces. Assuming that at each systole the left rentricle discharges all its blood, except perhaps a few drops, and that this quantity in an ordinarysized man is five ounces (for in the estimates of Robin and Hiffelsheim, the cavities were fully distended, and contained more than under the ordinary conditions of the circulation), it would require fifty-eight pulsations for the passage through the heart of the entire mass of blood. Assuming the pulsations to be seventy-two per minute, this would occupy about forty-eight seconds.

We have given elsewhere the opinions of various physiologists on the quantity of blood in the body, and the capacity of the cardiac cavities, and shall not discuss the discordant views on the "duration of the circulation," as each is based on different opinions regarding the two essential questions in the problem. As the quantity of blood in the body is subject to certain physiological variations, there should be corresponding variations in the duration of the circulation, which it is unnecessary to take up fully in this connection.

The almost instantaneous action of certain poisons, which must act through the blood, confirms our ideas with regard to the rapidity of the circulation. The intervals between the introduction of some agents (strychnine for example) into the circulation, and the characteristic effects on the system, have been carefully noted by Blake, ${ }^{1}$ whose observations coincide pretty closely in their results with the experiments of Hering.

The relation of the rapidity of the circulation to the frequency of the heart's action is a question of considerable interest, which was not neglected in the experiments of Hering. It is evident that if the charge of blood sent into the arteries be the same, or nearly the same, under all circum-

'Edinburgh Med. and Surgical Journal, 1840, vol. liii., p. 35, and 1841, rol. Ivi., p. 412. 
stances, any increase in the number of pulsations of the heart would produce a corresponding acceleration of the general current of blood. But this is a proposition which cannot be taken for granted; and there are many facts which favor a contrary opinion. It may be enunciated as a general rule, that when the acts of the heart increase in frequency, they diminish in force; which renders it probable that the ventricle is most completely distended and emptied when its action is moderately slow. When, however, the pulse is very much accelerated, the increased number of pulsations of the heart might be sufficient to overbalance the diminished force of each act, and increase the rapidity of the circulation.

Hering has settled these questions experimentally. His observations were made on horses by increasing the frequency of the pulse, on the one hand, physiologically, by exercise, and on the other hand, pathologically, by inducing inflammatory action. He found, in the first instance, that in a horse, with the heart beating at the rate of 36 per minute, with 8 respiratory acts, ferro-cyanide of potassium injected into the jugular appeared in the vessel on the opposite side after an interval of fiom 20 to 25 seconds. By exercise, the number of pulsations was raised to 100 per minute, and the rapidity of the circulation was from 15 to 20 seconds. The observations were made with an interval of 24 hours. The same results were obtained in other experiments. ${ }^{1}$ Here there is a considerable increase in the rapidity of the circulation following a physiological increase in the number of beats of the heart; but the value of each beat is materially diminished; otherwise the rapidity of the current would be increased about three times, as the pulse became three times as fiequent. In its tranquil action, with the pulse at 36 , the heart contracted thirteen times during one circuit of blood; while it required twenty-nine pulsations to send the blood over the same course, after exercise, with the pulse at 100 ; showing a 
diminution in the value of the ventricular systole of more than one-half.

In animals suffering under inflammatory fever, either spontaneous or produced by irritants, the same observer found a diminution in the rapidity of the circulation, accompanying acceleration of the pulse. In one observation, inflammation was produced in the horse by the injection of ammonia into the pericardium. At the commencement of the experiment, the pulse was from 72 to 84 per minute, and the duration of the circulation about 25 seconds. The next day, with the pulse at 90 , the circulation was accomplished in from 35 to 40 seconds; and the day following, with the pulse at 100 , the rapidity of the circulation was diminished to from 40 to 45 seconds.

If we are justified in applying these observations to the human subject (and there is no reason why this should not be done), it is shown that when the pulse is accelerated in disease, the value of the contractions ' of the heart, as represented by the quantity of blood discharged, bears an inverse ratio to their number; and is so much diminished as absolutely to produce a current of less rapidity than normal.

With regard to the relations between the rapidity of the heart's action and the general rapidity of the circulation, the following conclusions may be given as the result of experimental inquiry:

1. In physiological increase in the number of beats of the heart, as the result of exercise, for example, the general circulation is somewhat increased in rapidity, though not in proportion to the increase in the pulse.

2. In pathological increase of the heart's action, as in febrile movement, the rapidity of the general circulation is generally diminished, it may be, to a very great extent.

3. Whenever the number of beats of the heart is considerably increased from any cause, the quantity of blood discharged at each ventricular systole is very much diminished, 
either from lack of complete distention, or from imperfect emptying of the cavities. ${ }^{2}$

Phenomena in the Circulatory System after Death.-We do not believe that any one has proven the existence of a force in the capillaries or the tissues (capillary power) which materially assists the circulation during life, or produces any movement immediately after death; and shall not discuss further the extraordinary post-mortem phenomena of circulation, particularly those which have been observed by Dr. Dowler in subjects dead of yellow fever. ${ }^{2}$ But nearly every autopsy shows that after death the blood does not remain equally distributed in the arteries, capillaries, and veins. Influenced by gravitation, it accumulates in and discolors the most dependent parts of the body. The arteries are always found empty, and all the blood in the body accumulates in the venous system and capillaries; a fact which was observed by the ancients, and gave rise to the belief that the arteries, as their name implies, were air-bearing tubes.

This phenomenon has long engaged the attention of physiologists, who have attempted to explain it by various theories. Without discussing the views on this subject anterior to our knowledge of the great contractile power of the arteries as compared with other vessels, we may cite the experiment of Magendie, already referred to, ${ }^{2}$ as offering a satisfactory explanation. If the artery and vein of a limb be exposed in a living animal, and all the other ressels be tied, compression of the artery does not immediately arrest the current in the vein, but the blood will continue to flow until the artery is entirely emptied. The artery, when relieved

1 These great variations in the value of the ventricular systole, amounting even, in the experiment on the healthy animal, to a diminution of one-half, as the result of exercise, show the uncertainty of the basis of those estimates with regard to the time required for the entire mass of blood to pass through the heart, which are calculated from the entire quantity of blood, the quantity discharged from the heart at each pulsation, and the number of pulsations per minute.

2 See page 295. 
from the distending force of the heart, reacts on its contents by virtue of its contractile coat, and completely empties itself of blood. An action similar to this takes place after death throughout the entire arterial system. The vessels react on their contents, and gradually force all the blood into and through the capillaries, which are rery short, to the veins, which are capacious, distensible, and but slightly contractile. This begins immediately after death, while the irritability of the muscular coat of the arteries remains, and is seconded by the subsequent cadaveric rigidity, which affects all the in. voluntary, as well as the voluntary muscular fibres. Once in the venous system, the blood cannot return on account of the valves. Thus after death the blood is found in the veins and capillaries of dependent parts of the body. 


\title{
CHAPTER X.
}

\author{
RESPIRATION.
}

General considerations-Physiological anatomy of the respiratory organs-Respiratory movements of the larynx-Epiglottis-Trachea and bronchial tubesParenchyma of the lungs-Carbonaceous matter in the lungs-Movements of respiration-Inspiration-Muscles of inspiration-Action of the diaphragmAction of the scaleni-Intercostal muscles-Levatores costarum-Auxiliary muscles of inspiration.

THE characters of the blood are by no means identical in the great divisions of the vascular system; but thus far, physiologists have been able to investigate only the differences which exist between arterial and venous blood; for the capillaries are so short, eommunicating directly with the arteries on the one side and the veins on the other, that it has been impossible to obtain-a specimen of blood which can be said to belong to this system. In the capillaries, however, the nutritive fluid, which is identical in all parts of the arterial system, undergoes a remarkable change, rendèring it unfit for nutrition. It is then known as venous blood; and, as we have seen, the only office of the veins is to carry it back to the right side of the heart, to be sent to the lungs, where it loses the vitiating materials it has collected in the tissues, takes in a fresh supply of the vivifying oxygen, and goes to the left or systemic heart, again prepared for nutrition. As the processes of nutrition vary in different parts of the organism, necessarily, there are corresponding variations 
in the composition of the blood throughout the venous system.

The important principles which are given off by the lungs are exhaled from the blood; and the gas which disappears from the air is absorbed by the blood, mainly by its corpuscular elements.

A proper supply of oxygen is indispensable to nutrition, and even to the comparatively mechanical process of circulation; but it is no less necessary to the vital processes that carbonic acid, which the blood acquires in the tissues, should be given off.

Respiration may be defined to be the process by which the system receives nxygen, and is relieved of carbonic acid.

As it is almost exclusively through the blood that the tissues and organs are supplied with oxygen, and as the blood receives and exhales most of the earbonic acid, the respiratory process may be said to consist chiefly in the change of venous into arterial blood. But experiments have demonstrated that the tissues themselves, detached from the body and placed in an atmosphere of oxygen, will absorb this gas and exhale carbonic acid. ${ }^{2}$ Under these circumstances, they certainly respire, and it is evident, therefore, that in this process the intervention of the blood is not an absolute necessity.

The tide of air in the lungs does not constitute respiration, as we now understand it. These organs only serve to facilitate the introduction of air into the blood, and the exhalation of carbonic acid. If the system be drained of blood, or if the blood be rendered incapable of interchanging its gases with the air, respiration ceases, and all the phenomena of asphyxia are presented, though air be introduced into the lungs with

${ }^{1}$ G. Liebig demonstrated that the muscles of the frog, separated from the body and carefully freed from blood, will continue to absorb oxygen and exhale carbonic acid as long as they retain their irritability. (LenMann, Physiological Cluemistry, Philadelphia, 1855, vol. ii., pp. 247, 474.) 
the utmost regularity. It must be remembered that the essential processes of respiration take place in all the tissues and organs of the system, and not in the lungs. Respiration is a process similar to what are known as the processes of nutrition; and although it is much more active and uniform, as far as its products are concerned, than the ordinary nutritive acts, it is inseparably connected with, and strictly a part of the general process. As in the nutrition of the substance of tissues, certain principles of the blood, fibrin and albumen, for example, united with inorganic prineiples, are used up, transformed into the tissue itself, finally changed into excrementious products, such as urea or cholesterine, and discharged from the body, so the oxygen of the blood is appropriated, and carbonic acid, which is an excrementitious product, produced whenever tissues are worn out and regenerated. There is a necessary and inseparable connection between all these processes; and they must be considered, not as distinct functions, but as different parts of the one great function of nutrition. As we are as yet unable to follow out all the changes which take place between the appropriation of nutritive materials from the blood, and the production of effete or excrementitious substances, it is impossible to say precisely how the oxygen is used by the tissues, and how the carbonic acid is produced. We only know that more or less oxygen is necessary to the nutrition of all tissues, in all animals, high or low in the scale, and that they produce a certain quantity of carbonic acid. The fact that oxygen is consumed with much greater rapidity than any other nutritive principle, and that the production of carbonic acid is correspondingly active, as compared with other effete products, points pretty conclusively to a connection between the absorption of the one principle and the production of the other. In asphyxia, indeed, it is difficult to say which destroys life, the absence of oxygen or the accumulation of carbonic acid.

In some of the lowest of the inferior animals, there is 
no special respiratory organ, the interchange of gases being effected through the general surface. Higher in the animal scale, special organs are found, which are called gills, when the animals live under water and respire the air which is in solution in the water, and lungs, when the air is introduced in its gaseous form. ${ }^{1}$ Animals possessed of lungs have a tolerably perfect circulatory apparatus, so that the blood is made to pass continually through the respiratory organs. In the human subject and warm-blooded animals generally, the lungs are very complex, and present an immense surface by which the blood is exposed to the air, only separated from it by a delicate permeable membrane. These animals are likewise provided with a special heart, which has the duty of carrying on the pulmonary circulation. Though respiration is carried on to some extent by the general surface, the lungs are the important and essential organs in which the interchange of gases takes place.

The essential conditions for respiration in animals which have a circulating nutritive fluid are : air and blood, separated by a membrane which will allow the passage of gases. The effete products of respiration in the blood pass out and vitiate the air. The air is deprived of a certain portion of its oxygen, which passes into the blood, to be conveyed to the tissues. Thus the air must be changed to supply fresh oxygen and get rid of the carbonic acid. The rapidity of this change is in proportion to the nutritive activity of the animal and the rapidity of the circulation of the blood. ${ }^{2}$

${ }^{2}$ Insects have no lungs; but the air is disseminated throughout the organism by a system of air-bearing tubes (true arteries), or trachex, and is probably appropriated directly by the tissues, without the intervention of the blood.

${ }^{2}$ The manner in which this change of air is effected in the different classes of animals constitutes one of the most interesting subjects in comparative physiology. Its study has shown how, as we pass from the lower to the higher orders of animals, and the functions become more active, a division of labor takes place. Functions which in the lowest animals have no special organs, one part, as the integument or alimentary track, performing many offices, in the higher classes are assigned to special organs, which are brought to a high condition of develop- 
In treating in detail of the function of respiration, it will be convenient to make the following division of the subject:

1. The mechanical phenomena of respiration; or the processes by which the fresh air is introduced into the lungs (inspiration), and the vitiated air is expelled (expiration).

2. The changes which the air undergoes in respiration.

3. The changes which the blood undergoes in respiration.

4. The relations of the consumption of oxygen and the production of carbonic acid to the general process of nutrition.

5. The respiratory sense; a want, on the part of the system, which induces the respiratory acts (besoin de respirer).

6. Cutaneous respiration.

7. Asphyxia.

The study of these questions will be facilitated by a brief consideration of some points in the anatomy of the respiratory organs.

\section{Physiological Anatomy of the Respiratory Organs.}

Passing backward from the mouth to the pharynx, two openings present themselves: one, posteriorly, which leads to the œsophagus, and one, anteriorly, the opening of the larynx, which is the commencement of the passages deroted exclusively to respiration. The construction of the œsophagus and the air-tubes is entirely different. The œsophagus is flaccid, and destined to receive and convey to the stomach the articles of food which are introduced by the constrictions of the muscles above. The trachea and its ramifications are exclusively for the passage of air, which is taken in by a suction force produced by the enlargement of the thorax. The act of inhalation requires that the tubes should be kept open by

ment. The perfection of organization in the higher animals seems to consist in the multiplication of organs, for the more efficient performance of the varions functions. 
walls sufficiently rigid to resist the external pressure of the air.

Commencing with the larynx, it is seen that the cartilages of which it is composed are sufficiently rigid and unyielding to resist the pressure produced by any inspiratory effort. Across its superior opening are the vocal cords, which are four in number, and have a direction from before backwards. The two superior are called the false vocal cords, because they are not concerned in the production of the voice. The two inferior are the true vocal cords. They are ligamentous bands covered by folds of mucous membrane, which is quite thick on the superior cords, and very thin and delicate on the inferior. Anteriorly, they are attached to a fixed point between the thyroid cartilages, and posteriorly, to the movable arytenoid cartilages. Air is admitted to the trachea through an opening between the cords, which is called the rima glottidis. Little muscles, arising from the thyroid and cricoid, and attached to the arytenoid cartilages, are capable of separating and approximating the points to which the vocal cords are attached posteriorly, so as to open and close the rima glottidis.

If the glottis be exposed in a living animal, certain regular movements are presented which are synchronous with the acts of respiration. The larynx is widely opened at each inspiration by the action of the muscles referred to above, so that the air has a free entrance to the trachea. At the termination of the inspiratory act, these muscles are relaxed, the vocal cords fall together by their own elasticity, and in expiration, the chink of the glottis returns to the condition of a narrow slit. These respiratory movements of the glottis are constant, and essential to the introduction of air in proper quantity to the lungs. The expulsion of air from the lungs is rather a passive process, and tends in itself to separate the vocal cords; but inspiration, which is active and more violent, were it not for the movements of the glottis, would have a tendency to draw the vocal cords together. 
The muscles which are engaged in producing these movements are animated by the inferior laryngeal branches of the pneumogastric nerves. If these nerves be divided, the movements of the glottis are arrested, and respiration is very seriously interfered with. This is particularly marked in young animals, in which the walls of the larynx are comparatively yielding, when the operation is frequently followed by immediate death from suffocation. The morements of the glottis enable us to understand how foreign bodies of considerable size are sometimes accidentally introduced into the air-passages.

The respiratory movements of the larynx are entirely distinct from those engaged in the production of the voice, and are simply for the purpose of facilitating the entrance of air in inspiration.

Attached to the anterior portion of the larynx is the epiglottis; a little leaf-shaped lamella of fibro-cartilage, which, during ordinary respiration, projects upward, and lies against the posterior portion of the tongue. During the act of deglutition, respiration is momentarily interrupted, and the airpassages are protected by the tongue, which presses backward carrying the epiglottis before it, completely closing the opening of the larynx. Physiologists have questioned whether the epiglottis be necessary for the complete protection of the air-passages; and, repeating the experiments of Magendie, it has been frequently removed from the lower animals without apparently interfering with the proper deglutition of solids or liquids. We have been satisfied from actual experiment that a dog will swallow liquids and solids immediately after the ablation of the epiglottis, without allowing any to pass into the trachea; but it becomes a question whether this experiment can be absolutely applied to the human subject. In a case of entire loss of the epiglottis, which was observed in the Bellevue Hospital, the patient experienced slight difficulty in swallowing, from the passage of little particles into the larynx, which produced cough. This case 
seemed to show that the presence of the epiglottis, in the human subject at least, is necessary to the complete protection of the air-passages in deglutition. ${ }^{2}$

Passing down the neck from the larynx toward the lungs, is a tube, from four to four and a half inches in length, and about three-quarters of an inch in diameter, which is called the trachea. It is provided with cartilaginous rings, from sixteen to twenty in number, which partially surround the tube, leaving about one-third of its posterior portion occupied by fibrous tissue, mixed with a certain number of unstriped muscular fibres. Passing into the chest, the trachea divides into the two primitive bronchi; the right being shorter, larger, and more horizontal than the left. These tubes, provided, like the trachea, with imperfect eartilaginous rings, enter the lungs, divide and subdivide, until the minute ramifications of the bronchial tree open directly into the air-cells. After penetrating the lungs, the cartilages become irregular, and are in the form of angular plates, which are so disposed as to completely encircle the tubes. In tubes of very small size, these plates are less numerous than in the larger bronchi, until in tubes of a less diameter than $\frac{1}{50}$ of an inch, they are lost altogether.

The walls of the trachea and bronchial tubes are composed of two distinct membranes: an external membrane, between the layers of which the cartilages are situated, and a lining mucous membrane. The external membrane is composed of inelastic and elastic fibrous tissue. Posteriorly, in the space not covered by cartilaginous rings, these fibres are mixed with a certain number of unstriped or involuntary muscular fibres, which exist in two layers: a thick internal layer, in which the fibres are transverse, and a thinner longitudinal layer, which is external. This collection of muscular fibres is sometimes called the trachealis muscle. Throughout

2 This remarkable case, in which the epiglottis had sloughed entirely away leaving the parts completcly cicatrized, as demonstrated by a laryngoscopic examination, will be given in exterso in connection with the subject of deglutition. 
the entire system of bronchial tubes, there are circular fasciculi cf muscular fibres lying just beneath the mucous membrane, with a number of longitudinal elastic fibres. The character of the bronchi abruptly changes in tubes less than $\frac{1}{50}$ of an inch in diameter. They lose the cartilaginous rings, and the external and the mucous membranes become so closely united that they can no longer be separated by dissection. The circular muscular fibres continue down to the air-cells. The mucous membrane is smooth, covered by ciliated epithelium, the movements of the ciliæ being always from within outward, and it is provided with numerous mucous glands. These glands are of the racemose variety, and.in the larynx are of considerable size. In the trachea and bronchi, racemose glands exist in the membrane on the posterior surface of the tubes; but anteriorly are small follicles, terminating in a single, and sometimes a double, blind extremity. These follicles are lost in tubes less than $\frac{1}{50}$ of an inch in diameter.

It is the anatomy of the parenchyma of the lungs which possesses the most physiological interest, for here the essential processes of respiration take place. When moderately inflated, the lungs have the appearance of irregular cones, with rounded apices, and concave bases resting upon the diaphragn. They fill all of the cavity of the chest which is not occupied by the heart and great vessels, and are completely separated from each other by the mediastinum. In the human subject, the lungs are not attached to the thoracic walls, but are closely applied to them, each covered by a reflection of the serous membrane which lines the cavity on the corresponding side. They thus necessarily follow the movements of expansion and contraction of the thorax. Deep fissures divide the right lung into three lobes, and the left lung into two. The surtace of the lungs is divided into irregularly polygonal spaces, from $\frac{1}{4}$ of an inch to an inch in diameter, which mark what are sometimes called the pulmonary lobules, though this term is incorrect, as each of these divisions includes quite a number of the true lobules. 
Following out the bronchial tubes from the diameter of $\frac{1}{50}$ of an inch, the smallest, which are from $\frac{1}{120}$ to $\frac{1}{55}$ of an inch in diameter, open into a collection of oblong vesicles,

Fig. 10.

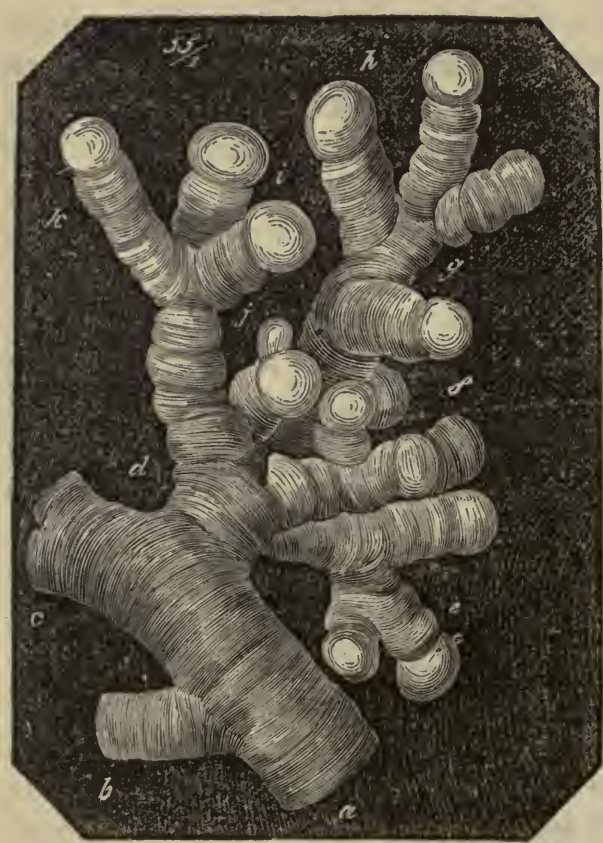

Mould of a terminal bronchus and a group of air-cells moderately distended by injection, from the human subject. (Aitter Robin.) which are the aircells. Each collection of resicles constitutes one of the true pulmonary lobules, and is from $\frac{1}{50}$ to $\frac{1}{12}$ of an inch in diameter. After entering the lobule, the tube forms a sort of tortuous central canal, sending off branches which terminate in groups of from eight to fifteen pulmonary cells. The cells are a little deeper than they are wide, and have a rounded blind extremity. Some are smooth, but many are marked by little circular constrictions, or rugæ. In the healthy lung of the adult, after death, they measure from $\frac{1}{200}$ to $\frac{1}{120}$ or $\frac{1}{70}$ of an inch in diameter, but are capable of very great distention. The smallest eells are in the deep portions of the lungs, and the largest are situated near the surface. By sections of lung inflated and dried, Magendie demonstrated, a number of years ago, that there is a considerable variation in the size of the cells at different periods of life; that the smallest cells are found in young children, and that they progres- 
sively increase in size with age. ${ }^{1}$ The air-cells are surrounded by a great number of elastic fibres, which do not form distinct bundles for each cell, but anastomose freely with each other, so that the same fibres belong to two or more. This structure is peculiar to the parenchyma of the lungs, and gives these organs their great distensibility and elasticity, properties which play an important part in expelling the air from the chest, as a consequence simply of cessation of the action of the inspiratory muscles. Interwoven with these elastic fibres is the richest plexus of capillary blood-vessels found in the economy. The vessels are larger than the capillaries in other situations, and the plexus is so close that the spaces between them are narrower than the ressels themselves. When distended, the blood-ressels furm the greater part of the walls of the cells.

'There is some difference of opinion among anatomists with regard to the lining of the air-cells. Some are of the opinion, with Rainey and Mandl, that the mucous membrane, and even the epithelium, cease in the small bronchial tubes, and the blood-vessels in the cells are uncovered. The presence of pavement epithelium has been demonstrated, however, in the cells, but the scales are detached soon after death, and cannot always be observed. All who contend for the existence of a mucous membrane admit that it is of excessive tenuity. Robin, Kölliker, and others have demonstrated in the aircells very thin scales of pavement epithelium, from $\frac{1}{2500}$ to $\frac{1}{2000}$ of an inch in diameter, which are applied directly to the walls of the blood-vessels." The epithelium here does not seem to be regularly desquamated, as in other situations. Examination of injected specimens shows that the blood-vessels are so situated between the cells, that the blood in the greater part of their circumference is exposed to the action of the air.

${ }^{1}$ Magennif, Mémoire sur la Structure du Poumon de l'Homme, etc. Journull de Physiologie, 1821, tome i., p. 78.

${ }^{2}$ Kölliker, Manual of Human Microscopic Anatomy, London, 1860, p. 387; and Podcher, Histologie Humaine, Paris, 1864, p. 286. 
The entire mass of venous blood is distributed in the lungs by the pulmonary artery for the purposes of aëration. Arterial blood is conveyed to these organs by the bronchial arteries, which ramify and subdivide on the bronchial tubes, and follow their course into the lungs, for the nourishment of these parts. It is possible that the tissue of the lungs may receive some nourishment from the blood conveyed there by the pulmonary artery; but as this vessel does not send any branches to the bronchial tubes, it is undoubtedly the bronchial arteries which supply the material for their nutrition and the secretion of the mucous glands. This is one of the anatomical reasons why inflammatory conditions of the bronchial tubes do not extend to the parenchyma of the lungs, and vice versâ.

The foregoing anatomical sketch shows the admirable adaptation of the trachea and bronchial tubes to the passage of the air by inspiration to the deep portions of the lungs, and the favorable conditions which it there meets with for an interchange of the elements of the air and blood. It is also evident, from the enormous number of air-cells, that the respiratory surface must be immense. ${ }^{1}$

Carbonaceous Matter in the Lungs.-The lungs of most of the inferior animals and the human subject, in early life, have a uniform rose tint; but in the adult, and particularly in old age, they contain a greater or less quantity of black matter, which may exist in little masses, deposited here and there in the pulmonary structure, or forming lines on the

${ }^{1}$ Hales estimated the combined surface of the air-cells at 289 square feet (Statical Essays, vol. i., p. 242); Keill at 21,906 square inches (Essays on Several Parts of the Animal Eiconomy, p. 122); and Lieberkühn at 1,500 square feet (Donglison's Human Physiology, 1856, vol. i., p. 278). There are not sufficient data on this point for us to form any thing like a reliable estimate. It is simply evident that the extent of surface must be very great. In passing from the lower to the higher orders of animals, it is seen that Nature provides for the necessity of an increase in the activity of the respiratory process, by a diminished size and a multiplication of the air-cells. 
surface of the organs. The deposit is generally most abundant at the summit of the lungs. This matter also exists in the lymphatic glands connected with the pulmonary structure, which are sometimes called the "bronchial glands." The nature of this deposit has been the subject of considerable discussion. Some have supposed that it was connected with melanotic deposits, and consisted of ordinary pigmentary matter ; but chemical investigations have now pretty conclusively demonstrated that it is nothing more nor less than carbon. It exists in great abundance in the lungs of miners, who inhale great quantities of carbonaceous particles, and of those who are much exposed to the inhalation of smoke. These facts, taken in connection with its absence in young persons and the inferior animals, and its small quantity, even in old age, in those who inhabit villages and are not exposed to a smoky atmosphere, point to its introduction from without. The subject has been most completely and ably investigated by Robin, who has come to the conclusion that the matter is really carbon ; that it is introduced in fine particles in the inspired air, and that, once in the lungs, it penetrates the tissue, not by absorption, but by mechanical action, until it finds its way beneath the pleura and into the intercellular substance. From the fact that carbon is insoluble, its penetration must be mechanical ; and, when found in the lymphatic glands, it is carried there by the absorbent vessels. When it has penetrated the substance of the tissues, it can no more be removed than the tattooing beneath the skin; indeed, the deposition in the lungs may be compared very aptly to the process of tattooing.

The mechanism of its introduction is the following: The little sharp, almost microseopic, particles are inhaled and come in contact with the delicate walls of the air-cells, in which they are imbedded under a certain pressure. When any part is subject to pressure, it is well known that it gives way by absorption, the pressure facilitating the removal of worn-out matter, but interfering with the deposition of new 
material. These particles thus penetrate the lung substance, from which they can never be remover. They may find their way into the lymphatic vessels, but become fixed in the lymphatic glands, in which the quantity is always proportionate to that which exists in the lungs. It has been shown that the particles introduced under the skin in tattooing may also be taken up by the lymphatics, but are arrested and fixed in the glands. ${ }^{2}$

There is no ground for the hypothesis that the carbonaceous matter of the lungs and bronchial glands is deposited as a residue of combustion of the hydrocarbons, in the process of respiration.

\section{Movements of Respiration.}

In man and the warm-blooded animals generally, the lungs attain their greatest degree of development, the surface which is exposed to the atmosphere is relatively greatest, and it is in these organs that nearly all of the process of interchange of gases takes place. In all animals of this class, inspiration takes place as a consequence of enlargement of the thoracic cavity, and the entrance of a quantity of air through the respiratory passages corresponding to the increased capacity of the lungs. In the mammalia, the chest is enlarged by the action of muscles; and in ordinary respiration, inspiration is an active process, while expiration is comparatively passive. In many birds, the chest is compressed by muscular action in expiration, and inspiration is effected in a measure by elastic ligaments. In both classes, the air is drawn into the chest to supply the space produced by its enlargement. In some of the lower orders of animals which have no ribs or sternum, or in which the thorax is immovable and there exists no division between its cavity and the abdomen, the air is forced into the lungs by an act like deglutition. In these animals (frogs, lizards, turtles,

'The results of the investigations of Robin are to be found in the Chimic Anatomique, by Robis and Verdeil, tome iii., p. 505 et seq. 
etc.) the respiratory acts are very infrequent; and in some, the oxidation of the blood is more effectually performed by the general surface than by the lungs.

A glance at the physiological anatomy of the thorax in the human subject makes it evident that the action of certain muscles will considerably increase its capacity. In the first place, the diaphragm mounts up into its cavity in the form of a vaulted arch. By contraction of its fibres, it is brought nearer a plane, and thus the vertical diameter of the thorax is increased. The walls of the thorax are formed by the dorsal vertebræ and ribs posteriorly, by the upper ten ribs laterally, and by the sternum and costal cartilages anteriorly. The direction of the ribs, their mode of connection with the sternum by the costal cartilages, and their articulation with the vertebral column, are such that by their movements the antero-posterior and transverse diameters of the chest may be considerably modified.

\section{Inspiration.}

The ribs are somewhat twisted upon themselves, and have a general direction forwards and downwards. The first rib is nearly horizontal, but the obliquity progressively increases from the upper to the lower parts of the chest. They are articulated with the bodies of the-vertebræ, so as to allow of considerable motion. The upper seven ribs are attached by the costal cartilages to the sternum, these cartilages running upwards and inwards. The cartilages of the eighth, ninth, and tenth ribs are joined to the cartilage of the seventh. The eleventh and twelfth are floating ribs, and are only attached to the vertebræ.

It may be stated in general terms that inspiration is effected by descent of the diaphragm and elevation of the ribs; and expiration by elevation of the diaphragm and descent of the ribs.

Arising severally from the lower border of each rib, and 
attached to the upper border of the rib below, are the eleven external intercostal muscles, the fibres of which have an oblique direction from above downwards and forwards. Attached to the inner borders of the ribs are the internal intercostals, which have a direction from above downwards and backwards, at right angles to the fibres of the external intercostals. There are also a number of muscles attached to the thorax and spine, thorax and head, upper part of humerus, etc., which are capable of elevating either the entire chest or the ribs. These must act as muscles of inspiration when the attachments to the thorax become the movable points. Some of them are called into action during ordinary respiration; others act as auxiliaries when respiration is a little exaggerated, as after exercise, and are called ordinary auxiliaries; while others, which ordinarily have a different function, are only brought into play when respiration is excessively difficult, and are called extraordinary auxiliaries.

The following are the principal muscles concerned in inspiration :

\section{Muscles of Inspiration.}

ORDINARY RESPIRATION.

Mruscle.

Attachments.

Diaphragm..................... Circumference of lower border of thorax. Scalenus Anticus............... Transverse processes of third, fourth, fifth, and sixth cervical vertebrætuberele of first rib.

Scalenus Medius ...............Transverse processes of six lower cervical vertebra-upper surface of first rib.

Scalenus Posticus................ Transverse processes of lower two or three cervical vertebræ-outer surface of second rib.

External Intercostals.............. Outer borders of the ribs. Sternal portion of Internal Intercostals..Borders of the costal cartilages. Twelve Levatores Costarum.......... Transverse processes of dorsal vertebræ -ribs, between the tubereles and angles. 


\section{Ordinary Auxiliaries.}

INusclo.

\section{Attachments.}

Serratus Posticus Superior..........Ligamentum nuchæ, spinous processes of last cervival and upper two or three dorsal vertebræ-upper borders of second, third, fourth, and fifth ribs just beyond the angles.

Sterno-mastoideus................ Upper part of sternum-mastoid process of temporal bone.

\section{Extraordinary Auxiliaries.}

Levator Anguli Scapulæ.............Transverse processes of upper three or four cervical vertebra-posterior border of superior angle of the scapula.

Trapezius (superior portion).......... Ligamentum nuchæ and seventh cervical vertebra - the upper border of the spine of the scapula.

Pectoralis Minor..................... Coracoid process of scapula -anterior surface and upper margins of third, fourth, and fifth ribs near the cartilages.

Pectoralis Major (inferior portion)..... Bicipital groove of humerus-costal cartilages and lower part of the sternum.

Serratus Magnus................ Inner margin of posterior border of scapula_-external surface and upper border of upper eight ribș.

Action of the Diaphragm.-The descriptive and general anatomy of the diaphragm gives a pretty correct idea of its functions in respiration. It arises, anteriorly, from the inner surface of the ensiform cartilage, laterally, from the inner surface of the lower borders of the costal cartilages and the six or seven inferior ribs, passes over the quadratus lumborum by the external arouate ligament, and the psoas magnus by the internal areuate ligament, and has two tendinous slips of origin, called crure of the diaphragm, from the bodies of the second, third, and fourth lumbar vertebræ and the intervertebral cartilages on the right side, and the second and third lumbar vertebræe and the intervertebral eartilages on the left side. From this origin, which extends around the lower cireunnfer- 
ence of the thorax, it mounts up into the eavity of the chest, forming a vaulted arch or dome, with its concavity toward the abdomen and its convexity toward the lungs. In the central portion there is a tendon of considerable size, and shaped something like the club on a playing card, with middle, right, and left leaflets. The remainder of the organ is composed of radiating fibres of the striped or voluntary muscular tissue. The cesophagus, aorta, and inferior vena cava pass through the diaphragm from the thoracic to the abdominal cavity, by three openings.

The opening for the œesophagus is surrounded by muscular fibres, by which it is partially closed when the diaphragm contracts in inspiration, as the fibres simply surround the tube, and none are attached to it.

The orifice for the aorta is bounded by the bone and aponeurosis posteriorly, and in front by a fibrous band to which the muscular fibres are attached; so that their contraction has rather a tendency to increase, than diminish, the caliber of the vessel.

The oritice for the vena cava is surrounded entirely by tendinous structure, and contraction of the diaphragm, though it might render the form of the orifice more nearly circular, can have no effect upon its caliber.

The action of the diaphragm can be easily studied in the inferior animals by vivisections. If the abdomen of a cat, which, from the conformation of the parts, is well adapted to this experiment, be largely opened, we can observe the descent of the tendinous portion, and the contraction of the muscular fibres. The action of this muscle may be rendered more apparent by compressing the walls of the chest with the hands, so as to interfere somewhat with the movements of the ribs. In ordinary respiration, the descent of the diaphragm and its approximation to a plane is the chief phenomenon observed; but as there is a slight resistance to the depression of the central tendon, it is probable that there is also a slight elevation of the inferior ribs, the diaphragm assisting, 
in a limited degree it is true, the action of the external intereostals.

The phenomena referable to the abdomen, which coincide with the descent of the diaphragm, can easily be observed in the human subject. As the diaphragm is depressed, it necessarily pushes the viscera before it, and inspiration is therefore accompanied by protrusion of the abdomen. This may be rendered very marked by a forced or deep inspiration.

The action of the diaphragm may be illustrated by a very simple yet striking experiment. In an animal just killed, after opening the abdomen, if we take hold of the structures which are attached to the central tendon, and make traction, we imitate, in a rough way, the movements of the diaphragm in respiration, and the air will pass into the lungs, sometimes with a distinctly audible sound.

The effects of the action of the diaphragm upon the size of its orifices are chiefly limited to the osophageal opening. The anatomy of the parts is such that contraction of the muscular fibres has a tendency to elose this orifice. When we come to treat of the digestive system, we shall see that this is auxiliary to the action of the muscular walls of the œsophagus itself, by which the cardiac opening of the stomach is regularly closed during inspiration. This may become important when the stomach is much distended; for descent of the diaphragm compresses all the abdominal organs, and might otherwise cause regurgitation of, a portion of its contents.

The contractions of the diaphragm are animated almost exclusively, if not exclusively, by the phrenie nerve; a nerve which, having the office of supplying the most important respiratory muscle, derives its filaments from a number of sources. It arises from the third and fourth cervical nerves, receiving a branch from the fifth, and sometimes from the sixth ; it passes through the chest, penetrates the diaphragm, and is distributed to its under surface. This nerve was the 
subject of numerous experiments by the earlier physiologists, who were greatly interested in the minutiæ of the action of the diaphragm, and other muscles, in respiration. Its galvanization produces convulsive contractions of the diaphragm, and its section paralyzes the muscle almost completely. It was noticed by Lower, that after section of both phrenic nerves the movements of the abdomen were reversed, and it became retracted in inspiration. ${ }^{1}$ This is explained and illustrated by voluntary suspension of the action of the diaphragm, and exaggeration of the costal movements. As the ribs are raised, the atmospheric pressure causes the diaphragm to mount up into the cavity of the thorax, and of course the abdominal organs follow.

From the great increase in the capacity of the chest produced by the action of the diaphragm, and its constant and universal action in respiration, it must be regarded as by far the most important and efficient of the muscles of inspiration.

Hiccough, sobbing, laughing, and crying are produced mainly by the action of the diaphragm, particularly hiccough and sobbing, which are produced by spasmodic contractions of this muscle, generally beyond the control of the will.

Action of the Muscles which elevate the Ribs.-Scatene Muscles.-In ordinary respiration, the ribs and the entire chest are elevated by the combined, action of a number of muscles. The three scalene muscles are attached to the cervical vertebræ and the first and second ribs. These muscles, which act particularly upon the first rib, must elevate with it, in inspiration, the rest of the thorax. The articulation of the first rib with the vertebral column is very movable, but it is joined to the sternum by a very short cartilage, which allows of very little movement, so that its elevation necessarily carries with it the sternum. This movement increases both the transverse and antero-posterior diameters of the

${ }^{2}$ Bérard, Cours de Physiologie, Paris, 1851, tome iii., p. 245. 
thorax, from the mode of articulation and direction of the ribs, which are somewhat rotated as well as rendered more horizontal.

Perhaps there is no set of muscular actions to which as much observation and speculation have been devoted as those concerned in respiration; and the actions of the muscles which are attached to the thorax are so complex and difficult of observation, that the views of physiologists concerning them are still somewhat conflicting. While some adopt the opinion of Haller, ${ }^{1}$ that the first rib is almost fixed and immovable, others contend, as did Magendie, that it is the most movable of all. ${ }^{2}$ With regard to this point there ean now, it seems, be no doubt. By putting the thumb and finger on either side of the neck over the scaleni, we can distinctly feel these muscles eontract with every tolerably deep inspiration (a movement which Magendie proposed to call the respiratory pulse, loc. cit.); and it is further evident that though in the male, in ordinary respiration, the sternum is almost motionless, in the female, and in the male in deep inspirations, the sternum is considerably elevated and projected, particularly at its lower part. This latter movement increases the antero-posterior diameter of the thorax, and can be measured with an appropriate instrument. The elevation of the sternum is necessitated by its close and almost immovable connection, through its short cartilage, with the first rib.

The action of the scaleni, while it is inconsiderable in ordinary respiration in the male, in all cases renders the first rib practically a fixed point, from which those intercostal muscles which raise the ribs can act.

Intercostal MIuscles.-Concerning the mechanism of the action of these muscles, there is great difference of opinion among physiologists; so much, indeed, that the author of a

${ }^{1}$ Elementa Physiologioe, Lausanne, 1761, tomus iii., p. 23.

${ }^{2}$ Précis Élémentaire de Physiologie, tome ii., p. 317. 
late elaborate work assumes that the question is still left in considerable uncertainty. ${ }^{2}$ The most elaborate researches on this point are those of Beau and Maissiat (Archives Générales de Médecine, 1843), and Sibson (Philosophical Transactions, 1846). The latter seem to settle the question of the mode of action of the intercostals, and explain satisfactorily certain points which even now are not generally appreciated. ${ }^{2}$

Let us first note the changes which take place in the direction of the ribs, and their relation to each other, in inspiration, before considering the way in which these movements are produced.

In the dorsal region, the spinal column forms an arch with its concavity toward the chest, and the ribs increase in length progressively, from above downwards, to the deepest portion of the arch, where they are longest, and then become progressively shorter. "During inspiration the ribs approach to or recede from each other according to the part of the arch with which they articulate; the four superior ribs approach each other anteriorly and recede from each other posteriorly ; the fourth and fifth ribs, and the intermediate set (sixth, seventh, and eighth), move further apart to a moderate, the diaphragmatic set (four inferior), to a great extent. The upper edge of each of these ribs glides toward the vertebræ in relation to the lower edge of the rib above, with the exception of the lowest rib, which is stationary." "

These movements, accurately and admirably described by Sibson, and illustrated by drawings of the chest, empty,

\section{${ }^{1}$ Longet, Traité de Physiologie, Paris, 1861, tome i., p. 629.}

"Sibson's article is the most complete ever published upon the mechanism of respiration. The action of the respiratory muscles was observed in vivisections, and the mechanism by which the capacity of the thorax is modified is illustrated in the most ingenious manner by mechanical contrivances, representing the position, etc., of the ribs, and their movements. By dilating the chest after death, also, he shows the change which takes place in the direction of the ribs and the consequent shortening of certain muscles, which, he assumes, must act as muscles of inspiration, a fact which he has taken care to verify by vivisections.

${ }^{3}$ Sibson, op. cit., p. 529. 
Fig. 11.

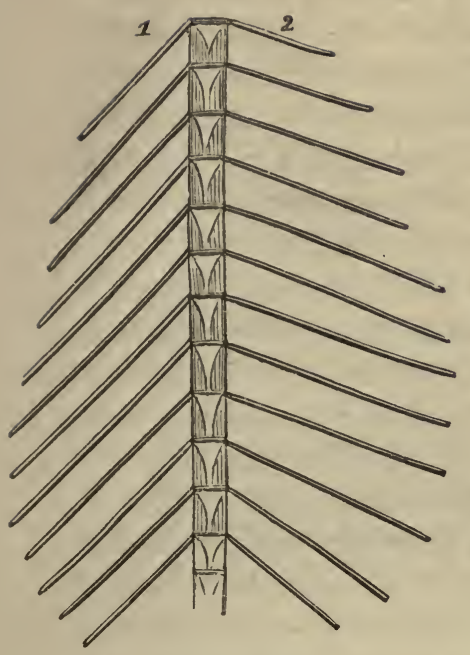

Dorsal Region.

\section{Expiration. Inspiration.}

Fเศ. 13.

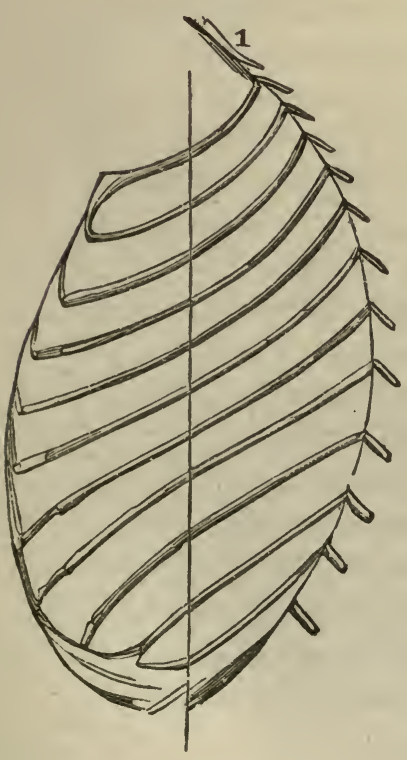

Expiration.
Fic. 12.

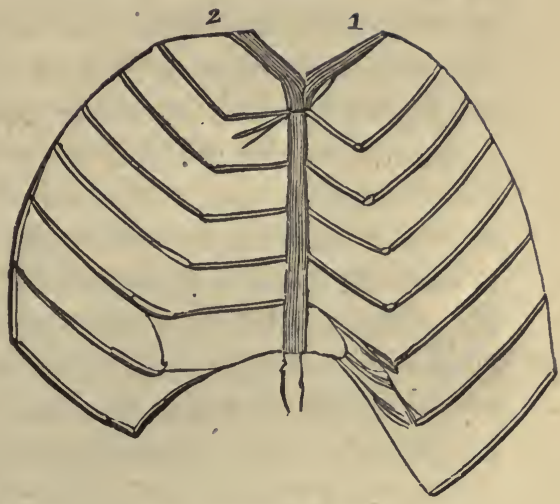

Anterior Region of the Thorax.
Inspiration.
Expiration.

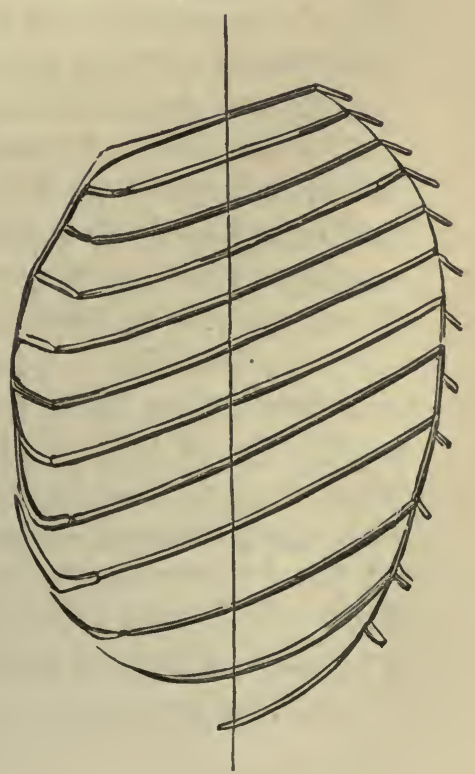

Inspiration. 
as in expiration, and distended with air, increase the anteroposterior and transverse diameters of the thorax. As the ribs are elevated and become more nearly horizontal, they must push forward the lower portion of the sternum. Their configuration and mode of articulation with the vertebræ are such, that they cannot be elevated without undergoing a considerable rotation, by which the concavity looking directly toward the lungs is increased, and with it the lateral diameter of the chest. All the intercostal spaces posteriorly are widened in inspiration.

These points are clearly illustrated in the accompanying diagrams. ${ }^{2}$

The ribs are elevated by the action of the external intercostals, the sternal portion of the internal intercostals, and the levatores costarum.

The external intercostals are situated between the ribs only, and are wanting in the region of the costal cartilages. As the vertebral extremities of the ribs are the pivots on which these levers move, and the sternal extremities are movable, the direction of the fibres of the intercostals, from above downwards and forwards, renders elevation of the ribs a necessity of their contraction; if it can be assumed that the first rib is fixed, or at least does not move downwards. The scalene muscles elevate the first rib in ordinary inspiration ; and in deep inspiration, this takes place to such an extent as to palpably carry with it the sternum and the lower ribs. Theoretically, then, the external intercostals can do nothing but render the ribs more nearly horizontal. The action of these muscles has, however, been the subject of considerable controversy, on theoretical grounds. We shall discuss the question chiefly from an experimental point of view.

If the external intercostals be exposed in a living animal, the dog for example, in which the costal type of respiration is very marked, close observation cannot fail to convince any one that these muscles enter into action in inspiration. This 
fact has been observed by Sibson and many other physiologists. If attention be now directed to the sternal portion of the internal intercostals, situated between the costal cartilages, their fibres having a direction from above downwards and backwards, it is equally evident that they enter into action with inspiration. By artificially inflating the lungs after death, Sibson confirmed these observations, and showed that when the lungs are filled with air, the fibres of these muscles are shortened. In inspiration the ribs are all separated posteriorly; but laterally and anteriorly, some are separated (all below the fourth), and some are approximated (all above the fourth). Thus all the interspaces, excepting the anterior portion of the upper three, are widened in inspiration. Sibson has shown by inflation of the chest, that though the ribs are separated from each other, the attachments of the intercostals are approximated. The ribs, from an excessively oblique position, are rendered nearly horizontal; and consequently the inferior attachments of the intercostals are brought nearer the spinal column, while the superior attachments on the upper borders of the ribs are slightly removed from it. Thus these muscles are shortened. If, by separating and elevating the ribs, the muscles are shortened, shortening of the muscles will elevate and separate the ribs. In the three superior interspaces, the constant direction of the ribs is nearly horizontal, and the course of the intercostal fibres is not as oblique as in those situated between the lower ribs. These spaces are narrowed in inspiration. The muscles between the costal cartilages have a direction opposite to that of the external intercostals, and act upon the ribs from the sternum, as the others do from the spinal column. The superior interspace is narrowed, and the remainder are widened, in inspiration.

The probable explanation of the great difference of opinion with regard to the action of the intercostals is the difficulty of comprehending, at the first blush, that the contraction of muscles situated between the ribs can separate them from each other; a phenomenon which is easily understood 
after a careful consideration of the relative position of the parts.

Levatores Costarum.-The action of these muscles cannot be mistaken. They have inmovable points of origin, the transverse processes of twelve vertebræ from the last cervical to the eleventh dorsal, and, spreading out like a fan, are attached to the upper edges of the ribs between the tubercles and the angles. In inspiration they contract and assist in the elevation of the ribs. They are more developed in man than in the inferior animals. .

Auxiliary Muscles of Inspiration.-The muscles which have just been considered are competent to increase the capacity of the thorax sufficiently in ordinary respiration; there are certain muscles, however, which are attached to the ches ${ }^{*}$ and the upper part of the spinal column, or upper extremities, which may. act in inspiration, though ordinarily the chest is the fixed point, and they move the head, neck, or arms. These muscles are brought into action when the movements of respiration are exaggerated. When this exaggeration is but slight and physiological, as after exercise, certain of them (ordinary auxiliaries) act for a time, until the tranquillity of the movements is restored. But when there is obstruction in the respiratory passages, or when respiration is excessively difficult from any cause, threatening suffocation, all the muscles which ean by any possibility raise the chest are brought into action. The principal ones are put down in the table under the head of extraordinary auxiliaries. Most of these muscles can voluntarily be brought into play to raise the chest, and the mechanism of their action can in this way be demonstrated.

Serratus Posticus Superior.-This muscle arises from the ligamentum nuchæ, the spinous processes of the last cervical and upper two or three dorsal vertebræ, its fibres passing 
obliquely downwards and outwards, to be attached to the upper borders of the second, third, fourth, and fifth ribs just beyond their angles. By reversing its action, as we have reversed the description of its origin and insertions, it is capable of increasing the capacity of the thorax. Sibson has seen this muscle contract in inspiration, in the dog and the ass. ${ }^{1}$

Sterno-mastoideus.-That portion of the muscle which is attached to the mastoid process of the temporal bone and the sternum, when the head is fixed, is capable of acting as a muscle of inspiration. It does not act in ordinary respiration, but its contractions ean be readily observed whenever respiration is hurried or exaggerated.

The following muscles, as a rule, only act as muscles of inspiration when respiration is exceedingly difficult or labored. In certain cases of capillary bronchitis, for example, the anxious expression of the countenance betrays the sense of impending suffocation; the head is thrown back and fixed, the shoulders are braced, and every available muscle is brought into action to raise the walls of the thorax. ${ }^{2}$

Levator Anguli Scapulce and Superior Portion of the Trapezins.-Movements of the scapula have often been observed in very labored respiration. Its elevation during inspiration is chiefly effected by the levator anguli scapulæ and the upper portion of the trapezius. The former arises from the transverse processes of the upper three or four cervical vertebræ, and is inserted into the posterior border of the scapula below the angle. It is a thick flat muscle, and when the neck is the fixed point, assists in the elevation of the thorax by raising the scapula. The trapezius is a broad flat muscle arising from the occipital protuberance, part of the superior curved line of the occipital bone, the ligamentum

1 Op. cit., p. 521.

${ }^{2}$ Under these circumstances, some muscles which we have not thought it necessary to enumerate may act indirectly as muscles of inspiration. 
nuchæ, and the spinous processes of the last cervical and all the dorsal vertebræ, to be inserted into the upper border of the spine of the scapula. Acting from its attachments to the occiput, the ligamentum nuchæ, the last cervical vertebra, and perhaps one or two of the dorsal vertebræ, this muscle may elevate the scapula and assist in inspiration.

Pectoralis Minor and Inferior Portion of the Pectoralis Major.-These muscles act together to raise the ribs in difficult respiration. The pectoralis minor is the more efficient. Tracing it from its attachment to the coracoid process of the scapula, its fibres pass downwards and forwards to be attached by three indigitations to the external surface and upper margins of the third, fourth, and fifth ribs, just posterior to the cartilages. With the coracoid process as the fixed point, this muscle is eapable of powerfully assisting in the elevation of the ribs. That portion of the pectoralis major which is attached to the lower part of the sternum and costal cartilages is capable of acting from its insertion into the bicipital groove of the humerus, when the shoulders are fixed, in concert with the pectoralis minor. In great dyspnœa, it is frequently observed that the shoulders are braced, the pectorals acting most vigorously to raise the walls of the chest.

Serratus Magnius. - This is a broad thin muscle covering a great portion of the lateral walls of the thorax. Attached to the inner margin of the posterior border of the scapula, its fibres pass forwards and downwards, and are attached to the external surface and upper borders of the eight superior ribs. Acting from the scapula, this muscle is capable of assisting the pectorals in raising the ribs, and becomes a powerful auxiliary in difficult inspiration.

We have thus considered the functions of the principal inspiratory muscles, without taking up those which have an insignificant or undetermined action. In many animals the nares are considerably distended in inspiration; and in the 
horse, which does not respire by the month, these movements are as essential to life as are the respiratory movements of the larynx. In man, as a rule, the nares undergo no movement unless respiration be somewhat exaggerated. In very difficult respiration the mouth is opened at each inspiratory act. We have not thought it necessary to treat of the action of those muscles which serve to fix the head, neck, or shoulders in dyspnœaa.

The division into muscles of ordinary inspiration, ordinary anxiliaries, and extraordinary auxiliaries, must not-be taken as absolute. In the male, in ordinary respiration, the diaphragm, intercostals, and levatores costarum are the great inspiratory muscles, and the action of the scaleni, with the consequent elevation of the sternum, is commonly very slight, or perhaps wanting. In the female, the movements of the upper parts of the chest are very marked, and the scaleni, the serratus posticus superior, and sometimes the sterno-mastoid, are brought into action in ordinary respiration. In the various types of respiration, the action of the muscles engaged in ordinary respiration necessarily presents considerable variations. 


\title{
CHAPTER XI.
}

\author{
MOVEMENTS OF EXPIRATION.
}

Influence of the elasticity of the pulmonary structure and walls of the chestMuscles of expiration-Internal intercostals-Infra-costales-Triangularis sterni-Action of the abdominal muscles in expiration-Types of respirationAbdominal type-Inferior costal type-Superior costal type-Frequency of the respiratory movements-Relations of inspiration and expiration to each otherThe respiratory sounds-Coughing-Sneezing-Sighing-Yawning-Laughing-Sobbing-Hiccough-Capacity of the lungs and the quantity of air changed in the respiratory acts-Residual air-Reserve air-Tidal, or breathing air-Complemental air-Extreme breathing capacity-Relations in volume of the expired to the inspired air-Diffusion of air in the lungs.

THE air is expelled from the lungs, in ordinary expiration, by a simple and comparatively passive process. The lungs contain a great number of elastic fibres surrounding the aircells and the smallest ramifications of the bronchial tubes, which give them great elasticity. We can form an idea of the extent of elasticity of these organs, by simply removing them from the chest, when they collapse and become many times smaller than the cavity which they before completely filled. The thoracic walls are also very elastic, particularly in young persons. After the muscles which increase the capacity of the thorax cease their action, the elasticity of the costal cartilages and the tonicity of muscles which have been put on the stretch, will restore the chest to what we may call its passive dimensions. This elasticity is likewise capable of acting as an inspiratory force when the chest has been com- 
pressed in any way. There are also certain muscles, the action of which is to draw the ribs downward, and which, in tranquil respiration, are antagonistic to those which elevate the ribs. Aside from this, many operations, such as speaking, blowing, singing, etc., require powerful, prolonged, or complicated acts of expiration, in which numerous muscles are brought into play.

Expiration may be considered as depending upon two causes:

1. The passive influence of the elasticity of the lungs and the thoracic walls.

2. The action of certain muscles, which either diminish the transverse and antero-posterior diameters of the chest by depressing the ribs and sternum, or the vertical diameter by pressing up the abdominal viscera behind the diaphragm.

Influence of the Elasticity of the Pulmonary Structure and Walls of the Chest.-It is easy to understand the influence of the elasticity of the pulmonary structure in expiration. From the collapse of the lungs when openings are made in the chest, it is seen that even after the most complete expiration, these organs have a tendency to expel part of their gaseous contents, which cannot be fully satisfied until the chest is opened. They remain partially distended, from the impossibility of collapse of the thoracic walls beyond a certain point; and by virtue of their elasticity, they exert a suction force upon the floor of the thorax, the diaphragm, causing it to form a vaulted arch or dome above the level of the lower circumference of the chest. When the lungs are collapsed, the diaphragm hangs loosely between the abdominal and thoracic cavities. In inspiration and in expiration, then, the relations between the lungs and diaphragm are reversed. In inspiration, the descending diaphragm exerts a suction force on the lungs, drawing them down; in expiration, the elastic lungs exert a suction force upon the diaphragm drawing it up. This antagonism is one of the causes of the great power 
of the diaphragm as an inspiratory muscle. Carson, in 1820, ${ }^{1}$ was the first to note the relation of the elasticity of the lungs to the expulsion of air. Introducing a $U$ tube partly filled with water into the trachea of an animal just killed, and securing it by a ligature, this observer noted a considerable pressure on opening the chest; equal in the calf, sheep, or dog to a column of water of from 12 to 18 inches, and in the cat or rabbit, from 6 to 10 inches. ${ }^{2}$

The elasticity of the lungs operates chicfly upon the diaphragm in reducing the capacity of the chest; for the walls of the thorax, by virtue of their own elasticity, have a reaction which succeeds the movements produced by the inspiratory muscles. A simple experiment, which we have often performed in public demonstrations, illustrates the chief expiratory influence of the elasticity of the lungs. If, in an animal just killed, we open the abdomen, seize hold of the rena cava as it passes through the diaphragm, and make traction, we imitate the action of this muscle sufficiently to produce at times an audible inspiration ; on loosing our hold, we have expiration, as it is in a measure accomplished in natural respiration, by virtue of the resiliency of the lungs, earrying the diaphragm up into the thorax.

Though this is the main action of the lungs themselves in expiration, their relations to the walls of the thorax are important. By virtue of their elasticity, they assist the passive collapse of the chest. When they lose this property to any considerable extent, as in vesicular emphysema, they offer a notable resistance to the contraction of the thorax; so much, indeed, that in old cases of this disease the movements are much restricted, and the chest presents a characteristic

\section{${ }^{1}$ Plilosophical Transactions, 1820.}

${ }^{2}$ If, after noting the elevation in the liquid due to the elasticity of the lungs, these organs be stimulated by means of a current of galvanism, the liquid will gradually rise, in obedience to the contractions of the muscular elements of the bronchial tubes. This slow contraction, characteristic of the non-striated muscular fibres, does not intervene in the physiological phenomena of expiration, but the action of these fibres is important in certain cases of discase. 
rounded and distended appearance. In some of these cases the elasticity of the lungs is so far lost, that when the chest is opened after death, they are actually protruded, instead of collapsed.'

Little more need be said concerning the passive movements of the thoracic walls. When the action of the inspiratory muscles ceases, the ribs regain their oblique direction, the intercostal spaces are narrowed, and the sternum, if it have been elevated and drawn forward, falls back to its place by the simple elasticity of the parts.

Action of Muscles in Expiration.-The following are the principal muscles concerned in expiration :

Muscles of Expiration.

ORDINARY RESPIRATION.

Mruscle.

Attachments.

Osseous portion of Internal Intercostals. . Inner borders of the ribs.

Infira-costales. . . . . . . . . . . . Inner surfaces of the

Triangularis Sterni...............nsiform cartilage, lower borders of sternum, lower three or four costal cartilages_cartilages of the second, third, fourth, and fifth ribs.

${ }^{1}$ In old cases of emphysema, the chest generally becomes rounded and distended, presenting constantly the appearance which it has in forced inspiration. This is explained in the following way: Emphysema is generally preceded and accompanied by a difficulty in respiration, from some cause which is more or less constant. This gives rise to frequent violent movements of inspiration, when the lungs and chest are distended to their utmost capacity. In this condition, expiration is difficult, and the chest collapses but imperfectly. Gradually, as the permanent dilatation of the air-cells gains ground, the lungs lose their elasticity, and offer considerable resistance to the collapse of the thoracic walls. But difficult breathing, and consequent violent elevation of the ribs, becomes more and more frequent; the chest is constantly dilated, the lungs following, of course, but refusing to collapse in expiration, until the chest becomes permanently distended. In this condition, the lungs press downward, as well as laterally, and the movements of the diaphragm are considerably restricted. 
Auxiliaries.

Muscle.

Attachments.

Obliquus Externus............... External surface and inferior borders of eight inferior ribs-the anterior half of the crest of the ileum, Poupart's ligament, linea alba.

Obliquus Internus................ Outer half of Poupart's ligament, anterior two-thirds of the crest of the ileum, lumbar fascia_- cartilages of four inferior ribs, lineal alba, crest of the pubis, pectineal line.

Transversalis.................. Outer third of Poupart's ligament, anterior two-thirds of the crest of the ileum, lumbar vertebræ, inner surface of cartilages of six inferior ribs -crest of the pubis, pectineal line, linea alba.

Sacro-lumbalis.................Sacrum-angles of the six inferior ribs.

Internal Intercostals. - The internal intercostals have different functions in different parts of the thorax. They are attached to the inner borders of the ribs and costal cartilages. Between the ribs they are covered by the external intercostals, but between the costal cartilages are simply covered by aponeurosis. Their direction is from above downwards and backwards, at right angles to the external intercostals. The function of that portion of the internal intercostals situated between the costal cartilages has already been noted. They assist the external intercostals in elevating the ribs in inspiration. Between the ribs these muscles are directly antagonistic to the external intercostals. They are more nearly at right angles to the ribs, particularly in that portion of the thorax where the obliquity of the ribs is greatest. The observations of Sibson have shown that they are elongated when the chest is distended, and shortened when the chest is collapsed. This fact, taken in connection with experiments on living animals, shows that they are muscles of expiration. Their contraction tends to depress the ribs, and consequently 
to diminish the capacity of the chest. If we bring an animal, a dog for example, completely under the influence of ether, expose the walls of the chest, dissect off the fascia from some of the external intercostals, then remove carefully a portion of one or two of these muscles so as to expose the fibres of the internal intercostals, it is not difficult, on close examination, to observe the antagonism between the two sets of muscles; one being brought into action in inspiration and the other in expiration.

Infra-costales.-These muscles, situated at the posterior. part of the thorax, are variable in size and number. They are most common at the lower part of the chest. Their fibres arise from the inner surface of one rib to be inserted into the inner surface of the first, second, or third rib below. The fibres follow the direction of the internal intercostals, and acting from their lower attachments, their contractions assist these muscles in drawing down the ribs.

Triangularis Sterni.-There has never been any doubt concerning the expiratory function of the triangularis sterni. From its origin, the ensiform cartilage, lower borders of the sternum, and lower three or four costal cartilages, it acts upon the cartilages of the second, third, fourth, and fifth ribs, to which it is attached, drawing them downwards, and thus diminishing the capacity of the chest.

The above-mentioned muscles are called into action in ordinary tranquil respiration, and their sole function is to diminish the capacity of the chest. In labored or difficult expiration, and in the acts of blowing, phonation, etc., other muscles, which are called auxiliaries, play a more or less important part. These muscles all enter into the formation of the walls of the abdomen, and their general action in expiration is to press the abdominal viscera and diaphragm into the thorax, and diminish its vertical diameter: Their action is voluntary; and by an effort of the will it may be 
opposed more or less by the diaphragm, by which means the duration or intensity of the expiratory act is regulated. They are also attached to the ribs or costal cartilages, and while they press up the diaphragm, depress the ribs, and thus diminish the antero-posterior and transverse diameters of the chest. In this action they may be opposed by the voluntary action of the muscles which raise the ribs, also for the purpose of regulating the character of the expiratory act. The importance of this kind of action in declamation, singing, blowing, etc., is evident; and the skill exhibited by vocalists and performers on wind instruments shows how delicately this may be regulated by practice.

In labored respiration in disease, and in the hurried respiration after violent exercise, the auxiliary muscles of expiration, as well as of inspiration, are called into action to a considerable extent.

Obliquus Externus.-This muscle, in connection with the obliquus internus and transversalis, is efficient in forced or labored expiration, by pressing the abdominal viscera against the diaphragm. Its fibres run obliquely from above downwards and forwards. Acting firom its attachments to the linea alba, crest of the ileum, and Poupart's ligament, by its attachment to the eight inferior ribs, it draws the ribs downwards.

Obliquus Internus.-This muscle also acts in forced expiration by compressing the abdominal viscera. The direction of its fibres is from below upwards and forwards. Acting from its attachments to the crest of the ileum, Poupart's ligament, and the lumbar fascia, by its attachments to the cartilages of the four inferior ribs, it draws them downwards. The direction of the fibres of this muscle is the same as that of the internal intercostals. By its action the ribs are drawn inwards as well as downwards.

Transversalis.-The expiratory action of this muscle is mainly in compressing the abdominal viscera. 
Sacro-lumbalis.-This muscle is situated at the posterior portion of the abdomen and thorax. Its fibres pass from its origin at the sacrum, upwards and a little outwards, to be inserted into the six inferior ribs at their angles. In expiration it draws the ribs downwards, acting as an antagonist to the lower levatores costarum.

There are some other muscles which may be brought into action in forced expiration, assisting in the depression of the ribs; such as the serratus posticus inferior, the superior fibres of the serratus magnus, the inferior portion of the trapezius; but their function is unimportant. ${ }^{\prime}$

Types of Respiration.-In the expansive movements of the chest, though all the muscles which have been classed as ordinary inspiratory muscles are brought into action to a greater or less extent, the fact that certain sets may act in a more marked manner than others has led physiologists to recognize different types of respiration. Following Beau and Maissiat, three types are generally given in works on physiology : ${ }^{2}$

1. The Abdominal type.-In this, the action of the diaphragm, and the consequent movements of the abdomen, are most prominent.

2. The Inferior Costal type.-In this, the action of the muscles which expand the lower part of the thorax, from the seventh rib inclusive, is most prominent.

3. The Superior Costal type.-In this, the action of the muscles which dilate the thorax above the seventh rib, and which elevate the entire chest, is most prominent.

${ }^{1}$ It is uncertain whether the straight muscles of the abdomen are ever concerned in expiration. From their situation, it might be supposed that they would have some action in the more violent phenomena of expiration, such as sneezing, coughing, crying, etc. ; but Beau and Maissiat, who have investigated these questions very carefully, state that in dogs they have never seen these muscles act, even in the most violent efforts. (Archives Générales, 4th series, vol. iii.)

2 Joc. cit. 
The abdominal type is most marked in children under the age of three years, irrespective of sex. In them, respira. tion is earried on almost exclusively by the diaphragm.

At a variable period after birth, a difference in the types of respiration in the sexes begins to show itself. In the male the abdominal, conjoined with the inferior costal type, is predominant, and continues thus through life. In the female the inferior costal type is insignificant, and the superior costal type predominates. Observers differ in their statements of the period when this distinction in the sexes becomes apparent. Haller states that he observed a difference in children less than a year old. Beau and Maissiat state that after the age of three years the superior costal type begins to be marked in the female. Sibson states that no great difference is observable before the age of ten or twelve years. ${ }^{1}$ Without discussing the nice question as to the exact age when this difference in the sexes first makes its appearance, it may be stated in general terms, that shortly before the age of puberty, in the female, the superior costal type becomes more marked, and soon predominates; while in the male, respiration continues to be carried on mainly by the diaphragm and lower part of the chest.

The cause of the excessive movements of the upper part of the chest in the female has been the subject of considerable discussion. It is evident that it is not due to the mode of dress now so general in civilized countries, which confines the lower part of the chest, and would render movements of expansion somewhat difficult, for the same phenomenon is observed in young girls, and others who have never made use of such appliances. But there is evidently a physiological condition, the enlargement of the uterus in gestation, which at certain times would nearly arrest all respiratory movements, excepting those of the upper part of the chest. The peculiar mode of respiration in the female is a provision of Nature against the mechanical difficulties which would other-

${ }^{1}$ Losget, Traité de Physiologie, Paris, 1861, tome i., p. 617. 
wise follow the physiological enlargement of the uterus. In pathology it is observed that, in consequence of this peculiarity, females are able to carry, without great inconvenience, immense quantities of water in the abdominal cavity; while a much smaller quantity, in the male, produces great distress from difficuity of breathing. ${ }^{1}$

Frequency of the Respiratory Movements.-In counting the respiratory acts, it is desirable that the subject be unconscious of the observation, otherwise their normal character is apt to be disturbed. Of all who have written on this subject, Hutchinson presents the most numerous and convincing collection of facts. This observer ascertained the number of respiratory acts per minute, in the sitting posture, in 1,897 males. The results of his observations, with reference to frequency, are given in the following table: ${ }^{2}$

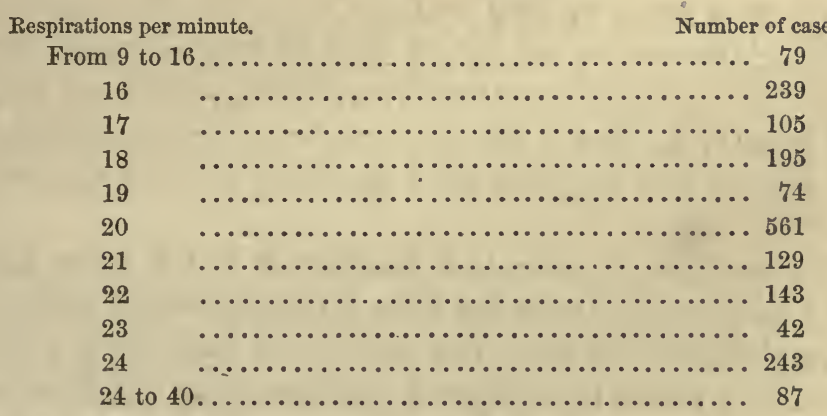

Though this table shows considerable variation in different individuals, the great majority $(1,731)$ breathed from sixteen to twenty-four times per minute. Nearly a third breathed twenty times per minute, a number which may be taken as the average.

${ }^{1}$ Modifications of the types of respiration by disease are frequently very marked. In peritonitis, when movements of the diaphragm would be produetive of excessive pain, the abdominal type may be wholly suppressed. In the early stages of acute plcurisy, the affected side may become nearly or quite motionless.

"Cyclopoedia of Anatomy and Physiology, vol. iv., part ii., p. 1085. 
The relations of the respiratory acts to the pulse are quite constant in health. It has been shown by Hutchinson that the proportion in the great majority of instances is one respiratory act to every four pulsations of the heart. The same proportion generally obtains when the pulse is accelerated in disease, except when the pulmonary organs are involved.

Age has an influence on the frequency of the respiratory acts, colresponding with what we have already noted with regard to the pulsations of the heart.

Quetelet gives the following as the results of observations on 300 males :

44 respirations per minute soon after birth;

26 , at the age of five years ;

20 , at the age of fifteen to twenty years;

19 , at the age of twenty to twenty-five years;

16 , about the thirtieth year;

18 , from thirty to fifty years.

The influence of sex is not marked in very young children. The same observer noted no difference between males and females at birth; but in young women the respirations are a little less frequent than in young men of the same age. $^{1}$

The various physiological conditions which have been noted as affecting the pulse have a corresponding influence on respiration. In sleep the number of respiratory acts is diminished about twenty per cent (Quetelet). Muscular effort accelerates the respiration pari passu with the movements of the heart.

Relations of Inspiration and Expiration to each other.The Respiratory Sounds.-In ordinary respiration, inspiration is produced by the action of muscles, and expiration, in greatest part, by the passive reaction of the elastic walls of the thorax and the lungs. The inspiratory and expiratory acts do not immediately follow each other. Commencing

${ }^{1}$ Milne-Edwards, Legons de Physiologie, tome ii., pp. 482, 483. 
with inspiration, it is found that this act maintains about the same intensity from its commencement to its termination; there is then a very brief interval, when expiration follows, which has its maximum of intensity at the commencement of the act, and gradually dies away. ${ }^{1}$ Between the acts of expiration and inspiration is an interval, somewhat longer than that which oceurs after inspiration.

The duration of expiration is generally somewhat greater than that of inspiration, though they may be nearly, or in some instances quite, equal.

After from five to eight oräinary respiratory acts, one generally occurs which is rather more profound than the rest, and by which the air in the lungs is more effectually changed. The temporary arrest of the acts of respiration in all violent muscular efforts, in straining, in parturition, etc., is familiar to all.

Ordinarily respiration is not accompanied by any sound which can be heard without applying the ear directly, or by the intervention of a stethoscope, to the respiratory organs; excepting when the mouth is closed, and breathing is carried on exclusively through the nasal passages, when a soft, breezy murmur accompanies both acts. If the mouth be sufficiently opened to adınit the free passage of air, no sound is to be heard in health. In sleep, the respirations are unusually profound; and if the mouth be closed, the sound is rather more intense than usual.

Snoring, a peculiar sound, more or less marked, which sometimes accompanies the respiratory acts during sleep, occurs when the air passes through both the mouth and the nose. It is more marked in inspiration, sometimes accompanying both acts, and sometimes not heard in expiration. It is not necessary to describe the characters of a sound so

${ }^{1}$ In listening to the respiratory murmur over the substance of the lungs, the expiratory follows the inspiratory sound without an interval (see p. 395). The interval between the acts of inspiration and expiration is only appreciated as the air passes in and out at the mouth. 
familiar. Snoring is an idiosyncrasy with many individuals, though those who do not snore habitually may do so when the system is unusually exhausted and relaxed. It only occurs when the mouth is open, and the sound is produced by a vibration, and sort of flapping, of the velum pendulum palati between the two currents of air from the mouth and nose, together with a vibration in the column of air itself.

The auscultatory phenomena which accompany the act of respiration have been made the subject of special experimental observations by Dr. Flint, who, from carefully recorded examinations of a large number of healthy persons, has arrived at the following conclusions : ${ }^{2}$

Applying the stethoscope over the larynx or trachea, a sound is heard, of a distinctly and purely tubular character, accompanying both acts of respiration. In inspiration, "it attains its maximum of intensity quickly after the development of the sound, and maintains the same intensity to the close of the act, when the sound abruptly ends, as if suddenly cut off." After a brief interval, the sound of expiration follows. This is also tubular in quality; it soon attains its maximum of intensity, but, unlike the sound of inspiration, gradually dies away and is lost imperceptibly. It is seen that these phenomena correspond with the nature of the two acts of respiration.

Sounds approximating in character to the foregoing are heard over the bronchial tubes before they penetrate the lungs.

Over the substance of the lungs, a sound may be heard entirely different in its character from that heard over the larynx, trachea, or bronchial tubes. In inspiration, the sound is much less intense than over the trachea, and has a breezy, expansive, or what is called in auscultation a vesicular character. It is much lower in pitch than the tracheal sound. It

1 Flist, Physical Exploration and Diagnosis of Diseascs affecting the Respiratory Organs, Philadelphia, 1856, p. 137 et seq. We give but a brief summary of these results, which are specially applied to auscultation in disease. 
is continuous, and rather increases in intensity from its commencement to its termination; ending abruptly, like the tracheal inspiratory sound. The sound is produced in part by the movement of air in the small bronchial tubes, but chiefly by the expansion of the innumerable air-cells of the lungs. It is followed, without an interval, by the sound of expiration, which is shorter, one-fifth to one-fourth as long, lower in pitch, and very much less intense. A sound is not always heard in expiration. In fifteen examinations recorded by Dr. Flint, five presented no expiratory sound.

The variations in the intensity of the respiratory sounds in different individuals are very considerable. As a rule they are more intense in young persons; which has given rise to the term puerile respiration, when the sounds are exaggerated in parts of the lung, in certain cases of disease. The sounds are generally more intense in females than in males, particularly in the upper regions of the thorax.

It is difficult by any description or comparison to convey an accurate idea of the character of the sounds heard over the lungs and air-passages; and it is supertluous to make the attempt, when they can be so easily studied in the living subject.

Coughing, Sneezing, Sighing, Yawning, Laughing, Sobbing, and Hiccough.

These peculiar acts demand a few words of explanation.

Coughing and sneezing are generally involuntary acts, produced by irritation in the air-tubes or nasal passages; though cough is often voluntary. In both of these acts there is first a deep inspiration, followed by convulsive action of the expiratory muscles, by which the air is violently expelled with a characteristic sound, in the one case by the mouth, and in the other by the mouth and nares. Foreign bodies lodged in the air-passages are frequently expelled in violent fits of coughing. In hypersecretion of the bronchial mucous 
membrane, the accumulated mucus is carried by the act of coughing either to the mouth, or well into the larynx, whence it is expelled by the act of expectoration. When either of these acts is the result of irritation, either from a foreign substance or secretions, it may be modified or partly smothered by the will, but is not completely under control. The exquisite sensibility of the mucous membrane at the summit of the air-passages, under most circumstances, protects them from the entrance of foreign matter, both liquid and solid; for the slightest impression received by the membrane gives rise to a violent and involuntary cough, by which the offending matter is removed. The glottis is also spasmodically closed.

In sighing, a prolonged and deep inspiration is followed by a rapid and generally audible expiration. This occurs, as a general rule, once in every five to eight respiratory acts, for the purpose of changing the air in the lungs more completely, and is due to an exaggeration of the cause which gives rise to the ordinary acts of respiration. When due to depressing emotions, it has the same cause; for at such times, respiration is less effectually performed. Yawning is an analogous process, but differs from sighing in the fact that it is involuntary, and cannot be produced by an effort of the will. It is characterized by a wide opening of the mouth, and a very profound inspiration. Yawning is generally assumed to be an evidence of fatigue, but it often occurs from a sort of contagion. When not the result of imitation, it has the same exciting cause as sighing, viz., defi. cient oxygenation of the blood, and is followed by a sense of satisfaction, which shows that it meets some decided want on the part of the system.

Laughing and sobbing, though expressing opposite conditions, are produced by very much the same mechanism. The characteristic sounds accompanying these acts are the result of short, rapid, and convulsive movements of the diaphragm, accompanied by contractions of the muscles of the 
face, which produce the expressions characteristic of hilarity or grief. Though to a certain extent under the control of the will, they are mostly involuntary. Violent and convulsive laughter may be excited in many individuals by titillation of certain portions of the surface of the body. Laughter and sometimes sobbing, like yawning, may be the result of involuntary imitation.

Hiccough is a peculiar modification of the act of inspiration, to which it is exclusively confined. It is produced by a sudden, convulsive, and entirely involuntary contraction of the diaphragm, accompanied by a spasmodic constriction of the glottis. The contraction of the diaphragm is more extensive than in laughing and sobbing, and occurs only once in four or five respiratory acts. The eauses which give rise to hiccough are numerous, and many of them are referable to the digestive system. Among these may be mentioned the rapid ingestion of a quantity of dry food, or of effervescing or alcoholic drinks. It occurs frequently in cases of disease.

\section{Capacity of the Lungs, and the Quantity of Air changed in the Respiratory Acts.}

Several points of considerable physiological interest arise in this connection. - It is evident from the simple experiment of opening the chest, when the elastic lungs collapse and expel a certain quantity of air which cannot be removed while the lungs are in situ, that a part of the gaseous contents of these organs necessarily remains after the most complete and forcible expiration. After an ordinary expiration, there is a certain quantity of air in the lnngs which can be expelled by a forced expiration. In ordinary respiration, a comparatively small volume of air is introduced with inspiration, which is expelled by the succeeding expiration. ${ }^{1}$ By the extreme action

${ }^{1}$ Experiments hare shown that a certain volume of air is lost in the lungs, the expired air being a little less in volume than the quantity inspired (from $\frac{1}{70}$ to $\frac{1}{60}$ ). This is not taken into account in this connection. 
of all the inspiratory muscles in a forced inspiration, a supplemental quantity of air may be introduced into the lungs, which then contain much more than they ever do in ordinary respiration. For convenience, many physiologists have adopted the following names, which are applied to these various volumes of air:

1. Residual Air; that which is not, and cannot be, expelled by a forced expiration.

2. Reserve Air; that which remains after an ordinary expiration, deducting the residual air.

3. Tidal, or ordinary Breathing Air; that which is changed by the ordinary acts of inspiration and expiration.

4. Complemental Air; the excess over the ordinary breathing air, which may be introduced by a forcible inspiration.

The questions relating to the abore divisions of the respired air have been made the subject of numerous investigations; but though at first it might seem easy to determine all of them by a sufficient number of experiments, the necessary observations are attended with considerable difficulty, and the sources of error are numerous. In measuring the air changed in ordinary breathing, it has been found that the acts of respiration are so easily influenced by the mind, and it is so difficult to experiment on any individual without his knowledge, that the results of many good observers are not to be relied upon. This is one of the most important of the questions under consideration. The difficulties in the way of estimating with accuracy the residual, reserve, or complemental volumes, will readily suggest themselves. The observations on these points, which may be taken as the most definite and exact, are those of Herbst of Göttingen, and Hutchinson of England. ${ }^{2}$ Those of the last-named observer

${ }^{1}$ A summary of the observations of Herbst, made in 1828 , is to be found in the Archives Générales de Médecine, tome xxi., p. 412. The observations of Hutch- 
are exceedingly elaborate, and were made on an immense number of subjects of both sexes, and of all ages and occupations. They are generally accepted by physiologists as the most extended and accurate.

Residual Air.-Perhaps there is not one of the questions under consideration more difficult to answer definitely than that of the quantity of air which remains in the lungs after a forced expiration; but fortunately it is not one of any great practical importance. The residual air remains in the lungs as a physical necessity. The lungs are always, in health, in contact with the walls of the thorax; and when this cavity is reduced to its smallest dimensions, it is impossible that any more air should be expelled. The volume which thus remains has been variously estimated at from 40 cubic inches (Fontana) to 220 cubic inches (Jurin). Dr. Hutchinson, who has carefully considered this point, estimates the residual volume at about 100 cubic inches, but states that it varies very considerably in different individuals. Taking every thing into consideration, we may assume this estimate to be as nearly correct as any. It is certain that the lungs of a man of ordinary size, at their minimum of distention, contain more than 40 cubic inches of air; and from measurements of the capacity of the thorax, deducting the estimated space occupied by the heart and vessels and the parenchyma of the lungs, it is shown that the residual air cannot amount to any thing like 200 cubic inches. ${ }^{1}$

There is no special division of the function of respiration connected with the residual air. It remains in the lungs merely as a physical necessity, and its volume must not be taken into account in considering the volumes

inson are contained in extenso in the Cyclopredia of Anatomy and Physiology, vol. iv., part 1, article Thorax.

${ }^{1}$ Hutchinson found the mean absolute capacity of the thorax to be 312 cubic inches. He allows 100 cubic inches for the heart and blood-vessels, and 100 for the parenchyma of the lungs, leaving about 100 for the residual volume. Op. cit., p. 1067 . 
which are changed in any of the operations connected with breathing.

Reserve Air.-This name is appropriately given to the volume of air which may be expelled and changed by a voluntary effort, but which remains in the lungs, added to the residual air, after an ordinary act of expiration. It may be estimated, without any reference to the residual air, by forcibly expelling air from the lungs, after an ordinary expiration. The average volume is 100 cubic inches. ${ }^{1}$

The reserve air is changed whenever we experience a necessity for a more complete renoration of the contents of the lungs than ordinary. It is encroached upon in the unusually profound inspiration and expiration which occur every five or six acts. It is used in certain prolonged vocal efforts, in blowing, etc.

Added to the residual air, it constitutes the minimum capacity of the lungs in ordinary respiration. As it is continually receiving watery vapor and carbonic acid, it is always more or less vitiated; and when reënforced by the breathing air, which enters with inspiration, is continually in circulation, in obedience to the law of the diffusion of gases. Those who are in the habit of arresting respiration for a time, as the pearl-diver, learn to change the reserve air as completely as possible by several forcible acts, and then fill the lungs with fresh air. In this way they are enabled to suspend the respiratory acts for from one to two minutes without inconvenience. The introduction of the fresh air with each inspiration, and the constant diffusion which is going on, and by which the proper quantity of oxygen finds its way to the aircells, gives, in ordinary breathing, a composition to the air in the deepest portions of the lungs which insures a constant aëration of the blood. The slight difference in the rapidity of oxidation between inspiration and expiration is only sufficient to give rise to the involuntary reflex acts of respiration,

${ }^{2}$ Hutchinson, loc. cit. 
and is not sufficiently marked to produce any sensation, such as is experienced when respiration is in the slightest degree interrupted.

Tidal, or Ordinary Breathing Air.-The volume of air which is changed in the ordinary acts of respiration is subject to immense physiological variations, and the respiratory movements, as regards intensity, are so easily influenced by the mind, that great care is necessary to avoid error in estimating the volume of ordinary breathing air. The estimates of Herbst and of Hutchinson are the results of very extended observations made with great care, and are generally acknowledged to be as nearly accurate as possible. As a mean of these observations, it has been found that the average volume of breathing air, in a man of ordinary stature, is 20 cubic inches. According to Hutchinson, in perfect repose, when the respiratory movements are hardly perceptible, not more than from 7 to 12 cubic inches are changed; while, under excitement, he has seen the volume increased to 77 cubic inches. Of course the latter is temporary. ${ }^{1}$ Herbst noted that the breathing volume is constantly increased in proportion to the stature of the individual, and bears no definite relation to the apparent capacity of the chest.

Complemental Air.-The thorax may be so enlarged by an extreme voluntary inspiratory effort, as to contain a quantity of air much larger than after an ordinary inspiration. The additional volume of air thus taken in may be estimated by measuring all the air which can be expelled from the lungs after the most profound inspiration, and deducting the sum of the reserve air and breathing air. This quantity has been found by Intchinson to vary in different individuals, bearing a close relation to stature. The mean complemental volume is 110 cubic inches.

The complemental air is drawn upon whenever an effort

${ }^{1}$ We have not thought it worth while to enumerate the raried estimates found in works on physiology, which are not based on extended experimental inquiry 
is made which requires a temporary arrest of respiration. Brief and violent muscular excrtion is generally preceded by a profound inspiration. In slcep, as the volume of breathing air is somewhat increased, the complemental air is encroached upon. A part or the whole of the complemental air is also used in certain vocal efforts, in blowing, in yawning, in the deep inspiration which precedes sneezing, in straining, etc.

Summary.-In a healthy male of medium stature, the residual air, which. cannot be expelled from the lungs, amounts to about 100 cubic inches.

The reserve air, which can be expelled, but which is not changed in ordinary respiration, amounts to about 100 cubic inches.

The tidal air, which is changed in ordinary respiration, amounts to about 20 cubic inches.

The complemental air, which may be taken into the lungs after the completion of an ordinary act of inspiration, amounts to about 110 cubic inches. ${ }^{3}$

${ }^{1}$ In Robin's Journal de l'Anatomie et de la Physiologie, Sept. 1864, p. 523 et seq., we find an article by Dr. Nestor Gréhant, on the physical phenomena of respiration in man, which contains some novel and interesting observations on the capacity of the lungs, volume of breathing air, etc. The volumes of air are estimated by a process which is exceedingly ingenions, and apparcntly accurate; but the number of observations is very small compared with those of Hutchinson, and in estimating the capacity of the lungs, he does not take into consideration the very decided influence of stature. The method employed is essentially the following:

It having been demonstrated by Regnault and Reiset that hydrogen introduced into the lungs is not absorbed by the blood, the author, taking advantage of the well-known property of gases, by which they form a uniform mixture when brought in contact with each other, caused the subjects of his experiments to respire a measured volume of hydrogen often enough to make the mixture uniform, and estimates, by analysis of the expired air, the quantity which remains in the lungs, which is necessarily represented by the volume of hydrogen lost. Ile ascertained by experiments that five respirations of the gas caused a perfect mixture.

By this method he estimates the normal capacity of the lungs after an ordinary expiration (the sum of the residual and reserve air), at from 133.65 to 191.51 cubic inches, in men between 17 and 30 yegars of age (p. 554). 
Extreme Breathing Capacity.-By the extreme breathing capacity is meant the volume of air which can be expelled from the lungs by the most forcible expiration, after the most profound inspiration. This has been called by Dr. Hutchinson the vital capacity, as signifying "the volume of air which can be displaced by living movements." Its volume is equal to the sum of the reserve air, the breathing air, and the complemental air, and represents the extreme capacity of the chest, deducting the residual air. Its physiological interest is due to the fact that it can readily be determined by an appropriate apparatus, the spirometer, ${ }^{1}$ and comparisons can thus be made between different individuals, both healthy and diseased. The number of observations on this point made by Dr. Hutchinson is enormous, amounting in all to little short of five thousand.

The extreme breathing capacity in health is subject to variations which have been shown to bear a very close relation to the stature of the individual. Hutchinson commences with the proposition that in a man of medium height (5 feet 8 inches), it is equal to two hundred and thirty cubic inches. He has shown that the extreme breathing capacity is constant in the same individual, and that it is not to be increased by habit or practice.

The most striking result of the experiments of Dr. Hutchinson, with regard to the modifications of the vital ca-

The tidal or breathing air, he estimates at 30 cubic inches.

The observations of Dr. Gréhant are as yet so few in number that we prefer to adhere to the results of the greatly extended observations of Hutchinson; though the new method is very ingenious, and further experiments will probably lead to important results.

1 The spirometer consists of a vessel containing water, out of which a receiver is raised by breathing into it through a tube; the height to which the receiver is raised indicating the volume of the vital eapacity (Cyclop. of Anat. and Phys., vol. iv., part 2, p. 1068). In all the observations of Dr. Hutchinson, he has taken care to see that the level of the water was the same in the receiver and the reservoir, and to carefully correct the volumes of air for temperature. All observations were made with the subject erect, and every thing earefully avoided which could interfere with the free action of the respiratory museles. 
pacity, is that it bears a definite relation to stature, without being affected in a very marked degree by weight, or the circumference of the chest. This is especially remarkable, as it is well known that height does not depend so much upon the length of the body, as the length of the lower extremities.

It has been ascertained that for every inch in hoight, between five and six feet, the extreme breathing capacity is increased eight cubic inches.

The following table shows the mean results of the immense number of observations on which this conclusion is based: ${ }^{1}$

Progression of the Vital Capacity Volume with the Stature.

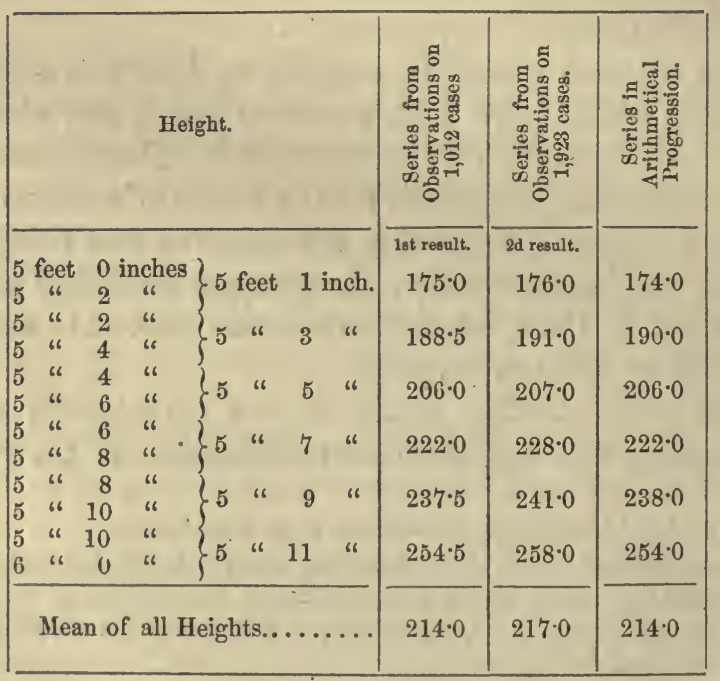

Age has an influence, though less marked than stature, upon the extreme breathing capacity. As the result of 4,800

${ }^{2}$ Op. cit., p. 1072. The increase in breathing capacity, pari passu with an increase in height, was mentioned by Herbst (loc. cit.), but Hutchinson was the first to make any extended observations, and gire any definite information on this point. 
observations (males), it was ascertained that the volume increases with age up to the thirtieth year, and progressively decreases, with tolerable regularity, from the thirtieth to the sixtieth year.

These figures, though necessarily subject to certain individual variations, may be taken as the basis for examinations of the extreme breathing capacity in disease, which frequently give important information. Of course, the breathing capacity is modified by any abnormal condition which interferes with the mobility of the thorax, or the dilatability of the lungs. Of all diseased conditions, phthisis pulmonalis is the most interesting in this connection. With regard to the significance of the variations in this disease, Dr. Hutchinson has arrived at the following conclusions :

"It has been found that ten cubic inches belaw the due quantity, i.e., 220 instead of 230 inches, need not excite. alarm; but there is a point of deficiency in the breathing volume at which it is difficult to say whether it is merely one of those physiological differences dependent on a certain irregularity in all such observations, or deficiency indicative of disease. A deficiency of 16 per cent. is suspicious. A man below 55 years of age breathing 193 cubic inches instead of 230 cubic inches, unless he is excessively fat, is probably the subject of disease.

"In phthisis pulmonalis the deficiency may amount to 90 per cent., and yet life be maintained. The vital capacity volume is likewise a measure of improvement. A phthisical patient may improve so as to gain 40 upon 220 cubic inches."

Herbst has shown ${ }^{2}$ that the extreme breathing capacity is diminished by obesity; that it is proportionally less in females than in males, and in children than in adults.

Relations in Volume of the Expired to the Inspired Air. $-A$ certain proportion of the inspired air is lost in respiration, so that the air expired is always a little less in volume 
than that which is taken into the lungs. All the older experimenters, except Magendie, were agreed upon this point. The loss was put by Davy at $\frac{1}{50}$, and by Cuvier at $\frac{1}{50}$ of the amount of air introduced. ${ }^{1}$ Observations on this point, to be exact, must include a considerable number of respiratory acts; and from the difficulty of continuing respiration in a perfeetly regular and normal manner, when the attention is directed to that function, the most accurate results may probably be obtained from experiments on animals. Despretz ${ }^{2}$ caused six young rabbits to respire for two hours in a confined space containing 299 cubic inches of air, and ascertained that the volume had diminished 61 cubic inches, or a little more than one-fiftieth. We may take the approximations of Davy and Cuvier, as applied to the human subject, as nearly correct, and assume that in the lungs, from $\frac{1}{70}$ to $\frac{1}{50}$ of the inspired air is lost.

Diffusion of Air in the Lungs.-When it is considered that with each inspiration but about twenty cubic inches of fresh air is introduced, sufficient only to fill the trachea and larger bronchial tubes, it is evident that some forces must act by which this fresh air finds its way into the air-cells, and the vitiated air is brought into the larger tubes, to be expelled with the succeeding expiration. The expired air may become so charged with noxious gases, by holding the breath for a few seconds, that when collected in a receiver under water, it is incapable of supporting combustion.

The interchange between the fresh air in the upper portions of the respiratory apparatus and the air in the deeper parts of the lungs is constantly going on, in obedience to the wellknown law of the diffusion of gases, aided by the active currents or impulses produced by the alternate movements of the chest. When two gases, or mixtures of gases, of different densities are brought in contact with each other, they diffuse

\footnotetext{
-1 Bérard, Cours de Physiologie, Paris, 1851, tome iii., p. 338.

${ }^{2}$ Idem.
} 
or mingle with great rapidity, until, if undisturbed, the whole mass has a uniform density and composition. This has been shown to take place between very light and very heavy gases in opposition to the laws of gravity, and eren when two reselvoirs are connected by a small tube many feet in length, though then it proceeds quite slowly. In the respiratory apparatus, at the termination of inspiration, the atmospheric air, composed of a mixture of oxygen and nitrogen, is introduced into the tubes with a considerable impetus, and is brought into contact with the gas in the lungs, which is much heavier, as it contains a considerable quantity of carbonic acid. Diffusion then takes place, aided by the elastic lungs, which are gradually forcing the gaseous contents out of the cells, until a certain portion of the air loaded with carbonic acid finds its way to the larger tubes, to be thrown off in expiration, its place being supplied by the fresh air.

In obedience to the law established by Graham, that the diffusibility of gases is inversely proportionate to the square root of their densities, the penetration of atmospheric air, which is the lighter gas, to the deep portions of the lings would take place with greater rapidity than the ascent of the air charged with carbonic acid; so that 81 parts of carbonic acid should be replaced by 95 of oxygen. ${ }^{3}$ It is found, indeed, that the volume of carbonic acid exhaled is always less than the volume of oxygen absorbed.

This diffusion is constantly going on, so that the air in the pulmonary vesicles, where the interchange of gases with the blood takes place, maintains a pretty uniform composition. The process of aëration of the blood, therefore, has none of that intermittent character which attends the mechanical processes of respiration, which would undoubtedly occur if the entire gaseous contents of the lungs were changed with every act.

There is no evidence sufficiently definite to show that the muscular fibres in the bronchial tubes, which are of the un-

${ }^{3}$ Cyclopoedia of Anatomy and Physiology, vol. iv., part 1, p. 362. 
striped variety, and slow and gradual in their contraction, have any thing to do with the diffusion of gases in the lungs; nor is it probable that any marked influence is exerted by the movements of the ciliæ which corer the mucous membrane. 


\section{CHAPTER XII.}

CHANGES WHICH THE AIR UNDERGOES IN RESPIRATION.

General considerations-Discovery of carbonic acid-Discovery of oxygen-Com. position of the air-Consumption of oxygen-Influence of temperature-Influence of sleep-Influence of an increased proportion of oxygen in the atmosphere-Temperature of the expired air-Exhalation of carbonic acid-Influence of age-Influence of sex-Influence of digestion-Influence of diet-Influence of sleep-Influence of muscular activity-Influence of moisture and temperature-Influence of seasons-Relations between the quantity of oxygen consumed and the quantity of carbonic acid exhaled-Exhalation of watery vapor-Exhalation of ammonia-Exhalation of organic matter-Exhalation of nitrogen.

From the allusions which have already been made to the general process of respiration, it is apparent, that before the discovery of the nature of the gases which compose the air and those which are exhaled from the lungs, it was impossible for physiologists to have any correct ideas of the nature of this important function. It is not surprising that the ancients, observing the regular introduction of air into the lungs, and noting the fact that the air is generally much cooler than the body, supposed the great object of respiration to be the cooling of the blood. It is also evident that no definite knowledge of any of the processes of respiration could exist prior to the discovery of the circulation of the blood.

Though it is foreign to our purpose to treat historically of the theories concerning any of the functions of the body, the facts relating to changes in the respired air, which from 
time to time have been developed, bear so close a relation to discoveries of the properties of certain gases, particularly carbonic acid and oxygen, that it seems desirable to give at least a rapid sketch of these discoveries, and follow the advances in our knowledge of the processes of respiration, with which they are necessarily connected. ${ }^{1}$

In the latter part of the fifteenth century, Leonardo da Vinci, the great painter, mathematician, and naturalist, made a discovery which conclusively proved the fallacy of the idea that the air simply cooled the blood in respiration. He discovered that fire consumed the air, and that animals could not live in a medium which was incapable of supporting combustion. This is the first statement in the history of science which points to the fact that the function of the air in respiration depends on its composition, and not on its physical properties.

About the middle of the seventeenth century, Van Helmont discovered some of the properties of what is now known as carbonic acid gas. He showed that a gas, the result of fermentation, or of the combustion of earbon, and formed by the action of vinegar on certain carbonates, was incapable of supporting combustion or maintaining animal life. He recognized this as the gas which is found in the lower part of the celebrated Grotto del Cane, near Naples, into which a man may enter with impunity, but which will asphyxiate a small animal, as it is brought under the influence of the lower strata.

A few years later (1670), Boyle, the founder of the Royal Society of London, by some experiments published in the Philosophical Transactions, attempted to show that air was necessary to the life of all animals, even those which live under water. In a remarkable paper entitled Suspicions about some Hidden Qualities of the Air, he pointed to the

1 The reader is referred to the elaborate work of MiLne-Edwards (Legons sur la Physiologie, tome i., p. 375 et seq.) for a complete and highly interesting history of the physiology of respiration, from which we have taken most of the historical facts to which reference will be made. 
probable existence of some unknown vital substance in the atmosphere. A few years later it was demonstrated by Bernoulli, that the existence of aquatic animals depends upon air held in solution in the water. About this time Robert Hooke performed his celebrated experiment of exposing the lungs of a living animal, and maintaining the vital processes by artificial respiration. He demonstrated that asphyxia occurred when he ceased to change the air in the lungs, though these organs were allowed to remain distended.

Fracassati also showed that the red color of the upper surface of a clot of blood was due to its exposure to the air; and Lower, examining the blood before and after its passage through the lungs, in artificial respiration, showed that the red color of arterial blood depends on the renewal of the atmosphere.

In 1674, Mayow published his work on Respiration, in which he advanced the view that the air contained a principle, capable of supporting combustion, which is absorbed in respiration, changes venous into arterial blood, and is the cause of the heat which is developed in animal bodies. ${ }^{1}$ The importance of this discovery was not appreciated by the physiologists of that day; and it was more than a century before it received its appropriate place in science.

In 1757, Joseph Black, of Glasgow, isolated and studied carbonic acid, which he called fixed air. He recognized this gas in the expired air, by passing the breath through lime-

${ }^{1}$ We find the following passage in an analysis of the work of Mayow on Respiration, published in the Philosophical Transactions, 1668, p. 833 :

"The author *** delivers his thoughts on the use of Respiration, waving those opinions, that would have respiration either to cool the heart, or make the Bloud pass through the Lungs out of the right ventricle of the heart to the left, or to reduce the thicker venal blood into thinner and finer parts; and affirming, That there is something in the Air, absolutely neeessary to life, which is conveyed into the Bloud; which, whatever it be, being exhausted, the rest of the air is made useless, and no more fit for Respiration. Where yet he doth not exclude this use, That with the expelled Air, the vapors also, steaming out of the Bloud, are thrown out together." 
water. It is evident that this was the gas which was observed so many years before by Van Helmont.

In 1775 , Priestley discovered that the air is composed of oxygen and nitrogen, though he did not make use of these names; and a few years later, showed that air which has been vitiated by the respiration of animals is consumed by vegetables, which return the elements necessary to the life of animals. In a paper published in the Philosophical Transactions for 1776 , he proved that the change in the color of the blood in the lungs is due to the absorption of the newly discovered oxygen; and showed, furthermore, that the interchange of gases between the air and the blood can take place through membranes, as readily as when the two fluids are brought directly in contact with each other. ${ }^{1}$

The discoveries above enumerated, though all bearing on the great question, were simply isolated facts, and failed to develop any definite idea of the changes of the air and blood in respiration. The application of these facts was made by the great chemist Lavoisier; who was the first to employ the delicate balance in chemical investigation, and whose observations mark the beginning of an accurate knowledge of the function of respiration. With the balance, Lavoisier showed the nature of the oxides of the metals; he discovered that carbonic acid is formed by a union of carbon and oxygen; and, noting the consumption of oxygen and the production of carbonic acid in respiration, advanced, for the first time, the view that the one was employed in the production of the

\footnotetext{
${ }^{1}$ BÉRARD attributes the discovery of oxygen to Bayen (op. cit., tome iii., p. 328). It is true that Bayen in 1774 evolved oxygen by heating the red oxide of mercury, but he simply saw a gas given off, the nature and properties of which he did not describe. Priestley first published his discovery of oxygen, with a description of certain of its important properties, in the same year; and because he thus described properties which distinguish this from every other gas, to Priestley is generally, and justly, ascribed the honor of its discovery. Schecle, in Sweden, obtained and described oxygen ("the air of fire") shortly after it had been obtained by Priestley, without the knowledge that his discovery had been anticipated. His work was published in 1777 .
} 
other. Though, as should naturally be expected, the doctrines of this great observer have been modified with the advances in science, he developed facts which will stand forever, and which have served as the starting point of all our knowledge on this subject. From that time physiologists began to look on respiration as consisting in the appropriation of oxygen and the exhalation of carbonic acid; and now the seat of this process is only changed from the lungs to the tissues. From the limited knowledge of the intimate phenomena of nutrition which obtained in his day, Lavoisier could not be expected to entertain any other view than that the carbonic acid produced was the result of the direct union of oxygen with carbon in the blood. It is only since investigations have made manifest the great complexity of the processes of nutrition, that some are unwilling to believe that carbonic acid is produced in as simple a way as it appeared to Lavoisier. ${ }^{1}$

Composition of the Air.-Pure atmospheric air is a mechanical mixture of 79.19 parts of nitrogen with 20.81 parts of oxygen (Dumas and Boussingault). ${ }^{2}$ It contains in addition a very small quantity of carbonic acid, about one part in 2,000 by volume, and traces of ammonia. The air is never free from moisture, which is very variable in quantity, being generally more abundant at a high than at a low temperature. In 1840 , Schönbein discovered in the air a peculiar odorous principle called ozone, which he conceived to be a compound of oxygen and hydrogen $\left(\mathrm{HO}_{3}\right)$, but which is now pretty well shown to be an allotropic form of oxygen. The

${ }^{1}$ The applications of the discoveries of Lavoisier to the production of animal beat will be taken up in connection with that phenomenon.

2 Some chemists suppose that the oxygen and nitrogen in the air are in a condition of feeble chemical combination. However that may be, it is certain that in respiration it is the oxygen which is absorbed by the blood, and which carries on the function. The nitrogen seems to act simply as a diluent, thus providing that the blood in the lungs shall be exposed to but a certain quantity of the respiratory principle. 
oxygen which is obtained by decomposing water by the Voltaic pile is in this condition. It exists in very small quantity in the air, and plays no part in the function of respiration. Its chief interest has been in a theoretical connection with epidemic diseases. ${ }^{1}$ Floating in the atmosphere are a number of excessively minute organic bodies. Various odorous and other gaseous matter may be present as accidental constituents.

In considering the function of respiration, it is not necessary to take account of any of the constituents of the atmosphere, except oxygen and nitrogen; the others being either inconstant, or existing in excessively minute quantity. It is necessary to the regular performance of the function that the air should contain about four parts of nitrogen to one of oxygen, and have about the density which exists on the general surface of the globe. When the density is very much increased, as in mines, respiration is somewhat, though not gravely, disturbed. By exposure to a rarefied atmosphere, as in the ascent of high mountains or in aërial voyages, respiration may be very seriously interfered with, from the fact that less oxygen than usual is presented to the respiratory surface, and the reduced atmospheric pressure diminishes the capacity of the blood for holding gases in solution.

Magendie and Bernard, in experimenting on the minimum proportion of oxygen in the air which is capable of sustaining life, found that a rabbit, confined under a bell-glass with an arrangement for removing the carbonic acid and water ex-

1 Ozone may be formed by passing electric discharges through the ordinary atmosphere, or through oxygen. Its proportion in the air is supposed to be much increased in storms which are accompanied by electric phenomena. Schönbein exposed animals to the action of this substance. A dog, confined for an hour in a bell-glass, into which ozone was passed, died, though it was estimated that he absorbed only about 03 of a grain. An examination showed the lungs in a condition of acute inflammation. M. de la Rive, who has also experimented upon it, compares its action on the respiratory organs to that of chlorine (BERNARD, Leguns sur les Effets des Substances Toxiques et Médicamenteuses, Paris, 1857, p. 150). 
haled, as fast as they were produced, died of asphyxia when the quantity of oxygen became reduced to from 3 to 5 per cent. $^{1}$

Following Lavoisier, the Abbé Spallanzani, ${ }^{2}$ by researches on a great number of animals of all classes, demonstrated the universal necessity of air, either in a gaseous condition or in solution in liquids, throughont the animal kingdom.

A few experiments are on record in which the human subject and animals have been made to respire for a time pure oxygen. Though this is the gas which is essential in ordinary respiration, the process being carried on about as well in a mixture of oxygen with hydrogen as with nitrogen, the functions do not seem to be much altered when the pure gas is taken into the lungs. Some authors state that its prolonged inhalation exaggerates the function for a time, and that inflammation of the lungs and death follow its prolonged use; while the experiments of others show that it is harmless. Allen and Pepys confined animals for twentyfour hours in an atmosphere of pure oxygen, without any notable results ; $^{3}$ but, as is justly remarked by Longet, these experiments do not show that it would be possible to respire unmixed oxygen indefinitely without inconvenience. As it exists in the air, oxygen is undoubtedly in the best form for the permanent maintenance of the respiratory function. The blood seems to have a certain capacity for the absorption of oxygen, which is not increased when the pure gas is presented.

The only other gas which has the power of maintaining respiration, even for a time, is nitrous oxide. This is absorbed by the blood-corpuscles with great avidity, and for a time produces an exaggeration of the vital processes, with delirium, etc.-properties which have given it the common

1 Bernard, op. cit., p. 115.

2 Spallanzani, Mémoires sur la Respiration, traduits en Français d'après son manuscrit inédit, 1803.

" Longet, Traité de Physiologie, Paris, 1861, tome i., p. 458. 
name of the "laughing gas"; but this condition is followed by anæsthesia, and finally asphyxia, probably because the gas has such an affinity for the blood-corpuscles as to remain to a certain extent fixed, interfering with the interchange of gases which is essential to life. Notwithstanding this, experimenters have confined rabbits and other animals in an atmosphere of nitrous oxide for a number of hours. In all cases they became asphyxiated, but in some instances were restored on being brought again into the atmosphere. ${ }^{1}$

Other gases which may be introduced into the lungs either produce asphyxia, negatively, from the fact that they are not absorbed by the blood and are incapable of carrying on respiration, like hydrogen or nitrogen, or positively, by a poisonous effect on the system. The most important of the gases which act as poisons are, carbonic oxide, sulphuretted hydrogen, and arseniuretted hydrogen. It is somewhat uncertain whether carbonic acid exerts its deleterious influence as a poison, or as merely taking the place of the oxygen in the blood-corpuscles: It is easily displaced from the blood by oxygen, and therefore does not seem to possess the properties of a poison, like carbonic oxide, and some other gases, which become fixed in the blood, and are not readily displaced when fresh air is introduced into the lungs.

Consumption of Oxygen.-The determination of the quantity of oxygen which is removed from the air by the process of respiration is a question of great physiological interest, and one which engaged largely the attention of Lavoisier and those who have followed in his line of observation. On this point there is an accumulated mass of observations which are comparatively unimportant, from the fact that they were made before the means of analysis of the gases were as perfect as they now are. Though many of the results obtained by the older experimenters are interesting and instructive, as showing the comparative quantities of

${ }^{1}$ Lovget, op. cit., tome i., p. 460. 
oxygen consumed under various physiological conditions, they are not to be compared with the more recent observations, particularly those of Regnault and Reiset, Valentin and Brunner, Dumas, Andral and Gavarret, Scharling, and Edward Smith, with regard to the absolute quantity of oxygen made use of in respiration. In the observations of Regnault and Reiset, the animal to be experimented upon was enclosed in a receiver filled with air, a measured quantity of oxygen was introduced as fast as it was consumed by respiration, and the carbonic acid was constantly removed and carefully estimated. In most of the experiments, the confinement did not appear to interfere with the functions of the animal, which ate and drank in the apparatus, and was in as good condition at the termination as at the beginning of the observation. This method is infinitely more accurate than that of simply causing an animal to breathe in a confined space, when the consumption of oxygen and accumulation of carbonic acid and other matters must interfere more or less with the proper performance of the respiratory function. This is known as the direct method of investigating the changes in the air produced by respiration. As employed by Regnault and Reiset, it is only adapted to experiments on animals of small size. These give but an approximative idea of the processes as they take place in the human subject, as it is natural to suppose that the relative quantities of gases consumed and produced in respiration vary in different orders of animals. ${ }^{1}$

${ }^{1}$ In Robin's Journal de l'Anatomie et de la Physiologie, July, 1864, tome i., p. 429, we find an analysis of researches on respiration by Dr. Max Pettenkofer, in which the couditions for accurate observations on the human subject seem to be fulfilled. Dr. I'ettenkofer has construeted a chamber large enough to admit a man, and allow perfect freedom of motion, eating, sleeping, etc., into which air is con. stantly introduced in definite quantity, and from which the products of respiration are constantly removed, and estimated. An incomplete series of obserrations is published, which has particular reference to the produets of respiration. Thus far the subject of consumption of oxygen has not been considered. Extended observations by Dr. Pettenkofer will undoubtedly settle many disputed questions regarding the changes of the air in respiration. This method was adapted to the 
The indirect method was first employed by Boussingault, but was particularly direeted to the exhalation of carbonic acid. This observer experimented upon large animals, such as the horse or cow, in the following way: Having first carefully regulated the diet, so that there was no change in weight during the experiments, he earefully weighed all that was introduced as food and drink, and all that was discharged as urine and feces. The excess in the quantity introduced, over that discharged in the way above mentioned, represents. necessarily, the amount lost by the skin and lungs. By a quantitative comparison of the elementary constituents of the food and excrements, tolerably accurate results were arrived at; though it must be admitted that this method would be considered of little value, did the results not correspond pretty closely with those obtained by direct analysis. ${ }^{1}$

Estimates of the absolute quantities of oxygen consumed, or of carbonic acid produced, which are based on analyses of the inspired and expired air, calculations from the average quantity of air changed with each respiratory act, and the average number of respirations per minute, are by no means as reliable as analyses showing the actual changes in the air, like those of Regnault and Reiset, provided the physiological conditions be fulfilled. When there is so much multiplication and calculation, a very slight and perhaps unavoidable inaccuracy in the quantities consumed or pro duced in a single respiration will make an immense error in the estimate for a day, or even an hour.

Bearing all these sources of error in mind, from the experiments of Valentin and Brunner, Dumas, and others, a sufficiently accurate approximation of the proportion of oxygen consumed by the human subject may be formed. The air,

human subject on a small scale in 1843 , by Scharling, but there was no arrangement for estimating the quantity of oxygen furnished (Milne-Edwards, Physiologie, tome ii., p. 498 , note.)

${ }^{1}$ Boussingatlt, Mémoires de Chimie Agricole et de Physiologie, Paris, 1854, pp. 1-12. 
which contains, when inspired, 20.81 parts of oxygen per 100 , is found on expiration to contain but about 16 parts per 100. In other words, the volume of oxygen absorbed in the lungs is five per cent. or $\frac{1}{20}$ of the volume of air inspired. ${ }^{1}$

It is interesting and useful to extend this estimate as far as possible to the quantity of oxygen absorbed in a definite time ; for the regulation of the supply of oxygen where many persons are assembled, as in public buildings, hospitals, etc., is a question of great practical importance. Assuming that the average respirations per minute are 18 , and that with each act 20 cubic inches of air are changed, 15 cubic feet of oxygen are consumed in the twenty-four hours, which represents 300 cubic feet of pure air. This is the minimum quantity of air which is actually used, making no allowance for the increase in the intensity of the respiratory processes, which is liable to occur from various causes. To meet all the respiratory exigencies of the system, in hospitals, prisons, etc., it has been found necessary to allow at least 800 cubic feet of air for each person, unless the situation is such that the air is changed with unusual frequency; for, beside the actual loss of oxygen in the respired air, constant emanations from both the pulmonary and cutaneous surfaces are taking place, which should be removed. In some institutions as much as 2,500 cubic feet of air is allowed to each person. ${ }^{2}$

The quantity of oxygen consumed is subject to great variations, depending upon temperature, the condition of the digestive system, muscular activity, etc. The following conclusions, the results of the observations of Lavoisier and Séguin, give at a glance the variations from the above-mentioned causes : ${ }^{3}$

${ }^{1}$ Mrluxe-Edwards, Physiologie, tome ii., p. 510.

2 Tond and Bowman, Physiological Anatomy and Physiology of Man, Philadelphia, 1857 , p. 728.

${ }^{3}$ Taken from Longet, Traité de Physiologie, Paris, 1861, tome i., p. 526. Though the absolute quantities obtained by Lavoisier and Séguin are not so reliable as those ubtained by later observers, yet the accurate employment of the 
"1. A man, in repose and fasting, with an external temperature of $90^{\circ} \mathrm{Fahr}$., consumes 1,465 cubic inches of oxygen per hour.

"2. A man, in repose and fasting, with an external temperature of $59^{\circ}$ Fahr., consumes 1,627 cubic inches of oxygen per hour.

"3. A man, during digestion, consumes 2,300 cubic inches of oxygen per hour.

"4. A man, fasting, while he accomplishes the labor necessary to raise, in fifteen minutes, a weight of $7 \cdot 343$ kil. (about $16 \mathrm{lb} .3 \mathrm{oz}$. av.) to the height of 656 feet, consumes 3,874 cubic inches of oxygen per hour.

"5. A man, during digestion, accomplishing the labor" necessary to raise, in fifteen minutes, a weight of $7 \cdot 343 \mathrm{kil}$. (about $16 \mathrm{lb} .3 \mathrm{oz}$. av.) to the height of 700 feet, consumes 5,568 cubic inches of oxygen per hour."

Influence of Temperature.-All who have experimented on the influence of temperature upon the consumption of oxygen, in the warm-blooded animals and in the human subject, have noted a marked increase at low temperatures. Cold-blooded animals always suffer a depression of the vital processes at low temperatures, with a corresponding diminution in the quantity of oxygen consumed, until they finally become torpid.

Immediately after birth, the consumption of oxygen in the warm-blooded animals is relatively very slight. Buffon ${ }^{1}$ and Legallois ${ }^{2}$ bave shown that just after birth, dogs and other animals will live for half an hour or more under water; and cases are on record where life has been restored in newlyborn children after seven, and, it has been stated, after twentythree hours of asphyxia. During the first periods of existence, the condition of the newly-born approximates to that of a

best means of investigation at their command leads us to place every confidence in the comparative results.

${ }^{3}$ Milne-Edwards, Physiolngie, tome ii., p. 559.

${ }^{2}$ Legallors, Euvres, Paris, 1824, tome i., p. 57. 
cold-blooded animal. The lungs are relatively very small, and it is some time before they fully assume their function. The muscular movements are hardly more than is necessary to take the small amount of nourishment consumed at that period, and nearly all of the time is passed in sleep. There is also very. little power of resistance to low temperature. Though accurate researches regarding the comparative quantities of oxygen in the venous and arterial blood of the foetus are wanting, it has been frequently observed that the difference in color is not as marked as it is after pulmonary respiration becomes established. The direct researches of W. F. Edwards have shown that the absolute consumption of oxygen by very young animals is very small $;^{1}$ and the observations of Legallois on rabbits, made every five days during the first month of existence, show a rapidly increasing demand for this principle with age. ${ }^{2}$

Regnault and Reiset have shown that the consumption of oxygen is greater in lean than in very fat animals, provided they be in perfect health. They have also shown that the consumption is much greater in carnivorous than in herbivorous animals; and in animals of different sizes, is relatively very much greater in those which are very small. In very small birds, such as the sparrow, the proportional quantity of oxygen absorbed was ten times greater than in the fowl. ${ }^{3}$

In sleep, the quantity of oxygen consumed is considerably

¿ De l'Influence des Agens Physiques sur la Vie, Paris, 1824, p. 178 et seq.

${ }^{2}$ Loc. cit. In his experiments on rabbits, Legallois found that immmediately after birth they would live for fifteen minutes deprived of air. "In asphyxiating rabbits of different ages, for example, every five days, from the moment of birth to the age of one month, it was constantly observed that the duration of sensation, of voluntary motion, in a word, the signs of life, always diminished in proportion as the animals advanced in age. Thus, in a rabbit newly born, sensation and voluntary movements were not extinet until the end of about fifteen minutes of asphyxia, while they were extinct in less than two minutes in a rabbit of the age of thirty days." Рp. 57, 58.

soc. cit. 
diminished; and in hibernation is so small, that Spallanzani could not detect any difference in the composition of the air in which a marmot, in a state of torpor, had remained for three hours. ${ }^{1}$ In experiments on a marmot in hibernation, Regnault and Reiset observed a reduction in the quantity of oxygen consumed to about $\frac{1}{30}$ of the normal standard. ${ }^{2}$

It has been shown by experiments, that the consumption of oxygen bears a pretty constant ratio to the production of carbonic acid; and as the observations on the influence of sex, number of respiratory acts, etc. on the activity of the respiratory processes, have been made chiefly with reference to the carbonic acid exhaled, we will consider these influences in connection with the products of respiration.

Experiments on the effect of increasing the proportion of oxygen in the air have led to varied results in the hands of different observers. Regnault and Reiset, whose observations on this point are generally accepted, did not discover any increase in the consumption of oxygen when this gas was largely in excess.

The results of confining an animal in an atmosphere composed of 21 parts of oxygen and 79 parts of hydrogen are very curious and instructive. When hydrogen is thus substituted for the nitrogen of the air, the consumption of oxygen is largely increased. Regnault and Reiset attribute this to the superior refrigerating power of the hydrogen; but a more rational explanation would seem to be in its superior diffusibility. Hydrogen is the most diffusible of all gases; and when introduced into the lungs in the place of the nitrogen of the air, the vitiated air, charged with carbonic acid, is undoubtedly more readily removed from the deep portions of the lungs, giving place to the mixture of hydrogen and oxygen; and it is probably for this reason that the quantity of oxygen consumed is increased. It is probable that the

'Spallaxzaxi, Mémoires sur la Respiration, traduites par Senebier, Genive, 1803, p. 334 .

Op. cit., p. 442. 
nitrogen of the air plays an important part in the phenomena of respiration by virtue of its degree of diffusibility.

In view of the great variations in the consumption of oxygen dependent on different physiological conditions, such as digestion, exercise, temperature, etc., it is impossible to fix upon any number which will represent, even approximatively, the average quantity consumed per hour. The estimate arrived at by Longet, ${ }^{1}$ from a comparison of the results obtained by different reliable observers, is perhaps as near the truth as possible. This estimate puts the hourly consumption at from 1,220 to 1,525 cubic inches, "in an adult male, during repose and in normal conditions of health and temperature."

In passing through the lungs, the air, beside losing a proportion of its oxygen, undergoes the following changes:

1. Increase in temperature.

2. Gain of carbonic acid.

3. Gain of watery vapor.

4. Gain of ammonia.

5. Gain of a small quantity of organic matter.

6. Gain, and occasionally loss, of nitrogen.

The elevation in temperature of the air which has passed through the lungs has been carefully observed by Dr. Gréhant. ${ }^{2}$ He found that with an external temperature of $72^{\circ}$, respiring 17 times per minute, the air taken in by the nares and expired by the mouth, through an apparatus containing a thermometer carefully protected from external influences, marked a temperature of $954^{\circ}$. Taking in the air by the mouth, the temperature of the expired air was $93^{\circ}$. At the commencement of the expiration, Dr. Gréhant noted a temperature of $94^{\circ}$. After a prolonged expiration, the temperature was $96^{\circ}$. In these observations the temperature taken beneath the tongue was $98^{\circ}$.

¿ Op. cit., p. 531 .

${ }^{2}$ Grémant, Recherches Physiques sur la Respiration de l'Homme. Journal de I Anatomie et de la Physiologie, 1864, tome i, p. 546. 
Valentin had previously made experiments on this point, and put the temperature of the expired air a little higher, i. e., about $99^{\circ}$, with an external temperature of $68^{\circ}$. He also showed that the temperature of the surrounding atmosphere exerted an important influence on the temperature of the expired air. In an observation made in winter, with an external temperature of $18^{\circ}$, the temperature of the expired air was only $85 \cdot 5^{\circ} .^{1}$

Exhalation of Carbonic Acid.-The production of carbonic acid in the respiratory process is as universal as the consumption of oxygen. Experiments have shown that all animals during life exhale this prineiple, as well as all tissues, so long as they retain their irritability. This takes place, not only when the animals or tissues are placed in an atmosphere of oxygen, or common air, but, as was observed by Spallanzani, ${ }^{2}$ in an atmosphere of pure nitrogen or hydrogen. This fact has since been noted by W. F. Edwards, J. Müller, G. Liebig, and others.

The study of the exhalation of carbonic acid presents several problems of great physiological interest:

1. What is the absolute quantity of carbonic acid exhaled by the lungs in a given time?

2. What are the variations in the exhalation of this principle due to physiological influences?

3. What is the relation between the quantity of carbonic acid produced and the quantity of oxygen consumed ?

On account of the variations in the quantities of carbonic acid exhaled at different periods of the day, and particularly the great influence of the rapidity of the respiratory movements, it is exceedingly difficult to fix upon any number which will represent the average proportion of this gas contained in the expired air. The same influences were found affecting the consumption of oxygen; and the same difficulties

${ }^{2}$ GréHaxt, Recherches Physiques sur la Respiration de l'Homme. Journal de ¿Anatomie et de la Physiologie, 1864, tome i., p. 545.

Op. cit., p. 343. 
were experienced in forming an estimate of the proportion of this gas consumed. As we assumed, after a comparison of the results obtained by different observers, that the volume of oxygen consumed is about five per cent. of the entire volume of air, it may be stated as an approximation, that in the intervals of digestion, in repose, and under normal conditions as regards the frequency of the pulse and respiration, the volume of carbonic acid exhaled is about four per cent. of the volume of the expired air. ${ }^{1}$ As the volume of the oxygen which enters into the composition of a definite quantity of carbonic acid is precisely equal to the volume of the carbonic acid, it is seen that a certain quantity of oxygen disappears in respiration, and is not represented in the carbonic acid exhaled.

There are great differences in the proportion of carbonic acid in the expired air, depending upon the time during which the air has remained in the lungs. This interesting point has been studied by Vierordt, in a series of 94 experiments made upon his own person, with the following results : ${ }^{2}$

"When the respirations are frequent, the quantity of carbonic acid expelled at each expiration is much less than in a slow expiration; but the quantity of carbonic acid produced during a given time by frequent respirations is greater than that which is thrown off by slow expirations." s

The air which escapes during the first period of an expiration is naturally less rich in carbonic acid than that which is last expelled and comes directly from the deeper portions of the lungs. Dividing, as nearly as possible, the expiration into two equal parts, Vierordt found, as the mean of twenty-

${ }^{1}$ Mrlne-Edwards, Physiologie, tome ii., p. 507. This approximation is taken from the observations of Valentin and Brunner, Dalton, Prout, Apjohn, Coathupe, Horn, and Vierordt. The experiments of Vierordt are, perhaps, entitled to the most credit, as he has studied very carefully the influence of the frequency of respiration upon the quantity of carbonic acid exhaled.

${ }^{2}$ Cited in Muxe-Entrands, Physiologie, tome i., p. 574, and Bérard, Cours de Physiologie, tome iii., p. 349.

' Bérard, loc. cit. 
one experiments, a percentage of 3.72 in the first part of the expiration, and 5.44 in the second part.

Temporary arrest of the respiratory morements, as we should expect, has a marked influence in increasing the proportion of carbonic acid in the expired air; though the absolute quantity exhaled in a given time is diminished. In a number of experiments on his own person, Vierordt ascertained that the percentage of carbonic acid becomes uniform in all parts of the respiratory organs, after holding the breath for 40 seconds. Holding the breath after an ordinary inspiration for 20 seconds, the percentage of carbonic acid in the expired air was increased 1.73 over the normal standard; but the absolute quantity exhaled was diminished by $2 \cdot 642$ cubic inches. After taking the deepest possible inspiration, and holding the breath for 100 seconds, the percentage was increased 3.08 above the normal standard; but the absolute quantity was diminished more than 14 cubic inches. ${ }^{2}$ Allen and Pepys state that air which has passed 9 or 10 times through the lungs contains $9 \cdot 5$ per cent. of carbonic acid. ${ }^{3}$

Vierordt gives the following formula as representing the influence of the frequency of the respirations on the production of carbonic acid: Taking 2.5 parts per hundred as representing the constant value of the gas exhaled by the blood, the increase over this proportion in the expired air is in exact ratio to the duration in the contact of the air and blood. This, though it may hold good in many instances, seems rather an excessive refinement. ${ }^{4}$

${ }^{1}$ Lehmans, Physiological Chemistry, Philadelphia, 1855, vol. ii., p. 439.

${ }^{2}$ Cyclopadia of Anatomy and Physiology, vol. iv., part 1, p. 352.

3 Ibid.

${ }^{4}$ The following table gives at a glance the most important results of these experiments :

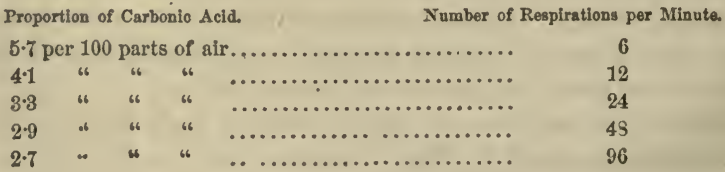


The absolute quantity of carbonic acid exhaled in a given time is a more important subject of inquiry than the proportion contained in the expired air ; for the latter is constantly varying with every modification in the number and extent of the respiratory acts, and the volume of breathing air is subject to great fluctuations, and is very difficult of determination. The direct method, in which the actual products of respiration are collected and estimated, has led to very important results, which have been confirmed to a certain extent by Boussingault, Barral, and others who have employed the indirect method. It is by the direct method, in the hands of Regnault and Reiset, Andral and Gavarret, and more recently Dr. Edward Smith, that we have learned so much regarding the physiological variations in the prodncts of respiration; one of the most important considerations connected with the subject.

Among the most reliable observations on the quantity of carbonic acid exhaled by the human subject in a definite time, and the variations to which it is subject, are those of Andral and Gavarret, ${ }^{1}$ and Dr. Edward Smith. ${ }^{2}$ The observations of Lavoisier and Séguin, Prout, Davy, Dumas, Allen and Pepys, Scharling, and others, have none of them seemed to fulfil the necessary experimental conditions so completely. Scharling's method was to enclose his subject in a tight box, with a capacity of about 27 cubic feet, to which air was constantly supplied; but the observations were comparatively few, being made on only six persons. In his observations, the quantities of gas exhaled must have been considerably modified by the elevation of temperature and exhalation of moisture in so small a space. ${ }^{3}$ The mental condition of the

${ }^{1}$ Recherches sur la Quantité d'Acide Carbonique exhalé par les Poumons dans l'Espèce Humaine. Annalcs de Chimie et de Physique, 3me série, tome viii., p. 129.

${ }^{2}$ Edward SuIth, Experimental Inquiries into the Chemical and other Phenomena of Respiration, and their Modifications by various Physical Agencies (Plilosophical Transactions, 1859, p. 681); and On the Action of Foods upon the Respiration during the Primary Processes of Digestion (Ibid., p. 715).

${ }^{3}$ Annales de Chim. et de Phys., tome viii., p. 488. Scharling recognized the 
subject of an experiment has an influence upon the products of respiration, and the function is sometimes modified from the mere fact that an experiment is being performed; an influence which Scharling did not fail to recognize, but which frequently cannot be guarded against.

The observations of Andral and Gavarret were made on sixty-two persons of both sexes and different ages, and under absolutely identical conditions as regards digestion, time of the day, barometric pressure, and temperature. The products of respiration were collected in the following way: A thin mask of copper covering the face, and large enough to contain an entire expiration, was fitted to the face by its edges, which were provided with India-rubber, so as to make it air-tight. At the upper part was a plate of glass for the admission of light, and at the lower part an opening, which allowed the entrance of air, but was provided with a valve preventing its escape. By another opening the mask was connected by a rubber tube with three glass balloons, capable of holding 8,544 cubic inches, in which a vacuum was previously established. With the mask fixed upon the face, and a stop-cock opened, connected with the balloons, so as to graduate the current of air, the subject respires freely in the current which comes from the exterior into the receivers. In this way, though the quantity of air respired is not measured, the vacuum in the receivers draws in the products of respiration. The current will continue for from 8 to 13 minutes, and is so regulated that the air is respired but once. The quantity of carbonic acid in the receivers represents the quantity produced during the time that the experiment has been going on.

By carefully fulfilling all the physiological conditions,

necessity of guarding against the influence of elevation of temperature and accumulation of moisture, and attempted to remove the latter by introducing a vessel of sulpluric acid. His greatest difficulty was in the analyses of the air. Though the results obtained are valuable, the process cannot claim the accuracy attained by Andral and Gavarret. 
regulating the number of respirations, as far as possible, to the normal standard, different observations on the same subject, at different times, under the same conditions, were attended with results so nearly identical, as to give every confidence in the accuracy of the process. But even then, these observers recognized such immense variations in the exhalation of carbonic acid with the constantly varying physiological conditions, that they did not feel justified in taking their observations as the basis for calculations of the entire quantity exhaled in the twenty-four hours.

The results of these observations on the male, between the ages of sixteen and thirty, between 1 and 2 P.M., under identical conditions of the digestive and muscular systems, each experiment lasting from eight to thirteen minutes, showed an exhalation of about 1,220 cubic inches of carbonic acid per hour.

Dr. Edward Smith, ${ }^{1}$ in his elaborate paper on the phenomena of respiration, employed a very rigorous method for the estimation of the carbonic acid exhaled. He used a mask, fitting closely to the face, which covered only the airpassages. The air was admitted after being measured by passing through an ordinary dry gas-meter. The expired air was passed through a drying apparatus, and the carbonic acid absorbed by a solution of potash, arranged in a number of layers, so as to present a surface of about 700 square inches, and carefully weighed. This apparatus was capable of collecting all the carbonic acid exhaled in an hour. The estimate was made for 18 waking hours and 6 hours of sleep. The observations for the 18 hours were made on four persons, namely: Dr. Smith, æt. 38 years, weighing 196 pounds, 6 feet high, with a vital capacity of 280 cubic inches; Mr. Chouls, æt. 48 years, 5 feet $9 \frac{1}{2}$ inches high, 175 pounds weight; Dr. Murie, æt. 26 years, 5 feet $7 \frac{1}{2}$ inches high, 133 pounds weight, vital capacity 250 cubic inches; Prof. Frankland, æt. 33 years, 5 feet $10 \frac{1}{2}$ inches high, and 136 pounds

${ }^{1}$ Loc. cit. 
weight. Breakfast was taken at $8 \frac{1}{2}$ A.M., dinner at $1 \frac{1}{2}$, tea at $5 \frac{1}{2}$, and supper at $8 \frac{1}{2}$ P.M. The observations occupied ten minutes, and were made every hour and half-hour for 18 hours. The average for the 18 hours gave 20,082 eubic inches of carbonic acid for the whole period. Observations during the 6 hours of sleep showed a total exhalation of 4,126 cubic inches. This, added to the quantity exhaled during the day, gives as the total exhalation in the twenty-four hours, during complete repose, 24,208 cubic inches (about 13.45 cubic feet), containing $7 \cdot 144 \mathrm{oz}$. av. of carbon. ${ }^{1}$

Considering the great variations in the exhalation of calbonic acid, this estimate can be nothing more than an approximation. One of the great modifying influences is muscular exertion, by which the production of carbonic acid is largely increased. This would indicate a larger quantity during ordinary conditions of exercise, and a much larger quantity in the laboring classes. Dr. Smith gives the following approximate estimates of these differences: ${ }^{2}$

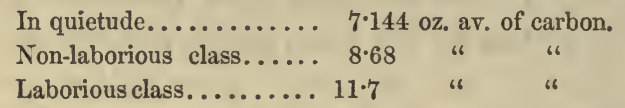

In studying the variations in the exhalation of carbonic acid, important information has been derived from experiments by many observers on the inferior animals, as well as from the observations of Dumas, Prout, Scharling, and others on the human subject. The principal conditions which influence the exhalation of this principle are:

Age and sex; activity or repose of the digestive system; form of diet; sleep; muscular activity ; fatigue; moisture, and surrounding temperature; season of the year.

${ }^{1}$ Op. cit., p. 692 . In these calculations there is a slight arithmetical error; but it makes a difference of only 40 cubic inches of gas in the estimate for the 24 hours. In the original paper, the quantity is given by weight. We have reduced it to cubic inches, assuming that 100 cubic inches of gas weigh $47 \cdot 26$ grains.

${ }^{2}$ Op. cit., p. 693. 
Influence of Age.-In treating of the consumption of oxygen, it was stated that during the first few days of extrauterine existence, the demand for oxygen on the part of the system is very slight. At this period there is a correspondingly feeble exhalation of carbonic acid. It is well known that during the first hours and days after birth the new being has little power of generating heat, needs constant protection from changes in temperature, and the voluntary movements are very imperfect. During the first few days, indeed, the infant does little more than sleep and take the small quantity of colostrum which is furnished by the mammary glands of the mother. While the animal functions are so imperfectly developed, and until the nourishment becomes more abundant and the child begins to increase rapidly in weight, the quantity of carbonic acid exhaled is very small.

After the respiratory function becomes fully established, it is probable, from the greater number of respiratory movements in early life, that the production of carbonic acid, in proportion to the weight of the body, is greater in infancy than in adult life. Direct observations, however, are wanting on this point.

The observations of Andral and Gavarret ${ }^{1}$ show the comparative exhalation of carbonic acid in the male, from the age of twelve to eighty-two, and give the results of a single observation at the age of one hundred and two years. They show an increase in the absolute quantity exhaled from the age of twelve to thirty-two; a slight diminution fiom thirty-two to sixty; and a considerable diminution from sixty to eightytwo. These results are given in the following table:

Carbonic acid exhaled per hour.

In boys from twelve to sixteen years........... 915 cubic inches.

In young men from seventeen to nineteen years...... " 1,220 " "

In men from twenty-five to thirty-two years........ 1,343 " "

In men from thirty-two to sixty years............ " "

In men from sixty-three to eighty-two years........ 933 " "

In an old man of one hundred and two years....... 671 " *

${ }^{1}$ Loc. cit. 
Taking into consideration the increase in the weight of the body with age, it is evident that the respiratory activity is much greater in youth than in, adult life. Andral and Gavarret do not give the weight of the subjects of their observations, but as the weight generally does not diminish after maturity, there can be no doubt that there is a rapid diminution in the relative quantity of carbonic acid produced in old age.

Scharling, in a series of observations on a boy nine years of age, weighing 48.5 pounds, an adult of twenty-eight, and one of thirty-five years, the latter weighing 163.6 pounds, showed that the respiratory activity in the child was nearly twice as great, in proportion to his weight, as the average in the adults. ${ }^{1}$ It is seen fiom the observations of Andral and Gavarret, that the absolute increase in the exhalation of carbonic acid from childhood to adult life is very slight in comparison with the natural increase in the weight of the body; showing that, proportionately, the exhalation of carbonic acid is greater in early life.

Influence of Sex.-All observers have found a marked difference between the sexes, in favor of the male, in the proportion of carbonic acid exhaled. Andral and Gavarret noted an absolute difference of about forty-five cubic inches per hour, but did not take into consideration the difference in the weight of the body. Scharling, taking the proportion exhaled to the weight of the body, noted a marked difference in favor of the male.

The difference in the degree of muscular activity in the sexes is sufficient to account for the greater evolution of carbonic acid in the male, for this principle is exhaled in pro-

${ }^{1}$ Scharlivg, Recherches sur la Quantité d'Acide Carbonıque expiré par l'Homme. Annales de Chimie et de Physique, 3me série, tome viii., p. 486.

Taking the proportion of earbonic acid exhaled per hour to the weight, in the man 28 years of age, as 1 , in the man 35 years of age the proportion was $1 \cdot 14$, and in the boy $9 \frac{1}{2}$ years of age, $2 \cdot 07$. P. 489 . 
portion to the muscular development of the individual; but there is an important difference connected with the variations with age, which depends upon the condition of the generative system of the female.

The absolute increase in the evolution of carbonic acid with age in the female is arrested at the time of puberty, and remains stationary during the entire menstrual period, provided the menstrual flow occur with regularity. During this time, the average exhalation per hour is 714 cubic inches. After the cessation of the menses, the quantity gradually increases, until at the age of sixty it amounts to 915 cubic inches per hour. From the age of sixty to eighty-two, the quantity diminishes to 793 , and finally to 670 cubic inches.

When the menses are suppressed, there is an increase in the exhalation of carbonic acid, which continues until the flow becomes reëstablished. In a case of pregnancy the exhalation was increased to about 885 cubic inches. ${ }^{1}$

Influence of Digestion.-Almost all observers agree that the exhalation of carbonic acid is increased during digestion. Lavoisier and Séguin found that in repose and fasting, the quantity exhaled per hour was 1,210 cubic inches; which was raised to 1,800 and 1,900 during digestion. ${ }^{2}$ Numerous experiments on animals have confirmed this statement. A very interesting series of observations on this point was made by Vierordt upon his own person. Taking his dinner at from 12.30 to 1 P. M., having noted the frequency of the pulse and respirations and the exhalation of carbonic acid at 12, he found at 2 P. M. the pulse and respirations increased in frequency, the volume of expired air augmented, and that the carbonic acid exhaled had increased from $15 \cdot 77$ to 18.22 cubic inches per minute. In order to ascertain that this

1 The above facts, showing the peculiar influence of the condition of the generative organs in the female, are among the most important results of the observations of Andral and Gavarret. Loc, cit.

2 Cyclopcedice of Anatomy and Physiology, vol. iv., part i., pp. 346, 347. 
variation did not depend upon the time of day, independently of the digestive process, he made a comparison at 12 M., at 1 and at 2 P. M., without taking food, which showed no notable variation, either in the pulse, number of respirations, volume of expired air, or quantity of carbonic acid exhaled. ${ }^{2}$

It is unnecessary to cite other observations on this point, unless we mention those of Prout and Coathupe, which seemed to show a diminution in the exhalation of carbonic acid during digestion. Dr. John Reid, in the Cyclopadia of Anatomy and Physiology, points out the source of error in these observations. ${ }^{2}$ Prout did not estimate the actual quantity of gas exhaled, but only its proportion in the expired air ; and it has been demonstrated that in digestion the volume of the expirations is notably increased. Coathupe, in the observations on his own person, took a pint of wine with his dinner. As it has been shown by experiment that alcohol has the effect of rapidly reducing the exhalation of carbonic acid, this observation does not represent the simple influence of digestion.

There can be no doubt, then, that the exhalation of carbonic acid is notably increased during the functional activity of the digestive system.

The effect of inanition is to gradually diminish the exhalation of carbonic acid. This fact was long since demonstrated by Spallanzani on caterpillars, and Marchand on frogs; but observations on the warm-blooded animals are more applicable to the human subject. Bidder and Schmidt noted the daily production of carbonic acid in a cat which was subjected to eighteen days of inanition, at the end of which time it died. The quantity diminished gradually from day to day, until just before death it was reduced a little more than one-half. Dr. Smith ${ }^{3}$ noted in his own person

1 Cyclopcedia of Anatomy and Physiology, vol. iv., part i., pp. 346, 347 .

${ }^{2}$ Ilid., article Respiration.

Op. cit., p. 696. 
the influence of a fast of twenty-seven hours. There was a marked diminution in the quantity of air respired, in the quantity of vapor exhaled, in the number of respirations, and in the rapidity of the pulse. The exhalation of carbonic acid, was diminished one-fourth. An interesting point in this observation was the fact that the quantity was as small four and a half hours after eating, as at the end of the twentyseven hours. "An increase of carbonic acid in the absence of food, at or near the periud when it is usually increased by food," was also noted in the experiment of Dr. Smith.

Influence of Diet.--Regnault and Reiset, in their experiments on animals, studied the effect of different kinds of diet upon the relations of the quantity of oxygen absorbed to the carbonic acid exhaled. About the only conclusive and extended series of investigations on the influence of diet upon the absolute quantity of carbonic acid exhaled are those of Dr. Smith. This observer made a large number of experiments on the influence of various kinds of food, and extended his inquiries into the influence of certain beverages, such as tea, coffee, cocoa, malt and fermented liquors. ${ }^{1} \mathrm{We}$ have already fully described the method employed in these experiments, and the conclusions, which are of great interest and importance, are very exact and reliable.

Dr. Smith divides food into two classes, one which increases the exhalation of carbonic acid, which he calls respiratory excitants, and the other, which diminishes the exhalation, which he calls non-exciters.

The following are the results of a large number of carefully conducted observations upon four persons :

"The excito-respiratory are nitrogeneous food, milk and its components, sugars, rum, beer, stout, the cereals, and potato.

"The non-exciters are starch, fat, certain alcoholic com-

${ }^{1}$ On the Action of Foods on the Respiration during the Primary Processes of Digestion. Philosophical Transactions, 1859, p. 715 
pounds, the volatile elements of wines and spirits, and coffee leaves.

"Respiratory excitants have a temporary action; but the action of most of them commences very quickly, and attains its maximum within one hour.

"The most powerful respiratory excitants are tea and sugar; then coffee, rum, milk, cocoa, ales, and chicory; then casein and gluten, and lastly, gelatin and albumen. The amount of action was not in uniform proportion to their quantity. Compound aliments, as the cereals, containing several of these substances, have an action greater than that of any of their elements.

"Most respiratory excitants, as tea, coffee, gluten, and casein, cause an increase in the evolution of carbon greater than the quantity which they supply, whilst others, as sugar, supply more than they evolve in this excess, that is, above the basis. No substance containing a large amount of carbon evolves more than a small portion of that carbon in the temporary action occurring above the basis line, and hence a large portion remains unaccounted for by these experiments."

The comparative observations of Dr. Smith upon the four persons who were the subjects of experiment demonstrated one very important fact: namely, that the action of different kinds of food upon respiration is modified by idiosyncrasies, and the tastes of different individuals. For example, in experiments on his own person, eertain articles which were agreeable to him excited the exhalation of carbonic acid; but in experimenting with the same articles upon Mr. Choul, to whom they were distasteful, he found the respiratory action diminished.

Quite a number of observers have noted the influence of alcohol upon the products of respiration; but the results of experiments have not been entirely uniform. Prout observed a constant diminution in the quantity of carbonic acid exhaled, under the influence of alcohol. This has been confirm 
ed by the observations of Horn, Vierordt, and many others; but Hervier and Saint-Lager assert that the use of alcohol increases the exhalation of carbonic acid. ${ }^{1}$ In the experiments of Prout, a small quantity of wine taken fasting caused the proportion of carbonic acid in the expired air to fall immediately from 4 to 3 parts per 100. During the four hours following, it oscillated between $3 \cdot 40,3 \cdot 10$, and 3 . The administration of a second dose, followed by some symptoms of intoxication, diminished the proportion to $2 \cdot 70$ per 100 . Dr. Fyfe, of Edinburgh, showed that the depressing effects of an alcoholic excess were continued into the following day. ${ }^{2}$ Dr. Hammond, in an elaborate and excellent paper on the effects of alcohol and tobacco on the human system, observed a diminished exhalation of carbonic acid following the ingestion of twelve drachms of alcohol daily for five days, both when the system was kept at the normal standard of weight, etc., by the ingestion of the habitual quantity of food, when the weight was diminished by an insufficient diet, and when the weight was increased by an excessive diet. ${ }^{3}$

The observations of Dr. Smith, which were all made fasting, show a certain variation in the effects of different alcoholic beverages. His results are briefly the following:

"Brandy, whiskey, and gin, and particularly the latter. almost always lessened the respiratory changes recorded, whilst rum as commonly increased them. Rum and milk had a very pronounced and persistent action, and there was no effect on the sensorium. Ale and porter always increased them, whilst sherry wine lessened the quantity of air inspired, but slightly increased the carbonic acid evolved.

${ }^{2}$ Milne-Edwards, Physiologie, Paris, 1857, tome ii., p. 535.

${ }^{2}$ Ibid. Prout took cognizance only of the proportion of carbonic acid in the expired air, and not of the absolute quantity exhaled in a given time.

' Wr. A. Hamond, M. D., The Physiological Effects of Alcohol and Tobacco upon the Human System. Physiological Memoirs, Philadelphia, 1863. In this valuable paper the author considers the general influence of alcohol and tobacco on nutrition, as indicated by the production of urea, carbonic acid, and other excrementitious principles, and the variations in the weight of the body. 
"The volatile elements of alcohol, gin, rum, sherry, and port-wine, when inhaled, lessened the quantity of carbonic acid exhaled, and usually lessened the quantity of air inhaled. The effect of fine old port-wine was very decided and uniform; and it is known that wines and spirits improve in aroma and become weaker in alcohol by age. The excitorespiratory action of rum is probably not due to its volatile elements."

From these facts, it would seem that the most constant effect of alcohol, and alcoholic liquors, such as wines and spirits, is to diminish the exhalation of earbonic acid. This effect is almost instantaneous, when the articles are taken into the stomach fasting; and when taken with the meals, the increase in carbonic acid which habitually accompanies the process of digestion is materially lessened. Rum, which Dr. Smith found to be a respiratory excitant, is an exception to this rule. Malt liquors seem to increase the exhalation of carbonic acid. With regard to alcohol itself, Dr. Smith says: "The action of pure álcohol was much more to increase than to lessen the respiratory changes, and sometimes the former effect was well pronounced." "

Regarding as one of the great sources of carbonic acid the development of this principle in the tissues, whence it is taken up by the blood, Dr. Smith attributes the grateful and soothing influence of tea, coffee, eau sucrée, and the other beverages which he classes as respiratory excitants, to their action in facilitating, the removal of this principle from the system. The presence of carbonic acid in the tissues and in the blood produces a sense of malaise, or depression, which we should suppose would be relieved by any thing which facilitates its elimination. It is undoubtedly this indefinite sense of discomfort which induces the act of sighing, by which the air in the lungs is more effectually renovated. This view is sustained by the fact that intellectual fatigue and mental emotions diminish the exhalation of carbonic acid. 
Apjohn cites an instance in which the proportion of carbonic acid in the expirations was reduced to 2.9 parts per 100 under the influence of mental depression. ${ }^{2}$

Dr. Hammond could not determine any modification in the exhalation of carbonic acid under the influence of tobacco. ${ }^{2}$

Influence of Sleep.-All who have directed attention to the influence of sleep upon the respiratory products have noted a marked diminution in the exhalation of carbonic acid; but we again recur to the experiments of Dr. Smith for exact information on this point. Dr. Smith estimated the quantity of carbonic acid exhaled during six hours of sleep, at night, at 4,126 cubic inches. According to this observer, the quantity during the night is to the quantity during the day, in complete repose, as 10 is to 18. During a light sleep, the exhalation was $10 \cdot 32$, and during profound sleep, 9.52 cubic inches per minute.

We have alluded to the great diminution in the quantity of oxygen consumed in hibernating animals, while in a torpid condition. Regnault and Reiset found that a marmot in hibernation consumed only $\frac{1}{30}$ of the oxygen which he used in his active condition. In the same animal they noted an exhalation of carbonic acid equal to but little more than half the weight of oxygen absorbed; so that in this condition the diminution in the exhalation of carbonic acid is proportionately even greater than in the consumption of oxygen."

Influence of Muscular Activity.-All observers, except Prout," agree that there is a considerable increase in the

${ }^{2}$ Hilne-Edwards, Physiologie, tome ii., p. 535.

${ }^{2}$ Op. cit.

${ }^{3}$ Regnault and Reiset, Annales de Chimie et de Physique, 3me série, tome זxvi., p. 446. The marmot consumed in five days 13,088 grammes of oxygen, and exhaled 7,174 grammes of carbonic acid.

4 Prout only noted the proportion of carbonic acid in the expired air; and as exercise has the effect of immediately and largely increasing the number of respi- 
exhalation of carbonic acid during and immediately following muscular exercise. In insects, Mr. Newport has found that a greater quantity is sometimes exhaled in an hour of violent agitation, than in twenty-four hours of repose. In a drone, the exhalation in twenty-four hours was 0.30 of a cubic inch, and during violent muscular exertion the exhalation in one hour was $0 \cdot 34 .^{\prime}$ Lavoisier recognized the great influence of muscular activity upon the respiratory changes. In treating of the consumption of oxygen, we have quoted his observations on the relative quantities of air vitiated in repose and activity.

Vierordt, in a number of observations on the human subject, ascertained that moderate exercise increased the average quantity of air respired per minute by nearly nineteen cubic inches, and that there was an increase of 1.197 cubic inches per minute in the absolute quantity of carbonic acid exhaled. ${ }^{2}$

The following results of the experiments of Dr. Edward Smith on this subject are very definite and satisfactory :

In walking at the rate of two miles an hour, the exhalation of carbonic acid during one hour was equal to the quantity produced during $1 \frac{4}{5}$ hour of repose, with food, and $2 \frac{1}{2}$ hours of repose, without food.

Walking at the rate of three miles per hour, one hour was equal to $2 \frac{8}{4}$ hours with, and $3 \frac{1}{2}$ hours without food.

One hour's labor at the tread-wheel, while actually working the wheel, was equal to $4 \frac{1}{2}$ hours of rest with food, and 6 hours without food.'s

The various observers we have cited have remarked that

ratory movements and the quantity of air passing through the lungs, and as we have seen the quantity of carbonic acid in the expired air is increased in proportion to the length of time that the air remains in the lungs, we can easily sce the source of error in his observations.

${ }^{1}$ Mrluse-Edwards, Physislogie, tome ii., p. 530.

${ }^{2}$ Cyclopredia of Anatomy and Physiology, vol. iv., part i., p. 348.

Op. cit., p. 713. 
when muscular exertion is carried so far as to produce great fatigue and exhaustion, the exhalation of carbonic acid is notably diminished.

Influence of Moisture and Temperature.-Lehmann has shown that the exhalation of carbonic acid is much greater in a moist than in a dry atmosphere. ${ }^{2}$ This conclusion was the result of a number of experiments on birds and animals confined in air at different temperatures and different degrees of moisture. He found that $35 \frac{1}{3} \mathrm{oz}$. av. weight of rabbits, at a temperature of about $100^{\circ} \mathrm{Fahr}$, exhaled during an hour before noon, in a dry air, about 15 cubic inches of carbonic acid; while in a moist air, at the same temperature, the exhalation was about 22 cubic inches.

Disregarding observations on the influence of temperature in cold-blooded animals, as inapplicable to the human subject, it has been ascertained that the exhalation of carbonic acid is much greater at low than at high temperatures, within the limits of heat and cold that are easily borne by the human subject; thus following the rule which governs the consumption of oxygen. Crawford, in his experiments on animal heat, was the first to call attention to this fact. ${ }^{2}$ Since then it has been confirmed by numerous observations on animals.

The experiments of Vierordt on the human subject show that there is an increase in the exhalation of carbonic acid of about one-sixth, under the influence of a moderate diminution in temperature. In these observations, the low temperatures ranged between $37.5^{\circ}$ and $59^{\circ}$, and the high temperatures between $60.5^{\circ}$ and $75.5^{\circ} \mathrm{Fahr}$. He found the quantity of air taken into the lungs slightly increased at low temperatures. The absolute quantity of carbonic acid exhaled per minute was $18 \cdot 27$ cubic inches for the low temperatures, and $15 \cdot 73$ cubic inches for the high temperatures. ${ }^{3}$

${ }^{2}$ Lehmany, Physiological Chemistry, Philadelphia, 1855, vol. ii., p. 444.

${ }^{2}$ Milne-Edwards, op. cit., p. 548.

s Ibid., p. 551. 
Influence of the Season of the Year.-It has been pretty well established by the researches of Dr. Smith, that spring is the season of the greatest, and fall the season of the least activity of the respiratory function.

The months of maximum are: January, February, March, and April.

The months of minimum are: July, August, and a part of September.

The months of decrease are: June and July.

The months of increase are: October, November, and December. ${ }^{1}$

W. F. Edwards, in 1819, showed in a marked manner this influence of the seasons upon the respiratory phenomena in birds. In a series of very curious observations, which he repeatedly verified, it was demonstrated that the increase in the activity of respiration during the winter was to a certain extent independent of the immediate influence of the surrounding temperature. In the month of January he confined six yellow-hammers in a receiver entaining 71.4 cubic inches of air, carrying the temperature to from $69^{\circ}$ to $70^{\circ} \mathrm{Fahr}$. The mean duration of their life was 62 minutes 25 seconds. In the months of August and September he repeated the experiment on thirteen birds of the same species, at the same temperature. The mean duration of life was 82 minutes. $^{2}$

These experiments have an important bearing on our views concerning the essential nature of the respiratory function. They seem to indicate that the respiratory processes are intimately connected with nutrition. Like the other nutritive phenomena, they undoubtedly vary at different seasons of the year, and are to a certain extent independent of sudden and transitory conditions. During the winter, more air is habitually used than in summer, and the respiratory

${ }^{1}$ Résumé de Recherches Expérimentales sur la Respiration. Journal de la Physiologie, 1860, tome iii., p. 519.

'W. F. Enwards, De l' Influence des Agens Physiques sur la Vie, Paris, 1824, p. 200. 
processes cannot be immediately brought down to the summer standard by a mere elevation of temperature.

Observations on the influence of barometric pressure are not sufficiently definite in their results to warrant any exact conclusions.

Some physiologists have attempted to fix certain hours of the day when the exhalation of carbonic acid is at its maximum, or at its minimum; but the respiratory activity is influenced by such a variety of conditions, that it is impossible to do this with any degree of accuracy.

\section{Relations between the Quantity of Oxygen consumed and the Quantity of Carbonic Acid exhaled.}

Oxygen unites with carbon in certain proportions, to form carbonic acid gas, the volume of which is precisely equal to the volume of the oxygen which enters into its composition. In studying the relations of the volumes of these gases in respiration, we have a guide in the comparison of the volumes of the inspired and expired air. It is now generally recognized that the volume of air expired is less, at an equal temperature, than the volume of air inspired. Assuming, then, that the changes in the expired air, as regards nitrogen, and all gases except oxygen or carbonic acid, are insigniticant, it must be admitted that a certain quantity of the oxygen consumed by the economy is unaccounted for by the oxygen which enters into the composition of the carbonic acid exhaled. We have already noted that from $\frac{1}{30}$ to $\frac{1}{50}$, or about 1.4 to 2 per cent. of the inspired air is lost in the lungs ; ${ }^{3}$ or it may be stated, in general terms, that the oxygen absorbed is equal to about five per cent. of the volume of air inspired, and the carbonic acid exhaled only about four per cent. A certain amount of the deficieney in volume of the expired air is then to be accounted for by a deficiency in the exhalation of carbonic acid. 
The experiments of Regnault and Reiset, to which frequent reference has been made, have a most important bearing on the question under consideration. $\Lambda$ s these observers were able to carefully measure the entire quantities of oxygen consumed and carbonic acid produced in a given time, the relation between the two gases was kept constantly in view. They found great variations in this relation, mainly dependent upon the reyimen of the animal. The total loss of oxygen was found to be much greater in carnivorous than in herbivorous animals; and in animals that could be subjected to a mixed diet, by regulating the food, this was made to vary between the two extremes. The mean of seven experiments on dogs showed that for every 1,000 parts of oxygen consumed, 745 parts were exhaled in the form of carbonic acid. In six experiments on rabbits, the mean was 919 for every 1,000 parts of oxygen. ${ }^{1}$

In animals fed on grains, the proportion of carbonic acid exhaled was greatest, sometimes passing a little beyond the volume of oxygen consumed.

"The relation is nearly constant for animals of the same species which are subjected to a perfectly uniform alimentation, as is easy to realize as regards dogs ; but it varies notably in animals of the same species, and in the same animal, submitted to the same regimen, but in which we cannot regulate the alimentation, as in fowls." "

When herbivorous animals were entirely deprived of food, the relation between the gases was the same as in carnivorous animals.

The final result of the experiments of Regnault and Reiset was, that the "relation between the oxygen contained in the carbonic acid and the total oxygen consumed, varies, in the same animal, from 0.62 to $1 \cdot 04$, according to the regimen to which he is subjected."

${ }^{1}$ Regnadlt and Reiset, Recherches Chimiques sur la Respiration. Annales de Chimie et de Physique, 3me série, tome xxvi.

${ }^{2}$ Ibid., p. 514. 
These observations on animals have been confirmed in the human subject by M. Doyère, who found a great variation in the relations of the two gases in respiration; the rolume of carbonic acid exhaled varying between 1.087 and 0.862 for 1 part of oxygen consumed. ${ }^{1}$

The destination of the oxygen which is not represented in the carbonic acid exhaled is obscure. Some have thought that it unites with hydrogen to form water; but there is not sufficient evidence of the formation of water in the economy, for researches have failed to show that there is more thrown off from the body than is taken in with food and drink.

The variations in the relative volumes of oxygen consumed and carbonic acid produced in respiration are not favorable to the hypothesis that the carbonic acid is the result of a direct action of oxygen upon earbonaceous matters. We should hardly expect a definite relation to exist between these two gases in respiration, when we find carbonic acid exhaled in the absence of oxygen, as has been shown by the experiments of W. F. Edwards and Geo. Liebig.

Sources of Carbonic Acid in the Expired Air.-All the carbonic acid in the expired air comes from the venous blood, where it exists in two forms : in a free state in simple solution, or at least in a state of very feeble combination, and in union with bases, forming the carbonates and bicarbonates. That which exists in solution in the blood is simply displaced by the oxygen of the air and exhaled. The alkaline carbonates and bicarbonates of the blood, coming to the lungs, meet with pneumic acid (discovered by Verdeil in 1851), and are decomposed, giving rise to a further evolution of gas. It is pneumic acid which gives the constant acid reaction to the tissue of the lungs. This principle is found in the pulmonary parenchyma at all periods of life, from which it may be extracted by the proper manipulations, and obtained

${ }^{3}$ Mrlde-Edwards, Physiologie, tome ii., p. 594. 
in a crystalline form. Its quantity is not very great. The lungs of a female who suffered death by decapitation contained about 0.77 of a grain. ${ }^{1}$

The action of pneumic acid upon the bicarbonates in the blood is exemplified in a marked manner by certain experiments of Bernard. When bicarbonate of soda is injected into the jugular of a living animal, a rabbit, for example, it is decomposed as fast as it gets to the lungs, and carbonic acid is evolved. This experiment produces no inconvenience to the animal when the bicarbonate is introduced slowly; but when it is injected in too great quantity, the evolution of gas in the lungs is so great as to fill the pulmonary structure and even the heart and great vessels, and death is the result. $^{2}$

Exhalation of Watery Vapor.-The fact that the expired air contains a considerable quantity of watery vapor has long been recognized; and most of the earlier experimenters who directed their attention to the phenomena of respiration made the estimation of the quantity exhaled, and the laws which regulate pulmonary transpiration, the subject of investigation. It is evident that there must be many circumstances materially influencing this process, such as the hygrometric condition of the atmosphere, temperature, extent of respiratory surface, etc., which are of sufficient importance to demand special consideration. In many points of view, also, it is interesting to know the absolute quantity of exhalation from the lungs.

${ }^{1}$ Robin and VerdeIL, Chimie Anatomique et Physiologique, Paris, 1853, tome ii., p. 460.

${ }^{2}$ Op. cit., tome i., p. 165. These experiments referred to the decomposition of cyanide of potassium in the lungs, as well as bicarbonate of soda. They were published in the Archives Générales in 1848, before the discovery of pneumic acid, and Bernard expressed surprise that the two substances experimented upon, which required an acid for their decomposition, should be decomposed in an alkaline fluid like the blood. Though made without a knowledge of the existence of pneumic acid, the observations none the less illustrated its physiological action. 
When the surrounding atmosphere has a temperature below $40^{\circ}$ or $43^{\circ} \mathrm{Fahr}$, a distinct cloud is produced by the condensation of the vapor of the breath. By breathing upon any polished surface, it is momentarily tarnished by the condensed moisture. Though the fact that watery vapor is contained in the breath is thus easily demonstrated, the estimation of its absolute quantity presents difficulties which.were not overcome by the older physiologists. Hales collected the vapor of the breath by expiring through wood ashes, ${ }^{1}$ which was the first attempt to estimate the amount of this exhalation by absorption. With the present improved methods of analysis there are many very accurate means of estimating watery vapor. One method is by the use of Liebig's bulbs filled with sulphuric acid, or tubes filled with chloride of calcium, both of which articles have a great avidity for water. From a large number of observations on his own person and eight others, collecting the water by sulphuric acid, Valentin makes the following estimate of the weight of water exhaled from the lungs in twenty-four hours:

In his own person, the exhalation in 24 hours was 6,055 grains.

In a young man of small size, the quantity was 5,042 grains.

In a student rather above the ordinary height, the quantity was 11,930 grains.

The mean of his observations gave a daily exhalation of 8,333 grains, or about $1 \frac{1}{6}$ lb. av. ${ }^{2}$

${ }^{1}$ Hales, Statical Essays, London, 1739, vol. ii., p. 326. Sanctorius, in 1614, was the first (Milse-Edwards, Physiologie, vol. ii., p. 602) to attempt the estimation of the exhalation of vapor of water from the body by comparing the gain in weight due to the ingestion of aliments with the loss by transpiration. We pass over the estimates of Lavoisier and Séguin, Keill, Abernethy, and others, and give only the more exact results obtained by Valentin. Dalton, estimating the quantity of air passing through the lungs in respiration, and assuming that it passes out of the lungs saturated with watery vapor, makes an estimate of the total exhalation in the twenty-four hours, which corresponds pretty closely with the results obtained by Valentin.

${ }^{2}$ Milise-Edwards, op. cit., p. 621. 
The extent of respiratory surface has a very marked influence on the quantity of watery vapor exhaled. This fact is very well shown by a comparison of the exhalation in the adult and in old age, when the extent of respiratory surface is much diminished. Barral found the exhalation in an old man less than half that of the adult. ${ }^{1}$

It is evident that the absolute quantity of vapor exhaled is increased when respiration is accelerated.

The quantity of water in the blood also exerts an important influence. Valentin found that the pulmonary transpiration was more than doubled in a man immediately after drinking a large quantity of water."

The vapor in the expired air is derived from the entire surface which is traversed in respiration, and not exclusively from the air-cells. The air which passes into the lungs derives a certain amount of moisture from the mouth, nares, and trachea. The great vascularity of the mucous membranes in these situations, as well as of the air-cells, and the great number of mucous glands which they contain, serve to keep the respiratory surfaces continually moist. This is important, for only moist membranes allow the free passage of gases, which is of course essential to the process of respiration.

Exhalation of Ammonia.-The most recent and extended observations on the exhalation of ammonia by the lungs, are those of Dr. Richardson, to which we have already alluded in treating of the coagulation of the blood. In more than a thousand experiments, made upon persons of both sexes, and on various of the inferior animals, with but one exception, a notable quantity of ammonia was found in the expired air. Dr. Richardson found the quantity very variable at different times of the day. At certain periods it is absent.

${ }^{1}$ Milne-Edwards, op. cit., p. 625, note.

Ibid., p. 607, note. It has not been thought necessary to discuss the in fluences of dry and moist atmosphere, barometric pressure, and temperature, which are purely physical in their character. 
In a number of observations made on his own person, the following variations were noted $:^{1}$

On rising in the morning, after a sound night's rest, the breath contained no ammonia.

In the evening, when fatigued and exhausted, and after exercise, the exhalation was generally considerable.

During a high temperature the exhalation is considerable, especially after exercise; but during cold weather the exhalation is very slight, or it may be absent altogether.

The amount of ammonia exhaled is greatest at the end of an expiration. If short and rapid expirations be made, the exhalation ceases until the respirations become deeper and more prolonged.

Ammonia has long been recognized as an exhalation from the human body in health, from the skin as well as the lungs. Dr. Richardson calls attention in his essay to the observations of Mr. Reade, Dr. Reuling, Viale and Latini, and others on this subject. Reuling has shown that the quantity of ammonia in the expired air is increased in certain diseases, particularly in uremia. ${ }^{2}$ Its characters in the expired air are frequently so marked, that patients who are entirely unacquainted with the pathology of uremia sometimes recognize an ammoniacal odor in their own breath.

Exhalation of Organic Matter, etc.-The pulmonary surface exhales a small quantity of organic matter. This has never been collected in sufficient quantity to enable us to recognize in it any peculiar or distinctive properties, but its presence may be demonstrated by the fact that a sponge completely saturated with the exhalations from the lungs, or the vapor from the lungs condensed in a glass vessel, will undergo putrefaction, a property distinctive of organic substances.

It is well known that certain substanees which are only

2 The Cause of the Coagulation of the Blood, London, 1857, p. $360 \mathrm{et}$ seq.

${ }^{2}$ In Lemuax's Physiological Chomistry, Philadelphia, 1855, vol. ii, p. 434. 
occasionally found in the blood may be eliminated by the lungs. Alcohol is partly removed from the system in this way; and its presence, with certain odorous principles, in the breath is pretty constant in those who take liquors habitually in considerable quantity. The odor of garlics, onions, turpentine, and many other principles which are taken into the stomach, may be recognized in the expired air. The lungs are among the important organs for the elimination of foreign matters from the system.

The action of the lungs in the elimination of certain gases, which are poisonous in very small quantities when they are absorbed in the lungs and carried to the general system in the arterial blood, is very well shown by the experiments of Bernard. Sulphuretted hydrogen, which produces death in a bird when it exists in the atmosphere in the proportion of 1 to 800 , may be taken into the stomach in solution with impunity, and even be injected into the venous system; in both instances being eliminated by the lungs with great promptness and rapidity. ${ }^{1} \quad$ Nysten showed that the carbonic oxide, one of the most violent and rapid in its effects of any of the poisonous gases when inhaled, could be injected into the veins with impunity, by simply taking care to introduce it only as rapidly as it is absorbed by the blood. ${ }^{2}$

The lungs, then, while they present an immense and rapidly absorbing surface for volatile poisonous substances, are capable of relieving the system of some of these substances by exhalation, when they find their way into the veins.

${ }^{1}$ Bernard, Leçons sur les Effets des Substances Toxiques et Médicamenteuses, Paris, 1857, p. 58. In an experiment on a dog of medium size, injecting a little more than a fluid drachm of water saturated with sulphuretted hydrogen into the jugular vein, the gas was aetected almost instantly in the expired air, and the animal suffered no inconvenience from the operation. The gas appeared in the breath in sixty-five seconds, when about an ounce of the solution was injected into the rectum. We have repcatedly verified the experiment of Bernard showing the almost instantaneous elimination of this gas by the lungs, when injected into the veins.

${ }^{2}$ Nysten, Recherches de Physiologie, etc., Paris, 1811, p. 81 et seq. 
Exhalation of Nitrogen.-The latest and most accurate direct experiments, particularly those of Regnault and Reiset, show that the exhalation of a small quantity of nitrogen is a pretty constant respiratory phenomenon. From a large number of experiments on dogs, rabbits, fowls, and birds, these observers came to the conclusion that when animals are subjected to their habitual regimen, they exhale a quantity of nitrogen equal in weight to from $\frac{1}{100}$ to $\frac{1}{50}$ of the weight of oxygen consumed. In birds, during inanition, they sometimes observed an absorption of nitrogen, but this was rarely seen in mam. mals. ${ }^{1} \quad$ Boussingault, by the indirect method, estimating the nitrogen taken into the body and comparing it with the entire quantity discharged, arrived at the same results in experiments upon a cow. ${ }^{2}$ Barral, by the same method, confirmed these observations by experiments on the human subject. $^{3}$

In spite of the conflicting testimony of the older physiologists, there can now be no doubt that, under ordinary physiological conditions, there is an exhalation by the lungs of a small quantity of nitrogen.

1 Regnault and Rerset, op. cit., Annales de Chimie et de Physique, 3me série, tome xxvi., pp. 510, 511.

${ }^{2}$ Boussingault, Mémoires de Chimie Agricole et de Physiologie, Paris, 1854, pp. 1-24.

${ }^{3}$ Longet, Physiologie, Paris, 1861, tome i., p. 543. 


\section{CHAPTER XIII.}

\section{CHANGES OF THE BLood in Respiration. (Hematosis.)}

Difference in color between arterial and venous blood-Comparison of the gases in venous and arterial blood-Observations of Magnus-Analysis of the blood for gases-Relative quantities of oxygen and carbonic acid in venous and arterial blood-Nitrogen of the blood-Condition of the gases in the bloodMechanism of the interchange of gases between the blood and the air in the lungs-General differences in the composition of arterial and venous blood.

IT is to be expected that the blood, receiving on the one hand all the products of digestion, and on the other the products of destructive assimilation or decay of the tissues, connected with the lymphatic system, and exposed to the action of the air in the lungs, should present important differences in composition in different parts of the vascular system.

In the first place, there is a marked difference in color, composition, and properties, between the blood in the arteries and in the veins; the change from venous to arterial blood being effected almost instantaneously in its passage through the lungs. The blood which goes to the lungs is a mixture of the fluid collected from all parts of the body; and we have seen that it presents great differences in its composition in different parts of the venous system. In some veins it is almost black, and in some nearly as red as in the arteries. In the hepatic vein it contains sugar, and its fibrin, albumen, and corpuscles are diminished; in the portal 
vein, during digestion, it contains materials absorbed from the alimentary canal; and finally, there is every reason to suppose that parts which require different materials for their nutrition, and produce different excrementitious principles, exert different influences on the constitution of the blood which passes through them. After this mixture of different kinds of blood has been collected in the right side of the heart and passed through the lungs, it is returned to the left side, and sent to the system, thoroughly changed and renovated, and, as arterial blood, has a uniform composition, as far as can be ascertained, in all parts of the system. This fact has been proven by the direct experiments of Béclard, who analyzed blood from the abdominal aorta, the carotid, temporal, occipital, crural, and epigastric arteries, in the same animal during life, and found the composition identical in all the specimens. His experiments were performed on horses and dogs, and care was taken to draw but a small quantity from each vessel, so as not to change the constitution of the fluid." The change, therefore, which the blood undergoes in its passage through the lungs, is the transformation of the mixture of venous blood from all parts of the organism into a fluid of uniform character, which is capable of nourishing and sustaining the function of every tissue and organ of the body.

The capital phenomena of respiration, as regards the air in the lungs, are loss of oxygen and gain of carbonic acid; the other phenomena being accessury and comparatively unimportant. As the blood is capable of holding gases in solution, in studying the essential changes which this fluid undergoes in respiration, we look for them in connection with the proportions of oxygen and carbonic acid before and after it has passed through the lungs. In respiration, the most marked effect on the venous blood is change in color.

${ }^{1}$ Archives G'énérales de Médecine, 4me série, tome xviii., p. 123; and Bérard, Physiologie, tome iii., p. 369 . 
Difference in Color between Arterial and Venous Blood. -We have already considered this in treating of the properties of the blood, and will only take up in this connection the cause of the remarkable change in the color of the blood in the lungs. This change is instantaneous, and, long before the discovery of oxygen by Priestley, was recognized by Lower, Goodwyn, and others, as due to the action of the air.

The celebrated experiment of Bichat showed the effect on the color of the blood in the arteries, of preventing the access of fresh air to the lungs. This observer adapted a stop-cock to the trachea of a dog, by which he could regulate the entrance of air into the lungs, and exposing the carotid artery, adapted a small one to this vessel. When he prevented the air from getting to the lungs by closing the stop-cock in the trachea, the blood became black in the artery, but regained its florid hue when air was readmitted to the lungs. ${ }^{1}$

The influence of air in changing the color of venous blood may be noted in blood which has been drawn from the body; as is exempiified by the red color of that portion of a clot, or the surface of defibrinated venous blood, which is exposed to the air. If we cut into a clot of venous blood, the interior is almost black, but becomes red on exposure to the air for a very few seconds.

We have been in the habit of illustrating the physiological influence of the air on renous blood by the following simple experiment: Removing the lungs of an animal (a dog) just killed, the nozzle of a syringe is secured in the pulmonary artery by a ligature, and a canula, connected with a rubber tube which empties into a glass vessel, is secured in the pulmonary vein. Adapting a bellows to the trachea, we imitate the process of respiration; and if defibrinated venous blood be carefully injected through the lungs, it will be return-

${ }^{1}$ Xıv. Bıchut, Recherches Physiologiques sur la Vie et la Mort, 5me édition, par F. Magende, Paris, 1829, p. 386. 
ed by the pulmonary vein with the bright red color of arterial blood. When the artificial respiration is interrupted, the blood passes through the lungs. without change. ${ }^{\prime}$ In exposing the thoracic organs, and keeping up artificial respiration, repeating the celebrated experiment of Robert Hooke, made before the Royal Society in 1664, we can see through the thin walls of the auricles the red color of the blood on the left side contrasting with the dark venous blood on the right.

Since the discovery of oxygen, it has been ascertained that this is the only constituent of the air which is capable of arterializing the blood. Priestley showed that venous blood is not changed in color by nitrogen, hydrogen, or carbonic acid ; while all these gases, by displacing oxygen, will change the arterial blood from red to black. ${ }^{2}$

The elements of the blood which absorb the greater part of the oxygen are the red corpuscles. While the plasma will absorb, perhaps, twice as much gas as pure water, it has been shown by Magnus and Gay-Lussac that the corpuscles will absorb from ten to thirteen times as much. ${ }^{3}$ By some the proportion is put much higher. The red corpuseles may be considered as the respiratory elements of the blood. It is

1 This demonstration is very striking, especially if we use a syringe with a double nozzle, one point secured in the pulmonary artery, and the other simply carrying the blood by a rubber tube into a glass vessel. Receiving the blood which passes through the lungs, and that which simply passes through the tube, into two tall glass vesssels, the one is of a bright red, and the other retains its dark color. In preparing for the experiment it is necessary, immediately after removing the lungs from the animal, to inject them with a little defibrinated blood, so as to remove the coagulating blood from the pulmonary capillaries, whiclı would otherwise become obstructed. The injection should be made gently and gradually, to avoid extravasation. Defibrinated ox-blood may be used. The most convenient way to secure the canulæe in the vessels is to push them into the pulmonary artery through the right ventricle, and into the pulmonary vein through the left auricle.

${ }^{2}$ Carbonic oxide and nitrous oxide have a strong affinity for the blood-corpuscles, and become fixed in them, the former giving the blood a vivid red color. Sugar and many salts will also redden venous blood. These agents, however, do not impart the physiological properties of arterial blood.

s RoBIN and VerdeIL, op. cit., tome i., p. 32. 
undoubtedly true that the corpuseles, deprived of their natural plasma, are not changed in color by being exposed to the air, or even to pure oxygen. Dr. Stevens, after removing the serum from a clot by repeated washings with pure water, found that the color remained black when exposed to the air, ${ }^{1}$ but was reddened by the addition of its serum, or certain saline solutions. From this he reasoned that the red color of arterial blood is due to the saline constituents of the plasma. This is true; but the saline constituents of the plasma affect the color indirectly, by maintaining the anatomical integrity of the corpuscles. If blood be received from a vein into pure water, it remains almost black, however long it may be exposed to the air, ${ }^{2}$ from the fact that the corpuseles are destroyed. These facts are only additional evidence of the function of the red corpuscles in absorbing oxygen and carrying it to the tissues. According to the late researches of Fernet, which have been confirmed by L. Meyer, the volume of oxygen fixed by the corpuscles is about twenty-five times that which is dissolved in the plasma. ${ }^{3}$

\section{Comparison of the Gases in Venous and Arterial Blood.-} The demonstration of the fact that free oxygen and carbonie acid exist in the blood, with a knowledge of the relative proportion of these gases in the blood before and after its passage through the lungs, is a point hardly second in importance to the relative composition of the air before-and after respiration. The idea enunciated by Mayow about two hundred years ago, that "there is something in the air, absolutely

${ }^{1}$ William Stevens, Observations on the Healthy and Diseased Properties of the Blood, London, 1832, p. 362; and Philosoplical Transactions, 1835.

${ }^{2}$ Mrlwe-Enwards, Physiologie, tome i., p. 475.

${ }^{3}$ Longer, Traité de Physiologie, Paris, 1861, tome i., p. 595. Fernet made a great number of experiments on the influence of the various salts contained in the serum on the absorbing power of the blood for gases. His observations had particular reference to carbonic acid, the solubility of which was influenced most by saline principles. These experiments were confirmed and extended by Lothar Meyer (Die Gase des Blutes). 
necessary to life, which is conveyed into the blood," "excepting that the vivifying principle is not named nor its other properties described, expresses what we now consider as one of the two great principles of respiration. This is even more strictly in accordance with fact than the idea of Lavoisier, who supposed that all the chemical processes of respiration took place in the lungs. Mayow also described the evolution of gas from blood placed in a vacuum. ' Many observers have since succeeded in extracting gases from the blood by various processes. Sir Humphry Davy induced the evolution of carbonic acid by raising arterial blood to the temperature of $200^{\circ}$ Fahr., and venous blood to a temperature of $112^{\circ} ;^{3}$ Stevens, ${ }^{4}$ and others, disengaged gas by displacement with hydrogen, nitrogen, or the ordinary atmosphere; but in spite of this, before the experiments of Magnus in 1837, many denied the existence in the blood of any free gas whatsoever. ${ }^{5}$

Magnus made some experiments upon the human blood, extracting the gases by displacement with hydrogen; but the observations which are most generally referred to by physiologists were made upon the blood of horses and calves, extracting the gases by the air-pump, and giving the comparative quantities existing in the arterial and venous blood. These experiments were of great value as settling the question of the existence of gases in the blood, either in a free state, or very loosely combined with some of its organic constituents; and until very recently they have been universally

${ }^{1}$ See page 411 , note.

${ }^{2}$ See quotation in Milne-Edwards, Physiologie, tome i., p. 438, note.

${ }^{3}$ Sir Homphry Davx, Works, London, 1839, vol. i., pp. 77-79. An Essay on Light, Heat, and the Combination of Light, with a new Theory of Respiration.

Loc. cit.

${ }^{5}$ Gmelin, Mitscherlich, and Tiedemann denied the existence of any free gases in the blood. At one time Dr. John Dary held the same opinion, though he finally recognized his error, and succeeded in extracting gas from the blood by means of the air-pump (Researches, Physiological and Anatomical, London, 1839 , vol. ii., p. 154). 
received by physiologists, as representing the relative proportions of the gases in the two kinds of blood, though Magnus states in his paper that he does not think he succeeded in extracting all the gas the blood contained. ${ }^{1}$ It is a question of the last importance, as bearing upon our comprehension of the essential processes of respiration, to be able to determine the relative proportion of oxygen and carbonic acid in the arterial and venous blood. Until very recently, our ideas on this subject have had for their sole experimental basis the observations of Magnus, and in discussing the accuracy of the modes of analysis of the blood for gases we need take no account of any experiments anterior to his.

Analysis of the Blood for Gases.-There are certain grave sources of error in the method employed by Magnus, which render his observations of little value, except as demonstrating that oxygen, carbonic acid, and nitrogen may be extracted by the air-pump from both arterial and venous blood. The only source of error in the results which he fully recognized lay in the difficulty in extracting the entire quantity of gas in solution; but a careful study of his paper shows another element of inaccuracy which is even more important. The relative quantities of oxygen and carbonic acid in any single specimen of blood present great variations, dependent upon the length of time that the blood has been allowed to stand before the estimate of the gases is made. As it is impossible to make this estimate immediately after the blood is drawn, on account of the froth produced by agitation with a gas, when the method by displacement is employed, ${ }^{2}$ and the bubbling of the gas when extracted by the air-pump, this

${ }^{1}$ The original article of Magnus is published in the Annalen der Physik und Chemie of Poggendorff, April, 1837, and is translated into French in the Ann. de Chimie et de Phys. of the same year.

${ }^{2}$ When a gas, such as hydrogen, which is not contained in the blood, is thoroughly mixed with it by agitation in a closed ressel, it will penetrate the liquid, and displace, or drive off, all the free gas which is held in solution. This is called the method of analysis by displacement. 
objection is fatal. It is necessary to wait until the froth has subsided before attempting to make an accurate estimate of the volume of gas given off. The following observation of Magnus illustrates this fact. The observation was on the human blood six hours after it had been thoroughly mixed with hydrogen: ${ }^{1}$

Blood of Man.

4.077 cubic inches.

$3 \cdot 650$ "

$3 \cdot 838$
Carbonio acid.

$1 \cdot 013$ cubic inches.

0.781

$1 \cdot 355$

After twenty-four hours, at the end of which time the blood had no odor:

4.077 cubic inches.
3.650 "
3.833

1.517 cubic inches.

$1 \cdot 456$

$2 \cdot 075$

The excess of carbonic acid fornd twenty-four hours after, over the quantity found six hours after, in the first and third specimens, is a little over 50 per cent.; while in the second specimen it is very nearly 100 per cent.

In these analyses the proportion of oxygen is not given. The question naturally arises as to the source of the carbonic acid which was evolved during the last eighteen hours of the observation. This is evident, when we consider one of the important properties of the blood. A number of years ago, Spallanzani demonstrated that, in common with other parts of the body, fresh blood removed from the body has, of itself, the property of consuming oxygen; and W. F. Edwards has shown that the blood will exhale carbonic acid. In 1856, Harley, by a series of ingenious experiments, found that blood, kept in contact with air in a closed vessel for twentyfour hours, consumed oxygen and gave off carbonic acid. ${ }^{2}$

${ }^{1}$ G. Magnos, Sur les Gas que contient le Sang: Oxygène, Azote et Acide Carbonique. Annales de Chimie et de Physique, 2me série, tome lxv., 1837, p. 174.

${ }^{2} \mathrm{G}$. HARLEY, The Chemistry of Respiration. The British and Foreign Mcdico-Chirurgical Revicw, July, 1856, p. 328. 
More recently, Bernard has shown that for a certain time after the blood is drawn from the vessels, it will continue to consume oxygen and exhale carbonic acid. If all the carbonic acid be removed from a specimen of blood, by treating it with hydrogen, and it be allowed to stand for twenty-four hours, another portion of gas can be removed by again treating it with hydrogen, and still another quantity by treating it with hydrogen a third time. ${ }^{1}$

From these facts it is clear that, in the experiment of Magnus, the excess of carbonic acid involved a post-mortem consumption of oxygen; and no analyses made in the ordinary way, by displacement with hydrogen, or by the airpump, in which the blood must necessarily be allowed to remain in contact with oxygen for a number of hours, can be accurate. The only process which can give us a rigorous estimate of the relative quantities of oxygen and carbonic acid in the blood is one in which the gases can be estimated without allowing the blood to stand, or in which the formation of carbonic acid in the specimen, at the expense of the oxygen, is prevented. All others will give a less quantity of oxygen and a greater quantity of carbonic acid than exists in the blood circulating in the vessels, or immediately after it is drawn from the body.

A solution of this important and difficult problem in analysis of the blood has been accomplished by Bernard. This observer made a great number of experiments, in the hope of discovering some means by which the consumption of axygen by the blood-corpuscles could be arrested. ${ }^{2}$ He found, finally, that carbonic oxide, one of the most active of the poisonous gases, had a remarkable affinity for the blood-corpuscles. When

${ }^{1}$ Bernarn, Leģons sur les Propriétés Physiologiques et les Altérations Pathologiques des Liquides de l'Organisme, Paris, 1859, tome i., p. 354 et seq.

${ }^{2}$ Harley (op. cit., p. 334) ascertained that a few drops of chloroform, added to the fresh blood, greatly diminished the activity of the change of oxygen into carbonic acid. It did not entirely arrest it, however, and the author does not sug. gest its use in quantitative analyses for gases. 
taken into the lungs, it is absorbed by and becomes fixed in the corpuscles, effectually preventing the consumption of oxygen and production of carbonic acid, which normally takes place in the capillary system, and which is one of the indispensable conditions of nutrition. We have already referred to the mechanism of poisoning by the inhalation of this gas, by its fixation in the blood-corpuscles, their consequent paralysis, and the arrest of their function as respiratory organs. As it is the continuance of this transformation of oxygen into carbonic acid, after the blood is drawn from the vessels, which interferes with the ordinary analysis of the blood for gases, we might expect to extract all the oxygen, if we could immediately saturate the blood with carbonic oxide. The preliminary experiments of Bernard on this point are conclusive. He ascertained that by mixing carbonic oxide in sufficient quantity with a specimen of fresh arterial blood, in about two hours, all the oxygen which it contained was displaced. Introducing a second quantity of carbonic oxide after two hours, and leaving it in contact with the blood for an hour, a quantity of oxygen was removed, so small that it might almost be disregarded. A third experiment on the same blood failed to disengage any oxygen or carbonic acid. ${ }^{\prime}$

The view entertained by Bernard of the action of carbonic oxide in displacing the oxygen of the blood is, that the former gas has a remarkable affinity for the blood-corpuscles, in which nearly all the oxygen is contained, and when brought in contact with them unites with the organic matter, setting free the oxygen, in the same way that the acid entering into the composition of a salt is set free by any other acid which has a stronger affinity for the base. There is every reason to suppose that this view is correct; as carbonic oxide is much less soluble than oxygen, and as it only has the property of disengaging this gas from the blood, leaving the other gases still in solution.

As carbonic oxide only displaces the oxygen, it is neces-

'Bernard, Liquides de l' Organisme, tome i., p. 373. 
sary to resort to some other process, in addition to this, to disengage the other gases contained in the blood. It is only necessary to arrest the action of the corpuscles upon the oxygen, and then the gases may be set free by the air-pump, or any method which may be convenient. The method adopted by Bernard for the disengagement of all the gases contained in the blood is first to displace the oxygen by carbonic oxide, using about two-thirds of gas by volume to one-third of blood, then to attach the tube to a tube of mercury, and subject the blood to the barometric vacuum, which sets free the carbonic acid and the nitrogen. The results obtained by this method correspond with our ideas concerning the nature of the respiratory process; and analyses of the blood taken at different periods show variations in the quantities of oxygen in the arterial, and carbonic acid in the venous blood, corresponding with some of the variations which we have noted in the loss of oxygen and gain of carbonic acid in the air, in respiration.

The analyses of Bernard, who obtained from fifteen to twenty per cent. of oxygen in volume from the arterial blood, show the great imperfection of the process employed by Magnus, who obtained from the arterial blood of horses and calves a mean of but $2 \cdot 44$ per cent. of oxygen. It does not seem necessary, therefore, to discuss the criticisms of the results obtained by Magnuis which were made by Gay-Lussac and Magendie, soon after their publication, and more recently by Harley and others. ${ }^{1}$

${ }^{1}$ To Magnus belongs the credit of demonstrating the important fact that oxygen, carbonic acid, and nitrogen can be extracted from the blood by removing the atmospheric pressure. Before his observations, Gmelin, Mitscherlich, and Tiedemanu placed venous blood in a tube over mercury, in the receiver of an air-pump, and by removing the pressure as far as possible, caused the mercury to descend. On admitting air into the receiver and restoring the pressure, the mercury ascended, with the blood, again filling the tube completely. From this they reasoned that there was no free carbonic acid in the blood. By passing up a little acetic acid, carbonic acid was set free, which led them to believe that all the carbonic acid was in combination. Magnus showed that the reason why other observers had failed to extract gas by means of the air-pump was, that the 


\section{Bernard's experiments were made chiefly on dogs, and} had especial reference to the proportion of oxygen in the

rarefaction of the air was not carried sufficiently far. J. Davy, in his second experiments, recognized this fault in his first observations. As the results obtained by Magnus are generally quoted and received in works on physiology, we give the table, which is taken from the translation of his original article in the Annales de Chimie et de Physique (loc. cit.). We have not thought it worth while to reduce the volumes from cubic centimetres to cubic inches, as we add the percentage of gas in volume, which is not given by Magnus.

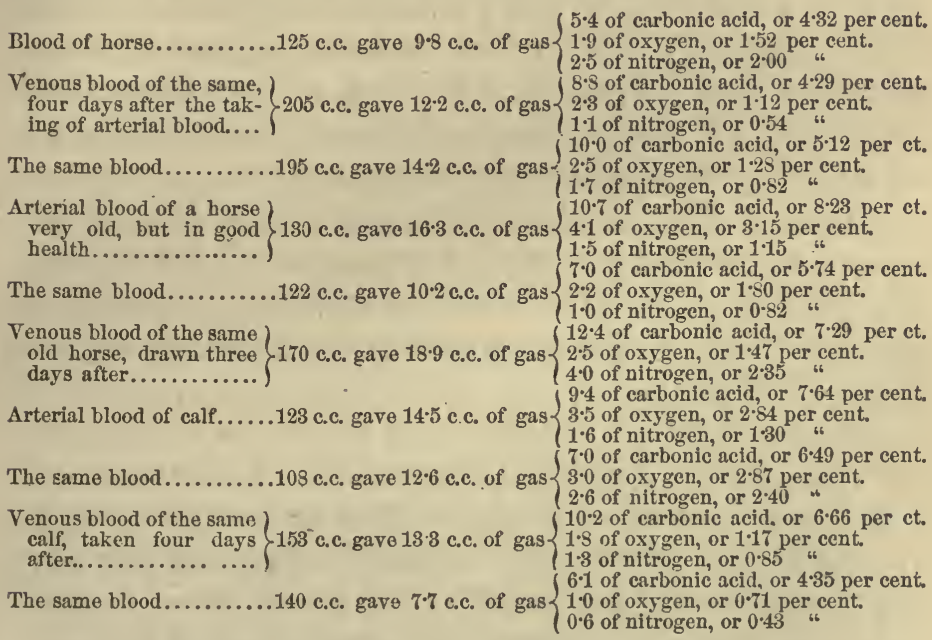

We have given this table in full, and calculated the percentage of gas to the blood in each observation, bccause it is a common impression that the observations of Magnus show a greater proportion of oxygen in the arterial blood, and a greatcr proportion of carbonic acid in the venous blood. This is not the fact. The table shows that the proportion of all gases is greater in the arterial blood, and that the proportion of carbonic acid to the oxygen is greater in the venous blood; but while the percentage of oxygen is greater in the arterial blood, there is also a larger percentage of carbonic acid. In the specimens of arterial blood examined, the mean proportion of oxygen was 2.44 per cent., and of carbonic acid 6.48 per cent. In the venous blood, the mean proportion of oxygen was 1.15 per cent., and of earbonic acid, 5.54 per cent. It is difficult to reconcile an analysis, showing a greater absolute quantity of carbonic acid in arterial than in venous blood, with our settled and well-sustained ideas regarding the processes of respiration. A glance at the wide differences in the different analyses of specimens of the same blood shows that there must have been some grave error in the process. 
blood. As far as we know, no analyses of the human blood have yet been made by his method. In two specimens taken from a $\operatorname{dog}$ in good condition, a specimen of arterial blood, drawn from the ressels by a syringe and put in contact with carbonic oxide without being exposed to the air, was found to contain 18.28 per cent., and a specimen of venous blood, taken in the same way, 8.42 per cent., in volume, of oxygen. ${ }^{1}$ The proportion of gases in the blood is found to vary very considerably under different conditions of the system, particularly with reference to the digestive process. The following are the general results of later observations, showing the differences and variations in the proportions of all the gases, in arterial and venous blood. ${ }^{2}$

Arterial Blood, while an animal is fasting, contains from nine to eleven parts per hundred of oxygen. In full digestion, the proportion is raised to seventeen, eighteen, or even twenty parts per hundred. The proportion varies in different animals; being much greater, for example, in birds than in mammals.

The quantity of carbonic acid is even more variable than the quantity of oxygen. During digestion there are from five to six parts per hundred of free carbonic acid in the arterial blood. During the intervals of digestion this quantity is reduced to almost nothing; and after fasting for twentyfour hours, frequently not a trace is to be discovered.

Venous Blood always contains a large quantity of carbonic acid, both free in solution, and combined in the form of carbonates and bicarbonates. This quantity varies in different parts of the venous system, and bears a relation to the color of the blood. It is well known that the venous blood coming from some glands is dark during the intervals of secretion, and nearly as red as arterial blood during their functional activity. In the renous blood from the sub-max-

${ }^{2}$ Loc. cit., p. 367 .

2 These results were given in a eourse of lectures which we had the privilege of hearing at the College of France in the summer of 1861, and which have not yet been published. 
illary gland of a dog, Bernard found 18.07 per cent. of carbonic acid during repose, and 10.14 per cent. during secretion. The blood coming from the muscles is the darkest in the body, and contains the greatest quantity of free carbonic acid.

The quantity of free earbonic acid is immensely increased in the venous blood during digestion. It is owing to this fact that the gas then exists in the arterial blood. During the intervals of digestion, the quantity is so small that the lungs are capable of completely eliminating it, and none passes into the arteries ; but during digestion, the proportion is so much greater, that for a time it cannot be entirely removed, and a part finds its way into the arterial system.

These facts coincide with the views which are. now held regarding the essential processes of respiration. The blood going to the lungs ordinarily contains carbonic acid, and no oxygen; for during the intervals of digestion, there is only enough oxygen taken up by the blood to supply the wants of the system. In the lungs, carbonic acid is given off, appearing in the expired air, and the oxygen which disappears from the air is carried away by the arterial blood. Under some conditions, and particularly during the height of the digestive process, the quantity of oxygen absorbed is largely increased, and so much may exist in the arterial blood that a small portion passes into the veins. At the same time the production of carbonic acid is increased in activity, and it may exist in such quantity in the venous blood, as temporarily to pass in small quantity into the arteries.

Nitrogen of the Blood.-As far as is known, nitrogen has no important office in the process of respiration. There is generally a slight exhalation of this gas by the lungs, and the analyses of Magnus and others have demonstrated its existence in solution in the blood. Maguus found generally a larger proportion in the arterial than in venous blood, though in one instance there was a larger proportion in the 
venous blood. It is not absolutely certain whether the nitrogen which exists in the blood is derived from the air or from the tissues. Its almost constant exhalation in the cxpired air would lead to the supposition that it is produced in small quantity in the system, or supplied by the food. According to Bernard; the quantity of nitrogen in the arterial blood is from two to five parts per thousand, but it is present in very much larger quantity in the venous blood. ${ }^{2}$ There is no evidence that nitrogen enters into combination with the blood-corpuscles; it exists simply in solution in the blood, which is capable of absorbing about ten times as much as pure water. ${ }^{2}$ Nothing is known with regard to the relations of the free nitrogen of the blood to the processes of nutrition.

Condition of the Gases in the Blood.-It is now pretty generally admitted that the oxygen of the blood exists, not in simple solution, but in a condition of feeble combination with certain of the constituents of the blood-corpuscles. ${ }^{3}$ It is clearly demonstrated that the corpuscles are the elements which fix the greatest quantity of this gas. Carbonic oxide, which has a great affinity for the corpuscles, displaces almost immediately all the oxygen which the blood contains. When the corpuscles are destroyed, as they may be readily by receiving fresh blood into a quantity of pure water, the red color is instantly changed to black. Oxygen in the blood bears a closer relation to the corpuscles than that of mere solu-

${ }^{2}$ Unpublished lectures delivered at the College of France in the summer of 1861 .

2 Magnus; loc. cit.

${ }^{3}$ It is not settled which of the constituents of the blood-corpuseles has the greatest affinity for oxygen. 'It has been supposed to be combined especially with the coloring matter; but experiments on this point are contradictory. Lehmann noticed no difference in the color of a solution of blood-crystals treated with oxygen, and the same solution treated with earbonic acid; the only difference was that the latter became turbid (Physiological Chem., Am. ed., vol. i., p. 573). Meckel made some expcriments in which "hæmatoglobulin" was changed to a bright red by oxygen, and to a bluish red by carbonie acid (Ibid., p. 574). 
tion. The proportion which they are capable of containing: is to a certain degree absolute, and not dependent upon physical conditions, such as pressure, which invariably have an influence on the proportion of gas merely held in solution by liquids. The proportion of oxygen in the blood cannot be increased by pressure, nor is it diminished by reduction of the pressure, until it approaches a vacuum. ${ }^{1}$ The fact that the blood-corpuscles are capable of consuming oxygen and giving off carbonic acid is an additional argument in favor of the union of these anatomical elements with the gas, though this union is very feeble and easily disturbed. The plasma will absorb a certain quantity of oxygen, and its action in respiration seems to be intermediate; it first takes oxygen from the air and then gives it up to the corpuscles.

Carbonic acid is more easily exhaled from the blood than oxygen. It was this principle which was obtained by those who first succeeded in extracting gas from the blood. While there is every reason to suppose that oxygen is in combination with the blood-corpuscles, carbonic acid seems to be in a condition of simple solution, and is contained more especially in the plasma. What may be considered as the free carbonic acid of the blood behaves in all regards like a gas simply held in solution. The view that it is held in solution chiefly in the plasma is sustained by the fact that serum will absorb more carbonic acid than an equal volume of defibrinated blood. ${ }^{2}$

Liebig has shown that the phosphate of soda, one of the constituents of the blood, influences to a remarkable degree the quantity of carbonic acid which can be held in solution by any liquid. One hundredth of a part of this salt in pure water will double its capacity for dissolving carbonic acid. ${ }^{3}$

1 The fact that oxygen is exhaled from the blood in vacuo is not an argument against the view that it enters into feeble combination with the blood-corpuscles; for it is well known that many distinctly recognized chemical combinations are disturbed by the same means. For example, a vacuum is capable of disengaging from some of the bicarbonates one equivalent of carbonic acid.

${ }^{2}$ Losaer, Traité de Physiologie, Paris, 1861, tome i., p. 494.

3 Milne-Edwards, Physiologie, tome i., p. 471. 
-When carbonic acid is formed by the blood, after it is drawn from the body, it is immediately exhaled, at least in part. When blood is in contact with a certain quantity of air, oxygen is consumed and carbonic acid is exhaled. The fact that carbonic oxide, which has such a remarkable affinity for the corpuscles, displaces oxygen almost exclusively, is another argument in favor of the view that the carbonic acid is contained mainly in the plasma.

A portion of the carbonic acid which is formed by the system unites with the carbonates in the blood, particnlarly the carbonate of soda, to form bicarbonates, is carried to the lungs, and there set free by the pneumic acid. It here exists in so loose a condition of combination, that it may be disengaged by treating the blood with inert gases, or putting it under the receiver of an air-pump.

The carbonic acid which is formed in the tissues, and taken up by the blood in its passage through the capillaries, exists in this fluid in two forms: one, in simple solution, chiefly in the plasma; and the other, in a state of such loose chemical combination in the bicarbonates, that it may be disengaged by displacement by another gas, and is readily set free by pneumic acid. This gas is a product of excretion, and is not engaged in any of the vital functions; while oxygen, which has an all-important function to perform, unites immediately with the blood-corpuscles, and is not easily disengaged, except when it undergoes transformation in the process of nutrition. It is certain that all the carbonicacid in the blood is not in combination with bases, for the proportion of salts is not sufficient to account for all the carbonic acid that can be disengaged.

In addition to this excrementitious carbonic acid, there is another portion which is a permanent constituent of the blood, in the carbonates, and cannot be set free without the use of reagents.

Nitrogen exists in the blood in the same condition of solution in the plasma as carbonic acid. 
Mechanism of the Interchange of Gases between the Blood and the Air, in the Lungs.-The gases from the air pass into the blood, and the gases of the blood are exhaled through the delicate membrane which separates these two fluids, in accordance with laws which are now well understood. The first to point out the power of gases thus to penetrate and pass through membranes was the late Dr. J. K. Mitchell, of Philadelphia. ${ }^{1}$ His attention was first directed to this subject by noticing the escape of gas from gum-elastic balloons filled with hydrogen. In order to satisfy himself that the gas passed through the membrane independently of pressure, he put different gases in wide-mouthed bottles covered with gumelastic, and by a series of ingenious experiments, which have become so common that it is unnecessary to describe them in detail, extended Dutrochet's law of endosmosis and exosmosis to the gases. He demonstrated the same phenomena when he used thin animal membranes instead of the gumelastic, and found that the more recent the membrane, the more rapid was the action. The rapidity of transmission was found to be very great in living animals. Observations on the lungs of the snapping turtle, filled with air and placed in an atmosphere of carbonic acid or nitrous oxide, showed a very rapid passage of gas from the exterior to the interior. Dr. Mitchell recognized the passage of gases through membranes into liquids, and the exhalation of gases which were in solution in these liquids. He noted this action in the absorption of oxygen and the exhalation of carbonic acid in the lungs; though he fell into the crror of supposing that there was no carbonic acid in solution in the blood, and that it was exhaled as soon as formed. ${ }^{2}$ A few years later, Dr. Rogers, of Philadelphia, enclosed a fresh pig's bladder, filled with

${ }^{1}$ On the Penetrativeness of Fluids. By J. K. MrxcheLL, M.D., Lecturer on Nedical Chemistry in the Philadelphia Medical Institute. American Journal of the Medical Sciences, Nov., 1830, p. 36.

${ }^{2}$ Ibid., p. 56. 
venous blood, in a bell-glass of oxygen.' In two hours a quantity of oxygen had disappeared, and a large quantity of carbonic acid had made its appearance. Dr. Rogers is frequently referred to as the first to demonstrate the passage of gases through animal membranes to and from the blood. The credit of this is due to Mitchell, whose paper was published in 1830, while the experiments of Rogers were published in 1836.

We have already seen that the blood is exposed to the air in the lungs, separated from it only by a very delicate membrane, over. an immense surface. The membrane, far from interfering with the interchange of gases, actually favors it ; and thus, in obedience to the laws which regulate endosmosis between gases and liquids, the oxygen is continually passing into the blood, and the free carbonic acid is exhaled.

General Differences in the Composition of Arterial and Venous Blood.-All observers agree that there are certain marked differences in the composition of arterial and venous blood, aside from their free gases. The arterial blood contains less water, and is richer in organic, and most inorganic, constituents than the venous blood. It also contains a greater proportion of corpuscles, fibrin, and inorganic salts. It is more coagulable, and offers a larger and firmer clot than venous blood. Numerous analyses have failed to detect a constant difference in the proportion of albumen; sometimes the proportion is greater in the venous, and sometimes in the arterial blood. The only principles which are constantly more abundant in venous blood are water and the alkaline carbonates. 10,000 parts of venous blood contained $12 \cdot 3$ parts of carbonic acid combined, and the same quantity of arterial blood contained but 8.3 parts. $^{2}$ The deficiency of water in the blood which comes from the lungs is readily explained by the escape of watery vapor in the expired air.

1 Experiments on the Blood, etc. By RoBert E. Rogers, M.D., of Philadelphia. American Journal of the Medical Sciences, August, 1836, p. 296.

${ }^{2}$ LoNGet, op. cit., tome i, p. 584. 
An important distinction between arterial and venous blood is one to which we have already incidentally alluded, viz., that the former has a uniform composition in all parts of the arterial system, while the composition of the latter varies very much in the blood coming from different organs. Arterial blood is capable of carrying on the processes of nutrition; while venous blood is not, and cannot even circulate freely in the systemic capillaries. 


\section{CHAPTER XIV.}

\section{RELATIONS OF RESPIRATION TO NUTRITION, ETC.}

Views of physiologists anterior to the time of Lavoisier-Relations of the consumption of oxygen to nutrition-Relations of the exhalation of carbonic acid to nutrition-Essential processes of respiration-The respiratory sense, or want on the part of the system which induces the respiratory movementsLocation of the respiratory sense in the general system-Sense of suffocation -Respiratory efforts before birth-Cutaneous respiration-Asphyxia.

IT has been demonstrated that all tissues, so long as they retain their absolute integrity of composition, have the property of appropriating oxygen and exhaling carbonic acid, independently of the presence of blood; and that the arterial blood carries oxygen from the lungs to the tissues, there gives it up, and receives carbonic acid, which is carried by the venous blood to the lungs, to be exhaled. From this fact alone, it is more than probable that respiration is inseparably connected with the general act of nutrition. Its processes must be studied, therefore, as they take place in the tissues and organs of the body. In the present state of the science, the questions which naturally arise in connection with the essential processes of respiration are:

1. In what way is oxygen consumed in the system?

2. How is carbonic acid produced by the system?

3. What is the nature of the processes which take place between the disappearance of oxygen and the evolution of carbonic acid?

When these questions are satisfactorily answered, we shall understand the essence of respiration; but in reasoning on this 
subject, we must not fall into the error of assimilating the respiratory phenomena too closely to those with which we are acquainted as they occur in inorganic bodies. It must be remembered that in the organism we are dealing with principles which have the remarkable property of self-regeneration; and which, as a simple condition of vital existence, consume oxygen, when it is presented to them, and exhale carbonic acid. Without a proper supply of oxygen, the tissues die, lose these peculiar properties, and finally disappear by putrefactive decomposition. This consumption of oxygen cannot be regarded in any other light than as the appropriation by a living part, of an element necessary to supply waste; in the same way as those materials which are ordinarily called nutritive are appropriated. That waste is continually going on there can be no doubt; and as the production of urea, creatine, creatinine, cholesterine, etc., is to a certain extent independent of the absorption of food, so the production of carbonic acid is to a certain extent independent of the absorption of oxygen. This has been fully demonstrated by the experiments of Spallanzani, Edwards, Geo. Liebig, and others, who have noted the exhalation of carbonic acid in atmospheres which contained no oxygen. How different are these phenomena from those which attend combinations and decompositions of inorganic matters! As an example, let oxygen be brought in contact, under proper conditions, with iron. Under these circumstances, a union of iron and oxygen takes place, and a new substance, oxide of iron, is formed, which has peculiar and distinct properties. In the same way, carbonic acid may be disengaged from its combinations by the action of a stronger acid, which unites with the base and forms a new substance, in no way resembling the original salt. To make the contrast still more striking, let a hy dro-carbon, like fat, be heated in oxygen or the air, until it undergoes combustion; it is then changed into carbonic acid and water, by a definite chemical reaction, and is utterly destroyed as fat. 
In the living body the organic nitrogenized principles are in a condition of continual change; breaking down, and forming various excrementitious principles, at the head of which may be placed carbonic acid. It is essential to life that these principles be maintained in their chemical integrity, which requires a supply of fresh matter as food, and above all a supply of oxygen. We put ourselves in the position of ignoring well-established facts and principles when, we assimilate without reserve the process of the consumption of oxygen and production of carbonic acid by living organic bodies, to simple combustion of sugar or fat. The ancients saw that the breath was warmer than the surrounding air, that in the lungs the air took heat from the body; and as they knew of no other changes in the air produced by respiration, they assumed that its object was simply to cool the blood. Lavoisier discovered that the air, containing oxygen, lost a portion of this principle in respiration, and gained carbonic acid and watery vapor. He saw that this might be imitated by the combustion of hydro-carbons, such as exist in the blood. He called respiration a slow combustion, and regarded as its principal office the maintenance of animal temperature. When it was shown by analyses of the blood for gases, that oxygen was not consumed in the lungs, but taken up by the circulating fluid, and carried all over the body, and that carbonic acid was brought from all parts by the blood to the lungs, these facts, taken in connection with the fact that the tissues have the property of consuming oxygen and exhaling carbonic acid, led physiologists to change the location of the combustive process from the lungs to the tissues.

We cannot stop at this point. Now it is known that the organic principles of the body, which form the basis of all tissues and organs, are continually undergoing change as a condition of existence; that they do not unite with any substance in definite chemical proportions, but their particles, after a certain period of existence, degenerate into excrementitious substances, and they are regenerated by an 
appropriation and change of materials furnished by the blood. As far as the respiration of these parts is concerned, we can only say, that in this process, carbonic acid is produced and oxygen is consumed. These facts show that respiration is essentially a phenomenon of nutrition, possessing a degree of complexity equal to that of the other nutritive processes. It must be acknowledged that thus far its cause and intimate nature have eluded investigation. In respiration by the tissues, no one has yet been able to give the cause of the absorption of oxygen or the exhalation of carbonic acid; or to demonstrate the condition in which oxygen exists when once appropriated, or the particular changes which take place, and the principles which are lost, in the formation of carbonic acid.

The views of physiologists with regard to the essential processes of respiration, before the time of Lavoisier, have barely an historical interest at the present day; except the remarkable idea of Mayow, which comprehended nearly the whole process, and which was unnoticed for about a hundred years. ${ }^{1}$ It is not our object to dwell upon the various theories which have been proposed from time to time, or even to fully discuss, in this connection, the combustion theory as proposed by Lavoisier, and modified by Liebig and others. Though this theory is nominally received by many physiologists of the present day, it will be found that most of them, in accordance with the facts which have since been developed, really regard respiration as connected with nutrition. They only differ from those who reject the combustion theory, in their definition of the term combustion. Lavoisier regarded respiration as a slow combustion of carbon and hydrogen; and if every rapid or slow combination of oxygen with any other body be considered a combustion, this view is absolutely correct, and was proven when it was shown that oxygen united with any of the tissues. Longet says that since the time of Lavoisier it is agreed to give the above signification 
to the word combustion $;^{1}$ but this must simply be for the purpose of retaining the name applied by Lavoisier to the respiratory process, while its signification is altered to suit the facts which have since taken their place in science. There is no doubt that combustion is generally regarded as signifying the direct and active union of oxygen with certain principles, which commonly contain carbon and hydrogen; and the immediate products of this union are carbonic acid, water, and incidentally heat and light. It is certain that oxygen does not unite in the body directly with carbon and hydrogen, though it is consumed, and carbonic acid and water are produced, in respiration. Important intermediate phenomena take place, and we do not therefore fully express the respiratory process by the term combustion. The researches of Spallanzani, W. F. Edwards, Collard de Martigny, ${ }^{2}$ and others, who have demonstrated the abundant exhalation of earbonic acid by animals and by tissues deprived of oxygen, show that it is not a product of combustion of any of the principles of the organism. ${ }^{3}$

Rejecting this hypothesis as insufficient to explain the intimate nature of the respiratory process, it remains to be seen how satisfactorily, in the present state of the science, it is possible to answer the several questions proposed at the . beginning of this chapter.

1. In what way is the oxygen consumed in the system?Oxygen, first taken from the air by the plasma of the blood, is immediately absorbed by, and enters into the composition of, the red corpuseles. Part of the oxygen disappears in the red corpuscles themselves, and carbonic acid is given off.

${ }^{1}$ Longet, Traité de Physiologıe, Paris, 1861, tome i., p. 392, note.

${ }^{2}$ Collard de Martigny, Recherches Expérimentales et Critiques sur I'Absorption et sur l'Exhalation Respiratoires. Journal de Physiologie, 1830, tome x., p. 111.

Sarious other considerations concerning the combustion theory of respiration, such as the so-called "respiratory, or calorific food," will be discussed in connection with the subject of animal heat. 
To how great an extent this takes place it is impossible to say; but it is evident, even from a study of the methods of analyses of the blood for gases, that the property of absorbing oxygen and giving off carbonic acid, which Spallanzani demonstrated to belong to the tissues, is possessed as well by the red corpuscles. During life it is not possible to determine how far this takes place in the blood, and how far in the tissues. Lagrange and Hassenfratz ${ }^{1}$ advanced the theory that all the respiratory change takes place in the blood as it circulates; but the avidity of the tissues for oxygen, and the readiness with which they exhale carbonic acid, leave no room for doubt that much of this change is effected in their substance. The late experiments of Bernard, ${ }^{2}$ showing that when blood is sent to the glands in large quantities, the oxygen is only imperfectly destroyed, the blood which is returned by the veins having nearly the color of arterial blood, are positive evidence against this view.

Oxygen, carried by the blood to the tissues, is appropriated and consumed in their substance, together with the nutritive materials with which the circulating fluid is charged. We are acquainted with some of the laws which regulate its consumption, but have not been able to follow it out and ascertain the exact nature of the changes which take place. Some have said that oxygen unites with the iron of the blood, or with the coloring matter of the corpuscles; but experiments on this point are contradictory and unsatisfactory. Some have said that it unites with the hydro-carbons of the blood and of the tissues; but there is more evidence that it enters into combination chiefly with the organic nitrogenized principles. All that we can say definitely on this point is,

\footnotetext{
${ }^{1}$ Hassenfratz, Mémoire sur la Combinaison de l'Oxygène avec le Carbone et l'Hydrogène du Sang, sur la Dissolution de l' Oxgyène dans le Sang, et sur la Manière dont le Calorique se dégage. Annales de Chimie, 1791, tome ix., p. 261.

${ }^{2}$ Liquides de l'Organisme, tome $i_{\text {. }}$; and unpublished lectures at the College of France, 1861. In the latter, Bernard gives comparative analyses of the renous blood from the submaxillary gland, showing a larger proportion of oxygen during its functional activity than during repose.
} 
that it unites with the organic principles of the system, satisfying the "respiratory sense," and supplying an imperative want which is felt by all animals, and extends to all parts of the organism. After being absorbed, it is lost in the intricate processes of nutrition. There is no evidence in favor of the view that oxygen unites directly with carbonaceous matters in the blood which it meets in the lungs, and, by direct union with carbon, forms carbonic acid.

2. How is carbonic acid produced by the system?That carbonic acid makes its appearance in the blood itself, produced in the red corpuscles, has been abundantly proven by observations already cited; though it is impossible to determine to what extent this takes place during life. It is likewise a product of the physiological decomposition of the tissues, whence it is absorbed by the blood circulating in the capillaries and conveyed by the veins to the right side of the heart. It has been experimentally demonstrated that its production is not immediately dependent upon the absorption of oxygen ; for it will go on in an atmosphere of hydrogen or of nitrogen. It is most reasonable to consider the carbonic acid thus formed as a product of excretion or destructive assimilation, like urea, creatine, or cholesterine. The fact that it may easily be produced artificially, out of the body, does not demonstrate that its formation in the body is as simple as when it is formed by the process of combustion. We may be able at some future time to produce artificially all the excrementitious principles, as has already been done in the case of urea $;^{1}$ but we are hardly justified in supposing that the mode of formation of this principle, as one of the phenomena of nutrition, is precisely the same as when it is made by our chemical manipulations.

${ }^{3}$ Wöller first formed urea artificially by a union of cyanic acid and ammonia. Since then it has been prepared by chemists by various processes (Lemmaxw, Physiological Chemistry, Philadelphia, 1855, vol. i., p. 147). 
As expressing nearly all that is known, even at the present day, regarding the mode of formation of carbonic acid in the economy, we may take the following concluding passage from the paper of Collard de Martigny, published in 1830: ${ }^{1}$

"The carbonic acid expired is a product of assimilative decomposition, secreted in the capillaries and excreted by the lungs."

The carbonic acid thus produced is taken up by the blood, part of it in a free state in solution, particularly in the plasma, and a part which has united with the carbonates to form bicarbonates. Carried thus to the lungs, the free gas is removed by simple displacement, and that which exists in combination is set free by the acids found in the pulmonary substance.

3. What is the nature of the intermediate processes, from the disappearance of oxygen to the evolution of carbonic acid?-A definite answer to this question would complete our knowledge of the respiratory process; but this, in the present state of the science, we are not prepared to give. We can only repeat what has already been so frequently referred to, that oxygen must be considered as a nutritive principle, and carbonic acid a product of excretion. The intermediate processes belong to the general function of nutrition, with the intimate nature of which we are unacquainted. We have not sufficient evidence for supposing that this process is identical with what is generally known as combustion.

The Respiratory Sense; or Want on the part of the System which induces the Respiratory Movements. (Besoin de Respirer.)

We are all familiar with the peculiar and distressing

${ }^{1}$ Loc. cit., p. 160. The author adds: "The chemical theory of Lavoisier, of respiration, is a gratuitous supposition. This function should be considered as a complete series of acts of general assimilation." 
sense of suffocation which attends an interruption in the respiratory process. Under ordinary conditions, the act of breathing takes place without our knowledge; but even when the air is but little vitiated, when its entrance into the lungs is slightly interfered with, or when a considerable portion of the pulmonary structure is involved by disease, we experience a certain sense of uneasiness, and become conscious of the necessity of respiratory efforts. This gradually merges into the sense of suffocation, and, if the obstruction be sufficient, is followed by convulsions, insensibility, and finally by death.

Though we are not sensible of any want of air under ordinary conditions, it was proven by the celebrated experiment of Robert Hooke, in 1664, that there is a want always felt by the system; and that if this want be effectually supplied, no respiratory movements will take place. We have often repeated the experiment demonstrating this fact. If a dog be brought completely under the influence of ether, the chest and abdomen opened, and artificial respiration be carefully kept up by means of a bellows fixed in the trachea, even after the animal has come from under the influence of the anæsthetic, so as to look around and wag his tail when spoken to, he will frequently cease all respiratory movements when the air is properly supplied to the lungs. This fact can be very satisfactorily observed, as the diaphragm and other important respiratory muscles are denuded, and exposed to view. If the artificial respiration be interrupted or imperfectly performed, the animal almost immediately feels the want of air, and the exposed respiratory muscles are thrown into violent but ineffectual contraction. ${ }^{1}$

It is generally admitted, indeed, that there exists in the

${ }^{1}$ For full details of these experiments the reader is referred to an article by the author, entitled Experimental Researches on Points connected with the Action of the Heart and with Respiration (American Journal of the Medical Scicnces, Oct., 1861). Since the publication of this paper, the experiments on respiration have been frequently repeated publicly, and the conclusions verified. 
system what may appropriately be called a respiratory sense, or, as it is called by the French, besoin de respirer, which is conveyed to the respiratory nervous centre and gives rise to the ordinary reflex and involuntary movements of respiration; that this sense is exaggerated by any thing which interferes with respiration, and is then carried on to the brain, where it is appreciated as dyspnœa, and finally as the overpowering sense of suffocation. An exaggeration of the respiratory sense constitutes an oppression, which is referred to the lungs. It has been demonstrated, however, that the sensation of hunger, which is felt in the stomach, and of thirst, which is felt in the throat and fauces, have their seat really in the general system, and are instinctively referred to the parts mentioned, because they are severally relieved by the introduction of food into the stomach, and the passage of liquid along the throat and œsophagus. It cannot therefore be assumed, from sensations only, that the sense of want of air is really located in the lungs. The question of its seat and its immediate cause is one of the most interesting of those connected with respiration.

Many physiologists accept the view of Marshall Hall, who first accurately described the reflex phenomena, that the respiratory sense is located in the lungs, is carried to the medulla oblongata by the pulmonary branches of the pneumogastric nerves, and is due to the accumulation of carbonic acid in the pulmonary vesicles; but there are facts in physiology and pathology which are inconsistent with such an exclusive view.

In cases of disease of the heart, when the system is imperfectly supplied with oxygenated blood, the sense of suffocation is trequently most distressing, though the lungs be unaffected, and receive a sufficient supply of pure air. This and other similar facts led Bérard to adopt the view that the respiratory sense has its point of departure in the right cavities of the heart, and is due to their distention as the result of obstruction to the passage of blood through the lungs. ${ }^{1}$ John 
Reid thought it was due in a measure to the circulation of venous blood in the medulla oblongata. ${ }^{1}$ What has been shown to be the correct explanation was given by Volkmann in 1841. He regarded the sense of want of air as dependent on a deficiency of oxygen in the tissues, producing an impression which is conveyed to the medulla oblongata by the nerves of general sensibility. By a series of experiments, this observer disproved the view that this sense resides in the lungs and is transmitted along the pneumogastric nerves; and by exclusion, he located it in the general system, and showed that such a supposition is competent to explain all the phenomena connected with the respiratory movements. ${ }^{2}$ In the hope of settling some of these questions, which might be regarded as somewhat uncertain, we instituted, a few years ago, a series of experiments, which were embodied in the paper already referred to ${ }^{3}$ In these observations, the following facts, some of which had been previously noted, were demonstrated; and their results leave no doubt as to the location and cause of the respiratory sense:

1. If the chest be opened in a living animal, and artificial respiration be carefully performed, inflating the lungs sufficiently but cautiously, and taking care to change the air in

${ }^{1}$ An Experimental Investigation into the Functions of the Eighth Pair of Nerves, etc. Part second. Anatomical and Physiological Researches, Edinburgh, 1848, p. 285; and Edinburgh Medical and Surgical Journal, April, 1839.

${ }^{2}$ Volkmand, in Schmidt's Jahrbücher, 1842, p. 290. Volkmann shows that after division of the pneumogastrics, an animal dies when deprived of air, not calmly, but with undoubted symptoms of distress from suffocation, as if it had been strangled without previous division of the vagi. He also made a number of experiments, in which respiratory efforts continued for many minutes after extirpation of the lungs, in eats and dogs, care being taken to leave the phrenic nerves intact. He goes on to reason that the sense of want of air must reside in the general system, that it is due to a deficieney of oxygen, and that its exaggeration constitutes the sense of suffocation. His observations do not show, however, that this is not due to the presence of carbonic acid, as has been supposed by many. Vierordt is of the opinion that the respiratory sense is due to the circulation of the renous blood in the substance of the nerves.

3 American Journal, October, 1861. 
the bellows every few moments, as long as this is continued, the animal will make no respiratory effort ; showing that, for the time, the respiratory sense is abolished.

2. When the artificial respiration is interrupted, the respiratory muscles are thrown into contraetion, and the animal makes regular, and at last violent efforts. If we now expose an artery, and note the color of the blood as it flows, it will be observed that the respiratory efforts only commenee when the blood in the vessel begins to be dark. When artificial respiration is resumed, the respiratory efforts cease only when the blood becomes red in the arteries. The invariable result of this experiment seems to show that the respiratory sense is connected with a supply of blood containing little oxygen and charged with carbonie acid to the systemic capillaries by the arteries, and that it varies in intensity with the degree of change in the blood.

3. It, while artificial respiration is regularly performed, a large artery be opened, and the system be thus drained of blood, when the hemorrhage has proceeded to a certain extent, the animal makes respiratory efforts, which become more and more violent, until they terminate, just before death, in general convulsions. The same result follows when the blood is prevented from getting to the system by applying a ligature to the aorta.

These facts, which may be successively observed in a single experiment, remain precisely the same if we previously divide both pneumogastric nerves in the neck; showing that these are by no means the only nerves which convey the respiratory sense to the medulla oblongata.

The conclusions which may legitimately be drawn from the above-mentioned facts are the following:

The respiratory sense has its seat in the system, and is transmitted to the medulla oblongata by the general sensory nerves. It is not located in the lungs, for it operates when the lungs are regularly filled with pure air, if the system be drained of the oxygen-carrying fluid. 
It is due to a want of oxygen on the part of the system, and not to any fancied irritant properties of carbonic acid; for when the lungs are filled with air, and the system is gradually drained of blood, though all the blood which finds its way to the capillaries is fully oxygenated, as the quantity becomes insufficient to supply the required amount of oxygen, the sense of want of air is felt, and respiratory efforts take place. The experimental results on which these conclusions are based are invariable, and have been demonstrated repeatedly; so that the location of the respiratory sense in the general system, and the fact that it is an expression of a want of oxygen, seem as certain as that oxygen is taken up by the blood from the lungs, and distributed to the tissues by the arteries. With this view we can explain all the reflex phenomena which are connected with the respiratory function. ${ }^{1}$

The supposition of Bérard that the respiratory sense is due to distention of the right.cavities of the heart is disproved by the simple experiment of sudden excision of this organ. In that case, as the system is drained of blood, efforts at respiration invariably take place, though the supply of air to the lungs be continued.

Sense of Suffocation.-We must separate, to a certain extent, the respiratory sense from the sense of distress from want of air, and its extreme degree, the sense of suffocation. The first is not a sensation, but an impression conveyed to the medulla oblongata, giving rise to involuntary reflex movements. The necessities on the part of the system for oxygen regulate the supply of air to the lungs. We have already seen that every five to eight respirations, or when the respi-

${ }^{1}$ There are many phenomena which physiologists found it impossible to explain on the supposition that the "besoin de respirer" was located in the lungs and conveyed to the medulla oblongata by the pneumogastrics; among which may he mentioned the effect of irritation of the general surface in the resuscitation of new-born children in which respiration is not established spontaneously. Dr. Marshall Ifall and John Reid thought that in these cases the sensory filaments distributed on the skin had something to do in transmitting impressions to the respiratory centre. 
ratory movements are a little restricted under the influence of depressing emotions, an involuntary deep or sighing inspiration is made, for the purpose of changing the air in the lungs more completely. The increased consumption of oxygen and a certain amount of interference with the mechanical process of respiration during violent muscular exercise put us "out of breath;" and for a time the respiratory movements are exaggerated. This is perhaps the first physiological way in which the want of air is appreciated by the senses. A deficiency in hematosis, either from a vitiated atmosphere, mechanical obstruction in the air-passages, or grave trouble in the general circulation, produces all grades of sensations, from the slight oppression which is felt in a crowded room, to the intense distress of suffocation. When hematosis is but slightly interfered with, only an indefinite sense of oppression is experienced; the respiratory movements are a little increased, the most marked effect being an inerease in the number and extent of sighing inspirations. In the experiments upon animals to which we have referred, when artificial respiration was interrupted, we first noticed regular and not violent contractions of the respiratory muscles; but as the sense of want of air increased, every muscle which could be used to raise the chest was brought into action. In the human subject in this condition, the countenance has a peculiar expression of anxiety and distress, and the movements soon extend to the entire muscular system, resulting in general convulsions, and, finally, insensibility.

Bearing in mind the fact, that though these sensations are referred to the lungs, indicating increased respiratory effort as the common means for their relief, they have their real point of departure in the general system, we can understand the operation of various abnormal conditions of the circulation, when the lungs are adequately supplied with fresh air. The first subjective symptom of air in the veins is a sense of impending suffocation. There is no want of air in the lungs, but the circulation is instantaneously inter- 
rupted, and oxygenated blood is not supplied to the tissues. The same effect, practically, follows abstraction of the circulating fluid, or the absorption of any poisonous agent which destroys the function of the corpuscles as carriers of oxygen; though in hemorrhage, the effects are not as marked, as generally the system is gradually debilitated by the progressive loss of blood. It was invariably noticed in the experiments above referred to, that after the division of a large artery, though artificial respiration was carefully performed, respiratory efforts took place when the system was nearly drained of blood. As the hemorrage continued, these efforts became more violent, and eventuated, just before death, in general convulsions. ${ }^{1}$ A comparison of this experiment with those in which artificial respiration was simply interrupted shows that in sudden hemorrhage there can be no doubt that the system feels the want of oxygen; and when the loss of blood is very great, this is increased until it amounts to a sense of suffocation. In gradual hemorrhage, there is a con-

1 "Expt. xxxiv., Feb. 19, 1861. A good-sized dog was etherized and the chest opened in the usual way. Artificial respiration was established, and Expt. xxix. verified. The blood was then allowed to flow freely from the femoral artery, while artificial respiration was actively continued. While the blood continued to flow, the respiratory muscles were carefully observed. During the first part of the bleeding no respiratory efforts took place; but when the blood had flowed for a considerable time, and the system was becoming drained, respiratory efforts commenced, feeble at first, but as the bleeding continued, becoming more violent until the whole muscular systern was affected by convulsive movements." (Am. Journ., loc. cit., p. 376.)

Convulsions after profuse hemorrhage have long been observed by physiologists, but no entirely satisfactory explanation of their oceurrence has ever been given. There now can be no doubt that they are due to a defieieney of oxygen. The experiments of Kusmaul and Tenner (On the Nature and Origin of Epileptiform Convulsions caused by Profuse Bleeding. New Sydenham Society, London, 1859) show that convulsions may be produced by ligature of the great vessels carrying blood to the brain. In this case they are probably due to a defieiency of oxygen in this vascular and highly organized part. In their experiments, which were made on rabbits, it was observed that "respiration is at first accelerated, but shortly afterwards, a little while before the approach of general convulsions, it becomes prolonged and deep." P. 14. 
servative provision of Nature, by which faintness and diminution in the force of the heart's action favor the arrest of the flow of blood.

Poisoning by carbonic oxide is generally accompanied with convulsions, which arise from the sense of suffocation, and are due to a fixation of this gas in the blood-corpuscles, by which they are rendered incapable of giving oxygen to the system. Convulsions also attend poisoning by hydrocyanic acid, in cases in which the system is not overpowered immediately by a large dose of this agent, and the muscular irritability destroyed.

Experiments have failed to show that the respiratory sense, or the sense of suffocation, is-due to irritation produced by carbonic acid in the non-oxygenated blood.

\section{Respiratory Efforts before Birth.}

It is generally admitted that one of the most important functions of the placenta, and the one which is most immediately connected with the life of the fœus, is a respiratory interchange of gases, analogous to that which takes place in the gills of aquatic animals. The vascular prolongations from the fœtus are continually bathed in the blood of the mother, and this is the only way in which it can receive oxygen. Notwithstanding the statements of those who have been unable to note any difference in color between the blood contained in the umbilical arteries and the vein, there are direct observations showing that such a difference does exist. Legallois frequently observed a bright red color in the blood of the umbilical vein; and on alternately compressing and releasing the ressel, he saw the blood change in color successively from red to dark, and dark to red. ${ }^{2}$ As oxygen is thus adequately supplied to the system, the fotus is in a condition similar to that of the animals in which artificial respiration was effectually performed. The want of oxygen is fully met, and therefore no respiratory

\footnotetext{
${ }^{1}$ Bérard, Cours de Physiologie, tome iii., p. 422.
} 
efforts take place. Respiratory movements will take place, however, even in very young animals, when there is a deficiency of oxygen in the system. It has been observed that the liquor amnii occasionally finds its way into the respiratory passages of the foetus, where it could only enter in efforts at respiration. Winslow, in the latter part of the last century, first noticed respiratory efforts in the foetuses of cats and dogs, in the uterus of the mother during life ${ }^{1}$ and many others have observed that when fotuses are removed from vascular connection with the mother, they will make vigorous efforts at respiration. This fact we have frequently had occasion to demonstrate in making operations upon pregnant animals. After the death of the mother, the fotus always makes a certain number of respiratory efforts, which are not uncertain in their character, but distinct, accompanied by great elevation of the ribs, opening of the mouth, and following each other at regular intervals, independently of irritation of the general surface."

From what has been experimentally demonstrated with regard to the location and cause of the respiratory sense after birth, it is evident that want of oxygen is the cause of respiratory movements in the fœetus. When the circulation in the maternal portion of the placenta is interrupted from any cause, or when the blood of the foetus is obstructed in its course to and from the placenta, the impression due to the want of oxygen is conveyed to the medulla oblongata, and efforts at respiration are the result. This cannot be due to an accumulation of carbonic acid in the lungs, and is entirely

${ }^{2}$ British and Foreign Medico-Chimurgical Review, April, 1864, p. 330.

${ }^{2}$ We take from our note-book the following observation showing respiratory efforts in a very young animal:

"Jan. 6, 1865. In operating to-day on a small-sized bitch, for the purpose of demonstrating the glyeogenic process in the lirer, I found her pregnant, and in the uterus were six pups, certainly not more than one-fourth the size which they attain before birth. (They were four inches long.) On removing them from the womb, and dividing the umbilical vessels, they all made a number of profound respiratory efforts at intervals of from two to three minutes." 
consistent with our views, locating the respiratory sense in the general system. ${ }^{1}$

\section{Cutaneous Respiration.}

This mode of respiration, though very important in many of the lower orders of animals, is insignificant in the human subject, and even more slight in animals covered with hair or feathers. ${ }^{2}$ Still, an appreciable quantity of oxygen is absorbed by the skin of the human subject, and an amount of carbonic acid, which is proportionately larger, is exhaled. Exhalation of carbonic acid, which is connected rather with the functions of the skin as a general excreting organ and is by no means an essential part of the respiratory process, will be more fully considered under the head of excretion. Carbonic acid is given off with the general emanations from the surface, being found at the same time in solution in the urine and in most of the secretions. It is well known that death follows the application of an impermeable coating to the entire cutaneous surface; but this is by no means due to a suppression of its respiratory function alone. The skin has other offices, particularly in connection with regulation of the animal temperature, which are infinitely more important.

An estimate of the extent of cutaneous, compared with pulmonary respiration, has been made by Scharling, ${ }^{3}$ by com-

${ }^{1}$ The physiological and pathological questions connected with the subject of "respiration before birth," are ably and exhaustively discussed in a review published in the Medico-Chirurgical Review, for April, 1864. A number of experiments by various observers are here detailed, fully establishing the facts we liave stated. Among the most interesting are those of Schwartz, showing respiratory movements in foetuses, when care was taken not to expose them to the cool air or any other irritation of the general surface, p. 333.

${ }^{2}$ Regracle and Reiset found the cutancous respiration so slight in the animals which they uscd for their experiments, that its influence upon the composition of the air in which they were confined could be disregarded. (Op. cit.)

s In Milne-Edwarns, Leçons sur la Physiologie, tome ii., p. 635. The reader will here find an account of the experiments of De Milly, Abernethy, and others, demonstrating the absorption of oxygen and exhalation of earbonic acid by the skin. 
paring the relative quantities of carbonic acid exhaled in the twenty-four hours. According to this observer, the skin performs from $\frac{1}{50}$ to $\frac{1}{40}$ of the respiratory function.

\section{Asphyxia.}

The effects of cutting off the supply of oxygen from the lungs are mainly referable to the circulatory system, and have already been considered under the head of the influence of respiration upon the circulation. ${ }^{1}$ It will be remembered that in asphyxia the non-aërated blood passes with so much difficulty through the systemic capillaries, as finally to arrest the action of the heart. It is the experience of those who lave experimented on this subject, that the movements of the heart, once arrested in this way, cannot be restored; but that while the slightest regular movements continue, its functions will gradually return if air be readmitted to the lungs.

A remarkable power of resisting asphyxia exists in newly born animals that have never breathed. This was noticed by Haller and others, and has been the subject of numerous experiments, among which we may mention those of Buffon, Legallois, and W. F. Edwards. Legallois found that young rabbits would live for fifteen minntes deprived of air by submersion, but that this power of resistance diminished rapidly with age. ${ }^{2}$ W. F. Edwards has shown that there exists a great difference in this regard in different classes of animals. Dogs and eats, that are born with the eyes shut, and in which there is at first a very slight development of animal heat, will show signs of life after submersion for more than half an hour; while Guinea pigs, which are born with the eyes open, are much more active, and produce a greater amount of heat, will not live more than seven minutes. ${ }^{3}$

${ }^{1}$ See page 290.

${ }^{3}$ W. F. Edwards, De l'Influence des Agens Physiques sur la Vie, Paris, 1824, pp. 171, 172. 
The cause of this peculiarity has been attributed to the existence of the foramen ovale, enabling the blood to get to the system without passing through the Jungs, by those who regard the arrest of the circulation in asphyxia as due to obstruction to the pulmonary circulation; but this explanation is not sufficient, as blood passes easily through the lungs in asphyxia, and is obstructed only in the systemic capillaries.

The true explanation seems to be, that in most warmblooded animals, during the very first periods of extra-uterine life, the demands on the part of the system for-oxygen are comparatively light. At this time there is very little activity in the processes of nutrition, and the actual consumption of oxygen and exhalation of earbonic acid are very much below the regular standard in animals of this class. In fact, their condition is somewhat like that of cold-blooded animals. The actual difference in the consumption of oxygen immediately after birth and at the age of a few days is sufficient to explain the remarkable power of resisting asphyxia just after birth. The comparative observations of Edwards on dogs, cats, and Guinea pigs, show that this power bears a definite relation to the respiratory activity.

One of the most interesting questions, in a practical point of view, connected with the subject of asphyxia, is the effect on the system of air vitiated from breathing in a confined space. There are here several points presented for consideration. The effect of respiration on the air is to take away a certain proportion of oxygen, and add certain principles which are regarded as deleterious. The emanation which is generally regarded as having the most decided influence upon the system is carbonic acid.

A careful review of the most reliable observations on this subject shows that the influence of earbonic acid is generally very much over-estimated. In poisoning by charcoal fumes, it is generally carbonic oxide which is the active princi- 
ple. Regnault and Reiset ${ }^{2}$ exposed dogs and rabbits for many hours to an atmosphere containing 23 parts per 100 of carbonic acid artificially introduced, and 30 to 40 parts of oxygen, without any ill effects. They took care, however, to keep up a constant supply of oxygen. These experiments are at variance with the results obtained by others, but Regnault and Reiset explain this difference by the supposition that the gases in other observations were probably impure, containing a little chlorine or carbonic oxide. There is no reason to doubt, from the high reputation of the observers for skill and accuracy, that their experiments are perfectly reliable; and in that case, they prove that carbonic acid does not act upon the system as a poison. This view is sustained by the mure recent observations of Dr. Hammond, which we give in his own words:

"I confined a sparrow under a large bell-glass, having two openings. Through one of these I introduced every hour 1,000 cubic inches of an atmosphere containing 45 parts of oxygen, 30 of nitrogen, and 25 of carbonic acid, allowing the vitiated air in which the animal had respired partially to escape. At the end of twelve hours the bird was in as good a condition as at the commencement of the experiment; and when the bell-glass was raised, it flew away as if nothing had happened to it. A mouse subjected to a similar experiment also suffered no inconvenience." ${ }^{3}$

In breathing in a confined space, the distress and finally fatal results are produced, in all probability, more from animal emanations and deficiency of oxygen, than from the presence of earbonic acid. When the latter gas is removed as fast as it is produced, the effects of diminution in the proportion of oxygen are soon very marked, and progressively increase till death occurs. Bernard has shown that birds enclosed in a confined space, from which the carbonic acid is carefully

1 Loc. cit.

' Hammond, Treatise on Hygiene, Philadelphia, 1863, p. 351. 
removed, will gradually consume oxygen, until, when death occurs, the proportion is reduced to from 3 to 5 parts per 100. When the carbonic acid is allowed to remain, the increased density of the atmosphere interferes with the diffusion between the gases of the blood and the air, and death supervenes with greater rapidity.

The influence on animals of emanations from the lungs and general surface, from which the carbonic acid and watery vapor have been removed, has been shown by Dr. Hammond to be very decided and rapid. He confined a mouse in a large glass jar, so arranged as to admit fresh air as the atmosphere became rarefied by respiration, causing the carbonic acid to be absorbed by sponges saturated with baryta-water, and the watery vapor by pieces of chloride of calcium. The animal died in forty-five minutes; when, by passing the gaseous contents of the jar through baryta-water, it was shown to contain no carbonic acid, and the presence of organic matter in large quantity was demonstrated. ${ }^{2}$

In crowded assemblages, the slight diminution of oxygen, the elevation of temperature, increase in moisture, and particularly the presence of organic emanations, com bine to produce unpleasant sensations. The terrible effects of this carried to an extreme were exemplified in the confinement of the 146 English prisoners, for eight hours only, in the "Black Hole" of Calcutta; a chamber eighteen feet square, with only two small windows, and those obstructed by a verandah. Out of this number, 96 died in six hours, and 123 at the end of the eight hours. Many of

${ }^{1}$ Bernard, Legons sur les Effets des Substances Toxiques et Médicamenteruses, Paris, 1857 , p. 116.

${ }^{3}$ Op. cit., p. 170. "For the detection of organic matter in the atmosphere, the permanganate of potassa affords a very sensitive reagent. A solution of this substance in water loses its brilliant red color, and the salt undergoes decomposition, when air containing organic matter is passed through it. By the extent to which the loss of color reaches we are enabled to form an approximative idea of the amount of such matter present in the air. The solution is placed in Liebig's bulbs, and the air is drawn through it by means of an aspirator." P. 172. 
those who immediately survived afterwards died of putrid fever. ${ }^{1}$ This frightful tragedy has frequently been repeated on emigrant and slave ships, by confining great numbers in the hold of the vessel, where they were entirely shut out from the fresh air. This subject possesses great pathological interest; the effects of an insufficient supply of air and the accumulation in the atmosphere of animal emanations being very important in connection with the cause and prevention of many diseases.

The condition of the system has a marked and important influence on the rapidity, with which the effects of vitiated atmosphere are manifested, as we should anticipate from what we know of the variations in the consumption of oxygen under different conditions. As a rule, the immediate effects of confined air are not as rapidly manifested in weak and debilitated persons, as in those who are active and powerful. It has sometimes been observed, in cases where a male and a female hare attempted suicide together by the fumes of charcoal, that the female may be restored some time after life is extinct in the male. This is probably owing to the greater demand for oxygen on the part of the male.

The following interesting fact is reported by Bernard, showing the relative power of resisting asphyxia in health and disease:

"Two young persons were in a chamber warmed by a stove fed with coke. One of them was seized with asphyxia and fell unconscious. The other, at that time suffering with typhoid fever and confined to the bed, resisted sufficiently to be able to call for help. We know already that this resistance to toxic influences is manifested in animals, when they are made sick; we here have the proof of the same phenomenon in man. As for the one who, in good health, had experienced the effects of the commencement of poisoning, she had a

${ }^{1}$ A full account of the sufferings of these unfortunate men, by one of the survivors, is to be found in the Annual Register, 1758, p. 278. 
paralysis of the left arm, which was not completely cured at the end of six months." 1

It is thought that the condition of syncope has an influence on the power of resistance to asphyxia. A case is quoted by Carpenter in which a woman, who had been submerged for fifteen minutes, was taken out of the water and recovered spontaneously. She stated that she was insensible at the moment of her submersion. ${ }^{2}$

When poisoning by confined air is gradual, the system becomes somewhat accustomed to the toxic influence; the temperature of the body is lowered, ${ }^{3}$ and an animal will live in an atmosphere which will produce instantaneous death in one that is fresh and vigorous. Bernard has made a number of curious and instructive experiments on this point. In one of them, a sparrow was confined under a bell-glass for one hour and a half, at the end of which time another was introduced, the first being still quite vigorous. The second became instantly much distressed, and died in tive minutes; but ten minutes after, the sparrow which had been confined for more than an hour and a half was released, and flew away."

This is simply demonstrating, with experimental accuracy, a fact of which we are all conscious; for it is well known, that going from the fresh air into a close room, we experience a malaise which is not felt by those who have been in the room for a length of time, and whose emanations have vitiated the atmosphere.

${ }^{2}$ Bernard, op. cit., p. 197.

${ }^{2}$ Carpenter, Principles of Human Physiology, Am. edit., 1853, p. 536.

${ }^{3}$ Bernard noted a diminution in the temperature in the rectum of a pigeon, from $105^{\circ}$ to $88^{\circ}$ Fahr., after four hours' sojourn in a confined space, containing 732 cubic inches of air. The animal was nearly dead when removed. (Loc. cit., p. 128.)

Op. cit., p. 119. 



\section{N D E X.}

Air, diffusion of, in the lungs, ... 406 composition of,.........4 413 changes of, in passage through the lungs, . . . . ........ 423

- increase in temperature of, in passage through the lungs, .... 423

Air-cells, anatomy of, . ........ 362

Albumen, situations and quantity of,....................

- mode of extraction and properties of, ................ - influence of, on polarized light, ................ — tests for,$\ldots \ldots \ldots \ldots \ldots \ldots$

- origin and functions of,.....

Albuminometer, .............

Albuminose,...............

Alcohol, exhalation of, by the lungs, ............... 450

Ammonia, exhalation of, in respiration,............... 448

Arteries, circulation in,....... 240

— physiological anatomy of,... 241 divisions of, .......... 243

- coats of ,................ 243

- nerves in walls of,....... 245

— blood-ressels in walls of,... 245

- elasticity of, ........... 246

_ experiments showing dilatation of ............... 247

- influence of elasticity of, on

the current of blood,....... 248

- contractility of, .......... 250 locomotion of, and produc-

tion of the pulse,.......... 252 variations in caliber of, at

different periods of the day,...2 261
Arterial pressure,........... 261

— in different vessels, ....... 266

_ influence of respiration on,.. 267 influence of hemorrhage on,. 269

Arterial circulation, rapidity of,.. 270

apparatus of Volkmann and

Hüttenheim for measuring rapidity of $\ldots \ldots \ldots \ldots \ldots \ldots 271$

$\longrightarrow$ apparatus of vierordt, . . . 272

apparatus of Chaureau,..... 273

— rapidity of, in different ves-

sels, ................ 274

Arterial murmurs,......... 276

Asphyxia,................ 490

- power of resistance to in the

newly-born, .........421, 490

__ from breathing in a confined

space,............ 491, 495

- from chareoal fumes,...... 491 influence of, on pulmonary circulation,

Besoin de respirer,....... 479-484

Bicarbonate of soda,......... 45

Bilirerdine,............. 93

Black Hole of Calcutta,......... 493

Blood, general considerations,.... 95

- immediate importance to

life, ................ 96

_ expcriment of withdrawing a

large quantity from the vessels, 97

— transfusion of, ......... 97

transfusion of, in discase,... 98

transfusion of, in experi-

ments on animals,......... 99 entire quantity of, in the

body,................. 100 
Blood, reaction, odor, and opacity of ................... 104

- temperature and specific gravity of,................ 105 color of,............ 106 color of, in reins of the glands,.................. - analyses of,.......... 127 inorganic constituents of,.... 128 organic nitrogenized constituents of ,............... 129 organic non-nitrogenized constituents of,............. 129 — quantitative analyses of, ... 130 - quantitative analysis of, by method of Becquerel and Rodier, .................. 131

- quantitative analysis of, by the author's method,....... 134 — table of composition of, ... 138 coagulation of,.......... 142 rapidity of coagulation of, . . 143 circumstances modifying co-

agulation of,........... 149 coagulation of, in the organ-

ism,................ 150 - office of coagulation in arrest

of hemorrhage,.......... 153 cause of coagulation of,.... 156 summary of properties and

functions of, .......... 167 changes of, in respiration,.. 452 difference in color between

venous and arterial,........ 454 - general differences between

arterial and venous, ........ 470 analyses of, for gases, . . 458-464 condition of gases in, ....4 466 Blood-corpuscles (red),........ 108 anatomical characters of, ... 109 table of measurements of,.. 113 chemical characters of,..... 117 development of,......... 118 functions of, ............ 120 (white) . .............. 121 elementary corpuscles, ..... 126 absorption of oxygen by,... 455

Blood-crystals,............. 117 Breathing capacity, extreme,.... 403 Bronchial tubes, anatomy of, . . 360

Calorific elements,........... 60

Capillaries, circulation in,...... 278

anatomy of, ........... 279 distribution of, ......... 281 course of blood in,....... 283

Capillary system, capacity of,.... 282
Capillary, circulation, microscopic examination of,........... 284 rapidity of,........... 289 - relations of, to respiration,.. 290 - causes of,............. 293 phenomena in patients dead

with yellow fever,......... 295 influence of temperature on, 297 influence of direct irritation on $. . . \ldots \ldots \ldots \ldots \ldots . . .298$

Carbonate of lime,.......... 42

- crystals of, in internal ear,.. 43

- formation of, in analysis by , incineration, ............... - quantity of (table), and function,................ 43 Carbonate of soda, quantity of (table), and function,.........

Carbonate of potassa, and carbonate of magnesia,.......... 45

Cartilagine,............... 91 Cardiometer of Magendie and Bernard,............ 263, 265 - of Marey (differential),..... 264 Carbonaceous matter in the lungs, 364 Carbonic acid, discovery of,..... 410 - exhalation of, in respiration, 424

- influence of arrest of respiratory movements on exhalation of, ................ 425 - quantity of, exhaled,....... 427 influence of age on exhala-

tion of,$\ldots \ldots \ldots \ldots \ldots \ldots . . .431$

— influence of sex,........ 432

- influence of digestion,......443 433

- influence of diet,........ 435

— influence of alcohol,....... 437

— influence of sleep,........ 439

- influence of moisture and

temperature,............441

— influence of seasons, ...... 442 sources of, in the expired air,................. 445 - proportion in arterial and venous blood,........... 464 - condition of, in the blood,.. 467 — effect of inhalation of,...... 492 - production of, in respiration, 478 Carbonic oxide, exhalatiou of, by the lungs when injected into the blood,................. 450 Cascine, extraction of, ete,..... 86 Catalysis, definition of,....... 74 Cephalo-rachidian fluid, uses of, . 334 Chloride of sodium,.......... 35 - quantity of (table),...... 35 function of,........... 36 
Chloride of sodium, desire of all animals for,.............. - effect of deprivation of, on nutrition, ...............

- quantity of in blood almost constant,...............

- removal of excess of by the kidneys,................

Chloride of potassium, .........

Chloride of ammonium,........

Circulation of the blood, discovery of,$\ldots \ldots \ldots \ldots \ldots \ldots \ldots . \ldots \ldots$

- general course of,........ 175 action of the heart in (see Heart),............... 177 - in the arteries (see Arteries),............... 240

- in the capillaries (see Capillaries),............... 278 - in the veins (see Veins),... 301 Circulation, derivative,........ 339

— pulmonary,............. 340

— general rapidity of,....... 343

- rapidity of, in different ani-

mals,...................... 346

- relations of rapidity of, to the frequency of the heart's action, 348 Circulatory system, phenomena in, after death,............. 351

Clot, characters of ,......... 144

Coloring matters,........... 92

Complemental air,...........4 401

Convulsions from hemorrhage,... 486

Coughing, ............... 395

Coagulation of the blopod (see

Blood),............... 142

Cranial cavity, circulation in,.... 332

-_ amorphous sheath of blood-

vessels of, ............. 336

Crystalline,.............. 90

Diabetic sugar,............ 50

Diaphragm, action of, in respira-

tion, ................. 369

Diffusion of air in the lungs, .... 406

Elasticine,............... 91

Emulsion,................ 63

Emphysema, changes of thorax in, 385

Epiglottis, action of, in deglutition, 359

Erectile tissues, circulation in,.... 336

Erection, mechanism of,....... 338

Expiration, movements of, ..... 382

- influence of elasticity of the

lungs and thoracic walls in,.... 383

— muscles of (table),...... 385
Expiration, action of internal intercostals in, ............ 386

- action of infra-costales and triangularis sterni in, ....... 387

- action of obliquus externus and internus in,........... 388

- action of transversalis in,... 388 action of sacro-lumbalis in, .. 389

Fats, varieties of, \&c........ 60

composition and properties

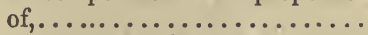

condition of, in nervous tissue and blood-corpuscles,........

- saponification of,$\ldots \ldots \ldots \ldots$ emulsion of,............. origin and functions of, .... formation of, in the organism,................... - average quantity of, in the body, and mechanical function of,$\ldots \ldots \ldots \ldots \ldots \ldots$. - changes which they undergo

in the organism,........... 60

Fatty acids, .............. 62, 66

Fermentation of sugar,........ 51

Fermentation-test for sugar,.... 56

Fibrin,.................. 76

— mode of extraction of, and condition in the organism,..... organization of,.......... distinctions from plastic

lymph,................. origin of, ............. function of, and destruction

by liver and kidneys,........

\section{6} (1) 76 77 78 80

Gases, as proximate principles,... 29

— in the alimentary canal,.... 29 proportions of, in renous and arterial blood,......456,464-470 of the blood, table of Magnus,............... 463 Gases, condition of, in the blood, . 466 Globuline,................ 90 Glucose,................ 50 Glycerine,.............. 62

Hæmatoidine, .............. 117

Heart, anatomy of,.......... 176

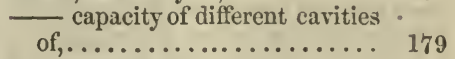
- valves of $, \ldots \ldots \ldots \ldots \ldots \ldots, 181$

- movements of,........ 183

— action of the auricles,..... 184 - action of the ventricles,... 185 
Heart, locomotion of ......... twisting, hardening, shortening, and elongation of,.....

- impulse of, ............. - succession of movements of, force of,............ 197 action of the valves,...... 199 - sounds of,............ 203

- cause of the sounds of,....

thelations of the sounds to

the blood-currents,......... 210

— frequency of action of, .... 211

- influence of age and sex on

frequency, .............. 212

- influence of posture and mus-

cular exertion, ............ 213

- influence of exercise,...... 215

— influence of sleep,....... 216

_ influence of temperature,... 216

- influence of respiration on

action of,.............. 217

cause of rhythmical contrac-

tions of $\ldots \ldots \ldots \ldots \ldots \ldots 220$

- irritability of,.......... 222

pulsations of, after removal

from the body,........... 223

- effect of ligature of coronary

arteries on pulsations of,..... 225

- effect of emptying the cavi-

ties, ..................

- influence of the nerrous sys-

tem on,$\ldots \ldots \ldots \ldots \ldots \ldots . . \ldots 228$

— influence of pneumogastrics

on, ..................... 231

effects of blows on epigastrium

on,$\ldots \ldots \ldots \ldots \ldots \ldots \ldots \ldots 238$

Hematine,.............. 92

Hematosis, .................. 452

Hemodynamometer of Poiseuille,...............262, 265

- registering instrument of

Ludwig (note),............ 264

differential instrument of $\mathrm{Ber}$ -

nard (note),.............. 266

Hydro-carbons, general considera-

tions, .............. 26, 48

Hydro-chlorate of ammonia,..... 47

Inorganic principles, general considerations, ................. table of,..............

— division into essential constituents of the tissues and those which influence nutrition,..... Inspiration, museles of (table),... .

- action of diaphragm in,.....

- action of scaleni,......... major, and serratus magnus,... Intercostals, internal, action of, in respiration, ............. 386

Infra-costales, action of, in respiration, .................

movements of, the ribs in,... 374

- action of levatores costarum, 378

— auxiliary muscles of, ....... 378 superior, .............. 378 - action of sterno-mastoideus, levator anguli scapulæ, and superior portion of trapezius,.... 379 action of pectoralis minor, inferior portion of pectoralis 380 387

Keratine,.............. 91

Lactic acid,............. 67

- sources and function of,.... 68

Larynx, anatomy and respiratory movements of,........... 358

Laughing, . . . . . . . . . . . . 398

Levatores costarum, action of, in respiration,............. 378

Levator anguli scapulæ, action of, in respiration, ........... 379

Leucocytes,................... 121

- development of . ............ 124

- proportion of, to red corpuscles, .................. 125

Liver, influence of respiration on circulation in ............. 322

Liver-sugar, .............. 50

Lungs, anatomy of parenchyma of, 361

— capacity of, ........... 397

- carbonaceous matter in,.... 364

— vital capacity of, ....... 403

Melanine,................ 93

Milk-sugar, ................ 50

Mucosine,................ 89

Musculine, ................... 90

Nitrogen, exhalation of, in respiration,................. 451 - of the blood, ........465, 468 Nitrogenized principles, general considerations,.........27, 69

Nitrous oxide, effects of respiration of, ............. 415 47 Non-nitrogenized principles,... 25, 48

Obliquus externus and internus, action of, in respiration,...... 388 
Odorous principles,.......... 66

exhalation of, by the lungs,. 450

Organic non-nitrogenized principles, general considerations, .. 27, 69

Organic nitrogenized principles, composition, properties, and condition of, in the organism,... table of,............... summary of properties of,...

Organic matter, exhalation of, in respiration, ..............

Osteine,.................. 91

Otoconies, or otoliths,......... 18

Oxygen, discovery of,......... 412

- minimum proportion in the air which will support life,.... 414

- effects of confining animals in atmosphere of,......... 415

- consumption of, in respiration, ............4 416, 476 __ influence of age on consumption of ................. - influence of temperature,.... 420

- consumption of, in hibernation, .................. - absorption of, by blood-corpuscles,................... proportion in arterial and venous blood,.............. - condition of, in the blood,...

Ozone,..................

Pancreatine,................

Pepsin, ....................

Pectoral muscles, action of, in respiration, ................

Phosphate of lime (table of quantity of $), \ldots \ldots \ldots \ldots \ldots \ldots$

Phosphates of magnesia, soda, and

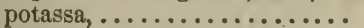

Physiology, definition of,........

Piezometer (note) ,.............

Pneumic acid,............... action of, on the bicarbonates in the blood,..............

Pneumate of soda,...........

Poisonous gases, exhalation of, by the lungs, .................

Proximate principles, general considerations, ............20-24

— inorganic,............ 25

- do. (table),.............

— organic non-nitrogenized,...

- organic nitrogenized,.......

Proteine,.................

Pulmonary artery, pressure of blood in .................. 455 464 466 414
Pulse, mechanism of production of, 252

— frequency of,$\ldots \ldots \ldots \ldots \ldots 212$

form of, ............ 254

- dierotic, ............. 257

— variations in character of,... 260 influence of temperature on, 260

Putrefaction,............ 73

Rennet,.............. 87

Respiration, influence of, on the

action of the heart,........ 217

- general considerations,..... 353

— movements of ......... 566

__ frequency of movements of,. 391

— movements of ribs in, ..... 374

- types of, .............. 389

— relations of inspiration and

expiration,.............. 392

- relations in volume of inspired and expired air,....... 405 - changes of air in (historical $421 \quad$ considerations), ........... consumption of oxygen in 409 (see Oxygen),............4 416 422 - effect of confining an animal in a mixture of oxygen and hydrogen,................ 422

- exhalation of carbonic acid

(see Carbonic Acid),........ 424

- relations between the quan-

tity of oxygen consumed and carbonic acid exhaled,.......443

_ exhalation of watery vapor, .. 446

- exhalation of ammonia,.... 448

__ exhalation of organic matter, 449

__ exhalation of alcohol,..... 450

exhalation of odorous princi-

ples,................. 450

- - exhalation of certain poison-

ous gases,.............. 450

—_ exhalation of nitrogen,.... 451

- changes of the blood in,.... 452

- absorption of oxygen by the

blood-corpuscles,........... 455

- proportions of gases in venous

and arterial blood,... 456, 464-470

-

—_ combustion-theory of, . . 473-476

- consumption of oxygen, ... 476

- production of carbonic acid, 478

— cutaneous, ............4 489

Respiratory organs, anatomy of,.. 357

Respiratory sounds (murmurs),.. 393

Respiratory sense, the sensation inducing respiratory movements,.............479-484

Respiratory efforts before birth,.. . 487 
Residual air,............. 399

Reserve air,............... 400

Saponification,.............. 62

Sacro-lumbalis, action of, in respiration,................ 389

Scalene muscles, action of, in respiration,............... 372

Serratus posticus superior, action of, in respiration, .......... 378

Serratus magnus, action of, in respiration,................. 380

Serum, characters of,$\ldots \ldots \ldots \ldots .146$

Sighing, ................. 396

Sleep, cerebral circulation in,.... 334

Snoring,................... 393

Sneezing, . . . . . . . . . . . . 395

Sobbing,.................. 396

Soaps,,$\ldots \ldots \ldots \ldots \ldots \ldots \ldots .62-66$

Sphygmograph of Marey,....... 255

— of Vierordt,.............. 256

Sterno-mastoideus, action of, in respiration, ................. 379

Sulphates of soda, potassa, and lime, .................

Sulphuretted hydrogen, exhalation of, by the lungs, ..........450

Sugar,................... 49

- varieties of, ............. 50

union of, with chloride of sodium,...$\ldots \ldots \ldots \ldots$.........

- composition and properties of,.....................

— fermentation of,........ . lactic-acid fermentation of,.. -influence of solution of on polarized light,............. tests for, Moore's or the potash test, Trommer's test,....

- Barreswill's test, ........... Maumené's test, fermentation test, Böttger's test,.......

— formation of torulæ,......... origin and functions of ,.... formation of, in the foetus, and influence on cell-development, .................. - destruction of, in the lungs,.

Suffocation, sense of,....... .

Tidal air
Torulæ cerevisix,..$\ldots \ldots \ldots \ldots$. 58

Transfusion of blood, ....... 97-99

Transversalis, action of, in respiration, .............. 388

Trapezius, action of, in respiration, 379

Trachea, anatomy of, ......... 360

Triangularis sterni, action of, in respiration, ............... 387

Urrosacine,............... 93

Valves of the veins, discovery of,. 172 Valves of the heart (see Heart),... 181

Vasa vasorum,............ 24z

Véins, anatomy of,.......... 301

— capacity of ............ 302

- strength of, ............ 306

— valves of, ............... 308

— function of valves of,..... 325

- _ course of blood in,...... 311

— pressure of blood in, ...... 314 rapidity and causes of circu-

lation in $. \ldots \ldots \ldots \ldots \ldots \ldots . \ldots . \ldots 15$

_ influence of muscular con-

traction on current of blood in, 317 influence of aspiration from

the thorax,.............. 319

- influence of gravitation, $324-330$

_ entrance of air into, ...... 323

conditions which impede cir-

culation in, .............. 328 influence of expiration on current of blood in,....... 328

Venous pulse,............. 813

Venous pulse, regurgitant,....... 329

Vital properties of organized structures,................ 18

Vital capacity of the lungs,.....4403

Water, as a proximate principle, . 30

- condition of, in the organism,................... 30

(table),.............. 33 entire quantity in the body,. 48

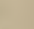




\section{RECENT PUBLICATIONS}

OF

D. APPLETON \& CO., 443 and 445 Broadway, New York.

\section{Mercantile Dictionary. A com-} plete vocabulary of the technicalities of Commercial Correspondence, names of Articles of Trade, and Marine Terms, in English, Spanish, and French; with Geographical Names, Business Letters, and Tables of the Abbreviations in common use in the three languages. By I. De Veitelle. Square 12mo. Half morocco. Price, $\$ 3.00$.

"A book of most decided necessity to all merchants, filling up a want long felt."-Journal of Commerce.

"It is undoubtedly a very important and serviceable work."-Indianapolis Journal.

\section{The Mystical Rose; or, Mary of}

Nazareth, the Lily of the House of David. "I am the Rose of Sharon and the Lily of the Valley."-Canticles. "Many daughters have done virtuously, but thou excellest them all."-Solomon. "Blessed art thou among women."-Gabriel. By Marie Josepilene. $12 \mathrm{mo}$. Cloth extra. Price $\$ 2.00$.

"This elegant and charming hook has just appeared; it is worthy of a place in every family." - Bellows Falls Times.

"Its strong devotional character will commend it to many readers." $I l l i$. nois Stute Journal.

\section{Cousin Alice: A Memoir of}

ALICE B. HAVEN. 12mo, pp. 392, with portrait. Cloth. $\$ 1.75$.

"This is a record of deep interest, compiled with taste, skill, and jidgment." Christian Times.

"Exceedingly interesting,"-Eastern Argus.

"Written with great vigor and simplicity."-Boston Post. 


\section{A Report of the Debates and}

Proceedings in the Secret Sessions of the Conference Convention for Proposing Amendments to the Constitution of the United States, held at Washington, D. C., in February, A. D. 1861. By L. E. Chittenden, one of the Delegates. Large 8vo. 626 pp. Cloth. $\$ 5.00$.

"The only authentic account of its proceedings."-Indianapolis Journal.

"It sheds floods of light upon the real causes and impulses of secession and rebellion."-Christian Times.

"Will be found an invaluable authority."-New York Tribune.

\section{The Conflict and the Victory of}

Life. Memoir of Mrs. CAROLINE P. KEITH, Missionary of the Protestant Episcopal Church to India. Edited by her brother, William C. Tenney. "This is the victory that overcometh the world, even our faith."-St. John. "Thanks be to God, who giveth us the victory through our Lord Jesus Christ." -St. Paul. With portrait. 12mo. Price, \$2.00.

"A work of real interest and instruction."-Buffalo Courier.

"Books like this are valuable as incentives to good, as monuments of Christian zeal, and as contributions to the practical history of the Church." - Con gregationalist.

\section{Sermons Preached at the Church}

of St. Paul the Apostle, New York, during the year 1864. Second Edition. 18mo. $406 \mathrm{pp}$. Cloth. Price, $\$ 1.50$.

"They are all stirring and vigorous, and possess more than usual merit." Methodist Protestant.

"These sermons are short and practical, and admirably calculated to improve and instruct those who read them."-Commercial Advertiser.

\section{The Trial. More Links of the}

Daisy Chain. By the Author of "The Heir of Redclyffe." Two volumes in one. Large $12 \mathrm{mo}$. $390 \mathrm{pp}$. Cloth, price $\$ 1.75$.

"The plot is well developed; the characters are finely sketched; it is a capital novel."-Providence Journal.

"It is the best novel we have seen for many months."-MIontreal Gozette.

"It is marked by all the fascinating qualities of the works that preceded it. *** Equal to any of her former novels."-Commercial. 





\section{UNIVERSITY OF CALIFORNIA MEDICAL SCHOOL LIBRARY}

\section{THIS BOOK IS DUE ON THE LAST DATE}

STAMPED BELOW

Books not returned on time are subject to a fine of $50 \mathrm{c}$ per volume after the third day overdue, increasing to $\$ 1.00$ per volume after the sixth day. Books not in demand may be renewed if application is made before expiration of loan period.

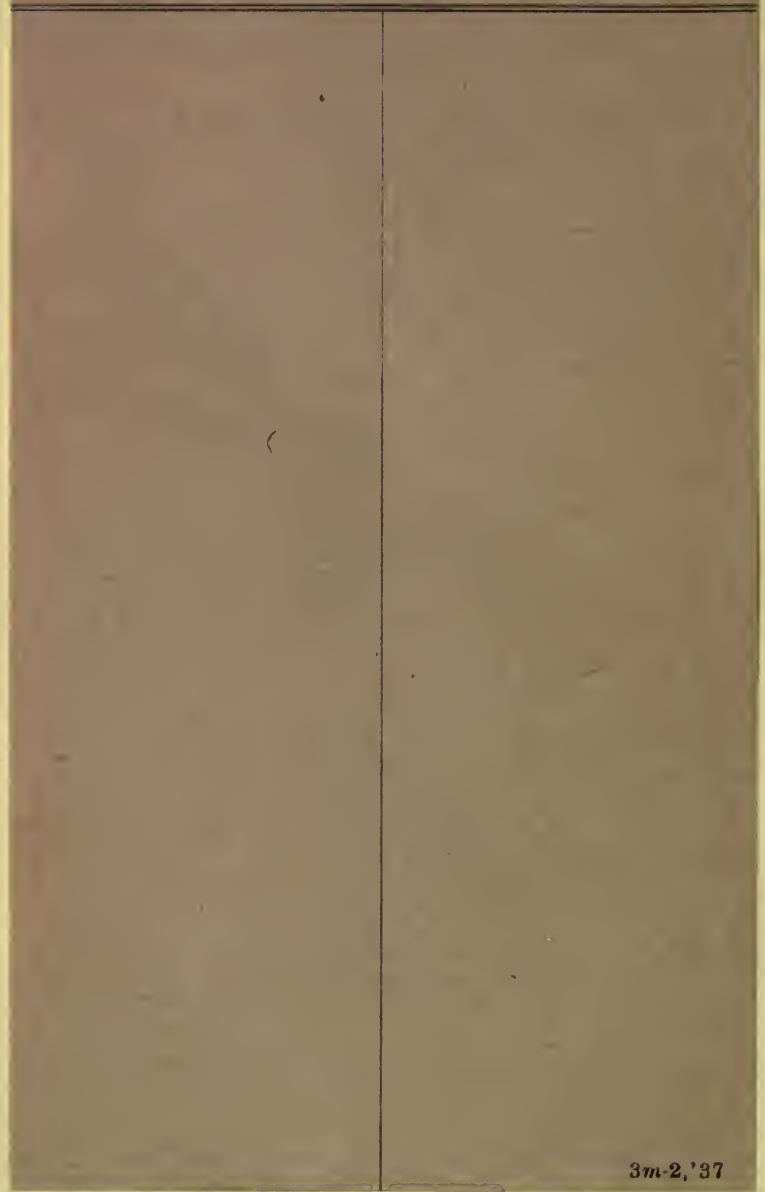




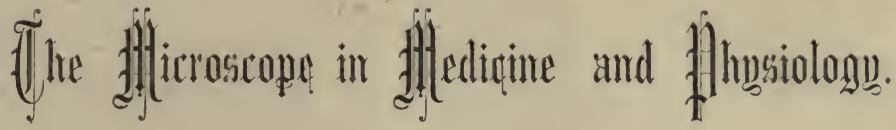

\section{DR. JAMES TYSON}

Will begin his Ninth Course of Lectures early in April, and continue two lectures per week, throughout A pril, May, and June, at the

\section{Jayne Street Medical Institute,}

(Opposite the University of Penna.)

The present Course will be supplemental to the Fall Course on Microscopy, at the University of Pennsylvania, and is designed especially for advanced students and graduates in medicine. It will include lectures on the physiology and pathology of the Blood and Urine, the minute structure of all important physiological and pathological tissues, with the theories of their development and growth, and the special methodsmequired for the proper lemonstration and preservation of each. Each subject will be illustrated by suitable microscopical preparations.

That the Members of the Class may have an opportunity of becoming familiar with manipulation, after a few lectures ha been delivered an adilitional hour will be assigned each weer laboratory practice, during which they will be enabled pare specimens of the solid and fluid tissues of the b the effect of reagents, and familiarize themselves $g$. the use of the Microscope in Practical Medieine.

\section{FEE-For the Lectures, $\$ 10.00 \ldots \ldots$ F'}

For further information apply to

\section{JAMES}


3.

- 35 - 3 -

Fos

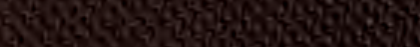

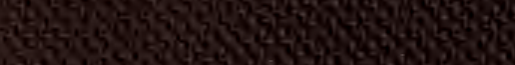

6.

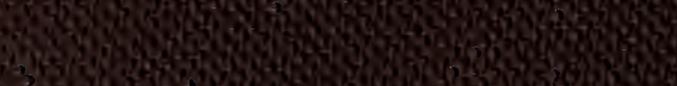

6.3.

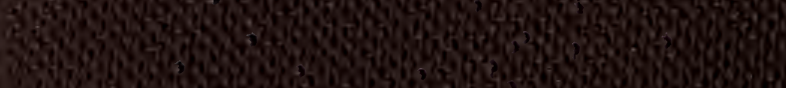

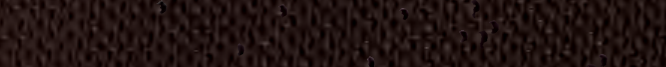

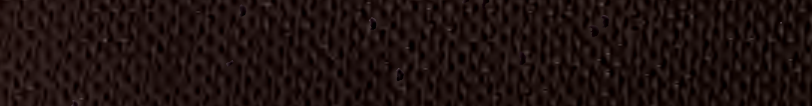

ong

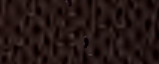

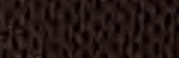

igengsing

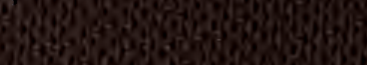

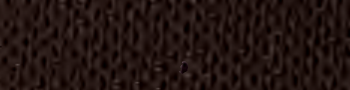

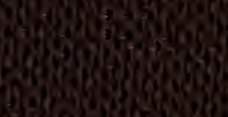

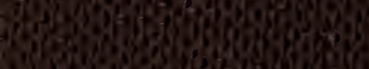

as

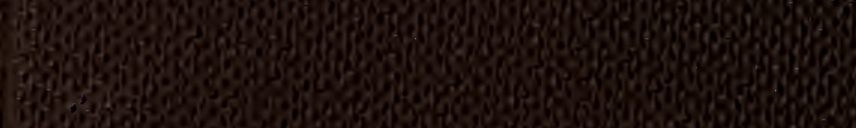

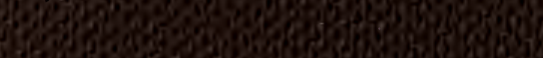

yoris

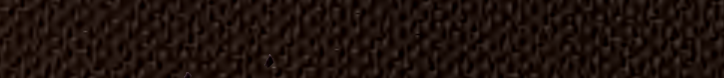

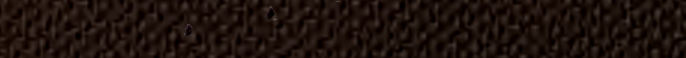

* 